Floods of November 1965 to

January 1966 in the Gila River

Basin, Arizona and New Mexico,

and Adjacent Basins in Arizona

GEOLOGICAL SURVEY WATER-SUPPLY PAPER 1850-C

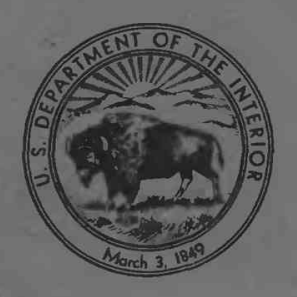




\section{Floods of November 1965 to}

January 1966 in the Gila River Basin, Arizona and New Merico, and Adjacent Basins in Arizona By B. N. ALDRIDGE

\section{FLOODS OF 1965 IN THE UNITED STATES}

GEOLOGICAL SURVEY WATER-SUPPLY PAPER 1850-C

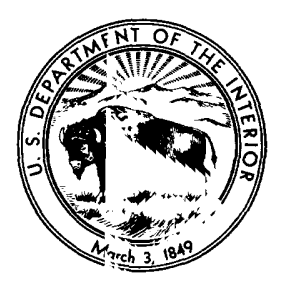


UNITED STATES DEPARTMENT OF THE INTERIOR

WALTER J. HICKEL, Secretary

\section{GEOLOGICAL SURVEY}

William T. Pecora, Director 


\begin{tabular}{|c|c|}
\hline \multicolumn{2}{|l|}{ 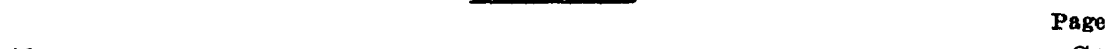 } \\
\hline Abstract & \\
\hline & \\
\hline & \\
\hline & \\
\hline weather conditions & \\
\hline er $22-25,1965$ & \\
\hline 1 and December 14-15, 1965 $\ldots \ldots \ldots$ & \\
\hline 23,1965 & \\
\hline 30,1965 & \\
\hline - & \\
\hline am. ...... & \\
\hline Dam and Salt River........ & \\
\hline low Salt River.............. & \\
\hline (2. & \\
\hline Stewart Mountain and Bartlett Dams... & \\
\hline $\begin{array}{l}\text { Stages and discharges downstream from Granite Reef Dam } \\
\text { during the Phoenix flood }\end{array}$ & \\
\hline lood & \\
\hline oy storage in reservoirs & \\
\hline Is_ & \\
\hline Is & \\
\hline miscellaneous measuring sites.. & \\
\hline (2. & \\
\hline harges. & \\
\hline - & \\
\hline (n- & \\
\hline & \\
\hline$\cdots$ & \\
\hline
\end{tabular}

\section{L L US T R A T ION S}

[Plates in pocket]

Prate 1. Map showing area of report and sites where hydrologic data were collected, Gila River basin, Arizona and New Mexico.

2. Maps showing precipitation, November and December 1.965, in the Gila River basin, Arizona and New Mexico.

3. Hydrographs showing discharge at selected gaging stations, Santa Cruz and Gila River basins, Arizona and New Mexico.

4. Graphs showing discharge and stage at selected stations on the Salt, Verde, and Gila Rivers, Ariz.

5. Photographs and flood map of the Salt River in the Phoenix metropolitan area, Arizona.

6. Maps and hydrographs showing daily mean discharge and rise in water levels at selected wells along Rillito and Tanque Verde Creeks and Salt and Gila Rivers, Ariz. 
Figure 1. Map showing major areas where large amounts cf runoff originated from storms of November and December 1965.2. Profile of flood crest on Rillito Creek and the Santa Cruz River_-

Page

C4

\section{T A B LES}

TABLE 1. Snowfall and snow on the ground, December 1965

2. Snow-survey data, December 1965

3. Times when the Phoenix flood reached different points on the

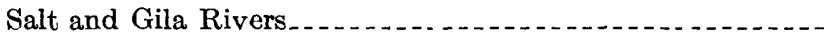

4. Runoff of the Salt and Gila Rivers downstream from Granite

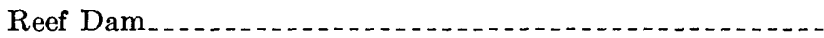

5. Summary of damages from the Phoenix flood along the Salt and Gila Rivers

6. Discharge data for major floods, Salt River and main tribu-

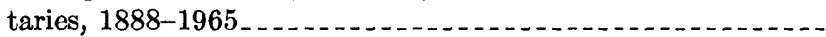

7. Sources of data for major floods, Salt River and main tribu-

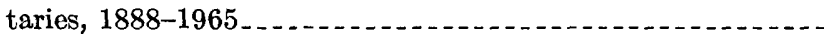

8. Representative rises in ground-water levels along major flood plains in the Gila River basin, spring 1965 to spring 1966...

9. Summary of flood stages and discharges........ 


\title{
FLOODS OF NOVEMBER 1965 TO JANUARY 1966 IN THE GILA RI'TER BASIN, ARIZONA AND NEW MEYICO, AND ADJACENT BASINS IN ARIZONA
}

\author{
By B. N. AtDridge
}

\begin{abstract}
Above-normal precipitation, which fell over much of Arizona and New Mexico in November and December 1965 during five distinct storm periods, caused flooding in several parts of the Gila River basin. The floods caused about $\$ 5$ million in damages in the Gila River basin upstream from Salt River anc about $\$ 6$ million in damages along the Salt River and Gila River below Salt River. The most significant flood was the Phoenix flood, which resulted from the release of water from storage reservoirs on the Salt and Verde Rivers. A large volume of fall and winter runoff added to a large carryover storage from the preceding spring raised the contents of the reservoirs to an unusually high level for December. The regulated discharge in the Salt River below Granite Ree. Dam was the highest since the reservoir system was completed-in 1939 ; however, several larger floods had occurred in earlier years.

The maximum discharge of the Salt River through the downtown $\varepsilon$ rea during the Phoenix flood was 66,000 efs (cubic feet per second) on December 31, 1965. Without the storage provided by the upstream reservoirs, the peat discharge during the flood would have been more than $80,000 \mathrm{cfs}$, and the peak discharge on December 23 might have exceeded $120,000 \mathrm{cfs}$. If the reservoirs were not present, a peak discharge of $66,000 \mathrm{cfs}$, equal to that of December 31, would occur on an average of about once every 6 years.

The peak discharge during the Phoenix flood was reduced only sl'ghtly as it traveled from Granite Reef Dam on the Salt River to Gillespie Dam on the Gila River; however, the volume of flow was reduced considerably in the same reach. More than 500,000 acre-feet of water passed over Granite Reef Dam, but only 37,000 acre-feet of water reached the mouth of the Gila River. Rises in groundwater levels in the flood plains indicate that a large amount of the streamflow loss went to ground-water recharge.
\end{abstract}




\section{INTRODUCTION}

Five major storms moved into Arizona and western New Mexico from the Pacific Ocean in November and December 1965. From the last week in November until the end of December, stcrms were a weekly occurrence, and rain or snow fell on about four out of every 10 days. The major storms occurred November 22-25, December 9-11, December 14-15, December 22-23, and December 29-30. Only the last two storms caused appreciable flooding, although the earlier storms did cause extremely high flows in some areas. The earlier storms set the stage for the floods later in December by thoroughly soaking the soil at low altitudes and by depositing snow in the mountains.

The most significant flood with respect to property damage began December 31, when water was released from storage reservoirs into the normally dry chaninel of the Salt River below Granite Reef Dam; the reservoirs were full because of the unusually heavy autumn and winter runoff and the above-normal carryover storage from the 1965 irrigation season. The flood, which is called the Phoenix flood, crused severe damage along the Salt River below Granite Reef Dam and along the Gila River below Salt River. Flood runoff continued until May 1966 in the lower reaches of the Gila River. Severe flooding also occurred in the Gila River basin upstream from the Salt River following the storm of December 22-23 and in the Gila River basin upstream from Coolidge Dam following the storm of December 29-30.

The purpose of the report is to summarize the conditions preceding the floods, to give a factual account of the floods, to describs, the floods in proper perspective to past floods, and to show the effects of the reservoirs on the floods in the Salt and Verde Rivers. Although the report deals primarily with the Phoenix flood, it contains data for the other floods (fig. 1).

\section{ACKNOWLEDGMENTS}

The author wishes to acknowledge the assistance given by L. C. Goldsmith, hydrology analyst, Salt River Valley Water Users'. Association; Paul Kangeiser, State climatologist, U.S. Weather Bureau; R. W. Enz, snow-survey supervisor, U.S. Soil Conservation Service; Ralph Edde, supervisor of county mapping, Photogrammetry and Mapping Division, Arizona Highway Department; and the Los Angeles district, U.S. Army Corps of Engineers, who supplied hydrologic data concerning the area. 


\section{GEOGRAPHIC SETTING}

The Mogollon Rim extends across the eastern half of Arizona and divides the north-flowing tributaries of the Little Colorado River from the south-flowing tributaries of the Verde, Salt, and Gila Rivers (pl. 1). The Mogollon Rim rises sharply above the surrounding area and is generally at altitudes between 6,500 and 7,500 feet. Tro. White Mountains in Arizona and the Gallo and Mangas Mountains in New Mexico form the eastern end of the divide between the Gila and Little Colorado Rivers. The floods of November 1965 to January 1966 originated along this divide, in the rugged mountainous area south of the divide, and in a few small areas in southern Arizona (fig. 1).

The Gila River heads in New Mexico and flows generally westward across Arizona to the Colorado River. The Gila River is joined by the south-flowing San Francisco River and Eagle Creek a few miles across the Arizona-New Mexico State line in Arizona. The Gila River is controlled at Coolidge Dam. Downstream from Coolidge Dam, the Gila is joined by the San Pedro and Santa Cruz Rivers, which flow northward from Mexico through southern Arizona.

The Salt River heads in the mountainous area of easterr Arizona and flows westward to its confluence with the Gila River west of Phoenix. The Salt River is joined by its main tributary-the Verde River-25 miles upstream from Phoenix. The drainage basin above the confluence of the Salt and Verde Rivers is 12,900 square miles and is from 1,400 to 11,490 feet above mean sea level; the altitude of most of the area is between 3,000 and 7,000 feet. Generall"r, six reservoirs-four on the Salt River and two on the Verde River-impound all the runoff from the drainage basin. Water is released at a slow rate and is diverted into irrigation canals, and very little flow passes Granite Reef Dam-a diversion dam on the Salt River 31/2 miles downstream from the Verde River. In the last several years the Salt River channel through Mesa, Tempe, Scottsdale, and Phoenix has been dry, except for occasional small flows from local runoff.

\section{STORMS AND WEATHER CONDITIONS}

Precipitation in November and December 1965 was above average in all of Arizona and most of western New Mexico. T'le largest amounts of precipitation were reported in the mountainous area that extends from central Arizona into New Mexico. Unusually large amounts of precipitation also fell over the isolated mountain ranges between Tucson and Safford, Ariz.

The number of stations that recorded record-high amounts of precipitation in December 1965 are too numerous to list. Several stations 


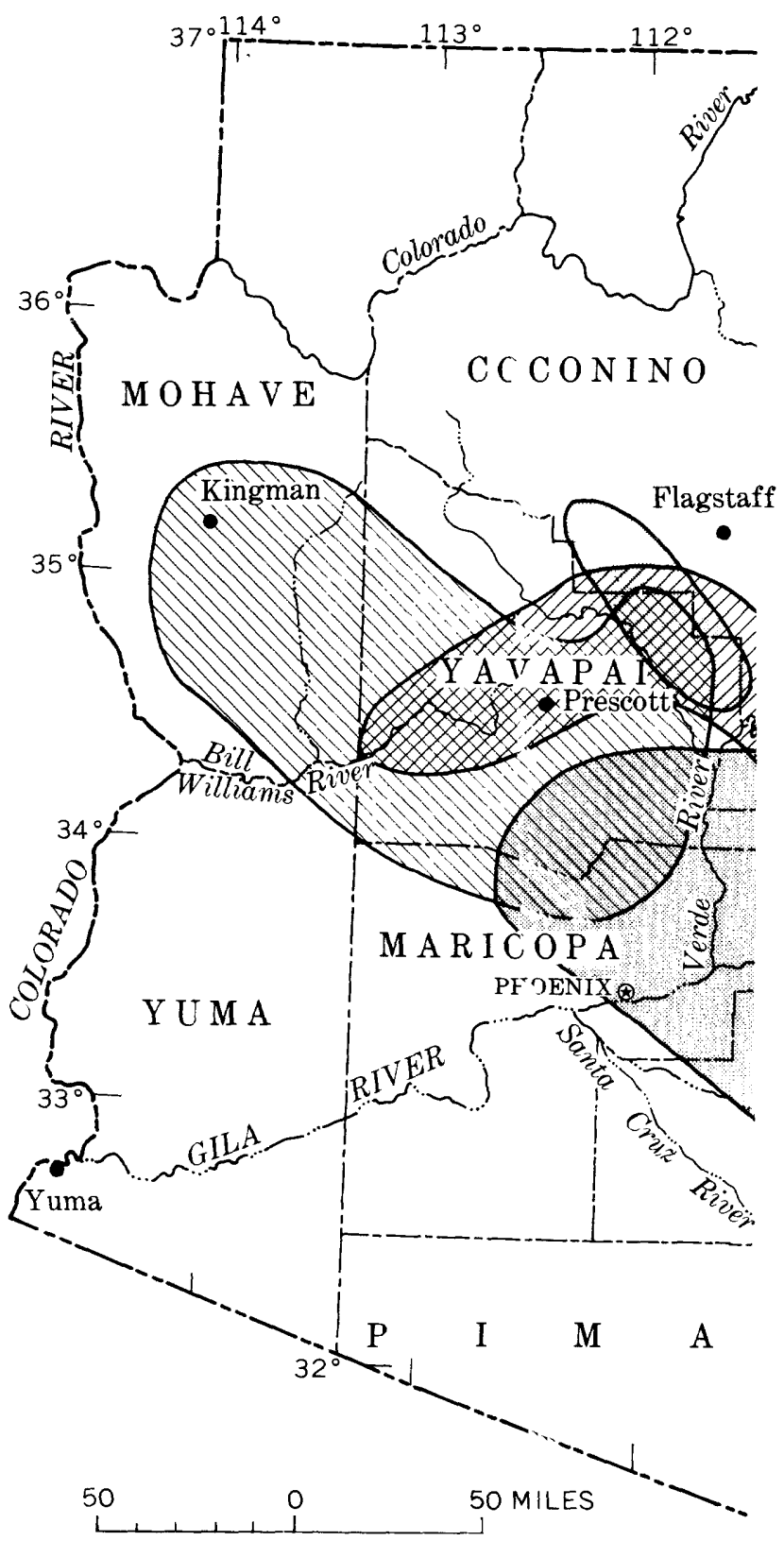

Figure 1.-Major areas where large amounts of runoff originated from 
having at least 50 years of record recorded more precipitation in December 1965 than in any previous month. The largest amount of precipitation measured in December was 16.32 inches at precipitation station 10, which is 20 miles north of Roosevelt ( $\mathrm{pl.1}$ ).

Although precipitation data were scanty in many areas, sufficient data were available to indicate the general distribution of the precipitation during the five storm periods. Isohyetal maps have been prepared for four of the storms by using the published climatological data of the U.S. Weather Bureau (1966a, 1966b, 1966c, 1966d, 1966e, 1966f) and unpublished data collected by personnel of the U.S. Geological Survey (pl. 2). In areas where no precipitation data were available, isohyets were based on runoff or on isohyets by the University of Arizona (1965) for winter precipitation.

\section{STORMS OF NOVEMBER 22-25, 1965}

During the storms of November 22-25, almost every precipitation station in Arizona recorded some precipitation. In general, less than 1 inch was recorded; however, several areas in the Salt River basin received from 3 to 5 inches of rain (pl. 2), and larger amounts were reported at individual stations in the Gila River basin. Precipitation station 10 (pl. 1) recorded 11.59 inches of rainfall in the 4-day period, and station 19 (pl. 1) at the head of Oak Creek recorded 7.58 inches. Most of the precipitation occurred during two periods- -1100 hours November 22 to 1800 hours November 23 and 0200 hours to 1700 hours November 25. Station 10 recorded 7.70 inches during the first period and 2.94 inches during the second period; station 19 recorded 4.40 inches during the first period and 2.82 inches during the second period.

The 4-day precipitation was related closely to altitude-the higher the altitude, the larger the amount of precipitation. Precipitation stations 11,12 , and 13 are within a few miles of station 10 (altitude 6,970 feet) but are at lower altitudes; records for the three stations show a sharp decrease in precipitation with a decrease in altitude--station 11 , altitude 5,550 feet, precipitation 5.73 inches; station 12, altitude 5,100 
feet, precipitation 4.36 inches; and station 13, altitude $\Sigma, 205$ feet, precipitation 2.35 inches.

The soil was quite dry at the time of the November 22-25 storm, because only a small amount of precipitation had fallen during October and the first 3 weeks of November. In most of the State much of the precipitation from the storm was either intercepted by plants or absorbed by the soil. Some snow fell above 6,500 feet, but most of it melted immediately. U.S. Weather Bureau stations at altitudes of as much as 8,000 feet had no snow remaining after November 27 .

\section{STORMS OF DECEMBER 9-11 AND DECEMBER 14-15, 1965}

The storm of December 9-11 was the largest in areal extent of any storm during the period of this report. Precipitation ranged from 2 to 5 inches over most of Arizona and New Mexico (pl. 2); most of the precipitation occurred in a period of about 20 to 30 hours-from midmorning of December 9 to midafternoon of December 10. Large amounts of snow fell at high altitudes, and on the evening of December 9 the snow level was at about 6,000 feet. Another storm began December 13; precipitation was intense December 14-15 and tapered off until cessation of the storm on December 18. The widespread precipitation increased the snow depth considerably in the mountainous areas. The snow level was down to 3,000 feet when the precipitation ceased on December 18, and there was 2 to 3 feet of snow in parts of the White Mountains. The snow depths at selected stations in the Gila Piver basin and adjacent area are shown in table 1 . Stations that received no snow during the month and those that failed to report are not included. Because of the unseasonably large amount of snow, the U.S. Soil Conservation Service made special snow surveys at the snow courses listed in table 2 (R. W. Enz, snow-survey supervisor, Soil Conservation Service, written commun., 1966). Warm daytime temperatures of December $20-21-$ generally in the $55^{\circ}$ to $70^{\circ} \mathrm{F}$ range-caused snow to melt at the lower altitudes, but cold nighttime temperstures prevented any sustained snowmelt in the mountains. 
TABLE 1.-Snowfall and snow

[Tr., trace; N, no record of snow on the ground. Data from respective datum was zero. $\mathrm{Da}$ ?h leaders indicate

\begin{tabular}{|c|c|c|c|c|c|c|c|c|c|}
\hline \multirow{2}{*}{$\begin{array}{l}\text { Site } \\
\text { on } \\
\text { pl. } 1\end{array}$} & \multirow{2}{*}{ Station } & \multirow{2}{*}{$\begin{array}{l}\text { Altitude } \\
\text { (feet } \\
\text { above } \\
\text { mean } \\
\text { sea level) }\end{array}$} & \multirow{2}{*}{ Snow (inches) } & \multicolumn{6}{|c|}{$D \geq y$} \\
\hline & & & & 9 & 10 & 11 & 12 & 13 & 14 \\
\hline 1 & Blue.... & 5,760 & $\begin{array}{l}\text { Fall } \\
\text { On the ground }\end{array}$ & & & & & & \\
\hline 2 & Alpine.... & 8,020 & Fall & & & & & $\begin{array}{l}6.0 \\
6\end{array}$ & $\begin{array}{l}2.0 \\
8\end{array}$ \\
\hline 3 & Maverick & 7,800 & Fall the ground.... & & $\begin{array}{l}8.0 \\
8\end{array}$ & 8.0 & & $\begin{array}{l}0.0 \\
15\end{array}$ & $\begin{array}{l}3.0 \\
18\end{array}$ \\
\hline 4 & Whiteriver... & 5,280 & Fall the ground.......... & & & Tr. & & .5 & 1.0 \\
\hline 5 & McNary . . . . . . & 7,320 & Fall the ground & & & $\begin{array}{l}6.0 \\
6\end{array}$ & 3 & $\begin{array}{l}1.0 \\
4\end{array}$ & 5.0 \\
\hline 6 & Lakeside Ranger Station. ....... & 6,700 & Fall & & .1 & $\begin{array}{l}0 \\
3.0 \\
3\end{array}$ & $\delta$ & & 2.0 \\
\hline 7 & Snowflake. . & 5,640 & Fall & & & & & & $\mathrm{Tr}^{3}$. \\
\hline 8 & Heber Ranger Station & 6,590 & Fall & & & & & .5 & 2.0 \\
\hline 9 & Chevelon Ranger Station....... & 7,006 & Fall the ground.................. & & & & & 4.0 & $\frac{2.5}{6}$ \\
\hline 12 & Sierra Ancha $\ldots . . .$. & 5,100 & Fall & & & & & & 7.0 \\
\hline 14 & Pinal Ranch.... & 4,520 & Fall & & & & & & 6 \\
\hline 15 & Payson $\ldots . . . . .$. & 4,910 & Fall the ground & & & & & & \\
\hline 16 & Natural Bridge . $_{\text {. }}$ & 4,607 & $\begin{array}{l}\text { On the ground.... } \\
\text { Fall } \\
\text { On the ground............... }\end{array}$ & & & & & $\begin{array}{l}2.0 \\
\text { Tr. }\end{array}$ & $\begin{array}{l}11 \\
3.3 \\
5\end{array}$ \\
\hline $\begin{array}{l}17 \\
18\end{array}$ & $\begin{array}{l}\text { Montezuma Castle } \\
\text { Sedona Ranger Station }\end{array}$ & $\begin{array}{l}3,180 \\
4,223\end{array}$ & Fall $(\mathrm{N})$ & & & & & Tr. & \\
\hline 19 & Junipine........................... & 5,124 & On the ground.... & & Tr. & & & 1.0 & 2.5 \\
\hline 20 & Burrus Ranch...... & 6,800 & $\begin{array}{l}\text { Fall } \\
\text { On the ground }\end{array}$ & & Tr. & & Tr. & $\dot{\operatorname{Tr}}$ & 4.0 \\
\hline 21 & Flagstaff (airport). & 6,993 & $\begin{array}{l}\text { Fall } \\
\text { On the ground } \\
\text { Water equivalent. }\end{array}$ & 2.2 & $\begin{array}{l}6.0 \\
5 \\
.6\end{array}$ & $\begin{array}{l}\text { Tr. } \\
4.6\end{array}$ & $3_{.6}^{.2}$ & $\begin{array}{l}2.3 \\
4.6\end{array}$ & $\begin{array}{l}3.6 \\
6 \\
.6\end{array}$ \\
\hline 22 & Fort Valley -. & 7,347 & Fall the ground & & $\begin{array}{l}.00 \\
4.5\end{array}$ & 1.0 & & 2.0 & 4.0 \\
\hline 23 & Jerome..... & 5,245 & Fall the ground & & & & $\stackrel{\stackrel{o}{T} r}{.}$ & & \\
\hline 24 & Williams.... & 6,750 & Fall & & 40 & $\begin{array}{l}6.0 \\
3\end{array}$ & $2^{5}$ & 11.5 & $\begin{array}{l}4.0 \\
18\end{array}$ \\
\hline 25 & Ash Fork & 5,200 & $\begin{array}{l}\text { Fall } \\
\text { on the ground }\end{array}$ & & & & & Tr. & 5.3 \\
\hline $\begin{array}{l}26 \\
27\end{array}$ & $\begin{array}{l}\text { Hillside } 4 \text { NNE } \\
\text { Chino Valley....... }\end{array}$ & $\begin{array}{l}3,320 \\
4,750\end{array}$ & $\begin{array}{l}\text { On the ground } \\
\text { Fall }(\mathrm{N}) \\
\text { On the ground }\end{array}$ & & & & & & $\begin{array}{l}\text { Tr. } \\
1.5 \\
1\end{array}$ \\
\hline 28 & Prescott (airport) & 5,017 & Fall & & & & $\operatorname{Tr}$. & $\mathrm{Tr}$. & 3. 0 \\
\hline 29 & Prescott $\ldots$ & 5,410 & Fall & & & & & $\mathrm{Tr}$ & 3.0 \\
\hline 30 & Crown King ... & 6,000 & Fall the ground & & & & & & 1. 2 \\
\hline 31 & Kitt Peak & 6,875 & $\begin{array}{l}\text { Fall } \\
\text { On the ground..... }\end{array}$ & & & Tr. & & & $\frac{1.5}{2}$ \\
\hline
\end{tabular}


on the ground, December 1965

U.S. Weather Bureau (1966a, b). A blank indicates that the that the item was not zero but was not recorded]

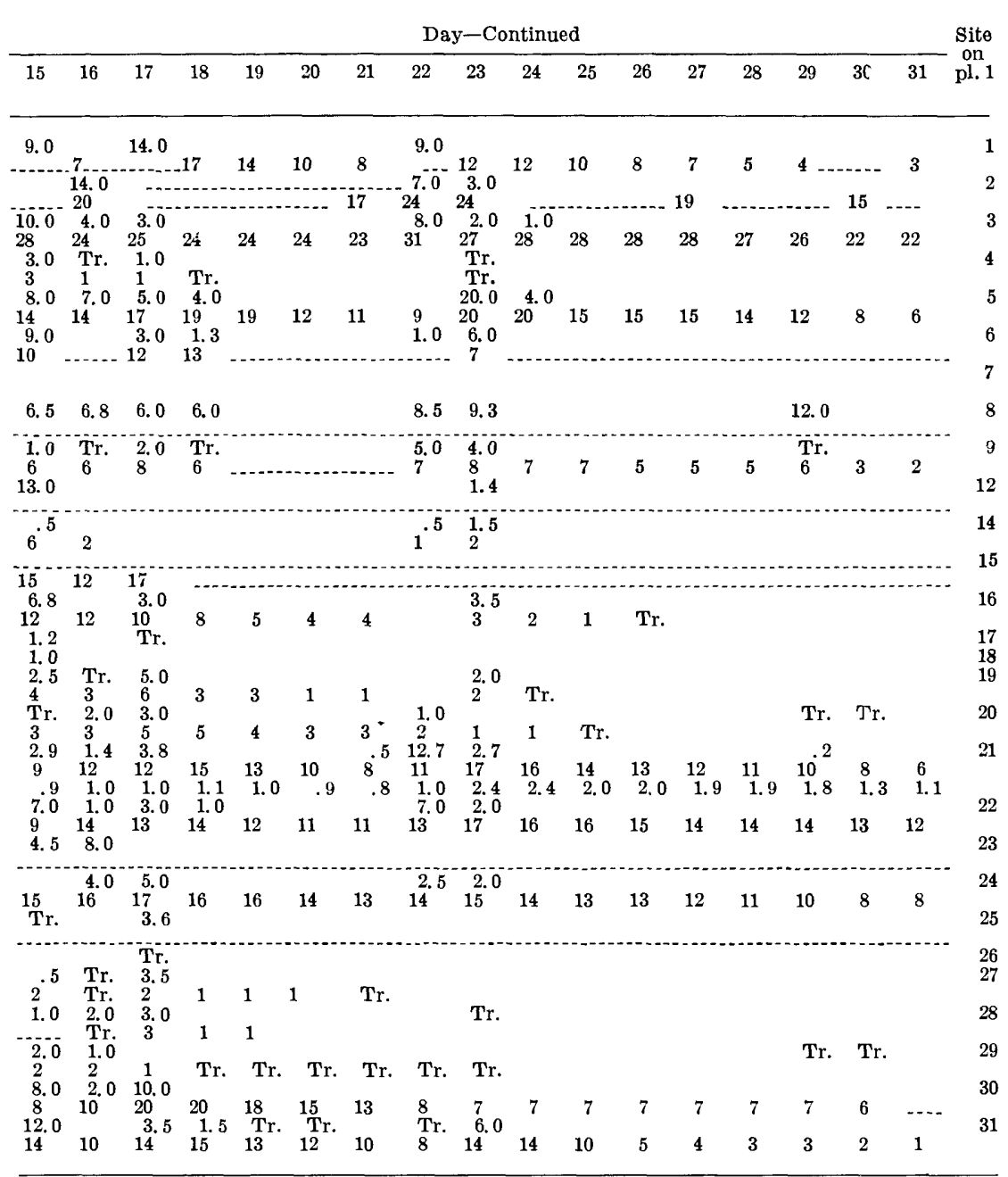


TABLE 2.-Snow-survey data, December 1965

[Data from R. W. Enz, snow-survey supervisor, U.S. Soil Conservation Service, written commun., 1966]

\begin{tabular}{|c|c|c|c|c|c|c|}
\hline $\begin{array}{c}\text { Site } \\
\text { (on pl. 1) }\end{array}$ & $\begin{array}{c}\text { Soil } \\
\text { Conservation } \\
\text { Service No. }\end{array}$ & Snow course & $\begin{array}{l}\text { Altitude } \\
\text { (feet } \\
\text { above } \\
\text { mean } \\
\text { sea level) }\end{array}$ & Day & $\begin{array}{l}\text { Snow } \\
\text { depth } \\
\text { (inches) }\end{array}$ & $\begin{array}{l}\text { Water } \\
\text { equiva- } \\
\text { lent } \\
\text { (inches) }\end{array}$ \\
\hline & $9 \mathrm{~S} 4$ & Nutrioso_ & 8,500 & 21 & 17 & 4. 0 \\
\hline 2 & $9 \mathrm{~S} 7$ & Coronado Trail & 8,000 & 21 & 24 & \\
\hline 3 & $9 \mathrm{~S} 11$ & Hannagan Meadow.- & 9,090 & 21 & 37 & 7. 5 \\
\hline 4 & $9 \mathrm{R} 6$ & Wilson Lake....... & 9,000 & 20 & 26 & 4.9 \\
\hline & $9 \mathrm{R} 9-\mathrm{A}^{1}$ & Mount Ord -- & 11,000 & 20 & 75 & 12 \\
\hline 6 & $9 \mathrm{R} 7-\mathrm{A}^{1}$ & Smith Cienega No. 1 . & 10,000 & 20 & 56 & 10 \\
\hline & $9 \mathrm{R} 7-\mathrm{A}^{1}$ & Smith Cienega No. 2 & 9,700 & 20 & $5 c$ & 9 \\
\hline $8 \ldots$ & (1) & Skeeter & 9,300 & 20 & 42 & 8 \\
\hline 9 & $9 \mathrm{R} 10$ & Hawley Lake. & 8,300 & 20 & 26 & 6. 0 \\
\hline 10 & $9 \mathrm{R} 2-\mathrm{M}$ & MeNary ...... & 7,200 & 21 & $1 \varepsilon$ & 4. 0 \\
\hline & $10 \mathrm{R} 4$ & Heber & 7,600 & 21 & 16 & 3. 6 \\
\hline $12_{-}$ & $11 \mathrm{R} 6$ & Baker Butte_ & 7,300 & 21 & 26 & 5. 0 \\
\hline 13. & $11 \mathrm{R5}$ & Happy Jack & 7,630 & 29 & 17 & 4. 5 \\
\hline $14_{-}$ & $11 \mathrm{P} 5-\mathrm{M}$ & Newman Park & 6,750 & 29 & 9 & 2.5 \\
\hline & $12 \mathrm{P} 1-\mathrm{M}$ & Chalender. & 7,100 & 30 & 12 & 2. 9 \\
\hline 16. & $12 \mathrm{R} 2$ & Iron Springs & 6,200 & 28 & 32 & \\
\hline & $12 \mathrm{R} 6$ & Copper Basin Divide & 6,720 & 27 & 13 & 3. 6 \\
\hline
\end{tabular}

1 Aerial markers; water equivalent estimated on basis of data from surrounding courses.

\section{STORM OF DECEMBER 22-23, 1965}

The storm that moved into Arizona on December 21 brought more snow. On December 22, 20 inches of snow fell at McNary (pl.1, precipitation station 5), and 13 inches fell at the Flagstaff airport (pl. 1, precipitation station 22). Observers at several U.S. Weatl ar Bureau precipitation stations reported about 10 inches of snow during the storm. Southerly winds brought warmer temperatures on December 22, and snow changed to rain at lower altitudes. The combination of rain and warm temperatures caused rapid melting of the snow that had accumulated during the preceding 10 days. By the afternoon of December 22, the snow level had risen several thousand feet, but more snow fell in the evening. After the storm, the depth of snow at an altitude of 6,500 feet was about equal to that before the storm; above 6,500 feet the depth was greater, below 6,500 feet it was considerably less, and very little snow remained below 5,500 feet. Mrny of the climatological stations in the Gila River basin reported from 1 to 3 inches of precipitation December 22-23, and a few reported as much as 4 inches. Isohyets for the December 22-23 storm are shown on plate 2.

\section{STORM OF DECEMBER 29-30, 1965}

Temperatures remained warm for several days after the December 22-23 storm, and snow continued to melt and increase in water con- 
tent or density. The average density of the snow at eight courses measured on December 20 or 21 was 21.3 percent whereas the average density of the snow at four courses measured December 27-29 was 27.5 percent. A light rain started to fall on December 29, and the intensity of the storm increased rapidly early on December 30. In a few hours 1 to 2 inches of precipitation, mostly rain, fell on most of the Salt River watershed, along the north side of the Mogollon Rim, and in the San Francisco River and Eagle Creek basins (pl. 2). The combination of rain and warm temperatures removed much of the remaining snow, and by December 31 there was very little snow left below 7,000 feet.

\section{FLOODS AND RUNOFF}

The only significant floods that resulted from the storms of November 22 to December 15 were those of November $23-25$ in small areas south and west of Flagstaff (fig. 1). Elsewhere, the storms and runoff in November and early December were significant only because of their wetting effect on soil and stream channels and their contribution to storage in reservoirs in the Salt River basin. As a result of the November storms Santa $\mathrm{Fe}$ Reservoir overflowed into the normally dry channel of Cataract Creek. The flood damaged several inexpensive homes and a few small bridges in Williams. Streams entering the Verde River from the north between Paulden and Camp Verde reached the highest stages in several years. On Thanksgiving Day (November 25), Oak Creek near Cornville peaked at 17,600 cfs (cubic feet per second), which was the highest discharge since 1938. The flood washed away several housetrailers and damaged trailer courts, forest camps, and minor road crossings. Several homes were isolated but received little damage. Streams in Beaver Creek basin had outstanding peaks on November 23 and November 25. Streams farther south and east in the Verde, Salt, and Gila River basins showed only moderate rises. Peak discharges at individual stations are shown in table 9.

The December 9-11 storm caused fairly large amounts of runoff in most of Arizona; however, the runoff was not excessive. Small streams in the western deserts of Arizona reached exceptionally high winter stages, but the peaks were small compared with those that have resulted from summer convective storms. The Bill Williams and Verde Rivers were the only large streams having high peaks. The marimum discharge during the flood period at Verde River near Clarkdale gaging station occurred on December 10. The storm of December 14-15 produced high flows in only a few low-lying desert areas, and the peak flows were not of sufficient magnitude to be called floods. In the mountains much of the precipitation fell as snow; although there was little in- 
crease in streamflow, the runoff remained above normal through the following week.

The storms of December 22-23 and December 29-30 eaused major flooding in much of the Gila River basin, and the December 29-30 storm also caused high flows in a strip about 10-15 miles wide along the north side of the Mogollon Rim between McNary and Flagstaff. On December 30, tributaries to the Little Colorado River had the highest flows since January 1952; on Clear and Chevelon Creeks, the peaks were the third highest in about 50 years. A few small streams overtopped roads and washed out shoulders and culverts, but in most places the high flows caused little damage because streams were confined to rock channels. Reservoirs in the Salt River basin had insufficient reserve storage to contain the high flows from the upper parts of the Verde and Salt River basins, and on December 31 water was released into the Salt River through Phoenix and other cities of the metropolitan area. The effects of this flood, hereafter referred to as the Phoenix flood, extended to the mouth of the Gila River and lasted until May 1966 in the lower reaches of the Gila.

In this report the floods in the Gila River basin that resulted from the storms of December 22-23 and December 29-30 are discussed under three geographical areas: (1) Gila River basin above Coolidge Dam, (2) Gila River basin between Coolidge Dam and Salt River, and (3) Salt River basin and Gila River below Salt River. Both stcrms caused unusually high peak flows in the Gila River basin above Conlidge Dam and the Salt River basin; only the first storm caused flooting in the Gila River basin between Coolidge Dam and Salt River. Although the Salt River basin and Gila River below Salt River area was affected by both floods, only the later flood had much effect below the reservoirs.

\section{GILA RIVER BASIN ABOVE COOLIDGE DAM}

The large amounts of precipitation that fell along the Arizona-New Mexico State line as rain and wet snow December 22-23 produced damaging floods in the lower reaches of the San Franciscr River, in the Gila River below San Francisco River, and in south-flowing tributaries to the Gila River between San Francisco River and Coolidge Dam. Flooding along tributaries to the Gila River above San Francisco River was minor except along Bear Creek, where irrigation-diversion headings, canals, dikes, levees, and crops were damaged. Minor flooding was reported along other tributaries in New Mexico, and some flooding occurred along the Gila River above San Francisco River, brt the peak discharge was small compared with that of other floods in recent years.

The gaging station on the San Francisco River at Cliftor and those on the Gila River between San Francisco River and Coolidge Dam recorded the highest stages since 1916. Records for San Francisco 
River, Eagle Creek, and San Carlos River show one crest late on December 22 and another late on the morning of December 23 (pl. 3). The first peak was higher at Eagle Creek above the pumping plant near Morenci, Gila River at the head of Safford Valley near Solomon, and San Carlos River near Peridot; the second peak was higl $\urcorner \mathbf{r}$ at the upper stations on Willow and Eagle Creeks and at San Francisco River at Clifton. The Gila River above San Francisco River peaked on December 24 and produced a third peak at the head of Safford Valley, where high flows continued for 2 days. By the time the flood reached the station at Calva, the first two peaks had combined into a single peak. Detailed tabulations of gage heights and discharges for the principal streamflow stations are given in the section "Streamflow data at gaging stations and miscellaneous measuring sites."

The recurrence intervals for the 1965 floods on the Gila River above Coolidge Dam are not as great as might be inferred from the number of years since the occurrence of similar floods; several floods in the late 1800's and early 1900's exceeded those of December 22-24. Curves developed by Patterson and Somers (1966) indicate average recurrence intervals of $12-17$ years for the 1965 . floods.

On December 30 the Gila River reached the second highest stage since 1916. Peak discharges were higher than those of Decernber 22-23 in the Eagle Creek basin and in the central part of the San Francisco River basin but were lower on the main stem of the Gila River. The peak discharge in Eagle Creek was the highest since at least, 1916.

From December 22 to January 5, 230,000 acre-feet of water was added to storage in the San Carlos Reservoir; the total amount of water in storage on January 5 was 330,000 acre-feet, which was the maximum since 1942 when the reservoir contained 819,200 acre-feet. By April 15, 1966, storage had increased to 504,700 acre-feet.

In the Gila River basin above Coolidge Dam, flood darages were about $\$ 1.6$ million in New Mexico (U.S. Geological Survey, New Mexico, written commun., 1967) and $\$ 1.8$ million in Arizona (D. E. Burkham, written commun., 1966). Most of the damage in New Mexico occurred near Cliff, Redrock, Virden, and Glenwood. Burkham (written commun., 1966) stated that in Arizona most of the damage from the floods was to farms, roads, and irrigation systems. Many of the farms were diked, and only a narrow band of the flood plain at the edge of the main river channel was uncultivated. The uncultivated part of the flood plain had become congested with a dense growth of saltcedar, which confined low and medium flows to a narrow main channel having a low conveyance capacity. As the flood wave moved downstream, the discharge exceeded the capacity of the main channel and overflowed onto the cultivated fields. After the fields were flooded to capacity, cross dikes between the fields broke, and the temporarily 
stored water spilled into the next downstream field. The movement of water through the cultivated fields breached many of the irrigation canals and scoured large channels in several fields. During the recession of the December 22-26 flood and during the flood of $\Gamma$-cember 30 to January 5, many of the fields carried more water than the main channel. Several farms and ranches were damaged by floots in tributaries of the Gila River. Agricultural damages were estimated at $\$ 465,000$ in Greenlee County (Greenlee County Agriculture Disaster Committee, written commun., Jan. 14, 1966) and $\$ 1,260,000$ in Graham County (Joint Graham County and Cities Civil Deferse, written commun., Jan. 14, 1966).

Cities and small communities in the area received relatively little damage. Many private homes in the northern part of Clifton were flooded, but those in the southern part were protected by floodwalls; South Clifton would have been flooded if the walls had been overtopped. The damage to sewage systems, roads, and parks in Clifton was estimated at $\$ 25,000$ (Tony Rodrigues, Mayor of Clifton, written commun., Jan. 28, 1966). In Safford the estimated flood c'amage was $\$ 25,000$ (Joint Graham County and Cities Civil Defense, written commun., Jan. 14, 1966). The damage was mostly to ros ds, sewers, and water systems. Many dwellings in Little Hollywood-a community of about 500 farmworkers near Safford-were flooded, but none were washed away. The San Carlos River flooded several homes in the village of San Carlos. The flood overtopped most of the roads in the flood plain, and many roadbeds were severely eroded. Bridges over the Gila River at Solomon, Safford, and Pima were overtopped and closed to traffic for several hours.

\section{GILA RIVER BASIN BETWEEN COOLIDGE DAM AND SAI.T RIVER}

After the storm of December 22-23, major tributaries to the Gila River between Coolidge Dam and Salt River had the highest flows in several years; however, compared with past floods the flov's were not as high as those above the dam. Records for the Gila Fiver below Coolidge Dam indicate that no water was released from the dam during the flood-the maximum flow at the gage during the flood period was 5 cfs. The San Pedro River upstream from Fenson contributed little flow to the flood; the peak at the San Pedro River gaging station at Charleston was only $690 \mathrm{cfs}$. Downstream from Penson, the peak on the San Pedro River increased rapidly as flow entered the river from the east slopes of the Rincon and Santa Catalina Mountains. A large flow in Aravaipa Creek washed out the approaches to a highway bridge near the mouth of the creek, swelled the San Pedro River to flood stage, and caused considerable flooding in the Winkelman area. The flood destroyed the gaging station on San Pedro River near 
Winkelman, inundated several hundred acres of farmland and part of the sewage-treatment plant at Kearny, and isolated a community near Kelvin; however, the amount of damage was generally small clong the Gila River from Coolidge Dam to the Santa Cruz River.

Near Tucson, the flood of December 22-23 did considerable damage along Rillito Creek and the Santa Cruz River below Rillito C"eek, but the magnitudes of peak discharges on the main streams were not unusual. The discharge of Rillito Creek was the highest since 1940 and the sixth highest since 1908. High flows prevailed for 3 days (pl. 3), and mean discharges for December 22-23 were more than the maximum instantaneous discharge of many peaks. The 3 -day volume for December 22-24 was the fourth greatest since 1908. The flow of Rillito Creek originated mainly in tributaries that drain to Tanque Verde Creek from the north slopes of the Tanque Verde Mountains and the south slopes of the Santa Catalina Mountains. The peak discharge of Tanque Verde Creek, which may be the highest since at least 1929, has a recurrence interval of about 27 years. Small streams that originate in the foothills had little or no flow. At the gage at Santa Cruz River at Tucson, the peak was the highest winter peak since 1916, but summer peaks of similar magnitude can be expected about once every 2 years. The peak at the gage at Santa Cruz River at Cortaro was the second highest since 1936 .

Rillito Creek eroded large sections from its banks, washel out the approaches to two bridges, undercut a trailer park, destroyed several trailers, and caused two major breaks in a main sewage-interceptor line. About $3.5 \mathrm{mgd}$ (million gallons per day) of raw sewage from Tucson poured into Rillito Creek over a period of several days. Arizona State Health Department officials found traces of contamination in wells within a quarter of a mile of the creek. The cost of repairing the sewerline was estimated at $\$ 1.3$ million. Damages to roads and bridge approaches in Pima County were estimated to be $\$ 156,000$ (D. A. DiCicco, Pima County Engineer, oral commun., 1966). Upstream from the gaging station near Tucson, Rillito Creek was confired to its channel, and there was little inundation damage; however, several small areas downstream from the gage were inundated. Much more of the flood plain probably would have been inundated if extensive lateral cutting had not occurred. The duration of the flood (pl. 3) may be the main reason why the flood was so destructive, but the place of origin of the flood also may have been a contributing factor. Large rock exposures exist in the mountains where the flood originated; therefore, the sediment-carrying potential of the water may lave been much greater than the load. When water reached the alluvial channel of Rillito Creek, it began picking up sediment from the banks. The relatively steep stream gradient (fig. 2) made it possible for the stream to carry large amounts of sediment. 


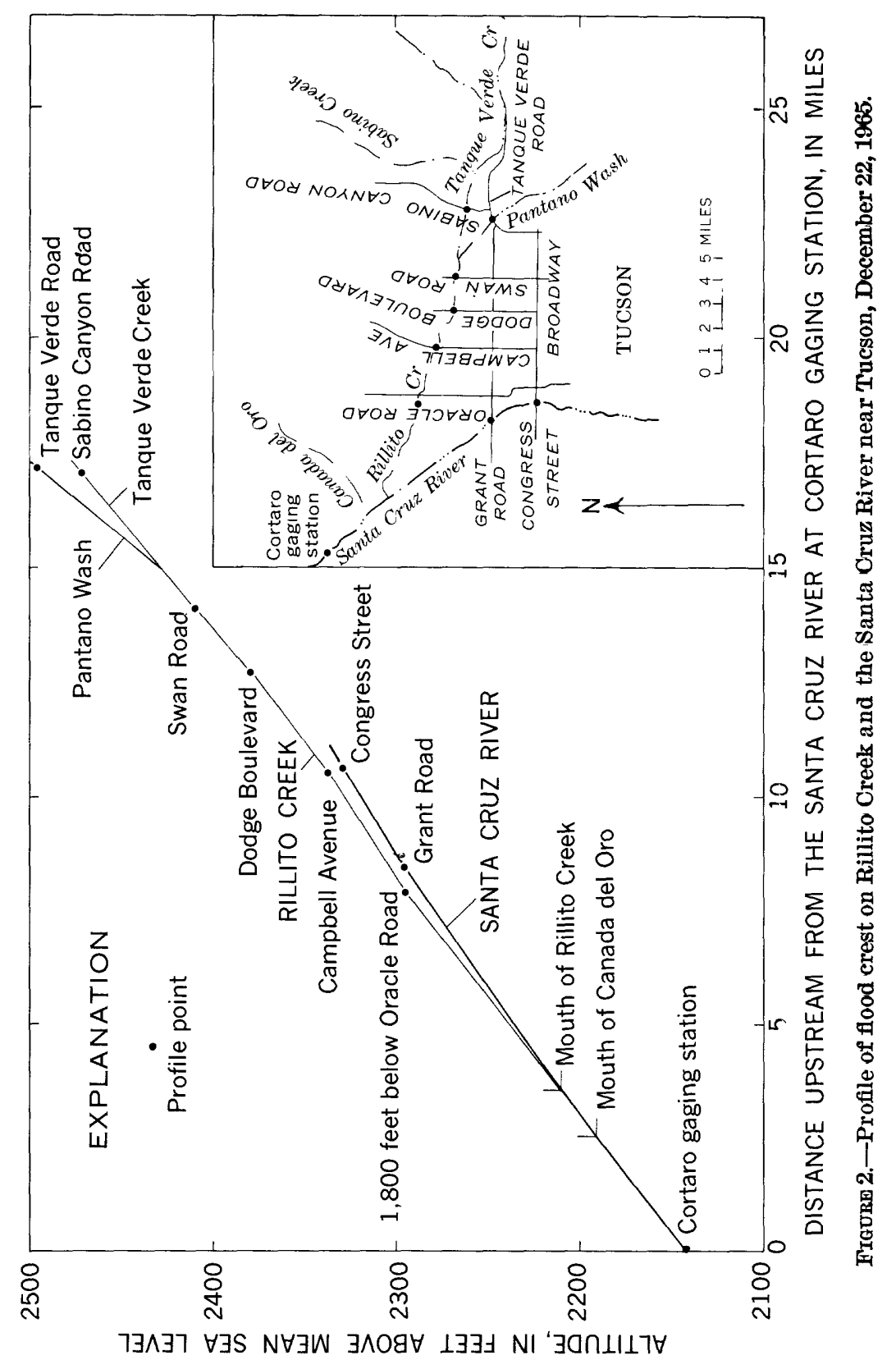


Flooding also occurred along Arivaca Creek, a tributary to Altar Wash at the head of Los Robles Wash. On December 24, a dam upstream from the community of Arivaca was washed out, snd about 2,000 acre-feet of water was released. Although the dam failure caused the highest known flow through the community of Arivaca, only a small part of the water reached the Santa Cruz River.

\section{SALT RIVER BASIN AND GILA RIVER BELOW SALT RJVER}

\section{RESERVOIR INFLOW}

Most of the inflow to Theodore Roosevelt Lake, hereafter referred to as Roosevelt Lake, is measured at gaging stations on the Falt River near Roosevelt (drainage area, 4,306 square miles) and Tonto Creek above Gun Creek near Roosevelt (drainage area, 675 square miles). During most of December 1965, the flow of the Salt River exceeded the previously recorded maximum flows for corresponding dates. On plate 4 the daily mean discharge of the Salt River near Roosevelt in November and December 1965 is compared with the previous maximum daily discharge for corresponding dates in the 30-year periot 1931-60 and with the daily discharge that was exceeded 10 and 50 percent (median value) of the time during the same period. Flow into the Verde River reservoir system was also above average. The amount of water in storage in the Salt and Verde reservoir systems increased 378,700 acre-feet between November 21 and December 21. On December 21 , there was more than $11 / 2$ million acre-feet of water in storage and only 537,700 acre-feet of reserve storage remained.

The large amounts of precipitation that fell on December 22-23 caused extremely high runoff from the East Verde River en stward to the lower reaches of the Black River. The Salt River, Tonto Creek, and Verde River had the highest peaks in several years. The contents of reservoirs on the Salt River increased 192,000 acre-feet in 2 days; the contents of reservoirs on the Verde River increased 69,300 acre-feet in the same period. Only a small amount of water was released from Roosevelt Lake into the three downstream reservoirs.

The measured flow at the two inflow stations and an estimated inflow from 843 square miles of ungaged drainage area were combined by the flood-routing method to define a hydrograph of total inflow to Roosevelt Lake (pl. 4). Routing coefficients were estimated from flond records for Salt River near Chrysotile and Salt River near Roosevelt and from velocities measured at these stations. The maximum rate of flow into Roosevelt Lake was computed as $88,000 \mathrm{cfs}$. Although the maximum inflow to Horseshoe Reservoir on the Verde River was not computed, it was probably about the same as that at the gaging station 
below Tangle Creek (peak flow 39,300 cfs), where most of the inflow to Horseshoe Reservoir is measured.

After the high flow of December 22-23, continued warm weather resulting in increased snowmelt caused above-normal runoff for the rest of the month. By December 29, the total amount of water in storage in the Salt and Verde reservoir systems-1,891,00C acre-feetwas more than in any preceding December. The reserve storage capacity had been reduced to 181,000 acre-feet- $-20,000$ acre-feet in the Verde River reservoirs and 161,000 in the Salt River reservoirs-and hydrologic conditions in the upper parts of the basins were conducive to more runoff. It was considered certain that the reservoirs would spill before the spring runoff season was over, but no one ant icipated the magnitude of the spill or how soon it would take place. There was little warning that a flood was in progress until streams k agan rising rapidly at about 0400 hours on December 30. Tributaries to the Salt River upstream from Cibecue Creek and to the Verde River upstream from the East Verde River had higher peak discharges on December 30 than on December 22-23. Downstream from Cibecue Creek $\varepsilon$ nd the East Verde River, however, peaks were lower on December 30 b'it of longer duration. The peak at Salt River near Roosevelt was only slightly lower than that of December 22-23, but at Tonto Creek above Gun Creek and Verde River below Tangle Creek, the peaks were considerably lower. The maximum rate of inflow to Roosevelt Lale was computed as 66,000 cfs on December 30 .

\section{REGULATED DISCHARGE AT STEWART MOUNTAIN AND BARTLETT DAMS}

The reservoirs contained most of the high flow of December 22-23. Water was released from Stewart Mountain Dam at a maximum rate of $4,360 \mathrm{cfs}$, but no water was released from Bartlett Dam. The water released from Stewart Mountain Dam and the flow from small tributaries below Stewart Mountain and Bartlett Dams produced a peak discharge below Granite Reef Dam of 9,500 cfs on December 23.

When the streams began rising rapidly on December 30, the reservoirs were nearly full, and it became obvious before the per.ks arrived that the entire flow could not be contained. At 1100 hours on December 30, the release of water at Bartlett Dam was increased by control valves from a few hundred to about $4,000 \mathrm{cfs}$, and the Phoenix flood was underway. The release of this amount of water was continued until the reservoir began to spill at about 1400 hours. Discharge increased rapidly and reached 35,600 cfs at 1900 hours on December 30. The flow was gradually reduced to a low of $28,000 \mathrm{cfs}$ at 1100 hours on December 31. The flow was held between 28,000 and 31,400 cfs 
until 1900 hours on December 31, when it was gradually decreased to slightly more than $3,000 \mathrm{cfs}(\mathrm{pl} .4)$.

Water began flowing over the Stewart Mountain Dam spillway at 1930 hours on December 30, and by 2100 hours the reservoir was spilling 39,600 cfs. Except for a 11/2-hour period on January 1, the flow from Stewart Mountain Dam was held between 35,000 and 4.1,000 cfs until 0230 hours on January 2. A low of 5,050 cfs occurred at 0800 hours on January 2. At 1300 hours on January 3, the flow again was increased to about $10,000 \mathrm{cfs}$ and was held to this amount f( ${ }^{\mathbf{r}}$ several days.

\section{STAGES AND DISGHARGES DOWNSTREAM FROM GRANITE REEF DAM DURING THE PHOENIX FLOOD}

The flow of the Salt River below Granite Reef Dam was derived by combining the flows of Salt River below Stewart Mountain Dam and Verde River near Scottsdale and subtracting diversions (pl. 4). Attenuation between the gaging stations and Granite Reef Dam was not computed, but such attenuation would be a small amount of the total flow. The combined maximum flow of the Salt and Verde Rivers was 67,500 cfs from 2000 to 2400 hours on December 31, and the flow was held within $3,000 \mathrm{cfs}$ of this amount from 0800 hours on December 31 to 0100 hours on January 1. During this time, canals at Granite Reef Dam were diverting about $500 \mathrm{cfs}$, which left a peak flow of $67,000 \mathrm{cfs}$ over Granite Reef Dam.

After 0100 hours on January 1, the discharge decreased rapidly until 1400 hours on January 1, when increased releases at Stewart Mountain Dam caused another sharp rise of short duration. On January 2 the discharge over Granite Reef Dam was less than 10,000 cfs. However, in order to lower the water in the Salt River reservoirs enough to provide some protection if another flood should occur, the dissharge at Stewart Mountain Dam was increased on January 3, thus bringing the flow at Granite Reef Dam to 13,000 cfs. The flow over Granite Reef Dam was held between 11,000 and 13,000 cfs until January 11, when the flow was shut off.

The first water reached the Phoenix area about 0300 hours c $n$ December 31 . The water rose rapidly to depths of from 10 to 15 fest, and in places the river was more than a mile wide. A gage-height record furnished by the Salt River Valley Water Users' Association (pl. 4) shows that at Jointhead Dam near 52d Street the river reached its maximum stage at about 0900 hours on December 31, dropped off 0.4 foot, and then rose slightly about midnight. The river began to recede about 0400 hours on January 1. 
Severe scour during the flood eliminated the possibility of making a reliable indirect measurement of the peak discharge through Phoenix. Personnel of the Geological Survey made several discharge measurements as the flow receded, however, and these measurements provide fair definition for a stage-discharge relation for Jointhead Dam below $43,000 \mathrm{cfs}$. The maximum discharge at Phoenix was computed to be 66,000 cfs by interpolating between the peak discharge at Gr`nite Reef Dam $(67,000 \mathrm{cfs})$ and that at Gillespie Dam $(64,200 \mathrm{cfs})$. In computing the discharges past the gage at Jointhead Dam, it was assumed that the maximum discharge $(66,000 \mathrm{cfs})$ occurred at the time of the second (lower) peak in stage. The assumption appears logical because a secondary channel that carried a large amount of bypass flow around the dam was enlarged during the flood and may have allowed the higher discharge of the second peak to pass at the lower stage. Also, the flood washed out several downstream street crossings, which may have affected the stage-discharge relation during the early part of the flood.

In the 74-mile reach between Granite Reef and Gillespie Dams, the peak was reduced only slightly - the peak discharge at Gillespie Dam was 64,200 cfs-but the volume of flow was reduced appreciably by infiltration and pondage in numerous gravel pits (pl. 4). The Gila River above Salt River did not contribute any flow to this flood, and most of the flood peaks in tributaries to the Salt and Gila Rivers below Granite Reef Dam were either dissipated or stored by reservoirs before reaching the main stream. The only tributary flow between Granite Reef and Gillespie Dams from December 30 to January 13 was from the Agua Fria River, which had a peak flow of $480 \mathrm{cfs}$ on December 30, and the Hassayampa River, which had a peak flow of about 1,000 cfs on the same day.

Water in the Painted Rock Reservoir rose to a depth of nearly 56 feet as a result of the flood. This was the first time that water had been stored behind the dam, which was built in 1959. Inflow to Painted Rock Reservoir (pl. 4) was computed using change in reservoir contents. The peak was quite sharp; therefore, the shape of the hydrograph, plotted on plate 4, and the magnitude of the peak ars sensitive to the increment of time used in computing the flow. A 1-hour increment gives a peak inflow rate of about $65,000 \mathrm{cfs}$; a 3 -hour increment, $62,000 \mathrm{cfs}$; and a 6 -hour increment, $55,900 \mathrm{cfs}$. The U.S. Army Corps of Engineers (1966) used the 6-hour increment in their report but qualified it as a 6 -hour a verage. They stated that wind-caused surge and a lack of refinement in the table of reservoir contents made the use of a shorter period infeasible. A 6-hour increment, however, is quite long for such a sharp peak, and a 3 -hour increment has been us?d in this 
report. According to the U.S. Army Corps of Engineers (oral commun., 1966) surface-water inflow ceased January 15.

Plate 4 shows a steepening of the rising limb of the hydrograph and a large reduction in duration and rolume of the flood peak but little reduction in peak discharge as the flood wave moved downstream. The peak traveled much faster than the front of the flood wave. The front took 10 hours to cover the 20 miles from Granite Reef Dam to Jointhead Dam and 36 hours to cover the 54 miles from Jointhead Dam to Gillespie Dam, whereas the traveltimes for the peak were 4 and 24 hours, respectively (table 3 ). The change in the shape of the hydrograph (pl. 4) as the flood moved downstream is typical for floods that occur in dry sand channels because the first water to reach each section of channel infiltrates into the dry sand. After the channel has been wetted, the water moves along the channel at a faster rate; therefore, the traveltime for the peak is less than that for the flood front.

TABLE 3.-Times when the Phoenix flood reached different points on the Salt and Gila Rivers

\begin{tabular}{|c|c|c|c|c|c|}
\hline \multirow{2}{*}{ Location } & \multirow{2}{*}{$\begin{array}{l}\text { Miles from } \\
\text { Granite } \\
\text { Reef Dam }\end{array}$} & \multicolumn{2}{|c|}{ Time main rise began } & \multicolumn{2}{|c|}{ Tin's of peak } \\
\hline & & Hour & Date & Hour & Date \\
\hline Reef $\mathrm{Da}$ & 0 & 2000 & $12-30$ & 2007 & $12-31$ \\
\hline Jointhead Dam-- & 20 & 0600 & $12-31$ & 2407 & $12-31$ \\
\hline Jack Rabbit Road & 51 & 1900 & $12-31$ & 0407 & $1-1$ \\
\hline $\begin{array}{l}\text { Gillespie Dam } \\
\text { Painted Rock Dam. }\end{array}$ & $\begin{array}{r}74 \\
103\end{array}$ & $\begin{array}{r}1800 \\
11200\end{array}$ & $\begin{array}{l}1-1 \\
1-2\end{array}$ & $\begin{array}{r}2407 \\
11707\end{array}$ & $\begin{array}{l}1-1 \\
1-2\end{array}$ \\
\hline
\end{tabular}

${ }^{1}$ Approximate time based on increments of reservoir storage (pl. 4).

Water was released from Painted Rock Dam at a maximum rate of $2,850 \mathrm{cfs}$. No serious flooding occurred downstream from the dam, but grade-level road crossings were inundated for 2 or 3 months, and many were not reopened to traffic until late in April. The Bureau of Reclamation installed a gaging station at Avenue 51E near Mohawk to measure infiltration in the Wellton-Mohawk Irrigation and Drainage District. The first water from the flood reached that gage on February 2 and reached the gaging station at Gila River near Dome on February 19 ( $\mathrm{pl} .4$ ). The maximum daily discharges were 1,200 cfs at the Bureau of Reclamation gage and 615 cfs at the Dome gage. The last of the impounded water was released from Painted Rock Dam on March 25, but ground-water discharge in the reservoir area sustained flow in the Gila River for several months. Less than half the water released from Granite Reef Dam reached Painted Rock Dam as surface flow, and less than 10 percent of the water reached the mouth of the Gila River (table 4). 
TABLE 4.-Runoff of the Salt and Gila Rivers downstream from Granite Reef Dam

\begin{tabular}{|c|c|c|}
\hline Location & Period of runoff 1 & $\begin{array}{l}\text { Discharge } \\
\text { (acre-feet) }\end{array}$ \\
\hline \multirow[t]{2}{*}{$\begin{array}{l}\text { Inflow, Granite Reef Dam to Gillespie Dam: } \\
\text { Salt River below Granite Reef Dam.... }\end{array}$} & $\begin{array}{r}12-22-65 \text { to } 1-11-66 \\
2-12-66 \text { to } 5-4-66\end{array}$ & $\begin{array}{r}509,000 \\
42,800\end{array}$ \\
\hline & & 551,800 \\
\hline $\begin{array}{l}\text { Total from Gila River near Laveen, Santa Cruz River near Laveen, } \\
\text { Agua Fria River at Avondale, and Hassayampa River near } \\
\text { Arlington. }\end{array}$ & $12-10-65$ to $2-2 \varepsilon-66^{2}$ & 60,000 \\
\hline Total & $12-10-65$ to $3-4-66$ & 611,800 \\
\hline $\begin{array}{l}\text { Gila River at Gillespie Dam } \\
\text { Gila River below Painted Rock Dam } \\
\text { Gila River at A venue } 51 \mathrm{E} \text { near Mohawk- } \\
\text { Diversions between Avenue 51E and Dome gage.. } \\
\text { Gila River near Dome. }\end{array}$ & $\begin{array}{r}12-10-65 \text { to } 3-30-66 \\
12-27-65 \text { to } 7-9-66 \\
2-2-66 \text { to } 4-8-66 \\
2-2-66 \text { to } 4-6-66 \\
2-19-66 \text { to } 5-6-66\end{array}$ & $\begin{array}{r}436,600 \\
257,350 \\
99,500 \\
-14,150 \\
37,600\end{array}$ \\
\hline
\end{tabular}

1 The closing date for period of runoff is the date on which the discharge at a particular location reached zero after the flood, except at Gila River at Avenue $51 \mathrm{E}$ near Mohawk, where records were stopped on Apr. 8 when discharge was $4 \mathrm{cfs}$, and Gila River near Dome, where flow continued for more than a year. The discharge of Gila River near Dome reached a low of 2.2 cfs on May 6, increased slightly, and then gradually dropped off to 0.5 cfs June 4-9. May 6 is the last date on whicli flow can be attrik rited solely to the flond.

${ }^{2}$ No flow Jan. 10 through Feb. 6, 1966.

\section{EFFECTS OF THE PHOENIX FLOOD}

Soon after the release of water at Bartlett Dam on Docember 30, about 8,000 persons were evacuated from the area along the Salt River channel in Phoenix. Most of them returned to their homes that night, however, and fewer than 400 were housed in emergency quarters. Transportation facilities were damaged badly by the floot. Within 6 hours after the first water reached Phoenix, every grade-level crossing in the metropolitan area was flooded. Floodwater cut into the south approach of the bridge on the Maricopa Freeway (Interstate Highway 10 ), which had been opened to traffic only a month earlier. About 1,000 feet of shoulder, curb, and one traffic lane was washer away, and highway officials closed the bridge to traffic. One pier of the Central Avenue Bridge failed, and the bridge was closed early on January 1. For a while, the U.S. Highway bridge at Tempe was the only usable crossing. Half the Maricopa Freeway bridge was reopened on the afternoon of January 1, and two lanes of the Central Avenue Bridge were opened on January 3. Other crossings remained closed for 2-3 weeks.

As much as 15 inches of water flooded 2,600 feet of runway at Sky Harbor Airport. Some power-transmission towers and poles in the river channel were washed out, and there were many pover failures. Several sewage-oxidation ponds at treatment plants serving Mesa, Tempe, and Scottsdale and a sewerline that crossed Salt River were washed out. The damaged facilities discharged large amounts of raw sewage into the river, but dilution by the large volume of floodwater 
minimized the health hazard. Gaslines also were damaged, and gravel companies and cattle feedlots in the river channel were inundated.

Aerial photographs of the Salt River from Phoenix to Poosevelt Lake were taken near the crest of the flood by personnel of the Geological Survey. The pictures of the metropolitan area (pl. 5) were taken between 1230 and 1245 hours on December 31 when stage and discharge were slightly below the maximum (pl. 4). Photographs not included in this report are available from files of the Geological Survey. The Arizona Highway Department also obtained excellent aerial photographic coverage of the flood, and their files contain pictures that cover the reach from the Verde River to Painted Rock Dam.

In a 25-mile reach through the metropolitan area from Ork Street near Mesa to 51st Avenue in Phoenix, the average width of the Salt River was slightly more than half a mile. The width ranged from 400 feet near 16th Street to 7,000 feet near Scottsdale Road. A lthough a large area was inundated ( $\mathrm{pl} .5$ ), most of it was within the natural river channel, and water spread out onto the flood plain in orly a few small areas. At Scottsdale Road (pl. 5), land half a mile from the main channel was inundated, but in most areas flooding was confined to a narrow strip within a few hundred feet of the main chann?l:

Throughout the metropolitan area, the channel was enlarged and cleaned out by the flood; a wide well-defined channel was formed in what previously had been a poorly defined channel. The "before" photograph on plate 5 shows a flat brushy area crisscrossed by roads and powerlines and pocked with gravel pits and sewage-treatment tanks. The area shown in the "before" photograph is typical of the channel before the flood in most of the metropolitan area. In places the entire channel was a series of large gravel pits, and in others it was constricted by manmade landfills and commercial development. At Central Avenue more than a third of the bridge opening had been blocked off by waste material from a gravel operation. Levels run to debris lines that had been left by the flood showed that during the peak there was $31 / 2$ feet of fall through the constriction formed by the fill. In the 25-mile reach through the metropolitan area, 1.7 gradelevel crossings, similar to those shown in the "before" photograph, were destroyed.

The after photograph on plate 5 shows a smooth sand channel that contains very little brush. The gravel pits have been filled, and the roads have been washed away. At the time this photograph was taken, temporary crossings had been constructed on Scottsdale and Hayden Roads, and a new dike had been built around the sewage-treatment beds between the roads.

Damage data were collected and evaluated by the U.S. Army Corps of Engineers (1966) in cooperation with many Federal, Statє, county, 
city, and local agencies. Damages were estimated to be $\$ 5,827,000$ along the Salt River from Mesa to the mouth and $\$ 250,000$ along the Gila River from Salt River to Gillespie Dam (table 5). Lit ${ }^{+}$le damage occurred in the first 10 miles of the Salt River channel c'ownstream from Granite Reef Dam, but in the 28-mile reach from Oak Street to the mouth of the Salt River the amount of damage was large. The damage along the Gila River was spotty and occurred mainly in agricultural areas and on streets and highways.

TABLE 5.-Summary of damages from the Phoenix flood along the Salt and Gila Rivers

[Data from U.S. Army Corps of Engineers, 1966]

\begin{tabular}{lll} 
Type of property & Damages & \\
\cline { 2 - 4 } & $\begin{array}{c}\text { Physical } \\
\text { damages } \\
\text { costs and } \\
\text { business } \\
\text { losses }\end{array}$ & Total \\
\hline
\end{tabular}

Salt River, Granite Reef Dam to mouth

\begin{tabular}{|c|c|c|c|}
\hline Residential & $\$ 5,000$ & $\$ 1,000$ & $\$ 6,000$ \\
\hline Commercial. & 65,000 & 38,000 & 103,000 \\
\hline Industrial_ & $2,041,000$ & 398,000 & $2,439,000$ \\
\hline Public & 230,000 & 138,000 & 368,000 \\
\hline Utilities_ & 892,000 & 268,000 & $1,160,000$ \\
\hline Streets, highways, and bridges_ & $1,326,000$ & 360,000 & $1,686,000$ \\
\hline Irrigation works & 35,000 & 2,000 & 37,000 \\
\hline Agricultural _. . & 15,000 & 2,000 & 17,000 \\
\hline Railroad & 3,000 & 1,000 & 4,000 \\
\hline Total & $4,612,000$ & $1,208,000$ & $5,820,000$ \\
\hline
\end{tabular}

Gila River, mouth of Salt River to Gillespie Dam

\begin{tabular}{|c|c|c|c|}
\hline Residential_ & $\$ 32,000$ & $\$ 3,000$ & $\$ 35,000$ \\
\hline Commercial & 0 & 3,000 & 3,000 \\
\hline Industrial_ & 27,000 & 8,000 & 35,000 \\
\hline Public_. - & 0 & 6,000 & 6,000 \\
\hline Utilities & 0 & 0 & \\
\hline Streets, highways, and bridges $\ldots \ldots$ & 85,000 & 6,000 & 91,000 \\
\hline Irrigation works & 0 & 0 & \\
\hline Agricultural_ _ & 72,000 & 8,000 & 80,000 \\
\hline Railroad & 0 & 0 & \\
\hline Total & 216,000 & 34,000 & 250,000 \\
\hline
\end{tabular}

Industrial damage comprised about 42 percent of the total along the Salt River; industrial damages were estimated to be $\$ 2,439,000$, of which $\$ 1,967,000$ was sustained by gravel companies located in the river bottom. The second largest loss was sustained by cattle feedlots, also located in the river bottom. The flood damage to the runway at Sky Harbor Airport was estimated to be $\$ 235,000$. Damages to the utilities along the Salt River were $\$ 1,160,000$. 


\section{REDUCTION IN PEAK DISCHARGE BY STORAGE IN RESERVOIRS}

Without the storage provided by the six reservoirs on the Verde and Salt Rivers, the peaks of December 22-23 would have reache the confluence of the two streams at about the same time, and the combined flow would have caused a much higher flood than the one that occurred on December 31. In order to obtain an estimate of what the peak discharges during the floods would have been without the dams, the floods were routed from the gaging stations above the reservoirs to Granite Reef Dam. The coefficients used to develop the estimated hydrograph (pl. 4) were determined in the same manner as those used to compute the flow into Roosevelt Lake, which has been discussed previously. Without the storage provided by the reservoirs, the peak discharge at Granite Reef Dam would have been about 120,000 cfs on December 23 and slightly more than $80,000 \mathrm{cfs}$ on December 31 . The duration of the December 31 peak would have been about the same as that for the regulated peak.

\section{COMPARISON WITH PREVIOUS FLOODS}

The peak discharge during the Phoenix flood is the highes' flood on the Salt River and Gila River below Salt River since the completion of Bartlett Dam on the Verde River in 1939. The flood was publicized as a major catastrophe because most residents were not aware of previous floods and because businesses that had encroached on the river channel sustained damage; however, hydrologically, the magnitude of the flood was relatively small. The recurrence interval for unregulated conditions for the peak discharge is about 6 years (Patterson and Somers, 1966). Several higher peaks occurred prior to construction of the upstream dams; in the 50-year period 1890 to 1939, at least 14 floods exceeded the 1965 Phoenix flood (tables 6 and 7 ). Early floods, prior to 1910 when filling of Roosevelt Lake began, were from unregulated flows from the Salt and Verde Rivers; the floods of 1916 and 1920 were a combination of unregulated flow from the Verde River and spill from Roosevelt Lake; the floods of 1923-38 resulted only from unregulated flow in the Verde River.

The largest known flood in the Salt River basin occurred in February 1891, when a peak of about $300,000 \mathrm{cfs}$ inundated mot of the present downtown area of Phoenix. Water reached the Arizona State Hospital, on Van Buren Street between 24th and 28th Streets. and the corner of Jefferson and Central Avenues in downtown Phoenix, which is 11/4 miles north of the Maricopa Freeway (pl. 5). The discharge of Salt River below Granite Reef Dam was more than 100,000 cfs on at least five occasions between 1905 and 1920 , and several peaks since 
TABLE 6.-Discharge data for major floods,

[Peaks are those for which daily flow of the Salt River was computed to be more than 30,000

\begin{tabular}{llcccc}
\hline \multicolumn{4}{c}{ Peak discharge, in cfs } \\
\cline { 2 - 5 } Date of flood & $\begin{array}{c}\text { Salt River } \\
\text { near } \\
\text { Roosevelt }\end{array}$ & $\begin{array}{c}\text { Tonto } \\
\text { Creek }\end{array}$ & $\begin{array}{c}\text { Salt River } \\
\text { at Roosevelt }\end{array}$ & $\begin{array}{c}\text { Salt Piver } \\
\text { abcre } \\
\text { Verde River }\end{array}$ & $\begin{array}{c}\text { Verde } \\
\text { River }\end{array}$ \\
\hline
\end{tabular}

Feb. 22-23, 1890

Feb. 19, 1891

Feb. 23-24, $1891^{2}$

Jan. 17-18, 1895

Feb. 4-5, 1905 .

Feb. 28 to Mar. 1, 1905

Mar. 13-14, 1905.

Mar. 17-18, 1905

Mar. 20-21, 1905

Apr. 12-13, 1905

Nov. 27,1905

Mar. 13-14, 1906

Mar. 27, 1906

Dec. $3-4,1906$

Mar. 6, 1907

Dec. 16-17, 1908

Jan. 30,1915

Jan. 19-20, 1916

Jan. 29-30, 1916

Nov. 28, 1919

Dec. 6,1919

Feb. 22-23, 1920

Dec. 28, 1923

Feb. 17-18, 1927

Feb. 17-18, 1927_.....

Feb. 7-8, 1937

Mar. 4, 1938

Mar. 14-15, 1941

Jan. 18-19, 1952

Dec. 25-26, 1959

Dec. 22-24, 1965

Dec. $30-31,1965$.

100,000

7120,000

868,900

150,000

$3 \overline{3}, 400$

336,400

348,000

354,000

${ }^{3} 54,000$

年

${ }^{3} 145,000{ }^{5} 138000 \quad 96,000$

\begin{tabular}{|c|c|c|c|c|c|}
\hline Dec. 28,1923 & 43,000 & & & & \\
\hline Feb. $17-18,1927$ & 40,000 & & & & \\
\hline $\begin{array}{l}\text { Feb. } 10-11,1932 \\
\text { Feb. } 7-8,1937\end{array}$ & $\begin{array}{l}57,000 \\
88,000\end{array}$ & & 100000 & 28 & 53,000 \\
\hline $\begin{array}{l}\text { Feb. } 7-8,1937 \\
\text { Mar. } 4,1938\end{array}$ & $\begin{array}{l}00,000 \\
24,100\end{array}$ & & & 53 & 95,000 \\
\hline Mar. $14-15,1941$ & 117,000 & 32,000 & 136,000 & 29 & 45,800 \\
\hline Jan. $18-19,1952$ & 111,000 & 45,400 & 139,000 & 10 & 237 \\
\hline Dec. $25-26,1959$ & 78,200 & 25,200 & 103,000 & 10700 & ${ }^{10} 620$ \\
\hline Dec. $22-24,196$ & 68,800 & 44,700 & 88,000 & 4. 360 & 8,540 \\
\hline Dec. $30-31,19$ & 59,900 & 22,100 & 66,000 & 50100 & 31,300 \\
\hline
\end{tabular}

\footnotetext{
1 Data not previously published; from J. H. Gardiner and S. O. Decker (written commun., 1948).

2 Figures for February 23-24, 1891, have been published previously as maxima and as daily mean discharges. J. H. Gardiner and S. O. Decker (written commun., 1948) have indicated that th $e$ published figures should be used as maxima; they gave a daily discharge of about 225,000 cfs at Arizona Dam. Daily mean discharge at Roosevelt not determined.

3 Data not previously published; obtained by applying an extension of 1905 rating to maximum stage noted by observer.

C. T. Newton (U.S. Army Corps of Engineers, written commun., 1957).
}

1920 would have exceeded $100,000 \mathrm{cfs}$ if they had not hen reduced greatly by storage in the reservoirs. At least three and possibly four of these floods would have exceeded $150,000 \mathrm{cfs}$; the two largest floods would have been on March 14-15, 1941, and January 18-19, 1952. Without regulation, the 1941 flood would have had a peak discharge of about $170,000 \mathrm{cfs}$ at Granite Reef Dam, and the 1952 peak would have been only slightly less. A maximum discharge of $45,800 \mathrm{cfs}$ was released at Bartlett Dam during the 1941 flood and no water, except for the normal amounts for irrigation, was released during the 1952 flood. 
Salt River and main tributaries, 1888-1965

cfs at Roosevelt or more than 50,000 cfs below Verde Rivei; sources of data given in table 7]

\begin{tabular}{|c|c|c|c|c|c|c|}
\hline \multirow{2}{*}{$\begin{array}{c}\begin{array}{c}\text { Peak discharge, } \\
\text { in cfs-Con. }\end{array} \\
\begin{array}{c}\text { Salt River } \\
\text { below } \\
\text { Verde River }\end{array}\end{array}$} & \multicolumn{6}{|c|}{ Maximum daily discharge associated with the flood, in cfs } \\
\hline & $\begin{array}{l}\text { Salt River } \\
\text { near } \\
\text { Roosevelt }\end{array}$ & $\begin{array}{l}\text { Tol } \\
\text { Cre }\end{array}$ & $\begin{array}{l}\text { Salt } \\
\text { at } R_{0}\end{array}$ & $\begin{array}{c}\text { Salt River } \\
\text { above } \\
\text { Verde River }\end{array}$ & $\begin{array}{l}\mathrm{Ve} \\
\mathrm{Ri}\end{array}$ & $\begin{array}{l}\text { Salt River } \\
\text { below } \\
\text { Verde River }\end{array}$ \\
\hline & & & 71,600 & & 64,500 & 14 \\
\hline 1267 & & & & & 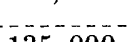 & 1225,000 \\
\hline 300 & & & & & 135,000 & ${ }^{1} 225,000$ \\
\hline 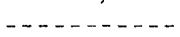 & $\ldots$ & - & 46,800 & 49,800 & 33,200 & 83,000 \\
\hline & - & - - & 31,400 & 32,300 & 33,000 & 65,000 \\
\hline - & - & - & 27, 600 & 29,100 & 25,100 & 54,000 \\
\hline 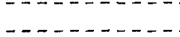 & $\cdots$ & - & 38,700 & 28,600 & 25,500 & 54,000 \\
\hline & - & & 39,800 & 34,800 & 29,400 & 64,000 \\
\hline & -- & 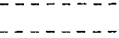 & 44,400 & 800 & 10,600 & 45,000 \\
\hline 4115 & & & 45,500 & 00 & 32,100 & 87,000 \\
\hline$>200,000$ & & - & 97,700 & ${ }^{6} 9$ & 61,500 & ${ }^{6} 150,000$ \\
\hline$-\ldots$ & 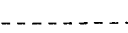 & $\ldots$ & 35,700 & 00 & 31 & 000 \\
\hline 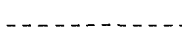 & . & -- & 28,200 & 00 & 30 & 00 \\
\hline & . & $\ldots$ & 36,600 & 00 & 15 & 00 \\
\hline & & - & 14,200 & 18,600 & 32,200 & 900 \\
\hline & & & & 35,000 & 51,600 & 63,000 \\
\hline & 37,500 & 11,000 & 48,500 & 79 & 15,700 & 16,000 \\
\hline 4120 & 91,000 & 18,000 & 109,000 & 000 & 53,400 & 000 \\
\hline 1105 & 69,500 & 8,690 & 78,200 & 50,000 & 22,600 & 73,000 \\
\hline & 36,500 & 6,430 & 42,900 & 294 & 46,800 & 47,000 \\
\hline & 600 & 8,920 & 59,500 & & 00 & 17,000 \\
\hline 130 & 000 & 9,880 & 65,900 & 28,000 & 48,200 & 76,000 \\
\hline & 200 & 20,000 & 52,200 & 0 & 40,800 & 41,000 \\
\hline$(\theta)$ & 600 & 12,600 & 40,9 & 1,110 & 00 & $(9)$ \\
\hline$(8)$ & 200 & 8, & 42,200 & 25 & 41,500 & (8) \\
\hline()$\left.^{8}\right)$ & 35,000 & 18,000 & 000 & 23 & 39,200 & (8) \\
\hline 185,000 & 17,800 & 6 & 0 & 14 & 700 & $(9)$ \\
\hline (9) & 60,200 & 16 & 0 & 29 & 32,700 & $(8)$ \\
\hline & & 21, & 0 & 5 & 189 & $-\ldots$ \\
\hline 88,800 & 800 & 14,300 & 0 & 134 & 182 & -1 \\
\hline & & 15,300 & & 3,750 & 2,950 & $9 \overline{0}$ \\
\hline 67,000 & 30,600 & 11,200 & 40,400 & 37,200 & 30,200 & 64,000 \\
\hline
\end{tabular}

5 Maximum observed; previously published as daily mean flow, but figure is inconsistent with records for Salt River at Roosevelt.

${ }^{6}$ Approximate; estimated from records for Salt River near Roosevelt and Verde River necr McDowell.

7 Approximate; estimated from records for Salt River near Roosevelt and Tonto Creek near Roosevelt.

8 From Salt River Valley Water Users' Association.

${ }^{9}$ Discharge approximately equai to that in the Verde River but may differ slightly because of inflow, attenuation, and time of travel.

10 Originated in tributaries below reservoir systems.

Upstream from the reservoirs, the flood resulting from the storm of December 29-30 generally had a larger recurrence interval than the flood below the reservoirs, but it was not a major flood except in a few small areas. Recurrence intervals of peak discharges at specific sites exceeded 50 years for sites on Carrizo and Corduroy Creeks and ranged from 9 to 19 years for sites on Tonto, Oak, and Dry Beaver Creeks, the headwaters of the East Verde River, and the lower reaches of the Black and Salt Rivers. Elsewhere in the Salt River k asin the recurrence intervals were 7 years or less. 
TABLE 7.-Sources of data for major floods, [Strean designations are

\begin{tabular}{|c|c|c|}
\hline Stream designation & Period & Location of gaging station \\
\hline Salt River near Roosevelt. . . . . . . . . . & $1915-65$ & 17 miles above Roosevelt Dam \\
\hline Tonto Creek... & $\begin{array}{l}1915-41 \\
1941-65\end{array}$ & 17 miles above Rooserelt Dam 26 miles above Roosevelt Dam \\
\hline Salt River at Roosevelt...................... & $\begin{array}{l}1890-95 \\
1905-7 \\
1915-65\end{array}$ & $\begin{array}{l}\text { A quarter of a mile above present site of Roosevelt } \\
\text { Dam. }\end{array}$ \\
\hline
\end{tabular}

Salt River above Verde River.

Verde River

Salt River below Verde River
1895-1910 Within 2 miles of Verde River

1915-23 At Roosevelt Dam.

1923-29 At Mormon Flat Dam

1930-49 Three-quarters of a mile below stowart Mountain Dam.

1950-65 31/2 miles below Stewart Mountair Dam

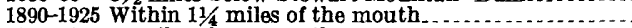

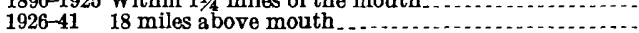

1942-61 21 miles above mouth.

1961-65 2.5 miles above mouth

1888-96 $11 / 4$ miles below Verde River

1896-1965

The December 22-23 storm produced unusually high flows in Sycamore and Tonto Creeks and the lower reaches of the East Verde and Black Rivers. On Tonto Creek, the peak was the second highest since 1941, when the gage was installed above Gun Creek, and ear'ier records collected 9 miles downstream indicate that it may have been the second highest since at least 1913. Recurrence intervals of peak discharges ranged from 9 to 14 years for sites on Corduroy Creek and the Black and Salt Rivers and from 20 to 25 years on Sycamore Creok and the East Verde River, and they were about 50 years for sites on Tonto Creek. Elsewhere in the basin, recurrence intervals were generally less than 5 years. The December 23 peak on the Salt and Verde Rivers has been exceeded by about 10 other floods in the last 80 years (table 6 ). At the Salt River near Roosevelt gage, the 1891 flood had a peak discharge of more than $150,000 \mathrm{cfs}$, and at least four other floods had peak discharges of more than $100,000 \mathrm{cfs}$. 
those used in table 6]

\begin{tabular}{|c|c|}
\hline Source of data during period shown & Discharge \\
\hline Published records for Salt River near Roosevelt & Natural discharge of the Salt River above Roosevclt \\
\hline $\begin{array}{l}\text { Published records for Tonto Creek near Roosevelt.- } \\
\text { Published records for Tonto Creek above Gun } \\
\text { Creek, near Roosevelt. } \\
\text { Published records computed from records above } \\
\text { and below Verde River. } \\
\text { Published records for Salt River at Roosevelt.....- }\end{array}$ & $\begin{array}{l}\text { Natural discharge of Tonto Creek. } \\
\text { Do. } \\
\text { Natural discharge of the Salt River below Tonto } \\
\text { Creek. } \\
\text { Do. }\end{array}$ \\
\hline $\begin{array}{l}\text { Unpublished records; from summation of flow of } \\
\text { Salt River near Roosevelt and Tonto Creek at } \\
\text { corresponding times. } \\
\text { Published records for Salt River at McDowell...... }\end{array}$ & $\begin{array}{l}\text { Natural discharge of the Salt River above the Verde } \\
\text { River. }\end{array}$ \\
\hline $\begin{array}{l}\text { Unpublished records from Salt River Valley Water } \\
\text { Users' Association. } \\
\text { Published records for Sait River below Stewart } \\
\text { Mountain Dam. }\end{array}$ & $\begin{array}{l}\text { Regulated discharge from the Salt River reservoir } \\
\text { system. } \\
\text { Do. } \\
\text { Do. }\end{array}$ \\
\hline $\begin{array}{l}\text { Published records for Verde River near McDowell-- } \\
\text { Published records for Verde River above Camp } \\
\text { Creek near McDowell. }\end{array}$ & $\begin{array}{l}\text { Natural discharge of the Verde River. } \\
\text { 1926-39: Natural discharge of the Verde River. } \\
\text { 1939-41: Regulated discharge from the Verde River } \\
\text { reservoir system; inflow between gag, and mouth } \\
\text { not included. }\end{array}$ \\
\hline $\begin{array}{l}\text { Published records for Verde River below Bartlett } \\
\text { Dam. }\end{array}$ & $\begin{array}{l}\text { Regulated discharge from the Verde } R \text { ver reservoir } \\
\text { system; inflow between gage and mouth not in- } \\
\text { cluded. }\end{array}$ \\
\hline Published records for Verde River near Scottsdale. & $\begin{array}{l}\text { Regulated discharge from the Verde R'ver reservoir } \\
\text { system plus natural inflow between Fartlett Dam } \\
\text { and the mouth of the Verde River. }\end{array}$ \\
\hline Published records for Salt $\mathbf{R}$ & $\begin{array}{l}\text { 1888-1908: Natural discharge of the Salt River below } \\
\text { the Verde River. }\end{array}$ \\
\hline $\begin{array}{l}\text { Unpublished records; from summation of flow of } \\
\text { Salt River above Verde River and Verde River } \\
\text { at corresponding times. }\end{array}$ & $\begin{array}{l}\text { 1915-38: Regulated discharge from the Salt River } \\
\text { reservoir system plus natural discharge of the } \\
\text { Verde River. } \\
\text { 1939-65: Regulated discharges from the Salt and Verde } \\
\text { River reservoir systems plus varying amounts of } \\
\text { inflow between the reservoirs and the mouth of the } \\
\text { Verde River. }\end{array}$ \\
\hline
\end{tabular}

\section{EFFECTS OF THE FLOODS ON GROUND-WATER LIVELS}

Although the floods were damaging to some parts of Arizor a's economy, they were beneficial to others. The arid lands affected by the floods depend, to a large extent, on ground water for their water supplies. Recharge to the ground-water reservoirs was beneficial except in the Wellton-Mohawk area, where drainage is a problem. Annual water-level measurements made in several hundred wells in Arizona provide a general index of changes in water levels. The water levels in wells along most of the major flood plains affected by the floods rose from spring 1965 to spring 1966 (table 8). Rises of more than 40 feet were measured in wells along Rillito Creek, and rises of 35 to 40 feet were measured in wells along the Salt River downstream from Granite Reef Dam.

Larger rises in water levels were recorded during the flood period. The water level in a well near Rillito Creek at Tucson rose 53 feet between December 7, 1965, and January 4, 1966 (University of Arizona, written commun., 1966). (See Well C on nl. 6.) The close correlation 
between rises in ground-water levels and streamflow in th. Salt and Gila Rivers indicates that a large part of the water lost from surface flow during the Phoenix flood infiltrated into the ground'-water reservoir (table 4, pls. 5 and 6). In general, water-level rises from spring 1965 to spring 1966 in other areas were less than 25 feet and extended from 1 to 3 miles away from the stream channels; between the flood plains and the mountain fronts, however, water levels continued to decline. Along a 40-mile reach of the Santa Cruz River from the international boundary northward, water levels were the highest ever measured, and rises of 10 feet were measured as much as 3 miles from the river (Clyma and Shaw, 1968). Although the floods of December 1965 to January 1966 were the main contributor to the rises in water levels, other periods of prolonged surface flow on unregulated streams and a reduction in pumping in some areas may have been contributing factors.

TABLE 8.-Representative rises in ground-water levels along major flood plains in the Gila River basin, spring 1965 to spring 1966

[Data from Hodges and others (1967)]

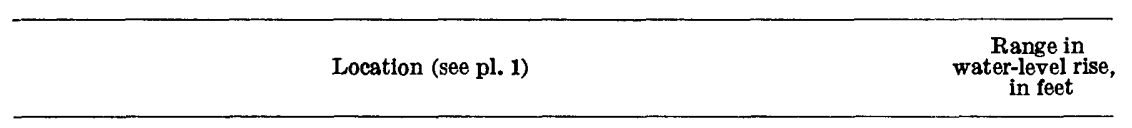

1. Duncan basin 1-3

2. Safford basin 4-12

3. Aravaipa Valley

4. Lower San Pedro River valley

5. Upper Santa Cruz basin:

Above Rillito Creek

Rillito Creek basin

6. Lower Santa Cruz basin

7. Salt River Valley

New River and Skunk Creek basin

8. Gila Bend area

9. Palomas Plain.

1 In T. 9 S., Rs. 7 and 8 E., rises of $12-30$ feet occurred.

Water-level rises were a detriment in the Wellton-Mohawk area, where waterlogging has been a problem for several years, and water is pumped to lower the water table. The high flows in February, March, and April 1966 practically nullified 5 years of pumping, and it was not until March 1967 that water levels were drawn down to about the same level as they had been before the flood. The U.S. Bureau of Reclamation (1967) reported that 54,140 acre-feet of water infiltrated to the ground-water reservoir within the bounderies of the Wellton-Mohawk Irrigation and Drainage District from the releases from Painted Rock Dam. The amount of land in the district having depths to water of less than 8 feet increased more than 300 percent. The 
U.S. Bureau of Reclamation (1967) also reported a total deposition of 47,150 tons of salts in the district.

\section{STREAMFLOW DATA AT GAGING STATIONS AND MISCELLANEOUS MEASURING SITES}

\section{DETERMINATION OF FLOOD DISCHARGES}

The operation of a stream-gaging station consists principally of the measurement of stage and discharge and the definition of the stagedischarge relation from which discharge can be calculated for a given stage. The development of a stage-discharge relation is based on current-meter measurements throughout the range of stage experienced or through a sufficient part of the range so that the discharge corresponding to the maximum stage can be obtained by a reasonable extension of the stage-discharge relation or rating curve. A short extension of a rating curve is usually made by logarithmic plot'ing or by the use of hydraulic principles. A long extension of a rating curve is based on indirect discharge measurements in which discharge is computed from channel geometry and high-water profiles. Techniques for making indirect measurements are given by Benson and Dalrymple (1967), Dalrymple and Benson (1967), Hulsing (1967), Matthai (1967), and Bodhaine (1968). Indirect measurements also rere made at miscellaneous sites where peak-discharge data were desired.

\section{SUMMARY OF FLOOD STAGES AND DISGHARGES}

Maximum stages and discharges at continuous-recording stations, crest-stage stations, and miscellaneous sites are summarized in table 9 . The reference numbers in the table correspond to those on plate 1.

The derivation of the maxima data is explained in the station descriptions for each site. The peak discharges in table 9 are those actually determined; that is, no adjustments for storage, regclation, or diversion have been made.

Explanation of data in the 12 columns in table 9 follows:

Number.-The number by which each station is identified on plate 1 . The numerical order follows the Geological Survey's standard downstream order of listing stations. Sites 108 and 131 are not plotted on plate 1 ; each is a system of reservoirs that cannot be plotted at any one place.

Permanent station number.-The number used in the Geological Survey's water-supply papers of surface-water supply in the United States and the annual reports of surface-water records of New Mexico and Arizona. Blank spaces in the column indicate a measurement at a miscellaneous site without a number. The number for each station in- 
cludes the part number, which locates the station in one of the Geological Survey's 14 major geographical divisions of principal river basins; all stations in this report are in Part 9, Colorado River basin. The station number is completed by a 4 - to 6 -digit number that is unique for each station.

Stream and place of determination.-The permanent name adopted for the site to which the listed data apply; each name is unique.

Drainage area.-The gross drainage area, in square miles. upstream from the station as determined from topographic maps.

The last eight columns in the table give data for the maximum known floods prior to November 1965 and for the maximum peaks of November 1965 to January 1966.

Period.-The period of known floods prior to November 1965. This period does not necessarily correspond to that in which continuous records of discharge were obtained, but for many records it extends back to an earlier date. In some instances it may start at a later date if early records are inadequate for determination of maximum discharge. For stations where part of the record represents natural flow and the remainder represents regulated flow and for the stations where it is desirable to show both the peak discharge during the period of record and one or more historical peaks, two or more concurrent periods have been used.

Year.-The calendar year in which the maximum stage or discharge during the period of known floods occurred.

Gage height and discharge.-Data in each pair of columns are associated with the year or date in the preceding column.

Date.-The date of peak discharge during the floods of November 1965 to January 1966.

Recurrence interval.-The average time interval in which a flood of a given discharge would be equaled or exceeded. The recurrence interval does not indicate the length of time between such floods; there may be decades between 10-year floods, and several 10-year floods may occur in a short period of time. The probability of a peak of a given magnitude occurring in any given year is equal to the re iprocal of the recurrence interval. Therefore, the probability of a 10-year flood occurring in any one year is 0.1 . Expressed as a percentage, there is a 10 percent chance of such a peak occurring in any given year. Recurrence intervals were computed from the flood-frequency curves developed by Patterson and Somers (1966), unpublished regional studies by the author, or the frequency curves for individual stations. Recurrence intervals generally have not been computed for regulated paaks or for short-term stations in areas for which flood-frequency relstions have not been defined. 


\section{EXPLANATION OF STATION DATA}

The data in this section of the report consist of descriptions of the stream-gaging stations or other measuring sites, daily and monthly discharges at gaging stations for November 1965 to January 1966 , and stages and discharges at indicated times for selected gaging stations. The station description gives information relative to location, size of drainage basin, nature of the gage-height record obtained during the period covered by this report, datum or altitude of gage, definition of the stage-discharge relation, maximum stage and discharge during the floods of November 1965 to January 1966 and the previous maximum, effect of regulation and diversion, and other pertinent general information.

Historical data from Senate Document 436 (Olmstead, 1919) are given for several stations in the Gila River basin above Coolidge Dam. Little is known about the accuracy of the data, but Olmstead (1919, p. 39) stated:

Of necessity the field work was carried out under considerable prissure, but the results obtained, it is believed, are sufficiently accurate to be used with full confidence in the general study of the hydrography of the Gila River mountain watershed.

* * * The record of fiood flows has been secured by careful obserration [of] the height of drift and high-water marks of all kinds in many places. The parallel measurements of present fiow and of crest the two weeks preceding give somewhat of a truthful account of the condition of the various minor drainage areas. *** The undertaking has been accomplished in the only arailable or practical way possible, and while some of the flood discharges may be 20 percent off, they are generally consistent and sufficiently close for the purposes for which the data have been used.

The table of stages and discharges at indicated times contains sufficient data for the construction of hydrographs. The time period included starts prior to the beginning of the major rise and ends at an arbitrary cutoff point on the recession; therefore, the time period is not the same for all stations. Beyond the cutoff point on the recession, daily discharges will give adequate definitions for a hydrogreph. More detailed information may be obtained from the Arizona district office of the U.S. Geological Survey in Tucson.

The stages and associated discharges should not be used in the preparation of a stage-discharge relation because, for many stations, the relation used to compute the discharge was shifted from the basic rating.

For many stations, the figures of daily mean discharge differ slightly from those that would be obtained from the tables of dissharge at indicated times in this report because the daily discharges were computed from additional data or were computed from mean c'ischarges 
for different increments of time. Unless otherwise noted, the daily mean discharges are those published in the annual streamflow reports of the U.S. Geological Survey (1967a, 1967b).

Records for several stations in the Gila River basin are not included in this report because (1) at many of the stations no flow was recorded during the period, and (2) at other stations the peak did not contribute to flooding in the Gila River and its tributaries either because of the small magnitude of the flow or because of the tim? when the peak occurred. Records for stations not included in this report are in the annual streamflow reports (U.S. Geological Survey, 1967a, 1967b).

The stations are numbered and arranged in downstream order from headwaters to mouth; stations on tributaries are inserted in corresponding order following the order in which the tributaries enter the main stream. The number preceding the permanent station number is the same as that used on plate 1 and will aid in identifying the site. 


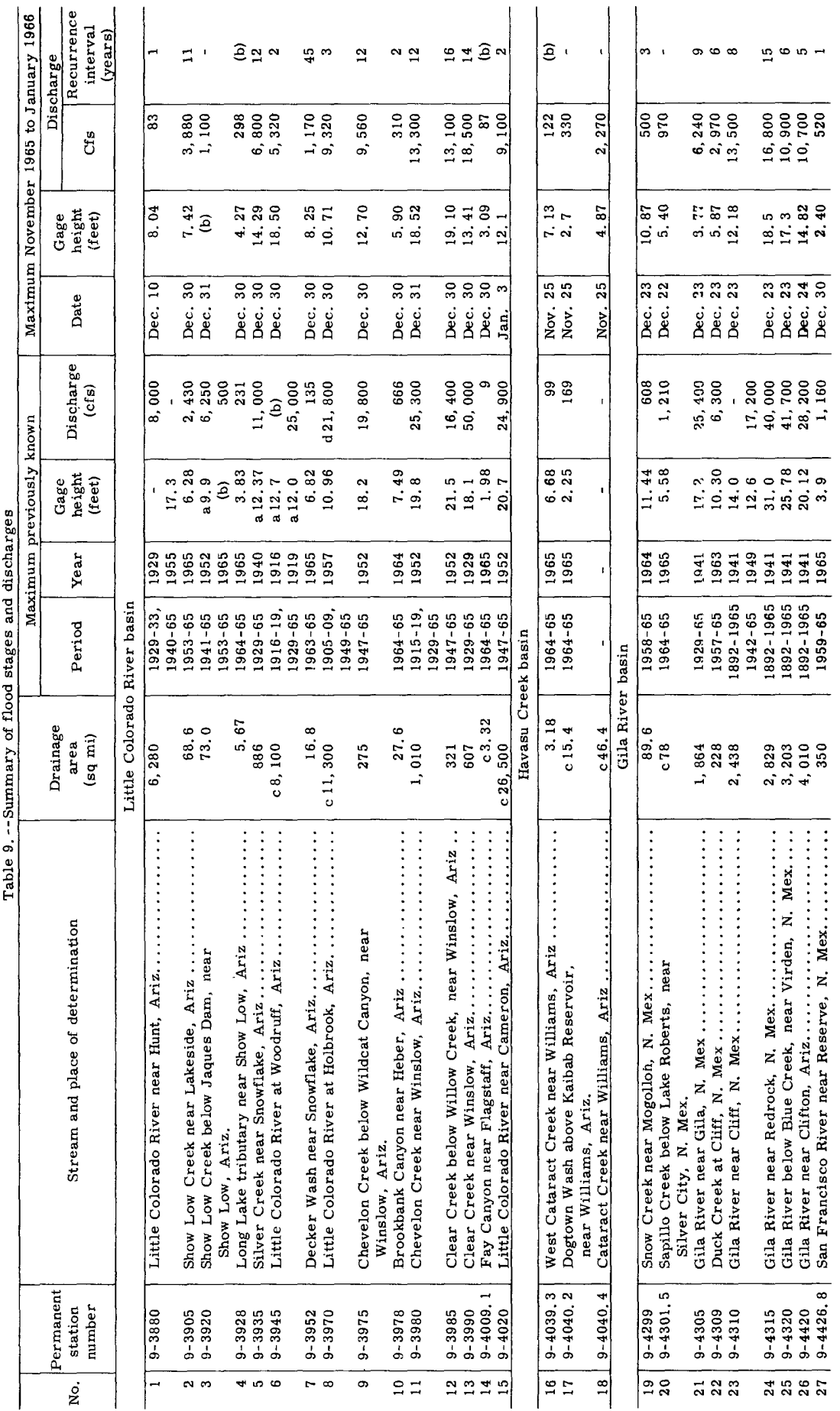


ー

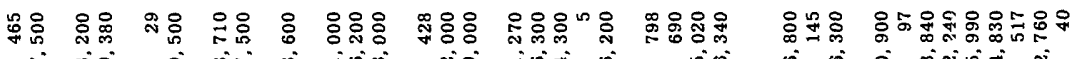

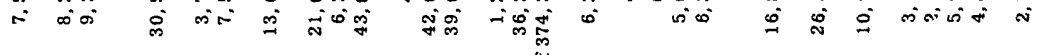

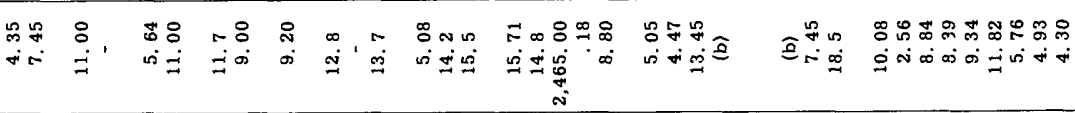

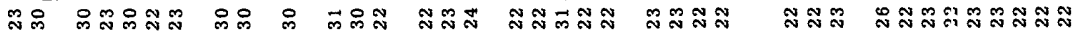

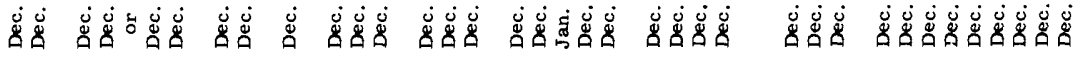

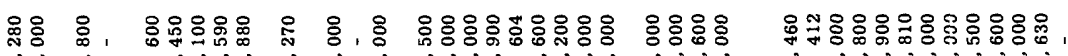

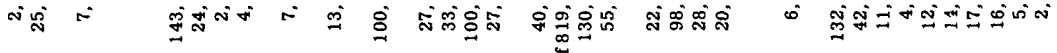

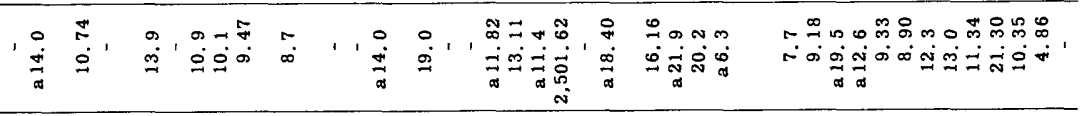

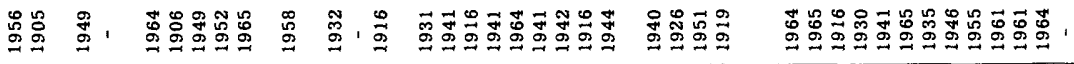

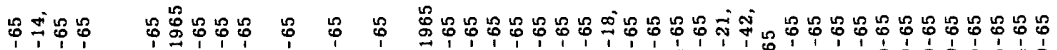

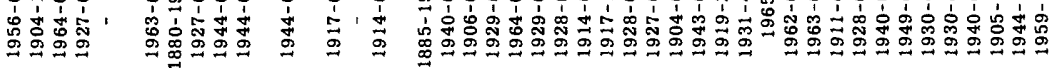

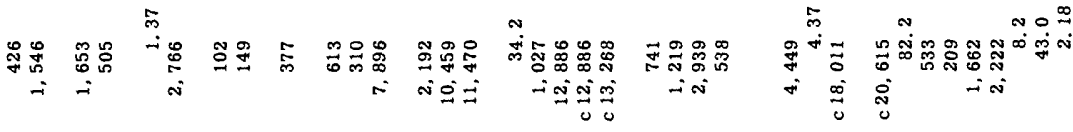

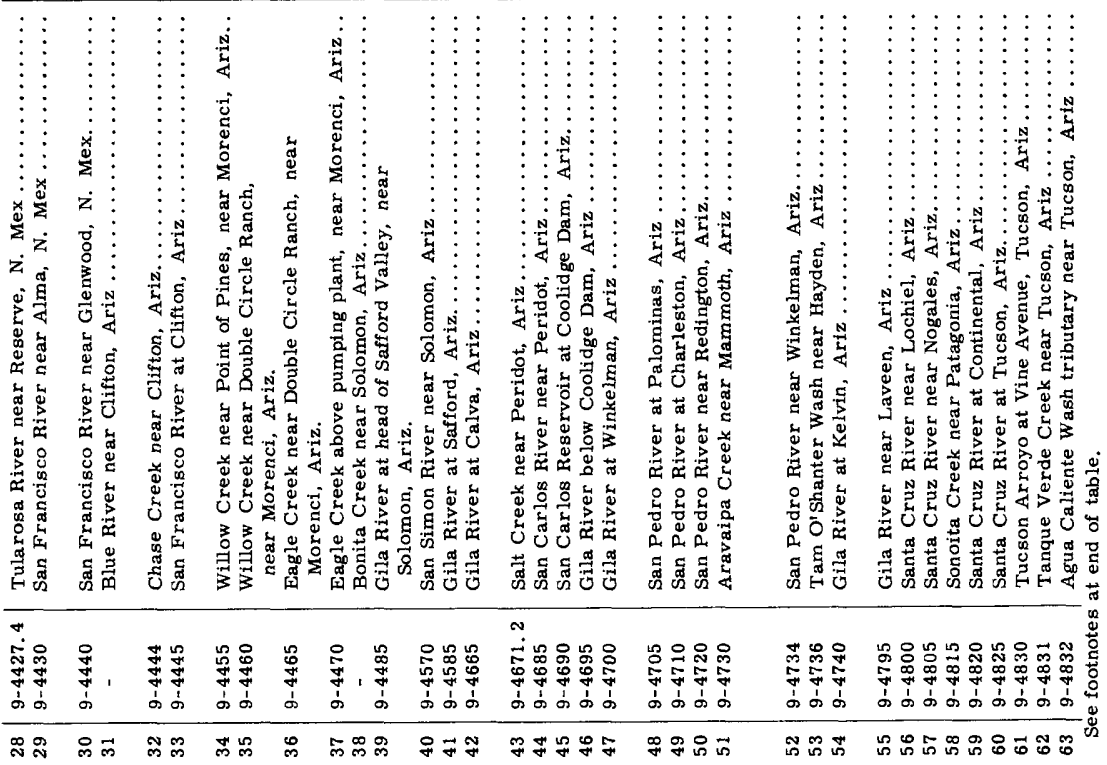









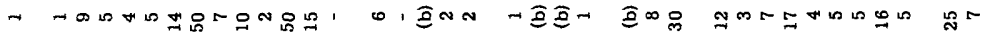

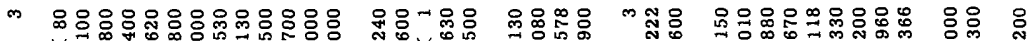

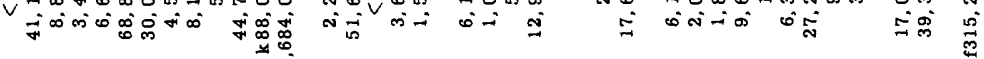

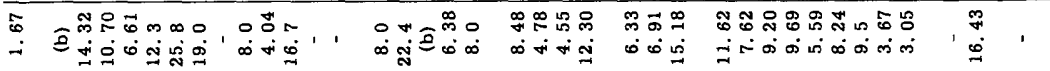

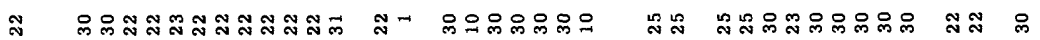

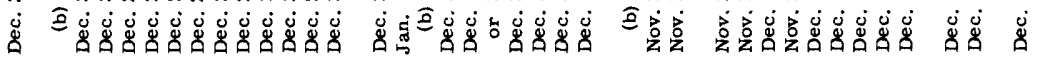

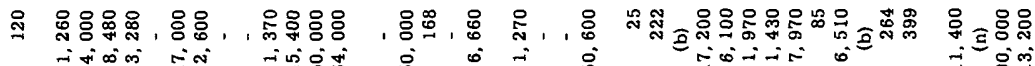

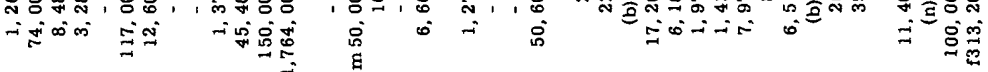

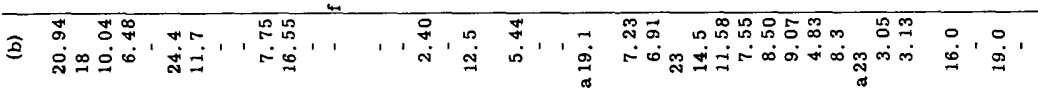

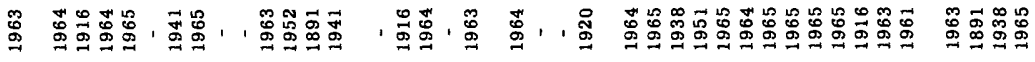

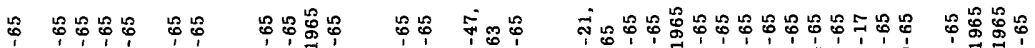

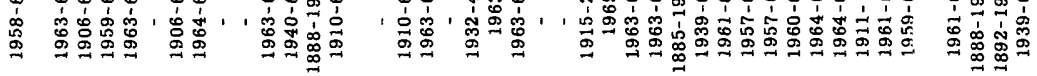

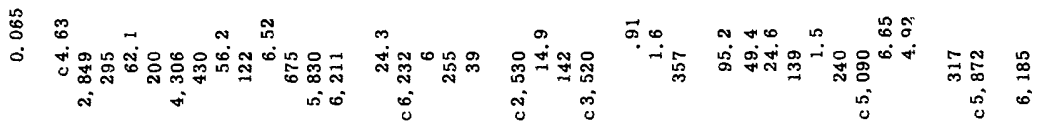

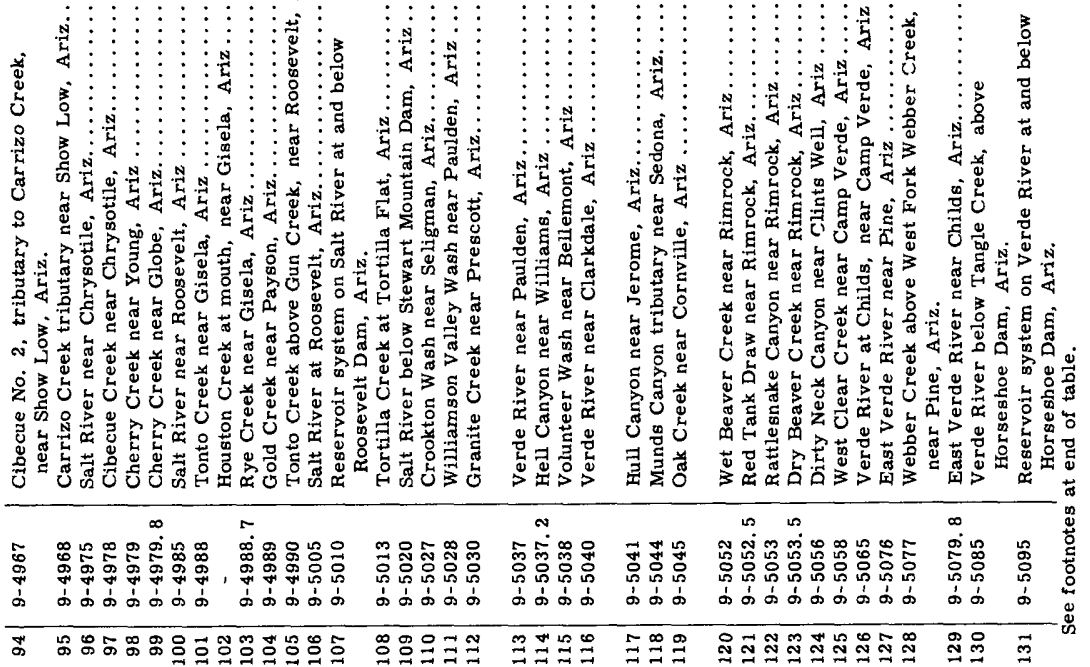




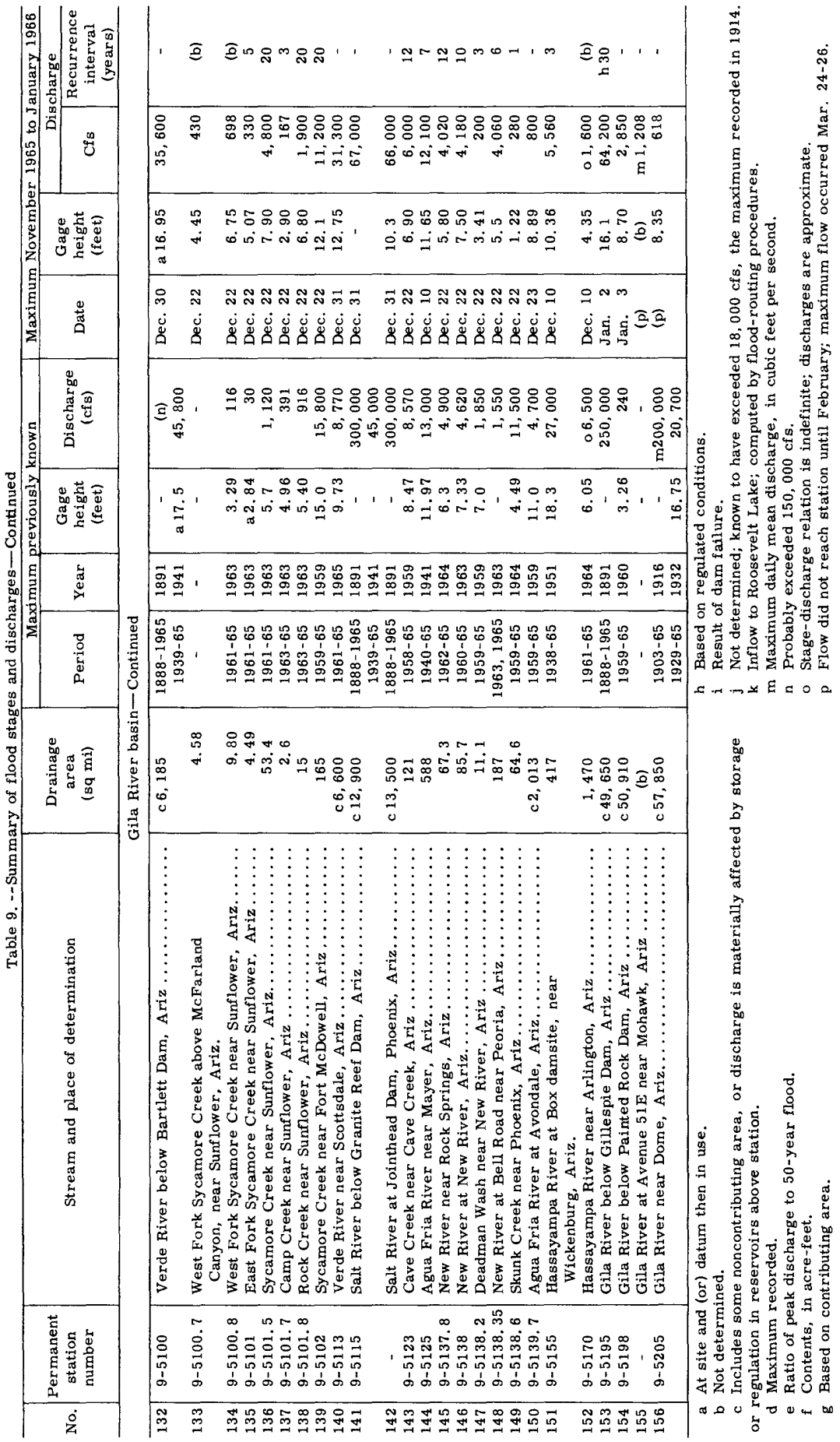




\section{STATION DATA}

\section{LITTLE COLORADO RIVER BASIN}

(1) 9-3880. Little Colorado River near Hunt, Ariz.

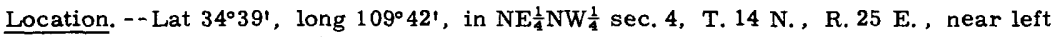
bank at U.S. Highway 180, 2 miles downstream from Zuni River, and 5 miles northwest of Hunt.

Drainage area. - -6,280 sq mi, approximately (about 2, $100 \mathrm{sq} \mathrm{mi}$, including $790 \mathrm{sq} \mathrm{mi}$ above Lyman Reservoir on Little Colorado River and 1, $320 \mathrm{sq} \mathrm{mi} \mathrm{above} \mathrm{Iribbel} \mathrm{Lake}$ and Blanes Lake in Carrizo Creek basin, is noncontributing, except duriro years of high runoff).

Gage-height record. --Water-stage recorder graph except Dec. 20-22, Jan. 3-19, 22-31 when float was frozen. Datum of gage is $5,371.59 \mathrm{ft}$ above mean sea leve', datum of 1929.

Discharge record. --Stage-discharge relation defined by current-meter measurements. Backwater from ice Nov. 28-30, Dec. 19, 23-29, Jan. 20-21. Discharge during periods of no record and backwater estimated on basis of records for nearby stations.

Maxima. --November 1965 to January 1966: Discharge, 83 cfs 0730 hours Lec. 10 (gage height, $8.04 \mathrm{ft}$ ).

1929-33, 1940 to October 1965: Discharge, 8,000 cf s July 28, 1929 (gage height, 16. $8 \mathrm{ft}$ ); gage height, 17. $3 \mathrm{ft}$ Aug. 8, 1955.

Remarks. -- Considerable regulation by many reservoirs (combined capacity about 59, 000 acre-ft). Minor peaks may be affected by storage in Zion Reservoir (capacity, 760 acre-ft in 1952) located on Little Colorado River 19 miles upstream from gaging station.

Mean discharge, in cubic feet per second, 1965-66

\begin{tabular}{|c|c|c|c|c|c|c|c|}
\hline Day & November & December & January & Day & November & December & January \\
\hline 1 & 3.2 & 7.9 & 7.9 & $16 \ldots$ & 4.5 & 9.6 & 6.0 \\
\hline $2 \ldots$ & 3.2 & 7.6 & 10 & $17 \ldots$ & 4.8 & 8.7 & 6.0 \\
\hline $3 \ldots$ & 4.5 & 6.8 & 10 & $18 \ldots$ & 5.2 & 9.0 & 6.0 \\
\hline $4 \ldots$ & 5.2 & 6.8 & 10 & $19 \ldots$ & 6.0 & 11 & 6.0 \\
\hline $5 \ldots$ & 5.0 & 6.8 & 9.0 & $20 \ldots$ & 6.2 & 10 & 6.5 \\
\hline $6 \ldots$ & 5.2 & 6.8 & .9 .0 & $21 \ldots$ & 6.2 & 10 & 6.0 \\
\hline $7 \ldots$ & 5.2 & 6.8 & 9.0 & $22 \ldots$ & 6.5 & 10 & 5.0 \\
\hline $8 \ldots$ & 4. 8 & 6.8 & 9.0 & $23 \ldots$ & 7.9 & 10 & 5.0 \\
\hline $9 \ldots$ & 4. 1 & 7. 6 & 8.0 & $24 \ldots$ & 9.0 & 7. 9 & 5.0 \\
\hline $10 \ldots$ & 3.4 & 35 & 8.0 & $25 \ldots$ & 8.7 & 7.5 & 6.0 \\
\hline $11 \ldots$ & 3. 0 & 26 & 8.0 & $26 \ldots$ & 11 & 8.0 & 6.0 \\
\hline $12 \ldots$ & 3.9 & 11 & 8.0 & $27 \ldots$ & 9.0 & 8.0 & 6.0 \\
\hline $13 \ldots$ & 4. 1 & 9.0 & 7.0 & $28 \ldots$ & 7.0 & 7.9 & 6.0 \\
\hline 14. & 4. 3 & 10 & 7.0 & $29 \ldots$ & 7.0 & 8.7 & 7. 0 \\
\hline $15 \ldots$ & 4. 8 & 9.5 & 7.0 & $30 \ldots$ & 4. 1 & 8.4 & 7. 0 \\
\hline & & & & $31 \ldots$ & $\ldots \ldots$ & 7.9 & 7.0 \\
\hline \multicolumn{5}{|c|}{ Monthly mean discharge, in cubic feet per second. . } & 5.57 & 9.90 & 7.21 \\
\hline \multicolumn{5}{|c|}{ Runoff, in acre-feet $\ldots \ldots \ldots \ldots \ldots \ldots \ldots \ldots$} & 331 & 609 & 443 \\
\hline
\end{tabular}


(2) 9-3905. Show Low Creek near Lakeside, Ariz.

Location. -- Lat $34^{\circ} 10^{\prime} 50^{\prime \prime}$, long $109^{\circ} 59^{\prime} 10^{\prime \prime}$, in NW $\frac{1}{4}$ sec. 14, T. 9 N. , R. 22 E., 1 mile upstream from pumping plant on Show Low Lake, $1 \frac{3}{4}$ miles northwest of I akeside, $2 \frac{1}{4}$ miles upstream from Jaques Dam, and $5 \frac{3}{4}$ miles southeast of Show Low.

Drainage area. $--68.6 \mathrm{sq} \mathrm{mi}$.

Gage-height record. --Water-stage recorder graph, except Dec. 25-29. Altitude of gage is $6,610 \mathrm{ft}$ (from topographic map).

Discharge record. --Stage-discharge relation defined by current-meter measurements. Slight backwater from ice Jan. 27-29. Discharge during period of no gag^-height record estimated.

Maxima. --November 1965 to January 1966: Discharge, 3, 880 cfs 1530 hours Dec. 30 (gage height, $7.42 \mathrm{ft}$ ).

1953 to October 1965: Discharge, 2,430 cfs Jan. 7, 1965 (gage height, 6. $28 \mathrm{ft}$ ); gage height, 6. $40 \mathrm{ft}$ Mar. 23, 1954.

Mean discharge, in cubic feet per second, 1965-66

\begin{tabular}{|c|c|c|c|c|c|c|c|}
\hline Day & November & December & January & Day & November & December & January \\
\hline $1 \ldots$ & 3.5 & 1.1 & 198 & 16. & 0.4 & 8.6 & 3.3 \\
\hline $2 \ldots$ & 3.5 & 1.0 & 88 & 17. & .6 & 7. 9 & 4. 0 \\
\hline $3 \ldots$ & 3.5 & .8 & 53 & $18 \ldots$ & .6 & 7.4 & 4. 8 \\
\hline $4 \ldots$ & 3.6 & .8 & 42 & $19 \ldots$ & .7 & 6.1 & 4. 9 \\
\hline $5 \ldots$ & 3.7 & .7 & 35 & $20 \ldots$ & .7 & 5. 6 & 6. 1 \\
\hline $6 \ldots$ & 4.0 & .6 & 28 & $21 \ldots$ & .6 & 5 & 6.6 \\
\hline $7 \ldots$ & 3.7 & .7 & 26 & $22 \ldots$ & .7 & 79 & 6.3 \\
\hline $8 \ldots$ & 3.6 & .6 & 25 & $23 \ldots$ & 1.0 & 134 & 7. 0 \\
\hline $9 \ldots$ & 3.6 & 1.2 & 24 & $24 \ldots$ & 1.2 & 50 & 7. 2 \\
\hline $10 \ldots$ & 3.6 & 96 & 24 & $25 \ldots$ & 3.0 & 25 & 7. 2 \\
\hline $11 \ldots$ & 7.2 & 50 & 18 & $26 \ldots$ & 7.5 & 20 & 6. 8 \\
\hline $12 \ldots:$ & 4.6 & 19 & 7.2 & $27 \ldots$ & 3.4 & 20 & 3 \\
\hline $13 \ldots$ & 1. 7 & 14 & 5.3 & $28 \ldots$ & 1.8 & 20 & 2 \\
\hline $14 \ldots$ & .7 & 11 & 4. 0 & $29 \ldots$ & 1.2 & 56 & 3 \\
\hline \multirow[t]{2}{*}{$15 \ldots$} & .4 & 11 & 3.6 & $30 \ldots$ & 1.1 & 2,140 & 11 \\
\hline & & & & $31 \ldots$ &.---- & 744 & 13 \\
\hline \multirow{2}{*}{\multicolumn{5}{|c|}{$\begin{array}{l}\text { Monthly mean discharge, in cubic feet per second. } \\
\text { Runoff, in acre-feet } \ldots \ldots \ldots \ldots \ldots \ldots \ldots \ldots \ldots \ldots\end{array}$}} & 2.51 & 114 & 21.8 \\
\hline & & & & & 150 & 7,020 & 1,340 \\
\hline
\end{tabular}

(3) 9-3920. Show Low Creek below Jaques Dam, near Show Low, Ariz.

Location. -- Lat $34^{\circ} 11^{\prime} 40^{\prime \prime}$, long $110^{\circ} 00^{\prime} 15^{\prime \prime}$, in NW $\frac{1}{4}$ sec. 10, T. 9 N. , R. 22 E. , on right bank just downstream from Jaques Dam, $3 \frac{1}{2}$ miles nor thwest of Lakeside, and $4 \frac{1}{2}$ miles southeast of Show Low.

Drainage area. $--73.0 \mathrm{sq} \mathrm{mi}$.

Gage-height record. --Water-stage recorder graph supplemented by once-daily staffgage readings at Jaques Dam during periods of spill from Show Low Lake. Altitude of gage is $6,530 \mathrm{ft}$ (from topographic map).

Discharge record. --Stage-discharge relation defined by current-meter measurements. Relation affected by backwater Dec. 30 to Jan. 31 during period of flow through spillway that enters a quarter of a mile downstream from gage; discharge during this period computed from head on spillway and records of inflow to Show Low Lake.

Maxima. --November 1965 to January 1966: Discharge, 1, 100 cfs 0300 hours Dec. 31. 1941 to October 1965: Discharge, $6,250 \mathrm{cfs}$ Jan. 18, 1952 (gage height, $9.9 \mathrm{ft}$, at site 5 miles downstream at Show Low, at datum then in use).

1953 to October 1965: Discharge, 500 cfs Apr. 12, 1965.

Remarks. --Flow regulated by Show Low Lake since 1953. 
Mean discharge, in cubic feet per second, 1965-66, of Show Low Creek below Jaques

\begin{tabular}{|c|c|c|c|c|c|c|c|}
\hline Day & November & December & January & Day & November & December & January \\
\hline 1 & 1.2 & 0.7 & 260 & $16 \ldots$ & 0.6 & 0.4 & 3.0 \\
\hline 2 & .9 & .5 & 110 & $17 \ldots$ & .6 & .4 & 3.5 \\
\hline 3 & .7 & .4 & 60 & $18 \ldots$ & .6 & .4 & 4 \\
\hline 4 & .7 & .4 & 45 & $19 \ldots$ & .6 & .4 & 4. 5 \\
\hline 5 & .6 & .4 & 35 & $20 \ldots$ & .7 & .4 & 5.5 \\
\hline 6 & .6 & .4 & 27 & $21 \ldots$ & .7 & .4 & 6 \\
\hline 7 & .6 & .4 & 25 & $22 \ldots$ & .7 & .4 & 5.5 \\
\hline 8 & .6 & .4 & 23 & $23 \ldots$ & .7 & .4 & 6.5 \\
\hline 9 & .6 & .4 & 22 & $24 \ldots$ & .7 & .4 & 6 \\
\hline 10 & .6 & .4 & 22 & $25 \ldots$ & .7 & .4 & 6 \\
\hline 11 & .6 & .4 & 20 & $26 \ldots$ & .7 & .4 & 5.5 \\
\hline 12 & .6 & .4 & 7 & $27 \ldots$ & .7 & .2 & 2 \\
\hline 13 & .6 & .4 & 5 & $\mid 28 \ldots$ & .7 & 0 & 1 \\
\hline 14 & .6 & .4 & 3.5 & $29 \ldots$ & .7 & 0 & 2 \\
\hline 15 & .6 & .4 & 3.0 & $30 \ldots$ & .7 & 15 & 9.5 \\
\hline & & & & $31 \ldots$ & $\ldots . .$. & 740 & 12 \\
\hline \multicolumn{5}{|c|}{ Monthly mean discharge, in cubic feet per second. . } & 0.67 & 24. 7 & 24.2 \\
\hline
\end{tabular}

(4) 9-3928. Long Lake tributary near Show Low, Ariz.

\section{(Crest-stage station)}

Location. - Lat $34^{\circ} 15^{\prime} 40^{\prime \prime}$, long $109^{\circ} 59^{\prime} 45^{\prime \prime}$, in SE⿺ sec. 15, T. 10 N., R. 22 E., at U.S. Highway 60, 1 mile east of Show Low.

Drainage area. $--5.67 \mathrm{sq} \mathrm{mi}$.

Gage-height record. --Crest stages only. Altitude of gage is $6,400 \mathrm{ft}$ (from topographic map).

Discharge record. --Stage-discharge relation defined by computations of flow through culvert.

Maxima. --November 1965 to January 1966: Discharge, $298 \mathrm{cfs}$ Dec. 30 (gage height, $4.27 \mathrm{ft}$ ).

1964 to October 1965: Discharge, 231 cfs Jan. 7, 1965 (gage height, 3. $83 \mathrm{ft}$ ). 
(5) 9-3935. Silver Creek near Snowflake, Ariz.

Location. -- Lat $34^{\circ} 40^{\prime} 00^{\prime \prime}$, long $110^{\circ} 02^{\prime} 30^{\prime \prime}$, in SW $\frac{1}{4} \mathrm{NW} \frac{1}{4}$ sec. 29, T. 15 N., R. 22 E., on left bank 6 miles upstream from mouth, and 11 miles north of Snowflake.

Drainage area. $--886 \mathrm{sq} \mathrm{mi}$.

Gage-height record. - - Water-stage recorder graph except Jan. 2-11. Datum of gage is $5,204.1 \mathrm{ft}$ above mean sea level, datum of 1929 .

Discharge record. --Stage-discharge relation defined by current-meter measurements below 4,400 cfs and extended above on basis of peak discharge during flcod of Jan. 19, 1952, at former station near Woodruff. Discharge for period of no gage-height record estimated.

Maxima. --November 1965 to January 1966: Discharge, 6, 800 cfs 1330 houns Dec. 30 (gage height, $14.29 \mathrm{ft}$ ).

1929 to October 1965: Discharge, 11,000 cfs July 26, 1940 (gage height, $12.37 \mathrm{ft}$, at site 9 miles downstream near Woodruff at datum then in use).

\begin{tabular}{|c|c|c|c|c|c|c|c|}
\hline Day & November & December & January & Day & November & December & January \\
\hline $1 \ldots$ & 0.7 & 4.2 & $\overline{314}$ & 16 & 2.5 & 3.7 & 43 \\
\hline $2 \ldots$ & .7 & 4. 6 & 200 & $17 \ldots$ & 2.4 & 2. 9 & 42 \\
\hline $3 \ldots$ & 1.0 & 3. 3 & 80 & $18 \ldots$ & 2.2 & 2. 9 & 43 \\
\hline 4 & 1.1 & 3.1 & 50 & 19 & 2.7 & 2. 9 & 44 \\
\hline & 1. 2 & 4. 6 & 50 & $20 \ldots$ & 2.5 & 2.4 & 42 \\
\hline & .9 & 3.9 & 50 & $\| 21 \ldots \ldots$ & 3.1 & 2.2 & 39 \\
\hline 7 & .8 & 2.5 & 50 & 22 & 3.3 & 2.4 & 31 \\
\hline 8 & .8 & 2.2 & 50 & 23 & 4.6 & 154 & 34 \\
\hline & .9 & 2.4 & 50 & 24 & 3.7 & 135 & 41 \\
\hline 10 . & .8 & 3.1 & 50 & 25 & 3.9 & 75 & 39 \\
\hline $11 \ldots$ & .7 & 167 & 50 & 26 . & 3.5 & 59 & 38 \\
\hline 12. & 1. 4 & 47 & 48 & 27 & 4.2 & 49 & 23 \\
\hline $13 \ldots$ & 2.9 & 26 & 46 & $28 \ldots$ & 3.9 & 39 & 23 \\
\hline $14 \ldots$ & 3.1 & 14 & 45 & $29 \ldots$ & 3.5 & 39 & 22 \\
\hline \multirow[t]{2}{*}{15} & 2.9 & 8.9 & 44 & $30 \ldots$ & 4.4 & 2,820 & 23 \\
\hline & & & & 31. & $\ldots-\ldots$ & 1,010 & 21 \\
\hline \multirow{2}{*}{\multicolumn{5}{|c|}{$\begin{array}{l}\text { Monthly mean discharge, in cubic feet per second. } \\
\text { Runoff, in acre-feet } \ldots \ldots \ldots \ldots \ldots \ldots \ldots \ldots \ldots \ldots\end{array}$}} & 2.34 & 151 & 55.6 \\
\hline & & & & & 139 & 9,310 & 3,420 \\
\hline
\end{tabular}


(6) 9-3945. Little Colorado River at Woodruff, Ariz.

Location. - Lat $34^{\circ} 47^{\prime}$, long $110^{\circ} 03^{\prime}$, in $\mathrm{NE}_{\frac{1}{4}} \mathrm{SW} \frac{1}{4}$ sec. 17, T. 16 N., R. $22 \mathrm{E}$, , on left bank at county bridge in Woodruff, $3 \frac{3}{4}$ miles downstream from Silver Creek.

Drainage area. $--8,100 \mathrm{sq} \mathrm{mi}$, approximately, of which about $2,100 \mathrm{sq} \mathrm{mi}$ is noncontributing except during years of high runoff.

Gage-height record. --Water-stage recorder graph except Dec. 10-14, 23-28, Jan. 1-12. Datum of gage is $5,130.3 \mathrm{ft}$ above mean sea level, datum of 1929 .

Discharge record. --Stage-discharge relation defined by current-meter meas'rements. Stage-discharge relation affected by ice Jan. 24-27. Discharge for period's of no gage-height record estimated.

Maxima. --November 1965 to January 1966: Discharge, 5, 320 cfs 1830 hours Dec. 30 (gage height, $18.50 \mathrm{ft}$ ).

1916-19, 1929 to October 1965: Discharge, 25, 000 cfs Dec. 5, 1919 (gage height, 12. $0 \mathrm{ft}$, at site $1 \frac{1}{2}$ miles downstream at different datum), from rating curve extended above $2,000 \mathrm{cfs}$.

Flood of Jan. 19, 1916, reached a stage of $12.7 \mathrm{ft}$ at site $1 \frac{1}{2}$ miles dow 1 stream.

Remarks. -- Considerable regulation by reservoirs above station (combined capacity about 73, 000 acre-ft).

\begin{tabular}{|c|c|c|c|c|c|c|c|}
\hline Day & November & December & January & Day & November & December & January \\
\hline$\overline{1}$ & 5.1 & 11 & 350 & 16 & 5.5 & 21 & 53 \\
\hline 2. & 4. 6 & 11 & 150 & 17 & 5.5 & 16 & 53 \\
\hline 3 & 5.1 & 11 & 100 & 18. & 5.5 & 17 & 55 \\
\hline 4 & 5.5 & 11 & 80 & 19. & 5.1 & 14 & 59 \\
\hline 5 & 5.5 & 10 & 80 & 20 & 5.1 & 9.9 & 55 \\
\hline 6. & 6.8 & 10 & 80 & 21. & 5.5 & 10 & 48 \\
\hline $7 \ldots$ & 6.8 & 10 & 70 & 22. & 5.5 & 13 & 43 \\
\hline $8 \ldots$ & 6.8 & 9.9 & 70 & $23 \ldots$ & 6.0 & 82 & 44 \\
\hline $9 \ldots$ & 6.8 & 9.9 & 70 & $24 \ldots$ & 6.4 & 150 & 40 \\
\hline $10 \ldots$ & 6.8 & 127 & 60 & $25 \ldots$ & 7.7 & 100 & 35 \\
\hline $11 \ldots$ & 6.4 & 150 & 60 & $26 \ldots$ & 9.2 & 70 & 37 \\
\hline $12 \ldots$ & 5.5 & 50 & 61 & $27 \ldots$ & 12 & 60 & 30 \\
\hline $13 \ldots$ & 5.1 & 40 & 53 & $28 \ldots$ & 14 & 50 & 33 \\
\hline $14 \ldots$ & 5.1 & 34 & 52 & 29. & 14 & 49 & 31 \\
\hline \multirow[t]{2}{*}{$15 \ldots$} & 5.5 & 25 & 52 & 30. & 11 & 1,980 & 32 \\
\hline & & & & 31 & $\ldots-\ldots$ & 1,430 & 34 \\
\hline \multirow{2}{*}{\multicolumn{5}{|c|}{$\begin{array}{l}\text { Monthly mean discharge, in cubic feet per second. } \\
\text { Runoff, in acre }- \text { feet } \ldots \ldots \ldots \ldots \ldots \ldots \ldots \ldots \ldots \ldots\end{array}$}} & 6.85 & 148 & 66.8 \\
\hline & & & & & 407 & 9,110 & 4,110 \\
\hline
\end{tabular}


(7) 9-3952. Decker Wash near Snowflake, Ariz.

\section{(Crest-stage station)}

Location. -- Lat $34^{\circ} 27^{\prime} 40^{\prime \prime}$, long $110^{\circ} 24^{\prime} 15^{\prime \prime}$, in SW $\frac{1}{4}$ sec. 2, T. 12 N., R. 18 E. , at State Highway 277, 19 miles west of Snowflake.

Drainage area. $--16.8 \mathrm{sq} \mathrm{mi}$.

Gage-height record. --Crest stages only. Altitude of gage is $6,300 \mathrm{ft}$ (from topographic map).

Discharge record. --Stage-discharge relation defined by computations of $f$.'ow through culvert.

Maxima. --November 1965 to January 1966: Discharge, 1,170 cfs Dec. 3C (gage height, $8.25 \mathrm{ft}$ ).

1963 to October 1965: Discharge, 135 cfs Jan. 7, 1965 (gage height, 6. $82 \mathrm{ft}$ ).

(8) 9-3970, Little Colorado River at Holbrook, Ariz.

Location. -- Lat $34^{\circ} 53^{\prime} 50^{\prime \prime}$, long $110^{\circ} 09^{\prime} 40^{\prime \prime}$, in SW $\frac{1}{4} \mathrm{SW} \frac{1}{4}$ sec. 6, T. 17 N., R. 21 E., near right bank on downstream side of bridge on U.S. Highway 180 at Holbronk, $2 \frac{1}{2}$ miles downstream from Puerco River.

Drainage area. $--11,300 \mathrm{sq} \mathrm{mi}$, approximately.

Gage-height record. - -Water-stage recorder graph except Nov. 25 to Dec. 10, Dec. 1319 , Dec. 31 to Jan. 11, Jan. 25-31. Datum of gage is $5,062.87 \mathrm{ft}$ above mean sea level, datum of 1929 .

Discharge record. --Stage-discharge relation defined by current-meter measurements made in 1964. Shift adjustments during flood period based on current-meter measurements below $67 \mathrm{cfs}$. Discharge for periods of no gage-height record estimated.

Maxima. - November 1965 to January 1966: Discharge, 9,320 cfs 1200 hours Dec. 30 (gage height, 10.71 ft).

1905-9, 1949 to October 1965: Discharge, 21, 800 cfs Aug. 5, 1957 (gage height, 10. $96 \mathrm{ft}$ ).

Remarks. - - Some regulation by reservoirs above station (combined capacity about 73,000 acre-ft).

Mean discharge, in cubic feet per second, 1965-66

\begin{tabular}{|c|c|c|c|c|c|c|c|}
\hline Day & November & December & January & Day & November & Decembor & January \\
\hline 1 & 4.0 & 40 & 1,000 & $16 \ldots$ & 5.7 & 21 & 97 \\
\hline 2 & 3.4 & 30 & 400 & $17 \ldots$ & 6.4 & 8.6 & 100 \\
\hline 3 & 3. 0 & 20 & 250 & $18 \ldots$ & 7.1 & 7.8 & 81 \\
\hline 4 & 2.6 & 10 & 150 & $19 \ldots$ & 6.4 & 2.6 & 68 \\
\hline 5 & 2.2 & 10 & 150 & $20 \ldots$ & 7.1 & 0 & 84 \\
\hline 6 & 1.8 & 10 & 90 & $21 \ldots$ & 7.1 & 0 & 87 \\
\hline 7 & 1. 8 & 5 & 85 & $22 \ldots \ldots$ & 7.8 & 1,740 & 78 \\
\hline 8 & 4 & 5 & 80 & $23 \ldots$ & 10 & 2,100 & 66 \\
\hline 9 & 5 & 5 & 75 & $24 \ldots \ldots$ & 36 & 690 & 61 \\
\hline 10 & 7.1 & 2,680 & 70 & $25 \ldots$ & 61 & 423 & 60 \\
\hline 11 & 5.7 & 788 & 74 & 26. & 108 & 278 & 60 \\
\hline 12 & 5.1 & 297 & 90 & 27. & 56 & 169 & 60 \\
\hline 13 & 3.4 & 151 & 94 & 28. & 100 & 74 & 50 \\
\hline 14. & 5.7 & 54 & 87 & 29. & 56 & 63 & 50 \\
\hline \multirow[t]{2}{*}{$15 \ldots$} & 7.1 & 17 & 94 & $30 \ldots$ & 78 & 4,840 & 50 \\
\hline & & & & $31 \ldots$ & $\ldots .$. & 1,860 & 40 \\
\hline \multirow{2}{*}{\multicolumn{5}{|c|}{ Monthly mean discharge, in cubic feet per second. }} & 20.5 & 529 & 125 \\
\hline \multicolumn{4}{|c|}{ Runoff, in acre-feet $\ldots \ldots \ldots \ldots \ldots \ldots \ldots$} & & 1,220 & 32,530 & 7,700 \\
\hline
\end{tabular}


(9) 9-3975. Chevelon Creek below Wildcat Canyon, near Winslow, Ariz.

Location. -- Lat $34^{\circ} 38^{\prime}$, long $110^{\circ} 43^{\prime}$, in SW $\frac{1}{4}$ sec. 36, T. 15 N. , R. 15 E. , on right bank three-eighths of a mile downstream from Wildcat Canyon, and 25 miles sorth of Winslow.

\section{Drainage area. $--275 \mathrm{sq} \mathrm{mi}$.}

Gage-height record. - -Water-stage recorder graph except 1200 hours Dec. 31 to Jan. 6. Gage height reconstructed 1200-2400 hours Dec. 31 . Datum of gage is 5,925.16 ft above mean sea level, datum of 1929 .

Discharge record. --Stage-discharge relation defined by current-meter measi rements below $6,300 \mathrm{cfs}$ and extended above on basis of slope-area measurement at $18.2 \mathrm{ft}$. Discharge for period of no gage-height record estimated.

Maxima. - -November 1965 to January 1966: Discharge, 9,560 cfs 1500 hours Dec. 30 (gage height, $12.70 \mathrm{ft}$ ).

1947 to October 1965: Discharge, $19,800 \mathrm{cfs}$ Jan. 18, 1952 (gage height. $18.2 \mathrm{ft}$ ).

Mean discharge, in cubic feet per second, 1965-66

\begin{tabular}{|c|c|c|c|c|c|c|c|}
\hline Day & November & December & January & Day & November & December & January \\
\hline 1 & 0 & 84 & 500 & 16. & 0 & 87 & 29 \\
\hline$\ldots$ & 0 & 77 & 200 & 17 & 0 & 86 & 29 \\
\hline$\ldots$ & 0 & 72 & 150 & 18 & 0 & 76 & 28 \\
\hline $4 \ldots$ & 0 & 72 & 120 & 19 & 0 & 66 & 23 \\
\hline $5 \ldots$ & 0 & 72 & 100 & 20 & 0 & 59 & 20 \\
\hline 6 & 0 & 71 & 90 & 21. & 0 & 65 & 18 \\
\hline 7 & 0 & 70 & 84 & 22 & 0 & 77 & 14 \\
\hline 8 & 0 & 70 & 76 & $23 \ldots$ & 0 & 81 & 14 \\
\hline 9 & 0 & 72 & 75 & 24 . & 0 & 84 & 11 \\
\hline 10. & 0 & 202 & 70 & $25 \ldots$ & 589 & 84 & 11 \\
\hline $11 \ldots$ & 0 & 206 & 68 & $26 \ldots$ & 351 & 81 & 12 \\
\hline $12 \ldots$ & 0 & 237 & 35 & $27 \ldots$ & 168 & 80 & 18 \\
\hline $13 \ldots$ & 0 & 201 & 24 & $28 \ldots$ & 121 & 77 & 31 \\
\hline $14 \ldots \ldots$ & 0 & 143 & 22 & $29 \ldots$ & 96 & 77 & 30 \\
\hline $15 \ldots$ & 0 & 114 & 26 & 30. & 86 & 4,280 & 30 \\
\hline & & & & 31 & $\ldots \ldots$ & 2,800 & 31 \\
\hline \multirow{2}{*}{\multicolumn{5}{|c|}{$\begin{array}{l}\text { Monthly mean discharge, in cubic feet per second. } \\
\text { Runoff, in acre-feet } \ldots \ldots \ldots \ldots \ldots \ldots \ldots \ldots \ldots\end{array}$}} & 47.0 & 320 & 64.2 \\
\hline & & & & & 2,800 & 19,680 & 3,950 \\
\hline
\end{tabular}

(10) 9-3978. Brookbank Canyon near Heber, Ariz.

(Crest-stage station)

Location. -- Lat $34^{\circ} 28^{\prime} 20^{\prime \prime}$, long $110^{\circ} 38^{\prime} 50^{\prime \prime}$, in $\mathrm{SE} \frac{1}{4}$ sec. 33, T. 13 N. , R. 16 F., at HeberWinslow road, 4. 0 miles northwest of Heber.

Drainage area. $-27.6 \mathrm{sq} \mathrm{mi}$.

Gage-height record. --Crest stages only. Altitude of gage is 6,600 ft (from topographic map).

Discharge record. --Stage-discharge relation defined by computations of flow through culverts.

Maxima. --November 1965 to January 1966: Discharge, 310 cfs Dec. 30 (gage height, $5.90 \mathrm{ft}$ ).

1964 to October 1965: Discharge, $666 \mathrm{cfs}$ Aug. 1964 (gage height, 7.49 ft). 
(11) 9-3980. Chevelon Creek near Winslow, Ariz.

Location. - - Lat $34^{\circ} 56^{\prime}$, long $110^{\circ} 31^{\prime}$, in SE $\frac{1}{4} \mathrm{SW} \frac{1}{4}$ sec. 27, T. 18 N., R. 17 E., on right bank 3 miles upstream from mouth, and 12 miles southeast of Winslow.

Drainage area. $--1,010 \mathrm{sq} \mathrm{mi}$, approximately, including some area which is probably noncontributing.

Gage-height record. - -Water-stage recorder graph except Jan. 3-4. Datum of gage is $4,899.5 \mathrm{ft}$ above mean sea level, datum of 1929 .

Discharge record. --Stage-discharge relation defined by current-meter measurements below 2,700 cfs and extended above on basis of slope-area measurement at $19.8 \mathrm{ft}$. Discharge for period of no gage-height record interpolated from adjoining record.

Maxima. --November 1965 to January 1966: Discharge, 13, 300 cfs 0030 h jurs Dec. 31 (gage height, $18.52 \mathrm{ft}$ ).

1915-19, 1929 to October 1965: Discharge, 25, 300 cfs Jan. 19, 1952 (gage height, $19.8 \mathrm{ft})$.

Mean discharge, in cubic feet per second, 1965-66

\begin{tabular}{|c|c|c|c|c|c|c|c|}
\hline Day & November & December & January & Day & November & Decembor & January \\
\hline 1 & 6.6 & 72 & 1,320 & 16 & 6.6 & 116 & 35 \\
\hline 2 & 6.6 & 65 & 592 & 17 & 6.6 & 97 & 38 \\
\hline 3 & 6.6 & 60 & 390 & 18 & 6.6 & 83 & 43 \\
\hline 4 & 6.6 & 58 & 270 & 19 & 6.6 & 78 & 44 \\
\hline 5 & 6.6 & 57 & 218 & 20 & 6.6 & 70 & 43 \\
\hline 6 & 6.6 & 56 & 175 & 21. & 6.6 & 58 & 36 \\
\hline 7 & 6.6 & 53 & 145 & $22 \ldots$ & 6. 0 & 69 & 29 \\
\hline $8 \ldots$ & 6. 6 & 53 & 127 & $23 \ldots$ & 6.3 & 70 & 29 \\
\hline $9 \ldots$ & 6.6 & 55 & 113 & $24 \ldots$ & 7.6 & 81 & 28 \\
\hline $10 \ldots$ & 6.6 & 58 & 108 & $25 \ldots$ & 6. 0 & 76 & 25 \\
\hline $11 \ldots$ & 6.6 & 138 & 102 & 26. & 74 & 81 & 22 \\
\hline $12 \ldots$ & 6.6 & 216 & 97 & 27. & 254 & 78 & 19 \\
\hline 13 & 6.6 & 229 & 81 & 28. & 155 & 77 & 19 \\
\hline 14 & 6.6 & 195 & 52 & 29 & 113 & 63 & 18 \\
\hline 15 . & 6.6 & 148 & 45 & 30. & 87 & 1,407 & 19 \\
\hline & & & & 31. & $\ldots \ldots$ & 6,750 & 36 \\
\hline \multirow{2}{*}{\multicolumn{5}{|c|}{$\begin{array}{l}\text { Monthly mean discharge, in cubic feet per second. } \\
\text { Runoff, in acre-feet } \ldots \ldots \ldots \ldots \ldots \ldots \ldots \ldots \ldots\end{array}$}} & 28.2 & 34.7 & 139 \\
\hline & & & & & 1,680 & 21,350 & 8,560 \\
\hline
\end{tabular}


(12) 9-3985. Clear Creek below Willow Creek, near Winslow, Ariz.

Location. --Lat $34^{\circ} 40^{\prime}$, long $111^{\circ} 00^{\prime}$, in NW $\frac{1}{4} \mathrm{NE} \frac{1}{4}$ sec. 30 , T. 15 N., R. 13 E., in Sitgreaves National Forest, on right bank 2 miles downstream from Willow Creek, and 30 miles southwest of Winslow.

Drainage area. $--321 \mathrm{sq} \mathrm{mi}$.

Gage-height record. --Water-stage recorder graph except Dec, 24-29, Jan. 3-27 when float was frozen. Altitude of gage is $6,000 \mathrm{ft}$ (from Forest Service map).

Discharge record. --Stage-discharge relation defined by current-meter meas'vrements below $6,000 \mathrm{cfs}$ and extended above by logarithmic plotting. Discharge for periods of no gage-height record estimated.

Maxima. - - November 1965 to January 1966: Discharge, 13, 100 cfs 1200 hours Dec, 30 (gage height, $19.10 \mathrm{ft}$ ).

1947 to October 1965: Discharge, 16, $400 \mathrm{cfs}$ Jan. 18, 1952 (gage height, 21. $5 \mathrm{ft}$ ).

Remarks. - -Some effect from storage in Blue Ridge Reservoir, which spilled during flood of Dec. 30, 1965.

Mean discharge, in cubic feet per second, 1965-66

\begin{tabular}{|c|c|c|c|c|c|c|c|}
\hline Day & November & December & January & Day & November & December & January \\
\hline $1 \ldots$ & 0 & 66 & 1,310 & $16 \ldots$ & 0 & 85 & 60 \\
\hline $2 \ldots$ & 0 & 52 & 680 & $17 \ldots$ & 0 & 90 & 50 \\
\hline$\ldots$ & 0 & 39 & 500 & $18 \ldots$ & 0 & 65 & 50 \\
\hline $4 \ldots$ & 0 & 32 & 350 & $19 \ldots$ & 0 & 50 & 40 \\
\hline 5 & 0 & 24 & 300 & $20 \ldots$ & 0 & 50 & 40 \\
\hline 6 & 0 & 20 & 250 & $21 \ldots$ & 0 & 55 & 30 \\
\hline $7 \ldots$ & 0 & 16 & 200 & 22. & 0 & 80 & 30 \\
\hline 8. & 0 & 14 & 150 & $23 \ldots$ & 0 & 80 & 25 \\
\hline 9 & 0 & 14 & 100 & 24. & 63 & 120 & 25 \\
\hline $10 \ldots$ & 0 & 684 & 90 & 25. & 2,390 & 115 & 20 \\
\hline $11 \ldots$ & 0 & 648 & 80 & $26 \ldots$ & 1,290 & 115 & 20 \\
\hline $12 \ldots$ & 0 & 352 & 80 & $27 \ldots$ & 425 & 105 & 20 \\
\hline $13 \ldots$ & 0 & 241 & 70 & $28 \ldots$ & 214 & 95 & 20 \\
\hline $14 \ldots$ & 0 & 184 & 70 & $29 \ldots$ & 126 & 95 & 15 \\
\hline \multirow[t]{2}{*}{$15 \ldots$} & 0 & 135 & 60 & $30 \ldots$ & 89 & 7,150 & 15 \\
\hline & & & & 31. & $\cdots \cdots-$ & 2,970 & 15 \\
\hline \multirow{2}{*}{\multicolumn{5}{|c|}{$\begin{array}{l}\text { Monthly mean discharge, in cubic feet per second. } \\
\text { Runoff in acre-feet }\end{array}$}} & 153 & 446 & 154 \\
\hline & & & & & 9,120 & 27,450 & 9,450 \\
\hline
\end{tabular}


(13) 9-3990. Clear Creek near Winslow, Ariz.

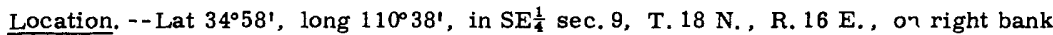
$1 \frac{1}{2}$ miles upstream from mouth, and 5 miles southeast of Winslow.

Drainage area. $--607 \mathrm{sq} \mathrm{mi}$.

Gage-height record. --Water-stage recorder graph except Jan. 2-4. Dat $\mathrm{m}$ of gage is $4,861,32 \mathrm{ft}$ above mean sea level, datum of 1929 .

Discharge record. --Stage-discharge relation defined by current-meter measurements below 13,500 cfs and extended above on basis of velocity-area studies, verified by slope-area measurement at gage height $13.4 \mathrm{ft}$. Discharge for period of no gageheight record computed on basis of normal recession.

Maxima. - -November 1965 to January 1966: Discharge, 18, 500 cfs 2330 hours Dec. 30 (gage height, $13.41 \mathrm{ft}$ ).

1929 to October 1965: Discharge, 50, $000 \mathrm{cfs}$ Apr. 4, 1929 (gage height, $18.1 \mathrm{ft}$ ). An earlier flood reached a stage $3 \mathrm{ft}$ higher than that of Apr. 4, 1969, at a site $1,850 \mathrm{ft}$ downstream.

Mean discharge, in cubic feet per second, 1965-66

\begin{tabular}{|c|c|c|c|c|c|c|c|}
\hline Day & November & December & January & Day & November & Decemher & January \\
\hline 1 & 3.5 & 88 & 2,400 & 16 & 0 & 166 & 62 \\
\hline $2 \ldots$ & 3.5 & 58 & 1,180 & 17 & 0 & 132 & 62 \\
\hline $3 \ldots$ & 3.5 & 44 & 650 & 18. & 0 & 111 & 58 \\
\hline $4 \ldots$ & 3.5 & 30 & 427 & $19 \ldots$ & 0 & 111 & 48 \\
\hline $5 \ldots$ & 3.5 & 26 & 366 & 20. & 0 & 77 & 44 \\
\hline $6 \ldots$ & 4.2 & 20 & 309 & 21. & 0 & 71 & 34 \\
\hline $7 \ldots$ & 4.2 & 19 & 232 & 22. & 0 & 65 & 26 \\
\hline $8 \ldots$ & 3.5 & 15 & 201 & 23 & 0 & 77 & 28 \\
\hline 9 & 3.5 & 13 & 166 & 24 & 0 & 94 & 22 \\
\hline 10 & 2.6 & 11 & 149 & 25 & 0 & 106 & 19 \\
\hline 11 & 1.3 & 300 & 117 & 26 & 1,570 & 123 & 17 \\
\hline 12 & .7 & 584 & 111 & 27 & 978 & 117 & 20 \\
\hline 13 & .4 & 402 & 100 & 28 & 455 & 111 & 22 \\
\hline 14 & .2 & 309 & 71 & 29 & 265 & 106 & 20 \\
\hline \multirow[t]{2}{*}{$15 \ldots$} & 0 & 221 & 65 & 30 & 149 & 1,600 & 19 \\
\hline & & & & $31 \ldots$ & $\ldots .$. & 8,620 & 17 \\
\hline \multirow{2}{*}{\multicolumn{5}{|c|}{ Monthly mean discharge, in cubic feet per second. }} & 115 & 446 & 228 \\
\hline & & & & & 6,850 & 27,430 & 14,010 \\
\hline
\end{tabular}

(14) 9-4009. 1. Fay Canyon near Flagstaff, Ariz.

(Crest-stage station)

Location. - - Lat $35^{\circ} 08^{\prime} 06^{\prime \prime}$, long $11^{\circ} 37^{\prime} 48^{\prime \prime}$, in NW $\frac{1}{4} N W \frac{1}{4}$ sec. 11, T. 20 N., R. 7 E. , at Lake Mary Road within the city limits of Flagstaff.

Drainage area. $--3.32 \mathrm{sq} \mathrm{mi}$, of which $0.56 \mathrm{sq}$ mi above Kellam Tank may be noncontributing.

Gage-height record. --Crest stages only. Altitude of gage is $6,850 \mathrm{ft}$ (from topographic map).

Discharge record. --Stage-discharge relation defined by computations of flow through culvert.

Maxima. --November 1965 to January 1966: Discharge, 87 cfs Dec. 30 (gage height, $3.09 \mathrm{ft}$ ).

1964 to October 1965: Discharge, $9 \mathrm{cfs}$ Sept. 18, 1965 (gage height, 1. $98 \mathrm{ft}$ ). 
(15) 9-4020. Little Colorado River near Cameron, Ariz.

Location. --Lat $35^{\circ} 55^{\prime} 35^{\prime \prime}$, long $111^{\circ} 34^{\prime} 00^{\prime \prime}$, in NW $\frac{1}{4}$ sec. 5 , T. 29 N. , R. 8 E. (unsurveyed), in Navajo Indian Reservation, on left bank 3 miles downstream from Coconino damsite, $9 \frac{1}{2}$ miles downstream from Moenkopi Wash, $9 \frac{1}{2}$ miles northwest of Cameron, and 45.5 miles upstream from mouth.

Drainage area. $--26,500 \mathrm{sq} \mathrm{mi}$, approximately.

Gage-height record. --Water-stage recorder graph except Dec. 15-24, 27-31, Jan. 4-5, 21-31. Datum of gage is 3,979. $2 \mathrm{ft}$ above mean sea level, datum of 1929 .

Discharge record. --Stage-discharge relation defined by current-meter measirements. Discharge for periods of no gage-height record estimated.

Maxima. - November 1965 to January 1966: Discharge, 9, 100 cfs 1300 hours Jan. 3 (gage height, $12.1 \mathrm{ft}$ ).

1947 to October 1965: Discharge, 24, 900 cfs Jan. 21, 1952 (gage height, $20.7 \mathrm{ft}$ ).

Mean discharge, in cubic feet per second, 1965-66

\begin{tabular}{|c|c|c|c|c|c|c|c|}
\hline Day & November & December & January & Day & November & December & January \\
\hline 1 & 0.5 & 262 & 2,670 & 16 & 1.4 & 590 & 216 \\
\hline $2 \ldots$ & .5 & 183 & 4,620 & 17. & 1.8 & 450 & 171 \\
\hline $3 \ldots$ & .6 & 143 & 8,290 & 18 & 2. 2 & 350 & 165 \\
\hline $4 \ldots$ & 4 & 110 & 4,430 & 19. & 2.4 & 300 & 162 \\
\hline $5 \ldots$ & .1 & 91 & 1,570 & 20. & 2.4 & 200 & 141 \\
\hline $6 \ldots$ & .2 & 75 & 912 & 21. & 2.2 & 150 & 135 \\
\hline $7 \ldots$ & .1 & 65 & 746 & 22 & 2.2 & 140 & 110 \\
\hline $8 \ldots$ & 6 & 56 & 608 & $23 \ldots$ & 2.8 & 130 & 110 \\
\hline $9 \ldots$ & .7 & 53 & 510 & $24 \ldots$ & 2.6 & 912 & 100 \\
\hline $10 \ldots$ & .8 & 270 & 415 & $25 \ldots$ & 5.0 & 1,460 & 95 \\
\hline $11 \ldots$ & .6 & 69 & 360 & $26 \ldots$ & 8.6 & 550 & 90 \\
\hline $12 \ldots$ & .8 & 1,440 & 324 & 27 . & 34 & 350 & 90 \\
\hline $13 \ldots$ & 1.0 & 1,480 & 279 & 28. & 839 & 300 & 85 \\
\hline $14 \ldots$ & 1.0 & 800 & 254 & 29. & 674 & 275 & 85 \\
\hline \multirow[t]{2}{*}{$15 \ldots$} & 1.0 & 620 & 238 & 30. & 385 & 250 & 80 \\
\hline & & & & 31. & $\ldots--\cdot$ & 225 & 80 \\
\hline \multirow{2}{*}{\multicolumn{5}{|c|}{$\begin{array}{l}\text { Monthly mean discharge, in cubic feet per second. } \\
\text { Runoff, in acre-feet } \ldots \ldots \ldots \ldots \ldots \ldots \ldots \ldots \ldots \ldots\end{array}$}} & 65.8 & 398 & 908 \\
\hline & & & & & 3,920 & 24,490 & 55,820 \\
\hline
\end{tabular}

\section{HAVASU CREEK BASIN}

(16) 9-4039.3. West Cataract Creek near Williams, Ariz.

(Crest-stage station)

Location. -- Lat $35^{\circ} 14^{\prime} 52^{\prime \prime}$, long $112^{\circ} 13^{\prime} 2^{\prime \prime}$, in NW $\frac{1}{4}$ sec. 31, T. 22 N., R. 2 E., at Country Club Road a quarter of a mile upstream from Cataract Lake, and 1.2 miles west of Williams city limits.

Drainage area. $--3.18 \mathrm{sq} \mathrm{mi}$.

Gage-height record. --Crest stages only. Altitude of gage is 6, $820 \mathrm{ft}$ (from topographic map).

Discharge record. --Stage-discharge relation defined by computations of flow through culvert and over road.

Maxima. --November 1965 to January 1966: Discharge, 122 cfs Nov. 25 (gag? height, 7. $13 \mathrm{ft}$ ).

1964 to October 1965: Discharge, $99 \mathrm{cfs}$ April 1965 (gage height, $6.68 \mathrm{ft}$ ). 
(17) 9-4040.2. Dogtown Wash above Kaibab Reservoir, near Williams, Ariz.

Location. - Lat $35^{\circ} 17^{\prime} 10^{\prime \prime}$, long $112^{\circ} 08^{\prime} 35^{\prime \prime}$, in $\mathrm{NE} \frac{1}{4} \mathrm{SE} \frac{1}{4} \mathrm{sec}, 14, \mathrm{~T}, 22 \mathrm{~N} ., \mathrm{R} .2 \mathrm{E}$, , in Kaibab National Forest, on left bank 1.1 miles upstream from dam on Kaibab Reservoir, 0.05 mile east of State Highway 64, and $3 \frac{1}{2}$ miles northeast of Williams.

Drainage area. $--15.4 \mathrm{sq} \mathrm{mi}$.

Gage-height record. --Digital recorder tape except Nov. 26 to Dec. 9, Dec. 13-24, Dec. 31 to Jan. 1 .

Discharge record. --Stage-discharge relation defined by current-meter measurements below $60 \mathrm{cfs}$ and extended above on basis of slope-area measurement of peak flow. Discharge for periods of no gage-height record estimated.

Maxima. - - November 1965 to January 1966: Discharge, 330 cfs 1400 hours Nov. 25 (gage height, $2.7 \mathrm{ft}$, from outside high-water marks).

1964 to October 1965: Discharge, 169 cfs Apr. 16, 1965 (gage height. $2.25 \mathrm{ft}$ ).

Remarks. --Flow partially regulated by Dogtown Reservoir (capacity 1, 100 acre-ft), which spilled during flood periods.

\begin{tabular}{|c|c|c|c|c|c|c|c|}
\hline Day & November & December & January & Day & November & December & January \\
\hline $1 \ldots$ & 0 & 0 & 10 & $16 \ldots$ & 0 & 0 & 0 \\
\hline $2 \ldots$ & 0 & 0 & 5.0 & $17 \ldots$ & 0 & 0 & 0 \\
\hline $3 \ldots$ & 0 & 0 & 0 & $18 \ldots$ & 0 & 0 & 0 \\
\hline $4 \ldots$ & 0 & 0 & 0 & $19 \ldots$ & 0 & 0 & 0 \\
\hline $5 \ldots$ & 0 & 0 & 0 & $20 \ldots$ & 0 & 0 & 0 \\
\hline $6 \ldots$ & 0 & 0 & 0 & $21 \ldots$ & 0 & 0 & 0 \\
\hline $7 \ldots$ & 0 & 0 & 0 & $22 \ldots$ & 0 & 0 & 0 \\
\hline $8 \ldots$ & 0 & 0 & 0 & $23 \ldots$ & 16 & 0 & 0 \\
\hline $9 \ldots$ & 0 & 8. 0 & 0 & $24 \ldots \ldots$ & 16 & 0 & 0 \\
\hline $10 \ldots$ & 0 & 96 & 0 & $25 \ldots$ & 172 & 0 & 0 \\
\hline $11 \ldots$ & 0 & 37 & 0 & $26 \ldots$ & 24 & 0 & 0 \\
\hline $12 \ldots$ & 0 & 20 & 0 & $27 \ldots$ & 5.0 & 0 & 0 \\
\hline $13 \ldots$ & 0 & 5.0 & 0 & $28 \ldots$ & 0 & 0 & 0 \\
\hline $14 \ldots$ & 0 & 2. 0 & 0 & $29 \ldots$ & 0 & 0 & 0 \\
\hline \multirow[t]{2}{*}{$15 \ldots$} & 0 & 1.0 & 0 & $30 \ldots$ & 0 & 120 & 0 \\
\hline & & & & 31. & $\ldots$ & 20 & 0 \\
\hline \multirow{2}{*}{\multicolumn{5}{|c|}{$\begin{array}{l}\text { Monthly mean discharge, in cubic feet per second. } \\
\text { Runoff, in acre-feet } \ldots \ldots \ldots \ldots \ldots \ldots \ldots \ldots \ldots \ldots \ldots\end{array}$}} & 7.77 & 9.97 & 0.484 \\
\hline & & & & & 462 & 613 & 30 \\
\hline
\end{tabular}


(18) 9-4040.4. Cataract Creek near Williams, Ariz.

Location. -- Lat $35^{\circ} 18^{\prime} 54^{\prime \prime}$, long $112^{\circ} 10^{\prime} 42^{\prime \prime}$, in NE $\frac{1}{4} \mathrm{SE} \frac{1}{4}$ sec. 4, T. 22 N. , R. 2 E. , in Kaibab National Forest, on right abutment of abandoned bridge on county r $>$ ad, 0.3 mile downstream from Dogtown Wash, and $4 \frac{1}{2}$ miles north of Williams.

Drainage area. $--46.4 \mathrm{sq} \mathrm{mi}$.

Gage-height record. - Water-stage recorder graph except Nov, 7-26, Dec. 14-29, Jan. 1-7.

Discharge record. --Stage-discharge relation defined by current-meter measurements below $620 \mathrm{cfs}$ and extended above on basis of slope-area measurement of poak flow. Stage-discharge relation affected by backwater from ice Jan. 8-31. Discharge for periods of no gage-height record estimated.

Maximum. - - November 1965 to January 1966: Discharge, 2, 270 cfs 0700 hours Nov. 25 (gage height, $4.87 \mathrm{ft}$ ).

Remarks. --Flow partially regulated by several reservoirs (combined capacit:x, 2, 750 acre-ft), all of which spilled during flood periods.

No flow from Kaibab Reservoir (drainage area, $16.6 \mathrm{sq} \mathrm{mi}$ ) during flood of Nov. 25, 1965.

Mean discharge, in cubic feet per second, 1965-66

\begin{tabular}{|c|c|c|c|c|c|c|c|}
\hline Day & November & December & January & Day & November & December & January \\
\hline $1 \ldots$ & 0.2 & 3.2 & 65 & $16 \ldots$ & 0.5 & 10 & 1.5 \\
\hline $2 \ldots$ & .4 & 2.2 & 45 & $17 \ldots$ & .5 & 5 & 1.5 \\
\hline $3 \ldots$ & 6 & 1. 1 & 35 & $18 \ldots$ & .5 & 4 & 1. 5 \\
\hline 4 & .8 & .8 & 15 & $19 \ldots$ & .5 & 3 & 1. 5 \\
\hline $5 \ldots$ & 6 & .6 & 8 & $20 \ldots$ & .5 & 2 & 1.5 \\
\hline $6 \ldots$ & .4 & .6 & 8 & $21 \ldots$ & .5 & 2 & 1. 0 \\
\hline $7 \ldots$ & 5.7 & .6 & 7 & $22 \ldots$ & .5 & 10 & 1. 0 \\
\hline & 5 & .2 & 4 & $23 \ldots$ & 1.0 & 80 & 1. 5 \\
\hline & 3 & 36 & 4 & $24 \ldots$ & 25 & 65 & 1. 0 \\
\hline $10 \ldots$ & 2 & 265 & 4 & $25 \ldots$ & 750 & 25 & 1.0 \\
\hline $11 \ldots$ & 1 & 87 & 3 & $26 \ldots$ & 47 & 5 & 1. 0 \\
\hline $12 \ldots$ & .5 & 46 & 2 & $27 \ldots$ & 22 & 4 & 1. 0 \\
\hline $13 \ldots \ldots$ & .5 & 30 & 2 & $28 \ldots$ & 13 & 2 & 1.0 \\
\hline $14 \ldots \ldots$ & 5 & 25 & 2 & $29 \ldots$ & 10 & 30 & .5 \\
\hline $15 \ldots$ & .5 & 20 & 1.5 & $30 \ldots$ & 5.7 & 746 & 1. 0 \\
\hline & & & & $31 \ldots$ &.---- & 179 & 1. 0 \\
\hline \multicolumn{5}{|c|}{$\begin{array}{l}\text { Monthly mean discharge, in cubic feet per second. } \\
\text { Runoff, in acre-feet }\end{array}$} & $\begin{array}{r}30.0 \\
1.780\end{array}$ & $\begin{array}{r}54.5 \\
3.350\end{array}$ & $\begin{array}{r}7.23 \\
444\end{array}$ \\
\hline
\end{tabular}




\section{GILA RIVER BASIN}

(19) 9-4299. Snow Creek near Mogollon, N. Mex.

\section{(Crest-stage station)}

Location. - - Lat $33^{\circ} 24^{\prime} 50^{\prime \prime}$, long $108^{\circ} 29^{\prime} 40^{\prime \prime}, 1,000 \mathrm{ft}$ below Gila Wilderness boundary and 17.5 miles east of Mogollon.

Drainage area. $--89.6 \mathrm{sq} \mathrm{mi}$.

Gage-height record. --Crest stages only. Altitude of gage is 7, $280 \mathrm{ft}$ (from topographic map).

Discharge record. --Stage-discharge relation defined by current-meter measurements below $10 \mathrm{cfs}$ and by slope-area measureinents at 276 and $608 \mathrm{cfs}$.

Maxima. --November 1965 to January 1966: Discharge, 500 cfs Dec. 23 (gage height, $10.87 \mathrm{ft}$ ).

1958 to October 1965: Discharge, $608 \mathrm{cfs}$ Aug. 15, 1964 (gage height, $11.44 \mathrm{ft}$ ).

(20) 9-4301.5. Sapillo Creek below Lake Roberts, near Silver City, N. Mex.

Location. -- Lat $33^{\circ} 01^{\prime} 55^{\prime \prime}$, long $108^{\circ} 10^{\prime} 10^{\prime \prime}$, in SE $\frac{1}{4} \mathrm{SE} \frac{1}{4}$ sec. 34, T. $14 \mathrm{~S}$. , R. $13 \mathrm{~W}$., on left bank $1,400 \mathrm{ft}$ below Lake Roberts Dam, about 1 mile upstream from former mining town of Meerschaum, and 18 miles north of Silver City.

Drainage area. $--78 \mathrm{sq} \mathrm{mi}$.

Gage-height record. --Water-stage recorder graph. Altitude of gage is $5,990 \mathrm{ft}$ (from topographic map).

Discharge record. --Stage-discharge relation defined by current-meter moasurements below $166 \mathrm{cfs}$ and extended above on basis of slope-area measurement of peak flow.

Maxima. --November 1965 to January 1966: Discharge, 970 cfs 1730 hours Dec. 22 (gage height, $5.40 \mathrm{ft}$ ).

1964 to October 1965: Discharge, 1, $210 \mathrm{cfs}$ Sept. 23, 1965 (gage height, $5.58 \mathrm{ft}$ ).

Remarks. --Flow regulated by Lake Roberts.

Mean discharge, in cubic feet per second, 1965-66

\begin{tabular}{|c|c|c|c|c|c|c|c|}
\hline Day & November & December & January & Day & November & December & January \\
\hline 1. & 4.2 & 3.1 & 90 & 16 & 3.5 & 5.0 & 7.0 \\
\hline $2 \ldots$ & 4.2 & 2. 9 & 50 & 17 & 3.5 & 5.4 & 6.2 \\
\hline $3 \ldots$ & 4.2 & 2.8 & 28 & $18 \ldots$ & 3.5 & 5.0 & 7.0 \\
\hline $4 \ldots \ldots$ & 4.1 & 2.8 & 21 & 19 & 3.5 & 4. 4 & 7. 0 \\
\hline $5 \ldots$ & 3.9 & 2.7 & 17 & 20 & 3.5 & 4.2 & 7.0 \\
\hline $6 \ldots$ & 3.9 & 2.6 & 14 & 21 & 3.4 & 4.4 & 7. 0 \\
\hline $7 \ldots$ & 3.9 & 2.6 & 13 & 22 & 3.4 & 248 & 6.2 \\
\hline $8 \ldots$ & 3.9 & 2.6 & 12 & 23 & 3.4 & 276 & 6.2 \\
\hline & 3.9 & 2. 7 & 11 & 24 & 3.2 & 94 & 6.2 \\
\hline 10 . & 3.9 & 111 & 9.2 & 25 & 3.5 & 55 & 6.2 \\
\hline $11 \ldots$ & 3.8 & 21 & 8.5 & 26 & 4.2 & 38 & 6.2 \\
\hline $12 \ldots$ & 3.6 & 7.6 & 8.5 & 27 & 3.8 & 28 & 5.7 \\
\hline $13 \ldots$ & 3.6 & 6.0 & 7.7 & $28 \ldots$ & 3.5 & 23 & 5.7 \\
\hline $14 \ldots$ & 3.5 & 5.4 & 7.0 & $29 \ldots$ & 3.4 & 20 & 5.7 \\
\hline \multirow[t]{2}{*}{$15 \ldots$} & 3.5 & 5.6 & 6.2 & 30 & 3.2 & 107 & 5.2 \\
\hline & & & & 31 & $\ldots \ldots$ & 188 & 5.2 \\
\hline \multicolumn{5}{|c|}{$\begin{array}{l}\text { Monthly mean discharge, in cubic feet per second. } \\
\text { Runoff, in acre-feet }\end{array}$} & 3. 69 & 41.5 & 13.0 \\
\hline
\end{tabular}


(21) 9-4305. Gila River near Gila, N. Mex.

Location. --Lat $33^{\circ} 03^{\prime} 45^{\prime \prime}$, long $108^{\circ} 32^{\prime} 20^{\prime \prime}$, in NWt sec. 30, T. 14 S. , R. 16 W. , on left bank at Hooker damsite, 1 mile upstream from Mogollon Creek, and 7 miles northeast of Gila.

Drainage area. $--1,864$ sq $\mathrm{mi}$.

Gage-height record. - Water-stage recorder graph. Datum of gage is 4,655.8 $\mathrm{ft}$ above mean sea level (river-profile survey).

Discharge record. --Stage-discharge relation defined by current-meter measurements below $3,900 \mathrm{cfs}$ and extended above on basis of velocity-area studies and logarithmic plotting.

Maxima. --November 1965 to January 1966: Discharge, 6, 240 cfs 0200 hours Dec. 23 (gage height, $8.77 \mathrm{ft}$ ).

1929 to October 1965: Discharge, 25,400 cfs Sept. 29, 1941 (gage height., 17. $2 \mathrm{ft}$ at site $200 \mathrm{ft}$ upstream at present datum).

Mean discharge, in cubic feet per second, 1965-66

\begin{tabular}{|c|c|c|c|c|c|c|c|}
\hline Day & November & December & January & Day & November & December & January \\
\hline $1 \ldots$ & 57 & 79 & 1,880 & $16 \ldots$ & 59 & 342 & 184 \\
\hline $2 \ldots$ & 55 & 75 & 1,090 & $17 \ldots$ & 55 & 358 & 178 \\
\hline $3 \ldots$ & 55 & 75 & 716 & $18 \ldots$ & 55 & 350 & 202 \\
\hline $4 \ldots$ & 55 & 77 & 538 & $19 \ldots$ & 57 & 298 & 217 \\
\hline $5 \ldots \ldots$ & 55 & 75 & 450 & $20 \ldots$ & 55 & 259 & 193 \\
\hline $6 \ldots$ & 57 & 72 & 386 & $21 \ldots$ & 55 & 259 & 205 \\
\hline $7 \ldots$ & 59 & 72 & 338 & $22 \ldots$ & 55 & 1,390 & 175 \\
\hline $8 \ldots$ & 57 & 70 & 301 & $23 \ldots$ & 55 & 4,020 & 151 \\
\hline $9 \ldots$ & 55 & 77 & 286 & $24 \ldots$ & 55 & 1,720 & 139 \\
\hline $10 \ldots$ & 55 & 1,410 & 262 & $25 \ldots$ & 55 & 1,060 & 136 \\
\hline 11. & 55 & 1,960 & 247 & 26 & 80 & 788 & 130 \\
\hline $12 \ldots$ & 55 & 900 & 235 & 27 & 90 & 645 & 125 \\
\hline 13 & 55 & 538 & 223 & 28 & 90 & 554 & 133 \\
\hline 14 & 55 & 438 & 214 & 29 & 85 & 498 & 136 \\
\hline \multirow[t]{2}{*}{$15 \ldots$} & 61 & 386 & 199 & $30 \ldots$ & 82 & 855 & 136 \\
\hline & & & & $31 \ldots$ & $\ldots-\ldots$ & 3,000 & 152 \\
\hline \multicolumn{5}{|c|}{$\begin{array}{l}\text { Monthly mean discharge, in cubic feet per second. } \\
\text { Runoff, in acre-feet } \ldots \ldots \ldots \ldots \ldots \ldots \ldots \ldots\end{array}$} & 60.8 & 732 & 321 \\
\hline
\end{tabular}


(22) 9-4309. Duck Creek at Cliff, N. Mex.

\section{(Crest-stage station)}

Location. --Lat $32^{\circ} 57^{\prime} 50^{\prime \prime}$ ', long $108^{\circ} 36^{\prime} 40^{\prime \prime}$, in SW $\frac{1}{4}$ SW $\frac{1}{4}$ sec. 28, T. 15 S., R. 17 W., at Cliff, below bridge on State Highway 211 , and 0.6 mile above mouth.

Drainage area. $-228 \mathrm{sq} \mathrm{mi}$.

Gage-height record. -- Crest stages only. Altitude of gage is 4, $490 \mathrm{ft}$ (from topographic map).

Discharge record. --Stage-discharge relation defined by current-meter moasurements below $3 \mathrm{cfs}$, by 4 slope-area measurements from 2,970 to $5,160 \mathrm{cfs}$, $\varepsilon$ nd by slopeconveyance study.

Maxima. --November 1965 to January 1966: Discharge, 2, 970 cfs Dec. 23 (gage height, $5.87 \mathrm{ft}$ ).

1957 to October 1965: Discharge, 6, $300 \mathrm{cfs}$ Aug. 21, 1963 (gage height, 10.30 ft).

Senate Document 436 (facing p. 71 , site 2) gives a discharge of 10,600 cfs for the flood of Oct. 16, 1916, and $41,800 \mathrm{cfs}$ for an earlier flood.

(23) 9-4310. Gila River near Cliff, N. Mex.

(Gaging station, discontinued 1951; crest-stage station)

Location. -- Lat $32^{\circ} 56^{\prime} 20^{\prime \prime}$, long $108^{\circ} 36^{\prime} 20^{\prime \prime}$, in $\mathrm{S} \frac{1}{2}$ sec. 4, T. 16 S., R. $17 \mathrm{~V}$., on downstream end of bridge pier on U.S. Highway 260, $1 \frac{1}{2}$ miles downstream from Bear Creek, $1 \frac{1}{2}$ miles south of Cliff, and $2 \frac{1}{2}$ miles southwest of Gila.

Drainage area. $--2,438 \mathrm{sq} \mathrm{mi}$.

Gage-height record. --Crest stages only. Datum of gage is $4,454.5 \mathrm{ft}$ above mean sea level, datum of 1929 .

Discharge record. --Stage-discharge relation defined by current-meter m?asurements below 7,140 cfs and by slope-area measurement at $12.6 \mathrm{ft}$.

Maxima. --November 1965 to January 1966: Discharge, 13,500 cfs Dec. 23 (gage height, $12.18 \mathrm{ft}$ ).

1942 to October 1965: Discharge, 17, $200 \mathrm{cfs}$ Jan. 14, 1949 (gage height, $12.6 \mathrm{ft}$ ).

(24) 9-4315. Gila River near Redrock, N. Mex.

Location. - - Lat $32^{\circ} 43^{\prime} 30^{\prime \prime}$ ', long $108^{\circ} 40^{\prime} 30^{\prime \prime}$, in W $\frac{1}{2}$ sec. 23, T. 18 S. , R. 18 W., on left bank 0.2 mile downstream from Copper Canyon, a quarter of a mile upstream from lower end of box canyon, 4.7 miles northeast of Redrock, and 14 miles downstream from Mangas Creek.

Drainage area. $--2,829 \mathrm{sq} \mathrm{mi}$.

Gage-height record. --Water-stage recorder graph. Altitude of gage is 4, $090 \mathrm{ft}$ (from planetable survey).

Discharge record. --Stage-discharge relation defined by current-meter measurements below 2,200 cfs and extended above on basis of logarithmic plotting.

Maxima. - November 1965 to January 1966: Discharge, 16, 800 cfs 0200 hours Dec. 23 (gage height, $18.5 \mathrm{ft}$ ).

Probably 1892 to October 1965: Discharge, about 40,000 cfs Sept. 29, 1941 (gage height, $31 \mathrm{ft}$, from floodmark). Computed on basis of peak flow at Gila River below Blue Creek, near Virden, N. Mex. 
Mean discharge, in cubic feet per second, 1965-66, of Gila River near Redrocl. N. Mex.

\begin{tabular}{|c|c|c|c|c|c|c|c|}
\hline Day & November & December & January & Day & November & December & January \\
\hline $1 \ldots$ & 59 & 98 & 2,880 & $16 \ldots$ & 67 & 525 & 340 \\
\hline $2 \ldots$ & 57 & 96 & 1,480 & $17 \ldots$ & 64 & 590 & 331 \\
\hline $3 \ldots$ & 60 & 90 & 1,120 & $18 \ldots$ & 61 & 626 & 340 \\
\hline $4 \ldots$ & 57 & 87 & 952 & $19 \ldots$ & 60 & 530 & 337 \\
\hline $5 \ldots$ & 57 & 87 & 805 & $20 \ldots$ & 65 & 466 & 316 \\
\hline $6 \ldots$ & 58 & 88 & 715 & $21 \ldots$ & 68 & 470 & 304 \\
\hline 7 & 59 & 84 & 620 & 22 & 72 & 3,130 & 280 \\
\hline 8 & 60 & 86 & 558 & 23 & 73 & 12,400 & 255 \\
\hline 9 & 57 & 88 & 514 & 24 . & 74 & 3,270 & 235 \\
\hline 10. & 57 & 1,410 & 462 & 25 . & 77 & 1,540 & 225 \\
\hline $11 \ldots$ & 59 & 3,320 & 426 & $26 \ldots$ & 106 & 1,260 & 218 \\
\hline $12 \ldots$ & 60 & 1,280 & 402 & $27 \ldots$ & 99 & 1,170 & 208 \\
\hline $13 \ldots$ & 62 & 722 & 378 & $28 \ldots \ldots$ & 97 & 1,090 & 205 \\
\hline $14 \ldots$ & 68 & 614 & 361 & 29. & 98 & 988 & 200 \\
\hline \multirow[t]{2}{*}{$15 \ldots$} & 69 & 565 & 352 & 30 . & 97 & 1,010 & 198 \\
\hline & & & & 31. & $\ldots \ldots$ & 5,360 & 205 \\
\hline \multirow{2}{*}{\multicolumn{5}{|c|}{$\begin{array}{l}\text { Monthly mean discharge, in cubic feet per second. } \\
\text { Runoff, in acre-feet } \ldots \ldots \ldots \ldots \ldots \ldots \ldots \ldots \ldots\end{array}$}} & 69.2 & 1,392 & 523 \\
\hline & & & & & 4,120 & 85,570 & 32,160 \\
\hline
\end{tabular}

(25) 9-4320. Gila River below Blue Creek, near Virden, N, Mex.

Location. - - Lat $32^{\bullet} 38^{\prime} 55^{\prime \prime}$, long $108^{\circ} 50^{\prime} 45^{\prime \prime}$, in $\mathrm{SE} \frac{1}{4} \mathrm{SW} \frac{1}{4}$ sec. 18 , T. $19 \mathrm{~S}$. , R. $19 \mathrm{~W}$., on left bank at head of canyon, $1 \frac{1}{4}$ miles downstream from Blue Creek, 10 miles east of Virden, and 16 miles upstream from New Mexico-Arizona State line.

Drainage area. $--3,203$ sq mi, excluding Animas River basin.

Gage-height record. - Water-stage recorder graph. Altitude of gage is $3,875 \mathrm{ft}$ (from river-profile map).

Discharge record. --Stage-discharge relation defined by current-meter measurements.

Maxima. --November 1965 to January 1966: Discharge, 10, 900 cfs 1000 hours Dec. 23 (gage height, $17.3 \mathrm{ft}$ ).

1892 to October 1965: Discharge, 41, 700 cfs Sept. 29, 1941 (gage height.. 25. $78 \mathrm{ft})$.

Mean discharge, in cubic feet per second, 1965-66

\begin{tabular}{|c|c|c|c|c|c|c|c|}
\hline Day & November & December & January & Day & November & December & January \\
\hline$\ldots$ & 60 & 99 & 3,040 & $16 \ldots$ & 69 & 670 & 334 \\
\hline $2 \ldots$ & 61 & 98 & 1,860 & $17 \ldots$ & 67 & 730 & 324 \\
\hline $3 \ldots$ & 65 & 95 & 1,270 & $18 \ldots$ & 67 & 838 & 320 \\
\hline $4 \ldots$ & 58 & 93 & 998 & $19 \ldots$ & 66 & 688 & 320 \\
\hline $5 \ldots$ & 57 & 93 & 838 & $20 \ldots$ & 66 & 515 & 312 \\
\hline $6 \ldots$ & 58 & 95 & 746 & $21 \ldots$ & 69 & 503 & 308 \\
\hline $7 \ldots$ & 61 & 88 & 662 & $22 \ldots$ & 73 & 2,920 & 302 \\
\hline $8 \ldots$ & 65 & 89 & 590 & $23 \ldots$ & 79 & 8,180 & 288 \\
\hline $9 \ldots$ & 63 & 94 & 527 & $24 \ldots$ & 80 & 4,870 & 276 \\
\hline $10 \ldots$ & 58 & 718 & 472 & $25 \ldots$ & 88 & 1,980 & 266 \\
\hline $11 \ldots$ & 57 & 2,910 & 442 & $26 \ldots$ & 100 & 1,320 & 260 \\
\hline 12 & 58 & 1,640 & 412 & $27 \ldots$ & 120 & 1,080 & 254 \\
\hline 13 & 58 & 842 & 390 & $28 \ldots \ldots$ & 106 & 990 & 248 \\
\hline 14. & 65 & 663 & 368 & $29 \ldots$ & 109 & 938 & 244 \\
\hline \multirow[t]{2}{*}{$15 \ldots$} & 69 & 688 & 352 & $30 \ldots$ & 102 & 906 & 242 \\
\hline & & & & $31 \ldots$ & $\ldots--\cdot-$ & 3,090 & 238 \\
\hline \multirow{2}{*}{\multicolumn{5}{|c|}{ Monthly mean discharge, in cubic feet per second. . }} & 72.5 & 1,243 & 568 \\
\hline & & & & & 4,310 & 76,410 & 34,720 \\
\hline
\end{tabular}


(26) 9-4420. Gila River near Clifton, Ariz.

Location. -- Lat $32^{\circ} 57^{\prime} 50^{\prime \prime}$, long $109^{\circ} 18^{\prime} 15^{\prime \prime}$, in SW $\frac{1}{4}$ SW $\frac{1}{4}$ sec. 30, T. 5 S. , R. 30 E., on left bank 1,100 ft upstream from bridge on county road, 6 miles upstream from San Francisco River, and 7 miles south of Clifton.

Drainage area. $--4,010 \mathrm{sq} \mathrm{mi}$.

Gage-height record. --Water-stage recorder graph except Dec, 26-28, Jan. 7-18, 28-31. Gage-height record reconstructed from normal recession and range in stage. Datum of gage is $3,339.50 \mathrm{ft}$ above mean sea level, datum of 1929 , supplementary adjustment of 1959 .

Discharge record. --Stage-discharge relation defined by current-meter reasurements.

Maxima. --November 1965 to January 1966: Discharge, 10, 700 cfs 0900 hours Dec. 24 (gage height, $14.82 \mathrm{ft}$ ).

1892 to October 1965: Discharge, 28, 200 cfs Sept. 29, 1941 (gage height, $20.12 \mathrm{ft}$, at site $1,100 \mathrm{ft}$ downstream at datum $4.03 \mathrm{ft}$ lower than present datur.

\begin{tabular}{|c|c|c|c|c|c|c|c|}
\hline Day & November & December & January & Day & November & December & January \\
\hline $1 \ldots$ & 20 & 81 & 2,960 & $16 \ldots$ & 35 & 668 & 420 \\
\hline $2 \ldots$ & 33 & 75 & 2,160 & $17 \ldots$ & 38 & 863 & 380 \\
\hline $3 \ldots$ & 34 & 75 & 1,420 & $18 \ldots$ & 40 & 961 & 356 \\
\hline $4 \ldots$ & 34 & 73 & 1,080 & $19 \ldots$ & 39 & 680 & 352 \\
\hline $5 \ldots$ & 35 & 70 & 885 & $20 \ldots$ & 33 & 530 & 342 \\
\hline $6 \ldots$ & 34 & 73 & 718 & $21 \ldots$ & 32 & 490 & 328 \\
\hline $7 \ldots$ & 34 & 72 & 610 & $22 \ldots$ & 33 & 1,340 & 330 \\
\hline 8 & 35 & 58 & 550 & $23 \ldots$ & 39 & 4,050 & 310 \\
\hline 9 & 34 & 60 & 530 & $24 \ldots$ & 44 & 7,170 & 300 \\
\hline 10 . & 32 & 160 & 510 & 25. & 52 & 3,590 & 290 \\
\hline 11. & 31 & 2,180 & 480 & $26 \ldots$ & 55 & 1,800 & 280 \\
\hline 12 & 29 & 2,040 & 470 & $27 \ldots$ & 73 & 1,400 & 280 \\
\hline $13 \ldots$ & 29 & 1,020 & 470 & $28 \ldots$ & 87 & 1,120 & 280 \\
\hline 14 & 29 & 662 & 460 & $29 \ldots$ & 81 & 950 & 260 \\
\hline 15 & 31 & 668 & 450 & $30 \ldots$ & 81 & 890 & 250 \\
\hline & & & & $31 \ldots$ & 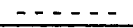 & 1,320 & 247 \\
\hline \multirow{2}{*}{\multicolumn{5}{|c|}{$\begin{array}{l}\text { Monthly mean discharge, in cubic feet per second. } \\
\text { Runoff, in acre-feet } \ldots \ldots \ldots \ldots \ldots \ldots \ldots \ldots \ldots\end{array}$}} & 41.2 & 1,135 & 605 \\
\hline & & & & & 2,450 & 69,800 & 37,210 \\
\hline
\end{tabular}

Gage height, in'feet, and discharge, in cubic feet per second, at indicated time, 1965-66

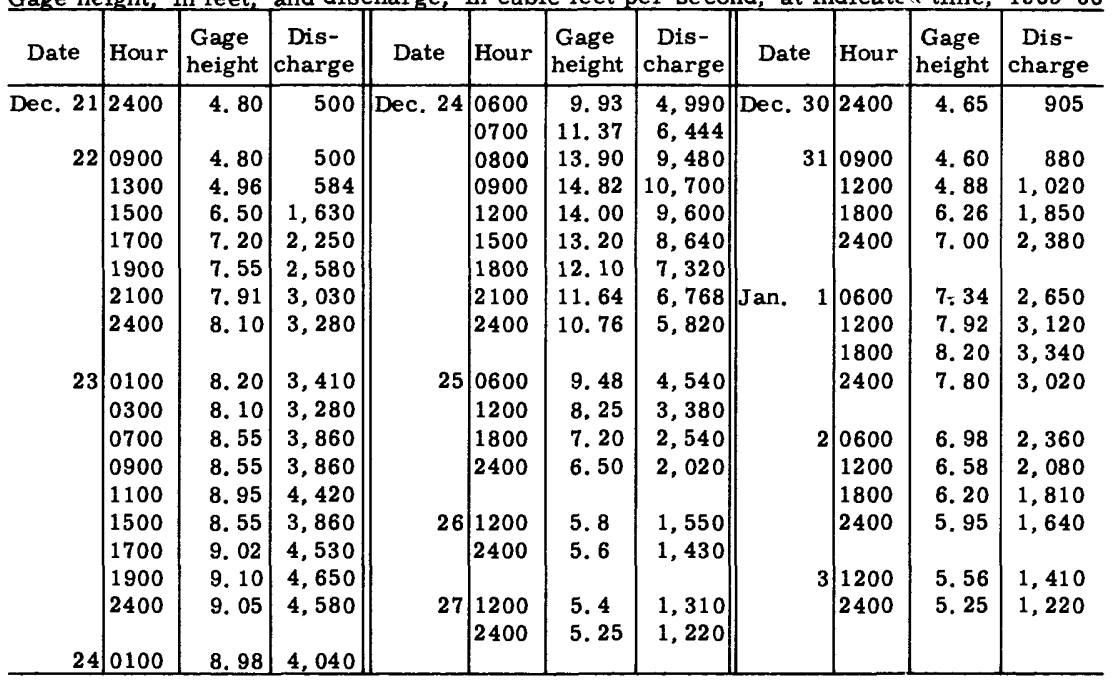


(27) 9-4426. 8. San Francisco River near Reserve, N. Mex.

Location. -- Lat $33^{\circ} 44^{\prime} 30^{\prime \prime}$, long $108^{\circ} 46^{\prime} 15^{\prime \prime}$, in SW $\frac{1}{4} \mathrm{NE} \frac{1}{4}$ Sec. 35, T. 6 S., R. 19 T. , on left bank $500 \mathrm{ft}$ upstream from mouth of Rainbow Bridge Canyon, and 2 miles northwest of Reserve.

Drainage area. $--350 \mathrm{sq} \mathrm{mi}$, approximately.

Gage-height record. - Water-stage recorder graph. Altitude of gage is 5, $850 \mathrm{f}^{\prime}$ (from topographic map).

Discharge record. --Stage-discharge relation defined by current-meter measur ments below $460 \mathrm{cfs}$ and extended above on basis of slope-area measurements at ga ge heights 2.92 and $3.05 \mathrm{ft}$.

Maxima. --November 1965 to January 1966: Discharge, 520 cfs 1000 hours Dec. 30 (gage height, 2.40 ft).

1959 to October 1965: Discharge, $1,160 \mathrm{cfs}$ July 26, 1965, estimated (gare height, $3.9 \mathrm{ft}$, inside, $4.9 \mathrm{ft}$, from floodmarks).

Old floodmarks, found in 1962 , show a stage of $15 \mathrm{ft}$; date of flood unknown. According to Senate Document 436 (p. 85, site 194), the flood of Oct. 16, 1916 (discharge, $17,850 \mathrm{cfs}$, at site about 6 miles upstream) is the maximum known.

Mean discharge, in cubic feet per second, 1965-66

\begin{tabular}{|c|c|c|c|c|c|c|c|}
\hline Day & November & December & January & Day & November & December & January \\
\hline $1 \ldots$ & 6.0 & 6.5 & 248 & 16 & 6.0 & 23 & 29 \\
\hline & 6.0 & 6. 3 & 153 & 17 & 6.1 & 23 & 29 \\
\hline 3 & 6.0 & 5.5 & 72 & $18 \ldots$ & 5.9 & 22 & 31 \\
\hline $4 \ldots$ & 6.0 & 5.3 & 60 & $19 \ldots$ & 5.9 & 16 & 32 \\
\hline $5 \ldots$ & 6.0 & 5.2 & 55 & $20 \ldots$ & 5.6 & 12 & 28 \\
\hline $6 \ldots$ & 6.0 & 5.3 & 52 & $21 \ldots$ & 5.6 & 13 & 27 \\
\hline $7 \ldots$ & 6.3 & 5.7 & 46 & $22 \ldots$ & 5.6 & 19 & 21 \\
\hline $8 \ldots$ & 6.2 & 5.9 & 46 & $23 \ldots$ & 5.9 & 156 & 22 \\
\hline $9 \ldots$ & 6.0 & 15 & 42 & $24 \ldots$ & 6. 0 & 43 & 21 \\
\hline $10 \ldots$ & 5.9 & 138 & 41 & $25 \ldots \ldots$ & 11 & 29 & 19 \\
\hline $11 \ldots$ & 5. 9 & 52 & 42 & $26 \ldots$ & 8.3 & 27 & 18 \\
\hline $12 \ldots$ & 5.5 & 32 & 36 & 27. & 5.9 & 26 & 18 \\
\hline $13 \ldots \ldots$ & 6.0 & 27 & 32 & $28 \ldots \ldots$ & 5.4 & 25 & 19 \\
\hline $14 \ldots$ & 6.1 & 23 & 31 & $29 \ldots$ & 4.5 & 29 & 18 \\
\hline $15 \ldots$ & 6.2 & 23 & 30 & $30 \ldots$ & 5.1 & 332 & 22 \\
\hline & & & & $31 \ldots$ & $\ldots-\cdots$ & 444 & 22 \\
\hline \multirow{2}{*}{\multicolumn{5}{|c|}{$\begin{array}{l}\text { Monthly mean discharge, in cubic feet per second. } \\
\text { Runoff, in acre-feet } \ldots \ldots \ldots \ldots \ldots\end{array}$}} & 6.10 & 51.4 & 44.0 \\
\hline & & & & & 363 & 3,160 & 2,710 \\
\hline
\end{tabular}

(28) 9-4427. 4. Tularosa River near Reserve, N. Mex.

(Crest-stage station)

Location. -- Lat $33^{\circ} 44^{\prime} 00^{\prime \prime}$, long $108^{\circ} 42^{\prime} 10^{\prime \prime}$, in SE⿺ sec. 33 , T. 6 S. , R. 18 W. , $150 \mathrm{ft}$ west of Eagle Peak Lookout Road and 3.3 miles northeast of Reserve.

Drainage area. $--426 \mathrm{sq} \mathrm{mi}$.

Gage-height record. --Crest stages only. Altitude of gage is 5,940 ft (from toxographic map).

Discharge record. --Stage-discharge relation defined by current-meter measurements below $100 \mathrm{cfs}$ and 5 slope-area measurements from 465 to $1,580 \mathrm{cfs}$.

Maxima. --November 1965 to January 1966: Discharge, 465 cfs Dec. 23 (gage height, $4.35 \mathrm{ft}$ ).

1956 to October 1965: Discharge, $2,280 \mathrm{cfs} J u l y ~ 1956$.

According to Senate Document 436 (following p. 64, site 45), the flood of Oct. 16 , 1916 (discharge, $16,420 \mathrm{cfs}$, at site about 2 miles downstream) is the maximum known. 
(29) 9-4430. San Francisco River near Alma, N. Mex.

Location. --Lat $33^{\circ} 21^{\prime} 50^{\prime \prime}$, long $108^{\circ} 54^{\prime} 50^{\prime \prime}$, in $\mathrm{SE}_{\frac{1}{4}} \mathrm{SW} \frac{1}{4}$ sec. $4, \mathrm{~T} .11 \mathrm{~S}$. , F, $20 \mathrm{~W}$. , on right bank $1 \frac{1}{4}$ miles downstream from Alma, 4 miles northwest of Glen wood, and 6 miles upstream from Whitewater Creek.

Drainage area. $--1,546$ sq $\mathrm{mi}$.

Gage-height record. - -Water-stage recorder graph. Altitude of gage is 4, $800 \mathrm{ft}$ (from topographic map).

Discharge record. - -Stage-discharge relation defined by current-meter m $\geqslant$ asurements below 4,500 cfs and extended above by logarithmic plotting.

Maxima. --November 1965 to January 1966: Discharge, 7,500 cfs 1900 hours Dec. 30 (gage height, $7.45 \mathrm{ft}$ ).

1904-14, 1964 to October 1965: Discharge, 25, 000 cf s Nov. 26, 1905 (gage height, $14 \mathrm{ft}$, at site three-quarters of a mile upstream at different datum), from float-area measurement of peak flow.

Senate Document 436 (following p. 64, site 27) gives a peak discharge of $31,800 \mathrm{cfs}$ for the flood of Oct. 16, 1916, and $49,400 \mathrm{cfs}$ for an earlier flood.

\begin{tabular}{|c|c|c|c|c|c|c|c|}
\hline Day & November & December & January & Day & November & Deceml sr & January \\
\hline $1 \ldots$ & 0 & 12 & 1,170 & $16 \ldots$ & 6.2 & 85 & 69 \\
\hline $2 \ldots$ & .1 & 13 & 672 & $17 \ldots$ & 6.6 & 125 & 65 \\
\hline $3 \ldots$ & 1.1 & 11 & 468 & $18 \ldots$ & 6.6 & 125 & 67 \\
\hline $4 \ldots$ & 1.4 & 9.8 & 312 & $19 \ldots$ & 6.6 & 95 & 67 \\
\hline $5 \ldots$ & 2.6 & 8.6 & 220 & $20 \ldots$ & 7.0 & 80 & 64 \\
\hline $6 \ldots$ & 3.0 & 7.5 & 174 & $21 \ldots$ & 7.0 & 85 & 60 \\
\hline $7 \ldots$ & 3.8 & 6.6 & 154 & $22 \ldots$ & 7.0 & 1,570 & 52 \\
\hline $8 \ldots$ & 4.6 & 6. 6 & 143 & $23 \ldots$ & 6.6 & 3,260 & 46 \\
\hline $9 \ldots$ & 5.4 & 8. 1 & 132 & $24 \ldots \ldots$ & 6.6 & 729 & 48 \\
\hline $10 \ldots$ & 5.8 & 663 & 118 & $25 \ldots$ & 9.8 & 388 & 44 \\
\hline $11 \ldots$ & 5.8 & 580 & 109 & $26 \ldots$ & 22 & 328 & 40 \\
\hline $12 \ldots$ & 6.6 & 312 & 109 & $27 \ldots$ & 18 & 225 & 39 \\
\hline $13 \ldots$ & 6.6 & 170 & 87 & $28 \ldots$ & 14 & 150 & 42 \\
\hline $14 \ldots$ & 6.2 & 110 & 81 & 29. & 14 & 90 & 38 \\
\hline $15 \ldots$ & 6.2 & 95 & 75 & $30 \ldots$ & 11 & 3,670 & 39 \\
\hline & & & & $31 \ldots$ & $\ldots-.-$. & 3,380 & 42 \\
\hline \multirow{2}{*}{\multicolumn{5}{|c|}{$\begin{array}{l}\text { Monthly mean discharge, in cubic feet per second } . \\
\text { Runoff, in acre-feet } \ldots \ldots \ldots \ldots \ldots \ldots \ldots \ldots \ldots\end{array}$}} & 6.94 & 529 & 156 \\
\hline & & & & & 413 & 32,530 & 9,610 \\
\hline
\end{tabular}

(30) 9-4440. San Francisco River near Glenwood, N. Mex.

Location. - - Lat $33^{\circ} 15^{\prime} 05^{\prime \prime}$, long $108^{\circ} 52^{\prime} 40^{\prime \prime}$, in NE $\frac{1}{4} N W^{\frac{1}{4}} \sec .23$, T. 12 S., R. 20 W. , on left bank a quarter of a mile upstream from hot springs, 5 miles south of Glenwood, and 6 miles downstream from Whitewater Creek.

Drainage area. $--1,653 \mathrm{sq} \mathrm{mi}$.

Gage-height record. --Water-stage recorder graph. Datum of gage is 4,552.06 ft above mean sea level, datum of 1929 .

Discharge record. --Stage-discharge relation defined by current-meter moasurements below 2,800 cfs and extended above on basis of slope-area measurement at gage height $10.74 \mathrm{ft}$.

Maxima. --November 1965 to January 1966: Discharge, 8, 200 cfs 2230 hcurs Dec. 30 (gage height, $11.00 \mathrm{ft}$ ).

1927 to October 1965: Discharge, 7, $800 \mathrm{cfs}$ Jan. 13, 1949 (gage height, 10.74 ft). 
Mean discharge, in cubic feet per second, 1965-66, of San Francisco River near Glenwood, N. Mex.

\begin{tabular}{|c|c|c|c|c|c|c|c|}
\hline Day & November & December & January & Day & November & December & January \\
\hline $1 \ldots$ & 8.0 & 30 & 1,430 & $16 \ldots$ & 23 & 105 & 104 \\
\hline $2 \ldots$ & 8.0 & 30 & 747 & $17 \ldots$ & 23 & 125 & 100 \\
\hline $3 \ldots$ & 9.0 & 32 & 510 & $18 \ldots$ & 21 & 125 & 104 \\
\hline $4 \ldots$ & 9.0 & 32 & 395 & $19 \ldots$ & 22 & 100 & 102 \\
\hline $5 \ldots$ & 9.5 & 32 & 320 & $20 \ldots$ & 24 & 88 & 98 \\
\hline $6 \ldots$ & 12 & 32 & 263 & $21 \ldots$ & 24 & 95 & 94 \\
\hline $7 \ldots$ & 11 & 32 & 230 & $22 \ldots \ldots$ & 23 & 1,710 & 83 \\
\hline $8 \ldots$ & 11 & 32 & 212 & $23 \ldots$ & 22 & 4,310 & 77 \\
\hline $9 \ldots$ & 15 & 36 & 200 & $24 \ldots \ldots$ & 24 & 908 & 84 \\
\hline $10 \ldots$ & 18 & 799 & 183 & $25 \ldots$ & 27 & 426 & 73 \\
\hline $11 \ldots$ & 21 & 620 & 177 & $26 \ldots$ & 58 & 258 & 67 \\
\hline $12 \ldots$ & 22 & 223 & 171 & $27 \ldots$ & 46 & 192 & 65 \\
\hline $13 \ldots$ & 22 & 145 & 157 & $28 \ldots \ldots$ & 38 & 130 & 65 \\
\hline $14 \ldots$ & 22 & 118 & 137 & $29 \ldots \ldots$ & 34 & 108 & 64 \\
\hline $15 \ldots$ & 23 & 112 & 114 & $30 \ldots$ & 33 & 3,030 & 62 \\
\hline & & & & $31 \ldots$ & $\cdots-\cdots$ & 4,350 & 69 \\
\hline \multirow{2}{*}{\multicolumn{5}{|c|}{$\begin{array}{l}\text { Monthly mean discharge, in cubic feet per second... } \\
\text { Runoff, in acre-feet } \ldots \ldots \ldots \ldots \ldots \ldots \ldots \ldots \ldots\end{array}$}} & 22.1 & 592 & 212 \\
\hline & & & & & 1,310 & 36,430 & 13,010 \\
\hline
\end{tabular}

Gage height, in feet, and discharge, in cubic feet per second, at indicated tim $\%, 1965-66$

\begin{tabular}{|c|c|c|c|c|c|c|c|c|c|c|c|c|}
\hline Date & Hour & $\begin{array}{l}\text { Gage } \\
\text { height }\end{array}$ & $\begin{array}{c}\text { Dis- } \\
\text { charge }\end{array}$ & Date & Hour & $\begin{array}{l}\text { Gage } \\
\text { height }\end{array}$ & $\begin{array}{l}\text { Dis- } \\
\text { charge }\end{array}$ & \multicolumn{2}{|c|}{ Date } & Hour & $\begin{array}{l}\text { Gage } \\
\text { height }\end{array}$ & $\begin{array}{l}\text { Dis- } \\
\text { charge }\end{array}$ \\
\hline Dec. 21 & 2400 & 2.46 & 95 & Dec. 23 & 1800 & 7.22 & 3,220 & Dec. & & 2230 & 11.00 & 8,200 \\
\hline & & & & & 2400 & 5.48 & 1,700 & & & 2400 & 10.72 & 7,840 \\
\hline 22 & 0600 & 2.50 & 105 & & & & & & & & & \\
\hline & 1000 & 2. 58 & 125 & 24 & 0600 & 4.60 & 1,080 & & 1 & 0600 & 9.05 & 5,820 \\
\hline & 1200 & 3.00 & 280 & & 1200 & 4.09 & 795 & & & 1200 & 7. 34 & 4,020 \\
\hline & 1400 & 3.96 & 730 & & 2400 & 3.79 & 645 & & & 1800 & 5. $\subseteq 1$ & 2,680 \\
\hline & 1500 & 5.35 & 1,600 & & & & & & & 2400 & 5. 19 & 2,100 \\
\hline & 1600 & 6.71 & 2,730 & 25 & 1200 & 3.24 & 388 & & & & & \\
\hline & 1800 & 8.00 & 4,050 & & 2400 & 3.13 & 338 & Jan. & 1 & 0800 & 4.39 & 1,540 \\
\hline & 2000 & 8.63 & 4,810 & & & & & & & 1600 & 3.86 & 1,170 \\
\hline & 2200 & 8.92 & 5,150 & 30 & 0100 & 2.47 & 106 & & & 2400 & 3.55 & 980 \\
\hline & 2400 & 8.50 & 4,650 & & 0600 & 2. 71 & 147 & & & & & \\
\hline & & & & & 1000 & 3.06 & 225 & & 2 & 1200 & 3. 10 & 720 \\
\hline 23 & 0230 & 8.07 & 4,130 & & 1130 & 5.90 & 2,510 & & & 2400 & 2. $\varepsilon 4$ & 590 \\
\hline & 0600 & 8. 74 & 4,940 & & 1230 & 5.85 & 2,470 & & & & & \\
\hline & 0900 & 9.28 & 5,610 & & 1600 & 7.56 & 4,240 & & 3 & 1200 & 2. $€ 4$ & 506 \\
\hline & 1100 & 10.14 & 6,860 & & 2030 & 10.70 & 7,810 & & & 2400 & 2.46 & 434 \\
\hline & 1400 & 9.03 & 5,290 & & 2100 & 10.55 & 7,620 & & & & & \\
\hline
\end{tabular}

(31) 9-4442. Blue River near Clifton, Ariz.

(Miscellaneous site)

Location. -- Lat $33^{\circ} 17^{\prime} 10^{\prime \prime}$, long $109^{\circ} 11^{\prime} 40^{\prime \prime}$; in NW $\frac{1}{4}$ sec. 6, T. 2 S., R. 31 E. (unsurveyed), in Apache National Forest, at county road, $7 \frac{1}{2}$ miles upstream from mouth, and 17 miles nor theast of Clifton.

Drainage area. $--505 \mathrm{sq} \mathrm{mi}$.

Maxima. --November 1965 to January 1966: Discharge, 9, 380 cfs Dec, 23 or 30, from slope-area measurement of peak flow.

According to Senate Document 436 the flood of Oct. 13, 1916 (discharge, 31, $900 \mathrm{cfs}$ at the mouth) is the maximum known.

Remarks. --Gaging station established Nov. 7, 1967. 
(32) 9-4444. Chase Creek near Clifton, Ariz. (Crest-stage station)

Location. -- Lat $33^{\circ} 10^{\prime} 20^{\prime \prime}$, long $109^{\circ} 22^{\prime} 10^{\prime \prime}$, in NW $\frac{1}{4}$ sec. 16, T. 3 S. , R. 29 E. , on U.S. Highway 666,9 miles northwest of Clifton.

Drainage area. $--1.37 \mathrm{sq} \mathrm{mi}$

Gage-height record. --Crest stages only. Altitude of gage is $6,050 \mathrm{ft}$ (from topographic map).

Discharge record. --Stage-discharge relation defined by computations of flow through culvert.

Maxima. --November 1965 to January 1966: Discharge, 29 cfs Dec. 22 (g7ge height, $5.64 \mathrm{ft}$ ).

1963 to October 1965: Discharge, $600 \mathrm{cfs}$ July 25, 1964 (gage height, $13.9 \mathrm{ft}$ ), from computation of flow through culvert and over roadway.

(33) 9-4445. San Francisco River at Clifton, Ariz.

Location. - - Lat $33^{\circ} 02^{\prime} 57^{\prime \prime}$, long $109^{\circ} 17^{\prime} 43^{\prime \prime}$, in SW $\frac{1}{4} \mathrm{SE} \frac{1}{4} \sec .30$, T. 4 S. , P. $30 \mathrm{E}$, on downstream side of right pier at Railroad Boulevard Bridge (U.S. High way 666) at Clifton, 9.9 miles upstream from mouth.

Drainage area. $--2,766 \mathrm{sq} \mathrm{mi}$

Gage-height record. --Water-stage recorder graph. Datum of gage is 3,436.16 ft above mean sea level, datum of 1929 .

Discharge record. --Stage-discharge relation defined by current-meter moasurements.

Maxima. --November 1965 to January 1966: Discharge, 30,500 cfs 1000 rours Dec. 23 (gage height, $11.00 \mathrm{ft}$ ).

1927 to October 1965: Discharge, $24,100 \mathrm{cfs}$ Jan. 13, 1949 (gage height, $10.9 \mathrm{ft}$, present datum).

Senate Document 436 lists the flood of Dec. 3, 1906, as the greatest known (discharge, $143,450 \mathrm{cfs}$ ), that of 1891 as the second highest (discharge not determined), and that of Oct. 14,1916, as third highest (discharge, 107, $870 \mathrm{cfs}$; gage height, 19. $7 \mathrm{ft}$, at site $60 \mathrm{ft}$ upstream at different datum). Methods used in computation of the se discharge figures are not known. The flood of $\mathrm{J}$ an. 19, 1916, reached a stage of 16. $8 \mathrm{ft}$, from floodmarks, at site $60 \mathrm{ft}$ upstream (discharge, $90,000 \mathrm{cfs}$, from slopeconveyance study).

\begin{tabular}{|c|c|c|c|c|c|c|c|}
\hline Day & November & December & January & Day & November & December & J anuary \\
\hline $1 \ldots$ & 33 & 74 & 5,230 & $16 \ldots$ & 46 & 335 & 332 \\
\hline $2 \ldots$ & 33 & 68 & 2,340 & $17 \ldots$ & 46 & 662 & 309 \\
\hline & 32 & 65 & 1,360 & $18 \ldots$ & 45 & 682 & 332 \\
\hline 4 & 33 & 63 & 1,050 & $19 \ldots$ & 44 & 558 & 340 \\
\hline & 34 & 63 & 844 & $20 \ldots$ & 44 & 450 & 340 \\
\hline $6 \ldots$ & 36 & 62 & 718 & $21 \ldots$ & 45 & 462 & 313 \\
\hline $7 \ldots$ & 36 & 60 & 616 & $22 \ldots \ldots$ & 45 & 6,130 & 274 \\
\hline $8 \ldots$ & 37 & 60 & 555 & $23 \ldots$ & 46 & 21,900 & 257 \\
\hline $9 \ldots$ & 36 & 77 & 528 & $24 \ldots \ldots$ & 46 & 6,650 & 240 \\
\hline 10. & 38 & 2,070 & 485 & $25 \ldots$ & 59 & 2,720 & 231 \\
\hline $11 \ldots$ & 40 & 2,000 & 446 & $26 \ldots$ & 130 & 1,680 & 224 \\
\hline $12 \ldots$ & 45 & 726 & 436 & $27 \ldots$ & 147 & 1,270 & 212 \\
\hline $13 \ldots$ & 46 & 439 & 396 & $28 \ldots$ & 101 & 1,040 & 203 \\
\hline $14 \ldots$ & 46 & 254 & 374 & $29 \ldots$ & 83 & 902 & 200 \\
\hline $15 \ldots$ & 46 & 368 & 348 & $30 \ldots$ & 78 & 6,370 & 191 \\
\hline & & & & $31 \ldots$ & ----- & 13,200 & 197 \\
\hline \multirow{2}{*}{\multicolumn{5}{|c|}{$\begin{array}{l}\text { Monthly mean discharge, in cubic feet per second. } \\
\text { Runoff, in acre-feet } \ldots \ldots \ldots \ldots \ldots \ldots \ldots \ldots\end{array}$}} & 52.5 & 2,456 & 643 \\
\hline & & & & & 3,130 & 151,000 & 39,510 \\
\hline
\end{tabular}


Gage height, in feet, and discharge, in cubic feet per second, at indicated tim\%, 1965-66,

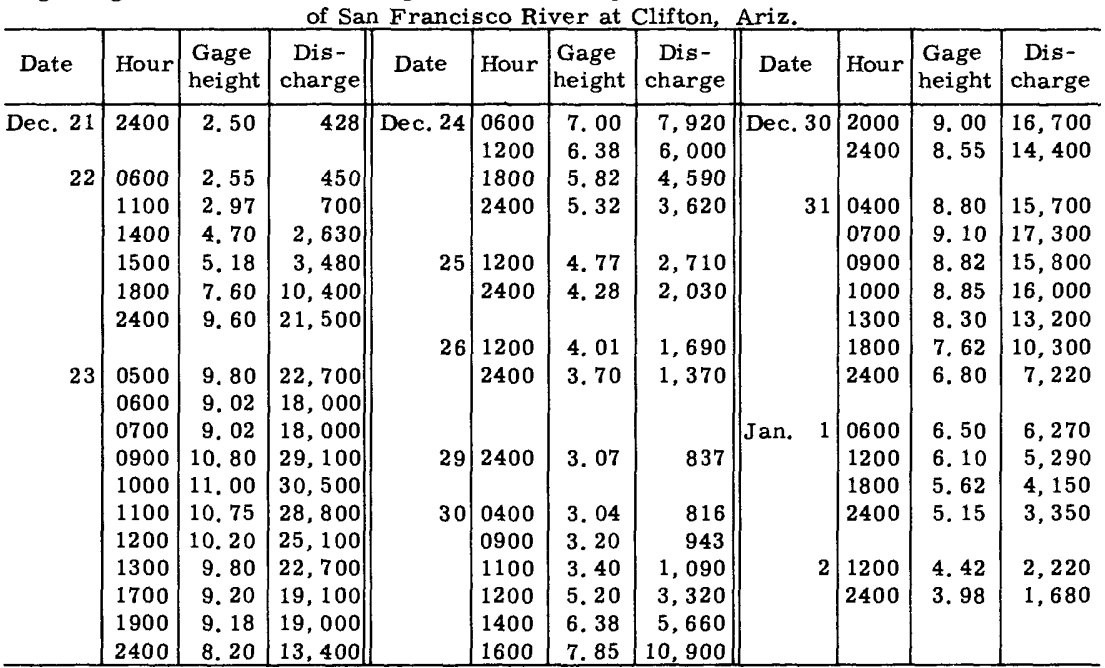

(34) 9-4455. Willow Creek near Point of Pines, near Morenci, Ariz.

Location. -- Lat $33^{\circ} 22^{\prime} 45^{\prime \prime}$, long $109^{\circ} 39^{\prime} 00^{\prime \prime}$, in NW $\frac{1}{4}$ sec. 2, T. 1 S., R. 26 E. (unsurveyed), in San Carlos Indian Reservation, on right bank at head of box canyon, 4 riiles east of Point of Pines, 10 miles west of Double Circle Ranch, and 23 miles northrest of Morenci.

Drainage area. $--102 \mathrm{sq} \mathrm{mi}$.

Gage-height record. - Water-stage recorder graph except Dec. 19 to 1030 hc urs Dec. 21, Jan, 4-11, and about half of each day Dec. 24-29 for which missing porticns were reconstructed. Altitude of gage is $5,804 \mathrm{ft}$ (by barometer).

Discharge record. --Stage-discharge relation defined by current-meter measurements below $80 \mathrm{cfs}$ and extended above on basis of slope-area measurements at gage heights 7. 96, 8.72, and 10.1 ft. Discharge for Dec. 19-20 and Jan. 4-11 estimated on basis of records for nearby streams.

Maxima. --November 1965 to January 1966: Discharge, 3,710 cfs 1200 hours Dec. 30 (gage height, $11.7 \mathrm{ft}$ ).

1944 to October 1965: Discharge, 2,590 cfs Jan, 13, 1952 (gage height. $10.1 \mathrm{ft}$ ).

Remarks. - Pumpage from Black River into Willow Creek above station for industrial and municipal use equaled 336 acre- $\mathrm{ft}$ in November and 319 acre-ft in December. 
Mean discharge, in cubic feet per second, 1965-66, of Willow Creek near Point of Pines, near Morenci, Ariz.

\begin{tabular}{|c|c|c|c|c|c|c|c|}
\hline Day & November & December & January & Day & November & December & January \\
\hline 1 & 0 & 4.2 & 213 & $16 \ldots$ & 12.0 & 12.0 & 5.5 \\
\hline $2 \ldots$ & 0 & 4.8 & 103 & $17 \ldots$ & 9.2 & 10.8 & 3.6 \\
\hline $3 \ldots$ & 0 & 8.3 & 83 & $18 \ldots$ & 8.7 & 9.7 & 4.8 \\
\hline $4 \ldots$ & 0 & 9.2 & 60 & $19 \ldots$ & 5.8 & 10 & 6.2 \\
\hline $5 \ldots$ & 0 & 9.2 & 40 & $20 \ldots$ & .2 & 10 & 4.5 \\
\hline 6 & 0 & 6.4 & 30 & $21 \ldots$ & .3 & 15.9 & 3.4 \\
\hline $7 \ldots$ & 0 & 10.2 & 30 & $22 \ldots$ & 4.2 & 279 & 2.4 \\
\hline $8 \ldots$ & 0 & 10.6 & 20 & $23 \ldots$ & 4.5 & 683 & 3.0 \\
\hline $9 \ldots$ & 0 & 9.6 & 20 & $24 \ldots$ & 4.5 & 223 & 3. 0 \\
\hline $10 \ldots$ & 8.1 & 155 & 15 & $25 \ldots$ & 4.8 & 112 & 2.6 \\
\hline $11 \ldots$ & 13.6 & 152 & 13.6 & $26 \ldots$ & 3.9 & 77 & 2.2 \\
\hline $12 \ldots$ & 13.6 & 55 & 10.6 & $27 \ldots$ & 3.4 & 59 & 2.2 \\
\hline $13 \ldots$ & 13.6 & 23.1 & 8.3 & $28 \ldots$ & 3.4 & 50 & 2. 1 \\
\hline $14 \ldots$ & 13.6 & 15.3 & 7.4 & $29 \ldots$ & 4.4 & 41 & 2.6 \\
\hline $15 \ldots$ & 13.6 & 10.5 & 7. 0 & $30 \ldots$ & 3.9 & 2,130 & 2.6 \\
\hline & & & & $31 \ldots$ & $\ldots \ldots$ & 647 & 3.0 \\
\hline \multicolumn{5}{|c|}{$\begin{array}{l}\text { Monthly mean discharge, in cubic feet per second.. } \\
\text { Runoff, in acre-feet } \ldots \ldots \ldots \ldots \ldots \ldots \ldots \ldots \ldots \ldots \ldots\end{array}$} & $\begin{array}{r}4.98 \\
296\end{array}$ & $\begin{array}{l}156.5 \\
9,630\end{array}$ & $\begin{array}{r}23.1 \\
1,420\end{array}$ \\
\hline
\end{tabular}

Gage height, in feet, and discharge, in cubic feet per second, at indicated time, 1965-66

\begin{tabular}{|c|c|c|c|c|c|c|c|c|c|c|c|}
\hline Date & Hour & $\begin{array}{l}\text { Gage } \\
\text { height }\end{array}$ & $\begin{array}{c}\text { Dis- } \\
\text { charge }\end{array}$ & Date & Hour & $\begin{array}{l}\text { Gage } \\
\text { height }\end{array}$ & $\begin{array}{c}\text { Dis- } \\
\text { charge }\end{array}$ & Date & Hour & $\begin{array}{c}\text { Gage } \\
\text { r eight }\end{array}$ & $\begin{array}{c}\text { Dis- } \\
\text { charge }\end{array}$ \\
\hline Dec. 21 & 2400 & 2.66 & 21.7 & Dec. 24 & 1500 & 37 & 240 & Dec. 3 & 0600 & 8.60 & 1,710 \\
\hline & & & & & 1700 & 4. 47 & 258 & & 1000 & 10 & 2,870 \\
\hline \multirow[t]{11}{*}{22} & 0400 & 2. 57 & 15.9 & & 2100 & 4.07 & 187 & & 1200 & 11.7 & 3,710 \\
\hline & 0800 & 2.66 & 21.7 & & 2400 & 3.69 & 131 & & 1300 & 11.4 & 3,500 \\
\hline & 1000 & 2.83 & 33.7 & & & & & & 1500 & 11.6 & 3,640 \\
\hline & 1200 & 3.23 & 75.3 & 25 & 0200 & 3.50 & 107 & & 1700 & 10.5 & 2,870 \\
\hline & 1400 & 4.02 & 179 & & 0800 & 3.3 & 83 & & 2000 & 9. 30 & 2,100 \\
\hline & 1500 & 4. 14 & 199 & & 1300 & 3. 69 & 131 & & 2400 & 7. 90 & 1,360 \\
\hline & 1530 & 6. 18 & 684 & & 1600 & 3.80 & 146 & & & & \\
\hline & 1730 & 6.21 & 693 & & 2100 & 3. 24 & 76 & & 0600 & 6.50 & 790 \\
\hline & 2100 & 5,90 & 600 & & & & & & 1200 & 5.71 & 493 \\
\hline & 2400 & 6.0 & 630 & & & & & & 1800 & 5.46 & 480 \\
\hline & & & & 28 & 2400 & 2.85 & 38.7 & & 2400 & 4. 97 & 347 \\
\hline \multirow[t]{8}{*}{23} & 0200 & 6.58 & 818 & & & & & & & & \\
\hline & 0400 & 6.93 & 947 & 29 & 0200 & 2. 79 & 33.7 & Jan. & 0600 & 4. 47 & 258 \\
\hline & 0530 & 7.23 & 1,070 & & 0600 & 2.7 & 27.1 & & 1200 & 4. 10 & 192 \\
\hline & 0800 & 6.80 & 895 & & 1000 & 2.72 & 28.4 & & 1800 & 3.93 & 165 \\
\hline & 1200 & 6.31 & 724 & & 1400 & 3.00 & 52.3 & & 2400 & 3.50 & 107 \\
\hline & 1800 & 5.65 & 530 & & 1600 & 2. 92 & 44.8 & & & & \\
\hline & 2400 & 4.80 & 325 & & 2200 & 3. 04 & 55.9 & & 0800 & 3.4 & 95 \\
\hline & & & & & 2400 & 3.37 & 91.4 & & 1600 & 3.60 & 119 \\
\hline \multirow[t]{3}{*}{24} & 0500 & 4.19 & 207 & & & & & & 2100 & 3. 37 & 91 \\
\hline & 0800 & 4. 1 & 192 & 30 & 0200 & 4. 07 & 187 & & 2400 & 3. 34 & 87 \\
\hline & 1300 & 4. 34 & 234 & & 0400 & 6. 50 & 790 & & & & \\
\hline
\end{tabular}


(35) 9-4460. Willow Creek near Double Circle Ranch, near Morenci, Ariz.

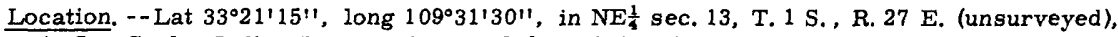
in San Carlos Indian Reservation, on left bank 1 mile upstream from lower end of box canyon, $2 \frac{1}{4}$ miles northwest of Double Circle Ranch, $2 \frac{1}{2}$ miles upstream from mouth, and 19 miles northwest of Morenci.

Drainage area. $-149 \mathrm{sq} \mathrm{mi}$.

Gage-height record. - -Water-stage recorder graph except Jan. 2-6, 17-18. Altitude of gage is $4,969 \mathrm{ft}$ (by barometer).

Discharge record. --Stage-discharge relation Nov. 1 to Dec. 29 defined by current-meter measurements below $50 \mathrm{cfs}$ and extended above on basis of slope-area measurement at gage height $9.42 \mathrm{ft}$. Stage-discharge relation Dec. 30 to Jan. 31 defined by current-meter measurements below $80 \mathrm{cfs}$ and extended above on basis of slope-area measurement of peak flow. Discharge for periods of no gage-height recorc estimated on basis of records at other stations on Willow and Eagle Creeks.

Maxima. - - November 1965 to January 1966: Discharge, 7,500 cfs 1000 hours Dec. 30 (gage height, $9.00 \mathrm{ft}$ ); outside stage, $12.65 \mathrm{ft}$ (result of pileup on bank).

1944 to October 1965: Discharge, 4, 880 cfs Aug. 1, 1965 (gage height, 9. $47 \mathrm{ft}$ ).

Remarks. -- Pumpage from Black River into Willow Creek above station for industrial and municipal use equaled 336 acre-ft in November and 319 acre-ft in December.

Mean discharge, in cubic feet per second, 1965-66

\begin{tabular}{|c|c|c|c|c|c|c|c|}
\hline Day & November & December & January & Day & November & December & January \\
\hline $1 \ldots$ & 1.2 & 7.4 & 239 & $16 \ldots$ & 15.1 & 12.7 & 7.7 \\
\hline $2 \ldots$ & 1.4 & 7. 1 & 100 & $17 \ldots$ & 11.5 & 14. 3 & 7 \\
\hline $3 \ldots$ & 1.5 & 8. 6 & 50 & $\mid 18 \ldots$ & 10.7 & 16. 0 & 7 \\
\hline $4 \ldots$. & 1.5 & 11.5 & 30 & $\mid 19 \ldots$ & 10.7 & 11.7 & 8. 3 \\
\hline$\ldots$ & 1.5 & 12.7 & 30 & $20 \ldots$ & 7.1 & 10.7 & 6. 7 \\
\hline & 1.6 & 12.1 & 25 & $21 \ldots$ & 3.6 & 13.9 & 5.7 \\
\hline 7 & 1.6 & 9.3 & 25.9 & $22 \ldots$. & 3.0 & 535 & 4.5 \\
\hline & 1.9 & 16. 3 & 22.8 & 23. & 6.8 & 1,340 & 3.4 \\
\hline $9 \ldots$ & 2.1 & 17.8 & 19.3 & 24. & 8.2 & 418 & 3. 8 \\
\hline $10 \ldots$ & 2.2 & 156 & 16.8 & 25 . & 9.9 & 237 & 3.8 \\
\hline $11 \ldots$ & 8.9 & 199 & 15.3 & $26 \ldots$ & 11.1 & 161 & 3.6 \\
\hline $12 \ldots$ & 14.5 & 82 & 13.9 & $27 \ldots$ & 8.2 & 121 & 3. 8 \\
\hline $13 \ldots$ & 14.5 & 25.9 & 12.3 & $28 \ldots$ & 7.1 & 96 & 3.6 \\
\hline $14 \ldots$ & 15. 1 & 18.2 & 10.7 & 29. & 7.1 & 87 & 3.6 \\
\hline $15 \ldots$ & 15.1 & 11.5 & 8.6 & 30. & 7.4 & 3,870 & 3. 8 \\
\hline & & & & 31 & $\ldots-\cdots$ & 923 & 4. 3 \\
\hline \multicolumn{5}{|c|}{ Monthly mean discharge, in cubic feet per second.. } & 7.07 & 273 & 22.6 \\
\hline \multicolumn{5}{|c|}{ Runoff, in acre-feet $\ldots \ldots \ldots \ldots \ldots \ldots \ldots \ldots$} & 421 & 16,790 & 1,390 \\
\hline
\end{tabular}


Gage height, in feet, and discharge, in cubic feet per second, at indicated time, 1965-66,

\begin{tabular}{|c|c|c|c|c|c|c|c|c|c|c|c|}
\hline Date & Hour & $\begin{array}{l}\text { Gage } \\
\text { height }\end{array}$ & $\begin{array}{c}\text { Dis- } \\
\text { charge }\end{array}$ & Date & Hour & $\begin{array}{l}\text { Gage } \\
\text { height }\end{array}$ & $\begin{array}{c}\text { Dis- } \\
\text { charge }\end{array}$ & Date & Hour & $\begin{array}{l}\text { Gage } \\
\text { height }\end{array}$ & $\begin{array}{c}\text { Dis- } \\
\text { charge }\end{array}$ \\
\hline \multirow[t]{2}{*}{$\overline{\text { Dec } . ~} 21$} & 2400 & 4.78 & 7.7 & Dec. 23 & 2400 & 6.85 & 725 & Dec. 30 & 0700 & 8.35 & 3,670 \\
\hline & & & & & & & & & 0900 & 8. 75 & 5,580 \\
\hline \multirow[t]{10}{*}{22} & 0600 & 4. 84 & 13.9 & 24 & 0600 & 6.52 & 450 & & 1000 & 9. 00 & 7,500 \\
\hline & 0800 & 4. 94 & 20.5 & & 1200 & 6.28 & 332 & & 1200 & 8.70 & 6,700 \\
\hline & 1000 & 5.30 & 57 & & 2000 & 6.45 & 415 & & 1300 & 8. 95 & 7,350 \\
\hline & 1200 & 6.12 & 268 & & 2400 & 6. 33 & 355 & & 1800 & 8.20 & 4,320 \\
\hline & 1300 & 6.77 & 669 & & & & & & 2400 & 7. 10 & 2,570 \\
\hline & 1730 & 7. 13 & 951 & 25 & 0600 & 6. 02 & 228 & & & & \\
\hline & 1800 & 7.65 & 1,500 & & 1400 & 5.83 & 169 & 31 & 0600 & 6.00 & 1,390 \\
\hline & 1900 & 7.48 & 1,300 & & 1800 & 6.13 & 272 & & 0800 & 5.30 & 850 \\
\hline & 2400 & 7. 45 & 1,260 & & 2400 & 6.02 & 228 & & 1200 & 4. 60 & 720 \\
\hline & & & & & & & & & 1800 & 4.20 & 515 \\
\hline \multirow[t]{5}{*}{23} & 0300 & 7. 79 & 1,570 & 29 & 2400 & 5. 76 & 134 & & 2400 & 3. 90 & 390 \\
\hline & 0430 & 8. 10 & 2,100 & & & & & & & & \\
\hline & 0900 & 7.75 & 1,620 & 30 & 0300 & 6. 06 & 285 & Jan. 1 & 0800 & 3.47 & 246 \\
\hline & 1200 & 7. 55 & 1,380 & & 0400 & 6. 70 & 720 & & 1600 & 3.25 & 187 \\
\hline & 1800 & 7. 23 & 1,050 & & 0600 & 8.55 & 3,200 & & 2400 & 3. 15 & 164 \\
\hline
\end{tabular}

(36) 9-4465. Eagle Creek near Double Circle Ranch, near Morenci, Ariz.

Location. -- Lat $33^{\circ} 18^{\prime} 00^{\prime \prime}$, long $109^{\circ} 29^{\prime} 30^{\prime \prime}$, in SW $\frac{1}{4}$ sec. 32 , T. 1 S. , R. 28 E. (unsurveyed), on left bank half a mile upstream from head of box canyon, $2 \frac{3}{4}$ miles downstream

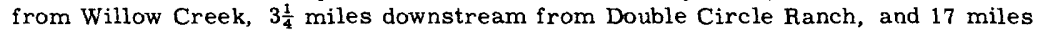
northwest of Morenci.

Drainage area. --377 sq $\mathrm{mi}$.

Gage-height record. --Water-stage recorder graph except Jan. 2-6. Altitıde of gage is $4,722 \mathrm{ft}$ (by barometer).

Discharge record. --Stage-discharge relation Nov. 1 to Dec. 21 defined by currentmeter measurements below $100 \mathrm{cfs}$ and extended above on basis of slope-area measurements at gage heights 7.44 and $8.51 \mathrm{ft}$. Stage-discharge relation Dec. 30 to Jan. 31 defined by current-meter measurements. Stage-discharge relation Dec. 22-29 estimated on basis of shape of ratings before and after. Discharge for Jan. 2-6 estimated on basis of records for other stations on Willow and Eagle Creek.

Maxima. -- November 1965 to January 1966: Discharge, 13,600 cfs 1400 hours Dec. 30 (gage height, $9.20 \mathrm{ft}$ ).

1944 to October 1965: Discharge, 7,270 cfs Sept. 10, 1958 (gage height, $8.7 \mathrm{ft}$ ), from rating curve extended above $900 \mathrm{cfs}$ on basis of slope-area measurement at gage height $8.51 \mathrm{ft}$.

Remarks. - - Pumpage from Black River and Eagle Creek Wells into Eagle Creek above station equaled 651 acre-ft in November and 319 acre-ft in December. 
Mean discharge, in cubic feet per second, 1965-66, of Eagle Creek near Double Circle Ranch, near Morenci, Ariz.

\begin{tabular}{|c|c|c|c|c|c|c|c|}
\hline Day & November & December & January & Day & November & December & January \\
\hline 1 & 18.0 & 8.4 & 795 & $16 \ldots$ & 18,0 & 45 & 45 \\
\hline $2 \ldots$ & 20.5 & 8.4 & 377 & $17 \ldots$ & 16.0 & 56 & 41 \\
\hline $3 \ldots$ & 20.5 & 8.6 & 230 & $18 \ldots$ & 14.2 & 60 & 42 \\
\hline $4 \ldots$ & 19.0 & 9.6 & 150 & $19 \ldots$ & 13.8 & 49 & 41 \\
\hline $5 \ldots$ & 17,0 & 10.1 & 120 & $20 \ldots$ & 11.9 & 43 & 38 \\
\hline $6 \ldots$ & 16.5 & 10.4 & 100 & $21 \ldots$ & 9.4 & 50 & 34.0 \\
\hline $7 \ldots$ & 17.0 & 9.4 & 90 & $22 \ldots \ldots$ & 8.1 & 1,180 & 30.1 \\
\hline $8 \ldots$ & 19.5 & 10.6 & 82 & $23 \ldots$ & 8.8 & 2,710 & 28.3 \\
\hline $9 \ldots$ & 21.5 & 12. 7 & 76 & 24 & 9.8 & 804 & 28.3 \\
\hline $10 \ldots$ & 21.0 & 122 & 74 & 25 & 10.6 & 427 & 27. 4 \\
\hline 11 & 21.5 & 219 & 69 & 26 & 10.8 & 316 & 26.5 \\
\hline 12 . & 24.0 & 112 & 68 & 27 . & 9.4 & 249 & 26.5 \\
\hline 13. & 19.0 & 62 & 60 & $28 \ldots$ & 8.6 & 211 & 25.6 \\
\hline 14. & 19.0 & 55 & 54 & $29 \ldots \ldots$ & 8.1 & 197 & 25.6 \\
\hline $15 \ldots$ & 18.5 & 50 & 50 & $30 \ldots$ & 8.4 & 6,350 & 24. 7 \\
\hline & & & & $31 \ldots$ &.---- & 2,100 & 21.4 \\
\hline \multicolumn{5}{|c|}{$\begin{array}{l}\text { Monthly mean discharge, in cubic feet per second.. } \\
\text { Runoff, in acre-feet } \ldots \ldots \ldots \ldots \ldots \ldots \ldots \ldots \ldots \ldots\end{array}$} & 15.3 & 502 & 93.6 \\
\hline
\end{tabular}

Gage height, in feet, and discharge, in cubic feet per second, at indicated time, 1965-66

\begin{tabular}{|c|c|c|c|c|c|c|c|c|c|c|c|}
\hline Date & Hour & $\begin{array}{l}\text { Gage } \\
\text { height }\end{array}$ & $\begin{array}{c}\text { Dis- } \\
\text { charge }\end{array}$ & Date & Hour & $\begin{array}{l}\text { Gage } \\
\text { height }\end{array}$ & $\begin{array}{c}\text { Dis- } \\
\text { charge }\end{array}$ & Date & Hour & $\begin{array}{l}\text { Gage } \\
\text { height }\end{array}$ & $\begin{array}{c}\text { Dis - } \\
\text { charge }\end{array}$ \\
\hline \multirow{12}{*}{$\begin{array}{r}\text { Dec. } 21 \\
22\end{array}$} & 2400 & 3.41 & 50 & \multirow[t]{3}{*}{ Dec. 23} & 2100 & 5.30 & 1,760 & \multirow[t]{13}{*}{ Dec. 30} & 0400 & 3.88 & 297 \\
\hline & & & & & 2400 & 5.12 & 1,540 & & 0500 & 4. 17 & 447 \\
\hline & 0300 & 3. 48 & 57 & & & & & & 0600 & 5.30 & 1,460 \\
\hline & 0600 & 3.65 & 74 & \multirow[t]{5}{*}{24} & 0600 & 4.70 & 1,690 & & 0800 & 7. 20 & 6,750 \\
\hline & 0900 & 4. 22 & 185 & & 1400 & 4. 33 & 661 & & 1000 & 7. 75 & 8,350 \\
\hline & 1200 & 5.67 & 1,320 & & 2100 & 4.42 & 737 & & 1200 & 8.73 & 11,700 \\
\hline & 1400 & 6.45 & 2,640 & & 2400 & 4. 37 & 612 & & 1300 & 8.90 & 12,400 \\
\hline & 1600 & 6. 17 & 2,060 & & & & & & 1400 & 9.20 & 13,600 \\
\hline & 1800 & 6.03 & 917 & \multirow[t]{6}{*}{25} & 0600 & 4. 18 & 483 & & 1500 & 8. 90 & 12,400 \\
\hline & 2000 & 6.10 & 980 & & 1200 & 3.98 & 370 & & 1700 & 8,00 & 9,100 \\
\hline & 2400 & 5.80 & 1,750 & & 1700 & 3. 94 & 349 & & 2000 & 7. 32 & 7,060 \\
\hline & & & & & 2000 & 4. 08 & 424 & & 2400 & 6. 25 & 4,500 \\
\hline \multirow[t]{9}{*}{23} & 0100 & 5.80 & 1,900 & & 2400 & 4. 05 & 408 & & & & \\
\hline & 0300 & 6.38 & 2,790 & & & & & \multirow[t]{5}{*}{31} & 0600 & 5.14 & 2,410 \\
\hline & 0500 & 6.80 & 3,600 & \multirow[t]{4}{*}{26} & 1800 & 3.75 & 262 & & 1200 & 4. 78 & 1,870 \\
\hline & 0630 & 7. 13 & 5,060 & & 2100 & 3.87 & 315 & & 1600 & 4. 44 & 1,450 \\
\hline & 0800 & 6.80 & 4,400 & & 2400 & 3. 86 & 359 & & 2400 & 4,23 & 1,230 \\
\hline & 1000 & 6.15 & 3,100 & & & & & & & & \\
\hline & 1200 & 5.80 & 2,500 & \multirow[t]{2}{*}{29} & 2400 & 3.65 & 204 & \multirow[t]{3}{*}{ Jan. 1} & 1200 & 3. 74 & 754 \\
\hline & 1500 & 5.67 & 2,300 & & & & & & 2400 & 3. 42 & 508 \\
\hline & 1800 & 5.52 & 2,080 & 30 & 0200 & 3.72 & 229 & & & & \\
\hline
\end{tabular}


(37) 9-4470. Eagle Creek above pumping plant, near Morenci, Ariz.

Location. -- Lat $33^{\circ} 04^{\prime} 12^{\prime \prime}$, long $109^{\circ} 27^{\prime} 0^{\prime \prime}$, in SE $\frac{1}{4} \mathrm{NE} \frac{1}{4}$ sec. 22, T. 4 S., P. $28 \mathrm{E}$., on right bank $2 \frac{1}{2}$ miles upstream from Phelps Dodge Corp. pumping plant, 5 miles west of Morenci, and 13 miles upstream from mouth.

Drainage area. $--613 \mathrm{sq} \mathrm{mi}$.

Gage-height record. --Water-stage recorder graph of stage in Parshall flume except Dec. 20-28 and 0500 hours Dec. 31 to Jan. 10 ; water-stage recorder graph of stage at auxiliary gage $80 \mathrm{ft}$ upstream from flume except Dec. 20 to Jan. 31. Datum of base gage at flume is $3,695 \mathrm{ft}$ above mean sea level. Gage heights shown are for base gage.

Discharge record. --Stage-discharge relation defined by current-meter measurements below 15,000 cfs and extended above on basis of slope-area measurement of peak flow. Discharge for 2400 hours Dec, 9 to 1000 hours Dec. 13, 0830 hours Der. 17 to 1730 hours Dec. 19 computed from stage at auxiliary gage. Discharge for poriods of no gage-height record estimated on basis of range in stage and record for Eagle Creek near Double Circle Ranch near Morenci.

Maxima. --November 1965 to January 1966: Discharge, 21, 000 cfs 1700 hours Dec. 30 (gage height, $8.9 \mathrm{ft}$ at base gage, $12.8 \mathrm{ft}$ at auxiliary gage).

1932 to October 1965: Discharge, 13,000 cfs Feb. 10, 1932 (gage height not determined), by slope-area measurement of peak flow.

Senate Document 436 (p. 77) gives a discharge of $36,000 \mathrm{cfs}$, probably during the flood of January 1916; other data indicate that August 1915 is a more lil - ily date for this peak.

Remarks. --Records above $250 \mathrm{cfs}$ are poor. Pumpage from Black River and Eagle Creek Wells into Eagle Creek above station equaled 651 acre-ft in November and 319 acre-ft in December. 
Mean discharge, in cubic feet per second, 1965-66, of Eagle Creek ak ove pumping plant, near Morenci, Ariz.

\begin{tabular}{|c|c|c|c|c|c|c|c|}
\hline Day & November & December & January & Day & November & December & January \\
\hline 1 & 19.7 & 14. 8 & 2,500 & 16 & 21.9 & 106 & 74 \\
\hline 2 & 22.8 & 14. 8 & 1,000 & 17 & 21.9 & 218 & 67 \\
\hline 3 & 24.6 & 14.8 & 750 & 18 & 20.1 & 261 & 72 \\
\hline . & 25.1 & 14. 8 & 300 & 19 & 18.9 & 197 & 72 \\
\hline$\ldots$ & 22.4 & 17.2 & 250 & $20 \ldots$ & 18.9 & 137 & 65 \\
\hline$\ldots$ & 21.5 & 17. 6 & 200 & $21 \ldots$ & 17.2 & 140 & 61 \\
\hline$\ldots$ & 21.0 & 17.6 & 150 & $22 \ldots \ldots$ & 14.1 & a 6,000 & 52 \\
\hline $8 \ldots$ & 21.0 & 16. 8 & 140 & $23 \ldots$ & 13.7 & a 7, 000 & 47 \\
\hline$\ldots$ & 23.3 & 30.0 & 120 & 24 & 15.6 & a 2, 500 & 43 \\
\hline $10 \ldots$ & 25.1 & 453 & 110 & 25 & 18.4 & 900 & 41 \\
\hline $11 \ldots$ & 23.7 & 1,880 & 104 & 26 & 19.7 & 400 & 40 \\
\hline $12 \ldots$ & 27.0 & 344 & 101 & 27 & 18.4 & 300 & 40 \\
\hline $13 \ldots$ & 27.0 & 156 & 94 & 28 & 16.4 & 260 & 39 \\
\hline 14 & 22.4 & 110 & 86 & 29 & 15.2 & 260 & 38 \\
\hline $15 \ldots$ & 22.4 & 125 & 81 & 30 & 14.8 & 8,000 & 37 \\
\hline & & & & $31 \ldots$ & $\ldots-\cdots$ & 4,000 & 44 \\
\hline \multirow{2}{*}{\multicolumn{5}{|c|}{$\begin{array}{l}\text { Monthly mean discharge, in cubic feet per second. } \\
\text { Runoff, in acre-feet } \ldots \ldots \ldots \ldots \ldots \ldots \ldots \ldots \ldots\end{array}$}} & 20.5 & 1,094 & 220 \\
\hline & & & & & 1,220 & 67,250 & 13,520 \\
\hline
\end{tabular}

a Revised.

Gage height, in feet, and discharge, in cubic feet per second, at indicated time, 1965

\begin{tabular}{r|c|c|r||r|r|r|r||r|r|r|r}
\hline Date & Hour & $\begin{array}{c}\text { Gage } \\
\text { height }\end{array}$ & $\begin{array}{c}\text { Dis- } \\
\text { charge }\end{array}$ & Date & Hour & $\begin{array}{c}\text { Gage } \\
\text { height }\end{array}$ & $\begin{array}{c}\text { Dis- } \\
\text { charge }\end{array}$ & Date & Hour & $\begin{array}{c}\text { Gage } \\
\text { height }\end{array}$ & $\begin{array}{c}\text { Dis - } \\
\text { charge }\end{array}$ \\
\hline Dec. 21 & 2400 & - & 150 & Dec. 23 & 1200 & - & 7,000 & Dec. 30 & 1400 & 5.4 & 5,800 \\
& & & & & 1800 & - & 4,300 & & 1600 & 8.2 & 18,000 \\
& 0400 & - & 150 & & 2400 & - & 3,000 & & 1700 & 8.9 & 21,000 \\
& 0900 & - & 600 & & & & & & 1800 & 8.6 & 20,000 \\
& 1200 & - & 3,500 & 24 & 0900 & - & 3,000 & & 2000 & 8.1 & 18,000 \\
& 1500 & - & 12,000 & & 2400 & - & 1,400 & & 2200 & 7.4 & 14,000 \\
& 1700 & 7.6 & 15,000 & & & & & & 2400 & 6.7 & 11,000 \\
& 2400 & - & 9,000 & 29 & 2400 & 2.68 & 155 & & & & \\
& & & & & & & & & & & \\
23 & 0200 & 6.4 & 8,800 & 30 & 0400 & 2.72 & 162 & & 1200 & - & 3,400 \\
& 0600 & - & 9,500 & & 0800 & 2.87 & 175 & & 2400 & - & 2,500 \\
\hline
\end{tabular}

(38) Bonita Creek :ear Solomon, Ariz.

(Miscellaneous site)

Location. -- Lat $32^{\circ} 56^{\prime} 30^{\prime \prime}$, long $109^{\circ} 30^{\prime} 20^{\prime \prime}$, in NW $\frac{1}{4}$ sec. 5 , T. 28 E., R. 6 S., at city of Safford water-supply galleries, 4 miles upstream from mouth, and $11 \frac{1}{2}$ miles northeast of Solomon.

Drainage area. $--310 \mathrm{sq} \mathrm{mi}$.

Maxima. --November 1965 to January 1966: Discharge, approximately 6, $200 \mathrm{cfs}$ Dec. 30 , from slope-area measurement of peak flow. Field data furnished by City of Safford Municipal Utilities.

According to Senate Document 436, the maximum flood known prior to 1919 occurred in August 1915 (discharge, 16,640 cfs at mouth). The peak of Oct. 13, 1916, was estimated as 8,960 cfs. The peak of January 1916 was less than that of Oct. 13 . 1916. 
(39) 9-4485. Gila River at head of Safford Valley, near Solomon, Ariz.

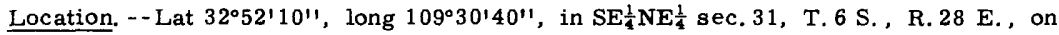
left bank 0.6 mile downstream from intake of Brown Canal, 8 miles northeast of Solomon, and 17 miles downstream from San Francisco River. Recorda include flow of Brown Canal, which is measured 2, $000 \mathrm{ft}$ downstream from intake.

Drainage area. $--7,896 \mathrm{sq} \mathrm{mi}$.

Gage-height record. - -Water-stage recorder graph. Datum of gage is 3,064. $88 \mathrm{ft}$ above mean sea level, datum of 1929, supplementary adjustment of 1959 .

Discharge record. --Stage-discharge relation defined by current-meter m€ asurements.

Maxima. --November 1965 to January 1966: Discharge, 43, 000 cfs 2400 hours Dec. 22 (gage height, $13,7 \mathrm{ft}$ ).

1914 to October 1965: Discharge, about $100,000 \mathrm{cfs}$ Jan. 19, 1916 (gage height, $14.0 \mathrm{ft}$, datum then in use), from rating curve extended above $18,000 \mathrm{cfs}$ on basis of velocity-area study.

\begin{tabular}{|c|c|c|c|c|c|c|c|}
\hline Day & November & December & January & Day & November & December & January \\
\hline $1 \ldots$ & 51 & 181 & 7,950 & $16 \ldots$ & 87 & 1,150 & 886 \\
\hline $2 \ldots$ & 55 & 169 & 5,310 & $17 \ldots$ & 94 & 1,450 & 820 \\
\hline $3 \ldots$ & 65 & 151 & 3,380 & $18 \ldots$ & 96 & 1,880 & 842 \\
\hline $4 \ldots$ & 63 & 146 & 2,420 & $19 \ldots$ & 89 & 1,540 & 864 \\
\hline $5 \ldots$ & 67 & 135 & 1,920 & 20. & 83 & 1,220 & 864 \\
\hline $6 \ldots$ & 69 & 132 & 1,610 & $21 \ldots$ & 83 & 1,110 & 800 \\
\hline $7 \ldots$ & 67 & 125 & 1,410 & $22 \ldots$ & 92 & 7,690 & 730 \\
\hline $8 \ldots$ & 69 & 115 & 1,310 & $23 \ldots$ & 91 & 30,800 & 675 \\
\hline $9 \ldots$ & 74 & 115 & 1,270 & 24. & 95 & 14,600 & 622 \\
\hline $10 \ldots$ & 74 & 2,120 & 1,210 & $25 \ldots$ & 116 & 6,640 & 570 \\
\hline $11 \ldots$ & 74 & 5,630 & 1,150 & 26. & 154 & 3,200 & 545 \\
\hline $12 \ldots$ & 74 & 3,160 & 1,110 & 27 & 224 & 2,580 & 497 \\
\hline $13 \ldots$ & 75 & 1,450 & 1,070 & 28. & 241 & 2,120 & 489 \\
\hline $14 \ldots$ & 77 & 1,110 & 990 & 29. & 204 & 1,890 & 473 \\
\hline \multirow[t]{2}{*}{$15 \ldots$} & 85 & 1,120 & 942 & $30 \ldots$ & 190 & 6,420 & 458 \\
\hline & & & & $31 \ldots$ & $\ldots-\ldots$ & 14,100 & 465 \\
\hline \multicolumn{5}{|c|}{$\begin{array}{l}\text { Monthly mean discharge, in cubic feet per second.. } \\
\text { Runoff, in acre-feet } \ldots \ldots \ldots \ldots \ldots \ldots \ldots\end{array}$} & $\begin{array}{r}99.3 \\
5,910\end{array}$ & $\begin{array}{r}3,6 \varepsilon 5 \\
226,600\end{array}$ & $\begin{array}{r}1,408 \\
86,580\end{array}$ \\
\hline
\end{tabular}

Gage height, in feet, and discharge, in cubic feet per second, at indicated time, 1965-66

\begin{tabular}{|c|c|c|c|c|c|c|c|c|c|c|c|}
\hline Date & Hour & $\begin{array}{l}\text { Gage } \\
\text { height }\end{array}$ & $\begin{array}{c}\text { Dis- } \\
\text { charge }\end{array}$ & Date & Hour & $\begin{array}{l}\text { Gage } \\
\text { height }\end{array}$ & $\begin{array}{c}\text { Dis- } \\
\text { charge }\end{array}$ & Date & Hour & $\begin{array}{l}\text { Gage } \\
\text { hoight }\end{array}$ & $\begin{array}{c}\text { Dis- } \\
\text { charge }\end{array}$ \\
\hline \multirow[t]{2}{*}{ Dec. 21} & 2400 & 5. 30 & 1,050 & Dec. 24 & 0500 & 9.35 & 15,000 & Dec. 30 & 1600 & 6.90 & 5,400 \\
\hline & & & & & 1000 & 8. 85 & 12,500 & & 1800 & 8.20 & 9,800 \\
\hline \multirow[t]{7}{*}{22} & 1200 & 5.42 & 1,200 & & 1400 & 9.80 & 17,300 & & 2100 & 10.35 & 20,200 \\
\hline & 1430 & 6.65 & 2,800 & & 1800 & 9.25 & 14 & & 2300 & 10. 72 & 22,300 \\
\hline & 1600 & 9.42 & 10,500 & & 2400 & 8. 60 & 11 , & & 2400 & 10.60 & 21,600 \\
\hline & 1900 & 10.3 & 15,800 & & & & & & & & \\
\hline & 2100 & 11.4 & 23,400 & 25 & 1200 & 7. 25 & 50 & 31 & 0600 & 9.60 & 300 \\
\hline & 2400 & 13.7 & 43,000 & & 2400 & 6. 35 & 3,840 & & 1200 & 9.25 & 14,600 \\
\hline & & & & & & & & & 1800 & 8.55 & 11,200 \\
\hline \multirow[t]{6}{*}{23} & 0400 & 13.1 & 38,500 & 26 & 1200 & 6.00 & 3,100 & & 2400 & 8. 08 & 9,320 \\
\hline & 1200 & 12. 1 & 31,200 & & 2400 & 5. 70 & 2,500 & & & & \\
\hline & 1500 & 12. 8 & 36,200 & & & & & Jan. & 1200 & 7. 63 & 7,700 \\
\hline & 1800 & 11.35 & 26,000 & & & & & & 2400 & 7.45 & 7,080 \\
\hline & 2400 & 10.3 & 20,000 & 29 & 2400 & 4.87 & 693 & & & & \\
\hline & & & & & & & & 2 & 1200 & 6.85 & 5,250 \\
\hline 24 & 0200 & 10.0 & 18,300 & 30 & 1400 & 4.80 & 630 & & 2400 & 6.40 & 3,960 \\
\hline
\end{tabular}


(40) 9-4570. San Simon River near Solomon, Ariz.

Location. -- Lat $32^{\circ} 48^{\prime} 06^{\prime \prime}$, long $109^{\circ} 38^{\prime} 19^{\prime \prime}$, in NW $\frac{1}{4} \mathrm{NE} \frac{1}{4}$ sec. 25, T. 7 S. , R. 29 E. , 1 mile southwest of Solomon, and $2 \frac{1}{4}$ miles upstream from mouth.

Drainage area. $--2,192 \mathrm{sq} \mathrm{mi}$.

Gage-height record. - Water-stage recorder graph except during short periods during recessions of Dec. 10,11, 15, 17, 23. Graph reconstructed. Datum of gage is 2, $960.15 \mathrm{ft}$ above mean sea level, datum of 1929 .

Discharge record. --Stage-discharge relation defined by current-meter measurements.

Maxima. - November 1965 to January 1966: Discharge, 428 cfs 1400 hours Dec. 22 (gage height, $5.08 \mathrm{ft}$ ).

1931 to October 1965: Discharge, 27,500 cfs Aug. 9, 1931 (gage height, 19.0 ft), by slope-area measurement of peak flow.

Flood of Aug. 9, 1931, is the maximum since at least the early 1880's. Senate Document 436 gives a peak discharge of $7,548 \mathrm{cfs}$ for the flood of October 1916 and $11,980 \mathrm{cfs}$ for the maximum flood known prior to 1919.

\begin{tabular}{|c|c|c|c|c|c|c|c|c|c|}
\hline Day & December & Day & December & Day & December & Day & Decermber & Day & December \\
\hline $\begin{array}{l}1 \ldots \\
2 \ldots \\
3 \ldots \\
4 \ldots \\
5 \ldots \\
6 \ldots\end{array}$ & $\begin{array}{l}0 \\
0 \\
0 \\
0 \\
0 \\
0\end{array}$ & 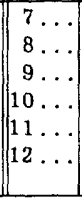 & $\begin{array}{r}0 \\
0 \\
71 \\
191 \\
163 \\
2.0\end{array}$ & $\left(\begin{array}{l}13 \ldots \\
14 \ldots \\
15 \ldots \\
16 \ldots \\
17 \ldots \\
18 \ldots\end{array}\right.$ & $\begin{array}{r}0 \\
0 \\
42 \\
0 \\
139 \\
0\end{array}$ & $\begin{array}{l}19 \ldots \\
20 \ldots \\
21 \ldots \\
22 \ldots \\
23 \ldots \\
24 \ldots\end{array}$ & $\begin{array}{r}0 \\
0 \\
0 \\
136 \\
40 \\
0\end{array}$ & $\begin{array}{l}25 \ldots \\
26 \ldots \\
27 \ldots \\
28 \ldots \\
29 \ldots \\
30 \ldots \\
31 \ldots\end{array}$ & $\begin{array}{l}0 \\
0 \\
0 \\
0 \\
0 \\
0 \\
0\end{array}$ \\
\hline \multicolumn{9}{|c|}{ 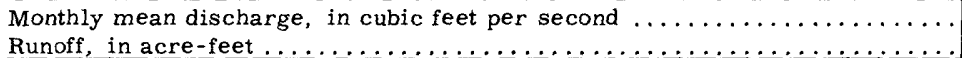 } & $\begin{array}{r}25.3 \\
1,550\end{array}$ \\
\hline
\end{tabular}

(41) 9-4585. Gila River at Safford, Ariz.

Location. - Lat $32^{\circ} 50^{\prime} 50^{\prime \prime}$, long $109^{\circ} 42^{\prime} 55^{\prime \prime}$, in SW $\frac{1}{4} \mathrm{SW} \frac{1}{4}$ sec. 5, T. 7 S. , R. 26 E., on downstream side of highway bridge 1 mile north of Safford, and $4 \frac{1}{2}$ miles downstream from San Simon River.

Drainage area. $--10,459$ sq mi.

Gage-height record. - -Water-stage recorder graph prior to Jan. 6 except for part of Dec. 31, Digital recorder tape Jan. 6-31.

Discharge record. - -Stage-discharge relation defined by current-meter measurements. Discharge computed for Dec. 22-27 only.

Maxima. --November 1965 to January 1966: Discharge, 42, 000 cfs 0600 hours Dec. 23 (gage height, $14.2 \mathrm{ft}$ ).

1940 to October 1965: Discharge, $33,000 \mathrm{cfs}$ Sept. 30,1941 ; gage height, $13.1 \mathrm{ft}$ Jan. 14, 1949.

Remarks. --Publication of records discontinued Sept. 30, 1965. 
Gage height, in feet, and discharge, in cubic feet per second, at indicated time, 1965, of Gila River at Safford, Ariz.

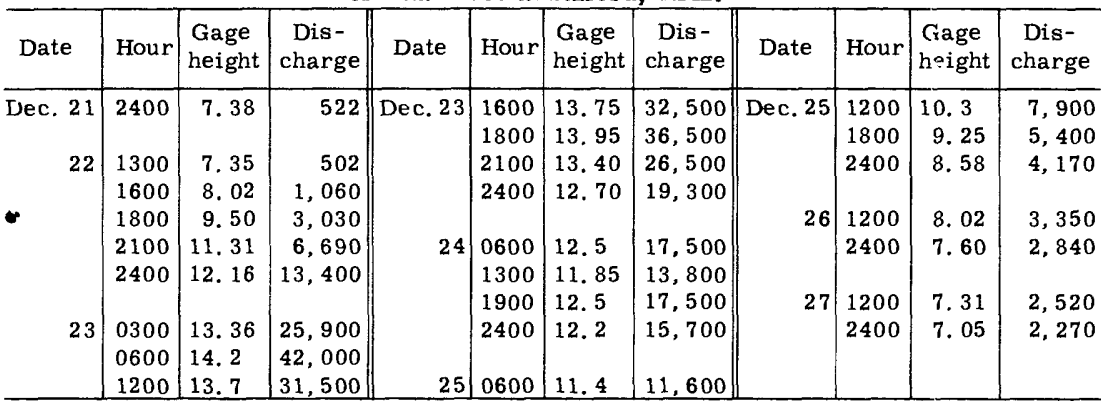

(42) 9-4665. Gila River at Calva, Ariz.

Location. --Lat $33^{\circ} 11^{\prime} 10^{\prime \prime}$, long $110^{\circ} 13^{\prime} 10^{\prime \prime}$, in SW $\frac{1}{4}$ sec. 8, T. 3 S. , R. 21 E. ('nsurveyed), in San Carlos Indian Reservation, on left bank $530 \mathrm{ft}$ downstream from Southern Pacific Railroad bridge at head of San Carlos Reservoir, $1 \frac{1}{2}$ miles northwest of Calva.

Drainage area. $--11,470 \mathrm{sq} \mathrm{mi}$.

Gage-height record. --From digital tape or analog recorder graph except 1200 hours Dec. 24 to 1220 hours Dec. 27, which was reconstructed on basis of unprblished records collected at sites 7 miles upstream ("near Bylas") and 4 miles downstream ("near Calva") and from high-water marks. Datum of gage is 2,513.64 ft above mean sea level, datum of 1929 .

Discharge record. --Stage-discharge relation defined by current-meter measurement below 5, $900 \mathrm{cfs}$ and extended above on basis of current-meter measurement of peak at site 7 miles upstream. Stage-discharge relation unstable during flood periods; discharges were computed from detailed study of records for "near Bylaa" and "near Calva" stations.

Maxima. - - November 1965 to January 1966: Discharge, 39, 000 cfs about 19:0 hours Dec. 24 (gage height, $15.5 \mathrm{ft}$ ).

1929 to October 1965: Discharge, 27, $900 \mathrm{cfs}$ Oct. 1, 1941 (gage height, $11.82 \mathrm{ft}$, site and datum then in use).

Flood of Jan. 20, 1916, probably exceeded 100, $000 \mathrm{cfs}$, determined on basis of peak discharge at stations near Solomon and at Kelvin.

Mean discharge, in cubic feet per second, 1965-66

\begin{tabular}{|c|c|c|c|c|c|c|c|}
\hline Day & November & December & January & Day & November & December & January \\
\hline 1 & 39 & 106 & 15,500 & 16 & 36 & 1,100 & 919 \\
\hline 2 & 36 & 90 & 10,200 & $17 \ldots$ & 38 & 1,430 & 867 \\
\hline . & 27 & 89 & 7,920 & $18 \ldots$ & 41 & 2,190 & 840 \\
\hline $4 \ldots$ & 35 & 85 & 4,310 & $19 \ldots$ & 46 & 1,740 & 836 \\
\hline$\ldots$ & 40 & 85 & 2,570 & 20 & 46 & 1,270 & 821 \\
\hline ( & 41 & 88 & 2,000 & $21 \ldots$ & 45 & 1,100 & 807 \\
\hline 7 & 41 & 78 & 1,700 & $22 \ldots$ & 40 & 1,780 & 774 \\
\hline 8 & 42 & 64 & 1,460 & 23. & 43 & 3,130 & 735 \\
\hline 9 & 38 & 62 & 1,340 & 24 & 46 & 17,600 & 715 \\
\hline 10 & 32 & 646 & 1,210 & 25 & 49 & 14,900 & 685 \\
\hline 11 & 37 & 2,800 & 1,130 & 26 & 50 & 8,900 & 655 \\
\hline 12 & 35 & 3,050 & 1,100 & 27 & 73 & 5,290 & 625 \\
\hline 13 & 36 & 2,800 & 1,070 & 28 & 127 & $3,07 \mathrm{C}$ & 590 \\
\hline 14 & 34 & 1,400 & 1,020 & 29 & 149 & $2,09 \mathrm{C}$ & 563 \\
\hline \multirow[t]{2}{*}{$15 \ldots$} & 36 & 1,160 & 976 & $30 \ldots$ & 112 & $1,93 C$ & 544 \\
\hline & & & & $31 \ldots$ & $\ldots-.-$ & $3,24 C$ & 539 \\
\hline \multicolumn{5}{|c|}{$\begin{array}{l}\text { Monthly mean discharge, in cubic feet per second } . \\
\text { Runoff, in acre-feet } \ldots \ldots \ldots \ldots \ldots \ldots \ldots \ldots \ldots \ldots \ldots\end{array}$} & 49.7 & $2,68 \mathrm{c}$ & 2,385 \\
\hline
\end{tabular}


Gage height, in feet, and discharge, in cubic feet per second, at indicated tirze, 1965-66,

\begin{tabular}{|c|c|c|c|c|c|c|c|c|c|c|c|c|}
\hline Date & Hour & $\begin{array}{l}\text { Gage } \\
\text { height }\end{array}$ & $\begin{array}{c}\text { Dis- } \\
\text { charge }\end{array}$ & Date & Hour & $\begin{array}{l}\text { Gage } \\
\text { height }\end{array}$ & $\begin{array}{c}\text { Dis- } \\
\text { charge }\end{array}$ & Date & & Hour & $\begin{array}{l}\text { Gage } \\
\text { height }\end{array}$ & $\begin{array}{c}\text { Dis- } \\
\text { charge }\end{array}$ \\
\hline Dec. 21 & 2400 & 3.82 & 1,080 & Dec. 25 & $\begin{array}{l}1200 \\
1400\end{array}$ & $\begin{array}{l}11.6 \\
11.25\end{array}$ & $\begin{array}{l}12,500 \\
11,800\end{array}$ & Dec. 3 & & 2400 & 8.75 & 6,500 \\
\hline \multirow[t]{11}{*}{22} & 0200 & 3.87 & 1,110 & & 1600 & 11.0 & 11,200 & Jan. & 1 & 0100 & 10.75 & 10,000 \\
\hline & 1200 & 3. 85 & 1,100 & & 1800 & 10.95 & 10,800 & & & 0200 & 12.00 & 15,000 \\
\hline & 1330 & 4.65 & 1,640 & & 2200 & 11.0 & 10,100 & & & 0230 & 13.05 & 16,500 \\
\hline & 1430 & 4. 55 & 1,580 & & 2400 & 10.9 & 9,900 & & & 0300 & 13.35 & 18,000 \\
\hline & 1600 & 5.05 & 1,980 & & & & & & & 0400 & 13.65 & 19,000 \\
\hline & 1800 & 5.50 & 2,400 & 26 & 0400 & 10.8 & 9,800 & & & 0430 & 13.73 & 19,500 \\
\hline & 2000 & 6.15 & 2,890 & & 0800 & 10.5 & 9,700 & & & 0500 & 13.72 & 19,800 \\
\hline & 2100 & 6.34 & 3,120 & & 1200 & 10.25 & 9,400 & & & 0530 & 13.74 & 20,000 \\
\hline & 2350 & 6. 40 & 3,130 & & 1600 & 9.95 & 8,600 & & & 0630 & $1 \varepsilon .70$ & 19,500 \\
\hline & 2400 & 6. 95 & 3,880 & & 2000 & 9.6 & 7,700 & & & 0800 & 12.55 & 18,500 \\
\hline & & & & & 2400 & 9.2 & 6,900 & & & 1000 & 12.30 & 17,500 \\
\hline \multirow[t]{8}{*}{23} & 0030 & 7. 02 & 3,980 & & & & & & & 1200 & 13. 05 & 16,000 \\
\hline & 0200 & 6.82 & 3,230 & 27 & 0600 & 8.6 & 6,000 & & & 1500 & 12.65 & 14,500 \\
\hline & 0400 & 7. 04 & 3,560 & & 1230 & 7. 95 & 5,200 & & & 1800 & 12.25 & 13,500 \\
\hline & 0800 & 6.85 & 2,990 & & 1800 & 7.40 & 4,700 & & & 2400 & 11. 32 & 12,000 \\
\hline & 1300 & 6. 90 & 3,060 & & 2200 & 6.98 & 3,890 & & & & & \\
\hline & 1800 & 7. 15 & 3,130 & & 2400 & 6.82 & 3,660 & & 2 & 0600 & 10.62 & 10,800 \\
\hline & 2400 & 7. 40 & 3,350 & & & & & & & 0900 & 10.37 & 10,400 \\
\hline & & & & 28 & 0300 & 6.60 & 3,420 & & & 1200 & 10.20 & 10,000 \\
\hline \multirow[t]{15}{*}{24} & 0200 & 7.48 & 3,620 & & 0600 & 6.42 & 3,300 & & & 1800 & 9.93 & 9,600 \\
\hline & 0400 & 7.65 & 4,200 & & 1200 & 6. 14 & 3,050 & & & 2400 & 9.71 & 9,000 \\
\hline & 0600 & 7. 95 & 5,200 & & 1800 & 5.85 & 2,760 & & & & & \\
\hline & 0800 & 8.35 & 6,100 & & 2400 & 5.58 & 2,760 & & 3 & 0300 & 9.53 & 8,800 \\
\hline & 1000 & 8.90 & 6,800 & & & & & & & 0600 & 9.32 & 8,700 \\
\hline & 1250 & 9.84 & 8,700 & 29 & 1200 & 5.25 & 2,070 & & & 1200 & 8.72 & 8,000 \\
\hline & 1400 & 13.0 & 17,000 & & 2400 & 4. 98 & 1,780 & & & 1800 & 8.27 & 7,300 \\
\hline & 1600 & 15.1 & 28,000 & & & & & & & 2400 & 7.79 & 6,400 \\
\hline & 1700 & 15.4 & 37,000 & 30 & 0600 & 4. 91 & 1,750 & & & & & \\
\hline & 1800 & 15.55 & 38,000 & & 1500 & 5.00 & 1,860 & & 4 & 1200 & 7.00 & 4,100 \\
\hline & 1900 & 15.57 & 39,000 & & 2400 & 5.4 & 2,210 & & & 2400 & 6.13 & 2,850 \\
\hline & 2100 & 15.50 & 37,500 & & & & & & & & & \\
\hline & 2200 & 15.3 & 35,500 & 31 & 0900 & 5.85 & 2,700 & & 5 & 0600 & 5.89 & 2,700 \\
\hline & 2400 & 14.7 & 32,000 & & 1200 & 6. 15 & 2,930 & & & 1200 & 5.70 & 2,600 \\
\hline & & & & & 1800 & 6.73 & 3,550 & & & 2400 & 5.30 & 2,200 \\
\hline \multirow[t]{4}{*}{25} & 0200 & 14.1 & 25,000 & & 2000 & 7.03 & 3,840 & & & & & \\
\hline & 0400 & 13.5 & 19,500 & & 2200 & 7. 50 & 4,500 & & 6 & 1200 & 5.06 & 2,000 \\
\hline & 0600 & 12.9 & 17,500 & & 2300 & 7. 90 & 5,100 & & & 2400 & 4. 88 & 1,800 \\
\hline & 0800 & 12.4 & 15,500 & & & & & & & & & \\
\hline
\end{tabular}

(43) 9-4671.2. Salt Creek near Peridot, Ariz. (Crest-stage station)

Lacation. --Lat $33^{\circ} 16^{\prime} 15^{\prime \prime}$, long $110^{\circ} 18^{\prime} 15^{\prime \prime}$, at U.S. Highway 70,4 miles above mouth, and 9.5 miles southeast of Peridot.

Drainage area. $--34.2 \mathrm{sq} \mathrm{mi}$.

Gage-height record. --Crest stages only. Altitude of gage is 2,800 ft (from topographic map).

Discharge record. --Stage-discharge relation defined by computations of flow through culverts.

Maxima. --November 1965 to January 1966: Discharge, 1, 270 cfs Dec. 22. (gage height, $15.71 \mathrm{ft}$ ).

1964 to October 1965: Discharge, $604 \mathrm{cfs}$ Aug. 13, 1964 (gage height, 13. $11 \mathrm{ft}$ ). 
(44) 9-4685. San Carlos River near Peridot, Ariz.

Location. -- Lat $33^{\circ} 19^{\prime} 20^{\prime \prime}$, long $110^{\circ} 26^{\prime} 50^{\prime \prime}$, in NW $\frac{1}{4}$ sec. 30, T. 1 S. , R. 19 E. (unsurveyed), in San Carlos Indian Reservation, at downstream side of concrete pier near right end of highway bridge, 2 miles downstream from San Carlos, and 2 miles upstream from Peridot.

Drainage area. $--1,027 \mathrm{sq} \mathrm{mi}$.

Gage-height record. - Water-stage recorder graph except Nov. 27-29, Dec. 20, 25-26, and short periods Dec. 12, 22-24, 30 which were reconstructed from adjoining record. Datum of gage is 2,579.49 ft above mean sea level. Prior to D?c. 22, datum of gage was $2.22 \mathrm{ft}$ higher.

Discharge record. --Stage-discharge relation defined by current-meter measurements below 11,000 $\mathrm{cfs}$ and extended above on basis of contracted-opening measurement of peak flow.

Maxima. --November 1965 to January 1966: Discharge, 36, 300 cfs 1400 hoi'rs Dec. 22 (gage height, $14.8 \mathrm{ft}$ ).

1929 to October 1965: Discharge, $40,600 \mathrm{cfs}$ Mar. 14, 1941 (gage height, 11. $4 \mathrm{ft}$, at site 4 miles downstream at different datum), from rating extended above $23,000 \mathrm{cfs}$ on basis of rate of change in storage in San Carlos Reservoir and verifiet by contracted-opening measurement of the Dec. 22, 1965, peak.

Mean discharge, in cubic feet per second, 1965-66

\begin{tabular}{|c|c|c|c|c|c|c|c|}
\hline Day & November & December & January & Day & November & December & January \\
\hline 1 & 3.2 & 8.5 & 732 & $16 \ldots$ & 4.0 & 547 & 26 \\
\hline $2 \ldots$ & 3.2 & 8. 2 & 380 & $17 \ldots$ & 4.2 & 1,100 & 24 \\
\hline $3 \ldots$ & 3.2 & 8.0 & 230 & $18 \ldots$ & 4.2 & 909 & 29 \\
\hline $4 \ldots$ & 3.2 & 8.0 & 182 & $19 \ldots$ & 4. 2 & 528 & 34 \\
\hline $5 \ldots$ & 3.4 & 8. 0 & 138 & $20 \ldots$ & 4.2 & 340 & 34 \\
\hline $6 \ldots$ & 3.4 & 8.2 & 105 & $21 \ldots$ & 4.4 & 308 & 37 \\
\hline $7 \ldots$ & 3.5 & 8.2 & 85 & $22 \ldots$ & 4.7 & 14,700 & 37 \\
\hline $8 \ldots$ & 3.4 & 8.2 & 69 & $23 \ldots$ & 6.0 & 12,200 & 31 \\
\hline $9 \ldots$ & 3.5 & 12 & 56 & $24 \ldots$ & 8.0 & 1,080 & 28 \\
\hline $10 \ldots$ & 3.5 & 2,810 & 48 & $25 \ldots$ & 269 & 380 & 26 \\
\hline $11 \ldots$ & 3.7 & 2,470 & 43 & 26 & 453 & 270 & 24 \\
\hline $12 \ldots$ & 3.7 & 389 & 38 & 27 & 80 & 234 & 22 \\
\hline $13 \ldots$ & 4. 0 & 296 & 32 & 28. & 30 & 185 & 22 \\
\hline $14 \ldots$ & 3.9 & 482 & 30 & $29 \ldots$ & 15 & 146 & 21 \\
\hline \multirow[t]{2}{*}{$15 \ldots$} & 4. 0 & 726 & 28 & 30 . & 10 & 4,980 & 20 \\
\hline & & & & 31 & $\ldots \ldots$ & 3,850 & 23 \\
\hline \multirow{2}{*}{\multicolumn{5}{|c|}{$\begin{array}{l}\text { Monthly mean discharge, in cubic feet per second.. } \\
\text { Runoff, in acre-feet } \ldots \ldots \ldots \ldots \ldots \ldots \ldots \ldots\end{array}$}} & 31.8 & 1,581 & 85.0 \\
\hline & & & & & 1,890 & 97,200 & 5,220 \\
\hline
\end{tabular}


Gage height, in feet, and discharge, in cubic feet per second, at indicated time, 1965-66, of San Carlos River near Peridot, Ariz.

\begin{tabular}{|c|c|c|c|c|c|c|c|c|c|c|c|}
\hline Date & Hour & $\begin{array}{c}\text { Gage } \\
\text { height }\end{array}$ & $\begin{array}{c}\text { Dis- } \\
\text { charge }\end{array}$ & Date & Hour & $\begin{array}{l}\text { Gage } \\
\text { height }\end{array}$ & $\begin{array}{c}\text { Dis- } \\
\text { charge }\end{array}$ & Date & Hour & $\begin{array}{c}\mathrm{G} \varepsilon \text { ge } \\
\text { height }\end{array}$ & $\begin{array}{c}\text { Dis- } \\
\text { charge }\end{array}$ \\
\hline Dec. 21 & 2400 & 5.26 & 250 & Dec. 23 & 2400 & 5.64 & 2,260 & Dec. 30 & 2400 & 8.3 & 7,400 \\
\hline \multirow[t]{10}{*}{22} & 0300 & 5. 23 & 238 & 24 & 0600 & 4.84 & 1,340 & 31 & 0200 & 9.6 & 11,200 \\
\hline & 0930 & 5.34 & 282 & & 1200 & 4. 31 & 888 & & 0500 & 8.7 & 8,500 \\
\hline & 1000 & 5.67 & 430 & & 1800 & 3.97 & 652 & & 0800 & 7. 3 & 5,100 \\
\hline & 1200 & 11.39 & 9,910 & & 2400 & 3.64 & 470 & & 1000 & 5. 76 & 2,440 \\
\hline & 1330 & 14.47 & 34,000 & & & & & & 1200 & 5.62 & 2,230 \\
\hline & 1400 & 14.80 & 36,300 & 29 & 2400 & 2.60 & 109 & & 1500 & 5. 04 & 1,540 \\
\hline & 1630 & 14.52 & 34,300 & & & & & & 1800 & 4. 80 & 1,290 \\
\hline & 1800 & 14. 31 & 32,900 & 30 & 0830 & .77 & 5 & & 2400 & 4.69 & 1,160 \\
\hline & 2400 & 14. 07 & 31,400 & & 0930 & 2.70 & 123 & & & & \\
\hline & & & & & 1030 & 4. 79 & 1,290 & Jan. 1 & 0500 & 4. 46 & 944 \\
\hline \multirow[t]{6}{*}{23} & 0300 & 13.64 & 28,800 & & 1130 & 8.5 & 7,900 & & 0900 & 4. 09 & 652 \\
\hline & 0915 & 9.84 & 11,900 & & 1230 & 11.00 & 16,500 & & 1200 & 3. 96 & 556 \\
\hline & 1300 & 7. 94 & 6,500 & & 1330 & 10.7 & 15,300 & & 2400 & 3. 79 & 455 \\
\hline & 1600 & 6. 89 & 4,280 & & 1500 & 9.6 & 11,200 & & & & \\
\hline & 1800 & 6. 14 & 3,010 & & 2000 & 7. 7 & 5,900 & 2 & 1200 & 3.60 & 342 \\
\hline & 2000 & 6.09 & 2,940 & & 2200 & 7.3 & 5,100 & & 2400 & 3.41 & 250 \\
\hline
\end{tabular}

(45) 9-4690. San Carlos Reservoir at Coolidge Dam, Ariz.

Location. -- Lat $33^{\circ} 10^{1} 30^{\prime \prime}$, long $110^{\circ} 31^{\prime 4} 45^{\prime \prime}$, in NW $\frac{1}{4}$ sec. 17, T. 3 S., R. 18 E. (unsurveyed), in San Carlos Indian Reservation, at right intake tower of Coolidge Dam on Gila River.

Drainage area. $--12,886 \mathrm{sq} \mathrm{mi}$.

Gage-height record. - Water-stage recorder graph except Nov. 15-17 which was reconstructed. Datum of gage is mean sea level, datum of 1929 .

Contents. - - Contents computed from capacity table based on 1947 survey by Corps of Engineers and Soil Conservation Service.

Maxima. --November 1965 to January 1966: Contents, 374, 300 acre-ft Jan. 31 (elevation, $2,465.00 \mathrm{ft}$ ).

1928 to October 1965: Contents, 819,200 acre-ft Mar, 18, 1942 (elevation, $2,501.62 \mathrm{ft}$ ).

Remarks. --Reservoir is formed by concrete multiple-dome dam completed Oct. 25, 1928. Storage began Nov. 15, 1928. Usable capacity, 1, 206, 000 acre-ft between elevations 2,382.63 (sill of outlet gate) and 2,523. $0 \mathrm{ft}$ (top of automatic spillway gates in raised position). Dead storage below elevation $2,382.63 \mathrm{ft}, 3,834$ acre-ft. Figures given herein represent usable contents. 
Contents, in acre-feet, at 2400 hours, 1965-66, of San Carlos Reservoir at Coolidge Dam, Ariz.

\begin{tabular}{|c|c|c|c|c|c|c|c|}
\hline Day & November & December & January & Day & November & December & January \\
\hline & 4,640 & 9,400 & 275,600 & 16 & 5,940 & 51,750 & 356,500 \\
\hline 2 & 4,730 & 9,490 & 300,900 & 17. & 6,030 & $56,2 \hat{0} 0$ & 357,700 \\
\hline$\ldots$ & 4,800 & 9,540 & 316,000 & $18 \ldots$ & 6,120 & 62,180 & 359,400 \\
\hline $4 \ldots$ & 4,880 & 9,530 & 324,900 & 19. & 6,220 & 67,300 & 360,900 \\
\hline $5 \ldots$ & 4,960 & 9,460 & 330,400 & 20 & 6,340 & $71,1 \approx 0$ & 362,300 \\
\hline & 5,040 & 9,380 & 334,400 & 21. & 6,440 & $73,7 \in 0$ & 363,700 \\
\hline & 5,140 & 9,310 & 337,600 & 22 & 6,610 & $90,0 \varepsilon 0$ & 364,900 \\
\hline 8 & 5,230 & 9,200 & 340,300 & 23 & 6,790 & 123,100 & 366,200 \\
\hline 9 . & 5,330 & 9,200 & 342,700 & 24 & 6,950 & $133,0 \mathrm{co}$ & 367,400 \\
\hline 10. & 5,430 & 11,840 & 345,200 & 25 & 7,310 & $172,1 C 0$ & 368,400 \\
\hline 11. & 5,500 & 24,540 & 347,300 & $26 \ldots$ & 8,220 & 199,400 & 369,500 \\
\hline 12 . & 5,560 & 31,890 & 349,200 & 27 . & 8,540 & 214,400 & 370,400 \\
\hline 13. & 5,660 & 38,800 & 351,000 & 28 & 8,740 & 222,200 & 371,400 \\
\hline 14 & 5,750 & 44,160 & 352,900 & 29 & 9,050 & 227,500 & 372,400 \\
\hline $15 \ldots$ & 5,850 & 48,050 & 354,600 & 30 & 9,290 & 241,000 & 373,500 \\
\hline & & & & 31 & $\ldots \ldots$ & 253,400 & 374,300 \\
\hline \multicolumn{5}{|c|}{ Change in contents, in acre-feet } & $+4,720$ & $+244,110$ & $+120,900$ \\
\hline
\end{tabular}

(46) 9-4695. Gila River below Coolidge Dam, Ariz.

Location. -- Lat $33^{\circ} 10^{\prime} 15^{\prime \prime}$, long $110^{\circ} 31^{\prime} 45^{\prime \prime}$, in SW $\frac{1}{4}$ sec. 17 , T. 3 S., R. 18 E. (unsurveyed), on left bank 2,200 ft downstream from Coolidge Dam.

Drainage area. $--12,886 \mathrm{sq} \mathrm{mi}$.

Gage-height record. - -Water-stage recorder graph except Dec. 29 to Jan. 4; graph was reconstructed. Datum of gage is $2,309.33 \mathrm{ft}$ above mean sea level, datum of 1929 .

Discharge record. --Stage-discharge relation defined by current-meter measurements.

Maxima. --November 1965 to January 1966: Discharge, 5 cfs 0830 hours Dic. 22 (gage height, $0.18 \mathrm{ft}$ ).

1914-27: Discharge, 130,000 cfs Jan. 20, 1916, estimated on basis of peak discharge near Solomon and at Kelvin.

1928 to October 1965: Discharge, 1, 350 efs July 28, 1952 (gage height, $4.64 \mathrm{ft}$ ).

Remarks. -- Flow regulated by San Carlos Reservoir at Coolidge Dam since 1928.

Mean discharge, in cubic feet per second, 1965-66

\begin{tabular}{|c|c|c|c|c|c|c|c|}
\hline Day & November & December & January & Day & November & December & January \\
\hline 1 & 1.6 & 72 & 2 & 16 & 1.6 & 3.2 & 3.7 \\
\hline $2 \ldots$ & 1. 6 & 51 & 2 & & 1.6 & 3.2 & 3.2 \\
\hline $3 \ldots$ & 1.6 & 55 & 1 & 18 & 1.6 & 3.2 & 2.4 \\
\hline $4 \ldots$ & 1.6 & 85 & 1.0 & 19 & 1.6 & 3.2 & 3. 7 \\
\hline $5 \ldots$ & 1.6 & 102 & 1. 0 & 20 & 1.6 & 3.2 & 2. 8 \\
\hline 6. & 1.6 & 102 & 1.6 & 21 & 1.6 & 3.2 & 3.2 \\
\hline 7 & 1.6 & 102 & 1.4 & 22. & 1.6 & 4.2 & 9.5 \\
\hline 8. & 1.6 & 102 & 1.4 & 23. & 1.6 & 3.7 & 4. 8 \\
\hline $9 \ldots$ & 1.6 & 102 & 1.4 & 24. & 1.6 & 3.7 & 8. 0 \\
\hline 10 . & 1.6 & 41 & 2.0 & $25 \ldots$ & 1.6 & 3.7 & 3.7 \\
\hline $11 \ldots$ & 1.6 & 3.2 & 2.4 & $26 \ldots$ & 1.6 & 3.7 & 2.4 \\
\hline $12 \ldots$ & 1.6 & 3.2 & 2.4 & $27 \ldots$ & 1.6 & 3.2 & 3.7 \\
\hline $13 \ldots$ & 1.6 & 3.2 & 2.0 & $28 \ldots \ldots$ & 1.6 & 3.7 & 3.7 \\
\hline $14 \ldots$ & 1.6 & 3.2 & 2.0 & $29 \ldots$ & 1.6 & 2.4 & 2. 8 \\
\hline \multirow[t]{2}{*}{$15 \ldots$} & 1.6 & 3.7 & 2.8 & $30 \ldots$ & 37 & 2 & 2. 8 \\
\hline & & & & 31 & $\ldots . .$. & 2 & 3.2 \\
\hline \multirow{2}{*}{\multicolumn{5}{|c|}{$\begin{array}{l}\text { Monthly mean discharge, in cubic feet per second.. } \\
\text { Runoff, in acre-feet } \ldots \ldots \ldots \ldots \ldots \ldots \ldots \ldots \ldots \ldots\end{array}$}} & 2.78 & 28.4 & 2. 92 \\
\hline & & & & & 165 & 1,750 & 179 \\
\hline
\end{tabular}


(47) 9-4700. Gila River at Winkelman, Ariz.

Location. - - Lat $32^{\circ} 59^{\prime} 05^{\prime \prime}$, long $110^{\circ} 46^{\prime} 20^{\prime \prime}$, in SW $\frac{1}{4} \mathrm{NW} \frac{1}{4} \sec .24$, T. $5 \mathrm{~S}$., R. $15 \mathrm{E}$., on bridge on State Highway 77 at Winkelman, half a mile upstream from San Pedro River, and 30 miles downstream from Coolidge Dam.

Drainage area. $--13,268 \mathrm{sq} \mathrm{mi}$, of which $382 \mathrm{sq} \mathrm{mi}$ is below Coolidge Dam.

Gage-height record. - - Water-stage recorder graph except 0600 hours Dec. 29 to 0300 hours Dec. 30 and Jan. 2-10, supplemented by once-daily staff-gage readings except on weekends. Datum of gage is $1,907.00 \mathrm{ft}$ above mean sea level, datum of 1927 .

Discharge record. --Stage-discharge relation defined by current-meter meas'rements below 1,600 cfs and extended above on basis of slope-area measurement of peak flow. Stage-discharge relation was affected by backwater from the San Pedro River Dec. $10-11,15-18,2000$ hours Dec. 22 to Dec. 25. Discharge computed by correcting for an estimated amount of backwater.

Maxima. --November 1965 to January 1966: Discharge, 6, 200 cfs 0330 hours Dec. 23 (gage height, $12.06 \mathrm{ft}$ ), from slope-area measurement of peak flow.

1917-18, 1928 to October 1965: Discharge, $55,000 \mathrm{cfs}$, of which $500 \mathrm{cfs}$ was released by Coolidge Dam, Aug. 9, 1944 (gage height, $18.40 \mathrm{ft}$, at base gage $1 \frac{1}{2}$ miles upstream), from rating curve extended above $2,900 \mathrm{cfs}$ on basis of slope-area measurement of peak flow.

Remarks. - Gage used during flood period is that shown as a supplementary gage in annual publication of surface-water records.

Mean discharge, in cubic feet per second, 1965-66

\begin{tabular}{|c|c|c|c|c|c|c|c|}
\hline Day & November & December & January & Day & November & December & January \\
\hline $1 \ldots$ & 3.7 & 31 & 246 & $16 \ldots$ & 5.0 & 200 & 64 \\
\hline $2 \ldots$ & 3.7 & 69 & 184 & $17 \ldots$ & 5.0 & 300 & 60 \\
\hline $3 \ldots$ & 3.7 & 57 & 140 & $18 \ldots$ & 5.0 & 200 & 74 \\
\hline $4 \ldots$ & 3.7 & 61 & 120 & $19 \ldots$ & 5.0 & 156 & 84 \\
\hline $5 \ldots$ & 3.0 & 91 & 110 & $20 \ldots$ & 5.8 & 109 & 76 \\
\hline $6 \ldots$ & 3.0 & 103 & 100 & 21 & 6.6 & 86 & 76 \\
\hline $7 \ldots$ & 3.7 & 103 & 90 & 22 & 7.4 & 1,270 & 71 \\
\hline $8 \ldots$ & 3.7 & 101 & 85 & 23 & 11 & 1,500 & 71 \\
\hline $9 \ldots$ & 3.7 & 105 & 82 & $24 \ldots$ & 12 & 600 & 65 \\
\hline $10 \ldots$ & 3.7 & 300 & 78 & 25. & 20 & 450 & 64 \\
\hline $11 \ldots$ & 4.3 & 800 & 76 & $26 \ldots$ & 61 & 285 & 59 \\
\hline $12 \ldots$ & 3.7 & 159 & 74 & $27 \ldots$ & 24 & 256 & 55 \\
\hline $13 \ldots$ & 4.3 & 74 & 71 & $28 \ldots$ & 16 & 211 & 52 \\
\hline $14 \ldots$ & 5.0 & 103 & 69 & $29 \ldots$ & 11 & 171 & 52 \\
\hline \multirow[t]{2}{*}{$15 \ldots$} & 5.8 & 350 & 65 & 30. & 8.3 & 536 & 52 \\
\hline & & & & 31 & $\ldots \ldots$ & 485 & 59 \\
\hline \multirow{2}{*}{\multicolumn{5}{|c|}{$\begin{array}{l}\text { Monthly mean discharge, in cubic feet per second. } \\
\text { Runoff, in acre-feet } \ldots \ldots \ldots \ldots \ldots \ldots \ldots \ldots \ldots\end{array}$}} & 8.73 & 301 & 84.6 \\
\hline & & & & & 519 & 18,490 & 5,200 \\
\hline
\end{tabular}


(48) 9-4705. San Pedro River at Palominas, Ariz.

Location. -- Lat $31^{\circ} 22^{\prime} 48^{\prime \prime}$, long $110^{\circ} 06^{\prime} 38^{\prime \prime}$, on section line between sec. $₹ 3$, T. $23 \mathrm{~S}$., R. 22 E. and sec. 4, T. 24 S., R. 22 E., near left bank on downstream gide of State Highway 92, 0.7 mile east of Palominas, $2 \frac{1}{2}$ miles upstream from Green Brush Draw, $4 \frac{1}{2}$ miles downstream from international boundary, and 12 miles southv'est of Bisbee.

Drainage area. $--741 \mathrm{sq} \mathrm{mi}$, of which $649 \mathrm{sq} \mathrm{mi}$ is in Mexico.

Gage-height record. --Water-stage recorder graph except Dec. 24 to Jan. 10. Datum of gage is $4,187.62 \mathrm{ft}$ above mean sea level (from Arizona Highway Department bench mark).

Discharge record. --Stage-discharge relation defined by current-meter miasurements. Discharge for period of no gage-height record estimated.

Maxima. --November 1965 to January 1966: Discharge, 798 cfs 1600 hours Dec. 23 (gage height, $5.05 \mathrm{ft}$ ).

1927 to October 1965: Discharge, 22, $000 \mathrm{cfs}$ Aug. 14, 1940 (gage height, 16. $16 \mathrm{ft}$, present datum), from rating curve extended above 5,600 cfs on basis of slope-area measurement.

Greatest flood known occurred Sept. 28, 1926 (gage height, about 23. $9 \mathrm{ft}$, present datum, from floodmarks; discharge not determined).

Mean discharge, in cubic feet per second, 1965-66

\begin{tabular}{|c|c|c|c|c|c|c|c|c|}
\hline Day & December & January & Day & December & January & Day & Decenther & January \\
\hline & 0 & 16 & 11 & 22 & 8.0 & 21 & 30 & 12 \\
\hline & 0 & 18 & 12 & 13 & 8. 0 & 22 & 103 & 10 \\
\hline 3 & 0 & 15 & 13 & 10 & 8. 0 & $23 \ldots$ & 698 & 9.6 \\
\hline $4 \ldots$ & 0 & 14 & $14 \ldots$ & 13 & 8. 0 & $24 \ldots$ & 350 & 9.6 \\
\hline $5 \ldots$ & 0 & 13 & $15 \ldots$ & 29 & 7.0 & $25 \ldots$ & 140 & 9.1 \\
\hline $6 \ldots$ & 0 & 13 & $16 \ldots$ & 27 & 7.0 & $26 \ldots$ & 80 & 8.6 \\
\hline $7 \ldots$ & 0 & 12 & $17 \ldots$ & 147 & 7. 0 & $27 \ldots$ & 45 & 9.6 \\
\hline $8 \ldots$ & 0 & 11 & 18 & 88 & 13 & 28 . & 30 & 10 \\
\hline $9 \ldots$ & 4.8 & 10 & 19 & 52 & 13 & 29 & 25 & 8. 6 \\
\hline \multirow[t]{2}{*}{$10 \ldots$} & 32 & 9.0 & $20 \ldots$ & 38 & 13 & 30 & 18 & 8.2 \\
\hline & & & & & & 31 & 15 & 8.2 \\
\hline \multirow{2}{*}{\multicolumn{7}{|c|}{$\begin{array}{l}\text { Monthly mean discharge, in cubic feet per second } \ldots \ldots \ldots \ldots \ldots \ldots \\
\text { Runoff in acre-feet } \ldots \ldots \ldots \ldots \ldots \ldots \ldots\end{array}$}} & 64.8 & 10.5 \\
\hline & & & & & & & 3,990 & 648 \\
\hline
\end{tabular}

(49) 9-4710. San Pedro River at Charleston, Ariz.

Location. -- Lat $31^{\circ} 37^{\prime} 40^{\prime \prime}$, long $110^{\circ} 10^{\prime} 30^{\prime \prime}$, in $\mathrm{NE}_{\frac{1}{4}} \mathrm{NE} \frac{1}{4}$ sec. 11 , T. $21 \mathrm{~S}$. , R. 21 E. , in Spanish land grant of San Juan de las Boquillas y Nogales, at downstream side of pier near center of highway bridge, a quarter of a mile south of Charleston, $1 \frac{1}{2}$ miles upstream from Charleston damsite, $8 \frac{1}{2}$ miles upstream from Babocomari River, and 29 miles upstream from Benson.

Drainage area. $--1,219 \mathrm{sq} \mathrm{mi}$, of which $696 \mathrm{sq} \mathrm{mi}$ is in Mexico.

Gage-height record. - Water-stage recorder graph. Datum of gage is 3,954.01 ft above mean sea level, datum of 1929 , supplementary adjustment of 1958 .

Discharge record. --Stage-discharge relation defined by current-meter measurements.

Maxima. - - November 1965 to January 1966: Discharge, 690 cfs 0830 hours Dec. 23 (gage height, $4.47 \mathrm{ft}$ ).

1904 to October 1965: Discharge, 98, $000 \mathrm{cfs}$ Sept. 28, 1926 (gage height, $21.9 \mathrm{ft}$, site and datum then in use), by slope-area measurement of peak flow. 
Mean discharge, in cubic feet per second, 1965-66, of San Pedro River

\begin{tabular}{|c|c|c|c|c|c|c|c|}
\hline Day & November & December & January & Day & November & December & January \\
\hline $1 \ldots$ & 4.2 & 8.0 & 32 & 16 & 6.5 & 25 & 17 \\
\hline $2 \ldots$ & 4.2 & 7. 7 & 34 & 17 & 6.5 & 92 & 18 \\
\hline $3 \ldots$ & 4.2 & 7.4 & 29 & $18 \ldots$ & 6.8 & 129 & 24 \\
\hline $4 \ldots$ & 4.4 & 7. 1 & 26 & $19 \ldots$ & 6.8 & 66 & 24 \\
\hline $5 \ldots$ & 4.6 & 7. 1 & 25 & $20 \ldots$ & 6.8 & 40 & 24 \\
\hline $6 \ldots$ & 4.8 & 7.7 & 25 & $21 \ldots$ & 6.8 & 30 & 26 \\
\hline $7 \ldots$ & 5.0 & 8. 0 & 24 & $22 \ldots$ & 7.4 & 33 & 25 \\
\hline $8 \ldots$ & 5.0 & 9.5 & 23 & 23 & 8.0 & 508 & 25 \\
\hline $9 \ldots$ & 5.3 & 30 & 22 & 24 & 8.0 & 382 & 23 \\
\hline $10 \ldots$ & 5.3 & 26 & 19 & 25 & 7.7 & 152 & 23 \\
\hline $11 \ldots$ & 5.3 & 26 & 18 & 26 & 7.4 & 97 & 22 \\
\hline $12 \ldots$ & 5.6 & 18 & 19 & $27 \ldots$ & 7.1 & 58 & 21 \\
\hline $13 \ldots$ & 5.9 & 16 & 19 & $28 \ldots \ldots$ & 7.4 & 45 & 22 \\
\hline $14 \ldots$ & 5.9 & 16 & 18 & $29 \ldots$ & 7.7 & 37 & 22 \\
\hline $15 \ldots$ & 6.2 & 19 & 17 & $30 \ldots$ & 8.0 & 30 & 21 \\
\hline & & & & 31. & $\ldots \ldots$ & 28 & 21 \\
\hline \multicolumn{5}{|c|}{$\begin{array}{l}\text { Monthly mean discharge, in cubic feet per second.. } \\
\text { Runoff, in acre-feet } \ldots \ldots \ldots \ldots \ldots \ldots \ldots \ldots \ldots \ldots\end{array}$} & $\begin{array}{r}6.16 \\
367\end{array}$ & $\begin{array}{r}63.4 \\
3,900 \\
\end{array}$ & $\begin{array}{r}22.8 \\
1,400\end{array}$ \\
\hline
\end{tabular}

(50) 9-4720. San Pedro River near Redington, Ariz.

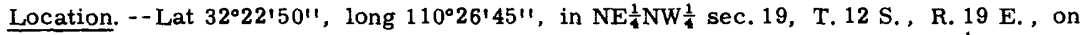
left bank, a quarter of a mile upstream from Cochise-Pima County line, $4 \frac{1}{2}$ miles upstream from Redington, and 30 miles north of Benson.

Drainage area. $--2,939 \mathrm{sq} \mathrm{mi}$, of which $696 \mathrm{sq} \mathrm{mi}$ is in Mexico.

Gage-height record. - -Water-stage recorder graph except Dec. 18 to 0800 hours Dec. 22, Dec. 25-28. Datum of gage is $2,930.04 \mathrm{ft}$ above mean sea level, datum of 1929 .

Discharge record. --Stage-discharge relation defined by current-meter measurements. Discharge for periods of no gage-height record estimated.

Maxima. - November 1965 to January 1966: Discharge, 5, 020 cfs 1500 hours Dec. 22 (gage height, $13.45 \mathrm{ft}$ ).

1943 to October 1965: Discharge, 28,600 cfs Aug. 2, 1951 (gage height, $20.2 \mathrm{ft}$, present datum):

Greatest discharge known, about 90,000 cfs Sept. 28, 1926 (gage height, $29.0 \mathrm{ft}$, present datum, from floodmark), computed on basis of records for San Pedro River at Charleston and Gila River at Kelvin.

Mean discharge, in cubic feet per second, 1965-66

\begin{tabular}{|c|c|c|c|c|c|c|c|}
\hline Day & November & December & January & Day & November & December & January \\
\hline $1 \ldots$ & 0.1 & 0.6 & 14 & $16 \ldots$ & 0.3 & 33 & 1.1 \\
\hline $2 \ldots$ & 1 & .6 & 4.2 & $17 \ldots$ & .3 & 274 & 1. 1 \\
\hline $3 \ldots$ & .1 & 6 & 3.2 & $18 \ldots$ & .2 & 83 & 1. 4 \\
\hline $4 \ldots$ & .1 & 6 & 2.9 & $19 \ldots$ & .3 & 103 & 1.4 \\
\hline . & .1 & .6 & 2.9 & $20 \ldots$ & .3 & 40 & 1.2 \\
\hline $6 \ldots$ & .2 & 6 & 2.9 & $21 \ldots$ & .3 & 30 & 1. 1 \\
\hline $7 \ldots$ & .1 & .6 & 2.9 & $22 \ldots$ & .4 & 1,580 & .8 \\
\hline 8 & .2 & 7 & 2.6 & $23 \ldots$ & .5 & 1,490 & .8 \\
\hline 9 . & .2 & 2.2 & 2.6 & $24 \ldots$ & .6 & 936 & .8 \\
\hline 10 . & .2 & 276 & 2.2 & 25 & .8 & 640 & .7 \\
\hline 11. & .3 & 972 & 2.0 & 26 . & .6 & 300 & .8 \\
\hline $12 \ldots$ & .3 & 110 & 1.8 & $27 \ldots$ & .6 & 125 & .8 \\
\hline $13 \ldots$ & .3 & 75 & 1.6 & $28 \ldots$ & .6 & 50 & 1. 0 \\
\hline $14 \ldots$ & .3 & 55 & 1.4 & $29 \ldots$ & .6 & 26 & 1.0 \\
\hline \multirow[t]{2}{*}{$15 \ldots$} & .3 & 52 & 1.2 & $30 \ldots$ & .6 & 85 & 1. 0 \\
\hline & & & & & $\cdots-\cdots$ & 83 & 5.1 \\
\hline \multirow{2}{*}{\multicolumn{5}{|c|}{$\begin{array}{l}\text { Monthly mean discharge, in cubic feet per second.. } \\
\text { Runoff, in acre-feet } \ldots \ldots \ldots \ldots \ldots \ldots \ldots \ldots \ldots \ldots\end{array}$}} & 0.33 & 240 & 2. 21 \\
\hline & & & & & 20 & 14,730 & 136 \\
\hline
\end{tabular}


(51) 9-4730. Aravaipa Creek near Mammoth, Ariz.

(Discontinued gaging station, previously published as "near Feldman")

Location. --Lat $32^{\circ} 50^{\prime} 40^{\prime \prime}$, long $110^{\circ} 37^{\prime} 45^{\prime \prime}$, in NW $\frac{1}{4} \mathrm{NE} \frac{1}{4}$ sec. 8, T. 7 S. , R. 17 E., 2 miles downstream from Holy Joe Canyon, 6 miles upstream from mouth, and $8 \frac{1}{2}$ miles north of Mammoth (half a mile downstream from former gaging station).

Drainage area. - $-538 \mathrm{sq} \mathrm{mi}$ at former gaging station half a mile upstream.

Maxima. --November 1965 to January 1966: Discharge, 6, 340 cfs Dec. 22, from slopearea measurement of peak flow.

1919-21, 1931-42, 1965: Discharge, 20, 000 cfs Aug. 2, 1919 (gage height, 6. 3 ft, at site 6 miles downstream at different datum), from rating curve extended above $5,100 \mathrm{cfs}$ on basis of velocity-area study.

(52) 9-4734. San Pedro River near Winkelman, Ariz.

Location. -- Lat $32^{\circ} 56^{\prime} 35^{\prime \prime}$, long $110^{\circ} 44^{\prime} 55^{\prime \prime}$, in SW $\frac{1}{4} \mathrm{NE} \frac{1}{4}$ sec. 6 , T. $6 \mathrm{~S}$. , R. 16 E., on right bank, $3 \frac{1}{2}$ miles southeast of Winkelman, and 4 miles upstream from mouth.

Drainage area. $--4,449 \mathrm{sq} \mathrm{mi}$, of which $696 \mathrm{sq} \mathrm{mi}$ is in Mexico.

Gage-height record. --Water-stage recorder graph until Dec, 13. Altitude of gage is $1,990 \mathrm{ft}$ (from topographic map).

Discharge record. --Stage-discharge relation defined by current-meter measurements. Discharge for period Dec. 13-31 estimated.

Maxima. --November 1965 to January 1966: Discharge, 16, 800 cfs about 2400 hours Dec. 22 (gage height unknown), from slope-area measurement of peak flow.

1962 to October 1965: Discharge, 6,460 cfs Aug. 15, 1964 (gage height, 7. $7 \mathrm{ft}$ ).

Remarks. --Gage was destroyed by flood of Dec. 22, 1965.

Mean discharge, in cubic feet per second, 1965

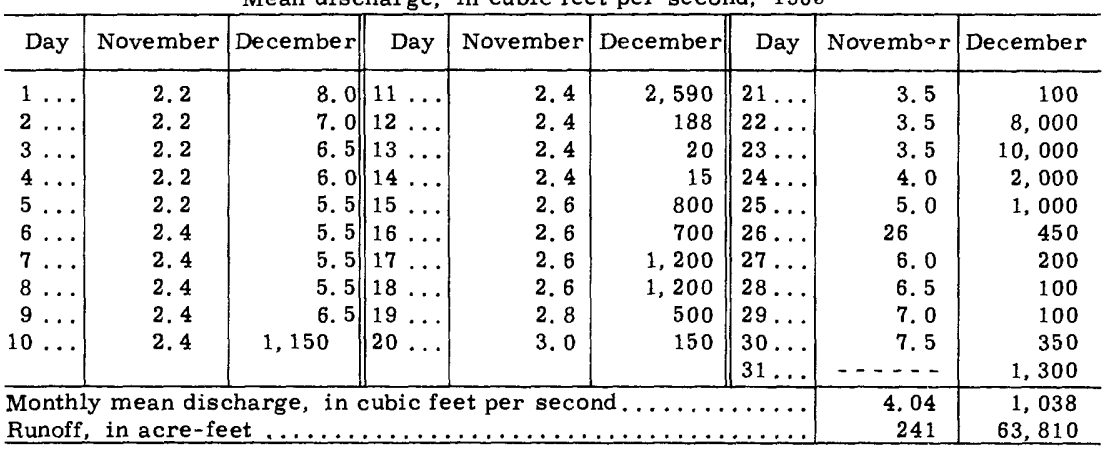


(53) 9-4736. Tam O'Shanter Wash near Hayden, Ariz.

\section{(Crest-stage station)}

Location. -- Lat $33^{\circ} 01^{\prime} 46^{\prime \prime}$, long $110^{\circ} 52^{\prime} 22^{\prime \prime}$, in $S E_{\frac{1}{4}} N W^{\frac{1}{4}}$ sec. 1, T. 5 S. , R. 14 E., at State Highway 177, 6 miles west of Hayden.

Drainage area. $--4.37 \mathrm{sq} \mathrm{mi}$.

Gage-height record. --Crest stages only. Altitude of gage is 1,900 ft (from tcpographic map).

Discharge record. --Stage-discharge relation defined by computations of flow through culvert.

Maxima. --November 1965 to January 1966: Discharge, $145 \mathrm{cfs}$ Dec. 22 (gage height, $7.45 \mathrm{ft}$ ).

1963 to October 1965: Discharge, $412 \mathrm{cfs}$ July 1965 (gage height, $9.18 \mathrm{ft}$ ).

(54) 9-4740, Gila River at Kelvin, Ariz.

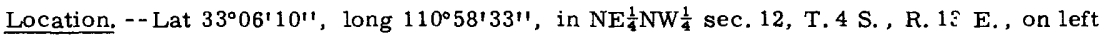
bank at Kelvin, $500 \mathrm{ft}$ downstream from Mineral Creek, 17 miles downstream from San Pedro River, and $19 \frac{1}{2}$ miles upstream from Ashurst-Hayden Dam.

Drainage area. $--18,011 \mathrm{sq} \mathrm{mi}$, of which $5,125 \mathrm{sq} \mathrm{mi}$ is below Coolidge Dam.

Gage-height record. - -Water-stage recorder graph except Nov. 28 to Dec. 2, 2400 hours Dec. 10 to 1700 hours Dec. 11 , and 2000 hours Dec. 22 to 1300 hours Dec. 25 when stage was above the operating range of recorder. Record for Dec. 11 and 22-25 reconstructed on basis of floodmarks and occasional staff-gage readings. D itum of gage is $1,745.02 \mathrm{ft}$ above mean sea level, datum of 1929 , supplementary adjustment of 1949 .

Discharge record. --Stage-discharge relation defined by current-meter measurements below 13,000 cfs and extended above on basis of slope-area measurement of peak flow. Discharge estimated Nov. 28 to Dec. 2.

Maxima. --November 1965 to January 1966: Discharge, 26, 300 cfs 1800 hours Dec. 23 (gage height, $18.5 \mathrm{ft}$, from floodmarks; gage height at former site, $17.5 \mathrm{ft}$, from from high-water mark in well).

1911-27: Discharge, about 132,000 cfs Jan. 20, 1916 (gage height, $19.5 \mathrm{ft}$, at site $900 \mathrm{ft}$ downstream at datum $1.80 \mathrm{ft}$ lower than present datum), from rating curve extended above slope-area measurement at gage height $16.2 \mathrm{ft}$.

1928 to October 1965: Discharge, $42,800 \mathrm{cfs}$ Aug. 8, 1930 (gage heigh ${ }^{+} .12 .6 \mathrm{ft}$, at site $900 \mathrm{ft}$ downstream at datum $1.80 \mathrm{ft}$ lower than present datum).

Remarks. --Records for flood period represent flow from area below Coolidge Dam (completed in 1928). 
Mean discharge, in cubic feet per second, 1965-66, of Gila River at Kelvin, Ariz.

\begin{tabular}{|c|c|c|c|c|c|c|c|}
\hline Day & November & December & January & Day & November & Decerrber & January \\
\hline $1 \ldots$ & 12 & 20 & 751 & $16 \ldots$ & 11 & 1,150 & 155 \\
\hline $2 \ldots$ & 13 & 57 & 443 & $17 \ldots$ & 11 & 2,130 & 144 \\
\hline $3 \ldots$ & 14 & 72 & 341 & $18 \ldots$ & 11 & 2,040 & 158 \\
\hline $4 \ldots$ & 11 & 70 & 276 & $19 \ldots$ & 12 & 738 & 180 \\
\hline $5 \ldots$ & 10 & 75 & 246 & $20 \ldots$ & 12 & 369 & 174 \\
\hline $6 \ldots$ & 11 & 96 & 218 & $21 \ldots$ & 11 & 251 & 168 \\
\hline $7 \ldots$ & 10 & 100 & 206 & $22 \ldots \ldots$ & 13 & 3,350 & 158 \\
\hline $8 \ldots$ & 9.4 & 100 & 197 & $23 \ldots$ & 18 & 14,600 & 151 \\
\hline 9 & 10 & 140 & 184 & 24 & 21 & 8,100 & 142 \\
\hline 10 & 11 & 3,670 & 178 & 25 & 311 & 1,710 & 136 \\
\hline 11. & 9.4 & 4,490 & 170 & 26 & 224 & 892 & 130 \\
\hline $12 \ldots$ & 11 & 1,000 & 164 & 27 & 100 & 565 & 124 \\
\hline $13 \ldots$ & 12 & 307 & 155 & $28 \ldots$ & 45 & 412 & 121 \\
\hline $14 \ldots$ & 9.7 & 407 & 155 & $29 \ldots$ & 30 & 331 & 120 \\
\hline \multirow[t]{2}{*}{$15 \ldots$} & 11 & 1,540 & 155 & $30 \ldots$ & 25 & 1,470 & 120 \\
\hline & & & & $31 \ldots$ & $\ldots \ldots$ & 1,980 & 136 \\
\hline \multicolumn{5}{|c|}{$\begin{array}{l}\text { Monthly mean discharge, in cubic feet per second. } \\
\text { Runoff, in acre-feet }\end{array}$} & 34.0 & $\begin{array}{r}1,685 \\
103,600\end{array}$ & $\begin{array}{rr}199 \\
12.210\end{array}$ \\
\hline
\end{tabular}

Gage height, in feet, and discharge, in cubic feet per second, at indicated time, 1965

\begin{tabular}{|c|c|c|c|c|c|c|c|c|c|c|c|}
\hline Date & Hour & $\begin{array}{l}\text { Gage } \\
\text { height }\end{array}$ & $\begin{array}{c}\text { Dis- } \\
\text { charge }\end{array}$ & Date & Hour & $\begin{array}{l}\text { Gage } \\
\text { height }\end{array}$ & $\begin{array}{c}\text { Dis- } \\
\text { charge }\end{array}$ & Date & Hour & $\begin{array}{l}\text { Gage } \\
\text { height }\end{array}$ & $\begin{array}{c}\text { Dis- } \\
\text { charge }\end{array}$ \\
\hline \multirow{13}{*}{$\begin{array}{r}\text { Dec. } 21 \\
22\end{array}$} & \multirow[t]{2}{*}{2400} & \multirow[t]{2}{*}{5.48} & \multirow[t]{2}{*}{216} & \multirow[t]{3}{*}{$\overline{\text { Dec. } 22}$} & 2100 & 13.68 & 8,560 & \multirow[t]{5}{*}{ Dec. 24} & 1500 & 11.50 & 5,200 \\
\hline & & & & & 2400 & 13.96 & 9,120 & & 1700 & 10. 19 & 3,510 \\
\hline & 0400 & 5.48 & 216 & & & & & & 2000 & 9.80 & 3,120 \\
\hline & 0800 & 5. 81 & 300 & \multirow[t]{8}{*}{23} & 0530 & 14.09 & 9,380 & & 2400 & 9.21 & 2,450 \\
\hline & 0900 & 6.16 & 421 & & 1230 & 13. 99 & 9,180 & & & & \\
\hline & 1000 & 8.61 & 1,870 & & 1600 & 18.00 & 23,100 & \multirow[t]{6}{*}{25} & 0100 & 8. 91 & 2,170 \\
\hline & 1100 & 11.02 & 4,520 & & 1800 & 18.50 & 26,300 & & 1000 & 8. 13 & 1,740 \\
\hline & 1230 & 11.57 & 5,220 & & 1930 & 18 & 25 , & & 1330 & 8. 37 & 1,730 \\
\hline & 1400 & 10.80 & 4,250 & & 2100 & 17.81 & 22,000 & & 2000 & 7.97 & 1,390 \\
\hline & 1630 & 8.87 & 2,140 & & 2400 & 16. 92 & 17,900 & & 2400 & 7.66 & 1,180 \\
\hline & 1800 & 11.00 & 4,550 & & & & & & & & \\
\hline & 1930 & 12.66 & 6,840 & \multirow[t]{2}{*}{24} & 0600 & 15. 11 & 11,700 & \multirow[t]{2}{*}{26} & 1200 & 7. 08 & 850 \\
\hline & 2000 & 13. 25 & 7,720 & & 1200 & 13. 19 & 7,640 & & 2400 & 6.73 & 686 \\
\hline
\end{tabular}


(55) 9-4795. Gila River near Laveen, Ariz.

Location. --Lat $33^{\circ} 15^{\prime} 25^{\prime \prime}$, long $112^{\circ} 09^{\prime} 59^{\prime \prime}$, in SW $\frac{1}{4} \mathrm{NW} \frac{1}{4}$ sec. 16, T. 2 S. , R. 2 E. , in Gila River Indian Reservation, on left abutment of highway bridge, 2.1 miles upstream from Santa Cruz River, 2.6 miles south of Komatke, and 7.3 miles south of Laveen.

Drainage area. $--20,615 \mathrm{sq} \mathrm{mi}$, of which $7,729 \mathrm{sq} \mathrm{mi}$ is below Coolidge Dam.

Gage-height record. - -Water-stage recorder graph for main channel. Auxiliary graph for overflow channel except 2200 hours Dec. 9 to 1400 hours Dec. 24; record for 2400 hours Dec. 22 to 1400 hours Dec. 24 reconstructed on basis of records for main channel. Datum of gage on main channel is $1,018.90 \mathrm{ft}$ above mean sea level, datum of 1929, supplementary adjustment of 1949. Gage heights are for main channel gage.

Discharge record. --Stage-discharge relation for both channels defined by current-meter measurements below $1,800 \mathrm{cfs}$ and extended above on basis of contracted-opening measurements of peak flows.

Maxima. - November 1965 to January 1966: Discharge, 10,900 cfs, of which 5, $720 \mathrm{cfs}$ flowed in overflow channel, 0300 hours Dec. 26 (gage height, $10.08 \mathrm{ft}$ ).

1940 to October 1965: Discharge, 11,900 cfs Jan. 2, 1941 (gage height, $9.33 \mathrm{ft}$ ).

Remarks. --Records include flow in main and overflow channels. Discharge represents runoff from drainage area below Coolidge Dam. No releases to Gila River at Coolidge Dam.

Mean discharge, in cubic feet per second, 1965-66

\begin{tabular}{|c|c|c|c|c|c|c|c|c|}
\hline Day & December & January & Day & December & January & Day & December & January \\
\hline $1 \ldots$ & 0 & 573 & $11 \ldots$ & 15 & 0 & $21 \ldots$ & 145 & 0 \\
\hline $2 \ldots$ & 0 & 627 & 12. & 431 & 0 & $22 \ldots$ & 115 & 0 \\
\hline $3 \ldots$ & 0 & 133 & $13 \ldots$ & 537 & 0 & $23 \ldots$ & 233 & 0 \\
\hline $4 \ldots$ & 0 & 11 & $14 \ldots$ & 351 & 0 & $24 \ldots$ & 775 & 0 \\
\hline . & 0 & 0 & $15 \ldots$ & 23 & 0 & $25 \ldots$ & 2,120 & 0 \\
\hline $6 \ldots$ & 0 & 0 & $16 \ldots$ & 143 & 0 & $26 \ldots$ & 7,040 & 0 \\
\hline $7 \ldots$ & 0 & 0 & 17 & 357 & 0 & 27. & 1,580 & 0 \\
\hline $8 \ldots$ & 0 & 0 & 18. & 128 & 0 & 28. & 288 & 0 \\
\hline & 0 & 0 & 19 & 599 & 0 & 29. & 32 & 0 \\
\hline $10 \ldots$ & .2 & 0 & $20 \ldots$ & 512 & 0 & 30. & 6.6 & 0 \\
\hline & & & & & & $31 \ldots$ & 13 & 0 \\
\hline \multirow{2}{*}{\multicolumn{7}{|c|}{$\begin{array}{l}\text { Monthly mean discharge, in cubic feet per second } \ldots \ldots \ldots \ldots \ldots \ldots \\
\text { Runoff, in acre-feet. } \ldots \ldots \ldots \ldots \ldots \ldots \ldots \ldots\end{array}$}} & 498 & 43.4 \\
\hline & & & & & & & 30,630 & 2,670 \\
\hline
\end{tabular}

Gage height, in feet, and discharge, in cubic feet per second, at indicated time, 1965-66

\begin{tabular}{|c|c|c|c|c|c|c|c|c|c|c|c|c|}
\hline Date & Hour & $\begin{array}{l}\text { Gage } \\
\text { height }\end{array}$ & $\begin{array}{c}\text { Dis- } \\
\text { charge }\end{array}$ & Date & Hour & $\begin{array}{c}\text { Gage } \\
\text { height }\end{array}$ & $\begin{array}{c}\text { Dis- } \\
\text { charge }\end{array}$ & \multicolumn{2}{|c|}{ Date } & Hour & $\begin{array}{l}\text { Gage } \\
\text { height }\end{array}$ & $\begin{array}{l}\text { Dis- } \\
\text { charge }\end{array}$ \\
\hline Dec. 23 & 2400 & 6.96 & 505 & Dec. 27 & $\begin{array}{l}1200 \\
1800\end{array}$ & $\begin{array}{l}7.89 \\
7.71\end{array}$ & $\begin{array}{l}1,320 \\
1,030\end{array}$ & \multicolumn{2}{|c|}{ Jan. 1} & 2400 & 7.57 & 828 \\
\hline \multirow[t]{2}{*}{24} & 1200 & 7. 66 & 764 & & 2400 & 7. 54 & 765 & & 2 & 0300 & 7. 58 & 864 \\
\hline & 2400 & 7. 89 & 1,050 & 30 & 2400 & 3.85 & 2.6 & & & $\begin{array}{l}0600 \\
1200\end{array}$ & $\begin{array}{l}\text { 7. } 56 \\
\text { 7. } 46\end{array}$ & $\begin{array}{l}837 \\
721\end{array}$ \\
\hline \multirow[t]{5}{*}{25} & 0600 & 8.05 & 1,270 & & & & & & & 1500 & 7. 24 & 634 \\
\hline & 1930 & 8. 35 & 1,920 & 31 & 1400 & 3. 81 & .1 & & & 1700 & 6.87 & 502 \\
\hline & 2200 & 9. 28 & 4,880 & & 2200 & 3.80 & 0 & & & 1900 & 6. 02 & 335 \\
\hline & 2400 & 9. 84 & 6,880 & & 2300 & 4. 04 & 172 & & & 2000 & 5.57 & 292 \\
\hline & & & & & 2400 & 5. 10 & 255 & & & 2400 & 4.52 & 218 \\
\hline \multirow[t]{6}{*}{26} & 0300 & 10.08 & 7,560 & \multirow{7}{*}{ Jan. 1} & & & & & & & & \\
\hline & 0800 & 9.87 & 6,830 & & 0100 & 6.20 & 351 & & 3 & 0200 & 4. 21 & 203 \\
\hline & 1400 & 9.37 & 5,630 & & 0200 & 6.60 & 413 & & & 0400 & 4.08 & 183 \\
\hline & 1800 & 9.02 & 4,200 & & 0400 & 6.85 & 468 & & & 2400 & 3. 97 & 55 \\
\hline & 2400 & 8. 53 & 2,850 & & 1300 & 7. 28 & 588 & & & & & \\
\hline & & & & & 1800 & 7. 41 & 638 & & 4 & 1400 & 3.87 & 5.3 \\
\hline 27 & 0600 & 8. 15 & 1,800 & & 2100 & 7. 50 & 727 & & & 2400 & 3.82 & 4 \\
\hline
\end{tabular}


(56) 9-4800. Santa Cruz River near Lochiel, Ariz.

Location. --Lat $31^{\circ} 21^{\prime} 20^{\prime \prime}$, long $110^{\circ} 35^{\prime} 25^{\prime \prime}$, in SW $\frac{1}{4}$ sec. 11 , T. 24 S., R. $17 \mathrm{E}$.

(unsurveyed), on southern border of Spanish land grant of San Rafael, near left bank on downstream side of county road, $1 \frac{1}{2}$ miles upstream from international boundary, and $2 \frac{1}{2}$ miles northeast of Lochiel.

Drainage area. $--82.2 \mathrm{sq} \mathrm{mi}$.

Gage-height record. - Water-stage recorder graph. Altitude of gage is $4,620 \mathrm{ft}$ (from topographic map).

Discharge record. --Stage-discharge relation defined by current-meter maasurements.

Maxima. --November 1965 to January 1966: Discharge, 97 cfs 2200 hours Dec. 22 (gage height, 2.56 ft).

1949 to October 1965: Discharge, 4,810 cfs Sept. 12, 1965 (gage height, 8. $90 \mathrm{ft}$ ), from rating curve extended above $2,300 \mathrm{cfs}$ on basis of slope-area measurement of peak flow.

Mean discharge, in cubic feet per second, 1965-66

\begin{tabular}{|c|c|c|c|c|c|c|c|}
\hline Day & Novernber & December & January & Day & November & Decemk ar & J anuary \\
\hline $1 \ldots$ & 0.1 & 0 & 1.3 & 16 & 0 & 0.7 & 3.9 \\
\hline $2 \ldots$ & 0 & 0 & 1.5 & $17 \ldots$ & 0 & 1. 7 & 3.7 \\
\hline $3 \ldots$ & 0 & 0 & 2.4 & $18 \ldots$ & 0 & .7 & 5.6 \\
\hline $4 \ldots$ & 0 & 0 & 2.6 & $19 \ldots$ & 0 & .7 & 5.4 \\
\hline $5 \ldots$ & 0 & 0 & 2.8 & $20 \ldots$ & 0 & .6 & 4.6 \\
\hline $6 \ldots$ & 0 & 0 & 3.0 & $21 \ldots$ & 0 & .6 & 4.6 \\
\hline $7 \ldots$ & 0 & 0 & 3.0 & $22 \ldots$ & 0 & 26 & 4. 6 \\
\hline $8 \ldots$ & 0 & 0 & 3.2 & 23 & 0 & 40 & 4.4 \\
\hline $9 \ldots$ & 0 & .4 & 3.5 & 24 & 0 & 5.5 & 4.1 \\
\hline $10 \ldots$ & 0 & 4. 7 & 3.7 & $25 \ldots$ & 0 & 2.1 & 3.9 \\
\hline $11 \ldots$ & 0 & 1.0 & 3.7 & 26 & 0 & 1.4 & 3.7 \\
\hline $12 \ldots$ & 0 & .4 & 3.7 & 27. & 0 & 1. 3 & 3.9 \\
\hline $13 \ldots$ & 0 & .4 & 3.7 & $28 \ldots$ & 0 & 1.2 & 4.2 \\
\hline $14 \ldots$ & 0 & .5 & 3.7 & $29 \ldots$ & 0 & 1. 2 & 3.7 \\
\hline $15 \ldots$ & 0 & .7 & 3.9 & $30 \ldots$ & 0 & 1.4 & 3.7 \\
\hline & & & & $31 \ldots$ & $\ldots-\ldots$ & 1.4 & 4.1 \\
\hline \multirow{2}{*}{\multicolumn{5}{|c|}{$\begin{array}{l}\text { Monthly mean discharge, in cubic feet per second.. } \\
\text { Runoff, in acre-feet } \ldots \ldots \ldots \ldots \ldots \ldots \ldots \ldots \ldots \ldots\end{array}$}} & 0.003 & 3.05 & 3.67 \\
\hline & & & & & 0.2 & $18 ?$ & 226 \\
\hline
\end{tabular}

(57) 9-4805. Santa Cruz River near Nogales, Ariz.

Location. --Lat $31^{\circ} 20^{\prime} 40^{\prime \prime}$, long $110^{\circ} 51^{\prime} 05^{\prime \prime}$, in NW $\frac{1}{4}$ sec. 18, T. 24 S. , R. $15 \mathrm{E}$. (unsurveyed), in Spanish land grant of Maria Santisima del Carmen, on left bank three-quarters of a mile downstream from international boundary, $5 \frac{1}{4}$ miles upstream from Yerba Buena damsite, and $5 \frac{1}{2}$ miles east of Nogales.

Drainage area. $-533 \mathrm{sq} \mathrm{mi}$, of which $348 \mathrm{sq} \mathrm{mi}$ is in Mexico.

Gage-height record. - Water-stage recorder graph. Datum of gage is 3,702.54 ft above mean sea level, datum of 1929 (levels by International Boundary and Water Commission).

Discharge record. --Stage-discharge relation defined by current-meter measurements below 3,700 cfs and extended above on basis of slope-area measurementis at 10.38 and $12.03 \mathrm{ft}$.

Maxima. --November 1965 to January 1966: Discharge, 3, 840 cfs 0700 houns Dec. 23 (gage height, $8.84 \mathrm{ft}$ ).

1930 to October 1965: Discharge, 12, 000 cfs Aug. 31, 1935 (gage height, 12. $3 \mathrm{ft}$ ), from rating curve extended above $2,300 \mathrm{cfs}$ on basis of slope-area measirements at 9.5 and 10.9 ft; gage height, 13. $71 \mathrm{ft}$ Aug. 20, 1955. 
Mean discharge, in cubic feet per second, 1965-66, of Santa Cruz River

\begin{tabular}{|c|c|c|c|c|c|c|c|}
\hline Day & November & December & January & Day & November & December & January \\
\hline $1 \ldots$ & 0 & 0.2 & 102 & $16 \ldots$ & 0 & 489 & 25 \\
\hline $2 \ldots$ & 0 & .2 & 74 & $17 \ldots$ & 0 & 1,340 & 27 \\
\hline $3 \ldots$ & 0 & .2 & 59 & $18 \ldots$ & 0 & 471 & 228 \\
\hline $4 \ldots$ & 0 & .2 & 48 & $19 \ldots$ & 0 & 250 & 370 \\
\hline $5 \ldots$ & 0 & .2 & 42 & $20 \ldots$ & 0 & 171 & 203 \\
\hline $6 \ldots$ & 0 & .2 & 36 & $21 \ldots$ & 0 & 126 & 182 \\
\hline $7 \ldots$ & 0 & .2 & 33 & $22 \ldots$ & 0 & 1,580 & 140 \\
\hline $8 \ldots$ & 0 & .3 & 31 & $23 \ldots$ & 0 & 2,640 & 110 \\
\hline $9 \ldots$ & 0 & .6 & 31 & $24 \ldots$ & 0 & 722 & 85 \\
\hline $10 \ldots$ & 0 & 643 & 27 & $25 \ldots$ & .1 & 341 & 72 \\
\hline $11 \ldots$ & 0 & 472 & 28 & 26. & .1 & 184 & 63 \\
\hline $12 \ldots$ & 0 & 40 & 27 & $27 \ldots$ & .1 & 118 & 59 \\
\hline $13 \ldots$ & 0 & 19 & 27 & $28 \ldots \ldots$ & .1 & 88 & 83 \\
\hline $14 \ldots$ & 0 & 32 & 26 & $29 \ldots$ & .1 & 63 & 81 \\
\hline \multirow[t]{2}{*}{$15 \ldots$} & 0 & 729 & 26 & $30 \ldots$ & .1 & 58 & 74 \\
\hline & & & & $31 \ldots$ & $\ldots-\cdots$ & 110 & 69 \\
\hline \multirow{2}{*}{\multicolumn{5}{|c|}{$\begin{array}{l}\text { Monthly mean discharge, in cubic feet per second. } \\
\text { Runoff, in acre-feet } \ldots \ldots \ldots \ldots \ldots \ldots \ldots \ldots \ldots \ldots\end{array}$}} & 0.02 & 345 & 80.3 \\
\hline & & & & & 1.2 & 21,200 & 4,930 \\
\hline
\end{tabular}

(58) 9-4815. Sonoita Creek near Patagonia, Ariz.

Location. -- Lat $31^{\circ} 30^{\prime} 00^{\prime \prime}$, long $110^{\circ} 49^{\prime} 00^{\prime \prime}$, in SE $\frac{1}{4} \mathrm{SW} \frac{1}{4} \sec .21, \mathrm{~T}, 22 \mathrm{~S} ., \mathrm{R} .15 \mathrm{E}$, on left abutment of former railroad bridge, 5 miles downstream from Patagonia.

Drainage area. --209 sq $\mathrm{mi}$.

Gage-height record. - -Water-stage recorder graph except Dec. 25 to Jan. 20. Datum of gage is $3,818.09 \mathrm{ft}$ above mean sea level, datum of 1929, supplementary arjustment of 1959 .

Discharge record. --Stage-discharge relation defined by current-meter measurements below $900 \mathrm{cfs}$ and extended above on basis of slope-area measurements at 8.70 and 10. $15 \mathrm{ft}$. Discharge for period of no gage-height record estimated.

Maxima. --November 1965 to January 1966: Discharge, 2, 240 cfs 1430 hours Dec. 22 (gage height, 6.39 ft).

1930 to October 1965: Discharge, 14,000 cfs Sept. 30, 1946 (gage height, $13.0 \mathrm{ft}$ ), from rating curve extended above $1,500 \mathrm{cfs}$ on basis of slope-area measurement of peak flow.

Mean discharge, in cubic feet per second, 1965-66

\begin{tabular}{|c|c|c|c|c|c|c|c|}
\hline Day & November & Dece mber & January & Day & November & December & January \\
\hline $1 \ldots$ & 0.9 & 2.5 & 45 & $16 \ldots$ & 1.8 & 66 & 10 \\
\hline $2 \ldots$ & 1.2 & 2.1 & 35 & $17 \ldots$ & 1.8 & 142 & 12 \\
\hline $3 \ldots$ & 1.2 & 2.1 & 30 & $18 \ldots$ & 1. 8 & 72 & 50 \\
\hline $4 \ldots$ & 1.5 & 1.8 & 27 & $19 \ldots$ & 1. 8 & 40 & 60 \\
\hline $5 \ldots$ & 1.5 & 1. 8 & 25 & $20 \ldots$ & 1. 4 & 28 & 50 \\
\hline $6 \ldots$ & 1.5 & 2,1 & 22 & $21 \ldots$ & 1.8 & 21 & 41 \\
\hline $7 \ldots$ & 1.8 & 2. 1 & 20 & $22 \ldots \ldots$ & 2,1 & 876 & 35 \\
\hline $8 \ldots$ & 1.8 & 2.5 & 20 & $23 \ldots$ & 2.5 & 740 & 26 \\
\hline $9 \ldots$ & 1.8 & 9 & 16 & $24 \ldots$ & 2.1 & 209 & 22 \\
\hline $10 \ldots$ & 1.8 & 238 & 15 & $25 \ldots$ & 3.4 & 130 & 17 \\
\hline $11 \ldots$ & 1.4 & 246 & 14 & $26 \ldots$ & 3,4 & 80 & 12 \\
\hline $12 \ldots \ldots$ & 1.6 & 14 & 13 & $27 \ldots$ & 3.4 & 60 & 12 \\
\hline $13 \ldots$ & 1.8 & 19 & 12 & $28 \ldots$ & 3.8 & 45 & 15 \\
\hline $14 \ldots \ldots$ & 1.8 & 52 & 11 & $29 \ldots$ & 2.9 & 35 & 12 \\
\hline \multirow[t]{2}{*}{$15 \ldots$} & 1.8 & 86 & 10 & $30 \ldots$ & 2.5 & 40 & 12 \\
\hline & & & & $31 \ldots$ & $\ldots . .-$ & 50 & 18 \\
\hline \multirow{2}{*}{\multicolumn{5}{|c|}{ Monthly mean discharge, in cubic feet per second. . }} & 2.00 & 107 & 23.2 \\
\hline & & & & & 119 & 6,580 & 1,430 \\
\hline
\end{tabular}


(59) 9-4820. Santa Cruz River at Continental, Ariz.

Location. -- Lat $31^{\circ} 51^{\prime} 10^{\prime \prime}$, long $110^{\circ} 58^{\prime} 40^{\prime \prime}$, in NE $\frac{1}{4} \mathrm{NE} \frac{1}{4}$ sec. 23, T. $18 \mathrm{~S}$, R. $13 \mathrm{E}$. (unsurveyed), in Spanish land grant of San Ignacio de la Canoa, near left bank on downstream side of pier of highway bridge at Continental.

Drainage area. $--1,662 \mathrm{sq} \mathrm{mi}$, of which $395 \mathrm{sq} \mathrm{mi}$ is in Mexico.

Gage-height record. - -Water-stage recorder graph except Dec. 12-15, D?c. 19 to 1300 hours Dec. 22, Jan. 23-31. Datum of gage is 2, 836. $35 \mathrm{ft}$ above mean sea level, datum of 1929, supplementary adjustment of 1958 .

Discharge record. --Stage-discharge relation defined by current-meter measurements below $5,500 \mathrm{cfs}$ and extended above on basis of indirect measurement at gage height $10.13 \mathrm{ft}$. Discharge for periods of no gage-height record estimated.

Maxima. --November 1965 to January 1966: Discharge, 5, 990 cfs 0900 hours Dec. 23 (gage height, $9.34 \mathrm{ft}$ ).

1940-46, 1951 to October 1965: Discharge, 17, 500 cfs Aug. 19, 1955 (gage height, $11.34 \mathrm{ft}$ ), from rating extended above $4,600 \mathrm{cfs}$ on basis of float-area measurements at gage heights 7.90 and $8.85 \mathrm{ft}$.

\begin{tabular}{|c|c|c|c|c|c|c|c|c|}
\hline & & & & & & & & \\
\hline Day & December & January & Day & December & January & Day & December & January \\
\hline & 0 & 0 & $11 \ldots$ & 598 & 0 & $21 \ldots$ & 0 & 106 \\
\hline & 0 & 0 & $12 \ldots$ & 39 & 0 & $22 \ldots$ & 1,930 & 84 \\
\hline 3 & 0 & 0 & $13 \ldots$ & 0 & 0 & 23. & 5,190 & 30 \\
\hline 4 & 0 & 0 & $14 \ldots$ & 0 & 0 & 24. & 1,710 & 5 \\
\hline 5 & 0 & 0 & 15 & 361 & 0 & 25. & 477 & 0 \\
\hline & 0 & 0 & & 337 & 0 & $26 \ldots$ & 84 & 0 \\
\hline & 0 & 0 & 17 & 1,390 & 0 & $27 \ldots$ & .8 & 0 \\
\hline & 0 & 0 & 18 & 619 & 9.5 & $28 \ldots$ & 0 & 0 \\
\hline 9 & 0 & 0 & 19 & 80 & 208 & $29 \ldots$ & 0 & 0 \\
\hline $10 \ldots$ & 202 & 0 & $20 \ldots$ & 0 & 123 & $30 \ldots$ & 0 & 0 \\
\hline & & & & & & $31 \ldots$ & 0 & 0 \\
\hline \multicolumn{7}{|c|}{ Monthly mean discharge, in cubic feet per second $\ldots \ldots \ldots \ldots \ldots$} & 422 & 18.2 \\
\hline \multicolumn{9}{|c|}{ 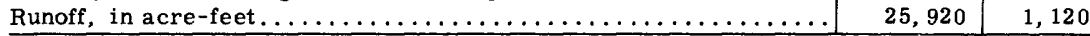 } \\
\hline
\end{tabular}

Gage height, in feet, and discharge, in cubic feet per second, at indicated time, 1965

\begin{tabular}{|c|c|c|c|c|c|c|c|c|c|c|c|}
\hline Date & Hour & $\begin{array}{l}\text { Gage } \\
\text { height }\end{array}$ & $\begin{array}{c}\text { Dis- } \\
\text { charge }\end{array}$ & Date & Hour & $\begin{array}{l}\text { Gage } \\
\text { height }\end{array}$ & $\begin{array}{c}\text { Dis- } \\
\text { charge }\end{array}$ & Date & Hour & $\begin{array}{l}\text { Gage } \\
\text { height }\end{array}$ & $\begin{array}{c}\text { Dis- } \\
\text { charge }\end{array}$ \\
\hline \multirow[t]{10}{*}{ Dec. 22} & 1330 & 4.75 & 32 & Dec. 23 & 0600 & 9.36 & 5,860 & \multirow[t]{4}{*}{ Dec. 24} & 1500 & 5.54 & 1,010 \\
\hline & 1440 & 7. 77 & 2,230 & & 0900 & 9.34 & 5,990 & & 1800 & 5.46 & 927 \\
\hline & 1500 & 8.08 & 2,700 & & 1100 & 9.20 & 5,880 & & 2400 & 5.20 & 640 \\
\hline & 1700 & 8.76 & 4,720 & & 1200 & 8. 92 & 5,450 & & & & \\
\hline & 1730 & 9.41 & 5,310 & & 1230 & 9.18 & 5,860 & \multirow[t]{4}{*}{25} & 1100 & 5.15 & 691 \\
\hline & 1800 & 9.53 & 5,430 & & 1430 & 9.23 & 5,930 & & 1800 & 4. 83 & 325 \\
\hline & 1930 & 9.16 & 5,060 & & 1700 & 8. 60 & 5,000 & & 2400 & 4. 57 & 165 \\
\hline & 2200 & 9.41 & 5,310 & & 1830 & 8.32 & 4,600 & & & & \\
\hline & 2400 & 9.35 & 5,250 & & 2400 & 7. 46 & 3,410 & 26 & 0700 & 4. 45 & 109 \\
\hline & & & & & & & & & 1200 & 4. 36 & 79 \\
\hline \multirow[t]{2}{*}{23} & 0230 & 9.57 & 5,470 & \multirow[t]{2}{*}{24} & 0600 & 6. 81 & 2,560 & & 1800 & 4. 26 & 49 \\
\hline & 0400 & 9.68 & 5,580 & & 1200 & 5.97 & 1,520 & & 2400 & 4. 12 & 18 \\
\hline
\end{tabular}


(60) 9-4825. Santa Cruz River at Tucson, Ariz.

Location, - - Lat $32^{\circ} 13^{\prime} 15^{\prime \prime}$, long $110^{\circ} 58^{\prime} 50^{\prime \prime}$, in $\mathrm{NE} \frac{1}{4} \mathrm{NE} \frac{1}{4}$ sec. 14 , T. $14 \mathrm{~S} .$, R. $13 \mathrm{E}$, on downstream side of pier of Congress Street Bridge in Tucson.

Drainage area. $--2,222 \mathrm{sq} \mathrm{mi}$, of which $395 \mathrm{sq} \mathrm{mi}$ is in Mexico.

Gage-height record. - -Water-stage recorder graph except part of each day Dec. 10-12, 16-18, 22, 25-28, Jan. 18-20 which was reconstructed, and Dec. 19-21. D tum of gage is $2,317,20 \mathrm{ft}$ above mean sea level, datum of 1929 .

Discharge record. --Stage-discharge relation defined by current-meter measurements below 4,200 cfs and extended above on basis of slope-area measurement at gage height 21. $30 \mathrm{ft}$ and a partial measurement at $19.0 \mathrm{ft}$, present datum. Discharge for Dec. 1921 estimated.

Maxima. --November 1965 to January 1966: Discharge, 4,830 cfs 1330 hours Dec. 23 (gage height, $11.82 \mathrm{ft}$ ).

1905 to October 1965: Discharge, 16,600 cfs Aug. 23, 1961 (gage height, 21. $30 \mathrm{ft}$, present datum).

Mean discharge, in cubic feet per second, 1965-66

\begin{tabular}{|c|c|c|c|c|c|c|c|}
\hline Day & November & December & January & Day & November & December & January \\
\hline $1 \ldots$ & 0 & 0 & 0 & $16 \ldots$ & 0 & 197 & 0 \\
\hline $2 \ldots$ & 0 & 0 & 0 & $17 \ldots$ & 0 & 712 & 2.4 \\
\hline $3 \ldots$ & 0 & 0 & 0 & $18 \ldots$ & 0 & 329 & 15 \\
\hline $4 \ldots$ & 0 & 0 & 0 & $19 \ldots$ & 0 & 50 & 62 \\
\hline $5 \ldots$ & 0 & 0 & 0 & $20 \ldots$ & 0 & 10 & 73 \\
\hline $6 \ldots$ & 0 & 0 & 0 & $21 \ldots$ & 0 & 0 & 0 \\
\hline$\ldots$ & 0 & 0 & 0 & $22 \ldots$ & 0 & 1,300 & 0 \\
\hline . & 0 & 0 & 0 & $23 \ldots$ & 0 & 3,680 & 0 \\
\hline 9 & 0 & 17 & 0 & 24. & 2. 7 & 1,460 & 0 \\
\hline 10. & 0 & 139 & 0 & $25 \ldots$ & 2.1 & 242 & 0 \\
\hline $11 \ldots$ & 0 & 861 & 0 & $26 \ldots$ & 0 & 24 & 0 \\
\hline 12 & 0 & 32 & 0 & $27 \ldots$ & 0 & 0 & 0 \\
\hline 13. & 0 & 0 & 0 & $28 \ldots \ldots$ & 0 & 0 & 0 \\
\hline $14 \ldots$ & 0 & 45 & 0 & $29 \ldots$ & 0 & 0 & 0 \\
\hline $15 \ldots$ & 0 & 144 & 0 & $30 \ldots$ & 0 & 1.9 & 0 \\
\hline & & & & $31 \ldots$ & $\ldots . .-$ & 0 & 2.8 \\
\hline \multirow{2}{*}{\multicolumn{5}{|c|}{ Monthly mean discharge, in cubic feet per second.. }} & 16 & 298 & 5.0 \\
\hline \multicolumn{3}{|c|}{ Runoff, in acre-feet $\ldots \ldots \ldots \ldots \ldots \ldots \ldots \ldots \ldots$} & & & 9.5 & 18,340 & 308 \\
\hline
\end{tabular}

Gage height, in feet, and discharge, in cubic feet per second, at indicated time, 1965

\begin{tabular}{|c|c|c|c|c|c|c|c|c|c|c|c|}
\hline Date & Hour & $\begin{array}{l}\text { Gage } \\
\text { height }\end{array}$ & $\begin{array}{c}\text { Dis- } \\
\text { charge }\end{array}$ & Date & Hour $r$ & $\begin{array}{l}\text { Gage } \\
\text { height }\end{array}$ & $\begin{array}{c}\text { Dis- } \\
\text { charge }\end{array}$ & Date & Hour & $\begin{array}{l}\text { Gage } \\
\text { height }\end{array}$ & $\begin{array}{c}\text { Dis- } \\
\text { charge }\end{array}$ \\
\hline \multirow[t]{10}{*}{$\overline{\text { Dec. } 22}$} & 0000 & 6.48 & 53 & \multirow[t]{10}{*}{ Dec. 22} & 1900 & $\overline{10.77}$ & $3, \overline{530}$ & \multirow[t]{10}{*}{ Dec. 23} & 1330 & 11.82 & 4,830 \\
\hline & 0900 & 6.40 & 42 & & 2100 & 11.12 & 4,440 & & 1800 & 11.50 & 4,310 \\
\hline & 1000 & 7. 36 & 266 & & 2300 & 10.72 & 3,440 & & 2200 & 11.12 & 3,740 \\
\hline & 1200 & 7. 57 & 348 & & 2400 & 10.42 & 2,920 & & 2400 & 10.92 & 3,470 \\
\hline & 1400 & 6. 41 & 43 & & & & & & & & \\
\hline & 1430 & 9.22 & 1,400 & & 0200 & 10.73 & 3,460 & & 0600 & 9.55 & 1,980 \\
\hline & 1530 & 10.47 & 3,000 & & 0400 & 10.02 & 2,330 & & 1200 & 8.52 & 1,120 \\
\hline & 1600 & 10.32 & 2,760 & & 0600 & 10.10 & 2,440 & & 1800 & 7.87 & 700 \\
\hline & 1730 & 8. 98 & 1,190 & & 0900 & 10.92 & 3,260 & & 2400 & 7.44 & 460 \\
\hline & 1800 & 10.02 & 2,330 & & 1100 & 11.62 & 4,170 & & & & \\
\hline
\end{tabular}


(61) 9-4830. Tucson Arroyo at Vine Avenue, Tucson, Ariz.

Location. -- Lat $32^{\circ} 13^{\prime} 00^{\prime \prime}$, long $110^{\circ} 57^{\prime} 00^{\prime \prime}$, in SW $\frac{1}{4} \mathrm{NE} \frac{1}{4} \sec .18$, T. $14 \mathrm{~S}$. , R. 14 E., on right bank at Vine Avenue in Tucson, a quarter of a mile downstream from Arroyo Chico, and $2 \frac{1}{2}$ miles upstream from mouth.

Drainage area. --Original area prior to August 1945, 27. 0 sq mi; subsequently reduced to the following areas by flood-control diversion structures: August 1945 to June $1953,23.4 \mathrm{sq} \mathrm{mi}$; June 1953 to June $1954,18.1 \mathrm{sq} \mathrm{mi}$; June 1954 to June $1956,15.9$ $\mathrm{sq} \mathrm{mi}$; since June $1956,6.2 \mathrm{sq} \mathrm{mi}$.

Gage-height record. --Water-stage recorder graph. Datum of gage is 2, $411.9 \mathrm{ft}$ above mean sea level (city of Tucson bench mark).

Discharge record. --Stage-discharge relation for artificial control defined by currentmeter measurements.

Maxima. - November 1965 to January 1966: Discharge, 517 cfs 1030 hours Dec. 22 (gage height, $5.76 \mathrm{ft}$ ).

1944 to October 1965: Discharge, 5, $000 \mathrm{cfs}$ Aug. 22, 1961 (gage height, 10. $35 \mathrm{ft}$ ), from rating curve extended above $2,100 \mathrm{cfs}$ on basis of slope-area mes surements at gage heights 10.13 and $10.35 \mathrm{ft}$.

Mean discharge, in cubic feet per second, 1965-66

\begin{tabular}{|c|c|c|c|c|c|c|c|}
\hline Day & November & December & January & Day & November & December & January \\
\hline $1 \ldots$ & 0 & 0.5 & 0 & $16 \ldots$ & 0 & 5.4 & 0 \\
\hline $2 \ldots$ & 0 & 0 & 0 & $17 \ldots$ & 0 & 17 & 15 \\
\hline $3 \ldots$ & 0 & 0 & 0 & $18 \ldots$ & 0 & 3.5 & 17 \\
\hline $4 \ldots$ & 0 & 0 & 0 & $19 \ldots$ & 0 & 0 & 0 \\
\hline $5 \ldots$ & 0 & 0 & 0 & $20 \ldots$ & 0 & 0 & 6 \\
\hline $6 \ldots$ & 0 & 0 & 0 & $21 \ldots$ & 0 & 0 & 0 \\
\hline $7 \ldots$ & 0 & 0 & 0 & $22 \ldots \ldots$ & 0 & 60 & 0 \\
\hline $8 \ldots$ & 0 & .4 & 0 & 23 & 0 & 18 & 0 \\
\hline 9. & 0 & 48 & 0 & 24 & 3. 8 & .1 & 0 \\
\hline $10 \ldots$ & 0 & 60 & 0 & 25 & 7.2 & 0 & 0 \\
\hline $11 \ldots$ & 0 & 6.4 & 0 & 26. & 0 & 0 & 0 \\
\hline $12 \ldots$ & 0 & 0 & 0 & $27 \ldots \ldots$ & 0 & 0 & 1 \\
\hline $13 \ldots$ & 0 & 0 & 0 & $28 \ldots \ldots$ & 0 & 0 & 0 \\
\hline $14 \ldots$ & 0 & 27 & 0 & $29 \ldots \ldots$ & 0 & 0 & 0 \\
\hline \multirow[t]{2}{*}{$15 \ldots$} & 0 & 16 & 0 & $30 \ldots$ & .6 & 1.7 & 3 \\
\hline & & & & 31 & $\ldots \cdots$ & 0 & 6 \\
\hline \multirow{2}{*}{\multicolumn{5}{|c|}{$\begin{array}{l}\text { Monthly mean discharge, in cubic feet per second. } \\
\text { Runoff, in acre-feet } \ldots \ldots \ldots \ldots \ldots \ldots \ldots \ldots\end{array}$}} & 0.39 & 8. 52 & 1.55 \\
\hline & & & & & 23 & 524 & 95 \\
\hline
\end{tabular}


(62) 9-4831. Tanque Verde Creek near Tucson, Ariz.

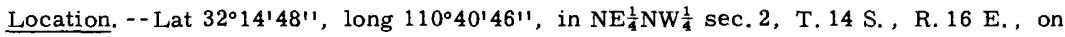
right bank, 4.4 miles east of Tanque Verde School, 7.4 miles upstream from Agua Caliente Wash, 7.8 miles northwest of Spud Rock, and 17.5 miles east of Tucson.

Drainage area. $--43.0 \mathrm{sq} \mathrm{mi}$.

Gage-height record. - -Water-stage recorder graph except 1200 hours Dec. 27 to 1600 hours Dec. 30. Altitude of gage is $2,720 \mathrm{ft}$ (from topographic map).

Discharge record. --Stage-discharge relation defined by current-meter measurements below $690 \mathrm{cfs}$ and extended above on basis of slope-area measurements at gage heights 3.85 and $4.86 \mathrm{ft}$. Discharge for period of no gage-height record estimated.

Maxima. - November 1965 to January 1966: Discharge, 2, 760 cfs 1200 hours Dec. 22 (gage height, $4.93 \mathrm{ft}$ ).

1959 to October 1965: Discharge, 2,630 cfs Sept. 10, 1964 (gage height, 4.86, from floodmark).

Mean discharge, in cubic feet per second, 1965-66

\begin{tabular}{|c|c|c|c|c|c|c|c|c|}
\hline Day & December & January & Day & December & January & Day & Decermber & January \\
\hline 1 & 0 & 78 & 11 & 655 & 11 & 21. & 58 & 81 \\
\hline $2 \ldots$ & 0 & 52 & 12 & 125 & 11 & 22. & 890 & 51 \\
\hline $3 \ldots$ & 0 & 39 & 13 & 70 & 10 & 23 . & 747 & 32 \\
\hline$\ldots$ & 0 & 29 & $14 \ldots$ & 93 & 7. 2 & 24. & 333 & 24 \\
\hline 5 & 0 & 24 & $15 \ldots$ & 244 & 6.8 & $25 \ldots$ & 192 & 19 \\
\hline$\ldots$ & 0 & 20 & $16 \ldots$ & 179 & 5.8 & $26 \ldots$ & 124 & 16 \\
\hline 7 & 0 & 18 & 17. & 424 & 5.4 & $27 \ldots$ & 78 & 14 \\
\hline 8 & 0 & 15 & & 234 & 56 & 28 & 65 & 18 \\
\hline 9 & 143 & 13 & 19 & 112 & 102 & & 60 & 15 \\
\hline \multirow[t]{2}{*}{$10 \ldots$} & 880 & 13 & $20 \ldots$ & 72 & 58 & 30 & 199 & 14 \\
\hline & & & & & & $31 \ldots$ & 156 & 178 \\
\hline \multirow{2}{*}{\multicolumn{7}{|c|}{ Monthly mean discharge, in cubic feet per second $\ldots \ldots \ldots \ldots \ldots$}} & 198 & 33.4 \\
\hline & & & & & & & 12,160 & 2,060 \\
\hline
\end{tabular}

Gage height, in feet, and discharge, in cubic feet per second, at indicated time, 1965

\begin{tabular}{r|c|c|c||r|r|r|r||r|r|r|r}
\hline Date & Hour & $\begin{array}{c}\text { Gage } \\
\text { height }\end{array}$ & $\begin{array}{c}\text { Dis- } \\
\text { charge }\end{array}$ & Date & Hour & $\begin{array}{c}\text { Gage } \\
\text { height }\end{array}$ & $\begin{array}{c}\text { Dis- } \\
\text { charge }\end{array}$ & Date & Hour & $\begin{array}{c}\text { Gago } \\
\text { height }\end{array}$ & $\begin{array}{c}\text { Dis- } \\
\text { charge }\end{array}$ \\
\hline Dec. 21 & 2400 & 1.66 & 60.1 & Dec. 22 & 1800 & 2.95 & 628 & Dec. 23 & 1700 & 2.70 & 440 \\
& & & & & 2000 & 2.83 & 544 & & 1800 & 2.85 & 537 \\
& 0200 & 1.83 & 92 & & 2300 & 2.85 & 558 & & 2400 & 2.69 & 434 \\
& 0500 & 2,40 & 291 & & 2400 & 3.99 & 1,540 & & & & \\
& 0600 & 2.68 & 446 & & & & & 24 & 1200 & 2.43 & 286 \\
& 0900 & 2.85 & 551 & 23 & 0100 & 4.30 & 1,890 & & 1600 & 2.65 & 410 \\
& 1000 & 4.35 & 1,960 & & 0200 & 4.45 & 2,080 & & 2400 & 2.37 & 260 \\
& 1200 & 4.93 & 2,760 & & 0300 & 4.13 & 1,690 & & & & \\
& 1300 & 4.43 & 2,060 & & 0400 & 3.95 & 1,500 & 25 & 1200 & 2.17 & 176 \\
& 1400 & 4.00 & 1,550 & & 0700 & 2.83 & 524 & & 2400 & 2.12 & 160 \\
\hline
\end{tabular}


(63) 9-4832. Agua Caliente Wash tributary near Tucson, Ariz.

(Crest-stage station)

Location. - Lat $32^{\circ} 16^{\prime} 07^{\prime \prime}$, long $110^{\circ} 44^{\prime} 15^{\prime \prime}$, in SW $\frac{1}{4}$ SW $\frac{1}{4}$ sec. 29, T. 13 S. , R. 16 E. , at Soldier Trail, 1.4 miles north of Tanque Verde Road, and 5 miles northeast of Tucson city limits.

Drainage area. $--2.18 \mathrm{sq} \mathrm{mi}$.

Gage-height record. --Crest stages only. Altitude of gage is 2, $700 \mathrm{ft}$ (fr $>\mathrm{m}$ topographic map).

Discharge record. --Stage-discharge relation defined by computation of flow through culvert.

Maxima. --November 1965 to January 1966: Discharge, 40 cfs Dec. 22 (gage height, 4. $30 \mathrm{ft}$ ).

August to October 1965: Discharge, 86 cfs August 1965 (gage heigh t., $5.48 \mathrm{ft}$ ).

(64) 9-4840. Sabino Creek near Tucson, Ariz.

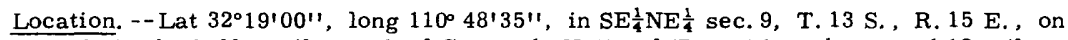
right bank, half a mile nor th of Coronado National Forest boundary, and 12 miles northeast of Tucson.

Drainage area. $--35.5 \mathrm{sq} \mathrm{mi}$.

Gage-height record. --Water-stage recorder graph. Altitude of gage is 2, $720 \mathrm{ft}$ (from topographic map).

Discharge record. --Stage-discharge relation defined by current-meter measurements below 3,000 cfs and extended above on basis of slope-area measurement of peak of Aug. 10,1966 , at gage height $9.65 \mathrm{ft}$.

Maxima. --November 1965 to January 1966: Discharge, 3, 920 cfs 1130 hours Dec. 22 (gage height, $8.00 \mathrm{ft}$ ).

1904-12, 1932 to October 1965: Discharge, 5, 100 cfs Mar. 23, 1954 (gage height, 8. $43 \mathrm{ft}$ ).

Mean discharge, in cubic feet per second, 1965-66

\begin{tabular}{|c|c|c|c|c|c|c|c|}
\hline Day & November & December & January & Day & November & December & January \\
\hline $1 \ldots$ & 0 & 3.3 & 138 & 16 & 0 & 95 & 28 \\
\hline 2 & 0 & 3.1 & 94 & $17 \ldots$ & 0 & 197 & 28 \\
\hline 3 & 0 & 2. 9 & 71 & 18 & 0 & 115 & 34 \\
\hline 4 & 0 & 2.8 & 58 & $19 \ldots$ & 0 & 78 & 38 \\
\hline 5 & 0 & 2.6 & 50 & $20 \ldots$ & 0 & 95 & 36 \\
\hline 6 & 0 & 2.5 & 45 & $21 \ldots$ & 0 & 101 & 36 \\
\hline $7 \ldots$ & 0 & 2.5 & 45 & $22 \ldots \ldots$ & 0 & 1,570 & 32 \\
\hline $8 \ldots$ & 0 & 2.3 & 47 & $23 \ldots \ldots$ & 0 & 1,050 & 32 \\
\hline $9 \ldots$ & 0 & 139 & 44 & $24 \ldots \ldots$ & 11 & 277 & 31 \\
\hline $10 \ldots$ & 0 & 941 & 43 & $25 \ldots \ldots$ & 145 & 184 & 30 \\
\hline $11 \ldots$ & 0 & 417 & 43 & $26 \ldots$ & 53 & 141 & 29 \\
\hline $12 \ldots$ & 0 & 136 & 38 & $27 \ldots$ & 16 & 112 & 28 \\
\hline 13 & 0 & 70 & 36 & $28 \ldots \ldots$ & 7.8 & 105 & 27 \\
\hline 14 & 0 & 84 & 33 & $29 \ldots$ & 4.6 & 111 & 28 \\
\hline \multirow[t]{2}{*}{$15 \ldots$} & 0 & 135 & 30 & $30 \ldots$ & 3.5 & 338 & 30 \\
\hline & & & & $31 \ldots$ & $\ldots \ldots$ & 214 & 39 \\
\hline \multirow{2}{*}{\multicolumn{5}{|c|}{ Monthly mean discharge, in cubic feet per second.. }} & 8.03 & 217 & 42.6 \\
\hline Runoff, & & & & & 478 & 13,340 & 2,620 \\
\hline
\end{tabular}


Gage height, in feet, and discharge, in cubic feet per second, at indicated time, 1965, of Sabino Creek near Tucson, Ariz.

\begin{tabular}{r|r|r|r||r|r|r|r|r|r|r|r}
\hline Date & Hour & $\begin{array}{c}\text { Gage } \\
\text { height }\end{array}$ & $\begin{array}{c}\text { Dis- } \\
\text { charge }\end{array}$ & Date & Hour & $\begin{array}{c}\text { Gage } \\
\text { height }\end{array}$ & $\begin{array}{c}\text { Dis- } \\
\text { charge }\end{array}$ & Date & Hour & $\begin{array}{c}\text { Gag } \\
\text { height }\end{array}$ & $\begin{array}{c}\text { Dis- } \\
\text { charge }\end{array}$ \\
\hline Dec. 21 & 2400 & 3.22 & 196 & Dec. 22 & 2000 & 5.96 & 1,240 & Dec. 23 & 2400 & 4.69 & 382 \\
& & & & & 2200 & 7.27 & 2,770 & & & & \\
& 0200 & 3.28 & 103 & & 2230 & 7.31 & 2,820 & 24 & 0600 & 4.48 & 300 \\
& 0400 & 3.52 & 134 & & 2400 & 7.17 & 2,640 & & 1200 & $4 . 乏 2$ & 248 \\
& 0600 & 3.94 & 201 & & & & & & 1400 & 4.21 & 242 \\
& 0800 & 4.57 & 347 & 23 & 0100 & 7.27 & 2,770 & & 1800 & $4 . 乏 7$ & 254 \\
& 0900 & 5.40 & 742 & & 0200 & 7.12 & 2,560 & & 2000 & 4.28 & 256 \\
& 1000 & 7.46 & 2,950 & & 0300 & 6.82 & 2,170 & & 2400 & 4.20 & 232 \\
& 1100 & 7.93 & 3,780 & & 0400 & 6.50 & 1,780 & & & & \\
& 1130 & 8.00 & 3,920 & & 0600 & 6.06 & 1,330 & 25 & 1500 & 4.02 & 163 \\
& 1230 & 7.84 & 3,670 & & 0800 & 5.72 & 1,040 & & 1700 & 4.02 & 163 \\
& 1400 & 7,27 & 2,760 & & 1000 & 5.56 & 917 & & 2100 & 4.11 & 176 \\
& 1600 & 6.65 & 1,970 & & 1200 & 5.35 & 768 & & 2400 & 4.09 & 171 \\
& 1800 & 6.22 & 1,500 & & 1800 & 4.92 & 495 & & & & \\
\hline
\end{tabular}

(65) 9-4842. Bear Creek near Tucson, Ariz.

Location. -- Lat $32^{\circ} 18^{\prime} 20^{\prime \prime}$, long $110^{\circ} 48^{\prime} 03^{\prime \prime}$, in NW $\frac{1}{4}$ sec. 15, T. 13 S., R. $15 \mathrm{E}$, on left bank 0.8 mile upstream from mouth, and 15 miles northeast of Tucson.

Drainage area. $--16.3 \mathrm{sq} \mathrm{mi}$.

Gage-height record. - -Water-stage recorder graph. Altitude of gage is 2, $670 \mathrm{ft}$ (from topographic map),

Discharge record. --Stage-discharge relation defined by current-meter measi rements below $300 \mathrm{cfs}$ and extended above on basis of computation of peak flow over dam.

Maxima. - -November 1965 to January 1966: Discharge, 1, 150 cfs 1200 hours Dec. 22 (gage height, recorded 4.1 ft, affected by drawdown; $4.90 \mathrm{ft}$ outside, from floodmark). 1959 to October 1965: Discharge, 575 cfs Jan. 11, 1960 (gage height, 2. $30 \mathrm{ft}$ ).

Mean discharge, in cubic feet per second, 1965-66

\begin{tabular}{|c|c|c|c|c|c|c|c|}
\hline Day & November & December & January & Day & November & December & January \\
\hline .. & 0 & 1.6 & 68 & $16 \ldots$ & 0 & 47 & 6.6 \\
\hline$\ldots$ & 0 & 1.4 & 41 & $17 \ldots$ & 0 & 90 & 5.3 \\
\hline 3 & 0 & 1.2 & 28 & $18 \ldots$ & 0 & 54 & 15 \\
\hline 4 & 0 & 1.0 & 21 & $19 \ldots$ & 0 & 40 & 20 \\
\hline 5 & 0 & .7 & 18 & $20 \ldots$ & 0 & 49 & 17 \\
\hline 6 & 0 & .6 & 15 & $21 \ldots$ & 0 & 54 & 18 \\
\hline 7 & 0 & .5 & 13 & $22 \ldots \ldots$ & 0 & 468 & 14 \\
\hline 8 & 0 & .6 & 13 & $23 \ldots$ & 0 & 374 & 12 \\
\hline 9 & 0 & 2. 3 & 13 & $24 \ldots$ & 0 & 122 & 12 \\
\hline 10 & 0 & 414 & 13 & $25 \ldots$ & 31 & 88 & 9.5 \\
\hline 11 & 0 & 218 & 13 & $26 \ldots$ & 40 & 69 & 9.0 \\
\hline 12 & 0 & 72 & 12 & 27 & 10 & 55 & 8.5 \\
\hline 13 & 0 & 35 & 12 & 28 & 4.4 & 49 & 10 \\
\hline 14 & 0 & 38 & 9.0 & 29 & 2.6 & 45 & 11 \\
\hline \multirow[t]{2}{*}{$15 \ldots$} & 0 & 54 & 7.7 & 30 & 1.5 & 179 & 12 \\
\hline & & & & 31 & $\ldots \ldots$ & 129 & 20 \\
\hline \multirow{2}{*}{\multicolumn{5}{|c|}{ Monthly mean discharge, in cubic feet per second.. }} & 2. 98 & 88.8 & 16.0 \\
\hline & & & & & 178 & 5,460 & 985 \\
\hline
\end{tabular}


Gage height, in feet, and discharge, in cubic feet per second, at indicated time, 1965, of Bear Creek near Tucson, Ariz.

\begin{tabular}{|c|c|c|c|c|c|c|c|c|c|c|c|}
\hline Date & Hour & $\begin{array}{l}\text { Gage } \\
\text { height }\end{array}$ & $\begin{array}{c}\text { Dis- } \\
\text { charge }\end{array}$ & Date & Hour & $\begin{array}{l}\text { Gage } \\
\text { height }\end{array}$ & $\begin{array}{c}\text { Dis- } \\
\text { charge }\end{array}$ & Date & Hour & $\begin{array}{l}\text { Gage } \\
\text { height }\end{array}$ & $\begin{array}{c}\text { Dis- } \\
\text { charge }\end{array}$ \\
\hline \multirow[t]{2}{*}{ Dec. 21} & 2400 & 1.52 & 55 & Dec. 22 & 1800 & 3.09 & 492 & Dec. 23 & 0800 & 2.76 & 423 \\
\hline & & & & & 2030 & 2.86 & 423 & \multirow{9}{*}{24} & 1200 & 2. 48 & 339 \\
\hline \multirow[t]{8}{*}{22} & 0400 & 1.69 & 83 & & 2200 & 3.54 & 649 & & 1800 & 2. 16 & 244 \\
\hline & 0800 & 2.02 & 155 & & 2300 & 3.84 & 754 & & 2400 & 1. 96 & 190 \\
\hline & 0900 & 2.49 & 288 & & 2400 & 3.71 & 709 & & & & \\
\hline & 1000 & 3.15 & 495 & \multirow{5}{*}{23} & & & & & 0600 & 1.82 & 130 \\
\hline & 1100 & 3.84 & 754 & & 0100 & 3.85 & 792 & & 1400 & 1. 73 & 111 \\
\hline & 1200 & 4. 14 & 1,150 & & 0230 & 3.77 & 764 & & 1700 & 1. 78 & 120 \\
\hline & 1400 & 3. 94 & 789 & & 0300 & 3.82 & 782 & & 2400 & 1.72 & 107 \\
\hline & 1600 & 3. 44 & 614 & & 0500 & 3.17 & 554 & & & & \\
\hline
\end{tabular}

(66) 9-4845. Tanque Verde Creek at Tucson, Ariz.

(Crest-stage station)

Location. - - Lat $32^{\circ} 15^{\prime} 57^{\prime \prime}$, long $110^{\circ} 50^{\prime} 27^{\prime \prime}$, in $\operatorname{SE} \frac{1}{4} \mathrm{SE} \frac{1}{4} \sec .30$, T. 13 S. , R. 15 E., at Sabino Canyon Road, 1.0 mile downstream from Sabino Creek, and $1 \frac{1}{4}$ miles northeast of Tucson city limits.

Drainage area. $--221 \mathrm{sq} \mathrm{mi}$.

Gage-height record. --Flood-hydrograph recorder graph 2300 hours Dec. 9 to 2000 hours Dec. 12, 1000 hours Dec. 15 to 1000 hours Dec. 19, 2400 hours Dec. 2.1 to 2000 hours Dec. 26, 0600 hours Dec. 30 to 1000 hours Dec. 31. Datum of gage is 2,464.33 ft above mean sea level (from Pima County bench mark).

Discharge record. --Stage-discharge relation defined by current-meter measurements.

Maxima. --November 1965 to January 1966: Discharge, 12, 200 cfs 1430 hours Dec. 22 (gage height, $7.89 \mathrm{ft}$ ).

1940 to October 1965: Discharge, 9, $000 \mathrm{cfs}$ Dec. 30, 1940 (gage height, $7.85 \mathrm{ft}$, at different datum).

Remarks. --Operated as continuous-record station 1940-45; published as Fillito Creek near Wrightstown.

Mean discharge, in cubic feet per second, December 1965
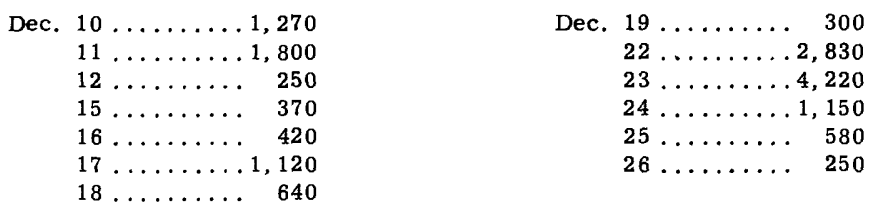
Gage height, in feet, and discharge, in cubic feet per second, at indicated time, 1965,

\begin{tabular}{|c|c|c|c|c|c|c|c|c|c|c|c|}
\hline Date & Hour & $\begin{array}{l}\text { Gage } \\
\text { height }\end{array}$ & $\begin{array}{c}\text { Dis- } \\
\text { charge }\end{array}$ & Date & Hour & $\begin{array}{l}\text { Gage } \\
\text { height }\end{array}$ & $\begin{array}{c}\text { Dis- } \\
\text { charge }\end{array}$ & Date & Hour & $\begin{array}{l}\text { Gage } \\
\text { height }\end{array}$ & $\begin{array}{c}\text { Dis- } \\
\text { charge }\end{array}$ \\
\hline \multirow[t]{2}{*}{ Dec. 21} & 2400 & 2. 20 & 220 & Dec. 22 & 2000 & 4. 79 & 2,270 & Dec. 24 & 0400 & 4. 29 & 1,490 \\
\hline & & & & & 2200 & 4. 57 & 1,800 & & 0800 & 4.03 & 1,140 \\
\hline \multirow[t]{14}{*}{22} & 0300 & 2. 20 & 220 & & 2400 & 5.97 & 4,880 & & 1200 & 3.89 & 960 \\
\hline & 0500 & 2.40 & 335 & & & & & & 1600 & 3.76 & 820 \\
\hline & 0800 & 2. 40 & 335 & 23 & 0100 & 5.51 & 3,740 & & 2000 & 3. 89 & 960 \\
\hline & 1000 & 2.75 & 575 & & 0200 & 6.75 & 7,280 & & 2400 & 3. 96 & 1,050 \\
\hline & 1100 & 3. 30 & 1,070 & & 0300 & 7. 07 & 8,480 & & & & \\
\hline & 1200 & 5.00 & 3,900 & & 0400 & 6. 96 & 8,040 & 25 & 0300 & 3. 69 & 750 \\
\hline & 1300 & 6.54 & 7,650 & & 0600 & 6.75 & 7,280 & & 0700 & 3. 69 & 750 \\
\hline & 1400 & 7. 49 & 10,200 & & 0800 & 5.97 & 4,880 & & 1500 & 3. 34 & 416 \\
\hline & 1430 & 7.89 & 12,200 & & 1000 & 5.51 & 3,740 & & 2400 & 3.27 & 348 \\
\hline & 1500 & 7. 49 & 10,200 & & 1200 & 5.27 & 3,220 & & & & \\
\hline & 1600 & 6.64 & 6,890 & & 1600 & 5.15 & 2,960 & 26 & 1200 & 3. 19 & 266 \\
\hline & 1700 & 5.97 & 4,870 & & 2000 & 4. 91 & 2,490 & & 1800 & 3. 19 & 266 \\
\hline & 1800 & 5.63 & 4,020 & & 2400 & 4. 61 & 1,970 & & & & \\
\hline & 1900 & 4. 97 & 3,600 & & & & & & & & \\
\hline
\end{tabular}

(67) 9-4845.1. Ventana Canyon Wash near Tucson, Ariz.

(Crest-stage station)

Location. --Lat $32^{\circ} 18^{\prime} 33^{\prime \prime}$, long $110^{\circ} 50^{\prime} 20^{\prime \prime}$, in SW $\frac{1}{4} \mathrm{SW} \frac{1}{4}$ sec. 8 , T. 13 S. , R. 15 E., at Sunrise Drive, half a mile upstream from Esperero Wash, and 4 miles northeast of Tucson city limits.

Drainage area. $--6.46 \mathrm{sq} \mathrm{mi}$.

Gage-height record. --Crest stages only. Altitude of gage is 2, $720 \mathrm{ft}$ (from tiopographic map).

Discharge record. --Stage-discharge relation defined by computations of flow through culvert.

Maxima. --November 1965 to January 1966: Discharge, 260 cfs Dec. 22 (gag : height, 11. $34 \mathrm{ft}$ ).

July to October 1965: Discharge, $93 \mathrm{cfs}$ Sept. 8 (gage height, $7.63 \mathrm{ft}$ ).

(68) 9-4846. Pantano Wash near Vail, Ariz.

Location. - Lat $32^{\circ} 02^{\prime} 09^{\prime \prime}$, long $110^{\circ} 40^{\prime} 37^{\prime \prime}$, in $\mathrm{SW} \frac{1}{4} \mathrm{SE} \frac{1}{4}$ sec. 14, T. $16 \mathrm{~S} .$, R. $16 \mathrm{E}$, , on right bank $60 \mathrm{ft}$ upstream from dam, 2.2 miles southeast of Vail, 2. 4 miles southwest of Pistol Hill, and 20 miles southeast of Tucson.

Drainage a: :a. --457 sq $\mathrm{mi}$.

Gage-height record. --Water-stage recorder graph except Dec. 13-15, 19-21, 24-26, and part of each day Dec. 12, 16, 22, 23, and 27. Graph reconstructed for missing periods. Altitude of gage is $3,205 \mathrm{ft}$ (from topographic map).

Discharge record. - -Stage-discharge relation defined by current-meter measurements below $2,000 \mathrm{cfs}=\mathrm{n}^{-1}=$ xtended above on basis of slope-area measurements at gage height 10.9.

Maxima. - - November 1965 to January 1966: Discharge, 1, 120 cfs 2300 hours Dec. 22 (gage height, $3.52 \mathrm{ft}$ ).

1958 to October 1965: Discharge, 38,000 cfs Aug. 11, 1958 (gage height, about 24 ft, from floodmarks). 
Mean discharge, in cubic feet per second, 1965-66, of Pantano Wash nec $r$ Vail, Ariz.

\begin{tabular}{|c|c|c|c|c|c|c|c|}
\hline Day & November & December & January & Day & November & Decemt or & January \\
\hline $1 \ldots$ & 0.3 & 1.7 & 7.2 & $16 \ldots$ & 0.4 & 2.0 & 1.7 \\
\hline $2 \ldots$ & .3 & 1. 7 & 5.9 & $17 \ldots$ & .4 & 9.6 & 1.7 \\
\hline $3 \ldots$ & .3 & 1.2 & 4.8 & $18 \ldots$ & .4 & 26 & 2.6 \\
\hline $4 \ldots$ & .3 & 1. 2 & 4.3 & $19 \ldots$ & .4 & 1. 7 & 2.3 \\
\hline $5 \ldots$ & .2 & 1. 2 & 3.4 & $20 \ldots$ & .7 & 1.5 & 1.7 \\
\hline $6 \ldots$ & .2 & 1. 2 & 3.0 & $21 \ldots$ & .7 & 1.5 & 2.3 \\
\hline $7 \ldots$ & .2 & 1. 2 & 3.0 & $22 \ldots$ & 1.7 & 502 & 2.3 \\
\hline $8 \ldots$ & .2 & 1.2 & 3.0 & $23 \ldots$ & 2.3 & 550 & 2.0 \\
\hline $9 \ldots$ & .3 & 18 & 3.0 & $24 \ldots$ & 2. 3 & 92 & 2.0 \\
\hline 10. & .3 & 31 & 2.6 & 25 & 3.0 & 50 & 2. 0 \\
\hline 11. & .3 & 154 & 2.6 & $26 \ldots$ & 2. 3 & 30 & 2. 0 \\
\hline 12 . & .3 & 9.9 & 2.6 & $27 \ldots$ & 2. 3 & 14 & 2.0 \\
\hline 13 & .2 & 1. 7 & 2.6 & $28 \ldots$ & 2. 3 & 10 & 2.3 \\
\hline $14 \ldots$ & .2 & 1.7 & 2.0 & $29 \ldots$ & 2.3 & 7.2 & 2. 3 \\
\hline $15 \ldots$ & .3 & 1.7 & 1.7 & $30 \ldots$ & 1.7 & 19 & 2.6 \\
\hline & & & & $31 \ldots$ & $\ldots . .$. & 13 & 3.0 \\
\hline \multirow{2}{*}{\multicolumn{5}{|c|}{$\begin{array}{l}\text { Monthly mean discharge, in cubic feet per second. } \\
\text { Runoff, in acre-feet } \ldots \ldots \ldots \ldots \ldots \ldots \ldots \ldots \ldots \ldots \ldots\end{array}$}} & 0.90 & 50.3 & 2. 79 \\
\hline & & & & & 54 & 3,090 & 172 \\
\hline
\end{tabular}

Gage height, in feet, and discharge, in cubic feet per second, at indicated time, 1965

\begin{tabular}{r|r|r|r||r|r|r|r||r|r|r|r}
\hline Date & Hour & $\begin{array}{c}\text { Gage } \\
\text { height }\end{array}$ & $\begin{array}{c}\text { Dis- } \\
\text { charge }\end{array}$ & Date & Hour & $\begin{array}{c}\text { Gage } \\
\text { height }\end{array}$ & $\begin{array}{c}\text { Dis- } \\
\text { charge }\end{array}$ & Date & Hour & $\begin{array}{c}\text { Gage } \\
\text { height }\end{array}$ & $\begin{array}{c}\text { Dis- } \\
\text { charge }\end{array}$ \\
\hline Dec. 21 & 2400 & 1.61 & 1.5 & Dec. 22 & 1645 & 2.77 & 507 & Dec. 23 & 0700 & 2.80 & 646 \\
& & & & & 1700 & 3.22 & 871 & & 0930 & 3.07 & 824 \\
& 0500 & 1.61 & 1.5 & & 1730 & 3.51 & 1,120 & & 1100 & 2.71 & 590 \\
& 0600 & 2.14 & 127 & & 1800 & 3.40 & 1,020 & & 1500 & 2.34 & 381 \\
& 0630 & 2.09 & 106 & & 1900 & 3.50 & 1,110 & & 1600 & 2.41 & 418 \\
& 0800 & 2.23 & 168 & & 2030 & 3.33 & 960 & & 1630 & 2.31 & 356 \\
& 0900 & 2.16 & 138 & & 2100 & 3.51 & 1,120 & & 1800 & 2.39 & 407 \\
& 1100 & 2.49 & 307 & & 2300 & 3.52 & 1,120 & & 2000 & 2.28 & 331 \\
& 1230 & 2.90 & 602 & & 2400 & 3.28 & 967 & & 2200 & 2.19 & 247 \\
& 1300 & 2.68 & 434 & & & & & & 2400 & 2.22 & 274 \\
& 1400 & 3.25 & 898 & 23 & 0130 & 2.90 & 711 & & & & \\
& 1500 & 3.22 & 871 & & 0400 & 3.15 & 877 & & & & \\
\hline
\end{tabular}

(69) 9-4850. Rincon Creek near Tucson, Ariz.

Location. --Lat $32^{\circ} 07^{\prime} 50^{\prime \prime}$, long $110^{\circ} 37^{\prime} 30^{\prime \prime}$, in NE⿺ sec. 17, T. 15 S., R. $17 \mathrm{E}$., on left bank, a quarter of a mile north of Sentinel Butte, 9 miles upstream from mouth, and 20 miles southeast of Tucson.

Drainage area. --44.8 sq mi.

Gage-height record. - Water-stage recorder graph. Altitude of gage is $3,120 \mathrm{ft}$ (from topographic map).

Discharge record. --Stage-discharge relation defined by current-meter me asurements below 1,800 cfs and extended above on basis of slope-area measurement of peak flow.

Maxima. --November 1965 to January 1966: Discharge, 3, 100 cfs 1230 hours Dec. 22 (gage height, $7.25 \mathrm{ft}$ ).

1952 to October 1965: Discharge, $8,250 \mathrm{cfs}$ Aug. 3, 1955 (gage height, $9.90 \mathrm{ft}$, from floodmarks). 
Mean discharge, in cubic feet per second, 1965-66, of Rincon Creek near Tusson, Ariz.

\begin{tabular}{|c|c|c|c|c|c|c|c|c|}
\hline Day & December & January & Day & December & January & Day & December & January \\
\hline & 0 & 134 & 11 & 374 & 27 & 21 & 55 & 40 \\
\hline & 0 & 80 & 12 & 74 & 25 & 22 . & 977 & 34 \\
\hline & 0 & 62 & & 46 & 22 & 23. & 658 & 30 \\
\hline & 0 & 50 & & 48 & 20 & $24 \ldots$ & 136 & 25 \\
\hline .. & 0 & 44 & $15 \ldots$ & 55 & 19 & 25 . & $9 \mathrm{C}$ & 22 \\
\hline $6 \ldots$ & 0 & 40 & 16 & 51 & 19 & 26. & 77 & 21 \\
\hline $7 \ldots$ & 0 & 36 & $17 \ldots$ & 70 & 19 & $27 \ldots$ & 72 & 22 \\
\hline $8 \ldots$ & 0 & 34 & $18 \ldots$ & 70 & 27 & $28 \ldots$ & $6 \varepsilon$ & 33 \\
\hline $9 \ldots$ & 12 & 30 & 19 & 58 & 42 & 29. & $6 \epsilon$ & 33 \\
\hline \multirow[t]{2}{*}{$10 \ldots$} & 405 & 27 & $20 \ldots$ & 58 & 40 & 30. & 251 & 32 \\
\hline & & & & & & $31 \ldots$ & 252 & 35 \\
\hline \multicolumn{7}{|c|}{ Monthly mean discharge, in cubic feet per second $\ldots \ldots \ldots \ldots \ldots$} & $13 \mathrm{C}$ & 36.3 \\
\hline \multicolumn{7}{|c|}{ 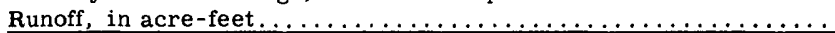 } & $7,98 \mathrm{C}$ & 2,230 \\
\hline
\end{tabular}

Gage height, in feet, and discharge, in cubic feet per second, at indicated time, 1965

\begin{tabular}{|c|c|c|c|c|c|c|c|c|c|c|c|}
\hline Date & Hour & $\begin{array}{l}\text { Gage } \\
\text { height }\end{array}$ & $\begin{array}{c}\text { Dis- } \\
\text { charge }\end{array}$ & Date & Hour & $\begin{array}{l}\text { Gage } \\
\text { height }\end{array}$ & $\begin{array}{c}\text { Dis- } \\
\text { charge }\end{array}$ & Date & Hour & $\begin{array}{l}\text { Gaq̧e } \\
\text { height }\end{array}$ & $\begin{array}{c}\text { Dis- } \\
\text { charge }\end{array}$ \\
\hline Dec. 21 & 2400 & 3.57 & 61 & Dec, 22 & $\begin{array}{l}1900 \\
1930\end{array}$ & $\begin{array}{l}5.68 \\
5.44\end{array}$ & $\begin{array}{l}832 \\
640\end{array}$ & Dec. 23 & $\begin{array}{l}1900 \\
2400\end{array}$ & $\begin{array}{l}\text { 4. } 74 \\
\text { 4. } 45\end{array}$ & $\begin{array}{l}281 \\
197\end{array}$ \\
\hline \multirow[t]{13}{*}{22} & 0200 & 4.02 & 105 & & 2100 & 5. 56 & 724 & & & & \\
\hline & 0400 & 4. 45 & 180 & & 2200 & 5.38 & 598 & 24 & 0000 & 4. 45 & 197 \\
\hline & 0500 & 4.85 & 313 & & 2400 & 5. 79 & 936 & & 0700 & 4. 11 & 133 \\
\hline & 0600 & 4. 86 & 317 & & & & & & 1300 & 3. 95 & 113 \\
\hline & 0730 & 4. 71 & 260 & 23 & 0100 & 6. 64 & 2,180 & & 1500 & 4.03 & 123 \\
\hline & 0900 & 5. 01 & 383 & & 0130 & 6.66 & 2,210 & & 1700 & 4. 13 & 135 \\
\hline & 1000 & 6.89 & 2,560 & & 0200 & 6.42 & 1,790 & & 1900 & 4. 14 & 137 \\
\hline & 1100 & 6. 95 & 2,670 & & 0400 & 6.04 & 1,220 & & 2400 & 3. 92 & 109 \\
\hline & 1230 & 7. 25 & 3,100 & & 0530 & 5.62 & 768 & & & & \\
\hline & 1300 & 7. 11 & 2,900 & & 0730 & 5.42 & 628 & 25 & 0700 & 3. 77 & 91 \\
\hline & 1400 & 6. 40 & 1,760 & & 0900 & 5.34 & 574 & & 1500 & 3.67 & 81 \\
\hline & 1500 & 6.24 & 1,500 & & 1200 & 5. 20 & 482 & & 2000 & 3. 74 & 88 \\
\hline & 1700 & 5.83 & 976 & & 1600 & 4. 93 & 352 & & 2400 & 3. 70 & 85 \\
\hline
\end{tabular}

(70) 9-4855. Pantano Wash at Tucson, Ariz.

(Crest-stage station)

Location. -- Lat $32^{\circ} 14^{\prime} 57^{\prime \prime}$, long $110^{\circ} 50^{\prime} 53^{\prime \prime}$, in NW $\frac{1}{4} \mathrm{NE} \frac{1}{4} \mathrm{sec} .6$, T. $14 \mathrm{~S}$. , R. $15 \mathrm{E}$., at Tanque Verde Road, 0.7 mile northeast of Tucson city limits, and $1 \frac{3}{4}$ miles above mouth.

Drainage area. $--602 \mathrm{sq} \mathrm{mi}$.

Gage-height record. --Flood-hydrograph recorder graph 1500-1900 hours De:. 22.

Discharge record. --Stage-discharge relation defined by current-meter measurements. Discharge for periods of no gage-height record estimated on basis of reccrds for nearby gaging stations.

Maxima. - November 1965 to January 1966: Discharge, 2, 830 cfs 1700 hours Dec. 22 (gage height, $1.9 \mathrm{ft}$ ).

1940-41, 1958 to October 1965: Discharge, 20,000 cfs Aug. 11 or 12, 1958 (gage height, $7.5 \mathrm{ft}$ ).

Remarks. -- Operated as continuous-record station 1940-41; published as "near Tucson." 
Mean discharge, in cubic feet per second, December 1965, of Panta7o Wash at Tucson, Ariz.

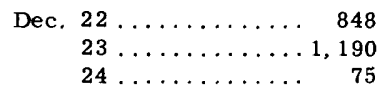

Gage height, in feet, and discharge, in cubic feet per second, at indicat?d time, 1965

\begin{tabular}{r|c|c|r||r|r|r|r||r|r|r|r}
\hline Date & Hour & $\begin{array}{c}\text { Gage } \\
\text { height }\end{array}$ & $\begin{array}{c}\text { Dis- } \\
\text { charge }\end{array}$ & Date & Hour & $\begin{array}{c}\text { Gage } \\
\text { height }\end{array}$ & $\begin{array}{c}\text { Dis- } \\
\text { charge }\end{array}$ & Date & Hour & $\begin{array}{c}\text { Gage } \\
\text { height }\end{array}$ & $\begin{array}{c}\text { Dis- } \\
\text { charge }\end{array}$ \\
\hline Dec. 21 & 2400 & - & 0 & Dec. 22 & 1700 & 1.89 & 2,830 & Dec. 23 & 0600 & - & 2,200 \\
& & & & & 1800 & 1.84 & 2,730 & & 1200 & - & 1,400 \\
& 0900 & - & 0 & & 1900 & 1.54 & 2,180 & & 1800 & - & 600 \\
& 1400 & - & 200 & & 2200 & - & 1,200 & & 2400 & - & 300 \\
& 1500 & 1.24 & 1,710 & & 2400 & - & 1,500 & & & & \\
& 1530 & 1.84 & 2,360 & & & & & 24 & 1200 & - & 0 \\
& 1600 & 1.74 & 2,530 & 23 & 0500 & - & 1,400 & & 2400 & - & 0 \\
\hline
\end{tabular}

(71) 9-4859. Pima Wash near Tucson, Ariz.

(Crest-stage station)

Location. - - Lat $32^{\circ} 20^{\prime} 15^{\prime \prime}$, long $110^{\circ} 57^{\prime} 35^{\prime \prime}$, in SW $\frac{1}{4} \mathrm{SW} \frac{1}{4} \sec .31, \mathrm{~T} .12 \mathrm{~S} ., \mathrm{R} .14 \mathrm{E}$, at Ina Road, 4 miles north of Tucson city limits.

Drainage area. $--4.93 \mathrm{sq} \mathrm{mi}$.

Gage-height record. --Flood-hydrograph recorder graph. Altitude of gage is 2,650 ft (from topographic map).

Discharge record. --Stage-discharge relation defined by computations of flow through culvert.

Maxima. -- November 1965 to January 1966: Discharge, $125 \mathrm{cfs}$ Dec. 22 (gage height, $9.93 \mathrm{ft}$ ).

1964 to October 1965: Discharge, $195 \mathrm{cfs}$ Sept. 6, 1964 (gage height, $11.12 \mathrm{ft}$ ).

(72) 9-4859.5. Geronimo Wash near Tucson, Ariz.

(Crest-stage station)

Location. - Lat $32^{\circ} 19^{\prime} 56^{\prime \prime}$, long $110^{\circ} 56^{\prime} 37^{\prime \prime}$, in $\mathrm{SE}_{\frac{1}{4}} \mathrm{NE} \frac{1}{4} \mathrm{sec} .6, \mathrm{~T} .13 \mathrm{~S}$, R. $14 \mathrm{E}$. , at Skyline Drive, 0.4 mile southeast of Ina Road, and $3 \frac{1}{2}$ miles north of Tucson city limits.

Drainage area. $--2.08 \mathrm{sq} \mathrm{mi}$.

Gage-height record. --Crest stages only. Altitude of gage is 2, $700 \mathrm{ft}$ (from topographic map).

Discharge record. --Stage-discharge relation defined by computations of flow through culvert.

Maxima. -- November 1965 to January 1966: Discharge, 65 cfs Dec. 22 (gage height, $5.04 \mathrm{ft}$ ).

1964 to October 1965: Discharge, $445 \mathrm{cfs}$ Sept. 6, 1964 (gage height, $11.9 \mathrm{ft}$ ). 
(73) 9-4860. Rillito Creek near Tucson, Ariz.

Location. --Lat $32^{\circ} 17^{\prime} 40^{\prime \prime}$, long $110^{\circ} 59^{\prime} 05^{\prime \prime}$, in SW $\frac{1}{4} \mathrm{SE} \frac{1}{4}$ sec. 14, T. $13 \mathrm{~S} .$, R. $13 \mathrm{E}$, on right bank 1, $100 \mathrm{ft}$ downstream from Pima Wash, 2, $300 \mathrm{ft}$ downstream from bridge on U.S. Highway $89,4 \frac{3}{4}$ miles upstream from mouth, and 5.4 miles north of Tucson city hall.

Drainage area. $--918 \mathrm{sq} \mathrm{mi}$.

Gage-height record. - - Water-stage recorder graph of stage at described location to 1600 hours Dec. 22. Flood-hydrograph recorder graph of stage $4 \frac{1}{2}$ miles upstream at Dodge Boulevard used as supplementary gage 1600 hours Dec. 22 to 1200 hours Dec. 24 and 1400 hours Dec. 30 to 2200 hours Dec. 31 . Wire-weight gage readings made at U.S. Highway 89 on Jan. 4, 7, 21, and 31. Datum of base gage is 2, 281.98 ft above mean sea level, datum of 1929 , supplementary adjustment of 1954 .

Discharge record. --Stage-discharge relation defined by current-meter measurements. Records for Dec. 25-27 and Dec. 31 to Jan. 31 estimated on basis of fragnentary gage-height record, discharge measurements, field estimates, observations of no flow, and records from other stations.

Maxima. --November 1965 to January 1966: Discharge, 12,400 cfs 1600 hours Dec. 22 (gage height, $10.36 \mathrm{ft}$ ).

1908 to October 1965: Discharge, 24, 000 cfs Sept. 23, 1929 (gage height, $24 \mathrm{ft}$, from floodmark, at site $1,800 \mathrm{ft}$ upstream at different datum).

Mean discharge, in cubic feet per second, 1965-66

\begin{tabular}{|c|c|c|c|c|c|c|c|c|}
\hline Day & lecember & January & Day & December & January & Day & December & January \\
\hline $\begin{array}{r}1 \ldots \\
2 \ldots \\
3 \ldots \\
4 \ldots \\
5 \ldots \\
6 \ldots \\
7 \ldots \\
8 \ldots \\
9 \ldots \\
10 \ldots \\
10 \ldots\end{array}$ & $\begin{array}{r}0 \\
0 \\
0 \\
0 \\
0 \\
0 \\
0 \\
0 \\
47 \\
1,130\end{array}$ & $\begin{array}{r}200 \\
80 \\
20 \\
0 \\
0 \\
0 \\
0 \\
0 \\
0 \\
0\end{array}$ & $\begin{array}{l}11 \ldots \\
12 \ldots \\
13 \ldots \\
14 \ldots \\
15 \ldots \\
16 \ldots \\
17 \ldots \\
18 \ldots \\
19 \ldots \\
20 \ldots \\
\end{array}$ & $\begin{array}{c}1,990 \\
163 \\
1.0 \\
51 \\
366 \\
348 \\
953 \\
499 \\
166 \\
109\end{array}$ & $\begin{array}{r}0 \\
0 \\
0 \\
0 \\
0 \\
0 \\
0 \\
0 \\
50 \\
50\end{array}$ & $\mid \begin{array}{l}21 \ldots \\
22 \ldots \\
23 \ldots \\
24 \ldots \\
25 \ldots \\
26 \ldots \\
27 \ldots \\
28 \ldots \\
29 \ldots \\
30 \ldots \\
31 \ldots \\
\end{array}$ & $\begin{array}{r}126 \\
3,520 \\
3,320 \\
1,160 \\
700 \\
400 \\
250 \\
350 \\
200 \\
600 \\
800 \\
\end{array}$ & $\begin{array}{r}15 \\
5 \\
0 \\
0 \\
0 \\
0 \\
0 \\
0 \\
0 \\
0 \\
6 \\
\end{array}$ \\
\hline \multicolumn{7}{|c|}{ 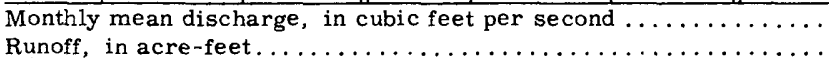 } & $\begin{array}{r}556 \\
34,210 \\
\end{array}$ & $\begin{array}{r}13.7 \\
845\end{array}$ \\
\hline
\end{tabular}

Gage height, in feet, and discharge, in cubic feet per second, at indicated t'me, 1965

\begin{tabular}{|c|c|c|c|c|c|c|c|c|c|c|c|}
\hline Date & Hour & $\begin{array}{l}\text { Gage } \\
\text { height }\end{array}$ & $\begin{array}{c}\text { Dis- } \\
\text { charge }\end{array}$ & Date & Hour & $\begin{array}{c}\text { Gage } \\
\text { height }\end{array}$ & $\begin{array}{c}\text { Dis- } \\
\text { charge }\end{array}$ & Date & Hour & $\begin{array}{l}\text { Gage } \\
\text { height }\end{array}$ & $\begin{array}{l}\text { Dis- } \\
\text { charge }\end{array}$ \\
\hline \multirow[t]{2}{*}{ Dec. 21} & 2400 & 2.89 & 81 & Dec. 22 & 1830 & - & 6,400 & Dec. 23 & 0730 & - & 6,000 \\
\hline & & & & & 1930 & - & 5,000 & \multirow{11}{*}{24} & 0900 & - & 4,600 \\
\hline \multirow[t]{10}{*}{22} & 0800 & 3. 39 & 194 & \multirow{10}{*}{23} & 2100 & - & 4,200 & & 1200 & - & 3,800 \\
\hline & 0930 & 4. 27 & 525 & & 2300 & - & 2,700 & & 1800 & - & 2,400 \\
\hline & 1030 & 5.40 & 1,230 & & 2400 & - & 4,400 & & 2400 & - & 2,000 \\
\hline & 1130 & 6.03 & 1,770 & & & & & & & & \\
\hline & 1200 & 7.24 & 3,280 & & 0100 & - & 5,600 & & 0400 & - & 1,450 \\
\hline & 1400 & 9.89 & 10,800 & & 0200 & - & 5,800 & & 0600 & - & 1,350 \\
\hline & 1430 & 9.86 & 10,700 & & 0400 & - & 7,600 & & 0800 & - & 1,160 \\
\hline & 1500 & 9.93 & 10,900 & & 0430 & - & 7,900 & & 1000 & - & 1,120 \\
\hline & 1600 & 10.36 & 12,400 & & 0500 & - & 8,200 & & 1200 & - & 1,080 \\
\hline & 1730 & - & 9,100 & & 0600 & - & 7,900 & & 2400 & - & 900 \\
\hline
\end{tabular}


(74) 9-4863. Canada del Oro near Tucson, Ariz.

Location. --Lat $32^{\circ} 22^{\prime} 25^{\prime \prime}$, long $111^{\circ} 00^{\prime} 30^{\prime \prime}$, in SW $\frac{1}{4} N W^{\frac{1}{4}}$ sec. 22, T. $12 \mathrm{~S} .$, R. 13 E., near right bank at upstream side of Overton Road, 5 miles upstream from mouth, and $10 \frac{1}{2}$ miles north of Tucson city hall.

Drainage area. $-\mathbf{- 2 5 0} \mathrm{sq} \mathrm{mi}$.

Gage-height record. --Water-stage recorder graph. Altitude of gage is 2, $380 \mathrm{ft}$ (from topographic map).

Discharge record. --Stage-discharge relation defined by current-meter measurements below $210 \mathrm{cfs}$ and extended above on basis of slope-area measurement cf peak flow.

Maximum. --November 1965 to January 1966: Discharge, 2, 290 efs 0100 hours Dec. 23 (gage height, $4.53 \mathrm{ft}$ ).

Remarks. --Flow occurred on Dec. 10-11 and 22-24 only.

Mean discharge, in cubic feet per second, December 1965

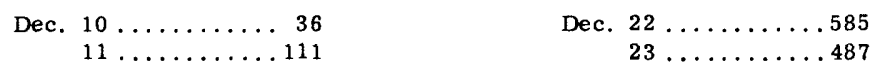

(75) 9-4865. Santa Cruz River at Cortaro, Ariz.

Location. --Lat $32^{\circ} 21^{\prime} 10^{\prime \prime}$, long $111^{\circ} 05^{\prime} 45^{\prime \prime}$, in SW $\frac{1}{4} \mathrm{SW} \frac{1}{4} \sec .26$, T. $12 \mathrm{~S}$. , R. 12 E., on downstream side of bridge pier half a mile southwest of Cortaro, 3 miles downstream from Canada del Oro, and 4 miles downstream from Rillito Creek.

Drainage area. $--3,503 \mathrm{sq} \mathrm{mi}$.

Gage-height record. - Water-stage recorder graph except Nov, 1-20, Dec, 13-14, 19-21, Dec. 24 to Jan. 20. Graph reconstructed for Dec. 13-14, 19-21. Fragmentary record for Jan, 20-21. Datum of gage is 2,137, $13 \mathrm{ft}$ above mean sea level (Arizona Highway Department bench mark).

Discharge record. --Stage-discharge relation defined by current-meter measurements. Discharge for periods of no gage-height record estimated on basis of records for upstream stations.

Maxima. -- November 1965 to January 1966: Discharge, 16, 800 efs 1800 hours Dec. 22 (gage height, $8.60 \mathrm{ft}$ ).

1936 to October 1965: Discharge, 17, 000 cfs Aug. 14, 1940 (gage height, 9. $9 \mathrm{ft}$, at site $4 \frac{3}{4}$ miles downstream at different datum).

Mean discharge, in cubic feet per second, 1965

\begin{tabular}{|c|c|c|c|c|c|c|c|c|}
\hline Day & December & January & Day & December & $J$ anuary & Day & December & January \\
\hline 1 & 0 & 0 & $11 \ldots$ & 2,230 & 0 & 21 & 10 & 1.4 \\
\hline$\ldots$ & 0 & 0 & $12 \ldots$ & 110 & 0 & 22 & 5,420 & 0 \\
\hline$\ldots$ & 0 & 0 & $13 \ldots$ & 0 & 0 & 23 & 8,460 & 0 \\
\hline 4 & 0 & 0 & 14 & 45 & 0 & 24 & 3,300 & 0 \\
\hline 5 & 0 & 0 & 15 & 328 & 0 & 25 & 1,000 & 0 \\
\hline & 0 & 0 & $16 \ldots$ & 536 & 0 & 26 & 100 & 0 \\
\hline 7 & 0 & 0 & $17 \ldots$ & 1,440 & 0 & 27 & 9 & 0 \\
\hline $8 \ldots$ & 0 & 0 & $18 \ldots$ & 1,500 & 0 & $28 \ldots$ & 2 & 0 \\
\hline $9 \ldots$ & 116 & 0 & $19 \ldots$ & 206 & 1.0 & 29 & 0 & 0 \\
\hline $10 \ldots$ & 1,290 & 0 & $20 \ldots$ & 50 & 16 & 30 & 0 & 0 \\
\hline & & & & & & 31 & 200 & 0 \\
\hline \multirow{2}{*}{\multicolumn{7}{|c|}{$\begin{array}{l}\text { Monthly mean discharge, in cubic feet per second. } \ldots \ldots \ldots \ldots \ldots \ldots \\
\text { Runoff, in acre-feet } \ldots \ldots \ldots \ldots \ldots \ldots \ldots \ldots \ldots \ldots\end{array}$}} & 850 & 0.59 \\
\hline & & & & & & & 52,270 & 36 \\
\hline
\end{tabular}


Gage height, in feet, and discharge, in cubic feet per second, at indicated time, 1965, of Santa Cruz River at Cortaro, Ariz.

\begin{tabular}{|c|c|c|c|c|c|c|c|c|c|c|c|}
\hline Date & Hour & $\begin{array}{c}\text { Gage } \\
\text { height }\end{array}$ & $\begin{array}{l}\text { Dis- } \\
\text { charge }\end{array}$ & Date & Hour & $\begin{array}{l}\text { Gage } \\
\text { height }\end{array}$ & $\begin{array}{c}\text { Dis- } \\
\text { charge }\end{array}$ & Date & Hour & $\begin{array}{l}\text { Grge } \\
\text { he ight }\end{array}$ & $\begin{array}{c}\text { Dis- } \\
\text { charge }\end{array}$ \\
\hline \multirow[t]{2}{*}{ Dec. 21} & 2400 & $(98.99)$ & 2 & Dec. 22 & 2100 & 6.13 & 9,300 & Dec. 23 & 0900 & 4.83 & 6,300 \\
\hline & & & & & 2200 & 5. 81 & 8,500 & & 0930 & 4.77 & 6,100 \\
\hline \multirow[t]{12}{*}{22} & 1130 & $(98.91)$ & 2 & & 2300 & 5.55 & 7,800 & & 1030 & 5.02 & 6,700 \\
\hline & 1200 & 3.27 & 2,620 & & 2400 & 5. 36 & 7,350 & & 1400 & 5.65 & 8,300 \\
\hline & 1300 & 5.50 & 7,800 & & & & & & 1600 & 5.51 & 8,000 \\
\hline & 1400 & 6.68 & 10,800 & 23 & 0130 & 6.62 & 10,700 & & 1800 & 5.29 & 7,400 \\
\hline & 1500 & 6.91 & 11,500 & & 0200 & 6. 70 & 10,900 & & 2000 & 4.90 & 6,430 \\
\hline & 1600 & 7. 22 & 12,400 & & 0300 & 7. 16 & 12,400 & & 2200 & 4.72 & 6,000 \\
\hline & 1700 & 8.02 & 14,900 & & 0330 & 7. 21 & 12,500 & & 2400 & 4.56 & 5,620 \\
\hline & 1730 & 8.44 & 16,300 & & 0400 & 6. 94 & 11,700 & & & & \\
\hline & 1800 & 8.60 & 16,800 & & 0500 & 7. 39 & 13,100 & 24 & 0100 & 4. 56 & 5,620 \\
\hline & 1830 & 8. 39 & 16,100 & & 0600 & 7. 31 & 12,900 & & 0600 & - & 4,200 \\
\hline & 1900 & 7. 70 & 14,500 & & 0630 & 7.22 & 12,600 & & 1200 & - & 2,800 \\
\hline & 2000 & 6.74 & 10,900 & & 0800 & 5.69 & 8,400 & & 2400 & - & 1,700 \\
\hline
\end{tabular}

(76) 9-4866. Arivaca Creek near Arivaca, Ariz.

(Miscellaneous site)

Location. -- Lat $31^{\circ} 36^{\prime} 15^{\prime \prime}$, long $111^{\circ} 23^{\prime} 00^{\prime \prime}$, in SW $\frac{1}{4} \mathrm{NW} \frac{1}{4}$ sec. 13, T. $21 \mathrm{~S}$, , R. 9 E., at county road 4 miles northwest of Arivaca.

Drainage area. $--78.4 \mathrm{sq} \mathrm{mi}$.

Maximum. --November 1965 to January 1966: Discharge, 15, $900 \mathrm{cfs}$ Dec, 24 (result of dam failure), from slope-area measurement of peak flow (outside stage at gage, 13. 3 $\mathrm{ft}$, from profile of floodmarks).

Remarks. --Gaging station established Mar. 23, 1967.

(77) 9-4872.5. Los Robles Wash near Marana, Ariz.

\section{(Miscellaneous site)}

Location. --Lat $32^{\circ} 26^{\prime} 16^{\prime \prime}$, long $111^{\circ} 18^{\prime} 13^{\prime \prime}$, in SE $\frac{1}{4} \mathrm{SE}_{4}^{\frac{1}{4}} \mathrm{sec} .27$, T. $11 \mathrm{~S} .$, R. $10 \mathrm{E}$. , at Trico Road, three-quarters of a mile downstream from confluence of Brawley Wash and China Draw, 3 miles upstream from Blanco Wash, and 5 miles soutr vest of Marana.

Drainage area. $--1,170 \mathrm{sq} \mathrm{mi}$.

Maxima. --November 1965 to January 1966: Discharge, 2, $040 \mathrm{cfs}$ probably Dec. 24 (gage height, $7.0 \mathrm{ft}$ ).

1962 to October 1965: Discharge, about $32,000 \mathrm{cfs}$ Sept. 26, 1962, from slopearea measurements at sites upstream and downstream from this site.

Remarks. --Crest-stage station established June 1967. 
(78) 9-4890. Santa Cruz River near Laveen, Ariz.

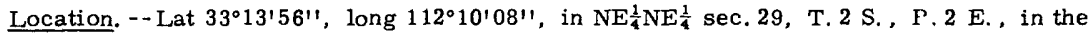
Gila River Indian Reservation, on downstream side of highway bridge, 3.4 miles upstream from mouth, 4.3 miles south of Komatke, and 9 miles south of Laveen.

Drainage area. $--8,581 \mathrm{sq} \mathrm{mi}$.

Gage-height record. --Water-stage recorder graph, except 0800 hours Dec. 31 to 0800 hours Jan. 3, Jan. 5-10, for which graph was reconstructed on basis of indicated recession for an adjacent peak. Datum of gage is $1,020.86 \mathrm{ft}$ above mean sea level, datum of 1929, Phoenix-Picacho supplementary adjustment of 1949 .

Discharge record. --Stage-discharge relation defined by current-meter measurements.

Maxima. --November 1965 to January 1966: Discharge, 2, 940 cfs 1100 ho'urs Dec. 26 (gage height, $15.50 \mathrm{ft}$ ).

1940 to October 1965: Discharge, 9, $200 \mathrm{cfs}$ Sept. 29, 1962 (gage height, 17, $50 \mathrm{ft}$ ).

Mean discharge, in cubic feet per second, 1965-66

\begin{tabular}{|c|c|c|c|c|c|c|c|}
\hline Day & November & December & January & Day & November & Decembor & January \\
\hline 1 & 0 & 0 & 0.4 & 16 & 0 & 172 & 0 \\
\hline 2. & 0 & 0 & .1 & 17 & 0 & 280 & 0 \\
\hline $3 \ldots$ & 0 & 0 & 4.5 & $18 \ldots$ & 0 & 262 & 4. 3 \\
\hline $4 \ldots$ & 0 & 0 & 1.8 & $19 \ldots$ & 0 & 270 & 2.0 \\
\hline$\ldots$ & 0 & 0 & .6 & $20 \ldots$ & 0 & 681 &. .6 \\
\hline 6 & 0 & 0 & .3 & $21 \ldots$ & 0 & 186 & .2 \\
\hline 7 & 0 & 0 & 1 & $22 \ldots$ & 0 & 218 & 1 \\
\hline 8 & 0 & 0 & 0 & $23 \ldots$ & 0 & 348 & .1 \\
\hline $9 \ldots$ & 0 & .3 & 0 & $24 \ldots$ & 0 & 641 & 0 \\
\hline $10 \ldots$ & 0 & 5. 3 & 0 & $25 \ldots$ & 2.6 & 2,170 & 0 \\
\hline 11. & 0 & 28 & 0 & $26 \ldots$ & 0 & 2,730 & 0 \\
\hline 12 & 0 & 77 & 0 & $27 \ldots$ & 0 & 1,250 & 0 \\
\hline 13 & 0 & 181 & 0 & $28 \ldots$ & 0 & 327 & 0 \\
\hline $14 \ldots$ & 0 & 290 & 0 & $29 \ldots \ldots$ & 0 & $3 c$ & 0 \\
\hline \multirow[t]{2}{*}{$15 \ldots$} & 0 & 163 & 0 & $30 \ldots$ & 0 & ล. 1 & 0 \\
\hline & & & & 31. & $\ldots \ldots$ & .8 & 0 \\
\hline \multicolumn{5}{|c|}{ Monthly mean discharge, in cubic feet per second. . } & 0.09 & 333 & 0.49 \\
\hline \multicolumn{5}{|c|}{ Runoff, in acre-feet $\ldots \ldots \ldots \ldots \ldots \ldots$} & 5.2 & 20,460 & 30 \\
\hline
\end{tabular}

Gage height, in feet, and discharge, in cubic feet per second, at indicated time, 1965

\begin{tabular}{|c|c|c|c|c|c|c|c|c|c|c|c|}
\hline Date & Hour & $\begin{array}{l}\text { Gage } \\
\text { height }\end{array}$ & $\begin{array}{c}\text { Dis- } \\
\text { charge }\end{array}$ & Date & Hour & $\begin{array}{l}\text { Gage } \\
\text { height }\end{array}$ & $\begin{array}{c}\text { Dis- } \\
\text { charge }\end{array}$ & Date & Hour & $\begin{array}{l}\text { Gage } \\
\text { height }\end{array}$ & $\begin{array}{c}\text { Dis- } \\
\text { charge }\end{array}$ \\
\hline \multirow[t]{2}{*}{ Dec. 21} & 2400 & 8.50 & 136 & Dec. 24 & 0500 & 11.50 & 566 & Dec. 27 & 0700 & 13.73 & 1,440 \\
\hline & & & & & 1200 & 11.45 & 554 & & 1500 & 12.87 & 1,020 \\
\hline \multirow[t]{8}{*}{22} & 0600 & 8.00 & 110 & & 1600 & 11.65 & 600 & & 2400 & 12.00 & 699 \\
\hline & 0900 & 9.20 & 188 & & 2400 & 13.00 & 1,080 & & & & \\
\hline & 1230 & 9.30 & 198 & & & & & 28 & 0600 & 11.15 & 486 \\
\hline & 1600 & 9.80 & 255 & 25 & 0600 & 14. 15 & 1,660 & & 1200 & 10.00 & 284 \\
\hline & 1730 & 10.22 & 314 & & 1330 & 15.28 & 2,660 & & 1800 & 8.70 & 148 \\
\hline & 1930 & 10.44 & 344 & & 2400 & 15.25 & 2,630 & & 2400 & 7. 50 & 80 \\
\hline & 2400 & 10.23 & 316 & & & & & & & & \\
\hline & & & & 26 & 0500 & 15.45 & 2,870 & 29 & 0500 & 6. 70 & 47 \\
\hline \multirow[t]{4}{*}{23} & 0630 & 10.40 & 339 & & 1100 & 15.50 & 2,940 & & 1200 & 5.98 & 23 \\
\hline & 1400 & 10.52 & 360 & & 1700 & 15.40 & 2,800 & & 2000 & 5.25 & 9.2 \\
\hline & 1830 & 10. 37 & 334 & & 2400 & 14.73 & 2,090 & & 2200 & 5.25 & 9.2 \\
\hline & 2400 & 10.77 & 406 & & & & & & 2400 & 5.10 & 6.9 \\
\hline
\end{tabular}


(79) 9-4890. 7. North Fork of East Fork Black River near Alpine, Ariz.

Location. -- Lat $33^{\circ} 54^{\prime} 10^{\prime \prime}$, long $109^{\circ} 19^{\prime} 20^{\prime \prime}$, in SW $\frac{1}{4} \mathrm{NE} \frac{1}{4}$ sec. 19, T. 6 N. , R. 29 E. (unsurveyed), in Apache National Forest, on right bank $1 \frac{1}{2}$ miles downstream from Crosby Crossing, and 12 miles northwest of Alpine.

Drainage area. $--38.1 \mathrm{sq} \mathrm{mi}$.

Gage-height record. --Water-stage recorder graph except Jan. 3-27. Altitude of gage is $8,650 \mathrm{ft}$ (from topographic map).

Discharge record. --Stage-discharge relation defined by current-meter meas arements. Discharge for period of no gage-height record estimated.

Maxima. --November 1965 to January 1966: Discharge, 76 cfs 2230 hours Nov. 25 (gage height, 2.12 ft).

June to October 1965: Discharge, $126 \mathrm{cfs}$ July 27 (gage height, $2.47 \mathrm{ft}$ ).

Mean discharge, in cubic feet per second, 1965-66

\begin{tabular}{|c|c|c|c|c|c|c|c|}
\hline Day & November & December & January & Day & November & December & January \\
\hline $1 \ldots$ & 1.5 & 3.6 & 10 & $16 \ldots$ & 1.3 & 2 & 1 \\
\hline $2 \ldots$ & 1.5 & 3.0 & 8 & $17 \ldots$ & 1.2 & 2 & 1 \\
\hline $3 \ldots$ & 1.6 & 2.2 & 6 & $18 \ldots$ & .9 & 2 & 1 \\
\hline$\ldots$ & 1.6 & 2.2 & 5 & 19 & .9 & 2 & 1 \\
\hline$\ldots$ & 1.6 & 2.2 & 4.5 & 20 & 1.0 & 2 & 1 \\
\hline $6 \ldots$ & 1.6 & 2.2 & 4 & 21 & 1.2 & 2 & 1 \\
\hline $7 \ldots$ & 1.8 & 2.6 & 3.5 & 22 & 1.2 & 2 & 1 \\
\hline $8 \ldots$ & 1.8 & 2.2 & 3 & $23 \ldots$ & 4.5 & 3 & 1 \\
\hline $9 \ldots$ & 1.8 & 7. 4 & 2.5 & $24 \ldots \ldots$ & 5.0 & 4 & 1 \\
\hline $10 \ldots$ & 1.7 & 6.4 & 2 & $25 \ldots$ & 22 & 3 & 1 \\
\hline $11 \ldots$ & 1.6 & 9.8 & 1.5 & $26 \ldots$ & 28 & 2 & 1 \\
\hline $12 \ldots$ & 1.4 & 11 & 1 & $27 \ldots$ & 11 & 2 & 1 \\
\hline $13 \ldots$ & 1.4 & 10 & 1 & $28 \ldots \ldots$ & 7.3 & 2 & 1 \\
\hline $14 \ldots$ & 1.3 & 6 & 1 & $29 \ldots \ldots$ & 4.2 & 2 & 1 \\
\hline $15 \ldots$ & 1.2 & 3 & 1 & $30 \ldots$ & 3.4 & 2 & 1 \\
\hline & & & & $31 \ldots$ & $\ldots-\cdots$ & 10 & 1 \\
\hline \multirow{2}{*}{\multicolumn{5}{|c|}{$\begin{array}{l}\text { Monthly mean discharge, in cubic feet per second } \ldots \\
\text { Runoff, in acre-feet } \ldots \ldots \ldots \ldots \ldots \ldots \ldots \ldots \ldots \ldots\end{array}$}} & 3. 88 & 3.80 & 2.26 \\
\hline & & & & & 231 & 234 & 139 \\
\hline
\end{tabular}

(80) 9-4890. 8. Hannagan Creek near Hannagan Meadow, Ariz.

(Crest-stage station)

Location. -- Lat $33^{\circ} 38^{\prime} 50^{\prime \prime}$, long $109^{\circ} 17^{\prime} 20^{\prime \prime}$, at U.S. Highway $666,2.5$ miles northeast of Hannagan Meadow.

Drainage area. $--1.61 \mathrm{sq} \mathrm{mi}$.

Gage-height record. --Crest stages only. Altitude of gage is $8,800 \mathrm{ft}$ (from topographic map).

Discharge record. --Stage-discharge relation defined by computation of flow through culvert.

Maxima. -- November 1965 to January 1966: Discharge, not determined, knov'n to have been less than $20 \mathrm{cfs}$.

1964 to October 1965: Discharge, $20 \mathrm{cfs}$ Apr. 22, 1965 (gage height, 4. $79 \mathrm{ft}$ ). 
(81) 9-4891. Black River near Maverick, Ariz.

Location. --Lat $33^{\circ} 42^{\prime} 30^{\prime \prime}$, long $109^{\circ} 26^{\prime} 50^{\prime \prime}$, in SW $\frac{1}{4}$ sec. 30, T. 4 N. , R. 28 E., in Apache National Forest, on right bank 1.1 miles upstream from Conklin Creek, 1.2 miles downstream from Fish Creek, and 6 miles southwest of Maverick.

Drainage area. $--315 \mathrm{sq} \mathrm{mi}$.

Gage-height record. - Water-stage recorder graph except Jan. 25-26 and fart of each day Jan. 2-24, when float was frozen. Graph reconstructed for missing periods. Altitude of gage is $6,850 \mathrm{ft}$ (from topographic map).

Discharge record. --Stage-discharge relation defined by current-meter measurements. Stage-discharge relation affected by backwater from ice part of each day Dec. 19-21.

Maxima. --November 1965 to January 1966: Discharge, 1, 290 cfs 2300 hours Dec. 30 (gage height, $3.55 \mathrm{ft}$ ).

1962 to October 1965: Discharge, 2, $010 \mathrm{cfs}$ Apr. 21, 1965 (gage height, 4. $20 \mathrm{ft}$ ).

Mean discharge, in cubic feet per second, 1965-66

\begin{tabular}{|c|c|c|c|c|c|c|c|}
\hline Day & November & December & January & Day & November & December & J anuary \\
\hline 1 & 23 & 95 & 742 & 16 & 21 & 127 & 150 \\
\hline $2 \ldots$ & 23 & 80 & 525 & $17 \ldots$ & 21 & 129 & 147 \\
\hline $3 \ldots$ & 22 & 69 & 400 & $18 \ldots$ & 21 & 122 & 139 \\
\hline $4 \ldots$ & 22 & 69 & 380 & $19 \ldots$ & 21 & 103 & 129 \\
\hline $5 \ldots$ & 22 & 64 & 315 & $20 \ldots$ & 20 & 110 & 115 \\
\hline 6 & 21 & 58 & 275 & $21 \ldots$ & 21 & 112 & 100 \\
\hline $7 \ldots$ & 22 & 61 & 240 & 22. & 22 & 142 & 80 \\
\hline $8 \ldots$ & 22 & 59 & 240 & 23 & 77 & 150 & 80 \\
\hline $9 \ldots$ & 22 & 90 & 240 & $24 \ldots$ & 113 & 122 & 90 \\
\hline $10 \ldots$ & 22 & 140 & 220 & $25 \ldots$ & 264 & 129 & 80 \\
\hline $11 \ldots$ & 22 & 157 & 220 & 26. & 550 & 134 & 70 \\
\hline $12 \ldots$ & 20 & 169 & 190 & 27 . & 224 & 132 & 77 \\
\hline 13. & 21 & 177 & 180 & 28 & 154 & 129 & 78 \\
\hline 14. & 21 & 147 & 180 & 29 & 107 & 147 & 69 \\
\hline \multirow[t]{2}{*}{$15 \ldots$} & 21 & 127 & 150 & 30 & 99 & 713 & 77 \\
\hline & & & & 31. & $---\cdot-$ & 1,130 & 73 \\
\hline \multirow{2}{*}{\multicolumn{5}{|c|}{ Monthly mean discharge, in cubic feet per second.. }} & 68.7 & 168 & 195 \\
\hline & & & & & 4,090 & 10,300 & 12,000 \\
\hline
\end{tabular}

(82) 9-4892. Pachete Creek at Maverick, Ariz.

Location. -- Lat $33^{\circ} 44^{\prime} 2^{\prime \prime \prime}$, long $109^{\circ} 32^{\prime} 25^{\prime \prime}$, at corner of secs, $28,29,32,33$, T. $4 \frac{1}{2}$ N. , R. 27 E., in Fort Apache Indian Reservation, on left bank, half a mile south of Maverick.

Drainage area. $--14.8 \mathrm{sq} \mathrm{mi}$.

Gage-height record. --Water-stage recorder graph except Dec. 19-27 and Jan. 1-13 when only range in stage was recorded. Altitude of gage is $7,850 \mathrm{ft}$ (by barometer).

Discharge record. --Stage-discharge relation defined by current-meter measurements. Discharge for periods of no gage-height record estimated.

Maxima. --November 1965 to January 1966: Discharge, 78 cfs (time unknown) Dec. 31 (gage height, $3.36 \mathrm{ft}$ ).

1957 to October 1965: Discharge, 312 cfs Apr. 22, 1958 (gage height. 4. $33 \mathrm{ft}$ ). 
Mean discharge, in cubic feet per second, 1965-66, of Pachete Creek at Mavarick, Ariz.

\begin{tabular}{|c|c|c|c|c|c|c|c|}
\hline Day & November & December & January & Day & November & December & January \\
\hline $1 \ldots$ & 1.1 & 3.5 & 40 & 16 & 1.1 & 10 & 12 \\
\hline $2 \ldots$ & 1.1 & 3.1 & 30 & 17 & 1.1 & 10 & 12 \\
\hline . & 1.1 & 2.7 & 25 & 18 & 1.1 & 8.4 & 13 \\
\hline $4 \ldots$ & 1.1 & 3.3 & 23 & 19 & 1.0 & 8 & 12 \\
\hline 5 & 1.1 & 3.5 & 22 & 20 & 1.0 & 8 & 11 \\
\hline 6 & 1.1 & 3. 8 & 21 & 21 & 1.1 & 8 & 9.9 \\
\hline 7 & 1.1 & 4.2 & 20 & 22 & 1.2 & 25 & 9 \\
\hline 8 & 1.0 & 4.2 & 20 & 23 & 4.0 & 35 & 9 \\
\hline $9 \ldots$ & 1.0 & 12 & 19 & 24 & 2.4 & 15 & 10 \\
\hline $10 \ldots$ & 1. 0 & 15 & 18 & $25 \ldots$ & 19 & 10 & 10 \\
\hline $11 \ldots$ & 1.0 & 11 & 17 & $26 \ldots$ & 13 & 9 & 11 \\
\hline $12 \ldots$ & 1.0 & 10 & 16 & 27 & 6.2 & 8 & 10 \\
\hline $13 \ldots$ & 1,0 & 13 & 15 & 28 & 4.4 & 7. 7 & 9 \\
\hline $14 \ldots$ & 1. 0 & 13 & 14 & 29 & 3.6 & 7. 0 & 8 \\
\hline \multirow[t]{2}{*}{$15 \ldots$} & 1.0 & 12 & 13 & 30. & 3.3 & 38 & 8 \\
\hline & & & & 31 & $\ldots-\cdots$ & 53 & 7 \\
\hline \multirow{2}{*}{\multicolumn{5}{|c|}{$\begin{array}{l}\text { Monthly mean discharge, in- cubic feet per second. } \\
\text { Runoff in acre-feet }\end{array}$}} & 2.64 & 12.1 & 15.3 \\
\hline & & & & & 157 & 741 & 940 \\
\hline
\end{tabular}

(83) 9-4895. Black River below pumping plant, near Point of Pines, Ariz.

Location. -- Lat $33^{\circ} 28^{\prime} 30^{\prime \prime}$ ', long $109^{\circ} 46^{\prime} 00^{\prime \prime}$, in W $\frac{1}{2}$ sec. 32, T. 2 N. , R. $25 \mathrm{E}$. (unsurveyed), in San Carlos Indian Reservation, on left bank 1 mile downstream from Fhelps Dodge Corp. pumping plant, $1 \frac{1}{2}$ miles downstream from Freezeout Creek, 7 miles northwest of Point of Pines, and 63 miles upstream from confluence with White Rivor.

Drainage area. $--560 \mathrm{sq} \mathrm{mi}$.

Gage-height record. - Water-stage recorder graph Nov. 1-11; digital recorder tape the reafter. Altitude of gage is $5,742 \mathrm{ft}$ (from river-profile map).

Discharge record. --Stage-discharge relation defined by current-meter measurements.

Maxima. --November 1965 to January 1966: Discharge, 6, 380 cfs 1130 hours Dec. 30 (gage height, $10.86 \mathrm{ft}$ ).

1953 to October 1965: Discharge, 5, 000 cfs Mar. 23, 1954 (gage height, 9. $35 \mathrm{ft}$ ).

\begin{tabular}{|c|c|c|c|c|c|c|c|}
\hline Day & November & December & January & Day & Noyember & December & January \\
\hline 1 & 42 & 150 & 1,760 & 16 & 23 & 249 & 265 \\
\hline 2 & 42 & 137 & 1,130 & 17 & 24 & 259 & 260 \\
\hline 3 & 41 & 115 & 874 & 18 & 24 & 240 & 255 \\
\hline 4 & 41 & 102 & 711 & 19 & 32 & 197 & 244 \\
\hline 5 & 41 & 94 & 586 & 20 & 37 & 207 & 219 \\
\hline $6 \ldots$ & 41 & 91 & 514 & $21 \ldots$ & 31 & 215 & 211 \\
\hline $7 \ldots$ & 41 & 80 & 461 & 22. & 30 & 557 & 186 \\
\hline $8 \ldots$ & 42 & 81 & 441 & 23. & 48 & 1,067 & 185 \\
\hline 9 & 38 & 91 & 409 & 24 & 146 & 442 & 188 \\
\hline $10 \ldots$ & 27 & 497 & 377 & $25 \ldots$ & 150 & 322 & 183 \\
\hline $11 \ldots$ & 22 & 600 & 357 & 26 & 811 & 273 & 175 \\
\hline 12 & 20 & 446 & 349 & 27 & 420 & 251 & 174 \\
\hline $13 \ldots$ & 20 & 386 & 314 & 28 & 274 & 233 & 201 \\
\hline $14 \ldots$ & 19 & 330 & 287 & 29 & 202 & 245 & 195 \\
\hline \multirow{2}{*}{$15 \ldots$} & 19 & 295 & 279 & 30 & 158 & 3,367 & 187 \\
\hline & & & & 31. & $\ldots-\cdots$ & 3,049 & 167 \\
\hline \multirow{2}{*}{\multicolumn{5}{|c|}{$\begin{array}{l}\text { Monthly mean discharge, in cubic feet per second } . \\
\text { Runoff, in acre-feet } \ldots \ldots \ldots \ldots \ldots \ldots \ldots \ldots \ldots \ldots \ldots\end{array}$}} & 96.9 & 473 & 392 \\
\hline & & & & & 5,760 & 29,067 & 24,090 \\
\hline
\end{tabular}


Gage height, in feet, and discharge, in cubic feet per second, at indicated time, 1965-66, of Black River below pumping plant, near Point of Pines, Ariz.

\begin{tabular}{|c|c|c|c|c|c|c|c|c|c|c|c|}
\hline Date & Hour & $\begin{array}{l}\text { Gage } \\
\text { height }\end{array}$ & $\begin{array}{c}\text { Dis- } \\
\text { charge }\end{array}$ & Date & Hour & $\begin{array}{l}\text { Gage } \\
\text { height }\end{array}$ & $\begin{array}{c}\text { Dis- } \\
\text { charge }\end{array}$ & Date & Hour & $\begin{array}{l}\text { Gage } \\
\text { height }\end{array}$ & $\begin{array}{l}\text { Dis- } \\
\text { charge }\end{array}$ \\
\hline \multirow[t]{2}{*}{ Dec. 21} & 2400 & 3.18 & 208 & Dec. 23 & 2400 & 4. 21 & 595 & Dec. 30 & 2000 & 9.11 & 4,390 \\
\hline & & & & & & & & & 2200 & 9.12 & 4,400 \\
\hline \multirow[t]{9}{*}{22} & 0600 & 3.21 & 214 & 24 & 1200 & 3.81 & 412 & & 2400 & 8. 83 & 4,110 \\
\hline & 1000 & 3.44 & 286 & & 2400 & 3.65 & 350 & & & & \\
\hline & 1200 & 3.71 & 384 & & & & & 31 & 0600 & 8.23 & 3,490 \\
\hline & 1400 & 4. 72 & 930 & 29 & 2400 & 3.56 & 290 & & 1200 & 7. 64 & 2,910 \\
\hline & 1600 & 4. 94 & 998 & & & & & & 1800 & 7. 22 & 2,540 \\
\hline & 2000 & 4. 81 & 925 & 30 & 0200 & 4. 09 & 492 & & 2400 & 7. 02 & 2,380 \\
\hline & 2200 & 4. 83 & 936 & & 0400 & 5.73 & 1,410 & & & & \\
\hline & 2400 & 5.39 & 1,270 & & 0600 & 7.53 & 2,830 & Jan. 1 & 1200 & 6. 17 & 1,690 \\
\hline & & & & & 0800 & 7. 60 & 2,890 & & 2400 & 5.77 & 1,410 \\
\hline \multirow[t]{6}{*}{23} & 0200 & 5. 84 & 1,560 & & 1000 & 8. 68 & 3,910 & & & & \\
\hline & 0415 & 5. 96 & 1,640 & & 1130 & 10.86 & 6,380 & 2 & 0800 & 5. 39 & 1,170 \\
\hline & 0600 & 5.71 & 1,470 & & 1200 & 9.52 & 4,850 & & 1600 & 5. 11 & 998 \\
\hline & 0800 & 5. 36 & 1,200 & & 1400 & 9.02 & 4,300 & & 2000 & 5.13 & 1,010 \\
\hline & 1200 & 4. 86 & 942 & & 1600 & 8. 69 & 3,970 & & 2400 & 5. 30 & 1,110 \\
\hline & 1800 & 4. 57 & 782 & & 1800 & 8. 76 & 4,040 & & & & \\
\hline
\end{tabular}

(84) 9-4897. Big Bonita Creek near Fort Apache, Ariz.

Location. --Lat $33^{\circ} 40^{\prime} 10^{\prime \prime}$, long $109^{\circ} 50^{\prime} 45^{\prime \prime}$, in NE⿺ $\frac{1}{4}$ sec. 28, T. 4 N., R. 24 F. (unsurveyed), in Fort Apache Indian Reservation, near right bank on downstream side of highway bridge, $1 \frac{3}{4}$ miles upstream from Tonto Creek, $3 \frac{1}{2}$ miles southeast of Chino Springs, and $11 \frac{1}{2}$ miles southeast of Fort Apache.

Drainage area. --119 sq $\mathrm{mi}$.

Gage-height record. - Water-stage recorder graph except 0400 hours Nov. 25 to 1200 hours Nov. 30 and 0700 hours Dec. 31 to Jan. 5. Gage heights estimated 0700-2400 hours Dec. 31. Altitude of gage is $5,910 \mathrm{ft}$ (by barometer).

Discharge record. --Stage-discharge relation defined by current-meter measurements. Discharge for periods of no gage-height record estimated.

Maxima. --November 1965 to January 1966: Discharge, 1,640 cfs 1230 hours Dec, 30 (gage height, 6.25 ft).

1957 to October 1965: Discharge, 1, $200 \mathrm{cfs}$ Aug. 15, 1959 (gage height, 6. $20 \mathrm{ft}$, from floodmark).

Mean discharge, in cubic feet per second, 1965-66

\begin{tabular}{|c|c|c|c|c|c|c|c|}
\hline Day & November & December & January & Day & November & December & January \\
\hline $1 \ldots$ & 10 & 70 & 320 & 16 & 11 & 97 & 90 \\
\hline $2 \ldots$ & 10 & 60 & 320 & 17 & 11 & 91 & 82 \\
\hline $3 \ldots$ & 11 & 55 & 230 & 18 & 11 & 91 & 81 \\
\hline $4 \ldots$ & 10 & 49 & 180 & 19 & 11 & 77 & 76 \\
\hline $5 \ldots$ & 10 & 45 & 160 & 20 & 11 & 93 & 70 \\
\hline $6 \ldots$ & 10 & 42 & 137 & $21 \ldots$ & 10 & 93 & 59 \\
\hline $7 \ldots$ & 11 & 41 & 128 & $22 \ldots$ & 12 & 204 & 48 \\
\hline $8 \ldots$ & 12 & 39 & 130 & $23 \ldots$ & 77 & $25 ?$ & 55 \\
\hline $9 \ldots$ & 13 & 50 & 124 & $24 \ldots$ & 89 & 147 & 55 \\
\hline $10 \ldots$ & 12 & 305 & 121 & $25 \ldots$ & 308 & 132 & 59 \\
\hline $11 \ldots$ & 11 & 219 & 121 & $26 \ldots$ & 450 & 121 & 65 \\
\hline 12 . & 11 & 156 & 114 & 27 . & 150 & 114 & 70 \\
\hline $13 \ldots$ & 10 & 145 & 103 & $28 \ldots$ & 120 & $10 ?$ & 65 \\
\hline 14. & 11 & 118 & 96 & 29 . & 85 & 114 & 60 \\
\hline \multirow[t]{2}{*}{$15 \ldots$} & 11 & 104 & 94 & 30 & 79 & $1,03 ?$ & 55 \\
\hline & & & & $31 \ldots$ & $\ldots \ldots$ & 756 & 52 \\
\hline \multirow{2}{*}{\multicolumn{5}{|c|}{$\begin{array}{l}\text { Monthly mean discharge, in cubic feet per second } . \\
\text { Runoff, in acre-feet } \ldots \ldots \ldots \ldots \ldots \ldots \ldots \ldots \ldots\end{array}$}} & 53.3 & $16 ?$ & 110 \\
\hline & & & & & 3,170 & $9,95 ?$ & 6,780 \\
\hline
\end{tabular}


Gage height, in feet, and discharge, in cubic feet per second, at indicated time, 1965, of Big Bonita Creek near Fort Apache, Ariz.

\begin{tabular}{r|c|c|c||c|c|c|c||c|c|c|c}
\hline Date & Hour & $\begin{array}{c}\text { Gage } \\
\text { height }\end{array}$ & $\begin{array}{c}\text { Dis- } \\
\text { charge }\end{array}$ & Date & Hour & $\begin{array}{c}\text { Gage } \\
\text { height }\end{array}$ & $\begin{array}{c}\text { Dis- } \\
\text { charge }\end{array}$ & Date & Hour & $\begin{array}{c}\text { Gage } \\
\text { height }\end{array}$ & $\begin{array}{c}\text { Dis- } \\
\text { charge }\end{array}$ \\
\hline Dec. 28 & 2400 & 2.93 & 114 & Dec. 30 & 0500 & 4.27 & 489 & Dec. 31 & 0100 & 5.76 & 1,282 \\
& & & & & 0700 & 4.58 & 612 & & 0200 & 5.88 & 1,370 \\
& 1500 & 2.88 & 106 & & 0900 & 5.25 & 950 & & 0300 & 5.82 & 1,320 \\
& 2300 & 3.00 & 126 & & 1100 & 5.96 & 1,420 & & 0800 & 5.25 & 950 \\
& 2400 & 3.12 & 149 & & 1230 & 6.25 & 1,640 & & 1200 & 4.7 & 665 \\
& & & & & 1600 & 6.08 & 1,500 & & 2400 & 3.9 & 360 \\
\hline
\end{tabular}

(85) 9-4905. Black River near Fort Apache, Ariz.

Location. --Lat $33^{\circ} 42^{\prime} 45^{\prime \prime}$, long $110^{\circ} 12^{\prime} 40^{\prime \prime}$, in NW $\frac{1}{4} \mathrm{sec}, 12$, T. 4 N. , R. 20 E. (unsurveyed), on downstream side of highway bridge, 5 miles upstream from ccnfluence with White River, and 14 miles west of Fort Apache.

Drainage area. $--1,232$ sq $\mathrm{mi}$.

Gage-height record. --Water-stage recorder graph. Altitude of gage is $4,345 \mathrm{ft}$ (from river-profile map).

Discharge record. --Stage-discharge relation defined by current-meter measurements below $8,900 \mathrm{cfs}$ and extended above on basis of slope-area measurement at gage height $14.70 \mathrm{ft}$ and logarithmic plotting.

Maxima. --November 1965 to January 1966: Discharge, 24, 800 cfs 1430 hours Dec. 30 (gage height, $20.05 \mathrm{ft}$ ).

1912-18, 1957 to October 1965: Recorded discharge, 18, $000 \mathrm{cfs}$ Dec. 20, 1914 (gage height, $15.9 \mathrm{ft}$, from floodmarks, at site 1 mile downstream at different datum), from rating curve extended above $1,000 \mathrm{cfs}$.

Floods of Jan, 28, 1915, Jan. 19, 1916, and Jan. 29, 1916, exceeded that of Dec. 20,1914 , by unknown amounts; that of Jan. 19, 1916, probably was greatest.

Mean discharge, in cubic feet per second, 1965-66

\begin{tabular}{|c|c|c|c|c|c|c|c|}
\hline Day & November & December & January & Day & November & December & January \\
\hline 1 & 56 & 251 & 4,130 & $16 \ldots$ & 42 & 875 & 477 \\
\hline$\ldots$ & 54 & 231 & 2,370 & $17 \ldots$ & 42 & $84 E$ & 454 \\
\hline 3 & 53 & 212 & 1,730 & $18 \ldots$ & 44 & 895 & 444 \\
\hline 4 & 53 & 183 & 1,400 & 19 & 47 & 716 & 431 \\
\hline 5 & 54 & 166 & 1,130 & $20 \ldots$ & 47 & 550 & 395 \\
\hline 6 & 54 & 158 & 955 & 21. & 50 & 577 & 355 \\
\hline 7 & 54 & 150 & 845 & 22. & 57 & $7,77 C$ & 302 \\
\hline 8 & 56 & 140 & 811 & 23. & 53 & 12,900 & 238 \\
\hline 9 & 57 & 144 & 788 & $24 \ldots \ldots$ & 105 & 2,770 & 238 \\
\hline 10 & 58 & 3,960 & 730 & $25 \ldots$ & 347 & 1,600 & 253 \\
\hline 11 & 57 & 3,760 & 685 & $26 \ldots$ & 1,130 & 1,170 & 238 \\
\hline 12 & 49 & 1,960 & 658 & $27 \ldots$ & 1,110 & 900 & 238 \\
\hline 13 & 45 & 1,450 & 604 & 28. & 616 & 766 & 251 \\
\hline 14 & 44 & 1,500 & 530 & 29. & 427 & 762 & 241 \\
\hline \multirow[t]{2}{*}{15} & 42 & 1,120 & 491 & 30 . & 308 & 14,800 & 241 \\
\hline & & & & 31. & $\ldots \ldots$ & 10,600 & 266 \\
\hline \multirow{2}{*}{\multicolumn{5}{|c|}{ Monthly mean discharge, in cubic feet per second. }} & 174 & 2,383 & 739 \\
\hline & & & & & 10,340 & 146,500 & 45,460 \\
\hline
\end{tabular}


Gage height, in feet, and discharge, in cubic feet per second, at indicated time, 1965-66, of Black River near Fort Apache, Ariz.

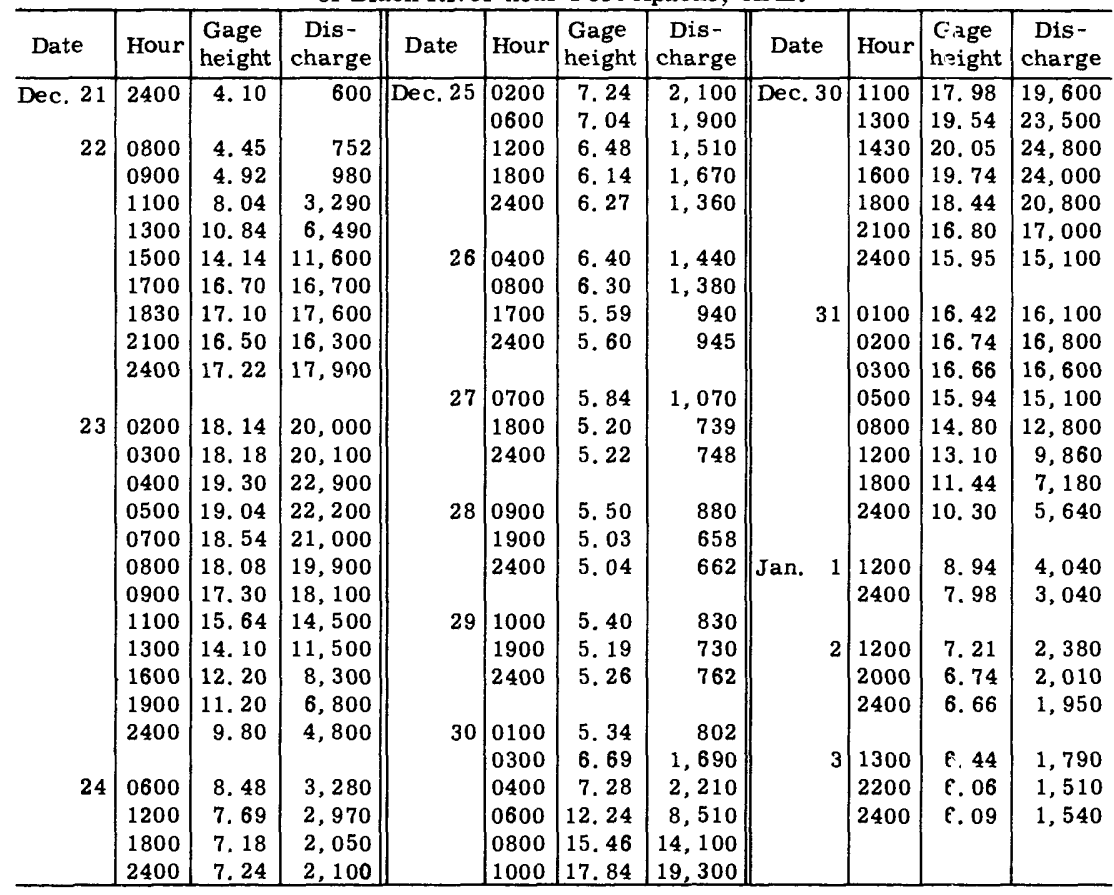


(86) 9-4908. North Fork White River near Greer, Ariz.

Location. --Lat $34^{\circ} 00^{\prime} 50^{\prime \prime}$, long $109^{\circ} 38^{\prime} 30^{\prime \prime}$, in SW $\frac{1}{4}$ sec. 7, T. 7 N., R. 26 E. (unsurveyed), in Fort Apache Indian Reservation, on right bank $300 \mathrm{ft}$ upstream from Bear Cienega Creek, and 11 miles west of Greer.

Drainage area. $--39 \mathrm{sq} \mathrm{mi}$, approximately.

Gage-height record. --Water-stage recorder graph except Nov. 9-13, Dec. 26-29, Dec. 31 to Jan. 31. Altitude of gage is $8,400 \mathrm{ft}$ (from topographic map).

Discharge record. --Stage-discharge relation defined by current-meter measurements below $85 \mathrm{cfs}$ and extended above by logarithmic plotting. Stage-discharge relation affected by ice Dec. 12-25, 30. Discharge for Nov. 9-13 and Dec. 12 to Jan. 31 estimated.

Maxima. --November 1965 to January 1966: Discharge, 191 cfs 1600 hours Nov. 25 (gage height, $2.94 \mathrm{ft}$ ).

June to October 1965: Discharge, $125 \mathrm{cfs}$ Aug. 1, 16, 1965 (gage height, $1.18 \mathrm{ft}$ ).

Mean discharge, in cubic feet per second, 1965-66

\begin{tabular}{|c|c|c|c|c|c|c|c|}
\hline Day & November & December & January & Day & November & December & January \\
\hline $1 \ldots$ & 12 & 27 & 25 & $16 \ldots$ & 12 & 15 & 13 \\
\hline $2 \ldots$ & 12 & 24 & 20 & $17 \ldots$ & 12 & 15 & 13 \\
\hline $3 \ldots$ & 11 & 23 & 18 & $18 \ldots$ & 12 & 15 & 13 \\
\hline $4 \ldots$ & 12 & 22 & 17 & $19 \ldots$ & 12 & 15 & 13 \\
\hline $5 \ldots$ & 11 & 20 & 16 & $20 \ldots$ & 12 & 15 & 13 \\
\hline $6 \ldots$ & 12 & 19 & 15 & $21 \ldots$ & 13 & 15 & 13 \\
\hline 7 & 12 & 18 & 14 & $22 \ldots \ldots$ & 17 & 30 & 13 \\
\hline 8 & 12 & 16 & 14 & 23. & 77 & 25 & 13 \\
\hline 9 & 12 & 19 & 14 & $24 \ldots \ldots$ & 53 & 18 & 13 \\
\hline $10 \ldots$ & 12 & 19 & 13 & $25 \ldots$ & 109 & 16 & 13 \\
\hline $11 \ldots$ & 12 & 15 & 13 & $26 \ldots$ & 84 & 15 & 13 \\
\hline $12 \ldots$ & 12 & 15 & 13 & $27 \ldots$ & 58 & 15 & 13 \\
\hline $13 \ldots$ & 13 & 15 & 13 & $28 \ldots \ldots$ & 44 & 15 & 13 \\
\hline $14 \ldots$ & 12 & 15 & 13 & $29 \ldots$ & 34 & 15 & 13 \\
\hline \multirow[t]{2}{*}{$15 \ldots$} & 12 & 15 & 13 & $30 \ldots$ & 35 & 50 & 13 \\
\hline & & & & 31. & $\ldots \ldots$ & 40 & 13 \\
\hline \multicolumn{5}{|c|}{$\begin{array}{l}\text { Monthly mean discharge, in cubic feet per second.. } \\
\text { Runoff, in acre-feet. }\end{array}$} & 25.4 & 19.7 & 14.2 \\
\hline
\end{tabular}

(87) 9-4910. North Fork White River near McNary, Ariz.

Location. -- Lat $34^{\circ} 02^{\prime} 45^{\prime \prime}$, long $109^{\circ} 44^{\prime} 15^{\prime \prime}$, in E $\frac{1}{2}$ sec. 31 , T. 8 N., R. 25 E. (unsurveyed), in Fort Apache Indian Reservation, on left bank 2 miles downstream from Paradise Creek, and $6 \frac{3}{4}$ miles southeast of McNary.

Drainage area. $--66 \mathrm{sq} \mathrm{mi,} \mathrm{approximately.}$

Gage-height record. --Water-stage recorder graph except Jan. 10-22. Alt' tude of gage is 7, $750 \mathrm{ft}$ (from Indian Irrigation Services river-profile map).

Discharge record. --Stage-discharge relation defined by current-meter me Discharge for period of no gage-height record estimated.

Maxima. --November 1965 to January 1966: Discharge, 250 cfs 1200 hours Dec. 30 (gage height, $2.77 \mathrm{ft}$ ).

1945 to October 1965: Discharge, 1,290 cfs Sept. 19, 1946 (gage height, 5. $36 \mathrm{ft}$ ), from rating curve extended above $350 \mathrm{cfs}$ by logarithmic plotting. 
Mean discharge, in cubic feet per second, 1965-66, of North Fork Wr ite River near McNary, Ariz.

\begin{tabular}{|c|c|c|c|c|c|c|c|}
\hline Day & November & December & January & Day & November & December & January \\
\hline 1 & 16 & 51 & 96 & 16 & 16 & 40 & 30 \\
\hline $2 \ldots$ & 16 & 46 & 86 & $17 \ldots$ & 16 & 40 & 28 \\
\hline $3 \ldots$ & 15 & 45 & 75 & 18 & 16 & 40 & 28 \\
\hline $4 \ldots$ & 15 & 41 & 65 & $19 \ldots$ & 16 & 43 & 28 \\
\hline $5 \ldots$ & 15 & 39 & 55 & 20 . & 16 & 45 & 25 \\
\hline 6 & 15 & 38 & 50 & $21 \ldots$ & 16 & 45 & 25 \\
\hline$\ldots$ & 17 & 37 & 45 & $22 \ldots \ldots$ & 17 & 90 & 25 \\
\hline $8 \ldots$ & 17 & 34 & 40 & $23 \ldots$ & 117 & 75 & 25 \\
\hline $9 \ldots$ & 17 & 43 & 37 & $24 \ldots$ & 88 & 50 & 25 \\
\hline $10 \ldots$ & 16 & 46 & 35 & $25 \ldots$ & 226 & 45 & 25 \\
\hline $11 \ldots$ & 16 & 45 & 35 & $26 \ldots$ & 176 & 45 & 22 \\
\hline 12 & 16 & 46 & 35 & $27 \ldots$ & 107 & 43 & 22 \\
\hline $13 \ldots$ & 16 & 43 & 30 & $28 \ldots \ldots$ & 78 & 42 & 22 \\
\hline $14 \ldots$ & 16 & 40 & 30 & 29. & 68 & 45 & 22 \\
\hline \multirow[t]{2}{*}{$15 \ldots$} & 15 & 37 & 30 & & 62 & 180 & 20 \\
\hline & & & & $31 \ldots$ & $\ldots \ldots$ & 150 & 20 \\
\hline \multirow{2}{*}{\multicolumn{5}{|c|}{$\begin{array}{l}\text { Monthly mean discharge, in cubic feet per second } \\
\text { Runoff in acre-feet } \ldots \ldots \ldots \ldots \ldots \ldots\end{array}$}} & 42.4 & 53.2 & 36.6 \\
\hline & & & & & 2,520 & 3,270 & 2,250 \\
\hline
\end{tabular}

(88) 9-4918. North Fork White River tributary near White River, Ariz. (Crest-stage station)

Location. -- Lat $33^{\circ} 55^{\prime} 45^{\prime \prime}$, long $109^{\circ} 56^{\prime} 30^{\prime \prime}$, in SE $\frac{1}{4}$ sec. 8, T. 6 N. , R. 23 E. (unsurveyed), at State Highway 73, 6.5 miles north of White River.

Drainage area. --About $2.7 \mathrm{sq} \mathrm{mi}$.

Gage-height record. --Crest stages only. Altitude of gage is 5, $700 \mathrm{ft}$ (from topographic map).

Discharge record. --Stage-discharge relation defined by computations of flow through culvert.

Maxima. --November 1965 to January 1966: Discharge, 24 cfs Dec. 22 (gage height, $5.40 \mathrm{ft}$ ).

1964 to October 1965: Discharge, not determined; no known flow occurred during the period, but a peak discharge of less than $24 \mathrm{cfs}$ could have occurred in August or September 1965.

(89) 9-4924. East Fork White River near Fort Apache, Ariz.

Location. - - Lat $33^{\circ} 49^{\prime} 20^{\prime \prime}$, long $109^{\circ} 48^{\prime} 50^{\prime \prime}$, in $\mathrm{SE} \frac{1}{4}$ sec. 16, T. 5 N., R. 24 E. (unsurveyed), in Fort Apache Indian Reservation, on left bank $600 \mathrm{ft}$ downstream from highway bridge, 0.1 mile upstream from Rock Creek, and $10 \frac{1}{4}$ miles east of Fort Apache.

Drainage area. $--38.8 \mathrm{sq} \mathrm{mi}$.

Gage-height record. - Water-stage recorder graph. Altitude of gage is $6,050 \mathrm{ft}$ (by barometer).

Discharge record. --Stage-discharge relation defined by current-meter measurements.

Maxima. - -November 1965 to January 1966: Discharge, 256 cfs 1630 hours Nov. 25 (gage height, $2.62 \mathrm{ft}$ ).

1957 to October 1965: Discharge, $663 \mathrm{cfs}$ Aug. 17, 1961 (gage height, $4.82 \mathrm{ft}$, present site and datum, from floodmark), from rating curve extended above 170 cfs on basis of slope-area measurement of peak flow. 
Mean discharge, in cubic feet per second, 1965-66, of East Fork White River near Fort Apache, Ariz.

\begin{tabular}{|c|c|c|c|c|c|c|c|}
\hline Day & November & December & January & Day & November & December & January \\
\hline 1 & 8.4 & 37 & 71 & 16 & 7.9 & 24 & 27 \\
\hline & 8.4 & 31 & 50 & $17 \ldots$ & 8.2 & 24 & 25 \\
\hline 3 & 8. 2 & 29 & 46 & $18 \ldots$ & 8.2 & 22 & 24 \\
\hline $4 \ldots$ & 8.2 & 27 & 40 & $19 \ldots$ & 7.9 & 22 & 24 \\
\hline $5 \ldots$ & 8.2 & 25 & 37 & 20 & 8.2 & 27 & 21 \\
\hline$\ldots$ & 8.2 & 24 & 35 & $21 \ldots$ & 8.4 & 27 & 19 \\
\hline 7 & 9.0 & 24 & 34 & $22 \ldots \ldots$ & 9.0 & 54 & 21 \\
\hline 8 & 9.0 & 24 & 35 & $23 \ldots$ & 79 & 45 & 26 \\
\hline$\ldots$ & 8.7 & 28 & 34 & $24 \ldots \ldots$ & 99 & 29 & 22 \\
\hline 10 & 8.4 & 42 & 34 & $25 \ldots$ & 155 & 28 & 22 \\
\hline $11 \ldots$ & 8. 2 & 34 & 34 & $26 \ldots$ & 180 & 27 & 24 \\
\hline 12 & 7. 3 & 28 & 32 & $27 \ldots$ & 91 & 25 & 22 \\
\hline 13 & 7. 9 & 29 & 31 & $28 \ldots \ldots$ & 66 & 24 & 19 \\
\hline 14 & 8. 7 & 27 & 29 & 29 & 50 & 27 & 20 \\
\hline \multirow[t]{2}{*}{15.} & 8.2 & 24 & 29 & & 42 & 173 & 18 \\
\hline & & & & & 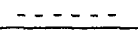 & 109 & 18 \\
\hline \multirow{2}{*}{\multicolumn{5}{|c|}{$\begin{array}{l}\text { Monthly mean discharge, in cubic feet per second. } \\
\text { Runoff, in acre-feet } \ldots \ldots \ldots \ldots \ldots \ldots \ldots \ldots \ldots \ldots\end{array}$}} & 31.5 & 36.1 & 29.8 \\
\hline & & & & & 1,870 & 2,220 & 1,830 \\
\hline
\end{tabular}

(90) 9-4940. White River near Fort Apache, Ariz.

Location. -- Lat $33^{\circ} 44^{\prime} 10^{\prime \prime}$, long $110^{\circ} 09^{\prime} 55^{\prime \prime}$, in $\mathrm{SE}_{\frac{1}{4}}^{\frac{1}{4}}$ sec. 32 , T. $4 \frac{1}{2}$ N., R. 21 E. (unsurveyed), in Fort Apache Indian Reservation, on right bank 2, $200 \mathrm{ft}$ downstream from road crossing, 5 miles upstream from mouth, and 11 miles west of Fort Apachs.

Drainage area. $--632 \mathrm{sq} \mathrm{mi}$.

Gage-height record. - Water-stage recorder graph. Datum of gage is 4,365. $99 \mathrm{ft}$ above mean sea level, datum of 1929.

Discharge record. --Stage-discharge relation defined by current-meter measurements below $3,300 \mathrm{cfs}$ and extended above on basis of slope-area measurement of peak flow.

Maxima. --November 1965 to January 1966: Discharge, 4, 360 cfs 1530 hours Dec. 30 (gage height, $8.71 \mathrm{ft}$ ).

1917-18, 1957 to October 1965: Discharge, 4, 900 cfs July 28, 1959 (gage height, $9.2 \mathrm{ft}$ ).

Mean discharge, in cubic feet per second, 1965-66

\begin{tabular}{|c|c|c|c|c|c|c|c|}
\hline Day & November & December & January & Day & November & December & January \\
\hline $1 \ldots$ & 41 & 242 & 994 & $16 \ldots$ & 43 & 208 & 224 \\
\hline $2 \ldots$ & 42 & 205 & 656 & $17 \ldots$ & 43 & 216 & 216 \\
\hline 3 & 42 & 178 & 525 & $18 \ldots$ & 45 & 199 & 219 \\
\hline 4 & 41 & 162 & 450 & $19 \ldots$ & 45 & 146 & 210 \\
\hline 5 & 41 & 152 & 398 & 20. & 43 & 158 & 180 \\
\hline 6 & 42 & 138 & 378 & 21. & 41 & 165 & 170 \\
\hline 7 & 42 & 136 & 346 & $22 \ldots$ & 45 & 1,070 & 127 \\
\hline 8 & 45 & 129 & 334 & 23. & 102 & 1,690 & 134 \\
\hline 9 & 48 & 141 & 320 & 24. & 428 & 405 & 143 \\
\hline 10 & 45 & 780 & 302 & $25 \ldots$ & 502 & 309 & 134 \\
\hline 11 & 43 & 504 & 298 & $26 \ldots$ & 1,350 & 274 & 127 \\
\hline 12 & 43 & 330 & 284 & $27 \ldots$ & 638 & 242 & 136 \\
\hline 13 & 41 & 288 & 251 & $28 \ldots$ & 414 & 224 & 138 \\
\hline $14 \ldots$ & 43 & 254 & 227 & $29 \ldots$ & 302 & 233 & 125 \\
\hline \multirow[t]{2}{*}{15} & 45 & 236 & 236 & $30 \ldots$ & 257 & 2,400 & 134 \\
\hline & & & & 31. & $\ldots \ldots$ & 1,820 & 136 \\
\hline \multicolumn{5}{|c|}{ Monthly mean discharge, in cubic feet per second.. } & 165 & 440 & 276 \\
\hline \multicolumn{5}{|c|}{ Runoff, in acre-feet $\ldots \ldots \ldots \ldots \ldots \ldots \ldots$} & 9,800 & 27,040 & 16,960 \\
\hline
\end{tabular}


Gage height, in feet, and discharge, in cubic feet per second, at indicated time, 1965-66, of White River near Fort Apache, Ariz.

\begin{tabular}{|c|c|c|c|c|c|c|c|c|c|c|c|}
\hline Date & Hour & $\begin{array}{l}\text { Gage } \\
\text { height }\end{array}$ & $\begin{array}{c}\text { Dis- } \\
\text { charge }\end{array}$ & Date & Hour & $\begin{array}{l}\text { Gage } \\
\text { height }\end{array}$ & $\begin{array}{c}\text { Dis- } \\
\text { charge }\end{array}$ & Date & Hour & $\begin{array}{l}\text { Gage } \\
\text { height }\end{array}$ & $\begin{array}{c}\text { Dis- } \\
\text { charge }\end{array}$ \\
\hline \multirow[t]{2}{*}{ Dec. 21} & 2400 & 3.70 & 180 & Dec. 23 & 0700 & 7.45 & 2,980 & Dec. 30 & 0600 & 5.50 & 1,120 \\
\hline & & & & & 0900 & 6.55 & 2,020 & & 0900 & 6.74 & 2,210 \\
\hline \multirow[t]{13}{*}{22} & 0830 & 3.80 & 213 & & 1200 & 5.67 & 1,220 & & 1100 & 7. 00 & 2,480 \\
\hline & 0930 & 4. 25 & 370 & & 1800 & 4.98 & 754 & & 1300 & 7.75 & 3,300 \\
\hline & 1130 & 5.00 & 760 & & 2400 & 4. 67 & 580 & & 1500 & 8.63 & 4,270 \\
\hline & 1230 & 5.55 & 1,140 & & & & & & 1530 & 8.71 & 4,360 \\
\hline & 1400 & 5.32 & 968 & 24 & 1200 & 4. 25 & 378 & & 1630 & 8. 63 & 4,270 \\
\hline & 1500 & 5.92 & 1,460 & & 1800 & 4. 10 & 316 & & 1800 & 8. 10 & 3,690 \\
\hline & 1600 & 6. 22 & 1,720 & & 2200 & 4.25 & 378 & & 2400 & 7. 26 & 2,770 \\
\hline & 1700 & 6.92 & 2,390 & & 2400 & 4. 26 & 378 & & & & \\
\hline & 1800 & 7.02 & 2,500 & & & & & 31 & 0500 & 6.75 & 2,220 \\
\hline & 1900 & 6.74 & 2,210 & 25 & 0200 & 4. 26 & 370 & & 0630 & 6.74 & 2,210 \\
\hline & 2000 & 6.84 & 2,310 & & 1700 & 3.98 & 267 & & 1200 & 6.25 & 1,740 \\
\hline & 2200 & 6. 92 & 2,390 & & 2400 & 4.08 & 302 & & 1800 & 5.88 & 1,430 \\
\hline & 2400 & 6.60 & 2,070 & & & & & & 2400 & 5. 70 & 1,290 \\
\hline \multirow[t]{3}{*}{23} & 0300 & 7. 87 & 3,440 & 29 & 2400 & 3. 89 & 239 & Jan. 1 & 1200 & 5.24 & 968 \\
\hline & 0430 & 8.00 & 3,580 & & & & & & 2400 & 4. 98 & 808 \\
\hline & 0530 & 7. 92 & 3,490 & 30 & 0300 & 4.20 & 346 & & & & \\
\hline
\end{tabular}

(91) 9-4943. Carrizo Creek above Corduroy Creek, near Show Lov', Ariz.

Location. --Lat $34^{\circ} 00^{\prime} 00^{\prime \prime}$, long $110^{\circ} 17^{\prime} 20^{\prime \prime}$, in sec. 13, T. 7 N., R. 19 E. (unsurveyed), in Fort Apache Indian Reservation, on left bank half a mile upstream from Corduroy Creek, and 23 miles southwest of Show Low.

Drainage area. $--225 \mathrm{sq} \mathrm{mi}$

Gage-height record. - - Water-stage recorder graph except Dec. 24-29, Jan. 1-5. Record for Dec. 30-31 uncertain because gage settled $1.36 \mathrm{ft}$ and tilted. Gage heights corrected to datum in use prior to settlement of gage. Altitude of gage is $4,800 \mathrm{ft}$ (from topographic map)

Discharge record. --Stage-discharge relation defined by current-meter me asurements. Discharge for periods of no gage-height record estimated.

Maxima. - - November 1965 to January 1966: Discharge, 10, 000 cfs 1000 hours Dec. 30; gage height, $9.85 \mathrm{ft}$ inside 0730 hours Dec, $30,10.3 \mathrm{ft}$ outside, from floodmark.

1953 to October 1965: Discharge, $3,260 \mathrm{cfs}$ Jan. 11, 1960 (gage height, 6. $95 \mathrm{ft}$ ).

Mean discharge, in cubic feet per second, 1965-66

\begin{tabular}{|c|c|c|c|c|c|c|c|}
\hline Day & November & December & January & Day & November & December & January \\
\hline 1 & 2.7 & 6.3 & 590 & 16 & 4.7 & 25 & 33 \\
\hline 2 & 2.7 & 5.1 & 285 & 17 & 4.7 & 25 & 31 \\
\hline$\ldots$ & 3.0 & 4.3 & 165 & 18 & 4.7 & 21 & 30 \\
\hline $4 \ldots$ & 3.0 & 4.3 & 110 & 19 & 4.7 & 17 & 29 \\
\hline $5 \ldots$ & 3.0 & 4. 3 & 65 & $20 \ldots$ & 4.7 & 15 & 27 \\
\hline $6 \ldots$ & 3.3 & 4.3 & 58 & $21 \ldots$ & 4.7 & 15 & 25 \\
\hline 7 & 3.6 & 4. 3 & 56 & $22 \ldots$ & 5.1 & 297 & 25 \\
\hline 8 & 3.6 & 4. 3 & 53 & $23 \ldots$ & 10 & 847 & 22 \\
\hline 9 & 3.6 & 16 & 50 & $24 \ldots$ & 9.3 & 160 & 20 \\
\hline $10 \ldots$ & 3.9 & 167 & 45 & $25 \ldots$ & 21 & 80 & 19 \\
\hline $11 \ldots$ & 3.9 & 174 & 43 & $26 \ldots$ & 12 & 50 & 19 \\
\hline $12 \ldots$ & 3.9 & 56 & 43 & $27 \ldots$ & 7.8 & 40 & 19 \\
\hline $13 \ldots$ & 4. 3 & 35 & 39 & $28 \ldots$ & 6.8 & 35 & 18 \\
\hline 14. & 4.3 & 30 & 35 & $29 \ldots$ & 5.9 & 45 & 18 \\
\hline \multirow[t]{2}{*}{$15 \ldots$} & 4.3 & 28 & 34 & 30 & 5.9 & 5,360 & 18 \\
\hline & & & & 31. & $\ldots \ldots$ & 1,750 & 17 \\
\hline \multirow{2}{*}{\multicolumn{5}{|c|}{$\begin{array}{l}\text { Monthly mean discharge, in cubic feet per second.. } \\
\text { Runoff, in acre-feet } \ldots \ldots \ldots \ldots \ldots \ldots \ldots \ldots\end{array}$}} & 5.50 & 301 & 65.8 \\
\hline & & & & & 327 & 18,500 & 4,050 \\
\hline
\end{tabular}


Gage height, in feet, and discharge, in cubic feet per second, at indicated time, 1965-66, of Carrizo Creek above Corduroy Creek, near Show Low, Ariz.

\begin{tabular}{|c|c|c|c|c|c|c|c|c|c|c|c|}
\hline Date & Hour & $\begin{array}{c}\text { Gage } \\
\text { height }\end{array}$ & $\begin{array}{c}\text { Dis- } \\
\text { charge }\end{array}$ & Date & Hour & $\begin{array}{c}\text { Gage } \\
\text { height }\end{array}$ & $\begin{array}{c}\text { Dis - } \\
\text { charge }\end{array}$ & Date & Hour & $\begin{array}{l}\text { Gage } \\
\text { height }\end{array}$ & $\begin{array}{c}\text { Dis- } \\
\text { charge }\end{array}$ \\
\hline Dec. 21 & 2400 & 5.57 & 17 & Dec. 23 & $\begin{array}{l}0700 \\
1200\end{array}$ & $\begin{array}{l}5.67 \\
5.02\end{array}$ & $\begin{array}{r}1,240 \\
762\end{array}$ & Dec. 30 & $\begin{array}{l}0900 \\
0930\end{array}$ & $\begin{array}{l}8.85 \\
8.0\end{array}$ & $\begin{array}{l}9,060 \\
8,650\end{array}$ \\
\hline \multirow[t]{12}{*}{22} & 0600 & 2.57 & 17 & & 1800 & 4. 35 & 450 & & 1000 & 7. 05 & 10,000 \\
\hline & 0800 & 2.65 & 24 & & 2400 & 3.95 & 318 & & 1300 & 6.38 & 7,990 \\
\hline & 1100 & 3.28 & 115 & & & & & & 1330 & 6. 26 & 7,540 \\
\hline & 1330 & 3.52 & 169 & & & & & & 1600 & 5. 75 & 5,640 \\
\hline & 1400 & 4. 30 & 425 & 28 & 2400 & - & 45 & & 1900 & 5.45 & 4,540 \\
\hline & 1500 & 4. 80 & 643 & & & & & & 2000 & 5. 48 & 4,600 \\
\hline & 1600 & 4.92 & 700 & 29 & 1800 & - & 45 & & 2400 & 5. 12 & 3,540 \\
\hline & 1800 & 4. 67 & 589 & & 2400 & 3.5 & 160 & & & & \\
\hline & 2000 & 4. 53 & 525 & & & & & 31 & 0600 & 4. 57 & 2,300 \\
\hline & 2200 & 4. 74 & 619 & 30 & 0100 & 4. 15 & 362 & & 1200 & 4.02 & 1,320 \\
\hline & 2400 & 5.45 & 507 & & 0300 & 5.50 & 1,180 & & 1800 & 3. 82 & 1,040 \\
\hline & & & & & 0500 & 6.90 & 2,900 & & 2400 & 3.52 & 823 \\
\hline \multirow[t]{3}{*}{23} & 0130 & 5. 92 & 1,480 & & 0700 & 7.75 & 4,460 & & & & \\
\hline & 0230 & 5.99 & 1,550 & & 0730 & 9.85 & 9,600 & Jan. 1 & 1200 & 3.37 & 571 \\
\hline & 0500 & 5.87 & 1,430 & & 0800 & 9.1 & 9,730 & & 2400 & 3.04 & 382 \\
\hline
\end{tabular}

(92) 9-4960. Corduroy Creek near mouth, near Show Low, Ariz.

Location, --Lat $34^{\circ} 01^{\prime} 06^{\prime \prime}$, long $110^{\circ} 14^{\prime} 30^{\prime \prime}$, in sec. 8, T. 7 N., R. 20 E. (unsurveyed), in Fort Apache Indian Reservation, on right bank 4 miles upstream from mot th, and 20 miles southwest of Show Low.

Drainage area. $--203 \mathrm{sq} \mathrm{mi}$.

Gage-height record. - -Water-stage recorder graph except Dec. 24-29, Dec. 31 to Jan. 4, Jan. 26-31. Record for Dec. 30 is poor. Altitude of gage is $5,000 \mathrm{ft}$ (from topographic map).

Discharge record. --Stage-discharge relation defined by current-meter meas urements. Discharge for periods of no gage-height record estimated.

Maxima. -- November 1965 to January 1966: Discharge, 10,900 cfs 1100 hours Dec, 30 (gage height, $11.75 \mathrm{ft}$ inside; approximately $12.5 \mathrm{ft}$ outside, from floodma rk).

1951 to October 1965: Discharge, $10,900 \mathrm{cfs}$ Jan. 18, 1952 (gage height, $11.1 \mathrm{ft}$ ).

Mean discharge, in cubic feet per second, 1965-66

\begin{tabular}{|c|c|c|c|c|c|c|c|}
\hline Day & November & December & January & Day & November & December & January \\
\hline $1 \ldots$ & 2. 0 & 4.5 & 270 & 16 & 2.2 & 68 & 23 \\
\hline$\ldots$ & 2.0 & 4. 1 & 160 & 17 & 2.2 & 57 & 22 \\
\hline 3 & 2.0 & 3.4 & 130 & 18 & 2.2 & 50 & 23 \\
\hline 4 & 2.0 & 3.1 & 114 & 19 & 2.2 & 51 & 26 \\
\hline 5 & 1. 7 & 3.1 & 89 & 20 & 2.2 & 53 & 23 \\
\hline 6 & 1.7 & 2.8 & 75 & $21 \ldots$ & 2.2 & 55 & 21 \\
\hline 7 & 1. 7 & 2.8 & 66 & $22 \ldots$ & 2.2 & 1,320 & 19 \\
\hline 8 & 2. 0 & 2.8 & 59 & 23 & 3.1 & 1,860 & 19 \\
\hline 9 & 2. 0 & 5.3 & 56 & 24 & 3.1 & 300 & 19 \\
\hline 10 & 2.0 & 567 & 46 & 25 & 4. 9 & 150 & 19 \\
\hline 11 & 2.2 & 324 & 43 & 26 & 7.8 & 80 & 18 \\
\hline 12 . & 2. 2 & 123 & 37 & 27 & 12 & 50 & 16 \\
\hline $13 \ldots$ & 2. 2 & 57 & 30 & 28 & 11 & 40 & 15 \\
\hline 14 & 2.2 & 48 & 27 & 29 & 6.6 & 50 & 14 \\
\hline \multirow[t]{2}{*}{$15 \ldots$} & 2.2 & 50 & 25 & 30 & 5.3 & 4,760 & 14 \\
\hline & & & & 31 & $\cdots$ & 600 & 15 \\
\hline \multirow{2}{*}{\multicolumn{5}{|c|}{$\begin{array}{l}\text { Monthly mean discharge, in cubic feet per second. } \\
\text { Runoff, in acre-feet } \ldots \ldots \ldots \ldots \ldots \ldots \ldots \ldots \ldots\end{array}$}} & 3.31 & 347 & 49.5 \\
\hline & & & & & 197 & 21,310 & 3,040 \\
\hline
\end{tabular}


Gage height, in feet, and discharge, in cubic feet per second, at indicated time, 1965, of Corduroy Creek near mouth, near Show Low, Ariz.

\begin{tabular}{|c|c|c|c|c|c|c|c|c|c|c|c|}
\hline Date & Hour & $\begin{array}{l}\text { Gage } \\
\text { height }\end{array}$ & $\begin{array}{c}\text { Dis- } \\
\text { charge }\end{array}$ & Date & Hour & $\begin{array}{l}\text { Gage } \\
\text { height }\end{array}$ & $\begin{array}{c}\text { Dis- } \\
\text { charge }\end{array}$ & Date & Hour & $\begin{array}{c}\text { Gage } \\
\text { height }\end{array}$ & $\begin{array}{l}\text { Dis- } \\
\text { charge }\end{array}$ \\
\hline$\overline{\text { Dec. } 21}$ & 2400 & 2.70 & 65 & Dec. 22 & $\begin{array}{l}2200 \\
2400\end{array}$ & $\begin{array}{l}7.10 \\
8.45\end{array}$ & $\begin{array}{l}2,600 \\
4,040\end{array}$ & Dec. 29 & 2400 & 5.0 & 960 \\
\hline \multirow[t]{11}{*}{22} & 0600 & 2. 75 & 73 & & & & & 30 & 0200 & 6.3 & 1,860 \\
\hline & 0900 & 2.86 & 91 & 23 & 0030 & 8. 68 & 4,300 & & 0400 & 8.0 & 3,540 \\
\hline & 1030 & 3. 25 & 199 & & 0200 & 8.42 & 4,000 & & 0600 & 10.75 & 7,150 \\
\hline & 1100 & 3. 75 & 384 & & 0400 & 8. 15 & 3,700 & & 0800 & 10.20 & 6,300 \\
\hline & 1200 & 5.50 & 1,280 & & 0500 & 7. 90 & 3,430 & & 1100 & 11.75 & 10,900 \\
\hline & 1300 & 6. 90 & 2,400 & & 0600 & 7. 35 & 2,850 & & 1300 & 9.65 & 7,120 \\
\hline & 1430 & 7. 38 & 2,880 & & 0800 & 6.34 & 1,890 & & 1400 & 8.65 & 5,620 \\
\hline & 1600 & 7.15 & 2,650 & & 1100 & 5.58 & 1,340 & & 1500 & 7. 90 & 4,500 \\
\hline & 1800 & 6.65 & 2,160 & & 1600 & 5.04 & 984 & & 1700 & 7. 10 & 3,420 \\
\hline & 2030 & 6.30 & 1,860 & & 2400 & 4. 5 & 696 & & 2000 & 6.00 & 2,300 \\
\hline & 2100 & 6.40 & 1,940 & & & & & & 2400 & 5.12 & 1,550 \\
\hline
\end{tabular}

(93) 9-4965. Carrizo Creek near Show Low, Ariz.

(Gaging station, discontinued 1960)

Location. -- Lat $33^{\circ} 59^{\prime} 15^{\prime \prime}$, long $110^{\circ} 17^{\prime} 00^{\prime \prime}$, in sec. 24 , T. 7 N., R. 19 E., at U. S. Highway 60,1 mile downstream from Cordoroy Creek, and $23 \frac{t}{2}$ miles southwert of Show Low.

Drainage area. $-459 \mathrm{sq} \mathrm{mi}$.

Gage-height record. --Crest stages only, from high-water mark in old well.

Discharge record. --Discharge obtained from rating curve in use prior to discontinuance of station in 1960; stage-discharge relation was defined by current-meter measurements below 2,000 cfs and extended above on basis of slope-area measi rement at gage height $12.08 \mathrm{ft}$.

Maxima. --November 1965 to January 1966: Discharge, approximately 23, 000 cfs (gage height, $13.0 \mathrm{ft}$, estimated mean of surge, based on high-water mark in well).

1951 to October 1965: Discharge, 20,500 cfs Jan. 18, 1952 (gage height, 12. $08 \mathrm{ft}$ ).

(94) 9-4967. Cibecue No. 2, tributary to Carrizo Creek, near Show I,ow, Ariz.

Location. --Lat $34^{\circ} 00^{\prime}$, long $110^{\circ} 19^{\prime}$, in $\mathrm{NE} \frac{1}{4}$ sec. 22 , T. 7 N., R. 19 E. (unsurveyed), in Fort Apache Indian Reservation, on an unnamed tributary to Carrizo Creek, on Cibecue Ridge, 1.9 miles upstream from mouth of main stem, and 25 miles southwest of Show Low.

Drainage area. $-0.065 \mathrm{sq} \mathrm{mi}(41.7$ acres $)$.

Gage-height record. -- Digital recorder tape. Altitude of gage is $5,200 \mathrm{ft}$ (from topographic map).

Discharge record. --Stage-discharge relation defined by laboratory rating of full-scale model of flume.

Maxima. - November 1965 to January 1966: Discharge, 3 cfs 1130 hours Гəc. 22 (gage height, $1.67 \mathrm{ft}$ ).

1958 to October 1965: Discharge, about $120 \mathrm{cfs}$ Aug. 20, 1963 (gage height not determined).

Mean discharge, in cubic feet per second, 1965
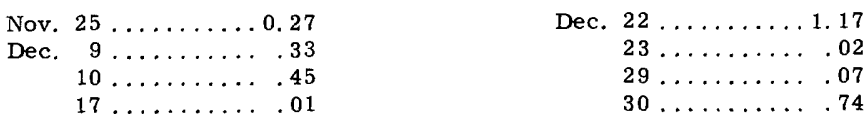

No flow during remainder of flood period. 
(95) 9-4968. Carrizo Creek tributary near Show Low, Ariz.

(Crest-stage station)

Location. -- Lat $33^{\circ} 57^{\prime} 30^{\prime \prime}$, long $110^{\circ} 20^{\prime} 00^{\prime \prime}$, at U.S. Highway 60,28 miles southwest of Show Low.

Drainage area. $--4.63 \mathrm{sq} \mathrm{mi}$, of which $2.08 \mathrm{sq} \mathrm{mi}$ above Wild Steer Tank is ge zerally noncontributing.

Gage-height record. --Crest stages only. Altitude of gage is 5, $400 \mathrm{ft}$ (from tonographic map).

Discharge record. --Stage-discharge relation defined by computations of flow through culvert.

Maxima. - - November 1965 to January 1966: Discharge, not determined, less than 80 cfs (minimum that will record on gage).

1963 to October 1965: Discharge, 1, 260 cfs July 31, 1964 (gage height, $20.94 \mathrm{ft}$ ).

(96) 9-4975. Salt River near Chrysotile, Ariz.

Location. -- Lat $33^{\circ} 48^{\prime}$, long $110^{\circ} 30^{\prime}$, in sec. 25 , T. 5 N., R. 17 E. (unsurveyed), in San Carlos Indian Reservation, on left bank 1, $200 \mathrm{ft}$ upstream from U.S. Highvay 60 , $5 \frac{1}{2}$ miles northeast of Chrysotile, 8 miles upstream from Cibecue Creek, and 33 miles downstream from confluence of Black and White Rivers.

Drainage area. $--2,849 \mathrm{sq} \mathrm{mi}$.

Gage-height record. - -Water-stage recorder graph except Jan, 24-31. Datum of gage is $3,354.57 \mathrm{ft}$ above mean sea level, datum of 1929 .

Discharge record. --Stage-discharge relation defined by current-meter measurements. Discharge for period of no gage-height record estimated.

Maxima. --November 1965 to January 1966: Discharge, 41, 100 cfs 1700 hours Dec. 30 (gage height, $14.32 \mathrm{ft}$ ).

1906 to October 1965: Discharge, 74,000 cfs probably Jan. 19, 1916 (gage height, $18.0 \mathrm{ft}$, from floodmark), from rating curve extended above 52,000 cfs by logarithmic plotting.

Mean discharge, in cubic feet per second, 1965-66

\begin{tabular}{|c|c|c|c|c|c|c|c|}
\hline Day & November & December & January & Day & November & December & January \\
\hline 1 & 144 & 599 & 7,820 & 16 & 134 & 1,390 & 802 \\
\hline$\ldots$ & 144 & 537 & 4,410 & 17 & 134 & 1,300 & 770 \\
\hline$\cdots$ & 144 & 491 & 2,870 & 18 & 134 & 1,320 & 730 \\
\hline$\cdots$ & 144 & 441 & 2,230 & 19 & 137 & 1,180 & 715 \\
\hline$\ldots$ & 144 & 412 & 1,780 & 20 & 137 & 882 & 685 \\
\hline$\ldots$ & 144 & 383 & 1,490 & 21 & 140 & 858 & 613 \\
\hline 7 & 144 & 366 & 1,360 & 22 & 144 & 9,480 & 565 \\
\hline 8 & 144 & 350 & 1,300 & 23 & 164 & 24,600 & 498 \\
\hline 9 & 147 & 356 & 1,250 & 24 & 376 & 5,520 & 500 \\
\hline 10 & 153 & 5,280 & 1,180 & 25 & 571 & 2,720 & 495 \\
\hline 11 & 153 & 7,020 & 1,110 & 26 & 2,420 & 2,060 & 490 \\
\hline 12 & 150 & 3,280 & 1,070 & 27 & 2,060 & 1,600 & 490 \\
\hline 13 & 140 & 1,810 & 989 & 28 & 1,300 & 1,360 & 485 \\
\hline 14 & 134 & 2,170 & 882 & 29 & 899 & 1,260 & 485 \\
\hline \multirow[t]{2}{*}{15} & 130 & 1,650 & 818 & 30 & 715 & 23,000 & 485 \\
\hline & & & & 31 & $\ldots \ldots$ & 19,800 & 485 \\
\hline \multirow{2}{*}{\multicolumn{5}{|c|}{$\begin{array}{l}\text { Monthly mean discharge, in cubic feet per second. } \\
\text { Runoff, in acre-feet } \ldots \ldots \ldots \ldots \ldots \ldots \ldots \ldots \ldots\end{array}$}} & 387 & 3,983 & 1,286 \\
\hline & & & & & 23,060 & 244,900 & 79,050 \\
\hline
\end{tabular}


Gage height, in feet, and discharge, in cubic feet per second, at indicated time, 1965-66, of Salt River near Chrysotile, Ariz.

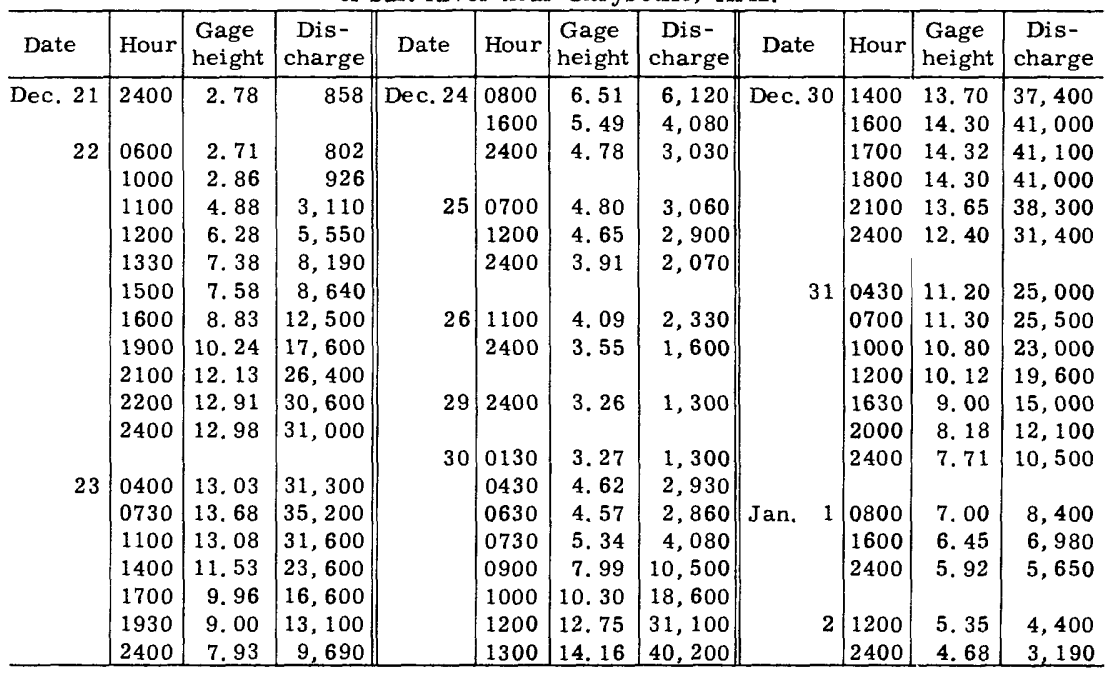

(97) 9-4978. Cibecue Creek near Chrysotile, Ariz.

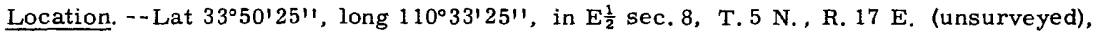
in Fort Apache Indian Reservation, on right bank half a mile upstream from mouth, and 7 miles north of Chrysotile.

Drainage area. $--295 \mathrm{sq} \mathrm{mi}$.

Gage-height record. - -Water-stage recorder graph except 2000-2100 hour 3 Dec. 22 for which graph was reconstructed. Altitude of gage is $3,200 \mathrm{ft}$ (from topographic map).

Discharge record. --Stage-discharge relation defined by current-meter measurements below 2,200 cfs and extended above on basis of slope-area measuremerts at gage heights 7.7 and $10.50 \mathrm{ft}$.

Maxima. --November 1965 to January 1966: Discharge, 8,800 cfs 0800 ho'rs Dec. 30 (gage height, $10.70 \mathrm{ft}$ ).

1959 to October 1965: Discharge, $8,480 \mathrm{cfs}$ July 29, 1964 (gage height, $10.04 \mathrm{ft}$ ); gage height, $10.5 \mathrm{ft}$ Aug. $31,1963$. 
Mean discharge, in cubic feet per second, 1965-66, of Cibecue Creek near Chrysotile, Ariz.

\begin{tabular}{|c|c|c|c|c|c|c|c|}
\hline Day & November & December & January & Day & November & December & January \\
\hline $1 \ldots$ & 12 & 26 & 379 & $16 \ldots$ & 12 & 65 & 48 \\
\hline $2 \ldots$ & 12 & 23 & 275 & $17 \ldots$ & 13 & 89 & 45 \\
\hline $3 \ldots$ & 12 & 19 & 221 & $18 \ldots$ & 13 & 65 & 48 \\
\hline $4 \ldots$ & 12 & 18 & 185 & $19 \ldots$ & 13 & 43 & 48 \\
\hline 5 & 12 & 17 & 136 & $20 \ldots$ & 13 & 36 & 43 \\
\hline $6 \ldots$ & 12 & 16 & 116 & $21 \ldots$ & 13 & 34 & 38 \\
\hline $7 \ldots$ & 12 & 15 & 100 & $22 \ldots$ & 14 & 2,130 & 38 \\
\hline $8 \ldots$ & 12 & 15 & 93 & $23 \ldots$ & 30 & 1,340 & 36 \\
\hline $9 \ldots$ & 12 & 90 & 82 & $24 \ldots \ldots$ & 26 & 275 & 32 \\
\hline $10 \ldots$ & 12 & 1,130 & 65 & $25 \ldots$ & 200 & 185 & 30 \\
\hline $11 \ldots$ & 12 & 253 & 56 & $26 \ldots$ & 59 & 149 & 30 \\
\hline $12 \ldots$ & 12 & 62 & 56 & $27 \ldots$ & 43 & 132 & 30 \\
\hline $13 \ldots$ & 12 & 59 & 53 & $28 \ldots$ & 56 & 124 & 28 \\
\hline $14 \ldots$ & 12 & 72 & 50 & 29 & 43 & 150 & 28 \\
\hline $15 \ldots$ & 12 & 93 & 50 & 30 & 32 & 3,890 & 30 \\
\hline & & & & 31 & $\cdots \cdots$ & 800 & 32 \\
\hline \multirow{2}{*}{\multicolumn{5}{|c|}{$\begin{array}{l}\text { Monthly mean discharge, in cubic feet per second } \ldots \\
\text { Runoff, in acre-feet } \ldots \ldots \ldots \ldots \ldots \ldots \ldots \ldots \ldots\end{array}$}} & 25.3 & 368 & 80.7 \\
\hline & & & & & 1,510 & 22,640 & 4,960 \\
\hline
\end{tabular}

Gage height, in feet, and discharge, in cubic feet per second, at indicated timo, 1965-66

\begin{tabular}{|c|c|c|c|c|c|c|c|c|c|c|c|}
\hline Date & Hour & $\begin{array}{l}\text { Gage } \\
\text { height }\end{array}$ & $\begin{array}{c}\text { Dis- } \\
\text { charge }\end{array}$ & Date & Hour & $\begin{array}{l}\text { Gage } \\
\text { height }\end{array}$ & $\begin{array}{c}\text { Dis- } \\
\text { charge }\end{array}$ & Date & Hour & $\begin{array}{l}\text { Gage } \\
\text { height }\end{array}$ & $\begin{array}{c}\text { Dis- } \\
\text { charge }\end{array}$ \\
\hline \multirow[t]{2}{*}{ Dec. 21} & 2400 & 2.65 & 32.0 & Dec. 23 & 1500 & 4.05 & 685 & Dec. 30 & 0600 & 7.9 & 4,900 \\
\hline & & & & & 1800 & 3.90 & 580 & & 0800 & 10.7 & 8,800 \\
\hline \multirow[t]{13}{*}{22} & 0500 & 2.65 & 32.0 & & 2400 & 3. 65 & 420 & & 0900 & 9.85 & 7,350 \\
\hline & 0700 & 2. 75 & 56.0 & & & & & & 1000 & 9.0 & 6,200 \\
\hline & 0800 & 3.83 & 601 & 24 & 1200 & 3.33 & 245 & & 1300 & 8.25 & 5,320 \\
\hline & 0900 & 5.00 & 1,540 & & 2400 & 3.30 & 230 & & 1600 & 7.04 & 3,720 \\
\hline & 1100 & 6.4 & 2,950 & & & & & & 2100 & 5,52 & 1,980 \\
\hline & 1300 & 7. 6 & 4,460 & & & & & & 2400 & 5.00 & 1,490 \\
\hline & 1400 & 6.8 & 3,430 & 28 & 2400 & 3. 06 & 124 & & & & \\
\hline & 1700 & 5.6 & 2,070 & & & & & 31 & 0230 & 4.70 & 1,220 \\
\hline & 2000 & 7.2 & 3,930 & 29 & 1600 & 3. 06 & 124 & & 0300 & 4. 75 & 1,260 \\
\hline & 2100 & 9.3 & 6,800 & & 2000 & 3. 14 & 158 & & 0600 & 4. 52 & 1,060 \\
\hline & 2300 & 8.5 & 5,580 & & 2030 & 3. 38 & 270 & & 1200 & 4.05 & 685 \\
\hline & 2400 & 8.00 & 5,050 & & 2200 & 3. 28 & 221 & & 1800 & 3.74 & 476 \\
\hline & & & & & 2300 & 3.40 & 280 & & & & \\
\hline \multirow[t]{3}{*}{23} & 0400 & 5.80 & 2,290 & & 2400 & 3. 98 & 636 & Jan. 1 & 1200 & 3.60 & 390 \\
\hline & 0700 & 4. 70 & 1,220 & & & & & & 2400 & 3.47 & 318 \\
\hline & 1100 & 4.35 & 920 & 30 & 0300 & 5.60 & 2,070 & & & & \\
\hline
\end{tabular}


(98) 9-4979. Cherry Creek near Young, Ariz.

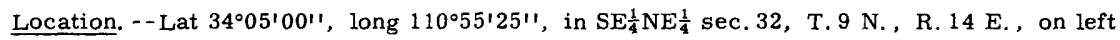
bank 0. 3 mile downstream from Deadman Canyon, and 2 miles southeast of Young.

Drainage area. $--62.1 \mathrm{sq} \mathrm{mi}$.

Gage-height record. --Water-stage recorder graph except Jan. 2-11. Alt tude of gage is 4, $950 \mathrm{ft}$ (from topographic map).

Discharge record. --Stage-discharge relation defined by current-meter msasurements below 22 cfs and extended above on basis of slope-area measurement at gage height $5.90 \mathrm{ft}$. Discharge for period of no gage-height record estimated.

Maxima. -- November 1965 to January 1966: Discharge, 3, 400 cfs 2100 hours Dec. 22 (gage height, $6.6 \mathrm{ft}$ ).

1963 to October 1965: Discharge, 3,280 cfs Aug. 17, 1965 (gage height, 6. $48 \mathrm{ft}$ ), from rating extended above $150 \mathrm{cfs}$ on basis of slope-area measurement at gage height $5.90 \mathrm{ft}$.

Mean discharge, in cubic feet per second, 1965-66

\begin{tabular}{|c|c|c|c|c|c|c|c|}
\hline Day & November & December & January & Day & November & December & January \\
\hline 1 & 0.8 & 1.8 & 87 & $16 \ldots$ & 1.1 & 57 & 13 \\
\hline 2 & .8 & 1. 8 & 55 & $17 \ldots$ & 1.2 & 60 & 13 \\
\hline$\ldots$ & .9 & 1. 6 & 45 & $18 \ldots$ & 1.4 & 55 & 14 \\
\hline 4 & .9 & 1. 6 & 40 & $19 \ldots$ & 1.4 & 47 & 13 \\
\hline 5 & .9 & 1. 6 & 35 & $20 \ldots$ & 1.2 & 44 & 14 \\
\hline 6 & .9 & 1. 6 & 30 & $21 \ldots$ & 1.2 & 44 & 13 \\
\hline 7 & 9 & 1.6 & 25 & $22 \ldots$ & 1.4 & 1,080 & 13 \\
\hline 8 & .9 & 1. 8 & 25 & $23 \ldots$ & 2.4 & 618 & 13 \\
\hline 9 & .9 & 28 & 25 & $24 \ldots$ & 1.6 & 188 & 13 \\
\hline 10 & .9 & 712 & 20 & $25 \ldots$ & 54 & 135 & 13 \\
\hline 11 & .9 & 221 & 20 & 26 & 49 & 107 & 12 \\
\hline 12 & .9 & 120 & 18 & $27 \ldots$ & 12 & 90 & 12 \\
\hline & .9 & 80 & 15 & $28 \ldots$ & 4.9 & 85 & 11 \\
\hline 14 & .9 & 70 & 14 & $29 \ldots$ & 2.8 & 118 & 11 \\
\hline \multirow[t]{2}{*}{$15 \ldots$} & 1. 1 & 68 & 14 & 30 . & 1.8 & 1,070 & 11 \\
\hline & & & & 31 & $\ldots-\cdots$ & 168 & 11 \\
\hline \multirow{2}{*}{\multicolumn{5}{|c|}{ Monthly mean discharge, in cubic feet per second. . }} & 5.03 & 170 & 21.5 \\
\hline & & & & & 299 & 10,470 & 1,320 \\
\hline
\end{tabular}


Gage height, in feet, and discharge, in cubic feet per second, at indicated time, 1965, of Cherry Creek near Young, Ariz.

\begin{tabular}{|c|c|c|c|c|c|c|c|c|c|c|c|}
\hline Date & Hour & $\begin{array}{l}\text { Gage } \\
\text { height }\end{array}$ & $\begin{array}{c}\text { Dis- } \\
\text { charge }\end{array}$ & Date & Hour & $\begin{array}{l}\text { Gage } \\
\text { height }\end{array}$ & $\begin{array}{c}\text { Dis- } \\
\text { charge }\end{array}$ & Date & Hour & $\begin{array}{c}\text { Gag? } \\
\text { height }\end{array}$ & $\begin{array}{l}\text { Dis- } \\
\text { charge }\end{array}$ \\
\hline Dec. 21 & 2400 & 1.61 & 46 & Dec. 22 & 2300 & 4.95 & 1,600 & Dec. 29 & 2200 & 2.19 & 282 \\
\hline & & & & & 2400 & 4.64 & 1,550 & & 2400 & 2. 73 & 472 \\
\hline \multirow[t]{16}{*}{22} & 0700 & 1. 67 & 55 & & & & & & & & \\
\hline & 0900 & 3.05 & 478 & 23 & 0400 & 3. 83 & 1,000 & 30 & 0200 & 3. $: 1$ & 725 \\
\hline & 0930 & 4. 95 & 1,770 & & 0800 & 3. 16 & 650 & & 0300 & 3.73 & 938 \\
\hline & 1000 & 5. 24 & 1,960 & & 1200 & 2. 72 & 468 & & 0600 & 4. 55 & 1,460 \\
\hline & 1030 & 4. 95 & 1,770 & & 1800 & 2. 37 & 340 & & 0800 & 4. 99 & 1,820 \\
\hline & 1200 & 4. 32 & 1,240 & & 2400 & 2. 06 & 249 & & 1000 & 5. 30 & 2,100 \\
\hline & 1300 & 4. 95 & 1,770 & & & & & & 1100 & 4. 99 & 1,620 \\
\hline & 1315 & 5.15 & 1,870 & 24 & 1200 & 1.78 & 170 & & 1300 & 4. 35 & 1,350 \\
\hline & 1330 & 4.95 & 1,770 & & 2400 & 1. 74 & 161 & & 1600 & 3. 56 & 850 \\
\hline & 1400 & 4. 65 & 1,510 & & & & & & 1900 & 2. 96 & 564 \\
\hline & 1630 & 3.88 & 934 & & & & & & 2400 & 2.31 & 318 \\
\hline & 1800 & 4.77 & 1,610 & 28 & 2400 & 1.35 & 84 & & & & \\
\hline & 1930 & 4.56 & 1,430 & & & & & 31 & 0600 & 1. 88 & 195 \\
\hline & 2000 & 4. 95 & 1,770 & 29 & 1600 & 1. 35 & 84 & & 1200 & 1. 66 & 144 \\
\hline & 2100 & 6.6 & 3,400 & & 1900 & 1. 38 & 89 & & 1800 & 1. 54 & 119 \\
\hline & 2200 & 5.50 & $2, .220$ & & 2030 & 1. 61 & 133 & & 2400 & 1. 48 & 107 \\
\hline
\end{tabular}

(99) 9-4979. 8. Cherry Creek near Globe, Ariz.

Location. -- Lat $33^{\circ} 49^{\prime} 40^{\prime \prime}$, long $110^{\circ} 51^{\prime} 20^{\prime \prime}$, in SW $\frac{1}{4}$ sec. 30, T. 6 N., R. $15 \mathrm{E}$. (unsurveyed), in Tonto National Forest, on left bank 0.2 mile upstream from Devils Chasm, 13 miles upstream from mouth, and 30 miles north of Globe.

Drainage area. $--200 \mathrm{sq} \mathrm{mi}$.

Gage-height record. --Water-stage recorder graph Nov. 1 to Dec. 10; digital recorder tape Jan. 7-31. Altitude of gage is $3,200 \mathrm{ft}$ (from topographic map).

Discharge record. --Stage-discharge relation defined by current-meter measurements below 75 cfs and extended above on basis of slope-area measurements at gage heights $5.85,6.70$, and $12.3 \mathrm{ft}$. Discharge for period of no gage-height record estimated on basis of record for Cherry Creek near Young.

Maxima. --November 1965 to January 1966: Discharge, 6, 620 cfs (time unknown) Dec. 22 (gage height, $12.3 \mathrm{ft}$ ), from slope-area measurement of peak flow.

May to October 1965: Discharge, 2, $280 \mathrm{cfs}$ Aug. 17, 1965 (gage height, 6. $70 \mathrm{ft}$ ).

Mean discharge, in cubic feet per second, 1965-66

\begin{tabular}{|c|c|c|c|c|c|c|c|}
\hline Day & November & December & January & Day & November & December & January \\
\hline 1 & 6.9 & 12 & 300 & 16 & 7.4 & 200 & 31 \\
\hline$\ldots$ & 6.9 & 11 & 200 & $17 \ldots$ & 7.9 & 180 & 31 \\
\hline$\ldots$ & 6.9 & 10 & 130 & $18 \ldots$ & 8.5 & 170 & 37 \\
\hline 4 & 6.9 & 9.1 & 110 & $19 \ldots$ & 8.5 & 160 & 38 \\
\hline $5 \ldots$ & 6.9 & 9.1 & 100 & $20 \ldots$ & 8.5 & 150 & 35 \\
\hline $6 \ldots$ & 7.4 & 7. 9 & 80 & $21 \ldots$ & 8.5 & 140 & 31 \\
\hline $7 \ldots$ & 7. 4 & 6. 9 & 74 & $22 \ldots$ & 11 & 3,500 & 29 \\
\hline $8 \ldots$ & 7.4 & 7.4 & 69 & $23 \ldots$ & 54 & 1,000 & 27 \\
\hline $9 \ldots$ & 7.4 & 217 & 64 & $24 \ldots$ & 20 & 500 & 25 \\
\hline $10 \ldots$ & 7. 4 & 2,300 & 56 & $25 \ldots$ & 246 & 450 & 24 \\
\hline $11 \ldots$ & 7.4 & 700 & 50 & 26 & 178 & 350 & 22 \\
\hline $12 \ldots$ & 7. 4 & 350 & 45 & 27 & 48 & 300 & 21 \\
\hline $13 \ldots$ & 7.4 & 300 & 41 & 28. & 23 & 280 & 20 \\
\hline 14 & 7.4 & 250 & 36 & $29 \ldots$ & 14 & 350 & 20 \\
\hline \multirow[t]{2}{*}{$15 \ldots$} & 7.4 & 220 & 33 & 30 & 13 & 3,500 & 20 \\
\hline & & & & $31 \ldots$ & $\ldots-\ldots$ & 1,000 & 22 \\
\hline \multirow{2}{*}{\multicolumn{5}{|c|}{$\begin{array}{l}\text { Monthly mean discharge, in cubic feet per second } . \\
\text { Runoff, in acre-feet } \ldots \ldots \ldots \ldots \ldots \ldots \ldots \ldots\end{array}$}} & 25.5 & 537 & 58.7 \\
\hline & & & & & 1,520 & 33,010 & 3,610 \\
\hline
\end{tabular}


(100) 9-4985. Salt River near Roosevelt, Ariz.

Location. --Lat $33^{\circ} 37^{\prime} 10^{\prime \prime}$, long $110^{\circ} 55^{\prime} 15^{\prime \prime}$, in $\mathrm{SE} \frac{1}{4} \mathrm{NE} \frac{1}{4}$ sec. 9, T. 3 N., R. 14 E.

(unsurveyed), in Tonto National Forest, on left bank $100 \mathrm{ft}$ downstream from State Highway 288, a quarter of a mile downstream from Pinal Creek, 14 miles east of village of Roosevelt, and 17 miles upstream from Roosevelt Dam.

Drainage area. $--4,306 \mathrm{sq} \mathrm{mi}$.

Gage-height record, - -Water-stage recorder graph. Datum of gage is 2,177.14 $\mathrm{ft}$ above mean sea level, datum of 1929 .

Discharge record. --Stage-discharge relation defined by current-meter measurements below $47,000 \mathrm{cfs}$ and extended above by logarithmic plotting.

Maxima. --November 1965 to January 1966: Discharge, 68, 800 cfs 0400 hours Dec. 23 (gage height, $25.8 \mathrm{ft}$ ).

1906 to October 1965: Discharge, 117, $000 \mathrm{cfs}$ Mar. 14, 1941 (gage height, 24. $4 \mathrm{ft}$ ), from rating curve extended above $55,000 \mathrm{cfs}$ on basis of velocity-area studies and float-area measurements at 66,000 and $102,000 \mathrm{cfs}$.

Mean discharge, in cubic feet per second, 1965-66

\begin{tabular}{|c|c|c|c|c|c|c|c|}
\hline Day & November & December & January & Day & November & December & January \\
\hline $1 \ldots$ & 165 & 845 & 10,500 & $16 \ldots$ & 162 & 2,110 & 1,110 \\
\hline $2 \ldots$ & 165 & 690 & 5,930 & $17 \ldots$ & 165 & 2,090 & 1,060 \\
\hline $3 \ldots$ & 165 & 616 & 4,060 & $18 \ldots$ & 165 & 2,040 & 1,060 \\
\hline $4 \ldots$ & 165 & 550 & 3,160 & $19 \ldots$ & 165 & 1,780 & 1,060 \\
\hline $5 \ldots$ & 165 & 490 & 2,500 & $20 \ldots$ & 167 & 1,430 & 1,020 \\
\hline $6 \ldots$ & 165 & 445 & 2,120 & 21. & 167 & 1,290 & 943 \\
\hline $7 \ldots$ & 167 & 417 & 1,920 & 22 & 172 & 13,200 & 853 \\
\hline 8 & 167 & 390 & 1,730 & 23 & 194 & 45,100 & 763 \\
\hline & 169 & 450 & 1,640 & 24 & 274 & 11,700 & 684 \\
\hline 10. & 172 & 9,410 & 1,560 & 25 & 919 & 5,170 & 684 \\
\hline $11 \ldots$ & 174 & 12,900 & 1,450 & $26 \ldots$ & 3,070 & 3,300 & 684 \\
\hline $12 \ldots$ & 174 & 5,660 & 1,380 & $27 \ldots$ & 2,820 & 2,400 & 644 \\
\hline 13 & 172 & 2,710 & 1,330 & 28. & 1,900 & 1,960 & 644 \\
\hline 14 & 167 & 2,860 & 1,240 & 29 & 1,330 & 1,740 & 644 \\
\hline \multirow[t]{2}{*}{$15 \ldots$} & 165 & 2,580 & 1,160 & 30 & 1,020 & 29,200 & 644 \\
\hline & & & & 31 & $\ldots--\cdots$ & 30,600 & 644 \\
\hline \multirow{2}{*}{\multicolumn{5}{|c|}{$\begin{array}{l}\text { Monthly mean discharge, in cubic feet per second. } \\
\text { Runoff, in acre-feet } \ldots \ldots\end{array}$}} & 507 & 6,326 & $1,7 \overline{70}$ \\
\hline & & & & & 30,160 & 389,000 & 108,700 \\
\hline
\end{tabular}

Gage height, in feet, and discharge, in cubic feet per second, at indicated time, 1965-66

\begin{tabular}{|c|c|c|c|c|c|c|c|c|c|c|c|}
\hline Date & Hour & $\begin{array}{l}\text { Gage } \\
\text { height }\end{array}$ & $\begin{array}{c}\text { Dis- } \\
\text { charge }\end{array}$ & Date & Hour & $\begin{array}{l}\text { Gage } \\
\text { height }\end{array}$ & $\begin{array}{c}\text { Dis- } \\
\text { charge }\end{array}$ & Date & Hour & $\begin{array}{l}\text { Gage } \\
\text { height }\end{array}$ & $\begin{array}{c}\text { Dis- } \\
\text { charge }\end{array}$ \\
\hline \multirow[t]{2}{*}{ Dec. 21} & 2400 & 9.25 & 1,260 & Dec. 23 & 1200 & 23.15 & 47,400 & Dec. 30 & 0900 & 17.50 & 18,100 \\
\hline & & & & & 1800 & 21.20 & 35,700 & & 1200 & 20.00 & \\
\hline \multirow[t]{13}{*}{22} & 0300 & 9. 35 & 1,360 & & 2400 & 18.30 & 21,300 & & 1500 & 21.00 & 34,500 \\
\hline & 0900 & 9.40 & 1,400 & & & & & & 1800 & 23.90 & 300 \\
\hline & 1000 & 9. 42 & 1,420 & 24 & 0600 & 16.50 & 14 , & & 2100 & 24.80 & 00 \\
\hline & 1100 & 10.25 & 2,210 & & 1200 & 15.30 & 10,600 & & 2400 & 24.30 & .900 \\
\hline & 1200 & 10.58 & 2,560 & & 1800 & 14.35 & 8,030 & & & & \\
\hline & 1300 & 12.22 & 4,890 & & 2400 & 13.75 & 6,570 & 3 & 0600 & 21.60 & 38 \\
\hline & 1400 & 13.58 & 7,460 & & & & & & 1000 & 20.00 & \\
\hline & 1530 & 15.75 & 12,800 & 25 & 0600 & 13.25 & 5,500 & & 1200 & 19.90 & 00 \\
\hline & 1800 & 20.35 & 31,200 & & 1800 & 12.85 & 4,700 & & 1800 & 18.25 & 21,100 \\
\hline & 1900 & 21.00 & 34,500 & & 2400 & 12.58 & 4,200 & & 2400 & 16.80 & 15,400 \\
\hline & 2000 & 21.33 & 36,500 & & & & & & & & \\
\hline & 2130 & 21.17 & 35,500 & & & & & Jan. 1 & 1200 & 14.95 & 9,660 \\
\hline & 2400 & 22.35 & 42,600 & 29 & 2400 & 10.82 & 1,780 & & 2400 & 14.10 & 7,400 \\
\hline \multirow[t]{3}{*}{23} & 0200 & 24.05 & 53,900 & 30 & 0400 & 11.60 & 2,680 & 2 & 1200 & 13.40 & 5, \\
\hline & 0400 & 25.8 & 68,800 & & 0500 & 13.50 & 6,020 & & 2400 & 12.85 & 4,700 \\
\hline & 1000 & 23.15 & 47,400 & & 0600 & 14.30 & 7,900 & & & & \\
\hline
\end{tabular}


(101) 9-4988. Tonto Creek near Gisela, Ariz.

Location. -- Lat $34^{\circ} 07^{\prime} 44^{\prime \prime}$, long $11^{\circ} 1^{\prime} 5^{\prime} 17^{\prime \prime}$, in NE⿺ sec. 18 , T. 9 N., R. $11 \mathrm{E}$., in Tonto National Forest, on left bank 0.2 mile upstream from Houston Creek, and $1 \frac{1}{2}$ miles northeast of Gisela.

Drainage area. $--430 \mathrm{sq} \mathrm{mi}$

Gage-height record. - -Water-stage recorder graph until Dec. 15. Altitude of gage is $2,940 \mathrm{ft}$ (from topographic map).

Discharge record. --Stage-discharge relation defined by current-meter measurements below 13,000 cfs and extended above on basis of slope-area measurement of peak flow below Houston Creek. Discharge after Dec. 15 estimated on basis of records for Tonto Creek above Gun Creek, near Roosevelt.

Maxima. - November 1965 to January 1966: Discharge, approximately 30, $000 \mathrm{cfs} 1900$ hours Dec. 22 (gage height, $19.0 \mathrm{ft}$ ), from slope-area measurement of peak flow adjusted for inflow from Houston Creek.

1964 to October 1965: Discharge, 12,600 cfs Jan. 7, 1965 (gage height, $11.7 \mathrm{ft}$ ).

\begin{tabular}{|c|c|c|c|c|c|c|c|}
\hline Day & November & December & January & Day & November & December & January \\
\hline 1 & 10 & 57 & 1,200 & 16 & 12 & 800 & 115 \\
\hline $2 \ldots$ & 10 & 50 & 700 & $17 \ldots$ & 12 & 900 & 120 \\
\hline $3 \ldots$ & 10 & 45 & 400 & $18 \ldots$ & 13 & 800 & 125 \\
\hline $4 \ldots$ & 10 & 41 & 350 & 19 & 13 & 700 & 120 \\
\hline $5 \ldots$ & 11 & 37 & 300 & 20 & 12 & 700 & 110 \\
\hline 6 & 11 & 36 & 250 & 21 & 12 & 700 & 100 \\
\hline 7 & 11 & 33 & 230 & $22 \ldots$ & 13 & 13,000 & 95 \\
\hline $8 \ldots$ & 11 & 32 & 210 & 23 & 45 & 8,000 & 85 \\
\hline$\ldots$ & 11 & 130 & 190 & $24 \ldots \ldots$ & 125 & 2,000 & 80 \\
\hline 10 & 11 & 10,400 & 180 & $25 \ldots$ & 1,900 & 1,100 & 75 \\
\hline $11 \ldots$ & 11 & 3,040 & 160 & $26 \ldots$ & 726 & 800 & 70 \\
\hline 12 & 11 & 1,400 & 150 & $27 \ldots$ & 168 & 500 & 68 \\
\hline 13 & 11 & 727 & 140 & $28 \ldots \ldots$ & 111 & 400 & 65 \\
\hline $14 \ldots$ & 12 & 904 & 130 & 29 & 83 & 350 & 62 \\
\hline 15 & 12 & 1,000 & 120 & 30 & 67 & 10,000 & 60 \\
\hline & & & & 31. & - - . - - - & 2,500 & 58 \\
\hline \multirow{2}{*}{\multicolumn{5}{|c|}{$\begin{array}{l}\text { Monthly mean discharge, in cubic feet per second. } \\
\text { Runoff, in acre-feet } \ldots \ldots \ldots \ldots \ldots \ldots \ldots \ldots\end{array}$}} & 116 & 1,974 & 197 \\
\hline & & & & & 6,890 & 121,400 & 12,130 \\
\hline
\end{tabular}

(102) Houston Creek near Gisela, Ariz.

(Miscellaneous site)

Location. - - Lat $34^{\circ} 07^{\prime} 32^{\prime \prime}$, long $111^{\circ} 15^{\prime} 26^{\prime \prime}$, in $\mathrm{SE} \frac{1}{4} \mathrm{NE} \frac{1}{4} \sec .18$, T. $9 \mathrm{~N}$, , R. $11 \mathrm{E} ., 300 \mathrm{ft}$ upstream from mouth, and 8. 7 miles southeast of Payson.

Drainage area. $--56.2 \mathrm{sq} \mathrm{mi}$.

Maximum. - - November 1965 to January 1966: Discharge, 4, 530 cfs Dec. 22, from slope-area measurement of peak flow. 
(103) 9-4988. 7. Rye Creek near Gisela, Ariz.

Location. -- Lat $34^{\circ} 02^{\prime} 15^{\prime \prime}$, long $111^{\circ} 17^{\prime} 40^{\prime \prime}$, in sec. 13 , T. 8 N., R. 10 E., in Tonto National Forest, on right bank a quarter of a mile upstream from moith, 0.7 mile downstream from bridge on county road, and $5 \frac{1}{2}$ miles south of Gisela.

Drainage area. --122 sq $\mathrm{mi}$.

Gage-height record. - Water-stage recorder graph after Dec. 8. Altitud? of gage is $2,730 \mathrm{ft}$ (from topographic map).

Discharge record. --Stage-discharge relation poorly defined by current-rzeter measurements below 3, $900 \mathrm{cfs}$ and extended above on basis of slope-area measurement of peak flow.

Maximum. --November 1965 to January 1966: Discharge, 8, 130 cfs 2100 hours Dec. 22 (gage height, $8.0 \mathrm{ft}$ ).

Mean discharge, in cubic feet per second, 1965-66

\begin{tabular}{|c|c|c|c|c|c|c|c|c|}
\hline Day & December & January & Day & December & January & Day & December & January \\
\hline$\ldots$ & - & 199 & 11 & 379 & 33 & 21. & 110 & 20 \\
\hline . . & - & 150 & 12 & 168 & 31 & $22 \ldots$ & 2,930 & 18 \\
\hline 3 & - & 110 & 13 & 102 & 28 & $23 \ldots$ & 1,960 & 17 \\
\hline 4 & - & 86 & 14 & 203 & 25 & 24 & 467 & 15 \\
\hline 5 & - & 68 & 15 & 197 & 23 & 25 & 280 & 15 \\
\hline 6 & - & 61 & 16 & 117 & 20 & 26 & 192 & 14 \\
\hline 7 & - & 53 & 17 & 219 & 18 & $27 \ldots$ & 152 & 14 \\
\hline$\ldots$ & - & 46 & 18 & 143 & 23 & $28 \ldots \ldots$ & 125 & 13 \\
\hline 9 & 5 & 42 & 19 & 105 & 24 & $29 \ldots$ & 141 & 13 \\
\hline 10 & 725 & 35 & 20 & 105 & 22 & 30 & 2,190 & 13 \\
\hline & & & & & & $31 \ldots$ & 486 & 13 \\
\hline \multirow{2}{*}{\multicolumn{7}{|c|}{ 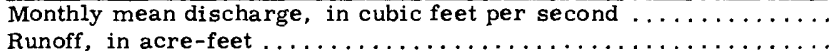 }} & - & 40.7 \\
\hline & & & & & & & - & 2,500 \\
\hline
\end{tabular}

(104) 9-4989. Gold Creek near Payson, Ariz.

\section{(Crest-stage station)}

Location. --Lat $34^{\circ} 00^{\prime} 10^{\prime \prime}$, long $111^{\circ} 21^{\prime} 30^{\prime \prime}$, in SW $\frac{1}{4}$ sec. 29, T. 8 N., R. 10 E., at State Highway 87, 16 miles south of Payson.

Drainage area. $--6.52 \mathrm{sq} \mathrm{mi}$.

Gage-height record. --Crest stages only. Datum of gage is 5, 763.7 ft above mean sea level (from Arizona Highway Department bench mark).

Discharge record. --Stage-discharge relation defined by current-meter moasurements below $20 \mathrm{cfs}$ and extended above on basis of computation of flow througl culvert.

Maxima. --November 1965 to January 1966: Discharge, $500 \mathrm{cfs}$ Dec. 22 (gage height, $4.04 \mathrm{ft}$ ).

1963 to October 1965: Discharge, 1, $370 \mathrm{cfs}$ Aug. 22, 1963 (gage height, $7.75 \mathrm{ft}$ ). 
(105) 9-4990. Tonto Creek above Gun Creek, near Roosevelt, Ariz.

Location. --Lat $33^{\circ} 59^{\prime}$, long $111^{\circ} 18^{\prime}$, in $\mathrm{NE}^{\frac{1}{4}} \mathrm{SW} \frac{1}{4} \mathrm{sec} .2$, T. 7 N., R. $10 \mathrm{E}$, in Tonto National Forest, on left bank $600 \mathrm{ft}$ upstream from Gun Creek, $23 \frac{1}{2}$ miles ncrthwest of village of Roosevelt, and 26 miles upstream from Roosevelt Dam.

Drainage area. --675 sq mi.

Gage-height record. - -Water-stage recorder graph. Datum of gage is 2,523.14 $\mathrm{ft}$ above mean sea level, datum of 1929 .

Discharge record. --Stage-discharge relation defined by current-meter measurements below 27,000 cfs.

Maxima. - - November 1965 to January 1966: Discharge, 44, 700 cfs 2100 hours Dec. 22 (gage height, $16.7 \mathrm{ft}$ ).

1940 to October 1965: Discharge, $45,400 \mathrm{cfs}$ Jan. 18, 1952 (gage height, 16. $55 \mathrm{ft}$ ).

Mean discharge, in cubic feet per second, 1965-66

\begin{tabular}{|c|c|c|c|c|c|c|c|}
\hline Day & November & December & January & Day & November & December & January \\
\hline$\ldots$ & 6.9 & 72 & 1,700 & 16. & 8.8 & 1,100 & 171 \\
\hline 2 & 8.1 & 51 & 1,030 & 17 & 10 & 1,190 & 158 \\
\hline $3 \ldots$ & 8.1 & 47 & 710 & 18. & 11 & 1,100 & 169 \\
\hline $4 \ldots$ & 7.5 & 37 & 564 & 19 & 11 & 908 & 174 \\
\hline . & 8.8 & 33 & 467 & 20 & 12 & 805 & 156 \\
\hline 6 & 8.8 & 28 & 401 & 21 & 13 & 789 & 153 \\
\hline 7 & 6.9 & 27 & 354 & 22 & 16 & 15,300 & 139 \\
\hline 8 & 6.3 & 24 & 322 & 23 & 66 & 10,900 & 136 \\
\hline 9 & 6.9 & 96 & 303 & 24 & 176 & 2,470 & 130 \\
\hline 10 & 6. 9 & 10,200 & 273 & 25 & 2,010 & 1,520 & 121 \\
\hline 11 & 6.9 & 3,280 & 249 & 26 & 936 & 1,070 & 112 \\
\hline 12 & 6.9 & 1,180 & 232 & 27 & 298 & 728 & 110 \\
\hline 13 & 6.9 & 910 & 213 & 28 & 186 & 572 & 104 \\
\hline 14 & 6.9 & 1,310 & 194 & 29 & 125 & 541 & 100 \\
\hline \multirow[t]{2}{*}{$15 \ldots$} & 7.5 & 1,410 & 186 & & 92 & 11,200 & 96 \\
\hline & & & & 31 & $\ldots-\cdots$ & 3,210 & 94 \\
\hline \multicolumn{5}{|c|}{ Monthly mean discharge, in cubic feet per second. } & 137 & 2,326 & 301 \\
\hline \multicolumn{5}{|c|}{ Runoff, in acre-feet $\ldots \ldots \ldots \ldots \ldots \ldots \ldots$} & 8,150 & 143,000 & 18,490 \\
\hline
\end{tabular}

Gage height, in feet, and discharge, in cubic feet per second, at indicated time, 1965-66,

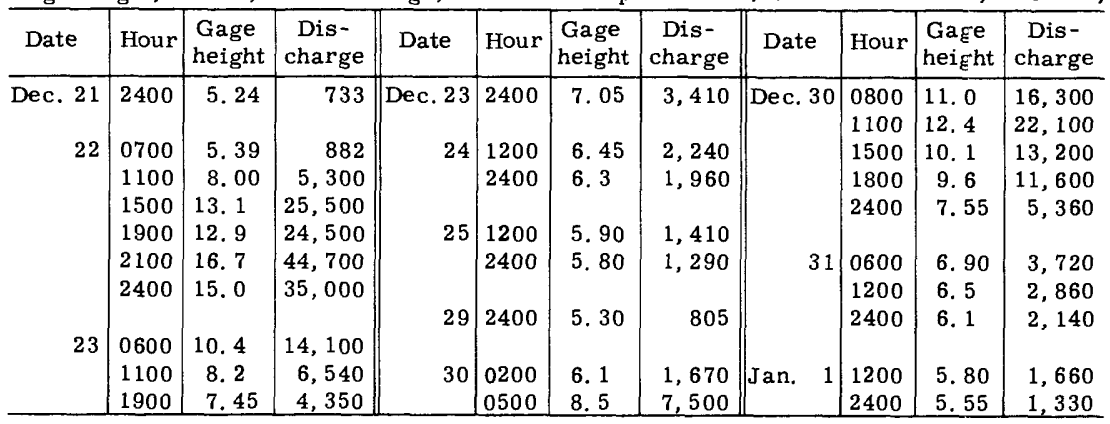


(106) 9-5005. Salt River at Roosevelt, Ariz.

(Former gaging-station site)

Location. --Lat $33^{\circ} 40^{\prime} 30^{\prime \prime}$, long $111^{\circ} 09^{\prime} 30^{\prime \prime}$, in sec. 20, T. 4 N., R. $12 \mathrm{E}$. (unsurveyed), at Roosevelt Dam, 1 mile west of Roosevelt.

Drainage area. $--5,830 \mathrm{sq} \mathrm{mi}$, approximately.

Maxima. --November 1965 to January 1966: Discharge, 88, 000 cfs 0300 hours Dec. 22, obtained by routing flow of Salt River near Roosevelt and Tonto Creek below Gun Creek near Roosevelt to Roosevelt Lake.

1888 to October 1965: Discharge, about 150, 000 cfs Feb. 22-23, 1891, estimated on basis of records for Salt River at McDowell.

(107) 9-5010. Reservoir system on Salt River at and below Roosevel: Dam, Ariz.

Location. - This system comprises 4 storage reservoirs created by $4 \mathrm{~d} \varepsilon \mathrm{ms}$ on Salt River: Roosevelt Lake, formed by Roosevelt Dam, in sec. 20, T. 4 N., R. 12 E. (unsurveyed); Apache Lake, formed by Horse Mesa Dam, 17 miles downstream from Roosevelt Dam; Canyon Lake, formed by Mormon Flat Dam, 27 miles downstre?m from Roosevelt Dam; and Saguaro Lake, formed by Stewart Mountain Dam, 37 miles downstream from Roosevelt Dam.

Drainage area. - -6, $211 \mathrm{sq} \mathrm{mi}$, at Stewart Mountain Dam.

Maxima. - November 1965 to January 1966: Contents, 1,684, 000 acre- $f^{\ddagger}$. Dec. 31. 1910 to October 1965: Contents, 1,764, 000 acre-ft May 22, 1941.

Remarks. --Records given herein represent usable contents. Dead storage negligible. Total capacity of the 4 reservoirs is $1,755,000$ acre-ft, divided as follows: Roosevelt Lake, 1, 382, 000 acre-ft; A pache Lake, 245, 000 acre-ft; Canyon Lake, 58, 000 acre$\mathrm{ft}$; Saguaro Lake, 70, 000 acre-ft. Dams forming these reservoirs were built as follows: Roosevelt 1905-11; Horse Mesa 1924-27; Mormon Flat 1923-26; Stewart Mountain 1928-30. Since 1910, spill over Roosevelt Dam because of capacity storage has occurred only during the following periods: Apr. 15 to June 21, 1915; Jan. 19 to May 30, 1916; Apr. 21 to June 7, 1917; Feb. 17 to June 3, 1920; Apr. 13 to July 24, 1941; Dec. 30, 1965, to Jan. 10, 1966. Records furnished by Salt River Valley Water Users' Association.

Contents, in thousands of acre-feet, at 2400, 1965-66

\begin{tabular}{r|r|r|r||r|r|r|r}
\hline Day & November & December & January & Day & November & December & January \\
\hline $1 \ldots$ & 1,140 & 1,164 & 1,647 & $16 \ldots$ & 1,134 & 1,313 & 1,558 \\
$2 \ldots$ & 1,140 & 1,165 & 1,628 & $17 \ldots$ & 1,134 & 1,322 & 1,560 \\
$3 \ldots$ & 1,140 & 1,166 & 1,624 & $18 \ldots$ & 1,134 & 1,329 & 1,564 \\
$4 \ldots$ & 1,139 & 1,167 & 1,614 & $19 \ldots$ & 1,134 & 1,335 & 1,567 \\
$5 \ldots$ & 1,139 & 1,168 & 1,602 & $20 \ldots$ & 1,134 & 1,340 & 1,569 \\
$6 \ldots$ & 1,138 & 1,169 & 1,590 & $21 \ldots$ & 1,134 & 1,343 & 1,574 \\
$7 \ldots$ & 1,138 & 1,169 & 1,577 & $22 \ldots$ & 1,134 & 1,413 & 1,577 \\
$8 \ldots$ & 1,137 & 1,167 & 1,564 & $23 \ldots$ & 1,137 & 1,535 & 1,582 \\
$9 \ldots$ & 1,137 & 1,178 & 1,548 & $24 \ldots$ & 1,137 & 1,560 & 1,583 \\
$10 \ldots$ & 1,136 & 1,224 & 1,539 & $25 \ldots$ & 1,142 & 1,570 & 1,585 \\
$11 \ldots$ & 1,135 & 1,260 & 1,543 & $26 \ldots$ & 1,149 & 1,576 & 1,587 \\
$12 \ldots$ & 1,135 & 1,273 & 1,546 & $27 \ldots$ & 1,155 & 1,581 & 1,588 \\
$13 \ldots$ & 1,134 & 1,281 & 1,550 & $28 \ldots$ & 1,158 & 1,587 & 1,589 \\
$14 \ldots$ & 1,135 & 1,292 & 1,553 & $29 \ldots$ & 1,161 & 1,593 & 1,591 \\
$15 \ldots$ & 1,134 & 1,305 & 1,556 & $30 \ldots$ & 1,162 & 1,679 & 1,592 \\
& & & $31 \ldots$. & $\ldots$ & 1,684 & 1,592 \\
\hline
\end{tabular}


(108) 9-5013. Tortilla Creek at Tortilla Flat, Ariz.

(Crest-stage station)

Location. -- Lat $33^{\circ} 31^{\prime} 38^{\prime \prime}$, long $111^{\circ} 23^{\prime} 13^{\prime \prime}$, NWt sec. 13, T. 2 N. , R. 9 E. (unsurveyed), $600 \mathrm{ft}$ upstream from State Highway 88 and Tortilla Flat Store, and 3.7 miles southeast of Mormon Flat Dam.

Drainage area. $--24.3 \mathrm{sq} \mathrm{mi}$.

Gage-height record. --Crest stages only, from floodmarks. Altitude of gage is $1,755 \mathrm{ft}$ (from topographic map).

Maximum. - November 1965 to January 1966: Discharge, 2, 440 cfs Dec. 22 (gage height, $8.0 \mathrm{ft}$ ), from computation of flow over irregular weir.

(109) 9-5020. Salt River below Stewart Mountain Dam, Ariz.

Location. -- Lat $33^{\circ} 33^{\prime} 00^{\prime \prime}$, long $111^{\circ} 34^{\prime} 35^{\prime \prime}$, in $\mathrm{N} \frac{1}{2} \mathrm{NW} \frac{1}{4} \sec .6, \mathrm{~T} .2 \mathrm{~N}$, , R. 8 E. (unsurveyed), on left bank $3 \frac{1}{2}$ miles downstream from Stewart Mountain Dam, and 6 miles upstream from Verde River.

Drainage area. $--6,232 \mathrm{sq} \mathrm{mi}$, of which $21 \mathrm{sq} \mathrm{mi}$ is below Stewart Mountain Dam.

Gage-height record. - -Water-stage recorder graph, except 1900 hours Dec. 30 to 1300 hours Jan. 2. Altitude of gage is 1, $370 \mathrm{ft}$ (from topographic map).

Discharge record. --Stage-discharge relation defined by current-meter measurements below $9,700 \mathrm{cfs}$ and extended above on basis of computation of peak flow oven dam. Discharge for period of no gage-height record computed on basis of records of releases at Stewart Mountain Dam by Salt River Valley Water Users' Associat'on.

Maxima. - November 1965 to January 1966: Discharge, 51,600 cfs 1900-2000 hours Jan. 1 (gage height, $22.4 \mathrm{ft}$, from floodmarks).

1921 to October 1965: Discharge, 11, $700 \mathrm{cfs}$ May 7, 1941 (gage height, 15.97 ft, at site $2 \frac{3}{4}$ miles upstream at different datum).

1910 to October 1965: Daily discharge, 50,000 cfs Jan. 20 and 29, 1916.

Remarks. --Flow regulated at Stewart Mountain Dam and three other dams (combined capacity, 1, 755, 000 acre-ft; see station 107, Reservoir system on Salt River at and below Roosevelt Dam). Storage began in Roosevelt Dam in 1910.

Mean discharge, in cubic feet per second, 1965-66

\begin{tabular}{|c|c|c|c|c|c|c|c|}
\hline Day & November & December & January & Day & November & December & January \\
\hline 1 & 221 & 4.7 & 38,600 & 16 & 175 & 7.9 & 10 \\
\hline 2 & 227 & 4.7 & 10,000 & $17 \ldots$ & 160 & 7.4 & 10 \\
\hline$\ldots$ & 273 & 4. 7 & 7,660 & $18 \ldots$ & 115 & 6.3 & 10 \\
\hline 4 & 345 & 4.4 & 9,960 & $.19 \ldots$ & 99 & 4. 7 & 10 \\
\hline 5 & 360 & 4.2 & 9,850 & $20 \ldots$ & 99 & 4. 7 & 10 \\
\hline 6 & 368 & 4.2 & 9,620 & $21 \ldots$ & 99 & 4.4 & 10 \\
\hline 7 & 368 & 3.9 & 9,700 & $22 \ldots$ & 94 & 203 & 10 \\
\hline $8 \ldots$ & 375 & 4.4 & 9,850 & 23 & 25 & 3,750 & 10 \\
\hline 9 & 398 & 6.3 & 9,720 & 24 & 16 & 3,660 & 10 \\
\hline 10 & 472 & 18 & 7,750 & 25 & 12 & 2,160 & 10 \\
\hline 11 & 510 & 10 & 10 & 26 & 7. 4 & 2,130 & 10 \\
\hline 12 & 352 & 6.8 & 10 & 27 & 6.8 & 1,040 & 10 \\
\hline 13 & 203 & 6.3 & 10 & 28 & 6.3 & 36 & 10 \\
\hline 14 & 165 & 6.3 & 10 & 29 & 5.2 & 20 & 10 \\
\hline \multirow[t]{2}{*}{$15 \ldots$} & 170 & 12 & 10 & 30 & 5. 2 & 6,180 & 10 \\
\hline & & & & $31 \ldots$ & $\ldots \ldots$ & 37,200 & 596 \\
\hline \multirow{2}{*}{\multicolumn{5}{|c|}{$\begin{array}{l}\text { Monthly mean discharge, in cubic feet per second. } \\
\text { Runoff, in acre-feet } \ldots \ldots \ldots \ldots \ldots \ldots \ldots \ldots \ldots\end{array}$}} & 191 & 1,823 & 3,984 \\
\hline & & & & & 11,370 & 112,100 & 245,000 \\
\hline
\end{tabular}


Gage height, in feet, and discharge, in cubic feet per second, at indicated time, 1965-66, of Salt River below Stewart Mountain Dam, Ariz.

\begin{tabular}{|c|c|c|c|c|c|c|c|c|c|c|c|c|}
\hline Date & Hour & $\begin{array}{l}\text { Gage } \\
\text { height }\end{array}$ & $\begin{array}{c}\text { Dis- } \\
\text { charge }\end{array}$ & Date & \multirow{2}{*}{$\frac{\text { Hour }}{2400}$} & \multirow{2}{*}{$\begin{array}{c}\begin{array}{c}\text { Gage } \\
\text { height }\end{array} \\
2.28\end{array}$} & \multirow{2}{*}{$\begin{array}{r}\begin{array}{c}\text { Dis- } \\
\text { charge }\end{array} \\
16\end{array}$} & \multicolumn{2}{|c|}{ Date } & \multirow{2}{*}{\begin{tabular}{|l|} 
Hour \\
2400
\end{tabular}} & $\begin{array}{l}\text { Gage } \\
\text { height }\end{array}$ & $\begin{array}{c}\text { Dis- } \\
\text { charge }\end{array}$ \\
\hline Dec. 21 & 2400 & 2.09 & 4.4 & Dec. 29 & & & & Jan. & 1 & & - & 40,000 \\
\hline \multirow{7}{*}{22} & 0100 & 2.09 & 4.4 & 30 & 1900 & 2.24 & 13 & & 2 & 0230 & - & 40,400 \\
\hline & 0200 & 2.67 & 86 & & 1930 & - & 9,120 & & & 0300 & - & 20,200 \\
\hline & 0800 & 2.82 & 145 & & 2000 & - & 9,120 & & & 0400 & - & 10,100 \\
\hline & 1600 & 2. 40 & 30 & & 2030 & - & 22,100 & & & 0500 & - & 10,200 \\
\hline & 2100 & 3. 23 & 412 & & 2100 & - & 39,600 & & & 0500 & - & 6,900 \\
\hline & 2400 & 4.08 & 1,140 & & 2300 & - & 39,600 & & & 0800 & - & 5,050 \\
\hline & & & & & 2400 & - & 38,000 & & & 2400 & - & 5,180 \\
\hline \multirow[t]{6}{*}{23} & 0400 & 5.00 & 2,260 & & & & & & & & & \\
\hline & 0600 & 6.30 & 3,980 & 31 & 0700 & - & 38,000 & & 3 & 1100 & 6.97 & 5,260 \\
\hline & 0800 & 6. 50 & 4,260 & & 1300 & - & 37,200 & & & 1200 & 7. 70 & 6,280 \\
\hline & 1600 & 6. 52 & 4,290 & & 1500 & - & 36,800 & & & 1230 & 9. 10 & 8,700 \\
\hline & 2400 & 6. 55 & 4,330 & & 2400 & - & $36 ; 000$ & & & 1300 & 9.82 & 10,100 \\
\hline & & & & & & & & & & 1400 & 9. 92 & 10,300 \\
\hline \multirow[t]{5}{*}{24} & 1530 & 6.57 & 4,360 & Jan. 1 & 0700 & - & 36,000 & & & 2400 & 9.78 & 10,100 \\
\hline & 1600 & 6.00 & 3,600 & & 0900 & - & 35,600 & & & & & \\
\hline & 1700 & 4. 99 & 2,250 & & 1400 & - & 35,500 & & 4 & 1200 & 9.71 & 9,920 \\
\hline & 2400 & 4.95 & 2,190 & & 1500 & - & 40,800 & & & 2400 & 9.70 & 9,900 \\
\hline & & & & & 1700 & - & 40,400 & & & & & \\
\hline \multirow[t]{2}{*}{25} & 1200 & 4.93 & 2,160 & & 1900 & 22.4 & 51,600 & & 5 & 1200 & 9.70 & 9,900 \\
\hline & 2400 & 4. 92 & 2,150 & & $\begin{array}{l}2000 \\
2030\end{array}$ & 22.4 & 51,600 & & & 2400 & 9.60 & 9,700 \\
\hline
\end{tabular}

(110) 9-5027. Crookton Wash near Seligman, Ariz.

(Crest-stage station)

Location. --Lat $35^{\circ} 17^{\prime} 15^{\prime \prime}$, long $112^{\circ} 43^{\prime} 55^{\prime \prime}$, in SE⿺ $\frac{1}{4}$ sec. 17, T. 22 N., R, 4 W. , at U.S. Highway 66, 9 miles east of Seligman, and 15 miles west of Ashfork.

Drainage area. - - About $6 \mathrm{sq} \mathrm{mi}$.

Gage-height record.--Crest stages only. Altitude of gage is 5,700 ft (from tcpographic map).

Maxima. --November 1965 to January 1966: Discharge estimated to be less than 1 cfs. 1963 to October 1965: Discharge, $168 \mathrm{cfs}$ Aug. 1, 1964 (gage heigh $\$$. 2. $40 \mathrm{ft}$ ), from computation of flow through culvert.

Remarks. --Station has been included in this report because it is the only station in Chino Valley. Record indicates that little flow came from upper end of the valley.

(111) 9-5028. Williamson Valley Wash near Paulden, Ariz.

Location. -- Lat $34^{\circ} 52^{\prime} 00^{\prime \prime}$, long $112^{\circ} 36^{\prime} 45^{\prime \prime}$, in $\operatorname{SE} \frac{1}{4} \mathrm{SE} \frac{1}{4}$ sec. 7, T, 17 N., P. 3 W. , on left bank, 3.6 miles north of Simmons, and 8.5 miles west of Paulden.

Drainage area. $--255 \mathrm{sq} \mathrm{mi}$.

Gage-height record. - -Water-stage recorder graph except Jan. 3-6 and short periods over the peaks of Nov. 25 and Dec. 10 which were reconstructed. Datim of gage is $4,447.38 \mathrm{ft}$ above mean sea level.

Discharge record. --Stage-discharge relation defined by current-meter measurements below $500 \mathrm{cfs}$ and extended above on basis of slope-area measurement of peak flow. Stage-discharge relation affected by backwater from ice Jan. 21-30. Cischarge for period of no gage-height record estimated.

Maxima. --November 1965 to January 1966: Discharge, 3,630 cfs 0400 ho'irs Dec. 30 (gage height, $6.38 \mathrm{ft}$ ).

March to October 1965: Discharge, $820 \mathrm{cfs}$ Apr. 10, 1965 (gage height, 3. $80 \mathrm{ft}$ ). 
Mean discharge, in cubic feet per second, 1965-66, of Williamson Valley Wash near Paulden, Ariz.

\begin{tabular}{|c|c|c|c|c|c|c|c|}
\hline Day & November & December & January & Day & November & December & January \\
\hline $1 \ldots$ & 1.7 & 3.0 & 52 & $16 \ldots$ & 2.8 & 33 & 3.6 \\
\hline $2 \ldots$ & 1. 7 & 2.8 & 35 & $17 \ldots$ & 3.0 & 32 & 3.6 \\
\hline $3 \ldots$ & 1. 7 & 2. 8 & 15 & $18 \ldots$ & 3.0 & 34 & 4. 2 \\
\hline $4 \ldots$ & 1. 8 & 2. 8 & 14 & $19 \ldots$ & 3.0 & 26 & 5.8 \\
\hline $5 \ldots$ & 1.8 & 2. 8 & 14 & $20 \ldots$ & 3.0 & 22 & 5.4 \\
\hline $6 \ldots$ & 1. 8 & 2. 8 & 14 & $21 \ldots$ & 3.0 & 40 & 4 \\
\hline $7 \ldots$ & 2. 0 & 2.8 & 13 & $22 \ldots$ & 3.4 & 686 & 3 \\
\hline $8 \ldots$ & 2. 0 & 3.0 & 12 & $23 \ldots$ & 4.0 & 691 & 3 \\
\hline $9 \ldots$ & 2.0 & 14 & 10 & $24 \ldots$ & 3.4 & 85 & 3 \\
\hline $10 \ldots$ & 2. 2 & 1,400 & 9.0 & $25 \ldots$ & 530 & 43 & 2 \\
\hline $11 \ldots$ & 2. 2 & 313 & 8.8 & $26 \ldots$ & 219.0 & 26 & 2 \\
\hline $12 \ldots$ & 2. 2 & 76 & 7.2 & $27 \ldots$ & 14.0 & 22 & 2 \\
\hline $13 \ldots$. & 2.4 & 65 & 5.4 & $28 \ldots \ldots$ & 4.0 & 19 & 3 \\
\hline $14 \ldots$ & 2. 6 & 72 & 4. 4 & $29 \ldots$ & 3.2 & 156 & 3 \\
\hline $15 \ldots$ & 2.6 & 41 & 4. 2 & $30 \ldots$ & 3.0 & 1,620 & 3 \\
\hline & & & & $31 \ldots$ & $--\cdot-\cdot$ & 192 & 3.4 \\
\hline \multirow{2}{*}{\multicolumn{5}{|c|}{$\begin{array}{l}\text { Monthly mean discharge, in cubic feet per second } \ldots \\
\text { Runoff, in acre-feet } \ldots \ldots \ldots \ldots \ldots \ldots \ldots \ldots\end{array}$}} & 27.8 & 185 & 8.77 \\
\hline & & & & & 1,650 & 11,370 & 540 \\
\hline
\end{tabular}

Gage height, in feet, and discharge, in cubic feet per second, at indicated time, 1965

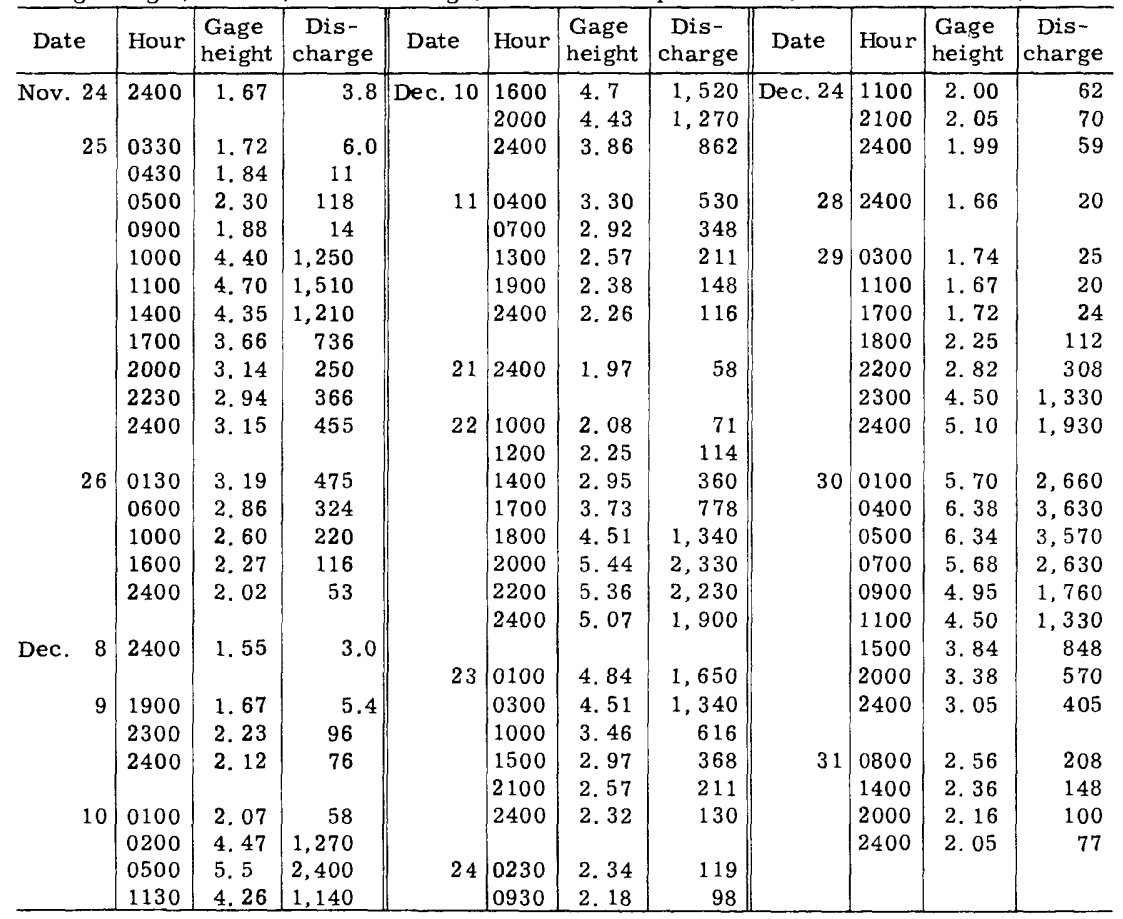


(112) 9-5030. Granite Creek near Prescott, Ariz.

(Gaging station, discontinued 1947)

Location. --Lat $34^{\circ} 34^{\prime}$, long $112^{\circ} 37^{\prime}$, in SW $\frac{1}{4}$ sec. 26, T. 14 N. , R. 2 W. , at old highway bridge, $1 \frac{1}{2}$ miles northeast of Prescott.

Drainage area. $--39 \mathrm{sq} \mathrm{mi}$.

Maxima. --November 1965 to January 1966: Discharge, about 1,500 cfs I ac. 10 or 30 (gage height, about 8.0, from floodmarks), from rating defined below 130 cfs by current-meter measurements made in 1946 and extended above on basis of contractedopening measurement at gage height $12.5 \mathrm{ft}$ made in 1963.

1932-47, 1963: Discharge, 6,660 cfs Aug. 19, 1963 (gage height, $12.5 \mathrm{ft}$, from floodmarks), from contracted-opening measurement of peak flow.

(113) 9-5037. Verde River near Paulden, Ariz.

Location. -- Lat $34^{\circ} 53^{\prime} 42^{\prime \prime}$, long $112^{\circ} 20^{\prime} 26^{\prime \prime}$, in SW $\frac{1}{4} \mathrm{SW} \frac{1}{4}$ sec. 35 , T. 18 N. , R. 1 W. , on right bank a quarter of a mile upstream from Verde Valley Ranch, $7 \frac{1}{4}$ miles east of Paulden, 8 miles upstream from Hell Canyon, $8 \frac{1}{2}$ miles downstream from Granite Creek, and $10 \frac{1}{2}$ miles downstream from Sullivan Lake.

Drainage area. $--2,530 \mathrm{sq} \mathrm{mi}$, approximately (including $373 \mathrm{sq} \mathrm{mi}$ in Aubrey Valley Playa, a closed basin).

Gage-height record. --Water-stage recorder graph except Dec. 11-17. Altitude of gage is $4,100 \mathrm{ft}$ (from topographic map).

Discharge record. --Stage-discharge relation defined by current-meter moasurements below 1,700 cfs and extended above on basis of slope-area measurements of peak flow. Discharge for period of no gage-height record estimated.

Maxima. --November 1965 to January 1966: Discharge, 6, 130 cfs 1700 hc urs Dec. 30 (gage height, $8.48 \mathrm{ft}$ ).

1963 to October 1965: Discharge, 1,270 cfs Aug. 5, 1964 (gage height, 5. $44 \mathrm{ft}$, from high-water mark).

Mean discharge, in cubic feet per second, 1965-66

\begin{tabular}{|c|c|c|c|c|c|c|c|}
\hline Day & November & December & January & Day & November & December & January \\
\hline 1 & 21 & 23 & 713 & 16 & 23 & 60 & 25 \\
\hline 2 & 21 & 23 & 229 & 17 & 23 & 46 & 25 \\
\hline 3 & 21 & 22 & 71 & 18 & 23 & 46 & 25 \\
\hline 4 & 21 & 22 & 48 & 19 & 23 & 43 & 25 \\
\hline 5 & 21 & 22 & 40 & 20 & 23 & 39 & 25 \\
\hline 6 & 21 & 22 & 36 & 21 & 23 & 37 & 25 \\
\hline $7 \ldots$ & 21 & 22 & 33 & 22 & 23 & 44 & 25 \\
\hline $8 \ldots$ & 21 & 22 & 29 & 23 & 24 & 1,460 & 25 \\
\hline $9 \ldots$ & 21 & 24 & 27 & 24 & 24 & 544 & 25 \\
\hline $10 \ldots$ & 21 & 636 & 26 & 25 & 24 & 379 & 25 \\
\hline $11 \ldots$ & 21 & 1,300 & 26 & $26 \ldots$ & 412 & 123 & 25 \\
\hline $12 \ldots$ & 22 & 500 & 25 & $27 \ldots$ & 65 & 66 & 25 \\
\hline $13 \ldots$ & 22 & 200 & 25 & 28 & 32 & 49 & 25 \\
\hline $14 \ldots$ & 22 & 150 & 25 & $29 \ldots$ & 26 & 42 & 25 \\
\hline \multirow[t]{2}{*}{$15 \ldots$} & 22 & 100 & 25 & 30 . & 24 & 1,750 & 26 \\
\hline & & & & 31 & ------ & 1,340 & 27 \\
\hline \multirow{2}{*}{\multicolumn{5}{|c|}{$\begin{array}{l}\text { Monthly mean discharge, in cubic feet per second } . \\
\text { Runoff, in acre-feet } \ldots \ldots \ldots \ldots \ldots \ldots \ldots \ldots \ldots \ldots\end{array}$}} & 37.0 & $2 £ 5$ & 57.5 \\
\hline & & & & & 2,200 & 18,160 & 3,530 \\
\hline
\end{tabular}


Gage height, in feet, and discharge, in cubic feet per second, at indicated time, 1965-66, of Verde River near Paulden, Ariz.

\begin{tabular}{|c|c|c|c|c|c|c|c|c|c|c|c|}
\hline Date & Hour & $\begin{array}{l}\text { Gage } \\
\text { height }\end{array}$ & $\begin{array}{c}\text { Dis- } \\
\text { charge }\end{array}$ & Date & Hour & $\begin{array}{c}\text { Gage } \\
\text { height }\end{array}$ & $\begin{array}{c}\text { Dis- } \\
\text { charge }\end{array}$ & Date & Hour & $\begin{array}{l}\text { Gage } \\
\text { height }\end{array}$ & $\begin{array}{l}\text { Dis- } \\
\text { charge }\end{array}$ \\
\hline \multirow[t]{19}{*}{ Nov. 25} & 2400 & 1. 63 & 24 & Dec. 21 & 2400 & 1. 85 & 37 & Dec. 30 & 1100 & 2.06 & 47 \\
\hline & & & & & & & & & 1200 & 2. 20 & 55 \\
\hline & 0100 & 2. 30 & 59 & 22 & 1000 & 1. 90 & 39 & & $: 300$ & 3.00 & 165 \\
\hline & 0200 & 4. 35 & 682 & & 1800 & 2. 13 & 50 & & 1400 & 5. 75 & 1,510 \\
\hline & 0300 & 4. 58 & 795 & & 2400 & 2. 27 & 59 & & 1500 & 7. 50 & 3,800 \\
\hline & 0400 & 4. 55 & 780 & & & & & & 1600 & 8. 38 & 5,690 \\
\hline & 0500 & 4. 45 & 730 & 23 & 0200 & 2. 32 & 62 & & 1700 & 8. 48 & 6,130 \\
\hline & 0900 & 3. 87 & 473 & & 0300 & 2. 55 & 80 & & 1800 & 8. 35 & 5,620 \\
\hline & 1200 & 3.62 & 373 & & 0400 & 3.05 & 180 & & 1900 & 8. 04 & 4,910 \\
\hline & 1700 & 3. 53 & 340 & & 0500 & 4. 95 & 980 & & 2100 & 7. 20 & 3,240 \\
\hline & 2100 & 3. 23 & 236 & & 0600 & 6. 65 & 2,440 & & 2400 & 6. 44 & 2,190 \\
\hline & 2400 & 2. 95 & 152 & & 0700 & 7. 06 & 3,020 & & & & \\
\hline & & & & & 0730 & 7. 09 & 3,060 & 31 & 0300 & 5.82 & 1,570 \\
\hline & 0300 & 2. 70 & 100 & & 0900 & 6. 98 & 2,890 & & 0700 & 5. 28 & 1,170 \\
\hline & 0600 & 2.49 & 74 & & 1500 & 5.86 & 1,590 & & 0900 & 5. 18 & 1,110 \\
\hline & 1200 & 2. 22 & 54 & & 1900 & 5. 19 & 1,110 & & 1100 & 5. 18 & 1,110 \\
\hline & 1800 & 2. 04 & 43 & & 2400 & 4. 78 & 895 & & 1300 & 5.28 & 1,170 \\
\hline & 2400 & 1. 92 & 37 & & & & & & 1530 & 5. 55 & 1,340 \\
\hline & & & & 24 & 1000 & 4. 05 & 548 & & 1800 & 5. 61 & 1,390 \\
\hline \multirow[t]{13}{*}{ Dec. } & 2400 & 1. 80 & 31 & & 1300 & 3. 77 & 433 & & 2000 & 5.60 & 1,380 \\
\hline & & & & & 2000 & 3.58 & 358 & & 2400 & 5.21 & 1,130 \\
\hline & 0300 & 1. 87 & 35 & & 2200 & 3. 65 & 385 & & & & \\
\hline & 1200 & 1. 80 & 31 & & 2400 & 3.90 & 485 & Jan. & 0300 & 4.92 & 965 \\
\hline & 1330 & 2. 05 & 44 & & & & & & 1000 & 4. 48 & 745 \\
\hline & 1400 & 2. 70 & 100 & 25 & 0200 & 3.99 & 521 & & 1500 & 4. 19 & 610 \\
\hline & 1500 & 5.50 & 1,310 & & 0300 & 4.00 & 525 & & 1900 & 3.96 & 509 \\
\hline & 1600 & 5. 88 & 1,610 & & 0600 & 3. 82 & 453 & & 2400 & 3. 77 & 433 \\
\hline & 1700 & 5. 91 & 1,640 & & 1200 & 3. 39 & 292 & & & & \\
\hline & 1800 & 5.86 & 1,590 & & 1400 & 3. 27 & 250 & 2 & 0900 & 3. 31 & 264 \\
\hline & 1900 & 5. 71 & 1,470 & & 1500 & 3. 27 & 250 & & 1300 & 2. 97 & 158 \\
\hline & 2200 & 5.73 & 1,480 & & 1600 & 3.33 & 272 & & 1700 & 2. 77 & 114 \\
\hline & & & & & 2400 & 3.20 & 225 & & 1830 & 2. 77 & 114 \\
\hline \multirow[t]{4}{*}{11} & 0200 & 6. 22 & 1,940 & & & & & & 2000 & 2. 88 & 136 \\
\hline & 0600 & - & 1,800 & 29 & 2400 & 1. 95 & 42 & & 2130 & 3.03 & 174 \\
\hline & 1200 & - & 1,200 & & & & & & 2400 & 2. 90 & 140 \\
\hline & 2400 & - & 850 & 30 & 0600 & 2. 05 & 46 & & & & \\
\hline
\end{tabular}

(114) 9-5037. 2 Hell Canyon near Williams, Ariz.

Location. --Lat $35^{\circ} 09^{\prime} 40^{\prime \prime}$, long $112^{\circ} 12^{\prime} 33^{\prime \prime}$, in NW $\frac{1}{4} \mathrm{NW} \frac{1}{4} \sec .32$, T. 21 N., R. 2 E. , in Kaibab National Forest, on right bank, 6 miles south of Williams.

Drainage area. $--14.9 \mathrm{sq} \mathrm{mi}$.

Gage-height record. --Water-stage recorder graph except Nov, 3-14, 23-28, Dec, 14 to Jan. 18. Altitude of gage is $6,750 \mathrm{ft}$ ( $\mathrm{from}$ topographic map).

Discharge record. --Stage-discharge relation defined by current-meter measurements. Discharge for periods of no gage-height record estimated.

Maxima. -- November 1965 to January 1966: Discharge, 1, 080 cfs Dec. 30 (gage height, $4.78 \mathrm{ft}$, from high-water mark in well corrected for an estimated amount of surge,

$5.4 \mathrm{ft}$, outside, from floodmarks, as result of pileup near gage).

August to October 1965: Discharge, 55 cfs Sept. 18, 1965 (gage height, 1. $80 \mathrm{ft}$ ). 
Mean discharge, in cubic feet per second, 1965-66, of Hell Canyon near Williams, Ariz.

\begin{tabular}{|c|c|c|c|c|c|c|c|}
\hline Day & November & December & January & Day & November & Deceraber & January \\
\hline $1 \ldots$ & 0 & 3.2 & 10 & $16 \ldots$ & 0 & 2.5 & 2 \\
\hline $2 \ldots$ & 0 & 2.4 & 8 & 17 & 0 & 2 & 2 \\
\hline$\ldots$ & 0 & 1.5 & 6 & $18 \ldots$ & 0 & 1.5 & 2 \\
\hline $4 \ldots$ & 0 & 1. 1 & 5 & $19 \ldots$ & 0 & 1 & 2 \\
\hline$\ldots$ & 0 & 1.0 & 4 & $20 \ldots$ & 0 & 1 & 1.5 \\
\hline $6 \ldots$ & 0 & .8 & 3 & $21 \ldots$ & 0 & 1 & 1. 5 \\
\hline . & 0 & .7 & 2 & 22 & 0 & 6 & 1.5 \\
\hline 8 & 0 & .6 & 2 & 23 & 150 & 25 & 1.5 \\
\hline 9 & 0 & 74 & 2 & 24 & 50 & 10 & 1.5 \\
\hline $10 \ldots$ & 0 & 112 & 2 & $25 \ldots$ & 400 & 3 & 1.5 \\
\hline $11 \ldots$ & 0 & 50 & 2 & $26 \ldots$ & 41 & 2 & 1.5 \\
\hline $12 \ldots$ & 0 & 28 & 2 & $27 \ldots$ & 10 & 1 & 1.5 \\
\hline $13 \ldots$ & 0 & 12 & 2 & $28 \ldots$ & 5.0 & 1 & 1.5 \\
\hline $14 \ldots$ & 0 & 6 & 2 & $29 \ldots$ & 3.6 & 3 & 1 \\
\hline \multirow[t]{2}{*}{$15 \ldots$} & 0 & 4 & 2 & $30 \ldots$ & 3.4 & 200 & 1 \\
\hline & & & & 31 & $\ldots-\ldots$ & 50 & 1 \\
\hline \multirow{2}{*}{\multicolumn{5}{|c|}{$\begin{array}{l}\text { Monthly mean discharge, in cubic feet per second.. } \\
\text { Runoff, in acre-feet } \ldots \ldots \ldots \ldots \ldots \ldots\end{array}$}} & 22.1 & 19.6 & 2.53 \\
\hline & & & & & 1,320 & 1,200 & 156 \\
\hline
\end{tabular}

(115) 9-5038. Volunteer Wash near Bellemont, Ariz.

Location. -- Lat $35^{\circ} 09^{\prime} 00^{\prime \prime}$, long $111^{\circ} 53^{\prime} 50^{\prime \prime}$, in $\mathrm{SE}_{\frac{1}{4}} \mathrm{SE} \frac{1}{4}^{\prime}$ sec. 31, T. 21 N. , R. 5 E. , in Kaibab National Forest, on right bank, 7 miles southwest of Bellemort, and 14 miles west of Flagstaff.

Drainage area. $--142 \mathrm{sq} \mathrm{mi}$.

Gage-height record. - -Water-stage recorder graph 1130 hours Nov. 25 to 2200 hours Nov. 26, 2000 hours Dec. 9 to 2000 hours Dec. 13, and 8 hours over peak of Dec. 30 . Altitude of gage is $6,820 \mathrm{ft}$ (from topographic map).

Discharge record. --Stage-discharge relation defined by current-meter measurements below $80 \mathrm{cfs}$ and extended above on basis of slope-area measurement of peak flow of Dec. 7,1966 , at gage height $5.73 \mathrm{ft}$. Discharge for periods of no gage-height record estimated.

Maxima. -- November 1965 to January 1966: Discharge, 578 cfs about 0270 hours Dec. 30 (gage height, $4.55 \mathrm{ft}$ ).

July to October 1965: Discharge, 92 cfs Sept. 19, 1965 (gage height, 2. 85 ft).

Mean discharge, in cubic feet per second, 1965-66

\begin{tabular}{|c|c|c|c|c|c|c|c|}
\hline Day & November & December & January & Day & November & December & January \\
\hline 1 & 0 & 0 & 20 & 16 & 0 & 0.2 & 0 \\
\hline . & 0 & 0 & 2.0 & $17 \ldots$ & 0 & 0 & 0 \\
\hline 3 & 0 & 0 & 0 & $18 \ldots$ & 0 & 0 & 0 \\
\hline $4 \ldots$ & 0 & 0 & 0 & $19 \ldots$ & 0 & 0 & 0 \\
\hline $5 \ldots$ & 0 & 0 & 0 & 20 & 0 & 0 & 0 \\
\hline $6 \ldots$ & 0 & 0 & 0 & $21 \ldots$ & 0 & 0 & 0 \\
\hline 7 & 0 & 0 & 0 & $22 \ldots$ & 0 & 0 & 0 \\
\hline 8 & 0 & 0 & 0 & 23. & 0 & 0 & 0 \\
\hline $9 \ldots$ & 0 & 27 & 0 & $24 \ldots$ & 0 & 0 & 0 \\
\hline $10 \ldots$ & 0 & 175 & 0 & $25 \ldots$ & 146 & 0 & 0 \\
\hline $11 \ldots$ & 0 & 112 & 0 & $26 \ldots$ & 43 & 0 & 0 \\
\hline 12 & 0 & 92 & 0 & $27 \ldots$ & 4.0 & 0 & 0 \\
\hline 13 & 0 & 45 & 0 & $28 \ldots$ & .2 & 0 & 0 \\
\hline 14 & 0 & 20 & 0 & $29 \ldots$ & 0 & .3 & 0 \\
\hline \multirow[t]{2}{*}{$15 \ldots$} & 0 & 3.0 & 0 & $30 \ldots$ & 0 & 300 & 0 \\
\hline & & & & $31 \ldots$ & $\ldots \ldots$ & 100 & 0 \\
\hline \multirow{2}{*}{\multicolumn{5}{|c|}{$\begin{array}{l}\text { Monthly mean discharge, in cubic feet per second } . \\
\text { Runoff, in acre-feet }\end{array}$}} & 6.44 & 28.2 & 0.71 \\
\hline & & & & & 383 & 1,730 & 44 \\
\hline
\end{tabular}


(116) 9-5040. Verde River near Clarkdale, Ariz.

Location. --Lat $34^{\circ} 51^{\prime} 05^{\prime \prime}$, long $112^{\circ} 03^{\prime} 55^{\prime \prime}$, in $\mathrm{SE} \frac{1}{4} \mathrm{NW} \frac{1}{4}$ sec. 17, T. $17 \mathrm{~N} .$, R. 2 E., on left bank 1,7 miles downstream of Sycamore Canyon, and 5,6 miles north of Clarkdale.

Drainage area. - -3, $520 \mathrm{sq} \mathrm{mi,} \mathrm{approximately} \mathrm{(including} 373 \mathrm{sq} \mathrm{mi}$ in Aubrey Valley Playa, a closed basin).

Gage-height record. - Water-stage recorder graph. Altitude of gage is 3, $520 \mathrm{ft}$ (from topographic map).

Discharge record. --Stage-discharge relation defined by current-meter measurements.

Maxima. --November 1965 to January 1966: Discharge, 12, 900 cfs 0700 hours Dec. 10 (gage height, $12.30 \mathrm{ft}$ ).

1915-21, April to October 1965: Discharge, 50,600 cfs Feb. 21, 1920 (gage height, 19. $1 \mathrm{ft}$, at site $2 \frac{1}{2}$ miles downstream at different datum).

Mean discharge, in cubic feet per second, 1965-66

\begin{tabular}{|c|c|c|c|c|c|c|c|}
\hline Day & November & December & January & Day & November & December & January \\
\hline 1 & 74 & 116 & 1,730 & 16 & 73 & 337 & 82 \\
\hline$\ldots$ & 74 & 106 & 675 & $17 \ldots$ & 74 & 295 & 82 \\
\hline$\ldots$ & 73 & 101 & 339 & $18 \ldots$ & 74 & 273 & 82 \\
\hline$\ldots$ & 73 & 99 & 227 & 19 & 74 & 235 & 82 \\
\hline 5 & 73 & 97 & 171 & 20 & 74 & 200 & 82 \\
\hline 6 & 73 & 96 & 143 & 21 & 74 & 197 & 82 \\
\hline $7 \ldots$ & 73 & 96 & 122 & $22 \ldots$ & 75 & 536 & 81 \\
\hline $8 \ldots$ & 73 & 94 & 109 & $23 \ldots$ & 1,370 & 2,880 & 80 \\
\hline$\ldots$ & 73 & 101 & 103 & 24. & 777 & 1,180 & 80 \\
\hline 10 & 73 & 5,880 & 101 & $25 \ldots$ & 4,130 & 686 & 79 \\
\hline 11 & 73 & 3,010 & 100 & 26 & 1,740 & 456 & 79 \\
\hline 12 & 73 & 1,380 & 97 & 27 & 618 & 328 & 79 \\
\hline 13 & 73 & 716 & 96 & 28. & 278 & 265 & 77 \\
\hline 14 & 73 & 556 & 87 & $29 \ldots$ & 182 & 232 & 77 \\
\hline \multirow[t]{2}{*}{$15 \ldots$} & 73 & 417 & 85 & $30 \ldots$ & 139 & 6,950 & 77 \\
\hline & & & & 31. & $\ldots \ldots$ & 4,080 & 80 \\
\hline \multirow{2}{*}{\multicolumn{5}{|c|}{ Monthly mean discharge, in cubic feet per second. . }} & 362 & 1,030 & 176 \\
\hline & & & & & 21,520 & 63,460 & 10,840 \\
\hline
\end{tabular}


Gage height, in feet, and discharge, in cubic feet per second, at indicated time, 1965-66, of Verde River near Clarkdale, Ariz.

\begin{tabular}{|c|c|c|c|c|c|c|c|c|c|c|c|c|}
\hline Date & Hour & $\begin{array}{l}\text { Gage } \\
\text { height }\end{array}$ & $\begin{array}{c}\text { Dis- } \\
\text { charge }\end{array}$ & Date & Hour & $\begin{array}{l}\text { Gage } \\
\text { height }\end{array}$ & $\begin{array}{c}\text { Dis- } \\
\text { charge }\end{array}$ & Date & & Hour & $\begin{array}{l}\text { Gage } \\
\text { height }\end{array}$ & $\begin{array}{c}\text { Dis- } \\
\text { charge }\end{array}$ \\
\hline \multirow[t]{11}{*}{ Nov。 22} & 2400 & 1.09 & 77 & Dec。10 & 0050 & 67 & 116 & Dec。 & & 1030 & 5.10 & 1,810 \\
\hline & & & & & 00 & & 690 & & & 1200 & & 20 \\
\hline & 3| $\begin{array}{l}1250 \\
1300\end{array}$ & $\begin{array}{l}1.12 \\
5.05\end{array}$ & \begin{tabular}{r||}
81 \\
760
\end{tabular} & & 0200 & 5.8 & 2,580 & & & $\begin{array}{l}1300 \\
1400\end{array}$ & $\begin{array}{l}6.80 \\
6.90\end{array}$ & $\begin{array}{l}3,680 \\
3,810\end{array}$ \\
\hline & 1400 & 6.75 & $\begin{array}{l}1,620 \\
3,620\end{array}$ & & 0400 & $\begin{array}{l}7.1 \\
7.95\end{array}$ & $\begin{array}{l}4,070 \\
5,230\end{array}$ & & & 1600 & & $\begin{array}{l}0,010 \\
3,420\end{array}$ \\
\hline & 1500 & 6.85 & 3,740 & & 0440 & 7.90 90 & 5,300 & & & 2000 & 5.90 & 2,580 \\
\hline & 1600 & 6.64 & 3,470 & & 0530 & 10.05 & 8,490 & & & 2400 & 5.35 & 2,040 \\
\hline & 1630 & 6.40 & 3,180 & & 0600 & 11.50 & 11,200 & & & & & \\
\hline & 1715 & 7.03 & 3,980 & & 0700 & 12.30 & 12,900 & & 24 & 0600 & 4.75 & 1,510 \\
\hline & 1900 & 6.40 & 3,180 & & 0800 & 11.50 & 11,200 & & & 1200 & 10 & 1,030 \\
\hline & 2200 & 5.10 & 1,810 & & 0900 & 10.85 & 9,930 & & & 1800 & 75 & 820 \\
\hline & 2400 & 4.50 & 1,310 & & 1100 & 10.05 & 8,490 & & & 2400 & 3.60 & 740 \\
\hline \multirow{6}{*}{24} & 0130 & 4. 80 & 1,550 & & $\begin{array}{l}1400 \\
1700\end{array}$ & $\begin{array}{l}8.30 \\
7.15\end{array}$ & $\begin{array}{l}5,750 \\
4\end{array}$ & & 25 & 0500 & 3.63 & 755 \\
\hline & 0400 & 4.25 & 1,140 & & & 6.75 & 3,620 & & & 0700 & 3 & $\begin{array}{l}755 \\
808\end{array}$ \\
\hline & 0700 & 3.87 & 892 & & 2000 & 6. & 3,480 & & & 0900 & 73 & 808 \\
\hline & 1500 & 3.08 & 497 & & & 6. & 3,620 & & & 1700 & & 05 \\
\hline & 1900 & 2.73 & 365 & & 2300 & 7.55 & 4,670 & & & 2400 & 3.15 & 525 \\
\hline & 2400 & 2.40 & 270 & & 2400 & 7.48 & 4,570 & & & & & \\
\hline \multirow[t]{17}{*}{25} & 0130 & & & & & & & & 26 & $\begin{array}{l}0400 \\
1000\end{array}$ & 17 & 533 \\
\hline & 0230 & & $\begin{array}{l}252 \\
288\end{array}$ & 11 & & & $\begin{array}{l}3,810 \\
3\end{array}$ & & & & & 89 \\
\hline & 0830 & 2.25 & 232 & & 1300 & 6.18 & 2,920 & & & 2400 & 2.71 & 358 \\
\hline & 0900 & 6.80 & 3,680 & & 1700 & 5. & 2,180 & & & & & \\
\hline & 1000 & 8.10 & 5,450 & & 100 & 5.25 & 1,940 & & 29 & 2400 & 2.30 & 245 \\
\hline & 1100 & 8.50 & 6,050 & & 2400 & 5.22 & 1,920 & & & & & \\
\hline & 1200 & 8.50 & 6,050 & & & & & & 30 & 0300 & 2.30 & 245 \\
\hline & 1300 & 9.60 & 7,760 & 12 & 0200 & 5.20 & 1,900 & & & 0550 & 2.42 & 72 \\
\hline & 1430 & 10.70 & 9660 & & 1200 & 455 & 1,350 & & & 0600 & 5.60 & 2,280 \\
\hline & 1530 & 11.50 & 11,200 & & 1800 & 4.20 & 1,100 & & & 0700 & 7.50 & 4,600 \\
\hline & 1600 & 10.75 & 9,750 & & 2400 & 3. 91 & 916 & & & 0800 & 10.45 & 9,210 \\
\hline & 1700 & 10.05 & 8,490 & & & & & & & & & \\
\hline & 1800 & 9. & 6,960 & 13 & 0600 & 3.' & 820 & & & 0930 & 1.45 & 11,100 \\
\hline & & 8. & 5,980 & & 0800 & 3. & 802 & & & & & 11,600 \\
\hline & 2100 & 7.55 & 4,670 & & 1800 & 3. & 595 & & & & $\therefore$ & 10,800 \\
\hline & 2400 & 6.56 & 3,370 & & 2100 & 3.22 & 554 & & & & & 8,490 \\
\hline & & & & & 2400 & 3.19 & 541 & & & 1800 & & 7,440 \\
\hline \multirow[t]{9}{*}{26} & 30500 & 38 & 2 & & & & & & & 0 & 0.05 & 8,490 \\
\hline & 0800 & 5. & 1,760 & 14 & 0200 & 3.1 & 537 & & & 2100 & 1.15 & 10,500 \\
\hline & 0 & 4. & 1, & & & & 568 & & & 2400 & 0.30 & 8,940 \\
\hline & 10 & 5. & 60 & & 00 & 3. & 650 & & & & & \\
\hline & & & & & & & 650 & & 31 & 0 & & 8,490 \\
\hline & 1300 & 5. & 1,720 & & 1800 & 3. & 501 & & & & & \\
\hline & & 4. & & & 2400 & 3. & 469 & & & & & \\
\hline & & 4. & 1,080 & & & & & & & & & \\
\hline & 0 & & & 21 & 2400 & 2.14 & 207 & & & $\begin{array}{l}1400 \\
1600\end{array}$ & $\begin{array}{l}5.95 \\
5.85\end{array}$ & $\begin{array}{l}2,640 \\
2,530\end{array}$ \\
\hline \multirow[t]{5}{*}{2} & 0400 & & 82 & 22 & 0 & & 222 & & & 1800 & 5 & 2,640 \\
\hline & & & & & & & 248 & & & & & \\
\hline & 1800 & 3. & 4 & & 1730 & 2. & 292 & & & 2400 & 5.95 & 2,640 \\
\hline & 2400 & & 36 & & & & 465 & & & & & \\
\hline & & & & & & & 545 & Jan. & 1 & & & \\
\hline & 30600 & 2. & 36 & & 2100 & 5. & 760 & & & & & 1,100 \\
\hline & 1200 & & & & 2300 & 5. & 2,380 & & & 2300 & 4.29 & 1,160 \\
\hline & & 2.40 & 270 & & 2400 & 0 & 3,000 & & & 2400 & 4.20 & 1,100 \\
\hline & 2400 & & 220 & & & & & & ? & $n_{2}$ & & \\
\hline \multirow{4}{*}{ ec. } & 312400 & & 94 & & & & 380 & & & 1600 & & 37 \\
\hline & $\angle 400$ & & $y_{4}$ & & & & 3,320 & & & 2400 & 2.85 & 404 \\
\hline & 120 & & 100 & & 0600 & 6. & 2,800 & & & & & \\
\hline & & & 10 & & 0800 & 5. & 280 & & & & & \\
\hline
\end{tabular}


(117) 9-5041. Hull Canyon near Jerome, Ariz.

\section{(Crest-stage station)}

Location. - - Lat $34^{\circ} 44^{\prime} 20^{\prime \prime}$, long $112^{\circ} 08^{\prime} 35^{\prime \prime}$, NW $\frac{1}{4}$ sec. 28, T. 16 N., R. 2 E., at U. S. Highway $89 \mathrm{~A}, 2$ miles west of Jerome.

Drainage area. $-0.91 \mathrm{sq} \mathrm{mi}$.

Gage-height record. --Crest stages only. Datum of gage is 6, $010 \mathrm{ft}$ above mean sea level (from Arizona Highway Department bench mark).

Discharge record. --Stage-discharge relation defined by computations of flow through culvert and estimates of low flows.

Maxima. --November 1965 to January 1966: Discharge, $3 \mathrm{cfs}$, date unknown (gage height, $6.33 \mathrm{ft}$ ).

1963 to October 1965: Discharge, about $25 \mathrm{cfs}$ Aug. 10, 1964 (gage height, 7.23 ft).

High-water lines in culvert indicate a peak of about $500 \mathrm{cfs}$ and a much $\mathrm{l}$ 'gher peak (discharge not determined).

(118) 9-5044. Munds Canyon tributary near Sedona, Ariz.

(Crest-stage station)

Location. -- Lat $34^{\circ} 55^{\prime} 10^{\prime \prime}$, long $111^{\circ} 38^{\prime} 50^{\prime \prime}$, in NW $\frac{1}{4} \sec .27$, T. 18 N., R. 7 E., at State Highway 79,7 miles northeast of Sedona.

Drainage area. $--1.6 \mathrm{sq} \mathrm{mi}$, approximately.

Gage-height record. --Crest stages only. Datum of gage is 6,486 ft above mean sea level (from Arizona Highway Department bench mark).

Discharge record. --Stage-discharge relation defined by computations of flow through culvert.

Maxima. --November 1965 to January 1966: Discharge, 222 cfs Nov. 25 (gage height, $6.91 \mathrm{ft}$ ).

1963 to October 1965: Discharge, 222 cfs Sept. 3, 1965 (gage height, 6. $91 \mathrm{ft}$ ). 
(119) 9-5045, Oak Creek near Cornville, Ariz.

Location. -- Lat $34^{\circ} 46^{\prime} 00^{\prime \prime}$, long $111^{\circ} 53^{\prime} 30^{\prime \prime}$, in SE $\frac{1}{4}$ sec. 23 , T. 16 N., R. 4 E., near left bank on downstream side of county highway bridge, 0.2 mile upstream from $\mathrm{Page}$ Springs, 4 miles northeast of Cornville, and 15 miles upstream from $m$ outh.

Drainage area. $--357 \mathrm{sq} \mathrm{mi}$.

Gage-height record. --Water-stage recorder graph except Jan. 14-21. Altitude of gage is $3,470 \mathrm{ft}$ (from topographic map).

Discharge record. --Stage-discharge relation defined by current-meter measurements below 7,700 cfs and extended above on basis of slope-area measurement of peak discharge. Discharge for period of no gage-height record estimated.

Maxima. -- November 1965 to January 1966: Discharge, 17,600 cfs 1400 hours Nov. 25 (gage height, $15.18 \mathrm{ft}$ in well, $16.89 \mathrm{ft}$ on left bank, from floodmarks).

1939 to October 1965: Discharge, 17, $200 \mathrm{cfs}$ Dec. 30, 1951 (gage height, 14. $5 \mathrm{ft}$ in gage well, $16.9 \mathrm{ft}$ on bank from floodmarks).

Maximum flood known since at least 1885 occurred in March 1938 (stage at upstream side of bridge, $23 \mathrm{ft}$, from floodmarks).

Mean discharge, in cubic feet per second, 1965-66

\begin{tabular}{|c|c|c|c|c|c|c|c|}
\hline Day & November & December & January & Day & November & Decembor & January \\
\hline 1 & 30 & 65 & 808 & 16 & 34 & 152 & 50 \\
\hline 2 & 29 & 57 & 414 & 17 & 37 & 136 & 50 \\
\hline 3 & 30 & 52 & 261 & 18 & 39 & 109 & 50 \\
\hline 4 & 31 & 48 & 192 & 19. & 36 & 83 & 45 \\
\hline 5 & 31 & 45 & 140 & $20 \ldots$ & 35 & 76 & 45 \\
\hline 6 & 31 & 44 & 120 & $21 \ldots$ & 35 & 78 & 45 \\
\hline . & 32 & 43 & 99 & $22 \ldots$ & 36 & 384 & 45 \\
\hline $8 \ldots$ & 32 & 42 & 96 & $23 \ldots$ & 3,170 & 1,207 & 45 \\
\hline 9 & 32 & 47 & 91 & $24 \ldots$ & 1,490 & 522 & 45 \\
\hline 10 & 33 & 2,300 & 86 & $25 \ldots$ & 6,160 & 357 & 44 \\
\hline 11 & 32 & 1,030 & 73 & 26 & 1,100 & 240 & 43 \\
\hline 12 & 32 & 643 & 75 & 27 & 436 & 165 & 43 \\
\hline 13 & 32 & 362 & 58 & 28 & 205 & 116 & 42 \\
\hline 14 & 32 & 251 & 55 & 29 & 115 & 110 & 42 \\
\hline \multirow[t]{2}{*}{$15 \ldots$} & 32 & 215 & 55 & 30 & 89 & $5,92 ?$ & 42 \\
\hline & & & & 31. & $\ldots-\cdots$ & 1,730 & 43 \\
\hline \multirow{2}{*}{\multicolumn{5}{|c|}{ Monthly mean discharge, in cubic feet per second. }} & 450 & 536 & 108 \\
\hline & & & & & 26,750 & 32,970 & 6,630 \\
\hline
\end{tabular}


Gage height, in feet, and discharge, in cubic feet per second, at indicated time, 1965-66, of Oak Creek near Cornville, Ariz.

\begin{tabular}{|c|c|c|c|c|c|c|c|c|c|c|c|}
\hline Date & Hour & $\begin{array}{l}\text { Gage } \\
\text { height }\end{array}$ & $\begin{array}{c}\text { Dis- } \\
\text { charge }\end{array}$ & Date & Hour & $\begin{array}{l}\text { Gage } \\
\text { height }\end{array}$ & $\begin{array}{c}\text { Dis- } \\
\text { charge }\end{array}$ & Date & Hour & $\begin{array}{c}\text { Gage } \\
\text { height }\end{array}$ & $\begin{array}{c}\text { Dis- } \\
\text { charge }\end{array}$ \\
\hline Nov。 22 & 2400 & 3.29 & 40 & Nov. 2 & $7 \begin{array}{l}1200 \\
2400\end{array}$ & $\begin{array}{l}4.52 \\
4.18\end{array}$ & $\begin{array}{l}423 \\
303\end{array}$ & Dec. 24 & $\begin{array}{l}1800 \\
2400\end{array}$ & $\begin{array}{l}4.55 \\
4.68\end{array}$ & $\begin{array}{l}415 \\
467\end{array}$ \\
\hline \multirow[t]{13}{*}{23} & 0900 & 3.38 & 47 & & & & & & & & \\
\hline & 0930 & 5.15 & 695 & Dec. & 82400 & 3.02 & 41 & 28 & 2400 & 3.62 & 89 \\
\hline & 1030 & 7.02 & 2,060 & & & & & & & & \\
\hline & 1130 & 8.88 & 4,060 & & \begin{tabular}{l|l}
9 & 1700
\end{tabular} & 3.21 & 56 & 29 & 0300 & 3.69 & 109 \\
\hline & 1230 & 9.28 & 4,540 & & 2400 & 3.34 & 71 & & 2100 & 3.72 & 115 \\
\hline & 1330 & 11,32 & 8,130 & & & & & & 2400 & 4.06 & 205 \\
\hline & $\mathrm{a} 1430$ & 11.59 & 8,760 & 1 & 00100 & 5.35 & 820 & & & & \\
\hline & 1530 & 11.47 & 8,360 & & 0300 & 7.02 & 2,020 & 30 & 0100 & 4.40 & 345 \\
\hline & 1630 & 10.91 & 7,520 & & 0500 & 8.35 & 3,400 & & 0200 & 5.25 & 770 \\
\hline & 1800 & 10.27 & 6,260 & & 0730 & 9.73 & 5,200 & & 0300 & 8.27 & 3,170 \\
\hline & 2100 & 8.72 & 3,860 & & 0800 & 9.00 & 4,260 & & 0400 & 8.73 & 3,880 \\
\hline & 2400 & 8.05 & 3,050 & & 1100 & 7.50 & 2,440 & & 0600 & 10.84 & 7,260 \\
\hline & & & & & 1700 & 6.50 & 1,580 & & 0700 & 12.03 & 9,790 \\
\hline \multirow[t]{5}{*}{24} & 0600 & 7.15 & 2,160 & & 2200 & 6.50 & 1,580 & & 0800 & 13.42 & 13,000 \\
\hline & 1200 & 6.01 & 1,260 & & 2400 & 6.25 & 1,400 & & 1100 & 12,03 & 9,790 \\
\hline & 1800 & 5.31 & 825 & & & & & & 1300 & 10.79 & 7,580 \\
\hline & 2400 & 4. 99 & 643 & 1 & 10400 & 6.20 & 1,370 & & 1400 & 10.53 & 6,800 \\
\hline & & & & & 1900 & 5.25 & 795 & & 1700 & 9.58 & 5,020 \\
\hline \multirow[t]{17}{*}{25} & 0100 & 5.27 & 805 & & 2400 & 5.33 & 835 & & 2000 & 8.97 & 4,160 \\
\hline & 0200 & 5.97 & 1,230 & & & & & & 2400 & 8.18 & 3,180 \\
\hline & 0300 & 7.02 & 2,060 & 1 & 2) 1200 & 4. 97 & 634 & & & & \\
\hline & 0600 & 8.12 & 3,120 & & 2400 & 4.57 & 443 & 31 & 0600 & 7.16 & 2,130 \\
\hline & 0800 & 8.42 & 3,420 & & & & & & 1200 & 6.40 & 1,510 \\
\hline & 0900 & 9.13 & 4,380 & 2 & 1) 2400 & 3.42 & 73 & & 1800 & 6,12 & 1,290 \\
\hline & 1000 & 10.28 & 6,260 & & & & & & 2400 & 5.85 & 1,130 \\
\hline & 1100 & 11.75 & 9,050 & 2 & 20600 & 3.50 & 86 & & & & \\
\hline & 1200 & 12. 99 & 11,700 & & 1200 & 3.70 & 130 & Jan. 1 & 1200 & 5.30 & 795 \\
\hline & 1300 & 14.42 & 15,500 & & 1500 & 4.00 & 215 & & 2400 & 4.98 & 607 \\
\hline & 1400 & 15.18 & 17,600 & & 1700 & 4.02 & 221 & & & & \\
\hline & 1500 & 14.42 & 15,500 & & 2000 & 5.50 & 920 & 2 & 1200 & 4.47 & 370 \\
\hline & 1700 & 11.73 & 9,030 & & 2100 & 6.30 & 1,400 & & 1800 & 4.32 & 317 \\
\hline & 2000 & 8.92 & 4,100 & & 2400 & 6.63 & 1,680 & & 2400 & 4.42 & 334 \\
\hline & 2200 & 8.00 & 3,000 & & & & & & & & \\
\hline & 2400 & 7.22 & 2,220 & 2 & 3) 0200 & 6.91 & 1,930 & 3 & 0300 & 4.45 & 345 \\
\hline & & & & & 0600 & 6.42 & 1,520 & & 1900 & 4.05 & 202 \\
\hline \multirow[t]{4}{*}{26} & 0600 & 6.23 & 1,370 & & 1200 & 5.78 & 1,060 & & 2400 & 4.07 & 208 \\
\hline & 1200 & 5.60 & 970 & & 1800 & 5.50 & 920 & & & & \\
\hline & 1800 & 5.22 & 755 & & 2400 & 5.25 & 745 & & & & \\
\hline & 2400 & 5.03 & 661 & & & & & & & & \\
\hline
\end{tabular}

a Corrected from hour published in annual report. 
(120) 9-5052. Wet Beaver Creek near Rimrock, Ariz.

Location. - - Lat $34^{\circ} 40^{\prime} 25^{\prime \prime}$, long $111^{\circ} 39^{\prime} 55^{\prime \prime}$, in center of $\mathrm{SE} \frac{1}{4} \mathrm{sec} .24, \mathrm{~T} .15 \mathrm{~N}$, , R. $6 \mathrm{E}$. (unsurveyed), in Coconino National Forest, on right bank, 5 miles northeast of Rimrock, and $5 \frac{3}{4}$ miles upstream from Red Tank Draw.

Drainage area. $--95.2 \mathrm{sq} \mathrm{mi}$.

Gage-height record. - - Water-stage recorder graph Nov. 1-9; digital recorder tape thereafter. Altitude of gage is $3,680 \mathrm{ft}$ (from topographic map).

Discharge record. --Stage-discharge relation defined by current-meter measurements.

Maxima. --November 1965 to January 1966: Discharge, 6, 150 cfs 1330 hours Nov. 25 (gage height, $11.62 \mathrm{ft}$ ).

1961 to October 1965: Discharge, 6,100 cfs Jan. 6, 1965 (gage height, 11. $58 \mathrm{ft}$ ).

Mean discharge, in cubic feet per second, 1965-66

\begin{tabular}{|c|c|c|c|c|c|c|c|}
\hline Day & November & December & January & Day & November & Decemk er & January \\
\hline 1 & 7.1 & 10 & 192 & 16 . & 7.4 & E2 & 10 \\
\hline . & 7. 1 & 9.2 & 87 & $17 \ldots$ & 7.8 & E7 & 9.5 \\
\hline . & 7. 1 & 8.3 & 57 & 18 & 7.6 & 48 & 9. 2 \\
\hline$\ldots$ & 7. 1 & 7.8 & 36 & 19 & 7.6 & $\varepsilon 3$ & 9.0 \\
\hline .. & 7. 3 & 7.6 & 26 & $20 \ldots$ & 7.6 & $£ 0$ & 8. 7 \\
\hline 6 & 7.3 & 7. 4 & 22 & $21 \ldots$ & 7.6 & 28 & 8.7 \\
\hline$\ldots$ & 7. 3 & 7. 4 & 20 & $22 \ldots$ & 8.0 & 1,110 & 8. 7 \\
\hline$\ldots$ & 7. 3 & 7. 3 & 20 & $23 \ldots$ & 531 & $5 € 4$ & 8.7 \\
\hline 9 & 7. 3 & 8. 2 & 21 & $24 \ldots$ & 211 & 159 & 8. 7 \\
\hline $10 \ldots$ & 7. 3 & 1,260 & 22 & $25 \ldots$ & 2,520 & 121 & 8. 7 \\
\hline $11 \ldots$ & 7. 3 & 367 & 23 & $26 \ldots$ & 270 & 88 & 8. 7 \\
\hline $12 \ldots$ & 7.4 & 149 & 24 & 27 & 82 & 64 & 8. 7 \\
\hline $13 \ldots$ & 7.4 & 82 & 18 & $28 \ldots$ & 34 & 55 & 8. 4 \\
\hline 14 & 7. 4 & 46 & 14 & 29 & 18 & 171 & 8.4 \\
\hline \multirow[t]{2}{*}{$15 \ldots$} & 7.4 & 68 & 12 & $30 \ldots$ & 13 & 2,460 & 8. 4 \\
\hline & & & & $31 \ldots$ & $\ldots \ldots$ & 536 & 8.3 \\
\hline \multirow{2}{*}{\multicolumn{5}{|c|}{$\begin{array}{l}\text { Monthly mean discharge, in cubic feet per second. } \\
\text { Runoff, in acre-feet } \ldots \ldots \ldots \ldots \ldots \ldots \ldots\end{array}$}} & 128 & 246 & 23.7 \\
\hline & & & & & 7.620 & 15,140 & 1,460 \\
\hline
\end{tabular}


Gage height, in feet, and discharge, in cubic feet per second, at indicated time, 1965, of Wet Beaver Creek near Rimrock, Ariz.

\begin{tabular}{|c|c|c|c|c|c|c|c|c|c|c|c|}
\hline Date & Hour & $\begin{array}{l}\text { Gage } \\
\text { height }\end{array}$ & $\begin{array}{c}\text { Dis- } \\
\text { charge }\end{array}$ & Date & Hour & $\begin{array}{c}\text { Gage } \\
\text { height }\end{array}$ & $\begin{array}{c}\text { Dis- } \\
\text { charge }\end{array}$ & Date & Hour & $\begin{array}{l}\text { Gage } \\
\text { height }\end{array}$ & $\begin{array}{c}\text { Dis- } \\
\text { charge }\end{array}$ \\
\hline \multirow[t]{2}{*}{ Nov. 22} & 2400 & 3. 18 & 9.0 & Nov. 26 & 0600 & 5.48 & 354 & Dec. 22 & 1830 & $9 . \overline{67}$ & 3,350 \\
\hline & & & & & 1200 & 4. 89 & 198 & & 2000 & 9.10 & 2,750 \\
\hline \multirow[t]{12}{*}{23} & 0200 & 3.21 & 9.8 & & 1800 & 4. 52 & 128 & & 2200 & 8. 12 & 1,870 \\
\hline & 1100 & 3.52 & 22 & & 2400 & 4. 51 & 126 & & 2400 & 7. 94 & 1,720 \\
\hline & 1200 & 4.91 & 202 & & & & & & & & \\
\hline & 1400 & 4. 31 & 99 & 27 & 1200 & 4. 19 & 84 & 23 & 0400 & 6. 60 & 820 \\
\hline & 1500 & 8. 39 & 1,800 & & 2400 & 3.81 & 41 & & 0800 & 5.81 & 468 \\
\hline & 1600 & 8. 56 & 1,930 & & & & & & 1200 & 5. 63 & 406 \\
\hline & 1700 & 8. 48 & 1,870 & Dec. 9 & 2400 & 3.21 & 9.8 & & 1600 & 5. 74 & 444 \\
\hline & 1800 & 8. 09 & 1,590 & & & & & & 2000 & 5. 41 & 333 \\
\hline & 2000 & 7. 22 & 1,040 & 10 & 0200 & 3.60 & 27 & & 2400 & 5.09 & 258 \\
\hline & 2200 & 6. 51 & 729 & & 0300 & 6. 26 & 650 & & & & \\
\hline & 2400 & 6.01 & 530 & & 0400 & 8. 21 & 1,940 & 24 & 1200 & 4. 40 & 111 \\
\hline & & & & & 0430 & 8.53 & 2,210 & & 2400 & 4. 74 & 163 \\
\hline \multirow[t]{10}{*}{24} & 0400 & 5.28 & 262 & & 0600 & 7.64 & 1,490 & & & & \\
\hline & 0800 & 4. 88 & 185 & & 0800 & 7. 04 & 1,080 & 25 & 1200 & 4. 22 & 88 \\
\hline & 1200 & 4. 61 & 142 & & 1000 & 7. 03 & 1,080 & & 2400 & 4. 05 & 67 \\
\hline & 1600 & 4. 40 & 111 & & 1200 & 7. 89 & 1,680 & & & & \\
\hline & 1800 & 4. 36 & 106 & & 1300 & 8. 03 & 1,790 & 28 & 2400 & 4. 19 & 84 \\
\hline & 2000 & 4. 81 & 173 & & 1400 & 8. 15 & 1,890 & & & & \\
\hline & 2200 & 5. 00 & 206 & & 1500 & 8.00 & 1,770 & 29 & 1600 & 4.02 & 63 \\
\hline & 2300 & 5. 33 & 272 & & 1600 & 7. 86 & 1,660 & & 1800 & 4. 71 & 157 \\
\hline & 2400 & 7. 50 & 1,350 & & 2000 & 7. 38 & 1,310 & & 2000 & 5. 71 & 434 \\
\hline & & & & & 2400 & 6.60 & 820 & & 2200 & 6. 10 & 580 \\
\hline \multirow[t]{17}{*}{25} & 0200 & 8. 38 & 2,070 & & & & & & 2400 & 7. 63 & 1,480 \\
\hline & 0400 & 8. 65 & 2,320 & 11 & 0600 & 5. 75 & 448 & & & & \\
\hline & 0600 & 8. 28 & 1,990 & & 1200 & 5. 29 & 298 & 30 & 0200 & 9. 35 & 3,000 \\
\hline & 0800 & 7. 92 & 1,710 & & 1800 & 5.09 & 248 & & 0400 & 9.85 & 3,540 \\
\hline & 1000 & 8. 61 & 2,280 & & 2400 & 5.26 & 290 & & 0500 & 10.10 & 3,840 \\
\hline & 1100 & 9.72 & 3,400 & & & & & & 0600 & 10.01 & 3,730 \\
\hline & 1200 & 10.54 & 4,370 & 12 & 1200 & 4. 61 & 142 & & 0800 & 9.63 & 3,300 \\
\hline & 1300 & 11.02 & 4,980 & & 2400 & 4. 40 & 117 & & 1000 & 9.41 & 3,060 \\
\hline & 1330 & 11. 62 & 6,150 & & & & & & 1200 & 8.81 & 2,460 \\
\hline & 1400 & 11. 53 & 5,920 & 21 & 2400 & 3.69 & 32 & & 1600 & 8. 23 & 1,950 \\
\hline & 1500 & 10.68 & 4,540 & & & & & & 2000 & 7.87 & 1,670 \\
\hline & 1600 & 9.69 & 3,370 & 22 & 0600 & 3. 97 & 58 & & 2400 & 7. 15 & 1,150 \\
\hline & 1800 & 8. 49 & 2,170 & & 0700 & 5.23 & 282 & & & & \\
\hline & 2000 & 7. 43 & 1,340 & & 0800 & 5. 71 & 434 & 31 & 0600 & 6. 31 & 675 \\
\hline & 2200 & 6.93 & 1,020 & & 1000 & 6. 10 & 580 & & 1200 & 5. 73 & 440 \\
\hline & 2400 & 6.68 & 868 & & 1200 & 6. 82 & 952 & & 1800 & 5.69 & 426 \\
\hline & & & & & 1400 & 7. 37 & 1,300 & & 2400 & 5.43 & 339 \\
\hline \multirow[t]{2}{*}{26} & 0200 & 6. 19 & 616 & & 1600 & 7. 59 & 1,450 & & & & \\
\hline & 0400 & 5.82 & 472 & & 1800 & 9.24 & 2,890 & & & & \\
\hline
\end{tabular}


(121) 9-5052.5. Red Tank Draw near Rimrock, Ariz.

Location. - Lat $34^{\circ} 41^{\prime} 45^{\prime \prime}$, long $111^{\circ} 42^{\prime} 50^{\prime \prime}$, in $\mathrm{SE}_{\frac{1}{4}} \mathrm{NE} \frac{1}{4} \mathrm{sec}, 16, \mathrm{~T} .15 \mathrm{~N} .$, R. 6 E. , in Coconino National Forest, on left bank 2. 6 miles downstream from confluence of Rarick and Mullican Canyons, and $3 \frac{1}{2}$ miles northeast of Rimrock.

Drainage area. $--49.4 \mathrm{sq} \mathrm{mi}$.

Gage-height record. --Digital recorder tape. Altitude of gage is 3, $950 \mathrm{ft}$ (from topographic map).

Discharge record. --Stage-discharge relation defined by current-meter measurements below $480 \mathrm{cfs}$ and extended above on basis of slope-area measurement at gage height $7.55 \mathrm{ft}$.

Maxima. --November 1965 to January 1966: Discharge, 2, 010 cfs 1300 hours Nov. 25 (gage height, $7.62 \mathrm{ft}$ ).

1957 to October 1965: Discharge, 1, 970 cfs Aug. 2, 1964 (gage height, 7. $55 \mathrm{ft}$ ).

Mean discharge, in cubic feet per second, 1965-66

\begin{tabular}{|c|c|c|c|c|c|c|c|}
\hline Day & November & December & January & Day & November & Decemter & January \\
\hline 1 & 0 & 0.79 & 28 & $16 \ldots$ & 0 & 28 & 0.50 \\
\hline 2 & 0 & .46 & 9.0 & $17 \ldots$ & 0 & 28 & .40 \\
\hline 3 & 0 & .27 & 5.2 & $18 \ldots$ & 0 & 18 & .40 \\
\hline 4 & 0 & .21 & 2. 8 & $19 \ldots$ & 0 & 8. 9 & .30 \\
\hline 5 & 0 & .15 & 2.1 & $20 \ldots$ & 0 & 6. 8 & .30 \\
\hline 6 & 0 & 11 & 1. 5 & 21 & 0 & 11 & .20 \\
\hline 7 & 0 & 11 & 1. 1 & 22 & 0 & 450 & .20 \\
\hline 8 & 0 & 11 & 90 & 23 & 215 & 323 & .20 \\
\hline 9 & 0 & .22 & 2.1 & $24 \ldots \ldots$ & 107 & 81 & .20 \\
\hline 10 & 0 & 705 & 2.8 & $25 \ldots$ & 710 & 48 & .10 \\
\hline 11 & 0 & 190 & 2.4 & $26 \ldots$ & 171 & 32 & .10 \\
\hline 12 & 0 & 62 & 1. 8 & $27 \ldots$ & 24 & 20 & .10 \\
\hline 13 & 0 & 25 & 1. 3 & $28 \ldots \ldots$ & 6.2 & 15 & .10 \\
\hline 14 & 0 & 30 & .90 & $29 \ldots \ldots$ & 2.8 & 79 & .10 \\
\hline 15 & 0 & 40 & .70 & $30 \ldots$ & 1. 4 & 913 & .10 \\
\hline & & & & $31 \ldots$ & $\ldots \ldots$ & 118 & .10 \\
\hline \multirow{2}{*}{\multicolumn{5}{|c|}{$\begin{array}{l}\text { Monthly mean discharge, in cubic feet per second } . \\
\text { Runoff, in acre-feet } \ldots \ldots \ldots \ldots \ldots \ldots \ldots \ldots \ldots \ldots\end{array}$}} & 41.2 & 104 & 2.13 \\
\hline & & & & & 2,450 & 6,410 & 131 \\
\hline
\end{tabular}


Gage height, in feet, and discharge, in cubic feet per second, at indicated time, 1965, of Red Tank Draw near Rimrock, Ariz.

\begin{tabular}{|c|c|c|c|c|c|c|c|c|c|c|c|}
\hline Date & Hour & $\begin{array}{l}\text { Gage } \\
\text { height }\end{array}$ & $\begin{array}{c}\text { Dis- } \\
\text { charge }\end{array}$ & Date & Hour & $\begin{array}{l}\text { Gage } \\
\text { height }\end{array}$ & $\begin{array}{c}\text { Dis- } \\
\text { charge }\end{array}$ & Date & Hour & $\begin{array}{l}\text { Gage } \\
\text { height }\end{array}$ & $\begin{array}{c}\text { Dis- } \\
\text { charge }\end{array}$ \\
\hline Nov. 22 & 2400 & - & 0 & Dec. 9 & 2400 & 1.89 & 0.6 & Dec. 23 & $\begin{array}{l}0400 \\
0600\end{array}$ & $\begin{array}{l}4.84 \\
4.36\end{array}$ & $\begin{array}{l}519 \\
368\end{array}$ \\
\hline \multirow[t]{10}{*}{23} & 1145 & - & 0 & 10 & 0100 & 3.25 & 110 & & 0800 & 4. 12 & 299 \\
\hline & 1200 & 4. 60 & 440 & & 0200 & 4. 88 & 533 & & 1400 & 3.78 & 212 \\
\hline & 1300 & 6. 12 & 1,130 & & 0400 & 6.59 & 1,390 & & 1700 & 3.96 & 256 \\
\hline & 1400 & 5. 76 & 940 & & 0500 & 7. 48 & 1,930 & & 2000 & 3.82 & 221 \\
\hline & 1600 & 4. 75 & 488 & & 0600 & 6. 90 & 1,580 & & 2400 & 3. 51 & 155 \\
\hline & 1800 & 4. 15 & 307 & & 0700 & 5.64 & 880 & & & & \\
\hline & 2000 & 3. 75 & 205 & & 0900 & 4. 70 & 470 & 24 & 1200 & 2.78 & 49 \\
\hline & 2200 & 3. 41 & 137 & & 1200 & 5.60 & 860 & & 2000 & 3.23 & 107 \\
\hline & 2400 & 3. 16 & 96 & & 1300 & 5. 64 & 880 & & 2400 & 3.05 & 80 \\
\hline & & & & & 1400 & 5.36 & 740 & & & & \\
\hline \multirow[t]{7}{*}{24} & 1000 & 2. 52 & 27 & & 1600 & 5. 16 & 647 & 25 & 1200 & 2. 59 & 32 \\
\hline & 1945 & 2. 41 & 19 & & 2000 & 4. 86 & 526 & & 2200 & 2. 97 & 69 \\
\hline & 2000 & 3. 05 & 80 & & 2400 & 4.37 & 371 & & 2400 & 2. 91 & 62 \\
\hline & 2100 & 3. 64 & 181 & & & & & & & & \\
\hline & 2200 & 4. 97 & 568 & 11 & 0600 & 3.92 & 245 & & & & \\
\hline & 2400 & 5. 01 & 584 & & 1200 & 3. 57 & 167 & 28 & 2400 & 2. 31 & 13 \\
\hline & & & & & 1800 & 3. 28 & 115 & & & & \\
\hline \multirow[t]{14}{*}{25} & 0200 & 5. 05 & 600 & & 2400 & 3. 35 & 126 & 29 & 1800 & 2.48 & 24 \\
\hline & 0400 & 4. 64 & 452 & & & & & & 2000 & 3. 86 & 230 \\
\hline & 0600 & 4. 19 & 328 & 12 & 1200 & 2.87 & 58 & & 2200 & 4. 19 & 318 \\
\hline & 0800 & 4. 15 & 307 & & 2400 & 2.56 & 30 & & 2400 & 4. 36 & 368 \\
\hline & 1000 & 5. 14 & 638 & & & & & & & & \\
\hline & 1200 & 6. 71 & 1,470 & & & & & 30 & 0200 & 5. 65 & 885 \\
\hline & 1300 & 7. 62 & 2,010 & 21 & 2400 & 2. 29 & 12 & & 0300 & 6. 96 & 1,620 \\
\hline & 1400 & 7. 17 & 1,680 & & & & & & 0400 & 7. 36 & 1,860 \\
\hline & 1600 & 5.83 & 975 & 22 & 0600 & 2.60 & 33 & & 0430 & 7. 49 & 1,930 \\
\hline & 1800 & 4. 89 & 536 & & 0800 & 4.47 & 401 & & 0600 & 6. 94 & 1,600 \\
\hline & 2000 & 4. 95 & 560 & & 1000 & 4.52 & 416 & & 0800 & 6. 39 & 1,280 \\
\hline & 2200 & 4. 74 & 484 & & 1200 & 4.34 & 362 & & 1200 & 5.68 & 900 \\
\hline & 2400 & 4. 54 & 422 & & 1400 & 4.51 & 413 & & 1600 & 5.08 & 612 \\
\hline & & & & & 1600 & 4. 81 & 508 & & 2000 & 4.68 & 464 \\
\hline \multirow[t]{5}{*}{26} & 0600 & 3. 94 & 250 & & 1800 & 5.22 & 674 & & 2400 & 4. 22 & 327 \\
\hline & 1200 & 3. 57 & 167 & & 1930 & 6. 48 & 1,330 & & & & \\
\hline & 1800 & 2. 92 & 63 & & 2000 & 6. 35 & 1,260 & 31 & 0200 & 3. 98 & 261 \\
\hline & 2400 & 2. 75 & 46 & & 2200 & 5. 46 & 790 & & 0600 & 3. 59 & 171 \\
\hline & & & & & 2400 & 5. 34 & 730 & & 1200 & 3.07 & 83 \\
\hline \multirow[t]{2}{*}{27} & 1200 & 2. 44 & 21 & & & & & & 1800 & 2. 92 & 62 \\
\hline & 2400 & 2. 25 & 10 & 23 & 0200 & 5.21 & 670 & & 2400 & 2. 83 & 54 \\
\hline
\end{tabular}


(122) 9-5053. Rattlesnake Canyon near Rimrock, Ariz.

Location. --Lat $34^{\circ} 46^{\prime} 00^{\prime \prime}$, long $111^{\circ} 40^{\prime} 20^{\prime \prime}$, in NW $\frac{1}{4} \mathrm{SW} \frac{1}{4} \sec .24$, T. 16 N. , R. $6 \mathrm{E}$., in Coconino National Forest, on left bank 2. 6 miles upstream from mouth. 7 miles northeast of Beaver Creek ranger station, and 9 miles northeast of Rirrock.

Drainage area. $--24.6 \mathrm{sq} \mathrm{mi}$.

Gage-height record. - -Water-stage recorder graph except Nov. 17 to 1300 hours Nov. 23, 0200-1800 hours Nov. 24, Jan, 2-7. Graph reconstructed for Nov. 23, 24 on basis of high-water mark in well. Altitude of gage is $5,100 \mathrm{ft}$ (from topographic map).

Discharge record. --Stage-discharge relation defined by current-meter measurements below $1,100 \mathrm{cfs}$ and extended above on basis of computations of flow over weir at gage height $8.50 \mathrm{ft}$. Stage-discharge relation affected by backwater from ice part of each day Jan. 8-18. Discharge for periods of no gage-height record estimated.

Maxima. --November 1965 to January 1966: Discharge, 1, 880 cfs 0230 hours Dec. 30 (gage height, $9.20 \mathrm{ft}$ ).

1957 to October 1965: Discharge, 1,430 cfs Jan. 6, 1965 (gage height, 8. $50 \mathrm{ft}$ ).

Mean discharge, in cubic feet per second, 1965-66

\begin{tabular}{|c|c|c|c|c|c|c|c|}
\hline Day & November & December & January & Day & November & December & January \\
\hline 1 & 0 & 1.1 & 35 & 16 & 0 & 24 & 0.2 \\
\hline $2 \ldots$ & 0 & .8 & 18 & $17 \ldots$ & 0 & 21 & .2 \\
\hline $3 \ldots$ & 0 & .5 & 16 & $18 \ldots$ & 0 & 15 & .2 \\
\hline $4 \ldots$ & 0 & .3 & 16 & $19 \ldots$ & 0 & 12 & .1 \\
\hline $5 \ldots$ & 0 & .2 & 8.0 & $20 \ldots$ & 0 & 16 & .07 \\
\hline $6 \ldots$ & 0 & .07 & 6.0 & $21 \ldots$ & 0 & 18 & .06 \\
\hline $7 \ldots$ & 0 & .06 & 5.0 & 22 & 0 & 32.3 & .06 \\
\hline 8 & 0 & .04 & 4.9 & $23 \ldots$ & 342 & 132 & .03 \\
\hline 9 & 0 & 49 & 5.9 & $24 \ldots$ & 235 & 47 & .02 \\
\hline 10 & 0 & 491 & 4.7 & $25 \ldots$ & 589 & 39 & .02 \\
\hline 11 & 0 & 128 & 5.1 & $26 \ldots$ & 65 & 25 & .01 \\
\hline 12 & 0 & 40 & 3.3 & 27 & 17 & 18 & .01 \\
\hline 13 & 0 & 26 & 1.9 & 28 & 7.2 & 18 & .01 \\
\hline 14 & 0 & 32 & 1.1 & 29 & 3.0 & 110 & .01 \\
\hline \multirow[t]{2}{*}{$15 \ldots$} & 0 & 31 & .4 & & 1. 9 & $7 \div 2$ & .01 \\
\hline & & & & 31 & $\ldots \ldots$ & 103 & .01 \\
\hline \multicolumn{5}{|c|}{ Monthly mean discharge, in cubic feet per second. . } & 42.0 & 79.8 & 4.27 \\
\hline \multicolumn{5}{|c|}{ Runoff, in acre-feet $\ldots \ldots \ldots \ldots \ldots \ldots \ldots \ldots \ldots$} & 2,500 & 4,910 & 262 \\
\hline
\end{tabular}


Gage height, in feet, and discharge, in cubic feet per second, at indicated time, 1965-66, of Rattlesnake Canyon near Rimrock, Ariz.

\begin{tabular}{|c|c|c|c|c|c|c|c|c|c|c|c|}
\hline Date & Hour & $\begin{array}{l}\text { Gage } \\
\text { height }\end{array}$ & $\begin{array}{c}\text { Dis- } \\
\text { charge }\end{array}$ & Date & Hour & $\begin{array}{c}\text { Gage } \\
\text { height }\end{array}$ & $\begin{array}{c}\text { Dis- } \\
\text { charge }\end{array}$ & Date & Hour & $\begin{array}{l}\text { Gage } \\
\text { height }\end{array}$ & $\begin{array}{l}\text { Dis- } \\
\text { charge }\end{array}$ \\
\hline \multirow[t]{10}{*}{ Nov. } & 2400 & - & 0 & Dec, 9 & 1600 & 2.01 & 0.2 & Dec. 23 & 1000 & 3.98 & 118 \\
\hline & & & & & 1630 & 2.96 & 28 & & 1430 & 4. 01 & 121 \\
\hline & a0800 & - & 0 & & 2000 & 2.91 & 26 & & 1900 & 3.72 & 90 \\
\hline & a1100 & 8.9 & 1,670 & & 2100 & 4.28 & 157 & & 2400 & 3.42 & 61 \\
\hline & 1300 & 8.1 & 1,190 & & 2200 & 5.22 & 322 & & & & \\
\hline & 1500 & 7.10 & 800 & & 2400 & 5.97 & 487 & 24 & 1000 & 2. 94 & 27 \\
\hline & 1700 & 5.55 & 391 & & & & & & 1800 & 3.59 & 77 \\
\hline & 2000 & 5.22 & 322 & 10 & 0200 & 7.07 & 791 & & 2400 & 3.15 & 40 \\
\hline & 2400 & 4.27 & 156 & & 0400 & 7.55 & 950 & & & & \\
\hline & & & & & 0500 & 6.80 & 710 & 28 & 2400 & 2.98 & 29 \\
\hline \multirow{10}{*}{24} & 0600 & 3.7 & 88 & & 0600 & 5.97 & 487 & & & & \\
\hline & 1500 & 3.2 & 43 & & 0830 & 5.34 & 346 & 29 & 1400 & 2.82 & 21 \\
\hline & 1800 & 4.1 & 132 & & 1100 & 6.42 & 602 & & 1700 & 4. 39 & 174 \\
\hline & 1900 & 6.30 & 570 & & 1130 & 6.52 & 630 & & 2000 & 4. 84 & 249 \\
\hline & 1930 & 5. 55 & 391 & & 1300 & 6.25 & 557 & & 2200 & 4.90 & 260 \\
\hline & 2000 & 7.13 & 810 & & 1900 & 5.30 & 338 & & 2400 & 6.60 & 652 \\
\hline & 2100 & 6.55 & 638 & & 2400 & 4.60 & 208 & & & & \\
\hline & 2300 & 7.70 & 1,000 & & & & & 30 & 0100 & 7.45 & 915 \\
\hline & 2400 & 7.77 & 1,030 & 11 & 1400 & 3.36 & 56 & & 0230 & 9.20 & 1,880 \\
\hline & & & & & 1800 & 4. 13 & 136 & & 0500 & 7.95 & 1,115 \\
\hline \multirow[t]{14}{*}{25} & 0100 & 7.50 & 932 & & 2400 & 3.62 & 80 & & 0600 & 7.75 & 1,025 \\
\hline & 0300 & 6. 35 & 583 & & & & & & 0730 & 8.10 & 1,190 \\
\hline & 0630 & 5.07 & 292 & 12 & 1800 & 2.93 & 26 & & 0900 & 7. 65 & 986 \\
\hline & 0900 & 7.43 & 908 & & 2400 & 3.03 & 32 & & 1100 & 6.87 & 731 \\
\hline & 1000 & 7.04 & 782 & & & & & & 1900 & 5.62 & 376 \\
\hline & 1100 & 7.58 & 961 & 21 & 2400 & 2.88 & 24 & & 2400 & 4.57 & 203 \\
\hline & 1130 & 8.29 & 1,300 & & & & & & & & \\
\hline & 1300 & 7.98 & 1,130 & 22 & 0400 & 2. 91 & 26 & 31 & 0600 & 3.98 & 118 \\
\hline & 1400 & 7.60 & 968 & & 0700 & 4. 32 & 163 & & 1200 & 3.71 & 89 \\
\hline & 1700 & 5,95 & 482 & & 0800 & 4.44 & 182 & & 1800 & 3.68 & 86 \\
\hline & 2100 & 4. 67 & 219 & & 0930 & 5.06 & 290 & & 2400 & 3.40 & 59 \\
\hline & 2200 & 4.69 & 222 & & 1100 & 4. 98 & 274 & & & & \\
\hline & 2400 & 4.49 & 190 & & 1500 & 5.23 & 324 & Jan. 1 & 0500 & 3.18 & 42 \\
\hline & & & & & 1700 & 6.04 & 504 & & 0900 & 3.15 & 40 \\
\hline \multirow[t]{4}{*}{26} & 0600 & 3.75 & 93 & & b1830 & 7.15 & 810 & & 1700 & 2. 90 & 25 \\
\hline & 1200 & 3.34 & 54 & & 2000 & 6.55 & 638 & & 2000 & 3.06 & 34 \\
\hline & 1800 & 3.03 & 32 & & 2230 & 5.79 & 444 & & 2400 & 2.85 & 22 \\
\hline & 2400 & 3.00 & 30 & & 2400 & 5.89 & 468 & & & & \\
\hline Dec. 8 & 2400 & 1. 94 & .1 & 23 & 0500 & 4. 28 & 157 & & & & \\
\hline
\end{tabular}

a Time approximate.

b Erroneously published as 1630 in annual report. 
(123) 9-5053.5. Dry Beaver Creek near Rimrock, Ariz.

Location. -- Lat $34^{\circ} 43^{\prime} 40^{\prime \prime}$, long $111^{\circ} 46^{\prime} 30^{\prime \prime}$, in NW $\frac{1}{4}$ sec. 1 , T. 15 N., R. 5 E., in Coconino National Forest, on upstream side of abandoned highway bridge, $300 \mathrm{ft}$ upstream from State Highway 179 , and $5 \frac{1}{2}$ miles north of Rimrock.

Drainage area. --139 sq mi.

Gage-height record. --Water-stage recorder graph Nov. 1-22; digital recorder tape thereafter. Altitude of gage is $3,690 \mathrm{ft}$ (based on elevation of bench mark on bridge $300 \mathrm{ft}$ downstream).

Discharge record. --Stage-discharge relation defined by current-meter measurements below $5,400 \mathrm{cfs}$ and extended above on basis of computations of peak flow over submerged weir at gage heights 9.07 and $9.69 \mathrm{ft}$.

Maxima. - - November 1965 to January 1966: Discharge, 9,670 cfs 1230 hours Nov. 23 (gage height, 9.69 ft).

1960 to October 1965: Discharge, 7,970 cfs Jan. 6, 1965 (gage height, 9. $07 \mathrm{ft}$ ).

Mean discharge, in cubic feet per second, 1965-66

\begin{tabular}{|c|c|c|c|c|c|c|c|}
\hline Day & November & December & January & Day & November & Decembor & January \\
\hline 1 & 0 & 8.2 & 290 & $16 \ldots$ & 0 & 83 & 5.8 \\
\hline$\ldots$ & 0 & 5.9 & 129 & 17. & 0 & 82 & 3.7 \\
\hline 3 & 0 & 3.5 & 73 & $18 \ldots$ & 0 & 64 & 2.9 \\
\hline$\ldots$ & 0 & 2.5 & 48 & $19 \ldots$ & 0 & 44 & 2.1 \\
\hline 5 & 0 & 1.8 & 33 & $20 \ldots$ & 0 & 45 & 1.6 \\
\hline$\ldots$ & 0 & 1.1 & 28 & $21 \ldots$ & 0 & 58 & 1.2 \\
\hline $7 \ldots$ & 0 & .60 & 26 & $22 \ldots$ & 0 & 685 & .80 \\
\hline $8 \ldots$ & 0 & .30 & 30 & $23 \ldots$ & 2,750 & 726 & .60 \\
\hline $9 \ldots$ & 0 & 130 & 35 & $24 \ldots$ & 894 & 226 & .35 \\
\hline $10 \ldots$ & 0 & 2,200 & 32 & $25 \ldots$ & 3,190 & 172 & .19 \\
\hline $11 \ldots$ & 0 & 599 & 30 & $26 \ldots$ & 441 & 119 & .12 \\
\hline $12 \ldots \ldots$ & 0 & 259 & 28 & $27 \ldots$ & 147 & 79 & .09 \\
\hline $13 \ldots$ & 0 & 150 & 18 & $28 \ldots$ & 65 & 59 & .07 \\
\hline $14 \ldots$ & 0 & 123 & 11 & $29 \ldots$ & 25 & 125 & .07 \\
\hline \multirow[t]{2}{*}{$15 \ldots$} & 0 & 120 & 7.7 & $30 \ldots$ & 13 & 3,870 & .06 \\
\hline & & & & 31. & ------ & 742 & .06 \\
\hline \multirow{2}{*}{\multicolumn{5}{|c|}{ Monthly mean discharge, in cubic feet per second.. }} & 251 & 349 & 27. 0 \\
\hline & & & & & 14,930 & 21,403 & 1,660 \\
\hline
\end{tabular}


Gage height, in feet, and discharge, in cubic feet per second, at indicated tim?, 1965, of Dry Beaver Creek near Rimrock, Ariz.

\begin{tabular}{|c|c|c|c|c|c|c|c|c|c|c|c|}
\hline Date & Hour & $\begin{array}{l}\text { Gage } \\
\text { height }\end{array}$ & $\begin{array}{c}\text { Dis- } \\
\text { charge }\end{array}$ & Date & Hour & $\begin{array}{l}\text { Gage } \\
\text { height }\end{array}$ & $\begin{array}{c}\text { Dis- } \\
\text { charge }\end{array}$ & Date & Hour & $\begin{array}{l}\text { Gage } \\
\text { height }\end{array}$ & $\begin{array}{l}\text { Dis- } \\
\text { charge }\end{array}$ \\
\hline Nov. 22 & 2400 & 0.75 & 0 & Nov. 26 & 1800 & 3.68 & 245 & Dec. 22 & 2000 & 5.85 & 2,110 \\
\hline \multirow{15}{*}{23} & & & & & 2400 & 3.62 & 223 & & 2100 & 5.65 & 1,860 \\
\hline & 0800 & .72 & 0 & & & & & & 2200 & 5.49 & 1,690 \\
\hline & 0900 & 2.84 & 31 & Dec. 8 & 2400 & 1. 81 & .2 & & 2400 & 5.33 & 1,500 \\
\hline & 0930 & 5.66 & 1,850 & & & & & & & & \\
\hline & 1000 & 7. 10 & 3,970 & 9 & 2045 & 1. 86 & .3 & 23 & 0400 & 5. 02 & 1,190 \\
\hline & 1100 & 8.22 & 6,160 & & 2100 & 4. 16 & $493^{\circ}$ & & 0800 & 4. 49 & 728 \\
\hline & 1200 & 9.38 & 8,750 & & 2200 & 4.53 & 752 & & 1200 & 4. 21 & 524 \\
\hline & 1230 & 9.69 & 9,670 & & 2300 & 4.69 & 880 & & 1600 & 4.18 & 511 \\
\hline & 1400 & 8.90 & 7,590 & & 2400 & 5. 51 & 1,690 & & 2000 & 4. 06 & 440 \\
\hline & 1600 & 7. 39 & 4,480 & & & & & & 2400 & 3.91 & 359 \\
\hline & 1800 & 6.18 & 2,510 & 10 & 0100 & 6. 16 & 2,500 & & & & \\
\hline & 2000 & 5.83 & 2,060 & & 0200 & 6.69 & 3,290 & 24 & 1000 & 3.47 & 170 \\
\hline & 2200 & 5.82 & 2,050 & & 0300 & 7. 13 & 4,040 & & 1600 & 3. 41 & 158 \\
\hline & 2400 & 5.40 & 1,560 & & 0400 & 7.24 & 4,210 & & 2000 & 3. 71 & 266 \\
\hline & & & & & 0500 & 7. 56 & 4,820 & & 2400 & 3.70 & 261 \\
\hline \multirow[t]{8}{*}{24} & 0600 & 4. 68 & 824 & & 0600 & 7. 17 & 4,110 & & & & \\
\hline & 1200 & 4. 09 & 417 & & 0700 & 6.30 & 2,700 & & & & \\
\hline & 2000 & 3.84 & 291 & & 0800 & 5.72 & 1,930 & 28 & 2400 & 2. 94 & 48 \\
\hline & 2100 & 5. 31 & 1,410 & & 1000 & 5. 33 & 1,490 & & & & \\
\hline & 2200 & 5.70 & 1,840 & & 1200 & 5.51 & 1,690 & 29 & 1945 & 3.07 & 71 \\
\hline & 2300 & 6. 16 & 2,420 & & 1400 & 5. 47 & 1,650 & & 2200 & 3. 94 & 374 \\
\hline & 2400 & 7.09 & 3,960 & & 1600 & 5.25 & 1,410 & & 2400 & 4. 15 & 493 \\
\hline & & & & & 1800 & 5.20 & 1,360 & & & & \\
\hline \multirow[t]{15}{*}{25} & 0130 & 7.64 & 4,960 & & 2400 & 4.87 & 1,030 & 30 & 0130 & 4. 68 & 880 \\
\hline & 0200 & 7.56 & 4,800 & & & & & & 0300 & 6.23 & 2,620 \\
\hline & 0400 & 6.58 & 3,110 & 11 & 0600 & 4.48 & 712 & & 0400 & 7. 57 & 4,860 \\
\hline & 0600 & 5. 77 & 1,980 & & 1200 & 4. 20 & 517 & & 0530 & 8.33 & 6,440 \\
\hline & 0800 & 5.55 & 1,720 & & 1800 & 4.04 & 423 & & 0700 & 8,11 & 5,970 \\
\hline & 0900 & 6. 67 & 3,240 & & 2200 & 4.22 & 530 & & 0830 & 9.08 & 8,040 \\
\hline & 1000 & 7.98 & 5,660 & & 2400 & 4. 13 & 475 & & 1000 & 8. 60 & 7,000 \\
\hline & 1030 & 7. 98 & 5,660 & & & & & & 1200 & 7. 46 & 4,650 \\
\hline & 1100 & 7.79 & 5,260 & & & & & & 1400 & 6.72 & 3,360 \\
\hline & 1200 & 7. 66 & 4,990 & 21 & 2400 & 2.95 & 34 & & 1600 & 6. 36 & 2,810 \\
\hline & 1400 & 7. 33 & 4,370 & & & & & & 2000 & 5.78 & 2,020 \\
\hline & 1600 & 6.58 & 3,110 & 22 & 0800 & 3. 12 & 82 & & 2400 & 5.20 & 1,370 \\
\hline & 1800 & 5.81 & 2,030 & & 1000 & 3.98 & 395 & & & & \\
\hline & 2400 & 4. 77 & 936 & & 1200 & 4. 33 & 610 & 31 & 0600 & 4.68 & 880 \\
\hline & & & & & 1700 & 4. 38 & 645 & & 1200 & 4.38 & 645 \\
\hline \multirow[t]{2}{*}{26} & 0600 & 4. 33 & 596 & & 1800 & 4.68 & 880 & & 1800 & 4. 28 & 575 \\
\hline & 1200 & 3.94 & 364 & & 1900 & 5.37 & 1,550 & & 2400 & 4. 11 & 469 \\
\hline
\end{tabular}

(124) 9-5056. Dirty Neck Canyon near Clints Well, Ariz.

(Crest-stage station)

Location. -- Lat $34^{\circ} 30^{\prime} 45^{\prime \prime}$, long $111^{\circ} 21^{\prime} 30^{\prime \prime}$, in $\mathrm{E} \frac{1}{2} \mathrm{sec} .14$, T. 13 N., R. $9 \mathrm{E}$, at county road, 4 miles southwest of Clints Well, and 18 miles north of Payson.

Drainage area. --About $1.5 \mathrm{sq} \mathrm{mi}$.

Gage-height record,--Crest stages only. Altitude of gage is 6, $800 \mathrm{ft}$ (from topographic map).

Discharge record. --Stage-discharge relation defined by computation of flow through culvert.

Maxima. --November 1965 to January 1966: Discharge, $115 \mathrm{cfs}$ Dec. 30 (grae height, $5.59 \mathrm{ft}$ ).

1964 to October 1965: Discharge, 85 cfs Jan. 6, 1965 (gage height, $4.83 \mathrm{ft}$ ). 
(125) 9-5058. West Clear Creek near Camp Verde, Ariz.

Location. -- Lat $34^{\circ} 32^{\prime} 20^{\prime \prime}$, long $111^{\circ} 41^{\prime} 35^{\prime \prime}$, in SW $\frac{1}{4}$ sec. 2 , T. 13 N., R. 6 E., in Coconino National Forest, on left bank at Bull Pen Ranch, $8 \frac{1}{2}$ miles upstream from mouth, and 9 miles east of Camp Verde.

Drainage area. $--240 \mathrm{sq} \mathrm{mi}$, approximately.

Gage-height record. - -Water-stage recorder graph prior to Nov. 16; digital recorder tape thereafter except 1000 hours Dec. 30 to Jan. 26. Altitude of gage is 3,650 ft (from topographic map).

Discharge record, --Stage-discharge relation defined by current-meter measurements. Discharge for period of no gage-height record estimated.

Maxima. --November 1965 to January 1966: Discharge, 6, 330 cfs 0300 hours Dec. 30 (gage height, $8.24 \mathrm{ft}$, from floodmark).

1964 to October 1965: Discharge, 6,510 cfs Jan. 6, 1965 (gage heigh t., $8,3 \mathrm{ft}$, from floodmark).

Mean discharge, in cubic feet per second, 1965-66

\begin{tabular}{|c|c|c|c|c|c|c|c|}
\hline Day & November & December & January & Day & November & December & January \\
\hline $1 \ldots$ & 14 & 39 & 300 & $16 \ldots$ & 15 & 97 & 25 \\
\hline $2 \ldots$ & 14 & 32 & 150 & $17 \ldots$ & 17 & 91 & 20 \\
\hline $3 \ldots$ & 14 & 28 & 100 & $18 \ldots$ & 16 & 80 & 20 \\
\hline $4 \ldots$ & 14 & 25 & 60 & $19 \ldots$ & 16 & 64 & 20 \\
\hline $5 \ldots$ & 14 & 23 & 45 & $20 \ldots$ & 15 & 56 & 20 \\
\hline 6 & 15 & 21 & 40 & $21 \ldots$ & 15 & 54 & 20 \\
\hline 7 & 15 & 21 & 35 & $22 \ldots \ldots$ & 15 & 1,520 & 20 \\
\hline 8 & 15 & 19 & 30 & 23 & 21 & 868 & 20 \\
\hline 9 & 15 & 23 & 25 & 24. & 22 & 259 & 20 \\
\hline $10 \ldots$ & 15 & 1,100 & 25 & 25 . & 1,210 & 200 & 20 \\
\hline $11 \ldots$ & 15 & 759 & 25 & $26 \ldots$ & 1,020 & 163 & 20 \\
\hline 12 & 15 & 362 & 25 & $27 \ldots$ & 197 & $12 \Omega$ & 20 \\
\hline $13 \ldots$ & 15 & 195 & 25 & $28 \ldots \ldots$ & 92 & 110 & 20 \\
\hline $14 \ldots$ & 15 & 129 & 30 & $29 \ldots$ & 58 & 311 & 20 \\
\hline $15 \ldots$ & 15 & 115 & 30 & $30 \ldots$ & 43 & $3,50 \mathrm{C}$ & 20 \\
\hline & & & & 31. & $\ldots-\ldots$ & $80 r$ & 20 \\
\hline \multirow{2}{*}{\multicolumn{5}{|c|}{$\begin{array}{l}\text { Monthly mean discharge, in cubic feet per second } . \\
\text { Runoff, in acre-feet } \ldots \ldots \ldots \ldots \ldots \ldots \ldots \ldots \ldots\end{array}$}} & 99.7 & 361 & 41.0 \\
\hline & & & & & 5,930 & $22,19 c$ & 2,520 \\
\hline
\end{tabular}


Gage height, in feet, and discharge, in cubic feet per second, at indicated t'me, 1965, of West Clear Creek near Camp Verde, Ariz.

\begin{tabular}{|c|c|c|c|c|c|c|c|c|c|c|c|}
\hline Date & Hour & $\begin{array}{l}\text { Gage } \\
\text { height }\end{array}$ & $\begin{array}{c}\text { Dis- } \\
\text { charge }\end{array}$ & Date & Hour & $\begin{array}{l}\text { Gage } \\
\text { height }\end{array}$ & $\begin{array}{c}\text { Dis- } \\
\text { charge }\end{array}$ & Date & Hour & $\begin{array}{c}\text { Gage } \\
\text { height }\end{array}$ & $\begin{array}{c}\text { Dis- } \\
\text { charge }\end{array}$ \\
\hline \multirow[t]{2}{*}{ Nov. 24} & 2400 & 3.31 & 23 & Dec. 10 & 1600 & 6.26 & 1,990 & Dec. 23 & 2400 & 4.55 & 402 \\
\hline & & & & & 1800 & 6.27 & 2,010 & & & & \\
\hline \multirow[t]{12}{*}{25} & 0200 & 3. 32 & 24 & & 2000 & 6.15 & 1,850 & 24 & 1600 & 3.96 & 194 \\
\hline & 0400 & 3.79 & 98 & & 2200 & 6.09 & 1,770 & & 2200 & 4. 19 & 240 \\
\hline & 0600 & 4. 41 & 304 & & 2400 & 5.70 & 1,280 & & 2400 & 4. 16 & 229 \\
\hline & 1000 & 4. 28 & 246 & & & & & & & & \\
\hline & 1200 & 5.77 & 1,310 & 11 & 0200 & 5. 48 & 1,060 & & & & \\
\hline & 1400 & 6.45 & 2,210 & & 0400 & 5. 40 & 990 & 28 & 2400 & 3.81 & 118 \\
\hline & 1600 & 6.51 & 2,300 & & 1200 & 5.02 & 686 & & & & \\
\hline & 1800 & 5.97 & 1,560 & & 1800 & 4.87 & 566 & 29 & 1800 & 4.03 & 184 \\
\hline & 2000 & 6. 19 & 1,850 & & 2400 & 4. 86 & 558 & & 1900 & 4.85 & 574 \\
\hline & 2200 & 6.95 & 3,030 & & & & & & 2000 & 5.13 & 798 \\
\hline & 2230 & 7.00 & 3,130 & 12 & 1200 & 4.50 & 362 & & 2200 & 5. 23 & 878 \\
\hline & 2400 & 6.91 & 2,950 & & 2400 & 4.20 & 232 & & 2400 & 6. 01 & 1,710 \\
\hline \multirow[t]{5}{*}{26} & 0600 & 5.88 & 1,430 & & & & & 30 & 0100 & 7. 13 & 3,530 \\
\hline & 1200 & 5. 17 & 766 & 21 & 2400 & 3.50 & 57 & & 0200 & 7.44 & 4,160 \\
\hline & 1800 & 4. 73 & 456 & & & & & & 0300 & a8. 24 & a6, 330 \\
\hline & 2400 & 4. 44 & 313 & 22 & 0800 & 3.58 & 70 & & 0400 & 7. 79 & 5,040 \\
\hline & & & & & 1000 & 4. 36 & 331 & & 0500 & 7. 53 & 4,380 \\
\hline \multirow[t]{4}{*}{27} & 1200 & 4.13 & 188 & & 1200 & 5.40 & 1,010 & & 0600 & 7. 66 & 4,660 \\
\hline & 2400 & 3.89 & 120 & & 1400 & 6.28 & 2,030 & & 0730 & 7. 46 & 4,160 \\
\hline & & & & & 1600 & 6.58 & 2,530 & & 0930 & 7. 98 & 5,510 \\
\hline & & & & & 1800 & 7.70 & 4,810 & & 1000 & 7.90 & 5,310 \\
\hline \multirow[t]{2}{*}{ Dec. 9} & 2400 & 3.29 & 26 & & 2000 & 7. 25 & 3,770 & & 1500 & - & 2,800 \\
\hline & & & & & 2200 & 6.44 & 2,300 & & 2000 & - & 1,800 \\
\hline \multirow[t]{5}{*}{10} & 0200 & 4. 05 & 180 & & 2400 & 6.35 & 2,160 & & 2400 & - & 1,200 \\
\hline & 0400 & 4. 21 & 236 & & & & & & & & \\
\hline & 0500 & 4. 99 & 662 & 23 & 0600 & 5. 39 & 1,010 & 31 & 1200 & - & 800 \\
\hline & 0800 & 5.22 & 854 & & 1200 & 4.92 & 630 & & 2400 & - & 500 \\
\hline & 1100 & 4.93 & 614 & & 1800 & 4. 84 & 566 & & & & \\
\hline
\end{tabular}

a Stage and discharge from outside high-water mark, published in annual report. 
(126) 9-5065. Verde River at Childs, near Camp Verde, Ariz.

(Discontinued gaging station)

Location. --Lat $34^{\circ} 20^{\prime} 45^{\prime \prime}$, long $111^{\circ} 41^{\prime} 45^{\prime \prime}$, $600 \mathrm{ft}$ downstream from Arizona Public Service Co. powerhouse at Childs, $6 \frac{3}{4}$ miles upstream from East Verde River, and 18 miles southeast of Camp Verde.

Drainage area. --5, $090 \mathrm{sq} \mathrm{mi}$, approximately (including $373 \mathrm{sq} \mathrm{mi}$ in Aubroy Valley Playa, a closed basin).

Gage-height record. - - Daily staff-gage readings except during flood period's, when gage was read more frequently. Records obtained from Arizona Public Service Co. for only those days shown below. Altitude of gage is 2,610 ft (from topographic map).

Discharge record. --Discharges at times of gage readings furnished by Arizona Public Service Co.; basis of stage-discharge relation unknown. Remaining discharges were estimated from hydrographic comparison with records for Verde River "near Clarkdale" and "below Tangle Creek, above Horseshoe Dam."

Maxima. --November 1965 to January 1966: Discharge observed, 27, 200 cfs 2000 hours Dec. 30 (gage height, $9.5 \mathrm{ft}$ ).

1911-17: Gage height, $23 \mathrm{ft} \mathrm{Jan} .19,1916$, at site and datum then ir use (discharge not determined).

Gage height, in feet, and discharge, in cubic feet per second, at indicated time, 1965-66

\begin{tabular}{|c|c|c|c|c|c|c|c|c|c|c|c|}
\hline Date & Hour & $\begin{array}{l}\text { Gage } \\
\text { height }\end{array}$ & $\begin{array}{c}\text { Dis- } \\
\text { charge }\end{array}$ & Date & Hour & $\begin{array}{l}\text { Gage } \\
\text { height }\end{array}$ & $\begin{array}{c}\text { Dis- } \\
\text { charge }\end{array}$ & Date & Hour & $\begin{array}{l}\text { Gage } \\
\text { height }\end{array}$ & $\begin{array}{c}\text { Dis- } \\
\text { charge }\end{array}$ \\
\hline Dec. 21 & 2400 & - & 450 & Dec. 24 & 2400 & - & 3,000 & Dec. 30 & $\begin{array}{l}1630 \\
2000\end{array}$ & $\begin{array}{l}8.6 \\
9.5\end{array}$ & $\begin{array}{l}21,600 \\
27,200\end{array}$ \\
\hline \multirow[t]{3}{*}{22} & $\begin{array}{l}1000 \\
1500\end{array}$ & $\begin{array}{c}0.80 \\
-\end{array}$ & $\begin{array}{l}480 \\
550\end{array}$ & 25 & $\begin{array}{l}1000 \\
2400\end{array}$ & $\begin{array}{c}2.0 \\
-\end{array}$ & $\begin{array}{l}2,080 \\
1,500\end{array}$ & & 2400 & - & 30,000 \\
\hline & 2000 & - & 3,000 & & & & & 31 & 0500 & - & 26,000 \\
\hline & 2400 & - & 11,000 & 29 & 2400 & - & 1,500 & & $\begin{array}{l}1000 \\
2400\end{array}$ & $\begin{array}{l}8.3 \\
-\end{array}$ & $\begin{array}{r}20,000 \\
7,500\end{array}$ \\
\hline \multirow[t]{2}{*}{23} & $\begin{array}{l}0400 \\
1000\end{array}$ & 5.8 & $\begin{array}{r}15,000 \\
9,880\end{array}$ & 30 & 0400 & - & 1,800 & Jan. & 1000 & 3. 8 & 5,270 \\
\hline & 2400 & - & 6,500 & & $\begin{array}{l}0700 \\
1000\end{array}$ & 5.8 & $\begin{array}{l}7,000 \\
9,880\end{array}$ & Sant & $\begin{array}{l}1300 \\
1500\end{array}$ & $\begin{array}{l}\text { 4. } 5 \\
\text { 3. } 8\end{array}$ & $\begin{array}{l}6,600 \\
5,270\end{array}$ \\
\hline \multirow[t]{2}{*}{24} & 0600 & - & 700 & & 1330 & 7. 6 & 16,300 & & 1900 & 3. 0 & 3,780 \\
\hline & 1000 & 4. 0 & 5,670 & & 1430 & 8.0 & 18,300 & & 2400 & - & 3,200 \\
\hline
\end{tabular}

(127) 9-5076. East Verde River near Pine, Ariz.

Location. --Lat $34^{\circ} 23^{\prime} 30^{\prime \prime}$, long $111^{\circ} 16^{\prime} 05^{\prime \prime}$, in SE $\frac{1}{4} \mathrm{SW} \frac{1}{4} \sec .26$, T. 12 N. , R. $10 \mathrm{E}$. (unsurveyed), on right bank 0.8 mile upstream from Dude Creek, 2.7 miles south of Washington Park, and $10 \frac{1}{2}$ miles east of Pine.

Drainage area. $--6.65 \mathrm{sq} \mathrm{mi}$.

Gage-height record. - -Water-stage recorder graph. Altitude of gage is 5, $400 \mathrm{ft}$ (from topographic map).

Discharge record. - -Stage-discharge relation defined by current-meter measurements below $40 \mathrm{cfs}$ and extended above on basis of slope-area measurements at gage heights 3.05 and $3.67 \mathrm{ft}$. Stage-discharge relations affected by backwater from ice part of each day Jan. 21-29.

Maxima. - - November 1965 to January 1966: Discharge, 960 cfs 0330 hours Dec. 30 (gage height, $3.67 \mathrm{ft}$ ).

1961 to October 1965: Discharge, 264 cfs Sept. 11, 1963 (gage height, 3. $05 \mathrm{ft}$ ). 
Mean discharge, in cubic feet per second, 1965-66, of East Verde River near Pine, Ariz.

\begin{tabular}{|c|c|c|c|c|c|c|c|}
\hline Day & November & December & January & Day & November & December & January \\
\hline 1 & 3.7 & 3.6 & 23 & $16 \ldots$ & 28 & 6.2 & 5.2 \\
\hline 2 & 9.6 & 3.6 & 16 & 17. & 28 & 8.4 & 5,2 \\
\hline 3 & 12 & 3.4 & 15 & 18 & 28 & 17 & 5.2 \\
\hline . & 26 & 6. 0 & 12 & 19 & 28 & 21 & 4.9 \\
\hline$\ldots$ & 26 & 5.2 & 10 & 20 & 28 & 5.8 & 4.9 \\
\hline$\ldots$ & 26 & 5.8 & 9.4 & 21 & 28 & 6.6 & 4.5 \\
\hline $7 \ldots$ & 26 & 2.6 & 9.8 & 22 & 29 & 65 & 4.0 \\
\hline$\ldots$ & 26 & 12 & 9.4 & 23 & 27 & 34 & 4.0 \\
\hline $9 \ldots$ & 24 & 17 & 8.9 & $24 \ldots$ & 15 & 15 & 4.0 \\
\hline $10 \ldots$ & 23 & 48 & 13 & 25 & 58 & 12 & 3.8 \\
\hline $11 \ldots$ & 24 & 12 & 7.5 & 26 & 15 & 11 & 3.8 \\
\hline 12 & 23 & 8.4 & 7.0 & 27 & 7. 5 & 11 & 3.8 \\
\hline 13 & 30 & 7. 9 & 6.6 & 28. & 5.5 & 11 & 3.8 \\
\hline $14 \ldots$ & 30 & 7. 0 & 6.2 & 29. & 4. 6 & 61 & 3.8 \\
\hline \multirow[t]{2}{*}{$15 \ldots$} & 28 & 6.6 & 5.5 & 30 & 3.8 & 260 & 3.6 \\
\hline & & & & 31 . & $\ldots . .$. & 38 & 3.6 \\
\hline \multirow{2}{*}{\multicolumn{5}{|c|}{$\begin{array}{l}\text { Monthly mean discharge, in cubic feet per second } . \\
\text { Runoff, in acre-feet } \ldots \ldots \ldots \ldots \ldots \ldots \ldots \ldots \ldots \ldots \ldots\end{array}$}} & 22.4 & 23.6 & 7.34 \\
\hline & & & & & 1,330 & 1,450 & 451 \\
\hline
\end{tabular}

(128) 9-5077. Webber Creek above West Fork Webber Creek, near Pine, Ariz.

Location. --Lat $34^{\circ} 24^{\prime} 40^{\prime \prime}$, long $111^{\circ} 22^{\prime} 20^{\prime \prime}$, in SW $\frac{1}{4}$ sec. 23, T. 12 N. , R. 9 E. (unsurveyed), in Tonto National Forest, on left bank 0.2 mile upstream from West Fork Webber Creek, and 4.9 miles northeast of Pine.

Drainage area. $--4.92 \mathrm{sq} \mathrm{mi}$.

Gage-height record. --Water-stage recorder graph. Altitude of gage is 5, $530 \mathrm{ft}$ (from topographic map).

Discharge record. --Stage-discharge relation defined by current-meter measurements below $33 \mathrm{cfs}$ and extended above on basis of slope-area measurement at gage height $3.13 \mathrm{ft}$.

Maxima. --November 1965 to January 1966: Discharge, $366 \mathrm{cfs} 0800$ hours L॰c. $30^{\circ}$ (gage height, $3.05 \mathrm{ft}$ ).

1959 to October 1965: Discharge, $399 \mathrm{cfs}$ Sept. 13, 1961 (gage height, $3.13 \mathrm{ft}$ ).

Mean discharge, in cubic feet per second, 1965-66

\begin{tabular}{|c|c|c|c|c|c|c|c|}
\hline Day & November & December & January & Day & November & December & J anuary \\
\hline 1 & 0.2 & 2.3 & 24 & 16 & 0.3 & 5.6 & 3.6 \\
\hline 2 & .3 & 2.0 & 16 & 17 & .3 & 5.6 & 3.6 \\
\hline $3 \ldots$ & .3 & 1.8 & 12 & 18 & .3 & 4. 6 & 3.4 \\
\hline $4 \ldots$ & .3 & 1.8 & 10 & 19 & .3 & 4. 6 & 3.1 \\
\hline $5 \ldots$ & .3 & 1.6 & 8.4 & $20 \ldots$ & 3 & 4. 6 & 2.9 \\
\hline $6 \ldots$ & .3 & 1.5 & 6.6 & $21 \ldots$ & .3 & 5.2 & 2.9 \\
\hline $7 \ldots$ & .3 & 1.5 & 6.8 & $22 \ldots$ & .5 & 29 & 2.7 \\
\hline $8 \ldots$ & .3 & 1.5 & 6.2 & $23 \ldots$ & 4. 7 & 20 & 2.7 \\
\hline $9 \ldots$ & .3 & 3.6 & 5.9 & $24 \ldots$ & 18 & 11 & 2. 7 \\
\hline $10 \ldots$ & 3 & 34 & 5.6 & $25 \ldots$ & 77 & 9.8 & 2.5 \\
\hline $11 \ldots$ & .3 & 16 & 5.6 & $26 \ldots$ & 15 & 9.3 & 2.5 \\
\hline $12 \ldots$ & .3 & 9.8 & 5.2 & 27 & 6.6 & 8. 4 & 2.5 \\
\hline $13 \ldots$ & .3 & 7. 6 & 4.6 & 28 & 4.4 & 8. 0 & 2.3 \\
\hline $14 \ldots$ & .3 & 7. 0 & 4.1 & 29 & 3.4 & 25 & 2.1 \\
\hline \multirow[t]{2}{*}{$15 \ldots$} & .3 & 6. 2 & 4.1 & 30 & 2. 7 & 200 & 2. 1 \\
\hline & & & & 31 & $\ldots \ldots$ & 50 & 2.1 \\
\hline \multirow{2}{*}{\multicolumn{5}{|c|}{ Monthly mean discharge, in cubic feet per second. }} & 4.62 & 16.1 & 5.45 \\
\hline & & & & & 275 & 990 & 335 \\
\hline
\end{tabular}


(129) 9-5079.8. East Verde River near Childs, Ariz.

Location. -- Lat $34^{\circ} 16^{\prime} 20^{\prime \prime}$, long $111^{\circ} 37^{\prime} 50^{\prime \prime}$, in sec. 21, T. 10 N. , R. 7 E. (unsurveyed), in Tonto National Forest, on right bank 2 miles upstream from mouth, and 7 miles southeast of Childs.

Drainage area. $--317 \mathrm{sq} \mathrm{mi}$.

Gage-height record. --Water-stage recorder graph to 1200 hours Dec. 16. Gage washed out during peak of Dec. 22. Altitude of gage is 2,600 ft (from topographic map).

Discharge record. --Stage-discharge relation defined by current-meter maasurements below $1,100 \mathrm{cf}$ s and extended above on basis of slope-area measurement at gage height $16.0 \mathrm{ft}$.

Maxima. --November 1965 to January 1966: Discharge, 17, 000 cfs about 2000 hours Dec. 22 (gage height unknown), from slope-area measurement of peak flow.

1961 to October 1965: Discharge, $11,400 \mathrm{cfs}$ Aug. 22, 1963 (gage həight, 16. $0 \mathrm{ft}$ ).

Mean discharge, in cubic feet per second, 1965

\begin{tabular}{rl|r|r||r|r|r||l|r|r}
\hline Day & November & December & Day & November & December & Day & Novemt er & December \\
\hline $1 \ldots$ & 1.4 & 27 & $11 \ldots$ & 24 & 855 & $21 \ldots$ & 31 & - \\
$2 \ldots$ & 1.5 & 22 & $12 \ldots$ & 24 & 201 & $22 \ldots$. & 32 & - \\
$3 \ldots$ & 1.6 & 20 & $13 \ldots$ & 27 & 121 & $23 \ldots$. & 52 & - \\
$4 \ldots$ & 1.6 & 17 & $14 \ldots$ & 25 & 171 & $24 \ldots$. & 82 & - \\
$5 \ldots$ & 5.4 & 16 & $15 \ldots$ & 29 & 209 & $25 \ldots$. & 730 & - \\
$6 \ldots$ & 17 & 16 & $16 \ldots$ & 29 & 156 & $26 \ldots$. & 419 & - \\
$7 \ldots$ & 21 & 16 & $17 \ldots$ & 29 & - & $27 \ldots$. & 107 & - \\
$8 \ldots$ & 22 & 18 & $18 \ldots$ & 31 & - & $28 \ldots$. & 60 & - \\
$9 \ldots$ & 22 & 16 & $19 \ldots$ & 30 & - & $29 \ldots$. & 42 & - \\
$10 \ldots$ & 24 & 2,200 & $20 \ldots$ & 30 & - & $30 \ldots$ & 32 & - \\
\hline
\end{tabular}


(130) 9-5085. Verde River below Tangle Creek, above Horseshoe Dam, Ariz.

Location. -- Lat $34^{\circ} 04^{\prime} 25^{\prime \prime}$, long $111^{\circ} 42^{\prime} 50^{\prime \prime}$, in sec. 35, T. 9 N., R, 6 E. (unsurveyed), in Tonto National Forest, on right bank $1 \frac{1}{4}$ miles downstream from Tangle Creek, and 9 miles upstream from Horseshoe Dam.

Drainage area. - -5,872 sq mi (including $373 \mathrm{sq} \mathrm{mi}$ in Aubrey Valley Playa, a closed basin).

Gage-height record. - -Water-stage recorder graph, except 1800 hours Nov. $2 \mathrm{~g}$ to 1400 hours Dec. 1, 2000 hours Dec. 25 to 1830 hours Dec. 28, and 1100 hours Jan. 2 to 1530 hours Jan. 6, for which graph was reconstructed on basis of indicated recession. Datum of gage is $2,029.0 \mathrm{ft}$ above mean sea level, datum of 1929 .

Discharge record. - -Stage-discharge relation defined by current-meter measurements.

Maxima. --November 1965 to January 1966: Discharge, 39, 300 cfs 2400 hours Dec, 22 (gage height, $16.43 \mathrm{ft}$ ).

1945 to October 1965: Discharge, $81,600 \mathrm{cfs}$ Dec. 31, 1951 (gage height, 17, $62 \mathrm{ft}$ ), from rating curve extended above 42,000 cfs by logarithmic plotting.

Greatest floods since 1888 occurred in February 1891 (discharge probab?y more than 150,000 cfs) and Mar. 3, 1938, discharge, 100, $000 \mathrm{cfs}$ (gage height, $19.0 \mathrm{ft}$, from floodmarks), computed from comparison of peak discharge at other stations on Verde River.

Mean discharge, in cubic feet per second, 1965-66

\begin{tabular}{|c|c|c|c|c|c|c|c|}
\hline Day & November & December & January & Day & November & December & January \\
\hline $1 \ldots$ & $\overline{179}$ & 710 & 7,710 & $16 \ldots$ & 220 & 2,040 & 515 \\
\hline 2 & 182 & 604 & 4,100 & 17 & 236 & 2,220 & 492 \\
\hline 3 & 171 & 522 & 2,600 & 18. & 246 & 1,870 & 488 \\
\hline 4 & 169 & 477 & 2,000 & 19 & 253 & 1,440 & 473 \\
\hline 5 & 175 & 440 & 1,500 & 20 & 274 & 1,230 & 469 \\
\hline 6 & 181 & 409 & 1,200 & 21 & 277 & 1,170 & 466 \\
\hline 7 & 192 & 386 & 978 & 22 & 298 & 12,400 & 451 \\
\hline 8 & 194 & 366 & 871 & 23 & 391 & 19,000 & 430 \\
\hline 9 & 202 & 392 & 795 & 24 & 6,420 & 6,880 & 420 \\
\hline 10 & 207 & 9,400 & 743 & $25 \ldots$ & 5,810 & 3,630 & 400 \\
\hline 11 & 216 & 14,700 & 702 & $26 \ldots$ & 16,400 & 2,600 & 390 \\
\hline 12 & 223 & 5,580 & 670 & 27 & 3,680 & 2,100 & 380 \\
\hline 13 & 221 & 3,080 & 625 & $28 \ldots$ & 1,900 & 1,700 & 370 \\
\hline 14 & 221 & 2,440 & 583 & $29 \ldots$ & 1,240 & 1,430 & 373 \\
\hline \multirow[t]{2}{*}{15} & 220 & 2,580 & 546 & 30 . & 930 & 18,900 & 370 \\
\hline & & & & 31 & $\ldots \ldots$ & 22,300 & 370 \\
\hline \multirow{2}{*}{\multicolumn{5}{|c|}{$\begin{array}{l}\text { Monthly mean discharge, in cubic feet per second } . \\
\text { Runoff, in acre-feet } \ldots \ldots \ldots \ldots \ldots \ldots \ldots \ldots \ldots \ldots\end{array}$}} & 1,384 & 4,613 & 1,048 \\
\hline & & & & & 82,370 & 283,600 & 64,420 \\
\hline
\end{tabular}


Gage height, in feet, and discharge, in cubic feet per second, at indicated time, 1965-66, of Verde River below Tangle Creek, above Horseshoe Dam, Ariz.

\begin{tabular}{|c|c|c|c|c|c|c|c|c|c|c|c|c|}
\hline Date & Hour & $\begin{array}{l}\text { Gage } \\
\text { height }\end{array}$ & $\begin{array}{c}\text { Dis- } \\
\text { charge }\end{array}$ & Date & Hour & $\begin{array}{c}\text { Gage } \\
\text { height }\end{array}$ & $\begin{array}{c}\text { Dis- } \\
\text { charge }\end{array}$ & Date & & Hour & $\begin{array}{l}\text { Gage } \\
\text { height }\end{array}$ & $\begin{array}{c}\text { Dis- } \\
\text { charge }\end{array}$ \\
\hline \multirow[t]{2}{*}{$\overline{\text { Nov. } 22}$} & 2400 & 2.66 & 321 & Dec. 10 & 0300 & 3.30 & 558 & Dec. 2 & & 0600 & 14.70 & 24,500 \\
\hline & & & & & 0500 & 5.00 & 1,480 & & & 1000 & 13.70 & 17,400 \\
\hline \multirow[t]{4}{*}{23} & 1900 & 2.96 & 416 & & 0600 & 6.00 & 2,150 & & & 1300 & 12.85 & 13,500 \\
\hline & 2100 & 3. 38 & 575 & & 0800 & 9. 90 & 4,810 & & & 1900 & 12.08 & 11,000 \\
\hline & 2400 & 3.25 & 522 & & 1000 & 12.10 & 10,600 & & & 2400 & 11.68 & 9,880 \\
\hline & & & & & 1200 & 11.55 & 9,310 & & & & & \\
\hline \multirow[t]{11}{*}{24} & 0400 & 3. 12 & 473 & & 1400 & 11.16 & 8,400 & & 24 & 0500 & 10.68 & 7,600 \\
\hline & 0500 & 9.50 & 5,250 & & 1600 & 12.46 & 11,700 & & & 1000 & 10.78 & 7,820 \\
\hline & 0700 & 11.11 & 7,860 & & 1800 & 13.65 & 16,400 & & & 1300 & 10.42 & 7,120 \\
\hline & 1000 & 11. 10 & 7,840 & & 2000 & 14. 28 & 20,000 & & & 1500 & 9.90 & 6,250 \\
\hline & 1300 & 11.90 & 9,680 & & 2200 & 13.90 & 17,600 & & & 2100 & 8.87 & 4,810 \\
\hline & 1400 & 12.04 & 10,000 & & 2400 & 14.18 & 19,300 & & & 2400 & 8.53 & 4,430 \\
\hline & 1600 & 11.86 & 9,580 & & & & & & & & & \\
\hline & 1800 & 11.25 & 8,130 & 11 & 0300 & 15.00 & 25,600 & & 25 & 1000 & 7. 70 & 3,660 \\
\hline & 2000 & 10.10 & 6,040 & & 0400 & 14. 96 & 25,300 & & & 1500 & 7. 47 & 3,470 \\
\hline & 2400 & 8.77 & 4,400 & & 0500 & 14.80 & 23,800 & & & 2400 & 6.87 & 2,960 \\
\hline & & & & & 0800 & 13. 90 & 17,600 & & & & & \\
\hline \multirow[t]{9}{*}{25} & 0600 & 7.55 & 3,290 & & 1200 & 12.53 & 11,900 & & 29 & 2400 & 5.45 & 1,800 \\
\hline & 1100 & 7.02 & 2,860 & & 1800 & 11. 60 & 9,430 & & & & & \\
\hline & 1300 & 9.00 & 4,650 & & 2400 & 11.00 & 8,040 & & 30 & 0200 & 6.35 & 2,480 \\
\hline & 1600 & 11. 24 & 8,150 & & & & & & & 0300 & 7. 40 & 3,330 \\
\hline & 1800 & 11. 84 & 9,520 & 12 & 0600 & 10.30 & 6,690 & & & 0400 & 9.10 & 5,040 \\
\hline & 2000 & 12.05 & 10,000 & & 1200 & 9.40 & 5,360 & & & 0500 & 10.87 & 7,930 \\
\hline & 2300 & 11.90 & 9,680 & & 1800 & 8.73 & 4,550 & & & 0600 & 12.57 & 12,400 \\
\hline & 2400 & 12.17 & 10,300 & & 2400 & 8. 12 & 3,940 & & & 0800 & 14.07 & 19,400 \\
\hline & & & & & & & & & & 1000 & 14.57 & 23,300 \\
\hline \multirow[t]{10}{*}{26} & 0300 & 13. 76 & 15,900 & 13 & 1200 & 7. 05 & 3,020 & & & 1300 & 14.87 & 25,700 \\
\hline & 0600 & 15.70 & 29,400 & & 2400 & 6. 45 & 2,560 & & & 2400 & 14.77 & 24,900 \\
\hline & 0700 & 15.94 & 31,400 & & & & & & & & & \\
\hline & 0800 & 15.66 & 29,100 & 14 & 1200 & 6. 12 & 2,300 & & 31 & 0100 & 15.13 & 27,700 \\
\hline & 1000 & 14. 98 & 23,700 & & 1900 & 6. 13 & 2,310 & & & 0530 & 15.58 & 31,600 \\
\hline & 1200 & 14.64 & 21,100 & & 2400 & 6. 72 & 2,760 & & & 1000 & 14. 92 & 26,100 \\
\hline & 1600 & 13.00 & 12,900 & & & & & & & 1200 & 14.27 & 20,900 \\
\hline & 2000 & 11.70 & 9,240 & 21 & 2400 & 4. 40 & 1,110 & & & 1800 & 13.68 & 17,000 \\
\hline & 2400 & 10. 24 & 6,360 & & & & & & & 2400 & 12.25 & 11,300 \\
\hline & & & & 22 & 0700 & 4.60 & 1,230 & & & & & \\
\hline \multirow[t]{5}{*}{27} & 0600 & 8.67 & 4,340 & & 0900 & 6.20 & 2,370 & Jan. & 1 & 0500 & 11.38 & 9,090 \\
\hline & 1200 & 7.63 & 3,410 & & 1200 & 9.70 & 5,880 & & & 1000 & 10.71 & 7,580 \\
\hline & 1800 & 7. 07 & 2,960 & & 1500 & 12.08 & 10,900 & & & 1800 & 10.18 & 6,600 \\
\hline & 2400 & 6. 50 & 2,530 & & 1700 & 13.68 & 17,300 & & & 2400 & 9.32 & 5,320 \\
\hline & & & & & 1800 & 14. 78 & 25,200 & & & & & \\
\hline \multirow[t]{2}{*}{28} & 1200 & 5.60 & 1,840 & & 1900 & 15.28 & 29,200 & & 2 & 1200 & 8.10 & 3,950 \\
\hline & 2400 & 5.02 & 1,460 & & 2400 & 16.43 & 39,300 & & & 2400 & 7. 10 & 3,080 \\
\hline ec. & 2400 & 3.06 & 466 & 23 & 0400 & 15.61 & 32,100 & & & & & \\
\hline
\end{tabular}


(131) 9-5095. Reservoir system on Verde River at and below Horseshoe Dam, Ariz.

Location. - This system comprises the two storage reservoirs created by Horseshoe and Bartlett Dams on Verde River: Horseshoe Reservoir, formed by Horseshoe Dam, lat $33^{\circ} 59^{\prime} 15^{\prime}$, long $111^{\circ} 42^{\prime} 45^{\prime \prime}$, in sec. 2, T. 7 N., R. 6 E. (unsurveyed); and Bartlett Reservoir, formed by Bartlett Dam, lat $33^{\circ} 49^{\prime}$, long $111^{\circ} 38^{\prime}$, in sec. 34, T. 6 N. , R. 7 E. (unsurveyed).

Drainage area. - -6, $185 \mathrm{sq} \mathrm{mi,} \mathrm{at} \mathrm{Bartlett} \mathrm{Dam.}$

Gage-height record. - Water-stage recorder graph. Datum of gage on Horsesłoe Reservoir is $1,900.00 \mathrm{ft}$ and on Bartlett Reservoir 1, 599.46 ft above mean sea level, datum of 1929 .

Maxima. --November 1965 to January 1966: Contents, 315, 200 acre-ft Dec. 3C. 1939 to October 1965: Contents, 313,200 acre-ft Apr. 20, 1965.

Remarks. - - Horseshoe Reservoir is formed by earth- and rock-fill dam, completed in November 1945. Bartlett Reservoir is formed by concrete multiple-arch dam, completed in May 1939. Total capacity of the two reservoirs is 317,700 acre-ft, divided as follows: Horseshoe Reservoir, 139, 200 acre-ft at elevation 2, 026. $0 \mathrm{ft}$ (top of spillway gates); Bartlett Reservoir, 178,500 acre-ft at elevation 1, 787. $46 \mathrm{f}^{+}$. (top of spillway gates). Capacity tables furnished by Salt River Valley Water Users' Association.

Contents, in acre-feet, at 2400, 1965-66

\begin{tabular}{r|r|r|r||c|c|c|c}
\hline Day & November & December & January & Day & November & December & January \\
\hline $1 \ldots$ & 15,330 & 91,690 & 307,200 & $16 \ldots$ & 20,680 & 174,900 & 274,500 \\
$2 \ldots$ & 16,610 & 92,200 & 306,700 & $17 \ldots$. & 21,040 & 180,400 & 274,600 \\
$3 \ldots$ & 16,880 & 92,850 & 306,200 & $18 \ldots$ & 21,430 & 184,400 & 274,700 \\
$4 \ldots$ & 17,120 & 93,220 & 304,200 & $19 \ldots$ & 21,820 & 187,300 & 275,000 \\
$5 \ldots$ & 17,390 & 94,560 & 299,400 & $20 \ldots$ & 22,260 & 189,900 & 275,900 \\
$6 \ldots$ & 17,660 & 93,550 & 292,800 & $21 \ldots$ & 22,680 & 192,400 & 276,500 \\
$7 \ldots$ & 17,920 & 93,790 & 286,000 & $22 \ldots$ & 23,200 & 221,100 & 276,300 \\
$8 \ldots$ & 18,220 & 93,870 & 279,100 & $23 \ldots$ & 23,930 & 261,700 & 275,900 \\
$9 \ldots$ & 18,510 & 94,240 & 271,900 & $24 \ldots$ & 34,690 & 275,000 & 275,300 \\
$10 \ldots$ & 18,800 & 112,400 & 266,300 & $25 \ldots$ & 45,200 & 283,200 & 274,600 \\
$11 \ldots$ & 19,040 & 141,700 & 267,800 & $26 \ldots$ & 76,020 & 288,800 & 273,700 \\
$12 \ldots$ & 19,340 & 151,900 & 269,800 & $27 \ldots$ & 83,580 & 292,800 & 272,700 \\
$13 \ldots$ & 19,690 & 158,400 & 271,100 & $28 \ldots$. & 87,090 & 295,100 & 271,200 \\
$14 \ldots$ & 20,040 & 164,000 & 272,300 & $29 \ldots$ & 89,250 & 297,800 & 270,500 \\
$15 \ldots$ & 20,380 & 170,700 & 273,500 & $30 \ldots$ & 90,750 & 315,200 & 269,400 \\
& \\
\hline
\end{tabular}


(132) 9-5100. Verde River below Bartlett Dam, Ariz.

Location. --Lat $33^{\circ} 48^{\prime} 50^{\prime \prime}$, long $111^{\circ} 38^{\prime} 10^{\prime \prime}$, in sec. 34, T. 6 N. , R. 7 E. (unsurveyed), on right bank 0.2 mile downstream from Bartlett Dam, $5 \frac{1}{2}$ miles upstream from Camp Creek, and 18 miles east of town of Cave Creek.

Drainage area. $--6,185 \mathrm{sq} \mathrm{mi}$.

Gage-height record. - -Water-stage recorder graph except 0400 hours Jan. 1 to 1700 hours Jan. 2 for which graph was reconstructed on the basis of adjoining record and records of releases from Bartlett Dam by Salt River Valley Water Users' Association. Altitude of gage is $1,600 \mathrm{ft}$ (from topographic map). Gage heights subsequent to 1600 hours Dec. 30 are for auxiliary gage located 2 miles downstream from base gage. Datum of auxiliary gage is $1,572.34 \mathrm{ft}$ above mean sea level, datum of 1929 .

Discharge record. --Stage-discharge relation defined by current-meter reasurements below $8,800 \mathrm{cf}$ s for base gage and below $32,000 \mathrm{cfs}$ for auxiliary gage.

Maxima. --November 1965 to January 1966: Discharge, 35, 600 cfs 1900 hours Dec. 30 (gage height, $16.95 \mathrm{ft}$ at auxiliary gage).

1888-1938: Discharge, not determined but probably over 150, $000 \mathrm{c}$ s Feb. 24, 1891.

1939 to October 1965: Discharge, 45,800 cfs Mar. 15, 1941 (gage height, $17.5 \mathrm{ft}$ at auxiliary gage).

Remarks. --Flow regulated by Bartlett Reservoir since Feb. 5, 1939, and by Horseshoe Reservoir since Nov. 15, 1945 (combined capacity, 317, 700 acre-ft; sie station 131).

Mean discharge, in cubic feet per second, 1965-66

\begin{tabular}{|c|c|c|c|c|c|c|c|}
\hline Day & November & December & January & Day & November & December & January \\
\hline 1 & 38 & 168 & 7,800 & $16 \ldots$ & 45 & 0 & 179 \\
\hline 2 & 42 & 204 & 4,600 & $17 \ldots$ & 43 & 0 & 360 \\
\hline 3 & 42 & 204 & 3,240 & $18 \ldots$ & 42 & 0 & 528 \\
\hline 4 & 42 & 204 & 3,160 & $19 \ldots$ & 42 & 0 & 411 \\
\hline 5 & 42 & 246 & 3,750 & $20 \ldots$ & 41 & 0 & 200 \\
\hline 6 & 42 & 337 & 4,200 & $21 \ldots$ & 43 & 0 & 286 \\
\hline 7 & 42 & 395 & 4,180 & $22 \ldots$ & 42 & 0 & 548 \\
\hline 8 & 42 & 375 & 4,150 & 23 & 41 & 0 & 630 \\
\hline 9 & 58 & 285 & 4,120 & 24. & 41 & 0 & 695 \\
\hline 10 & 50 & 80 & 3,370 & 25. & 41 & 0 & 752 \\
\hline 11 & 50 & 37 & 182 & $26 \ldots$ & 42 & 0 & 812 \\
\hline 12 & 48 & 36 & 179 & $27 \ldots$ & 42 & 0 & 932 \\
\hline 13 & 47 & 40 & 176 & $28 \ldots \ldots$ & 42 & 75 & 967 \\
\hline 14 & 43 & 37 & 176 & $29 \ldots$ & 42 & 339 & 953 \\
\hline 15 & 46 & 21 & 176 & 30 & 76 & 12,10 า & 600 \\
\hline & & & & 31. & $\ldots \ldots$ & 28,70 ר & 170 \\
\hline \multirow{2}{*}{\multicolumn{5}{|c|}{$\begin{array}{l}\text { Monthly mean discharge, in cubic feet per second } \ldots \\
\text { Runoff, in acre-feet } \ldots \ldots \ldots \ldots \ldots \ldots \ldots \ldots\end{array}$}} & 44.6 & 1,416 & 1,693 \\
\hline & & & & & 2,660 & 87,040 & 104,100 \\
\hline
\end{tabular}


Gage height, in feet, and discharge, in cubic feet per second, at indicated time, 1965-66, of Verde River below Bartlett Dam, Ariz.

\begin{tabular}{|c|c|c|c|c|c|c|c|c|c|c|c|c|}
\hline Date & Hour & $\begin{array}{l}\text { Gage } \\
\text { height }\end{array}$ & $\begin{array}{c}\text { Dis- } \\
\text { charge }\end{array}$ & Date & Hour & $\begin{array}{l}\text { Gage } \\
\text { height }\end{array}$ & $\begin{array}{c}\text { Dis- } \\
\text { charge }\end{array}$ & \multicolumn{2}{|l|}{ Date } & Hour & $\begin{array}{l}\text { Gage } \\
\text { heigh t. }\end{array}$ & $\begin{array}{c}\text { Dis- } \\
\text { charge }\end{array}$ \\
\hline \multirow[t]{5}{*}{ Dec. 28} & 1700 & - & 0 & \multirow[t]{8}{*}{ Dec. 31} & 1100 & 15.85 & 28,000 & Jan. & 2 & 1900 & 6.42 & 3,360 \\
\hline & 1700 & 1. 30 & 118 & & 1200 & 16. 35 & 31,400 & & & 2400 & 6. 39 & 3,240 \\
\hline & 1800 & 1. 78 & 267 & & 1900 & 16.15 & 30,000 & & & & & \\
\hline & 2400 & 1.78 & 267 & & 2100 & 15.45 & 25,400 & & 3 & 1200 & 6.31 & 3,240 \\
\hline & & & & & 2200 & 15.45 & 25,400 & & & 2400 & 6. 37 & 3,240 \\
\hline \multirow[t]{4}{*}{29} & 1700 & 1. 79 & 271 & & 2300 & 13.50 & 14,000 & & & & & \\
\hline & 1730 & 2.27 & 504 & & 2400 & 13.48 & 13,900 & & 4 & 1000 & 6.27 & 3,200 \\
\hline & 2400 & 2. 29 & 514 & & & & & & & 1400 & 6. 11 & 3,020 \\
\hline & & & & \multirow[t]{9}{*}{ Jan. } & 0300 & 13.50 & 14,000 & & & 1700 & 6.23 & 3,150 \\
\hline \multirow[t]{8}{*}{30} & 1000 & 2. 29 & 514 & & 0400 & 12.80 & 11,500 & & & 2400 & 6.21 & 3,130 \\
\hline & 1100 & 5.37 & 4,240 & & 0700 & 12.60 & 10,900 & & & & & \\
\hline & 1200 & 5.39 & 4,270 & & 0900 & 10.00 & 6,870 & & 5 & 1100 & 6. 21 & 3,130 \\
\hline & 1400 & 5. 14 & 3,910 & & 1200 & 8. 50 & 5,380 & & & 1130 & 7. 80 & 4,680 \\
\hline & 1600 & 11.3 & 14,400 & & 2400 & 8.40 & 5,270 & & & 1200 & 7. 40 & 4,300 \\
\hline & 1800 & 16.8 & 34,600 & & & & & & & 2400 & 7. 33 & 4,220 \\
\hline & 1900 & 16.95 & 35,600 & & 1500 & 8.30 & 5,170 & & & & & \\
\hline & 2400 & 16. 4 & 31,800 & & 1700 & 6.60 & 3,530 & & & & & \\
\hline
\end{tabular}

(133) 9-5100.7. West Fork Sycamore Creek above McFarland Canyon, near Sunflower, Ariz.

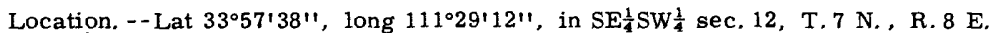
(unsurveyed), in Tonto National Forest, on left bank 0.2 mile upstream frcm McFarland Canyon, 2.7 miles upstream from mouth, and 6.8 miles north of Sunflower.

Drainage area. $--4.58 \mathrm{sq} \mathrm{mi}$.

Gage-height record. - Water-stage recorder graph. Altitude of gage is 4, $380 \mathrm{ft}$ (from topographic map).

Discharge record. - - Stage-discharge relation defined by current-meter measurements below $28 \mathrm{cfs}$ and extended above on basis of weir computation of peak flow.

Maximum. - - November 1965 to January 1966: Discharge, $430 \mathrm{cfs}$ Dec. 22 (ge ge height, $4.45 \mathrm{ft}$ ), from computation of flow over weir.

Gage height, in feet, and discharge, in cubic feet per second, at indicated time, 1965

\begin{tabular}{|c|c|c|c|c|c|c|c|c|c|c|c|}
\hline Date & Hour & $\begin{array}{l}\text { Gage } \\
\text { height }\end{array}$ & $\begin{array}{c}\text { Dis- } \\
\text { charge }\end{array}$ & Date & Hour & $\begin{array}{l}\text { Gage } \\
\text { height }\end{array}$ & $\begin{array}{l}\text { Dis- } \\
\text { charge }\end{array}$ & Date & Hour & $\begin{array}{l}\text { Gage } \\
\text { height }\end{array}$ & $\begin{array}{c}\text { Dis- } \\
\text { charge }\end{array}$ \\
\hline Dec. 21 & 2400 & 2.43 & 8.1 & Dec. 22 & 2400 & 3.78 & 172 & Dec, 29 & $\begin{array}{l}2200 \\
2400\end{array}$ & $\begin{array}{l}2.66 \\
2.81\end{array}$ & $\begin{array}{l}18 \\
27\end{array}$ \\
\hline \multirow[t]{14}{*}{22} & 0300 & 2. 44 & 8.4 & 23 & 0400 & 3. 40 & 91 & & & & \\
\hline & 0500 & 2. 52 & 11 & & 0700 & 3.26 & 78 & 30 & 0200 & 3.05 & 46 \\
\hline & 0700 & 2. 77 & 24 & & 1300 & 3.12 & 54 & & 0300 & 3. 12 & 54 \\
\hline & 0815 & 3.23 & 68 & & 1900 & 2. 99 & 40 & & 0500 & 3.21 & 65 \\
\hline & 0900 & 3. 40 & 91 & & 2400 & 2. 91 & 34 & & 0700 & 3.55 & 120 \\
\hline & 1030 & 3. 68 & 148 & & & & & & 0830 & 3.65 & 141 \\
\hline & 1100 & 3.86 & 194 & 24 & 1100 & 2.76 & 24 & & 1300 & 3.50 & 110 \\
\hline & 1145 & 3. 92 & 217 & & 1600 & 2.76 & 24 & & 1500 & 3. 33 & 81 \\
\hline & 1215 & 3.65 & 141 & & 2400 & 2.72 & 21 & & 1800 & 3.29 & 76 \\
\hline & 1645 & 3.67 & 145 & & & & & & 2400 & 3. 12 & 54 \\
\hline & 1730 & 4.05 & 255 & 28 & 2400 & 2.54 & 12 & & & & \\
\hline & 1845 & 4. 20 & 315 & & & & & 31 & 0600 & 3.03 & 44 \\
\hline & 1900 & 4. 45 & 430 & 29 & 1100 & 2.53 & 11 & & 1800 & 2. 87 & 31 \\
\hline & 2100 & 4.01 & 239 & & 2100 & 2. 62 & 16 & & 2400 & 2.81 & 27 \\
\hline
\end{tabular}


(134) 9-5100.8. West Fork Sycamore Creek near Sunflower, f riz.

Location. - - Lat $33^{\circ} 56^{\prime} 45^{\prime \prime}$, long $111^{\circ} 29^{\prime} 05^{\prime \prime}$, in SE $\frac{1}{4}$ sec. 13, T. 7 N., R. 8 E. , in Tonto National Forest, 1.2 miles upstream from mouth, and 5. 7 miles north of Sunflower.

Drainage area. $--9.80 \mathrm{sq} \mathrm{mi}$.

Gage-height record. - -Water-stage recorder graph except Dec. 24-29 and Jan. 1-14. Altitude of gage is 4,000 ft (from topographic map).

Discharge record. --Stage-discharge relation defined by current-meter masurements below $420 \mathrm{cfs}$ and extended above on basis of slope-area measurement of peak flow. Discharge for periods of no gage-height record estimated.

Maxima. - November 1965 to January 1966: Discharge, 698 cfs 1800 hours Dec. 22 (gage height, $6.75 \mathrm{ft}$ ).

1961 to October 1965: Discharge, $116 \mathrm{cfs}$ Feb. 11, 1963; gage heigl t, $3.29 \mathrm{ft}$ Aug. 22, 1963.

Mean discharge, in cubic feet per second, 1965-66

\begin{tabular}{|c|c|c|c|c|c|c|c|}
\hline Day & November & December & January & Day & November & Decemk er & J anuary \\
\hline 1 & 0 & 0.6 & 50 & 16 & 0 & 7.4 & 2.3 \\
\hline$\ldots$ & 0 & .5 & 35 & $17 \ldots$ & 0 & 9.6 & 2.3 \\
\hline$\ldots$ & 0 & .5 & 25 & $18 \ldots$ & 0 & 8. 1 & 2.8 \\
\hline $4 \ldots$ & 0 & .4 & 20 & $19 \ldots$ & .01 & 7. 7 & 2.8 \\
\hline $5 \ldots$ & 0 & .4 & 15 & $20 \ldots$ & .01 & 10 & 2.6 \\
\hline $6 \ldots$ & 0 & .3 & 10 & $21 \ldots$ & .01 & 14 & 2.3 \\
\hline $7 \ldots$ & 0 & .3 & 9 & $22 \ldots$ & .02 & 264 & 2. 0 \\
\hline 8 & 0 & .3 & 7 & $23 \ldots$ & .7 & 131 & 1.9 \\
\hline 9 & 0 & 1.4 & 6 & $24 \ldots$ & .3 & 50 & 1.9 \\
\hline 10 & 0 & 62 & 5 & $25 \ldots$ & 1.6 & 35 & 1.9 \\
\hline 11 & 0 & 24 & 4 & $26 \ldots$ & 2.2 & 25 & 1.9 \\
\hline 12 & 0 & 10 & 4 & $27 \ldots$ & 1. 7 & 20 & 1.9 \\
\hline 13 & 0 & 7. 7 & 3 & $28 \ldots$ & 1.2 & 15 & 1.7 \\
\hline 14 & 0 & 9.6 & 3 & $29 \ldots$ & .8 & 15 & 1.6 \\
\hline \multirow[t]{2}{*}{$15 \ldots$} & 0 & 9.6 & 2.6 & $30 \ldots$ & .7 & 196 & 1.6 \\
\hline & & & & $31 \ldots$ & $\ldots \ldots$ & 94 & 1.6 \\
\hline \multicolumn{5}{|c|}{$\begin{array}{l}\text { Monthly mean discharge, in cubic feet per second } \\
\text { Runoff, in acre-feet } \ldots \ldots \ldots \ldots \ldots \ldots \ldots\end{array}$} & $\begin{array}{r}0.308 \\
18\end{array}$ & $\begin{array}{r}33.2 \\
2.04 n\end{array}$ & $\begin{array}{r}7.47 \\
460\end{array}$ \\
\hline
\end{tabular}

Gage height, in feet, and discharge, in cubic feet per second, at indicated time, 1965

\begin{tabular}{|c|c|c|c|c|c|c|c|c|c|c|c|}
\hline Date & Hour & \begin{tabular}{|c|} 
Gage \\
height
\end{tabular} & $\begin{array}{c}\text { Dis- } \\
\text { charge }\end{array}$ & Date & Hour & \begin{tabular}{|l} 
Gage \\
height
\end{tabular} & $\begin{array}{c}\text { Dis- } \\
\text { charge }\end{array}$ & Date & Hour & $\begin{array}{c}\text { Gage } \\
\text { height }\end{array}$ & $\begin{array}{c}\text { Dis- } \\
\text { charge }\end{array}$ \\
\hline \multirow[t]{2}{*}{ Dec. 21} & 2400 & 1.69 & 14 & Dec. 22 & 1900 & 6.58 & 656 & Dec. 30 & 0100 & 2.70 & 121 \\
\hline & & & & & 2000 & 6.25 & 610 & & 0200 & 2. 95 & 147 \\
\hline \multirow[t]{13}{*}{22} & 0400 & 1. 72 & 16 & & 2200 & 4.00 & 253 & & 0400 & 3.06 & 159 \\
\hline & 0600 & 1. 95 & 33 & & 2400 & 3. 65 & 218 & & 0600 & 4. 12 & 263 \\
\hline & 0700 & 3.50 & 203 & & & & & & 0730 & 4.60 & 315 \\
\hline & 0830 & 3. 95 & 248 & 23 & 0300 & 3. 30 & 183 & & 1100 & .95 & 246 \\
\hline & 0930 & 4 & 283 & & 0600 & 3.00 & 153 & & 1500 & 35 & 187 \\
\hline & 1030 & & 293 & & 1200 & 2. 67 & 117 & & 1800 & .08 & 160 \\
\hline & 1130 & 4.42 & 295 & & 2400 & 2.36 & 80 & & 2400 & 2. 82 & 134 \\
\hline & 1200 & 4. 75 & 335 & & & & & & & & \\
\hline & 1330 & 4. 32 & 285 & 28 & 2400 & - & 13 & 31 & 0600 & 2.62 & 111 \\
\hline & 1500 & 4.67 & 325 & & & & & & 0900 & 2.50 & 97 \\
\hline & 1600 & 4. 67 & 325 & 29 & 1200 & - & 12 & & 1500 & 2. 40 & 84 \\
\hline & 1700 & 5. 80 & 500 & & 2000 & - & 14 & & 2400 & 2. 23 & 63 \\
\hline & 1800 & 6.75 & 698 & & 2400 & 2.55 & 103 & & & & \\
\hline
\end{tabular}


(135) 9-5101. East Fork Sycamore Creek near Sunflower, Ariz.

Location. - - Lat $33^{\circ} 56^{\prime} 58^{\prime \prime}$, long $111^{\circ} 27^{\prime} 39^{\prime \prime}$, in NE $\frac{1}{4} S E \frac{1}{4}$ sec. 18, T. 7 N. , R. 9 F., in Tonto National Forest, on left bank 1.7 miles upstream from West Fork, and 6 miles north of Sunflower.

Drainage area. $--4.49 \mathrm{sq} \mathrm{mi}$.

Gage-height record. - -Water-stage recorder graph except 1800 hours Dec. 23 to Dec. 29 and Dec. 31 to Jan, 14. Graph reconstructed for Dec. 23. Altitude of gage is 4,020 ft (from topographic map).

Discharge record. --Stage-discharge relation defined by current-meter measurements below $130 \mathrm{cfs}$ and extended above on basis of slope-area measurement of peak flow. Discharge for periods of no gage-height record estimated.

Maxima. - November 1965 to January 1966: Discharge, 330 cfs 1630 hours Dec, 22 (gage height, $5.07 \mathrm{ft}$ ).

1961 to October 1965: Discharge, $30 \mathrm{cfs}$ Sept. 1, 1963 (gage height, 2. $84 \mathrm{ft}$, at site 0.2 mile downstream at different datum).

Mean discharge, in cubic feet per second, 1965-66

\begin{tabular}{|c|c|c|c|c|c|c|c|}
\hline Day & November & December & January & Day & November & December & January \\
\hline 1 & 0 & 0.04 & 20 & $16 \ldots$ & 0 & 3.6 & 1.1 \\
\hline 2 & 0 & .04 & 10 & $17 \ldots$ & 0 & 5.4 & 1.1 \\
\hline 3 & 0 & .04 & 8 & 18 & 0 & 4.3 & 1.3 \\
\hline 4 & 0 & .03 & 6 & 19 & 0 & 4.0 & 1.4 \\
\hline 5 & 0 & .03 & 4 & 20 & 0 & 4.3 & 1.3 \\
\hline 6 & 0 & .03 & 3 & 21 & 0 & 5.4 & 1.2 \\
\hline $7 \ldots$ & 0 & .04 & 2 & 22 & 0 & 134 & 1.0 \\
\hline $8 \ldots$ & 0 & .04 & 2 & $23 \ldots$ & .9 & 53 & 1.0 \\
\hline $9 \ldots$ & 0 & .8 & 2 & $24 \ldots$ & .06 & 30 & 1.0 \\
\hline $10 \ldots$ & 0 & 41 & 1.5 & $25 \ldots$ & 9.0 & 20 & .9 \\
\hline $11 \ldots$ & 0 & 12 & 1.5 & $26 \ldots$ & 1.8 & 15 & .9 \\
\hline 12 & 0 & 3.7 & 1.4 & 27 . & .3 & 10 & .8 \\
\hline 13 & 0 & 2.7 & 1.3 & $28 \ldots$ & .1 & 10 & .8 \\
\hline$\cdots$ & 0 & 4.6 & 1.2 & 29. & .06 & 20 & .8 \\
\hline \multirow[t]{2}{*}{15} & 0 & 4.4 & 1.2 & 30 . & .05 & 84 & .8 \\
\hline & & & & $31 \ldots$ & $\ldots--\cdots$ & 30 & .8 \\
\hline \multicolumn{5}{|c|}{ Monthly mean discharge, in cubic feet per second. . } & 0.409 & 16.2 & 2.62 \\
\hline \multicolumn{5}{|c|}{ Runoff, in acre-feet $\ldots \ldots \ldots \ldots \ldots \ldots \ldots$} & 24 & 997 & 161 \\
\hline
\end{tabular}

Gage height, in feet, and discharge, in cubic feet per second, at indicated time, 1965

\begin{tabular}{|c|c|c|c|c|c|c|c|c|c|c|c|}
\hline Date & Hour & $\begin{array}{l}\text { Gage } \\
\text { height }\end{array}$ & $\begin{array}{c}\text { Dis- } \\
\text { charge }\end{array}$ & Date & Hour & $\begin{array}{l}\text { Gage } \\
\text { height }\end{array}$ & $\begin{array}{l}\text { Dis- } \\
\text { charge }\end{array}$ & Date & Hour & $\begin{array}{c}\text { Gase } \\
\text { height }\end{array}$ & $\begin{array}{c}\text { Dis- } \\
\text { charge }\end{array}$ \\
\hline \multirow[t]{2}{*}{ Dec. 21} & 2400 & 1. 62 & 5.6 & Dec. 22 & 1715 & 4. 81 & 299 & Dec. 29 & 2000 & - & 30 \\
\hline & & & & & 1900 & 4. 30 & 238 & & 2400 & 2. 68 & 52 \\
\hline \multirow[t]{13}{*}{22} & 0200 & 1.64 & 5.9 & & 2000 & 3.95 & 196 & & & & \\
\hline & 0300 & 1.70 & 7.1 & & 2200 & 3.53 & 146 & 30 & 0200 & 2.70 & 54 \\
\hline & 0400 & 1.95 & 14 & & 2400 & 3. 29 & 117 & & 0300 & 2.95 & 78 \\
\hline & 0500 & 2. 23 & 25 & & & & & & 0400 & 3.50 & 142 \\
\hline & 0700 & 3.32 & 120 & 23 & 0200 & 3.07 & 91 & & 0530 & 3.82 & 180 \\
\hline & 0800 & 3.38 & 128 & & 0400 & 2.85 & 68 & & 0630 & 3.74 & 171 \\
\hline & 0845 & 3.36 & 125 & & 0700 & 2.69 & 53 & & 0800 & 3.40 & 130 \\
\hline & 1030 & 3. 50 & 142 & & 1000 & 2. 61 & 47 & & 1115 & 3.00 & 83 \\
\hline & 1145 & 3.40 & 130 & & 2400 & 2. 45 & 36 & & 1430 & 2.77 & 60 \\
\hline & 1330 & 3. 70 & 166 & & & & & & 1900 & 2.65 & 50 \\
\hline & 1430 & 3. 66 & 161 & 28 & 2400 & - & 12 & & 2400 & 2.57 & 44 \\
\hline & 1530 & 4. 15 & 220 & & & & & & & & \\
\hline & 1630 & 5.07 & 330 & 29 & 1200 & - & 10 & & & & \\
\hline
\end{tabular}


(136) 9-5101.5. Sycamore Creek near Sunflower, Ariz.

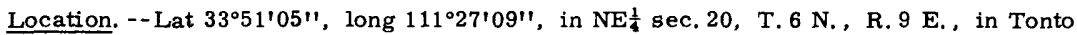
National Forest, on right bank 1. 1 miles upstream from Boulder Creek, 1.2 miles north of Crabtree Butte, and 1.2 miles southeast of Sunflower.

Drainage area. $--53.4 \mathrm{sq} \mathrm{mi}$.

Gage-height record. - -Water-stage recorder graph. Datum of gage is $3,307.9 \mathrm{ft}$ above mean sea level.

Discharge record. --Stage-discharge relation defined by current-meter reasurements below $260 \mathrm{cfs}$ and extended above on basis of slope-area measurements at gage heights 5.7 and $7.90 \mathrm{ft}$.

Maxima. --November 1965 to January 1966: Discharge, 4,800 cfs 1900 hours Dec. 22 (gage height, $7.90 \mathrm{ft}$ ).

1961 to October 1965: Discharge, 1,120 cfs Feb. 11, 1963 (gage height, $5.7 \mathrm{ft}$ ).

Mean discharge, in cubic feet per second, 1965-66

\begin{tabular}{|c|c|c|c|c|c|c|c|}
\hline Day & November & December & January & Day & November & December & January \\
\hline 1 . & 0.1 & 5.9 & 112 & 16 . & 0.2 & 90 & 9.6 \\
\hline $2 \ldots$ & .1 & 5. 9 & 75 & $17 \ldots$ & .2 & 177 & 8.5 \\
\hline $3 \ldots$ & .1 & 5.9 & 56 & $18 \ldots$ & .2 & $n$ & 13 \\
\hline $4 \ldots$ & .1 & 5.9 & 43 & $19 \ldots$ & .2 & 65 & 19 \\
\hline $5 \ldots$ & 1 & 5.9 & 36 & $20 \ldots$ & .2 & 58 & 17 \\
\hline $6 \ldots$ & .1 & 5. 9 & 29 & $21 \ldots$ & .2 & 56 & 14 \\
\hline $7 \ldots$ & .1 & 5.9 & 25 & $22 \ldots$ & .4 & $1,2 \sum 0$ & 11 \\
\hline $8 \ldots$ & 1 & 5.9 & 21 & $23 \ldots$ & 50 & 628 & 11 \\
\hline $9 \ldots$ & 1 & 88 & 19 & $24 \ldots$ & 2.2 & $2 £ 1$ & 10 \\
\hline $10 \ldots$ & .1 & 728 & 16 & $25 \ldots$ & 223 & $16 ?$ & 9.6 \\
\hline $11 \ldots$ & .2 & 156 & 14 & $26 \ldots$ & 41 & 120 & 8.5 \\
\hline $12 \ldots$ & .2 & 59 & 13 & $27 \ldots$ & 13 & $£ 8$ & 8.5 \\
\hline $13 \ldots$ & .2 & 84 & 11 & $28 \ldots \ldots$ & 7.9 & $\varepsilon 4$ & 8.2 \\
\hline $14 \ldots$ & .2 & 162 & 11 & $29 \ldots$ & 6.6 & $\varepsilon 7$ & 7.9 \\
\hline \multirow[t]{2}{*}{$15 \ldots$} & .2 & 176 & 10 & $30 \ldots$ & 6.1 & $5 E 5$ & 7.9 \\
\hline & & & & $31 \ldots$ & $\ldots \ldots$ & 180 & 7. 6 \\
\hline \multirow{2}{*}{\multicolumn{5}{|c|}{$\begin{array}{l}\text { Monthly mean discharge, in cubic feet per second. } \\
\text { Runoff, in acre-feet }\end{array}$}} & 11.8 & 175 & 21.4 \\
\hline & & & & & 701 & 10,740 & 1,310 \\
\hline
\end{tabular}

Gage height, in feet, and discharge, in cubic feet per second, at indicat ad time, 1965

\begin{tabular}{|c|c|c|c|c|c|c|c|c|c|c|c|}
\hline Date & Hour & $\begin{array}{c}\text { Gage } \\
\text { height }\end{array}$ & $\begin{array}{c}\text { Dis- } \\
\text { charge }\end{array}$ & Date & Hour & $\begin{array}{l}\text { Gage } \\
\text { height }\end{array}$ & $\begin{array}{c}\text { Dis- } \\
\text { charge }\end{array}$ & Date & Hour & $\begin{array}{l}\text { Gage } \\
\text { height }\end{array}$ & $\begin{array}{c}\text { Dis- } \\
\text { charge }\end{array}$ \\
\hline Dec. 21 & 2400 & 2.87 & 58 & Dec. 22 & 2400 & 6.00 & 1,400 & Dec. 29 & 1200 & 3,00 & 72 \\
\hline & & & & & & & & & 2400 & 3. 31 & 131 \\
\hline \multirow[t]{14}{*}{22} & 0600 & 3. 10 & 90 & 23 & 0100 & 6. 22 & 1,620 & & & & \\
\hline & 0700 & 4. 70 & 572 & & 0200 & 6.00 & 1,400 & 30 & 0200 & 3. 82 & 251 \\
\hline & 0715 & 6.49 & 1,930 & & 0300 & 5.45 & 985 & & 0400 & 4. 10 & 331 \\
\hline & 0800 & 6.00 & 1,400 & & 0400 & 5.60 & 1,080 & & 0600 & 4. 90 & 670 \\
\hline & 0900 & 5.45 & 985 & & 1100 & 4.50 & 482 & & 0630 & 6.20 & 1,570 \\
\hline & 1130 & 6.30 & 1,720 & & 1800 & 4.20 & 364 & & 0800 & 6.15 & 1,530 \\
\hline & 1330 & 5. 60 & 1,400 & & 2400 & 3.95 & 286 & & 1130 & 4.83 & 630 \\
\hline & 1500 & 6. 42 & 1,860 & & & & & & 1600 & 4.20 & 361 \\
\hline & 1600 & 5. 58 & 1,060 & 24 & 1200 & 3.65 & 180 & & 2400 & 3.85 & 254 \\
\hline & 1800 & 7.00 & 2,670 & & 1800 & 3.80 & 245 & & & & \\
\hline & 1900 & 7. 90 & 4,800 & & 2400 & 3.63 & 200 & 31 & 1200 & 3.56 & 180 \\
\hline & 2000 & 7. 00 & 2,670 & & & & & & 2400 & 3.45 & 157 \\
\hline & 2100 & 6.00 & 1,400 & 28 & 2400 & 3.03 & 80 & & & & \\
\hline & 2230 & 5. 35 & 920 & & & & & & & & \\
\hline
\end{tabular}


(137) 9-5101. 7. Camp Creek near Sunflower, Ariz.

Location. - - Lat $33^{\circ} 45^{\prime} 35^{\prime \prime}$, long $111^{\circ} 29^{\prime} 44^{\prime \prime}$, in SW $\frac{1}{4}$ sec. 24, T. 5 N., R. 8 E., on right bank at upstream side of culvert on State Highway 87, half a mile upstream from mouth, and 7 miles south of Sunflower.

Drainage area. $--2.6 \mathrm{sq} \mathrm{mi}$, approximately.

Gage-height record. - -Water-stage recorder graph except Jan. 2-11. Datum of gage is 2,186. $61 \mathrm{ft}$ above mean sea level (Arizona Highway Department bench mark).

Discharge record. --Stage-discharge relation defined by current-meter measurements below $26 \mathrm{cfs}$ and extended above on basis of computation of flow through culvert at gage height $4.96 \mathrm{ft}$. Discharge for period of no gage-height record estimated.

Maxima. --November 1965 to January 1966: Discharge, 167 cfs 1830 hours Der. 22 (gage height, 2.90 ft).

1963 to October 1965: Discharge, 391 cfs Aug. 16, 1963 (gage height, $4.96 \mathrm{ft}$, from high-water mark in gage well).

\begin{tabular}{|c|c|c|c|c|c|c|c|}
\hline Day & November & December & January & Day & November & December & January \\
\hline $1 \ldots$ & 0 & 0 & 3.5 & $16 \ldots$ & 0 & 5.2 & 0.6 \\
\hline $2 \ldots$ & 0 & 0 & 3 & $17 \ldots$ & 0 & 4. 4 & .4 \\
\hline $3 \ldots$ & 0 & 0 & 2.5 & $18 \ldots$ & 0 & 2. 9 & .8 \\
\hline $4 \ldots$ & 0 & 0 & 2 & $19 \ldots$ & 0 & 1. 3 & .8 \\
\hline $5 \ldots$ & 0 & 0 & 1.5 & $20 \ldots$ & 0 & .8 & .6 \\
\hline $6 \ldots$ & 0 & 0 & 1.5 & $21 \ldots$ & 0 & .5 & .6 \\
\hline $7 \ldots$ & 0 & 0 & 1.5 & $22 \ldots$ & 0 & 54 & .6 \\
\hline $8 \ldots$ & 0 & 0 & 1.5 & $23 \ldots$ & 1.7 & 53 & .8 \\
\hline $9 \ldots$ & 0 & 0 & 1.5 & $24 \ldots$ & 0 & 12 & .8 \\
\hline $10 \ldots$ & 0 & 27 & 1 & $25 \ldots$ & 4.7 & 6.8 & .8 \\
\hline $11 \ldots$ & 0 & 14 & 1 & $26 \ldots$ & 0 & 4.0 & .8 \\
\hline $12 \ldots$ & 0 & 1.8 & 1.0 & $27 \ldots$ & 0 & 3.2 & .8 \\
\hline $13 \ldots$ & 0 & 1.8 & .9 & $28 \ldots$ & 0 & 2.6 & .8 \\
\hline $14 \ldots$ & 0 & 3.2 & .9 & $29 \ldots$ & 0 & 2.1 & .8 \\
\hline $15 \ldots$ & 0 & 13 & .8 & $30 \ldots$ & 0 & 21 & .8 \\
\hline & & & & 31 & $\ldots-\cdots$ & 6. 8 & .7 \\
\hline \multirow{2}{*}{\multicolumn{5}{|c|}{$\begin{array}{l}\text { Monthly mean discharge, in cubic feet per second.. } \\
\text { Runoff, in acre-feet } \ldots \ldots \ldots \ldots \ldots \ldots \ldots \ldots \ldots \ldots\end{array}$}} & 0.21 & \multirow{2}{*}{$\begin{array}{r}7.79 \\
479\end{array}$} & 1.15 \\
\hline & & & & & 13 & & 71 \\
\hline
\end{tabular}

(138) 9-5101.8. Rock Creek near Sunflower, Ariz.

Location. -- Lat $33^{\circ} 43^{\prime} 49^{\prime \prime}$, long $111^{\circ} 30^{\prime} 28^{\prime \prime}$, in $\mathrm{SE}_{4}^{\frac{1}{4}}$ sec. 35, T. $5 \mathrm{~N}$, R. $8 \mathrm{E}$, , on left bank $300 \mathrm{ft}$ upstream from State Highway $87,0.3$ mile upstream from mouth, and 9.9 miles south of Sunflower.

Drainage area. - 15 sq mi, approximately.

Gage-height record. --Water-stage recorder graph except Dec, 24-30, Jan. 4-11. Record for Dec. 30 reconstructed. Datum of gage is $2,051.59 \mathrm{ft}$ above mean sea lovel (Arizona Highway Department bench mark).

Discharge record. - - Stage-discharge relation defined by current-meter measurements below $70 \mathrm{cfs}$ and extended above on basis of slope-area measurements at gage heights $4.49,5.40$, and $6.80 \mathrm{ft}$. Discharge for periods of no gage-height record eatimated.

Maxima. --November 1965 to January 1966: Discharge, 1,900 cfs 1900 hours Dec. 22 (gage height, $6.80 \mathrm{ft}$ ).

1963 to October 1965: Discharge, $916 \mathrm{cfs}$ Aug. 1, 1964 (gage height, 5. $40 \mathrm{ft}$ ). 
Mean discharge, in cubic feet per second, 1965-66, of Rock Creek near Sunflower, Ariz.

\begin{tabular}{|c|c|c|c|c|c|c|c|}
\hline Day & November & December & January & Day & November & December & January \\
\hline 1 & 0 & 0 & 26 & $16 \ldots$ & 0 & 21 & 2.8 \\
\hline $2 \ldots$ & 0 & 0 & 21 & $17 \ldots$ & 0 & 24 & 2.5 \\
\hline $3 \ldots$ & 0 & 0 & 20 & $18 \ldots$ & 0 & 18 & 5. 3 \\
\hline$\ldots$ & 0 & 0 & 17 & $19 \ldots$ & 0 & 15 & 6. 1 \\
\hline $5 \ldots$ & 0 & 0 & 13 & $20 \ldots$ & 0 & 13 & 5.3 \\
\hline $6 \ldots$ & 0 & 0 & 11 & $21 \ldots$ & 0 & 13 & 4. 0 \\
\hline 7 & 0 & 0 & 9 & $22 \ldots$ & 0 & 450 & 3. 6 \\
\hline 8 & 0 & 0 & 8 & 23. & 0 & 240 & 4. $C$ \\
\hline 9 & 0 & 0 & 6 & 24. & 0 & 60 & 3.2 \\
\hline 10 & 0 & 109 & 5 & 25. & 4.3 & 40 & 2.8 \\
\hline 11 & 0 & 54 & 4.5 & $26 \ldots$ & 2.0 & 30 & 2.8 \\
\hline 12 & 0 & 21 & 4.0 & $27 \ldots$ & 0 & 25 & 2.8 \\
\hline $13 \ldots$ & 0 & 18 & 3. 6 & $28 \ldots$ & 0 & 20 & 2. 8 \\
\hline $14 \ldots \ldots$ & 0 & 21 & 3.6 & $29 \ldots$ & 0 & 20 & 2. 8 \\
\hline \multirow{2}{*}{$15 \ldots$} & 0 & 28 & 2.8 & $30 \ldots$ & 0 & 115 & 3.2 \\
\hline & & & & $31 \ldots$ & $\ldots-\ldots$ & 42 & 3.2 \\
\hline \multicolumn{5}{|c|}{ Monthly mean discharge, in cubic feet per second. . } & 0.21 & 45.1 & 6.83 \\
\hline \multicolumn{5}{|c|}{ Runoff, in acre-feet $\ldots \ldots \ldots \ldots \ldots \ldots \ldots$} & 12 & 2,770 & 420 \\
\hline
\end{tabular}

(139) 9-5102. Sycamore Creek near Fort McDowell, Ariz.

Location. - - Lat $33^{\circ} 41^{\prime} 39^{\prime \prime}$, long $111^{\circ} 32^{\prime} 28^{\prime \prime}$, in sec. 16, T. 4 N., R. 8 E. (unsurveyed), in Tonto National Forest, on right bank, 0.7 mile southwest of Sugarloaf Mountain, $8 \frac{1}{2}$ miles northeast of Fort McDowell, 10 miles upstream from mouth, and $25 \frac{1}{2}$ miles northeast of Scottsdale.

Drainage area. --165 sq $\mathrm{mi}$.

Gage-height record. - -Water-stage recorder graph except Dec. 1-9, 2300 hours Dec. 11 to 1700 hours Dec. 14 for which graph was reconstructed on basis of adjoining good record and typical recession for the station. Datum of gage is $1,759.17 \mathrm{ft}$ above mean sea level, datum of 1929 .

Discharge record. --Stage-discharge relation defined by current-meter measurements below $3,600 \mathrm{cfs}$ and by slope-area measurement at $15,800 \mathrm{cfs}$.

Maxima. --November 1965 to January 1966: Discharge, 11, 200 cfs 2030 hours Dec. 22 (gage height, $12.1 \mathrm{ft}$ ).

1959 to October 1965: Discharge, 15, 800 cfs Dec. 25 or 26, 1959 (gage height, 15. $0 \mathrm{ft}$, from floodmarks), from rating curve extended above 1,600 cfs on basis of slope-area measurement of peak flow.

Mean discharge, in cubic feet per second, 1965-66

\begin{tabular}{|c|c|c|c|c|c|c|c|}
\hline Day & November & December & January & Day & November & December & January \\
\hline 1 & 0 & 0.4 & 293 & 16 & 0 & 235 & 40 \\
\hline 2 & 0 & .4 & 210 & 17 & 0 & 310 & 42 \\
\hline 3 & 0 & .3 & 163 & 18 & 0 & 216 & 45 \\
\hline 4 & 0 & .3 & 137 & 19 & 0 & 148 & 53 \\
\hline 5 & 0 & .2 & 120 & $20 \ldots$ & 0 & 110 & 55 \\
\hline$\ldots$ & 0 & .2 & 103 & $21 \ldots$ & 0 & 102 & 49 \\
\hline$\ldots$ & 0 & .2 & 86 & $22 \ldots$ & 0 & 3,030 & 42 \\
\hline $8 \ldots$ & 0 & .2 & 71 & $23 \ldots$ & 7.8 & 2,280 & 42 \\
\hline 9 & 0 & .9 & 63 & $24 \ldots$ & 8.6 & 618 & 40 \\
\hline 10 & 0 & 1,760 & 58 & 25 & 234 & 368 & 40 \\
\hline 11 & 0 & 648 & 53 & 26 & 52 & 263 & 40 \\
\hline 12 & 0 & 170 & 49 & 27 & 6.4 & 190 & 39 \\
\hline 13 & 0 & 90 & 47 & 28 & 1.2 & 157 & 37 \\
\hline 14 & 0 & 200 & 43 & $29 \ldots$ & .8 & 135 & 37 \\
\hline \multirow[t]{2}{*}{$15 \ldots$} & 0 & 448 & 42 & $30 \ldots$ & .5 & 1,240 & 37 \\
\hline & & & & $31 \ldots$ & $---\cdot-$ & 482 & 39 \\
\hline \multicolumn{5}{|c|}{ Monthly mean discharge, in cubic feet per second. } & 10.4 & 426 & 71.5 \\
\hline \multicolumn{5}{|c|}{ Runoff, in acre-feet $\ldots \ldots \ldots \ldots \ldots \ldots \ldots \ldots \ldots \ldots$} & 617 & 26,190 & 4,390 \\
\hline
\end{tabular}


Gage height, in feet, and discharge, in cubic feet per second, at indicated time, 1965-66, of Sycamore Creek near Fort McDowell, Ariz.

\begin{tabular}{|c|c|c|c|c|c|c|c|c|c|c|c|}
\hline Date & Hour & $\begin{array}{l}\text { Gage } \\
\text { height }\end{array}$ & $\begin{array}{c}\text { Dis- } \\
\text { charge }\end{array}$ & Date & Hour & $\begin{array}{c}\text { Gage } \\
\text { height }\end{array}$ & $\begin{array}{c}\text { Dis- } \\
\text { charge }\end{array}$ & Date & Hour & $\begin{array}{l}\text { Gage } \\
\text { height }\end{array}$ & $\begin{array}{c}\text { Dis- } \\
\text { charge }\end{array}$ \\
\hline \multirow[t]{2}{*}{ Dec. 21} & 2400 & 1.55 & 78 & Dec. 23 & 0400 & 6.14 & 3,350 & Dec. 30 & 0330 & $1.8 \mathrm{~g}$ & 182 \\
\hline & & & & & 0600 & 6.02 & 3,230 & & 0500 & 2. $0 \varepsilon$ & 252 \\
\hline \multirow[t]{18}{*}{22} & 0300 & 1. 55 & 78 & & 0700 & 6.24 & 3,450 & & 0700 & 3.15 & 800 \\
\hline & 0600 & 1. 63 & 99 & & 0830 & 5. 39 & 2,600 & & 0800 & 4. $8 \mathrm{C}$ & 2,070 \\
\hline & 0800 & 1.72 & 126 & & 1200 & 4. 59 & 1,880 & & 0900 & $5.7 \varepsilon$ & 2,990 \\
\hline & 0900 & 2. 30 & 340 & & 2000 & 3.85 & 1,290 & & 1000 & 5. $8 \mathrm{C}$ & 3,010 \\
\hline & 1000 & 3.20 & 830 & & 2400 & 3.38 & 950 & & 1100 & 5. $6 \mathrm{C}$ & 2,810 \\
\hline & 1030 & 4.69 & 1,970 & & & & & & 1330 & 4. 45 & 1,770 \\
\hline & 1100 & 7. 14 & 4,450 & 24 & 0600 & 3.00 & 710 & & 1700 & 3. $8 i$ & 1,270 \\
\hline & 1200 & 6.52 & 3,760 & & 1200 & 2.70 & 540 & & 2300 & 3. 12 & 780 \\
\hline & 1630 & 5. 74 & 2,950 & & 1800 & 2. 57 & 470 & & 2400 & 3. 04 & 734 \\
\hline & 1800 & 6.64 & 3,890 & & 2130 & 2.70 & 540 & & & & \\
\hline & 1900 & 7. 94 & 5,410 & & 2400 & 2. 63 & 502 & 31 & 0300 & 2. 65 & 622 \\
\hline & 2000 & 11.1 & 9,690 & & & & & & 1000 & 2. $5 ?$ & 460 \\
\hline & 2030 & 12.1 & 11,200 & 25 & 0400 & 2. 45 & 410 & & 1800 & 2. 41 & 390 \\
\hline & 2100 & 11.6 & 10,400 & & 1200 & 2.33 & 354 & & 2400 & 2. 32 & 349 \\
\hline & 2200 & 10.45 & 8,720 & & 1800 & 2. 25 & 320 & & & & \\
\hline & 2300 & 7. 94 & 5,410 & & 2400 & 2.25 & 320 & Jan. 1 & 1200 & 2. 17 & 288 \\
\hline & 2400 & 7. 14 & 4,450 & & & & & & 2400 & 2. 07 & 248 \\
\hline & & & & 29 & 2400 & 1.83 & 160 & & & & \\
\hline 23 & 0200 & 6.54 & 3,780 & & & & & & & & \\
\hline
\end{tabular}

(140) 9-5113. Verde River near Scottsdale, Ariz.

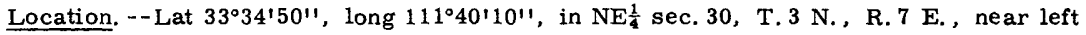
bank on downstream side of bridge on State Highway 87, in Fort McDowell Indian Reservation, 2.5 miles upstream from mouth, 3.8 miles downstream from Fort McDowell, and 16 miles northeast of Scottsdale.

Drainage area. $--6,600 \mathrm{sq} \mathrm{mi}$, approximately.

Gage-height record. - -Water-stage recorder graph, except 2000 hours Dec. 11 to 1800 hours Dec. 14, 1200 hours Dec. 19 to 0700 hours Dec. 22, 1800 hours Dec. 25 to 1130 hours Dec. 27, and 0200-1030 hours Dec. 31 for which graph was reconstructed on basis of indicated recession, peak stage in well, adjoining good record, and records for upstream stations. Datum of gage is $1,351.35 \mathrm{ft}$ above mean sea level, datum of 1929 .

Discharge record. --Stage-discharge relation defined by current-meter measurements below $8,000 \mathrm{cfs}$ and extended above on basis of two poor current-meter measurements that include estimate of about 30 percent of the flow.

Maxima. -- November 1965 to January 1966: Discharge, 31, 300 cfs 1900-2300 hours Dec. 31 (gage height, $12.75 \mathrm{ft}$ ).

1961 to October 1965: Discharge, 8, $770 \mathrm{cfs}$ Apr. 20, 1965 (gage height, 9. $73 \mathrm{ft}$ ).

Remarks. --Flow regulated by Bartlett and Horseshoe Reservoirs (station 131). 
Mean discharge, in cubic feet per second, 1965-66, of Verde $R$ :ver near Scottsdale, Ariz.

\begin{tabular}{|c|c|c|c|c|c|c|c|}
\hline Day & November & December & January & Day & November & Decermber & January \\
\hline $1 \ldots$ & 69 & 78 & 14,000 & $16 \ldots$ & 22 & 138 & 79 \\
\hline $2 \ldots$ & 47 & 168 & 6,280 & $17 \ldots$ & 23 & 90 & 186 \\
\hline $3 \ldots$ & 37 & 190 & 4,040 & $18 \ldots$ & 22 & 95 & 553 \\
\hline $4 \ldots$ & 33 & 197 & 3,290 & $19 \ldots$ & 21 & 40 & 605 \\
\hline $5 \ldots$ & 30 & 201 & 3,430 & $20 \ldots$ & 20 & 30 & 217 \\
\hline $6 \ldots$ & 27 & 268 & 4,510 & $21 \ldots$ & 18 & 20 & 86 \\
\hline $7 \ldots$ & 26 & 335 & 4,500 & $22 \ldots \ldots$ & 21 & 2,050 & 495 \\
\hline $8 \ldots$ & 25 & 360 & 4,420 & $23 \ldots$ & 34 & 2,950 & 760 \\
\hline $9 \ldots$ & 24 & 310 & 4,300 & $24 \ldots$ & 28 & 610 & 880 \\
\hline $10 \ldots$ & 32 & 954 & 4,170 & $25 \ldots$ & 59 & 334 & 922 \\
\hline $11 \ldots$ & 31 & 410 & 889 & $26 \ldots$ & 55 & 210 & 982 \\
\hline $12 \ldots$ & 27 & 100 & 212 & $27 \ldots$ & 37 & 150 & 1,020 \\
\hline $13 \ldots$. & 26 & 70 & 138 & $28 \ldots$ & 34 & 112 & 1,080 \\
\hline $14 \ldots \ldots$ & 25 & 120 & 109 & $29 \ldots$ & 33 & 217 & 1,070 \\
\hline $15 \ldots \ldots$ & 23 & 273 & 92 & $30 \ldots$ & 33 & 2,590 & 1,000 \\
\hline & & & & $31 \ldots$ & $\ldots \ldots$ & 30,270 & 492 \\
\hline \multirow{2}{*}{\multicolumn{5}{|c|}{$\begin{array}{l}\text { Monthly mean discharge, in cubic feet per second... } \\
\text { Runoff, in acre-feet } \ldots \ldots \ldots \ldots \ldots \ldots \ldots \ldots\end{array}$}} & 31.4 & 1,415 & 2,090 \\
\hline & & & & & 1,870 & 87,070 & 128,500 \\
\hline
\end{tabular}

Gage height, in feet, and discharge, in cubic feet per second, at indicated time, 1965-66

\begin{tabular}{|c|c|c|c|c|c|c|c|c|c|c|c|c|}
\hline Date & Hour & $\begin{array}{l}\text { Gage } \\
\text { height }\end{array}$ & $\begin{array}{c}\text { Dis- } \\
\text { charge }\end{array}$ & Date & Hour & $\begin{array}{l}\text { Gage } \\
\text { height }\end{array}$ & $\begin{array}{c}\text { Dis- } \\
\text { charge }\end{array}$ & \multicolumn{2}{|c|}{ Date } & Houn & $\begin{array}{l}\text { Gage } \\
\text { height }\end{array}$ & $\begin{array}{c}\text { Dis- } \\
\text { charge }\end{array}$ \\
\hline Dec. 21 & 2400 & - & 20 & Dec. 25 & 1700 & 2.77 & 286 & Jan. & 1 & 0400 & 11.95 & 20,800 \\
\hline & & & & & 2400 & 2. 70 & 250 & & & 0600 & 80 & 000 \\
\hline \multirow[t]{14}{*}{22} & 0700 & - & 20 & & & & & & & 1000 & 11.38 & 14,700 \\
\hline & 0800 & 2.22 & 116 & 29 & 2400 & 2. 78 & 291 & & & 1300 & 10.55 & 10,400 \\
\hline & 1030 & 2.50 & 201 & & & & & & & 1600 & 9.30 & 7,300 \\
\hline & 1230 & 2.38 & 162 & 30 & 0200 & 3. 09 & 445 & & & 1700 & 9.15 & 6,990 \\
\hline & 1245 & 4. 90 & 1,640 & & 0900 & 3.30 & 550 & & & 2000 & 9.03 & 6,740 \\
\hline & 1300 & 5.76 & 2,540 & & 1000 & 3.53 & 665 & & & 2400 & 8.95 & 6,600 \\
\hline & 1400 & 6.57 & 3,500 & & 1030 & 3. 82 & 832 & & & & & \\
\hline & 1600 & 6.22 & 3,040 & & 1100 & 4. 80 & 1,550 & & 2 & 0600 & 8. 90 & 6,510 \\
\hline & 1830 & 6.44 & 3,320 & & 1200 & 5.91 & 2,620 & & & 1200 & 8.85 & 6,420 \\
\hline & 2100 & 7.28 & 4,550 & & 1330 & 6. 30 & 3,030 & & & 2000 & 8. 79 & 6,310 \\
\hline & 2200 & 8.14 & 5,810 & & 1500 & 6.01 & 2,730 & & & 2100 & 8. 58 & 5,930 \\
\hline & 2330 & 9.67 & 8,540 & & 1600 & 6.46 & 3,230 & & & 2200 & 8. 18 & 5,290 \\
\hline & 2400 & 9.60 & 8,410 & & 1700 & 6.63 & 3,450 & & & 2400 & 7. 77 & 4,690 \\
\hline & & & & & 2000 & 6.86 & 3,780 & & & & & \\
\hline \multirow[t]{8}{*}{23} & 0200 & 8.15 & 5,830 & & 2100 & 7. 24 & 4,330 & & 3 & 0200 & 7. 58 & 4,410 \\
\hline & 0300 & 7. 67 & 5,140 & & 2200 & 7.96 & 5,390 & & & 0800 & 7. 37 & 4,100 \\
\hline & 0530 & 6.85 & 3,920 & & 2300 & 9. 36 & 7,880 & & & 2400 & 7. 08 & 3,680 \\
\hline & 0600 & 6.55 & 3,470 & & 2400 & 11.70 & 17,800 & & & & & \\
\hline & 0900 & 6.57 & 3,500 & & & & & & 4 & 1200 & 6.81 & 3,300 \\
\hline & 1400 & 5.08 & 1,810 & 31 & 0100 & 12.10 & 22,600 & & & 1600 & 6.65 & 3,100 \\
\hline & 2400 & 4. 12 & 1,020 & & 0300 & 12.35 & 25,700 & & & 1930 & 6.53 & 2,970 \\
\hline & & & & & 0500 & 12.50 & 27,800 & & & 2200 & 6.63 & 3,080 \\
\hline \multirow[t]{6}{*}{24} & 0130 & 3.93 & 900 & & 0800 & 12.57 & 28,800 & & & 2400 & 6. 62 & 3,060 \\
\hline & 1100 & 3.37 & 585 & & 1300 & 12.50 & 27,800 & & & & & \\
\hline & 1800 & 3.08 & 440 & & 1700 & 12.70 & 30,600 & & 5 & 0600 & 6.60 & 3,040 \\
\hline & 2130 & 3.03 & 415 & & 1900 & 12.75 & 31,300 & & & 1500 & 6.58 & 3,020 \\
\hline & 2400 & 3.08 & 440 & & 2300 & 12.75 & 31,300 & & & 1700 & 7. 26 & 3,950 \\
\hline & & & & & 2400 & 12.70 & 30,600 & & & 2000 & 7.53 & 4,330 \\
\hline \multirow[t]{2}{*}{25} & 0130 & 3.09 & 445 & & & & & & & 2400 & 7. 57 & 4,380 \\
\hline & 1000 & 2. 87 & 335 & Jan. 1 & 0100 & 12.60 & 29,200 & & & & & \\
\hline
\end{tabular}


(141) 9-5115. Salt River below Granite Reef Dam, Ariz.

(Discontinued gaging station)

Location. -- Lat $33^{\circ} 30^{\prime} 57^{\prime \prime}$, long $11^{\circ} 41^{\prime} 28^{\prime \prime}$, in $\mathrm{SE} \frac{1}{4}$ sec. 13 , T. 2 N., R. 6 E., at Granite Reef Dam, 3.4 miles downstream from Verde River, and 10.7 miles northeast of Mesa city hall.

Drainage area. $--12,900 \mathrm{sq} \mathrm{mi}$, approximately.

Discharge record. --Discharge computed by combining the flow for Salt River below Stewart Mountain Dam and Verde River near Scottsdale, adjusted for flow diverted into canals at Granite Reef Dam. Elevation of crest of dam is $1,310,0 \mathrm{ft}$ above mean sea level.

Maxima. --November 1965 to January 1966: Discharge, 67, 000 cfs 2000 hours Dec, 31. 1888 to October 1965: Discharge, 300,000 cfs Feb. 24, 1891, computed from weir formula for Arizona Dam.

1939 to October 1965: Discharge, about 45, $000 \mathrm{cfs}$ Mar. 14, 1941, estimated from records for Verde River below Bartlett Dam.

Remarks. - - Records of discharge include flow over Granite Reef Dam, but do not include flow in Arizona and South Canals which divert from river immediately above the dam. Flow regulated by reservoirs on the Salt River since 1910 (capacity, 1, 755, 000 acreft; see station 107) and by reservoirs on the Verde River since 1939 (capacity, 317, 700 acre-ft; see station 131).

A gaging station was operated at the former Arizona Dam $2 \frac{1}{2}$ miles upstream from Granite Reef Dam 1888-91 and 1895-96, Records published as "at Arizona Dam" and as "below Verde River."

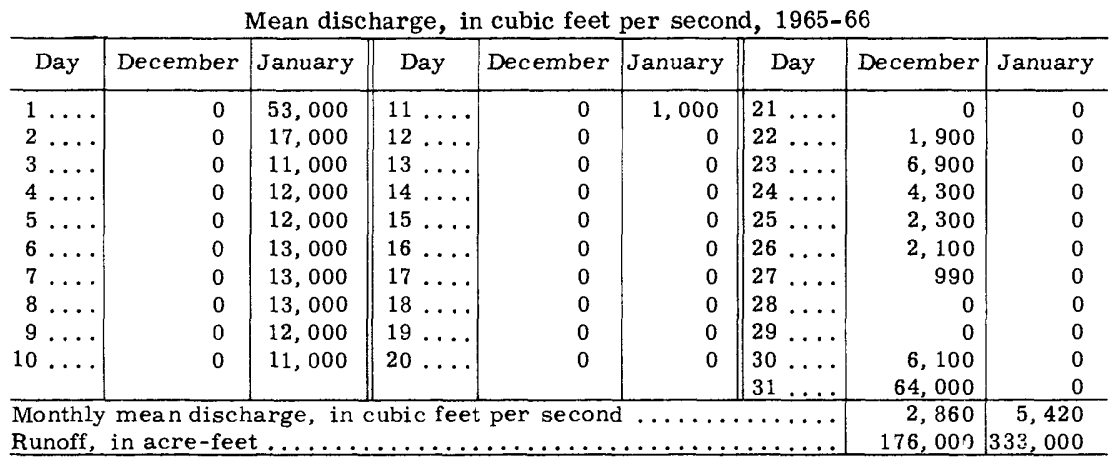


Discharge, in cubic feet per second, at indicated time, 1965-66, of Salt River below

\begin{tabular}{|c|c|c|c|c|c|c|c|c|c|c|c|c|}
\hline Date & Hour & $\begin{array}{c}\text { Dis- } \\
\text { charge }\end{array}$ & Date & Hour & $\begin{array}{c}\text { Dis- } \\
\text { charge }\end{array}$ & Date & Hour & $\begin{array}{c}\text { Dis- } \\
\text { charge }\end{array}$ & \multicolumn{2}{|l|}{ Date } & Hour & $\begin{array}{c}\text { Dis- } \\
\text { charge }\end{array}$ \\
\hline c. 21 & 2400 & 0 & Dec. 24 & 1600 & 4,700 & Dec. 30 & 2100 & 12,000 & Jan. & 1 & 2130 & 57,000 \\
\hline & & & & 1800 & 2,600 & & 2130 & 26,000 & & & 2130 & 46,000 \\
\hline \multirow[t]{12}{*}{22} & 0300 & 80 & & 2230 & 2,500 & & 2200 & 43,000 & & & 2400 & 46,000 \\
\hline & 0900 & 240 & & 2400 & 2,500 & & 2400 & 47,000 & & & & \\
\hline & 1130 & 270 & & & & & & & & 2 & 0330 & 46,000 \\
\hline & 1330 & 200 & 25 & 0230 & 2,500 & 31 & 0100 & 55,000 & & & 0400 & 26,000 \\
\hline & 1345 & 1,700 & & 1100 & 2,300 & & 0200 & 60,000 & & & 0500 & 16,000 \\
\hline & 1400 & 2,600 & & 2400 & 2,200 & & 0400 & 63,000 & & & 0600 & 16,000 \\
\hline & 1500 & 3,500 & & & & & 0600 & 65,000 & & & 0600 & 13,000 \\
\hline & 1700 & 3,000 & 29 & 2400 & 0 & & 0900 & 66,000 & & & 0900 & 11,000 \\
\hline & 1930 & 3,400 & & & & & 1400 & 64,000 & & & 2100 & 11,000 \\
\hline & 2200 & 4,900 & 30 & 0100 & 0 & & 1800 & 66,000 & & & 2300 & 9,900 \\
\hline & 2400 & 8,300 & & 0300 & 60 & & 2000 & 67,000 & & & 2400 & 9,700 \\
\hline & & & & 1000 & 80 & & 2400 & 67,000 & & & & \\
\hline \multirow[t]{9}{*}{23} & 0030 & 9,500 & & 1100 & 180 & & & & & 3 & 0300 & 9,200 \\
\hline & 0100 & 9,300 & & 1130 & 340 & Jan. 1 & 0200 & 65,000 & & & 0900 & 8,800 \\
\hline & 0400 & 6,900 & & 1200 & 1,000 & & 0500 & 56,000 & & & 1200 & 8,700 \\
\hline & 0700 & 7,400 & & 1300 & 2,100 & & 0700 & 54,000 & & & 1300 & 9,600 \\
\hline & 0900 & 7,700 & & 1400 & 2,400 & & 1100 & 50,000 & & & 1330 & 11,000 \\
\hline & 1000 & 7,700 & & 1600 & 2,200 & & 1400 & 45,000 & & & 1400 & 13,000 \\
\hline & 1500 & 6,000 & & 1700 & 2,700 & & 1500 & 44,000 & & & 1500 & 13,000 \\
\hline & 2400 & 5,300 & & 1800 & 2,900 & & 1600 & 48,000 & & & 2400 & 12,000 \\
\hline & & & & 2000 & 3,200 & & 1800 & 47,000 & & & & \\
\hline 24 & 0230 & 5,200 & & 2030 & 12,000 & & 2000 & 58,000 & & & & \\
\hline
\end{tabular}

(142) Salt River at Jointhead Dam, Phoenix, Ariz.

Location. --Lat $33^{\circ} 26^{\prime} 25^{\prime \prime}$, long $111^{\circ} 58^{\prime} 22^{\prime \prime}$, in SW $\frac{1}{4}$ sec. 8, T. 1 N., R. 4 E, , 1, $500 \mathrm{ft}$ upstream from 48 th Street in Phoenix, 1.7 miles downstream from Tempe bridge, and 5.8 miles east of Phoenix Post Office.

Drainage area. $--13,500 \mathrm{sq} \mathrm{mi}$, approximately.

Gage-height record. - -Water-stage recorder graph 1200 hours Dec. 23 to 1900 hours Dec. 28, 0500 hours Dec. 31 to 1200 hours Jan. 11, furnished by the Salt River Valley Water Users' Association. Altitude of gage is 1, $130 \mathrm{ft}$ (from topographic map).

Discharge record. --Stage-discharge relation defined by current-meter measurements below 10,000 cfs and extended above on basis of peak discharge at Granite Reef Dam and Gila River below Gillespie Dam. Discharge for 1900 hours Dec. 28 to 0500 hours Dec. 31 estimated on basis of field estimates and adjoining good record.

Maxima. --November 1965 to January 1966: Discharge, about 66, 000 cfs 2400 hours Dec. 31 (gage height, $10.3 \mathrm{ft}$ ).

1888 to October 1965: Discharge, about 300, 000 cfs Feb. 24, 1891, estimated on basis of records for former station at Arizona Dam.

Remarks. --Large diversions above station for irrigation, municipal, and industrial use. Flow regulated by 4 dams on Salt River (capacity, 1, 755, 000 acre-ft; sfe station 107) and 2 dams on Verde River (capacity, 317, 700 acre-ft; see station 131). 
Mean discharge, in cubic feet per second, 1965-66, of Salt River at Jointhead Dam, Phoenix, Ariz.
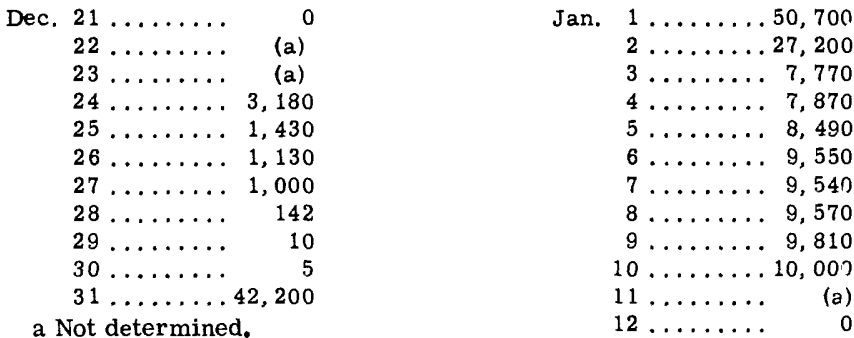

Gage height, in feet, and discharge, in cubic feet per second, at indicated time, 1965-66

\begin{tabular}{|c|c|c|c|c|c|c|c|c|c|c|c|c|}
\hline Date & Hour & $\begin{array}{l}\text { Gage } \\
\text { height }\end{array}$ & $\begin{array}{c}\text { Dis- } \\
\text { charge }\end{array}$ & Date & Hour & $\begin{array}{l}\text { Gage } \\
\text { height }\end{array}$ & $\begin{array}{c}\text { Dis- } \\
\text { charge }\end{array}$ & \multicolumn{2}{|c|}{ Date } & Hour & $\begin{array}{l}\text { Grge } \\
\text { hei. ght }\end{array}$ & $\begin{array}{c}\text { Dis- } \\
\text { charge }\end{array}$ \\
\hline \multirow[t]{6}{*}{$\overline{\text { Dec. } 23}$} & 1200 & 4.6 & 6,750 & Dec. 27 & 1200 & 2.49 & 1,050 & \multirow{6}{*}{\multicolumn{2}{|c|}{ Jan. }} & 0400 & 10.20 & 64,200 \\
\hline & 1300 & 4. 18 & 5,080 & & 1900 & 2. 44 & 1,000 & & & 0800 & 9.60 & 54,500 \\
\hline & 1500 & 4. 10 & 4,800 & & 2100 & 2.33 & 890 & & & 1400 & 9.10 & 46,900 \\
\hline & 1700 & 4. 13 & 4,900 & & \multirow[t]{2}{*}{2400} & \multirow[t]{2}{*}{1.95} & \multirow[t]{2}{*}{550} & & & 2200 & 8. 35 & 36,700 \\
\hline & 2400 & 3.81 & 3,850 & \multirow{7}{*}{28} & & & & & & \multirow[t]{2}{*}{2400} & \multirow[t]{2}{*}{8.80} & \multirow[t]{2}{*}{42,700} \\
\hline & & & & & 0300 & 1.60 & 310 & & & & & \\
\hline \multirow[t]{7}{*}{24} & 0700 & 3.67 & 3,380 & & 1200 & 1. 10 & 74 & \multirow{8}{*}{\multicolumn{2}{|c|}{2}} & 0100 & 9.00 & 45,500 \\
\hline & 0900 & 3.65 & 3,340 & & 1600 & .98 & 41 & & & 0400 & 8.60 & 39,900 \\
\hline & 1100 & 3. 58 & 3,120 & & 1900 & .79 & 16 & & & 0800 & 8.50 & 38,500 \\
\hline & 1500 & 3.50 & 2,940 & & 2400 & .4 & 10 & & & 1100 & 7.50 & 27,500 \\
\hline & 1900 & 3.50 & 2,940 & & & & & & & 1500 & 6.55 & 18,900 \\
\hline & 2400 & 3. 40 & 2,680 & 30 & 2400 & -.10 & 5 & & & 1900 & 6.00 & 14,800 \\
\hline & & & & & & & & & & 2400 & 5,50 & 11,400 \\
\hline \multirow[t]{6}{*}{25} & 0100 & 3.30 & 2,440 & \multirow{10}{*}{31} & 0300 & -.10 & 5 & & & & & \\
\hline & 0400 & 2. 90 & 1,600 & & 0500 & 3.00 & 1,620 & \multirow{7}{*}{\multicolumn{2}{|c|}{3}} & 0700 & 5.00 & 8,600 \\
\hline & 0700 & 2. 77 & 1,380 & & 0700 & 4.50 & 6,300 & & & 1200 & 4. 73 & 7,340 \\
\hline & 1100 & 2.70 & 1,270 & & 0800 & 7.00 & 22,500 & & & 1600 & 4.53 & 6,440 \\
\hline & 2400 & 2.61 & 1,170 & & 0900 & 10.60 & 64,000 & & & 1700 & 4.1 & 4,800 \\
\hline & & & & & 1200 & 10.35 & 63,000 & & & 2000 & 4.5 & 6,300 \\
\hline \multirow[t]{4}{*}{26} & 0600 & 2.60 & 1,160 & & 1500 & 10.20 & 62,000 & & & 2400 & 4.68 & 7,110 \\
\hline & 1200 & 2.58 & 1,140 & & 2100 & 10.20 & 63,000 & & & & & \\
\hline & 2400 & 2.52 & 1,080 & & 2400 & 10.30 & 66,000 & & 4 & 1300 & 4.90 & 8,100 \\
\hline & & & & & & & & & & 2400 & 4.93 & 8,250 \\
\hline
\end{tabular}

(143) 9-5123. Cave Creek near Cave Creek, Ariz.

Location. -- Lat $33^{\circ} 47^{\prime} 07^{\prime \prime}$, long $112^{\circ} 00^{\prime} 24^{\prime \prime}$, in SW $\frac{1}{4}$ sec. 12 , T. 5 N., R. $3 \mathrm{E}$, on left bank $200 \mathrm{ft}$ upstream from power transmission line, $4 \frac{3}{4}$ miles southwest of the town of Cave Creek, and 5.0 miles upstream from Cave Creek Dam.

Drainage area. $--121 \mathrm{sq} \mathrm{mi}$.

Gage-height record. - Water-stage recorder graph except Dec. 11-14, 24-29, Jan. 1-10. Altitude of gage is $1,800 \mathrm{ft}$ (from topographic map).

Discharge record. --Stage-discharge relation defined by current-meter measurements below $720 \mathrm{cfs}$ and extended above on basis of slope-area measurement at gage height $6.79 \mathrm{ft}$ and logarithmic plotting. Discharge for periods of no gage-height record estimated.

Maxima. -- November 1965 to January 1966: Discharge, 6, 000 cfs 1830 hours Dec. 22 (gage height, 6.90 ft).

1958 to October 1965: Discharge, 8,570 cfs Oct. 29, 1959 (gage height, $8.47 \mathrm{ft}$ ).

Remarks. -- All flow stored in Cave Creek Reservoir and released slowly to prevent flooding in Phoenix. 
Mean discharge, in cubic feet per second, 1965-66, of Cave Creek near Cave Creek, Ariz.

\begin{tabular}{|c|c|c|c|c|c|c|c|}
\hline Day & November & December & January & Day & November & December & January \\
\hline $1 \ldots$ & 0 & 0 & 1 & $16 \ldots$ & 0 & 67 & 0 \\
\hline $2 \ldots$ & 0 & 0 & 0 & 17 & 0 & 324 & 0 \\
\hline $3 \ldots$ & 0 & 0 & 0 & $18 \ldots$ & 0 & 101 & 0 \\
\hline $4 \ldots \ldots$ & 0 & 0 & 0 & $19 \ldots$ & 0 & 30 & 0 \\
\hline $5 \ldots$ & 0 & 0 & 0 & $20 \ldots$ & 0 & 8.0 & 0 \\
\hline $6 \ldots$ & 0 & 0 & 0 & $21 \ldots$ & 0 & 5.9 & 0 \\
\hline $7 \ldots$ & 0 & 0 & 0 & $22 \ldots$ & 0 & 886 & 0 \\
\hline $8 \ldots$ & 0 & 0 & 0 & $23 \ldots$ & 0 & 266 & 0 \\
\hline $9 \ldots$ & 0 & 2 & 0 & $24 \ldots$ & 0 & 100 & 0 \\
\hline $10 \ldots$ & 0 & 435 & 0 & $25 \ldots$. & 30 & 20 & 0 \\
\hline $11 \ldots$ & 0 & 95 & 0 & $26 \ldots$ & 8.1 & 1 & 0 \\
\hline $12 \ldots$ & 0 & 2 & 0 & $27 \ldots$ & 0 & 0 & 0 \\
\hline $13 \ldots$ & 0 & 1 & 0 & $28 \ldots \ldots$ & 0 & 0 & 0 \\
\hline $14 \ldots$ & 0 & 45 & 0 & $29 \ldots \ldots$ & 0 & 0 & 0 \\
\hline $15 \ldots$ & 0 & 184 & 0 & 30 . & 0 & 402 & 0 \\
\hline & & & & 31 & $\cdots \cdots$ & 50 & 0 \\
\hline \multirow{2}{*}{\multicolumn{5}{|c|}{$\begin{array}{l}\text { Monthly mean discharge, in cubic feet per second.. } \\
\text { Runoff, in acre-feet } \ldots \ldots \ldots \ldots \ldots \ldots \ldots \ldots \ldots \ldots\end{array}$}} & 1.27 & 97.6 & 0.03 \\
\hline & & & & & 76 & 6,000 & 2 \\
\hline
\end{tabular}

(144) 9-5125. Agua Fria River near Mayer, Ariz.

Location. -- Lat $34^{\circ} 19^{\prime}$, long $112^{\circ} 04^{\prime}$, in NW $\frac{1}{4} \mathrm{SE} \frac{1}{4}$ sec. 20 , T. 11 N. , R. $3 \mathrm{E}$, , on left bank at Sycamore damsite, $700 \mathrm{ft}$ downstream from Bigbug Creek, and 12 miles southeast of Mayer.

Drainage area. --588 sq $\mathrm{mi}$.

Gage-height record. - -Water-stage recorder graph except Nov. 26 to Dec. 1 and Jan. 410. Datum of gage is $3,434 \mathrm{ft}$ (levels by Maricopa County Municipal Water Conservation District No. 1).

Discharge record. --Stage-discharge relation defined by current-meter measurements below 1,600 cfs and extended above on basis of slope-area measurement at gage height $11.97 \mathrm{ft}$. Discharge for periods of no gage-height record estimated.

Maxima. --November 1965 to January 1966: Discharge, 12, 100 cfs 0530 hours Dec. 10 (gage height, $11.65 \mathrm{ft}$ ).

1940 to October 1965: Discharge, 13,000 cfs Mar. 1, 1941 (gage height, 11.97 ft).

Remarks. --All flow stored in Lake Pleasant above Waddell Dam for irrigation.

Mean discharge, in cubic feet per second, 1965-66

\begin{tabular}{|c|c|c|c|c|c|c|c|}
\hline Day & November & December & January & Day & November & December & January \\
\hline 1 & 1.0 & 2.4 & 225 & $16 \ldots$ & 1.0 & 67 & 15 \\
\hline $2 \ldots$ & 1. 0 & 2. 6 & 81 & $17 \ldots$ & 1.0 & 304 & 15 \\
\hline $3 \ldots$ & .9 & 2.9 & 44 & $18 \ldots$ & 1.0 & 72 & 15 \\
\hline $4 \ldots$ & .9 & 3.4 & 30 & $19 \ldots$ & 1.0 & 42 & 15 \\
\hline 5 & .9 & 3.2 & 21 & $20 \ldots$ & 1.0 & 30 & 15 \\
\hline$\ldots$ & .9 & 2.9 & 20 & $21 \ldots$ & 1.0 & 34 & 15 \\
\hline $7 \ldots$ & 1.0 & 3.2 & 18 & $22 \ldots$ & 1.7 & 3,400 & 14 \\
\hline $8 \ldots$ & 1.1 & 3.4 & 17 & $23 \ldots$ & 35 & 1,270 & 14 \\
\hline $9 \ldots$ & 1. 1 & 202 & 17 & $24 \ldots$ & 8.9 & 502 & 14 \\
\hline $10 \ldots$ & 1.3 & 4,290 & 16 & $25 \ldots$ & 865 & 151 & 14 \\
\hline $11 \ldots$ & 1. 4 & 678 & 16 & $26 \ldots$ & 32 & 84 & 14 \\
\hline $12 \ldots$ & 1.4 & 67 & 16 & $27 \ldots$ & 5 & 50 & 13 \\
\hline $13 \ldots$ & 1. 1 & 30 & 16 & $28 \ldots \ldots$ & 3 & 33 & 13 \\
\hline 14 & 1.1 & 61 & 16 & $29 \ldots$ & 3 & 34 & 13 \\
\hline \multirow[t]{2}{*}{$15 \ldots$} & 1.0 & 134 & 16 & 30. & 2.5 & 1,800 & 13 \\
\hline & & & & 31. & $\ldots \ldots$ & 678 & 13 \\
\hline \multirow{2}{*}{\multicolumn{5}{|c|}{$\begin{array}{l}\text { Monthly mean discharge, in cubic feet per second.. } \\
\text { Runoff, in acre-feet } \ldots \ldots \ldots \ldots \ldots \ldots \ldots \ldots \ldots \ldots\end{array}$}} & 32.6 & 453 & 25.6 \\
\hline & & & & & 1,940 & 27,840 & 1,570 \\
\hline
\end{tabular}


(145) 9-5137.8. New River near Rock Springs, Ariz.

Location. -- Lat $33^{\circ} 58^{\prime} 27^{\prime \prime}$, long $112^{\circ} 05^{\prime} 54^{\prime \prime}$, in SW $\frac{1}{4} \mathrm{SW} \frac{1}{4}$ sec. 6, T. 7 N., R. 3 E., on right bank $180 \mathrm{ft}$ upstream from road crossing, and 6 miles southeast of Ro:k Springs.

Drainage area. $--67.3 \mathrm{sq} \mathrm{mi}$.

Gage-height record. - Water-stage recorder graph except Dec, 24-27 and Jan. 6-10. Altitude of gage is 2, $300 \mathrm{ft}$ (from topographic map).

Discharge record. --Stage-discharge relation defined by current-meter measurements below $380 \mathrm{cfs}$ and extended above on basis of slope-area measurements at gage heights $2.6,3.73$, and $6.3 \mathrm{ft}$. Discharge for periods of no gage-height record estimated.

Maxima. --November 1965 to January 1966: Discharge, 4, 020 cfs 1800 hours Dec. 22 (gage height, $5.80 \mathrm{ft}$ ).

1962 to October 1965: Discharge, 4, $900 \mathrm{cfs}$ Aug. 2, 1964 (gage height, 6. $3 \mathrm{ft}$, from floodmarks).

Mean discharge, in cubic feet per second, 1965-66

\begin{tabular}{|c|c|c|c|c|c|c|c|}
\hline Day & November & December & January & Day & November & December & January \\
\hline $1 \ldots$ & 0 & 0 & 100 & $16 \ldots$ & 0 & 146 & 1.6 \\
\hline $2 \ldots$ & 0 & 0 & 72 & $17 \ldots$ & 0 & 545 & .4 \\
\hline $3 \ldots$ & 0 & 0 & 56 & $18 \ldots$ & 0 & 175 & 4.0 \\
\hline $4 \ldots$ & 0 & 0 & 42 & $19 \ldots$ & 0 & 104 & 8.5 \\
\hline $5 \ldots$ & 0 & 0 & 33 & $20 \ldots$ & 0 & 69 & 6.4 \\
\hline $6 \ldots$ & 0 & 0 & 25 & $21 \ldots$ & 0 & 79 & 5.6 \\
\hline $7 \ldots$ & 0 & 0 & 20 & $22 \ldots \ldots$ & 0 & 1,190 & 4.0 \\
\hline $8 \ldots$ & 0 & 0 & 15 & $23 \ldots$ & 0 & 468 & 3.2 \\
\hline $9 \ldots$ & 0 & 0 & 10 & $24 \ldots \ldots$ & 0 & 200 & 2.4 \\
\hline $10 \ldots$ & 0 & 623 & 9 & $25 \ldots$ & 67 & 100 & 1.6 \\
\hline $11 \ldots$ & 0 & 163 & 6.8 & $26 \ldots$ & 24 & 70 & .4 \\
\hline $12 \ldots$ & 0 & 44 & 5.6 & $27 \ldots$ & 10 & 40 & .4 \\
\hline $13 \ldots$ & 0 & 33 & 4.8 & $28 \ldots \ldots$ & 4.4 & 31 & .4 \\
\hline $14 \ldots \ldots$ & 0 & 130 & 4.0 & $29 \ldots$ & 0 & 28 & 0 \\
\hline \multirow[t]{2}{*}{$15 \ldots$} & 0 & 261 & 3.6 & $30 \ldots$ & 0 & 628 & 0 \\
\hline & & & & $31 \ldots$ & $-\cdots---$ & 175 & 0 \\
\hline \multirow{2}{*}{\multicolumn{5}{|c|}{$\begin{array}{l}\text { Monthly mean discharge, in cubic feet per second } . \\
\text { Runoff, in acre-feet } \ldots \ldots \ldots \ldots \ldots \ldots \ldots \ldots \ldots\end{array}$}} & 3.51 & 171 & 14.4 \\
\hline & & & & & 209 & 10,520 & 884 \\
\hline
\end{tabular}

(146) 9-5138. New River at New River, Ariz.

(Formerly published as "near Black Canyon")

Location. -- Lat $33^{\circ} 54^{\prime} 29^{\prime \prime}$, long $112^{\circ} 08^{\prime} 37^{\prime \prime}$, in SW $\frac{1}{4} \mathrm{NE} \frac{1}{4}$ sec. 34, T. 7 N. , R. 2 E. , on right bank 0.3 mile downstream from bridge on State Highway 69, 0.75 mile southwest of village of New River, and 10 miles south of Rock Springs.

Drainage area. $--85.7 \mathrm{sq} \mathrm{mi}$.

Gage-height record.--Water-stage recorder graph except Dec. 26-29 which was reconstructed. Datum of gage is $1,973.16 \mathrm{ft}$ above mean sea level, datum of 1929 .

Discharge record. - Stage-discharge relation defined by current-meter measurements below $1,300 \mathrm{cfs}$ and extended above on basis of slope-area measurements at gage heights 5.57 and $7.33 \mathrm{ft}$.

Maxima. --November 1965 to January 1966: Discharge, 4, 180 cfs 1900 hours Dec. 22 (gage height, $7.50 \mathrm{ft}$ ).

1960 to October 1965: Discharge, 4, 620 cfs Aug. 16, 1963 (gage height, 7. $33 \mathrm{ft}$ ). 
Mean discharge, in cubic feet per second, 1965-66, of New River at New River, Ariz.

\begin{tabular}{|c|c|c|c|c|c|c|c|}
\hline Day & November & December & January & Day & November & Decembar & January \\
\hline 1 & 0 & 0 & 77 & $16 \ldots$ & 0 & $1: 9$ & 5.9 \\
\hline $2 \ldots$ & 0 & 0 & 52 & $17 \ldots$ & 0 & 553 & 5.7 \\
\hline $3 \ldots$ & 0 & 0 & 41 & $18 \ldots$ & 0 & $1 \varepsilon 4$ & 7. 1 \\
\hline $4 \ldots$ & 0 & 0 & 32 & $19 \ldots$ & 0 & 101 & 9.4 \\
\hline $5 \ldots$ & 0 & 0 & 25 & $20 \ldots$ & 0 & 64 & 10 \\
\hline $6 \ldots$ & 0 & 0 & 21 & $21 \ldots$ & 0 & 67 & 8.4 \\
\hline $7 \ldots$ & 0 & 0 & 17 & $22 \ldots$ & 0 & $1,1 \varepsilon 0$ & 7. 1 \\
\hline $8 \ldots$ & 0 & 0 & 14 & $23 \ldots$ & 3.9 & 42.8 & 6. 3 \\
\hline $9 \ldots$ & 0 & 4.1 & 13 & $24 \ldots$ & .2 & $1 \varepsilon 4$ & 5.9 \\
\hline $10 \ldots$ & 0 & 686 & 11 & $25 \ldots$ & 39 & 111 & 5.7 \\
\hline $11 \ldots$ & 0 & 174 & 10 & $26 \ldots$ & 19 & $\varepsilon 0$ & 5.4 \\
\hline $12 \ldots$ & 0 & 40 & 9.2 & $27 \ldots$ & .8 & $E 0$ & 5.4 \\
\hline $13 \ldots$ & 0 & 24 & 8.4 & $28 \ldots$ & .1 & $\Sigma 5$ & 5.4 \\
\hline 14. & 0 & 138 & 7.1 & 29. & 0 & so & 5.4 \\
\hline $15 \ldots$ & 0 & 265 & 6.3 & 30 & 0 & $6 € 0$ & 5.4 \\
\hline & & & & 31 & $\ldots----$ & 144 & 5.2 \\
\hline \multirow{2}{*}{\multicolumn{5}{|c|}{ Monthly mean discharge, in cubic feet per second.. }} & 2.10 & 172 & 14.4 \\
\hline & & & & & 125 & $10,5 \subseteq 0$ & 888 \\
\hline
\end{tabular}

(147) 9-5138. 2. Deadman Wash near New River, Ariz.

(Crest-stage station)

Location. -- Lat $33^{\circ} 50^{\prime} 30^{\prime \prime}$, long $112^{\circ} 08^{\prime} 40^{\prime \prime}$, in NW $\frac{1}{4}$ sec. 27 , T. 6 N., R. 2 E., at State Highway $69,4 \frac{1}{2}$ miles south of New River.

Drainage area. $--11.1 \mathrm{sq} \mathrm{mi}$.

Gage-height record. - Crest stages only. Datum of gage is $1,782.82 \mathrm{ft}$ above mean sea level.

Discharge record. --Stage-discharge relation defined by current-meter me asurements below $60 \mathrm{cfs}$ and extended above on basis of slope-area measurement at gage height $5.92 \mathrm{ft}$.

Maxima. --November 1965 to January 1966: Discharge, $200 \mathrm{cfs}$ Dec. 22 (gage height, $3.41 \mathrm{ft})$.

1959 to October 1965: Discharge, $1,850 \mathrm{cfs}$ Dec. 25, 1959 (gage height, 7.0 ft).

(148) 9-5138. 35. New River at Bell Road near Peoria, Ariz.

\section{(Crest-stage station)}

Location. -- Lat $33^{\circ} 38^{\prime} 18^{\prime \prime}$, long $112^{\circ} 14^{\prime} 22^{\prime \prime}$, in NE $\frac{1}{4}$ NE $\frac{1}{4}$ sec. 3, T. 3 N., R. 1 E. , at Bell Road, 3. 1 miles north of Peoria.

Drainage area. $--187 \mathrm{sq} \mathrm{mi}$.

Gage-height record. --Crest stages only. Datum of gage is 1, 195.00 ft above mean sea level.

Discharge record. --Stage-discharge relation defined by current-meter measurements below 1,800 cfs and extended above on basis of slope-area measurement of peak flow.

Maxima. - November 1965 to January 1966: Discharge, 4, 060 cfs Dec. 22 (gage height, $5.5 \mathrm{ft}$ ).

1963, 1965: Discharge, 1, 550 cfs Aug. 17, 1963. 
(149) 9-5138. 8. Skunk Creek near Phoenix, Ariz.

(Crest-stage station)

Location. --Lat $33^{\circ} 43^{\prime} 40^{\prime \prime}$, long $112^{\circ} 07^{\prime} 10^{\prime \prime}$, in SE $\frac{2}{4}$ sec. 35, T. 5 N., R. 2 E., at State Highway 69, 3 miles north of Adobe, and $8 \frac{1}{2}$ miles north of Phoenix city limits.

Drainage area. $--64.6 \mathrm{sq} \mathrm{mi}$.

Gage-height record. --Crest stages only. Datum of gage is 1, 466. $00 \mathrm{ft}$ above mean sea level.

Discharge record. --Stage-discharge relation defined by current-meter measurements.

Maxima. -- November 1965 to January 1966: Discharge, 280 cfs Dec. 22 (gage height, $1.22 \mathrm{ft}$ ).

1959 to October 1965: Discharge, 11,500 cfs Aug. 1, 1964 (gage height, $4.49 \mathrm{ft}$ ).

(150) 9-5139.7. Agua Fria River at Avondale, Ariz.

(Crest-stage station)

Location. -- Lat $33^{\circ} 26^{\prime} 06^{\prime \prime}$, long $112^{\circ} 19^{\prime} 29^{\prime \prime}$, in NW $\frac{1}{4}$ sec. 14 , T. 1 N., R. 1 W. , at Buckeye Road, half a mile east of Avondale.

Drainage area. $--2,013 \mathrm{sq} \mathrm{mi}$ (including 1,459 $\mathrm{sq} \mathrm{mi}$ above Lake Pleasant that is noncontributing, but not including $247 \mathrm{sq} \mathrm{mi}$ above McMicken Dam from which flow has been diverted into Agua Fria River since 1956).

Gage-height record. --Crest stages only. Datum of gage is $950.00 \mathrm{ft}$ above mean sea level.

Discharge record. --Stage-discharge relation defined by current-meter measurements.

Maxima. --November 1965 to January 1966: Discharge, 800 cfs Dec. 23 (gaร̧e height, $8.89 \mathrm{ft}$ ).

1959 to October 1965: Discharge, 4,700 cfs December 1959 (gage height, $11.0 \mathrm{ft}$ ).

(151) 9-5155. Hassayampa River at Box damsite, near Wickenburg, Ariz.

Location. --Lat $34^{\circ} 02^{\prime} 35^{\prime \prime}$, long $112^{\circ} 42^{\prime} 35^{\prime \prime}$, in $\mathrm{SE} \frac{1}{4}$ sec. 7, T. 8 N., R. 4 W. (unsurveyed), - on right bank at Box damsite, $7 \frac{1}{2}$ miles upstream from Wickenburg.

Drainage area. $--417 \mathrm{sq} \mathrm{mi}$.

Gage-height record. --Water-stage recorder graph except Nov. 29 to Dec. 9 when float was on mud. Datum of gage is $2,236.12 \mathrm{ft}$ above mean sea level, datum of 1929 .

Discharge record. --Stage-discharge relation defined by current-meter measurements below $2,000 \mathrm{cfs}$ and extended above on basis of slope-area measurements at gage heights 9.16 and $18.3 \mathrm{ft}$. Discharge for period of no gage-height record estimated.

Maxima. --November 1965 to January 1966: Discharge, 5, 560 cfs 0530 hours Dec. 10 (gage height, $10.36 \mathrm{ft}$ ).

1938 to October 1965: Discharge, 27, 000 cfs Aug. 29, 1951 (gage height, 18. $3 \mathrm{ft}$ ). 
Mean discharge, in cubic feet per second, 1965-66, of Hassayampa Piver at Box damsite, near Wickenburg, Ariz.

\begin{tabular}{|c|c|c|c|c|c|c|c|}
\hline Day & November & December & January & Day & November & Decerrber & January \\
\hline 1 & 2 & 40 & 300 & 16 & 2 & 210 & 110 \\
\hline $2 \ldots$ & 2 & 30 & 260 & $17 \ldots$ & 2 & 330 & 110 \\
\hline $3 \ldots$ & 2 & 30 & 240 & $18 \ldots$ & 2 & 260 & 100 \\
\hline $4 \ldots$ & 2 & 20 & 200 & $19 \ldots$ & 2 & 220 & 100 \\
\hline $5 \ldots$ & 2 & 20 & 145 & $20 \ldots$ & 2 & 190 & 100 \\
\hline $6 \ldots$ & 2 & 20 & 135 & $21 \ldots$ & 2 & 180 & 100 \\
\hline $7 \ldots$ & 2 & 10 & 130 & $22 \ldots$ & 2 & 1,220 & 100 \\
\hline $8 \ldots$ & 2 & 10 & 130 & $23 \ldots$ & 410 & $£ 10$ & 100 \\
\hline $9 \ldots$ & 2 & 50 & 120 & $24 \ldots$ & 300 & 830 & 90 \\
\hline $10 \ldots$ & 2 & 1,700 & 120 & $25 \ldots$ & 710 & 300 & 90 \\
\hline $11 \ldots$ & 2 & 370 & 120 & $26 \ldots$ & 160 & 240 & 90 \\
\hline $12 \ldots$ & 2 & 140 & 120 & $27 \ldots$ & 81 & 200 & 90 \\
\hline 13. & 2 & 110 & 110 & $28 \ldots$ & 60 & 190 & 90 \\
\hline 14. & 2 & 110 & 110 & $29 \ldots$ & 50 & 180 & 90 \\
\hline \multirow[t]{2}{*}{$15 \ldots$} & 2 & 160 & 110 & $30 \ldots$ & 40 & 980 & 80 \\
\hline & & & & $31 \ldots$ & $\cdots-\cdots$ & 470 & 80 \\
\hline \multirow{2}{*}{\multicolumn{5}{|c|}{ Monthly mean discharge, in cubic feet per second.. }} & 61.8 & $2^{n} 8$ & 125 \\
\hline & & & & & 3,680 & $18,3 า 0$ & 7,680 \\
\hline
\end{tabular}

(152) 9-5170. Hassayampa River near Arlington, Ariz.

(Crest-stage station)

Location. - Lat $33^{\circ} 20^{\prime} 50^{\prime \prime}$, long $112^{\circ} 43^{\prime} 30^{\prime \prime}$, in NW $\frac{1}{4}$ sec. 13, T. 1 S., R. 5 W. , 0.2 mile east of Hassayampa Store, 1.8 miles upstream from mouth, and 2.8 miles northeast of Arlington.

Drainage area. $--1,470 \mathrm{sq} \mathrm{mi}$.

Gage-height record. --Water-stage recorder graph. Datum of gage is $831.91 \mathrm{ft}$ above mean sea level.

Discharge record. --Stage-discharge relation poorly defined by current-moter measurements below 1, $700 \mathrm{cfs}$ and extended above on basis of logarithmic plotting. Stagedischarge relation not adequately defined for determination of daily discharges.

Maxima. --November 1965 to January 1966: Discharge, about 1,600 cfs 1400 hours Dec. 10 (gage height, 4. $35 \mathrm{ft}$ ).

1961 to October 1965: Discharge, 6,500 cfs Aug. 14, 1964 (gage hejght, 6. $05 \mathrm{ft}$ ). 
(153) 9-5195. Gila River below Gillespie Dam, Ariz.

Location. --Lat $33^{\circ} 13^{\prime} 45^{\prime \prime}$, long $112^{\circ} 46^{\prime} 00^{\prime \prime}$, in $\mathrm{SE} \frac{1}{4} \mathrm{NE} \frac{1}{4}$ sec. 28, T. 2 S. , R. 5 W. , at left end of Gillespie Dam, 8 miles downstream from Hassayampa River.

Drainage area. $--49,650 \mathrm{sq} \mathrm{mi}$.

Gage-height record. -- Water-stage recorder graph except 1500 hours Jan, 3 to 1100 hours Jan. 4 for which graph was reconstructed on the basis of Corp of Engineers' telemark readings at Gillespie Dam. Datum of gage is $743.5 \mathrm{ft}$ above mean sea level, datum of 1929.

Discharge record. --Stage-discharge relation defined by current-meter measurements below 46,000 cfs.

Maxima. --November 1965 to January 1966: Discharge, 64, 200 cfs 0030 hours Jan. 2 (gage height, $16.1 \mathrm{ft}$ ).

1921 to October 1965: Discharge observed, 85, $000 \mathrm{cfs} \mathrm{Dec}, 28,1923$ (gage height, 16. $0 \mathrm{ft}$, present datum). The greatest flood since construction of Bartlett Dam on the Verde River in 1939 occurred Mar. 16, 1941, discharge, 45, 800 cfs (gago height, $9.45 \mathrm{ft}$ ).

Greatest discharge known, 250,000 cfs (estimated) February 1891.

Remarks. - Discharge records include flow over crest and through sluice gates of Gillespie Dam but do not include flow in Gila Bend and Enterprise Canals which divert from river immediately above dam. Flow is regulated by San Carlos Reservoir on Gila River (station 45), by a series of reservoirs on Salt River (station 107) and Verde River (station 131), and by Lake Pleasant on Agua Fria River.

\begin{tabular}{|c|c|c|c|c|c|c|c|c|}
\hline Day & December & January & Day & December & January & Day & Decembor & January \\
\hline 1 & 0 & 13,300 & & 574 & 12,000 & 21 & 555 & 20 \\
\hline $2 \ldots$ & 0 & 48,800 & 12 & 300 & 8,780 & $22 \ldots$ & 555 & 20 \\
\hline $3 \ldots$ & 0 & 26,500 & $13 \ldots$ & 68 & 3,130 & $23 \ldots$ & 640 & 20 \\
\hline $4 \ldots$ & 0 & 10,400 & $14 \ldots$ & 63 & 1,410 & $24 \ldots \ldots$ & 910 & 20 \\
\hline $5 \ldots$ & 0 & 9,410 & $15 \ldots$ & 205 & 590 & $25 \ldots \ldots$ & 730 & 20 \\
\hline $6 \ldots$ & 0 & 10,600 & $16 \ldots$ & 255 & 310 & $26 \ldots$ & 730 & 20 \\
\hline 7 & 0 & 11,400 & $17 \ldots$ & 330 & 160 & $27 \ldots \ldots$ & 730 & 20 \\
\hline 8 & 0 & 12,100 & 18 & 330 & 120 & 28 & 760 & 20 \\
\hline 9 & 0 & 11,900 & 19 & 330 & 80 & 29 & 1,030 & 20 \\
\hline \multirow[t]{2}{*}{10} & 12 & 12,000 & 20 & 480 & 20 & 30. & 1,590 & 20 \\
\hline & & & & & & 31. & $1,3 ? 0$ & 20 \\
\hline \multirow{2}{*}{\multicolumn{7}{|c|}{ Monthly mean discharge, in cubic feet per second $\ldots \ldots \ldots \ldots \ldots$}} & 425 & 6,230 \\
\hline & & & & & & & 24,910 & 383,300 \\
\hline
\end{tabular}


Gage height, in feet, and discharge, in cubic feet per second, at indicated time, 1965-66, of Gila River below Gillespie Dam, Ariz.

\begin{tabular}{|c|c|c|c|c|c|c|c|c|c|c|c|}
\hline Date & Hour & $\begin{array}{l}\text { Gage } \\
\text { height }\end{array}$ & $\begin{array}{c}\text { Dis- } \\
\text { charge }\end{array}$ & Date & Hour & $\begin{array}{c}\text { Gage } \\
\text { height }\end{array}$ & $\begin{array}{c}\text { Dis- } \\
\text { charge }\end{array}$ & Date & Hour & $\begin{array}{l}\text { Gage } \\
\text { height }\end{array}$ & $\begin{array}{c}\text { Dis- } \\
\text { charge }\end{array}$ \\
\hline \multirow[t]{2}{*}{ Dec. 31} & 2400 & 10.43 & 1,300 & Jan. 2 & 0800 & 15.65 & 52,600 & \multirow[t]{20}{*}{ Jan. 4} & 0200 & 12.3 & 12,400 \\
\hline & & & & & 1000 & 15.5 & 48,200 & & 0400 & 12. 2 & 11,800 \\
\hline \multirow[t]{14}{*}{ Jan. 1} & 0500 & 10.40 & 1,170 & & 1200 & 15.3 & 45,100 & & 0600 & 12.1 & 11,200 \\
\hline & 1500 & 10.29 & 827 & & 1500 & 15.2 & 43,200 & & 0800 & 12.0 & 10,600 \\
\hline & 1700 & 10.40 & 1,170 & & 1800 & 15.1 & 41,400 & & 1000 & 12.0 & 10,600 \\
\hline & 1730 & 10.50 & 1,530 & & 2400 & 15.05 & 40,600 & & 1200 & 11.99 & 10,500 \\
\hline & 1800 & 12.4 & 13,000 & & & & & & 1400 & 11.91 & 10,100 \\
\hline & 1830 & 14.1 & 27,400 & 3 & 0200 & 15.0 & 39,700 & & 1600 & 11.84 & 9,640 \\
\hline & 1900 & 14.6 & 33,700 & & 0400 & 14.9 & 38,100 & & 1800 & 11. 77 & 9,220 \\
\hline & 1930 & 15.0 & 39,700 & & 0600 & 14.7 & 35,100 & & 2000 & 11.71 & 8,860 \\
\hline & 2000 & 15.3 & 45,100 & & 0800 & 14.5 & 32,300 & & 2200 & 11.66 & 8,560 \\
\hline & 2100 & 15.7 & 53,700 & & 1000 & 14.35 & 30,400 & & 2400 & 11.62 & 8,320 \\
\hline & 2200 & 16.0 & 61,300 & & 1200 & 13.9 & 25,300 & & & & \\
\hline & 2300 & 16.05 & 62,800 & & 1400 & 13.65 & 22,800 & & 0300 & 11.60 & 8,200 \\
\hline & 2400 & 16.09 & 64,200 & & 1600 & 13.25 & 19,200 & & 0800 & 11.70 & 8,800 \\
\hline & & & & & 1800 & 13.0 & 17,200 & & 1100 & 11.80 & 9,400 \\
\hline \multirow[t]{4}{*}{2} & 0030 & 16. 1 & 64,200 & & 2000 & 12. 9 & 16,500 & & 1500 & 11.90 & 10,000 \\
\hline & 0300 & 16.0 & 61,300 & & 2200 & 12.6 & 14,400 & & 1800 & 11.94 & 10,200 \\
\hline & 0500 & 15.85 & 57,400 & & 2400 & 12.45 & 13,400 & & 2400 & 11.98 & 10,500 \\
\hline & 0600 & 15.8 & 56,100 & & & & & & & & \\
\hline
\end{tabular}

(154) 9-5198. Gila River below Painted Rock Dam, Ariz.

Location. --Lat $33^{\circ} 04^{\prime} 30^{\prime \prime}$, long $113^{\circ} 00^{\prime} 50^{\prime \prime}$, in $\mathrm{SE}_{4}^{\frac{1}{4}}$ sec. 18, T. 4 S., R. 7 W. , on left bank a quarter of a mile downstream from Painted Rock Dam, and 19 miles northeast of Sentinel.

Drainage area. $--50,910 \mathrm{sq} \mathrm{mi}$, approximately.

Gage-height record. --Water-stage recorder graph. Datum of gage is $518.69 \mathrm{ft}$ above mean sea level (levels by Corps of Engineers).

Discharge record. --Stage-discharge relation defined by current-meter measurements. Relation indefinite Jan. 2-4 and Mar. 24-31; discharge computed on the basis of gate openings at Painted Rock Dam furnished by Corps of Engineers.

Maxima. --November 1965 to January 1966: Discharge, 2, 850 cfs 1730 hours Jan. 3 (gage height, $8.70 \mathrm{ft}$ ).

1959 to October 1965: Discharge, 240 cfs Jan. 5, 1960 (gage height, $3.26 \mathrm{ft}$ ), but may have been more during period of backwater Jan. 18 to Apr. 11, 1960.

Remarks. -- Regulated at Painted Rock Dam since 1959. 
Mean discharge, in cubic feet per second, 1966, of Gila River below

Painted Rock Dam, Ariz.

\begin{tabular}{|c|c|c|c|c|c|c|c|}
\hline Day & January & February & March & Day & January & February & March \\
\hline 1 & 1.6 & 1,640 & 1,670 & 16 & 1,680 & 1,670 & 1,580 \\
\hline$\cdots$ & 552 & 1,650 & 1,680 & $17 \ldots$ & 1,690 & 1,630 & 1,600 \\
\hline$\ldots$ & 2,480 & 1,640 & 1,640 & $18 \ldots$ & 1,700 & 1,610 & 1,610 \\
\hline $4 \ldots$ & 598 & 1,620 & 1,680 & 19 & 1,680 & 1,600 & 1,570 \\
\hline .. & 622 & 1,610 & 1,700 & $20 \ldots$ & 1,670 & 1,570 & 1,510 \\
\hline $6 \ldots$ & 636 & 1,600 & 1,660 & $21 \ldots$ & 1,630 & 1.610 & 1,570 \\
\hline 7 & 782 & 1,610 & 1,630 & $22 \ldots$ & 1,610 & 1,630 & 1,620 \\
\hline & 1,210 & 1,620 & 1,620 & 23. & 1,580 & 1,610 & 1,590 \\
\hline & 1,230 & 1,630 & 1,620 & 24 & 1,630 & 1,640 & 700 \\
\hline $10 \ldots$ & 1,540 & 1,660 & 1,620 & 25 & 1,680 & 1,660 & 210 \\
\hline 11 . & 1,730 & 1,640 & 1,600 & 26 & 1,700 & 1,640 & 110 \\
\hline 12 & 1,750 & 1,630 & 1,600 & 27 . & 1,680 & 1,620 & 75 \\
\hline 13 & 1,770 & 1,610 & 1,540 & $28 \ldots \ldots$ & 1,640 & 1,630 & 60 \\
\hline 14 & 1,700 & 1,630 & 1,570 & $29 \ldots$ & 1,620 & $\cdots-\cdots$ & 46 \\
\hline \multirow[t]{2}{*}{$15 \ldots$} & 1,680 & 1,680 & 1,600 & $30 \ldots$ & 1,610 & $\cdots \cdots$ & 41 \\
\hline & & & & 31 & 1,610 & $-\cdots--\cdots$ & 39 \\
\hline \multirow{2}{*}{\multicolumn{5}{|c|}{$\begin{array}{l}\text { Monthly mean discharge, in cubic feet per second.. } \\
\text { Runoff, in acre-feet } \ldots \ldots \ldots \ldots \ldots \ldots \ldots \ldots \ldots\end{array}$}} & 1,432 & 1,628 & 1,231 \\
\hline & & & & & 88,640 & 90,430 & 75,670 \\
\hline
\end{tabular}

(155) Gila River at Avenue $51 \mathrm{E}$ near Mohawk, Ariz.

Location. -- Lat $32^{\circ} 47^{\prime} 20^{\prime \prime}$, Iong $113^{\circ} 45^{\prime} 50^{\prime \prime}$, at west line of sec. 25, T. 7 S. , R. 15 W., 5 miles northeast of Mohawk.

Drainage area. -- Not determined.

Gage-height record. --Water-stage recorder graph through Apr. 8. Altitude of gage is $310 \mathrm{ft}$ (from topographic map).

Discharge record. --Furnished by U.S. Bureau of Reclamation; basis for stage-discharge relation unknown. Probably no flow Apr. 9-30.

Maximum. --November 1965 to April 1966: Daily discharge, 1, $208 \mathrm{cfs}$ Mar. 25-26.

Remarks. - Flow completely regulated at Painted Rock Dam. Water released from Painted Rock Dam first reached this station Feb. 3.

Mean discharge, in cubic feet per second, 1966

\begin{tabular}{|c|c|c|c|c|c|c|c|c|}
\hline Day & February & March & Day & February & March & Day & February & March \\
\hline $1 \ldots$ & 0 & 961 & $11 \ldots$ & 730 & 1,156 & $21 \ldots$ & 853 & 1,188 \\
\hline $2 \ldots$ & 0 & 978 & $12 \ldots$ & 745 & 1,162 & $22 \ldots$ & 858 & 1,182 \\
\hline $3 \ldots$ & 53 & 1,002 & $13 \ldots$ & 770 & 1,175 & $23 \ldots$ & 858 & 1,142 \\
\hline $4 \ldots$ & 217 & 1,032 & $14 \ldots$ & 775 & 1,162 & $24 \ldots$ & 875 & 1,156 \\
\hline 5 & 329 & 1,068 & $15 \ldots$ & 775 & 1,162 & $25 \ldots$ & 898 & 1,208 \\
\hline 6 & 401 & 1,092 & $16 \ldots$ & 775 & 1,162 & $26 \ldots$ & 898 & 1,208 \\
\hline 7 & 477 & 1,110 & $17 \ldots$ & 795 & 1,156 & $27 \ldots$ & 904 & 848 \\
\hline & 578 & 1,136 & $18 \ldots$ & 826 & 1,168 & $28 \ldots$ & 921 & 508 \\
\hline & 662 & 1,142 & $19 \ldots$ & 836 & 1,175 & $29 \ldots$ & $-\cdots-\cdots$ & 202 \\
\hline $10 \ldots$ & 696 & 1,142 & $20 \ldots$ & 848 & 1,188 & $30 \ldots$ & $\ldots-\cdots$ & 174 \\
\hline \multirow{2}{*}{\multicolumn{7}{|c|}{ Monthly mean discharge, in cubic feet per second $\ldots \ldots \ldots \ldots \ldots$}} & $\frac{--}{656}$ & $\frac{149}{1.009}$ \\
\hline & & & & & & & 36,440 & 62,070 \\
\hline
\end{tabular}

Mean discharge, in cubic feet per second, April 1966

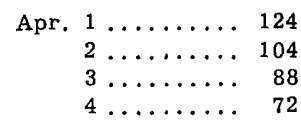

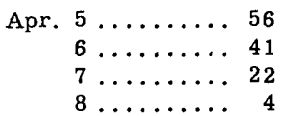


(156) 9-5205. Gila River near Dome, Ariz.

Location. --Lat $32^{\circ} 45^{\prime} 40^{\prime \prime}$, long $11^{\circ} 25^{\prime} 10^{\prime \prime}$, in SW $\frac{1}{4}$ sec. 4, T. 8 S., R. 21 W., on right bank $440 \mathrm{ft}$ upstream from State Highway 95, 3 miles west of Dome, and 12 miles upstream from mouth.

Drainage area. $--57,850 \mathrm{sq} \mathrm{mi}$, approximately.

Gage-height record. --Water-stage recorder graph. Datum of gage is $148.18 \mathrm{ft}$ above mean sea level, datum of 1929 .

Discharge record. --Stage-discharge relation defined by current-meter measurements.

Maxima. --November 1965 to April 1966: Discharge, 618 cfs 1200 hours Mar. 24 (gage height, $8.35 \mathrm{ft}$ ).

1929 to October 1965: Discharge, 20, $700 \mathrm{cfs}$ Feb. 15, 1932 (gage height, 16. $75 \mathrm{ft}$ ).

Greatest flood since 1903 occurred Jan. 22, 1916 (approximate daily discharge, $200,000 \mathrm{cfs})$.

Remarks. - Flow completely regulated at Painted Rock Dam. Water released from Painted Rock Dam first reached this station on Feb. 18.

Mean discharge, in cubic feet per second, 1966

\begin{tabular}{|c|c|c|c|c|c|c|c|}
\hline Day & February & March & April & Day & February & March & April \\
\hline 1 & 3.8 & 348 & 168 & 16 & 4.8 & 575 & 20 \\
\hline $2 \ldots$ & 3.8 & 366 & 124 & $17 \ldots$ & 5.1 & $5: 0$ & 16 \\
\hline $3 \ldots$ & 4.0 & 394 & 106 & $18 \ldots$ & 4.0 & 592 & 13 \\
\hline $4 \ldots$ & 4.3 & 422 & 93 & $19 \ldots$ & 14 & $5 \% 5$ & 11 \\
\hline $5 \ldots$ & 4.7 & 442 & 80 & $20 \ldots$ & 25 & 590 & 9.7 \\
\hline $6 \ldots$ & 5.1 & 460 & 70 & $21 \ldots$ & 42 & 598 & 8.9 \\
\hline $7 \ldots$ & 5.6 & 476 & 69 & $22 \ldots$ & 85 & 595 & 8.2 \\
\hline $8 \ldots$ & 4. 8 & 498 & 69 & $23 \ldots$ & 112 & 635 & 7.4 \\
\hline $9 \ldots$ & 2.4 & 515 & 59 & $24 \ldots$ & 137 & 615 & 6.7 \\
\hline $10 \ldots$ & 4.0 & 528 & 55 & 25. & 182 & 612 & 5.8 \\
\hline 11. & 4.6 & 548 & 42 & 26 & 230 & 592 & 5.2 \\
\hline 12. & 4.7 & 558 & 35 & 27. & 271 & 575 & 4.7 \\
\hline 13. & 5.0 & 560 & 30 & 28. & 316 & 590 & 4.4 \\
\hline 14. & 5.4 & 568 & 25 & $29 \ldots$ & $\ldots \ldots$ & 608 & 4.0 \\
\hline \multirow[t]{2}{*}{$15 \ldots$} & 5.4 & 570 & 23 & $30 \ldots$ & $\ldots \ldots$ & 5.28 & 3.8 \\
\hline & & & & $31 \ldots$ & $\ldots \ldots$ & 284 & $-\ldots-\cdot$ \\
\hline \multirow{2}{*}{\multicolumn{5}{|c|}{$\begin{array}{l}\text { Monthly mean discharge, in cubic feet per second.. } \\
\text { Runoff in acre-feet } \ldots \ldots \ldots \ldots \ldots \ldots \ldots \ldots \ldots\end{array}$}} & 53.4 & 527 & 39.2 \\
\hline & & & & & 2,970 & 32,420 & 2,330 \\
\hline
\end{tabular}




\section{REFERENCES CITED}

Aldridge, B. N. , 1966, Floods of December 1965 to January 1966 in the Salt and Gila Rivers downstream from Granite Reef Dam, Arizona: U.S. Geol. Survey open-file ropt. , 78 p.

Benson, M. A., and Dalrymple, Tate, 1967, General field and office procedvres for indirect discharge measurements: U.S. Geol. Survey Techniques Water-Resc urces Inv. , book 3 , chap. A1, 30 p.

Bodhaine, G. L., 1968, Measurement of peak discharge at culverts by indirect methods: U.S. Geol. Survey Techniques Water-Resources Inv., book 3, chap. A3, E0 p.

Clyma, Wayne, and Shaw, R. J., 1968, Natural recharge in Tucson basin: Frogressive Agriculture in Arizona, v. 20, no. 2, p. 14-15.

Dalrymple, Tate, and Benson, M. A., 1967, Measurement of peak discharge by the slopearea method: U.S. Geol. Survey Techniques Water-Resources Inv., book $乏$, chap. A2, $12 \mathrm{p}$.

Hodges, E. B., and others, 1967, Annual report on ground water in Arizona, spring 1965 to spring 1966: Arizona State Land Dept. Water-Resources Rept. 32, $61 \mathrm{p}$

Hulsing, Harry, 1967, Measurement of peak discharge at dams by indirect methods: U.S. Geol. Survey Techniques Water-Resources Inv., book 3, chap. A5, 29 p.

Matthai, H. F., 1967, Measurement of peak discharge at width contractions by indirect methods: U.S. Geol. Survey Techniques Water-Resources Inv., book 3, chao. A4, 44 p.

Olmstead, F. H. , 1919, Gila River flood control-a report on flood control of the Gila River in Graham County, Arizona: U.S. 65th Cong., 3d sess., Senate Doc. 436, 94 p.

Patterson, J. L., and Somers, W. P., 1966, Magnitude and frequency of floods in the United States, Part 9, Colorado River basin: U.S. Geol. Survey Water-Sipply Paper $1683,475 \mathrm{p}$.

U. S. Army Corps of Engineers, 1966, Flood-damage report on flood of December 1965January 1966, Salt and Gila Rivers, Granite Reef Dam to Gillespie Dam, Arizona: Corps of Engineers, U.S. Army Engineers District, Los Angeles, 33 p.

U.S. Bureau of Reclamation, 1967, Supplement No. 1 to Report on operation of the main outlet drain extension, Minute No. 218 with Mexico: Boulder City, Nev. , 10 p.

U.S. Geological Survey, 1967a, Water resources data for Arizona, 1966-Part 1: Surfacewater records: U.S. Geol. Survey open-file rept., 237 p.

1967b, Water resources data for New Mexico, 1966-Part 1, Surface-wat?r records: U.S. Geol. Survey open-file rept., 262 p.

U.S. Weather Bureau, 1966a, Climatological data, Arizona, November 1965: U. S. Dept. Commerce, v. 69, no. 11, p. 206-223.

1966b, Climatological data, Arizona, December 1965: U.S. Dept. Commerce, v. 69, no. 12 , p. $226-248$.

1966c, Climatological data, New Mexico, November 1965: U.S. Dept. Commerce, v. 69 , no. 11 , p. $192-207$.

1966 d, Climatological data, New Mexico, December 1965; U. S. Dept. Commerce, v. 69, no. 12 , p. 209-230.

1966e, Hourly precipitation data, Arizona, November 1965: U.S. Dept. Commerce, v. 15 , no. 11,4 p. 
1966f, Hourly precipitation data, Arizona, December 1965: U.S. Dept. Commerce, v. 15 , no. 12,7 p.

University of Arizona, 1965, Normal annual precipitation-normal Octob $\mathrm{r}-\mathrm{April}$ precipitation-1931-1960, State of Arizona: Univ. Arizona map. 
Aerial photographs

Agua Caliente Wash tributary near Tucson, Ariz-..-...-.

Agua Fria River at Avondale, Ariz near Mayer, Ariz -............

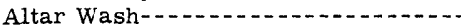

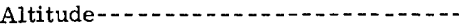
Aravaipa Creek near Mammoth, Ariz

Arivaca, Ariz -..... Arivaca Creek-1...

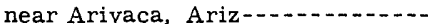

Bartlett Dam $18,22,25$

Bear Creek near Tucson, Ariz ...... Bear Creek (N. Mex.) Beaver Creek basin Benson, Ariz-a

Big Bonita Creek near Fort Apache, Ariz-............ 104

Bill Williams River-............ 11

Black River -..... 17, 27, 28

below pumping plant, near Point of Pines, Ariz-_......... 103

East Fork, North Fork of, near Alpine, Ariz ...............

near Fort Apache, Ariz-..... near Maverick, Ariz -......

Blue River near Clifton, Ariz ...... Bonita Creek near Solomon, Ariz--.Brookbank Canyon near Heber, Ariz -

Camp Creek near Sunflower, Ariz --Camp Verde, Ariz ............ Canada del Oro near Tucson, Ariz---

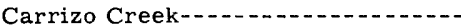
above Corduroy Creek, near Show Low, Ariz near Show Low, Ariz

Carrizo Creek tributary near Show Low, Ariz -

Cataract Creek -

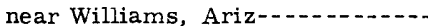

Cave Creek near Cave Creek, Ariz-Chase Creek near Clifton, Ariz -..--

Cherry Creek, near Globe, Ariz .... near Young, Ariz

Chevelon Creek below Wildcat Canyon, near Winslow, Ariz-.................. near Winslow, Ariz Cibecue Creek-...
Page

Cibecue Creek near Chrysotile, A riz- C114 Cibecue No. 2, tributary to Carrizo Creek, near Show Low, A riz- 112

Clear Creek ......... 12

below Willow Creek, near Winslow, Ariz -................ 49

near Winslow, Ariz - 50

Cliff, N. Mex

Clifton, Ariz-... 14

Coolidge Dam -....... 2, 3

Corduroy Creek -

near mouth, near Show Low, A riz- 111

Crookton Wash near Seligman, Ariz - 124

Daily mean discharge -.......... 33

Damages, Clifton, Ariz - .

Graham County, Ariz -....-. 14

Greenlee County, Ariz ....... 14

New Mexico--...... 13

Phoenix flood-......

Safford, Ariz-1.-1 14

Tucson, Ariz--.- 15

Deadman Wash near New River, A riz- 164 Decker Wash near Snowflake, Ariz-- 46 Dirty Neck Canyon near Clints Well, Ariz -..... 141

Dogtown Wash above Kaibab Reservoir, near Williams, Ariz-- 52

Dry Beaver Creek-........... 27

near Rimrock, Ariz-....... 140

Duck Creek at Cliff, N. Mex--.- 56

Eagle Creek -.... 3,11, 13 above pumping plant, near Morenci, Ariz - .

near Double Circle Ranch, near Morenci, Ariz -.... 66

East Verde River-_...... 17, 18, 27, 28 near Childs, Ariz-_.... 146 near Pine, Ariz - 144

Fay Canyon near Flagstaff, Ariz--- 50 Flagstaff, Ariz-......... 10, 11, 12

Gallo Mountains - 3

Geronimo Wash near Tucson, Ariz-- 96 Gila River, at Avenue $51 \mathrm{E}$ near Mohawk, Ariz-_-_-_ 21, 169 at Calva, Arizat head of Safford Valley, near Solomon, Ariz .........13,70 at Kelvin, Ariz 81 at Safford, Ariz 
Page

Gila River, at Winkelman, Ariz-.-- C77 below Blue Creek, near Virden, $\mathrm{N}$. Mex-1............. 57 below Coolidge Dam, Ariz -...--14, 76 below Gillespie Dam, Ariz-_...- 167 below Painted Rock Dam, Ariz --- 168 near Cliff, N. Mex - 56 near Clifton, Ariz-.... 58 near Dome, Ariz-....... 21, 170 near Gila, N. Mex -.... 55 near Laveen, Ariz -........ 83 near Redrock, N. Mex-.... 56

Gila River basin, above Coolidge Dam-12-14 below Salt River -........- 12, 20-22 Coolidge Dam to Salt River-. 12, 14-17 precipitation--...- 6-11 snow-_.......... 7-11 temperatures -... 7

Gillespie Dam -.........20,21

Glenwood, N. Mex....... 13

Gold Creek near Payson, Ariz -..-- 120

Graham County, Ariz ......... 14

Granite Creek near Prescott, Ariz -- 126

Granite Reef Dam-..-- 3, 18, 19, 20, 21, 25

Gravel pits-.... 20

Greenlee County, Ariz - ....... 14

Ground-water recharge-- - - 2 - 29-30

Hannagan Creek near Hannagan Meadow, Ariz-...-.

Hassayampa River -............. at Box damsite, near Wickenburg, Ariz-..-..-near Arlington, Ariz .......

Hell Canyon near Williams, Ariz -

Horseshoe Reservoir-...........-17, 18

Houston Creek near Gisela, Ariz ---- 119

Hull Canyon near Jerome, Ariz -..- 131

Interstate Highway 10 Inundated areas-...-

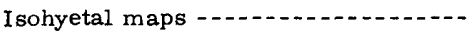

Jointhead Dam-_... 19, 20, 21

Kearny, Ariz-... 15

Kelvin, Ariz - 15

Largest flood known, Salt River-_-_ 25

Little Colorado River -_-_._-_._- 3,12

at Holbrook, Ariz-... 46

near Cameron, Ariz-.... 51

near Hunt, Ariz-....... 41

near Woodruff, Ariz-_..- 45

Little Hollywood, Ariz -.......- 14

Long Lake tributary near Show Low, Ariz-.-...-

Los Robles Wash near Marana, Ariz-.....

Mangas Mountains -

Maricopa Freeway--......-. 22

McNary, Ariz
Page

Mesa, Ariz -... C3, 22

Mogollon Rim -............ 3, 11, 12

Munds Canyon tributary near Sedona,

$$
\text { Ariz - - } 131
$$

New River, at Bell Road near Peoria, Ariz - ..... 164

at New River, Ariz - A 163

near Rock Springs, Ariz--_---- 163

Oak Creek-_....... 6,27 near Cornville, Ariz -...... 11, 132

Pachete Creek at Maverick, Ariz -- 102

Painted Rock Reservoir -..... 20 releases -........ 21

Pantano Wash, at Tucson, Ariz---- 95 near Vail, Ariz-...- 93

Paulden, Ariz-.............. 11

Peak discharges--_-_._-_.-. $31,36-40$

Phoenix, Ariz_...... 3, 12, 20, 22-24, 25 streets - .

Phoenix flood -.......... 2, 12, 18-25 damages -.................. 23, 24 effects--.-...--

inundated areas...... 23

traveltimes -............... 21

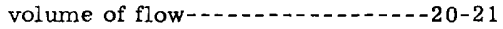

Pima, Ariz - 14

Pima County, Ariz-..- 15

Pima Wash near Tucson, Ariz---.- 96

Precipitation-..... 3-11

Rattlesnake Canyon near Rim rock, Ariz -........ 138

Recharge to ground-water reservoirs--.--

Recurrence intervals --13, 15, 25, 27-28, 32

Red Tank Draw near Rimrock, Ariz - 136

Redrock, N. Mex-_......... 13

Released water, from Bartlett Dam - 18

from Painted Rock Dam -......- 21

from Stewart Mountain Dam-.--- 18

Reservoirs, Cataract Creek-_..- 11

Gila River -

Salt River-........ 12, 17, 18, 19, 25

Verde River-........... 17, 18,25

Rillito Creek-.............. 29

near Tucson, Ariz - .

Rincon Creek near Tucson, Ariz-.- 94

Rock Creek near Sunflower, Ariz -- 155

Roosevelt, Ariz - . - 6

Roosevelt Lake-_-_._-_._-17, 25

Rye Creek near Gisela, Ariz -...-- 120

Sabino Creek near Tucson, Ariz --- 90

Safford, Ariz -

Safford Valley-..... 13

Salt Creek near Peridot, Ariz--.-- 73

Salt River, at Jointhead Dam, Phoenix,

Ariz -.... 19, 160

at Roosevelt, Ariz -

below Granite Reef Dam, Ariz - 19, 159 
Salt River, below Stewart Mountain Dam, Ariz -....- C19, 123 largest known flood--_-_-_-_- 25 near Chrysotile, Ariz -...-. 17, 113 near Roosevelt, Ariz-.---. 17, 28, 118 reservoir system on, at and below Roosevelt Dam, Ariz--.-.- 122

Salt River basin -............ flooding - precipitation-.- 6-11 previous floods - - 25-29 reservoirs - -

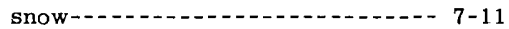
temperatures -

San Carlos, Ariz -... 14

San Carlos Reservoir -........ 13 at Coolidge Dam, Ariz-... 75

San Carlos River near Peridot, Ariz -

San Francisco River -...-. 3,11 at Clifton, Ariz near Alma, N. Mex -... 60 near Glenwood, N. Mex-..... 60 near Reserve, N. Mex-..... 59

San Pedro River -..- 3,14 at Charleston, Ariz at Palominas, Ariz-...- 78 near Redington, Ariz-_..... 79 near Winkelman, Ariz -..... 14, 15, 80 San Simon River near Solomon, Ariz - 71 Santa Catalina Mountains --..-.-.- 14 Santa Cruz River -...-.... 3, 15, 30 at Continental, Ariz -..- 86 at Cortaro, Ariz at Tucson, Ariznear Laveen, Ariz -........ 100 near Lochiel, Ariz-....... 84 near Nogales, Ariz-........ 84

Santa Fe Reservoir -...-_..... 11

Sapillo Creek below Lake Roberts, near Silver City, N. Mex-...- 54

Scottsdale, Ariz - 3, 22

Scour, Salt River channel-....... 20

Senate Document 436

Sewage - -

Show Low Creek, below Jaques Dam, near Show Low, Ariz-.... 42

near Lakeside, Ariz-_-_....- 42

Silver Creek near Snowflake, Ariz--- 44 Skunk Creek near Phoenix, Ariz----- 165 Sky Harbor Airport, Phoenix, Ariz--22, 24 Snow -....................... 7-11

Snow Creek near Mogollon, N. Mex-- 54 Soil moisture-1-_-_-_-_- 7

Solomon, Ariz-..... 14

Sonoita Creek near Patagonia, Ariz-- 85

Station data - - 41-170

Stewart Mountain Dam---_---18, 19

Storage, Salt River system ---- 12, 17, 18

San Carlos Reservoir........... 13

Verde River system -
Page

Storms - -

Sycamore Creek-_.-_-_-_._-_-_- 28

East Fork, near Sunflower, Ariz-- 153

near Fort McDowell, Ariz -...- 156

near Sunflower, Ariz_........ 154

West Fork, above McFarland Canyon, near Sunflower, Ariz -- 151

West Fork, near Sunflower, Ariz - 152

Tam O'Shanter Wash' near Hayden, Ariz -...... 81

Tanque Verde Creek -............- 15 at Tucson, Ariz near Tucson, Ariz -....... 89 Tanque Verde Mountains -...... 15 Tempe, Ariz-... 3,22 Temperatures--.... 7, 10 Theodore Roosevelt Lake-_...... 17 Tonto Creek - above Gun Creek, near Roosevelt, Ariz - 17, 18, 28, 121 near Gisela, Ariz-....... 119 Tortilla Creek at Tortilla Flat, Ariz- 123 Transportation facilities -..... 22 Traveltimes -.......... 21 Tucson, Ariz Tucson Arroyo at Vine Avenue, Tucson, Ariz -..... 88

Tularosa River near Reserve, N. Mex- 59

Ventana Canyon Wash near Tucson, Ariz - 93

Verde River ............... 3,11, 18 at Childs, near Camp Verde, Ariz - 144 below Bartlett Dam, Ariz-.....- 150 below Tangle Creek, above Horseshoe Dam, Ariz--.-- 17-18, 147 near Clarkdale, Ariz-...... 11, 129 near Paulden, Ariz -........ 126 near Scottsdale, Ariz -..... 19, 157

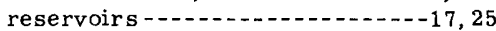
reservoir system on, at and below Horseshoe Dam, Ariz-...- 149

Virden, N. Mex-13

Volume of runoff -........... 21

Volunteer Wash near Bellemont, Arj z- 128

Webber Creek above West Fcrk Webber Creek, near Pine, Ariz -....... 145

Wellton-Mohawk a rea -..-- 29,30

Irrigation and Drainage District-- 21

We st Cataract Creek near Williars, Ariz - 51

We st Clear Creek near Camp Verde, Ariz--...... 142

Wet Beaver Creek near Rimrock, Ariz -.... 134

White Mountains-..... 3, 7 snow depths water content of snow 10 


\begin{tabular}{|c|c|c|c|}
\hline & Page & & Page \\
\hline $\begin{array}{l}\text { hite River, East Fork, near Fort } \\
\text { Apache, Ariz- }\end{array}$ & C108 & $\begin{array}{l}\text { Williamson Valley Wash near Paulden, } \\
\text { Ariz }\end{array}$ & C124 \\
\hline near Fort Apache, Ariz & 109 & Willow Creek & 13 \\
\hline North Fork, near Greer, Ariz---- & 107 & near Double Circle Ranch, near & \\
\hline $\begin{array}{l}\text { North Fork, near McNary, Ariz-- } \\
\text { North Fork tributary, near White }\end{array}$ & 107 & $\begin{array}{l}\text { Morenci, Ariz } \\
\text { near Point of Pines, near Morenci, }\end{array}$ & 65 \\
\hline River, Ariz- & 108 & Ariz $\ldots$ & 63 \\
\hline
\end{tabular}




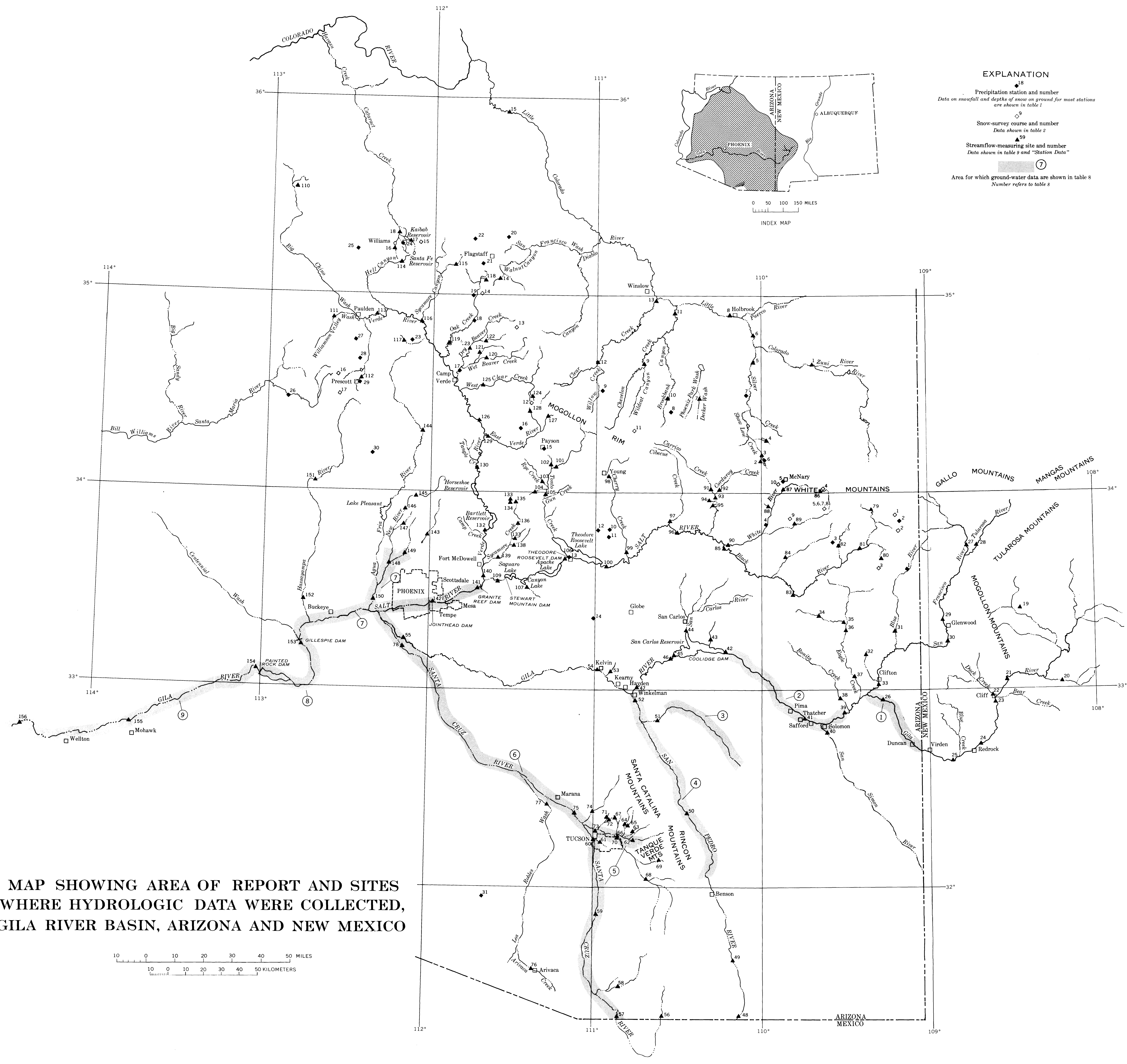



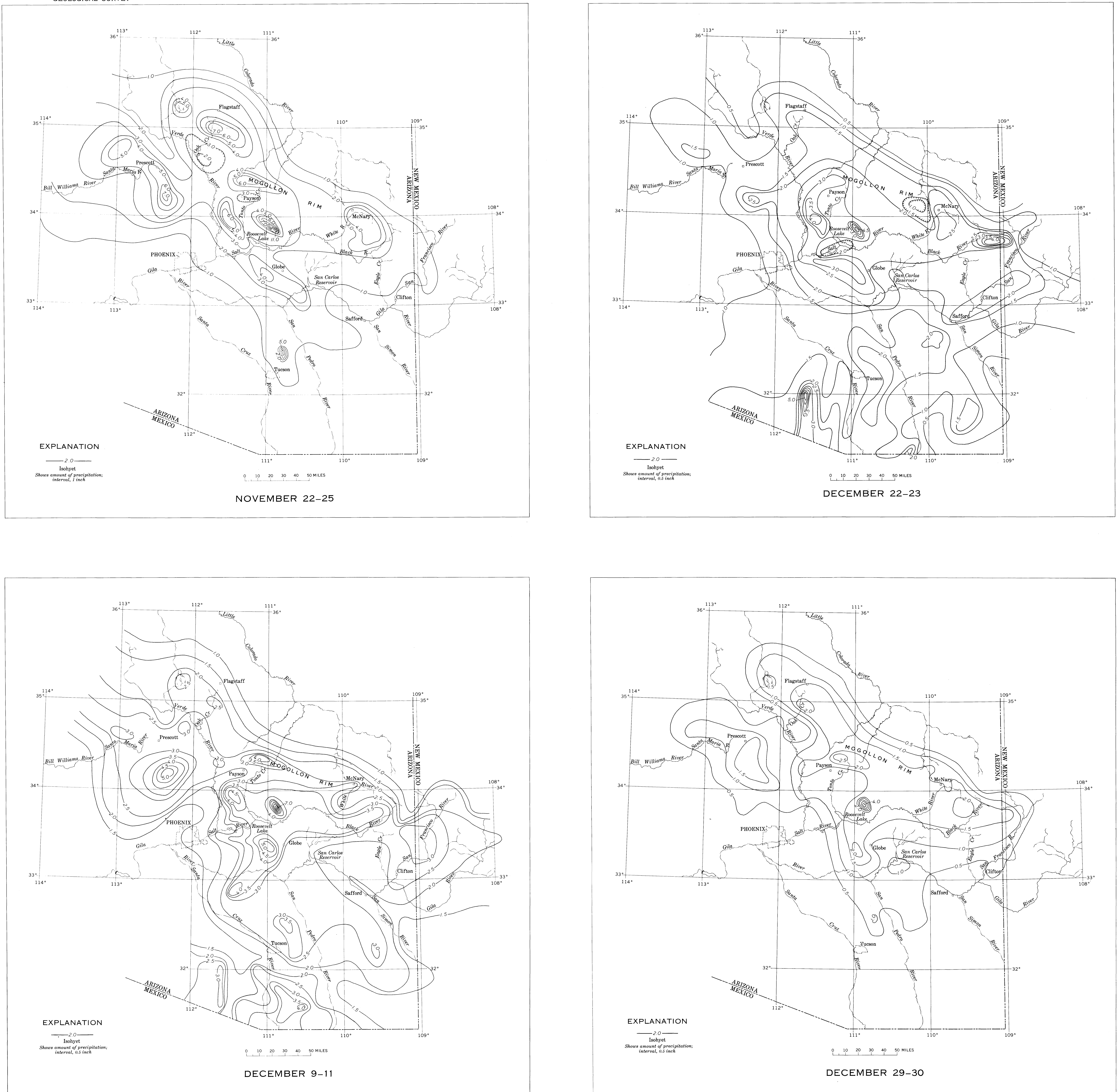

MAPS SHOWING PRECIPITATION, NOVEMBER AND DECEMBER 1965, IN THE GILA RIVER BASIN, ARIZONA AND NEW MEXICO 


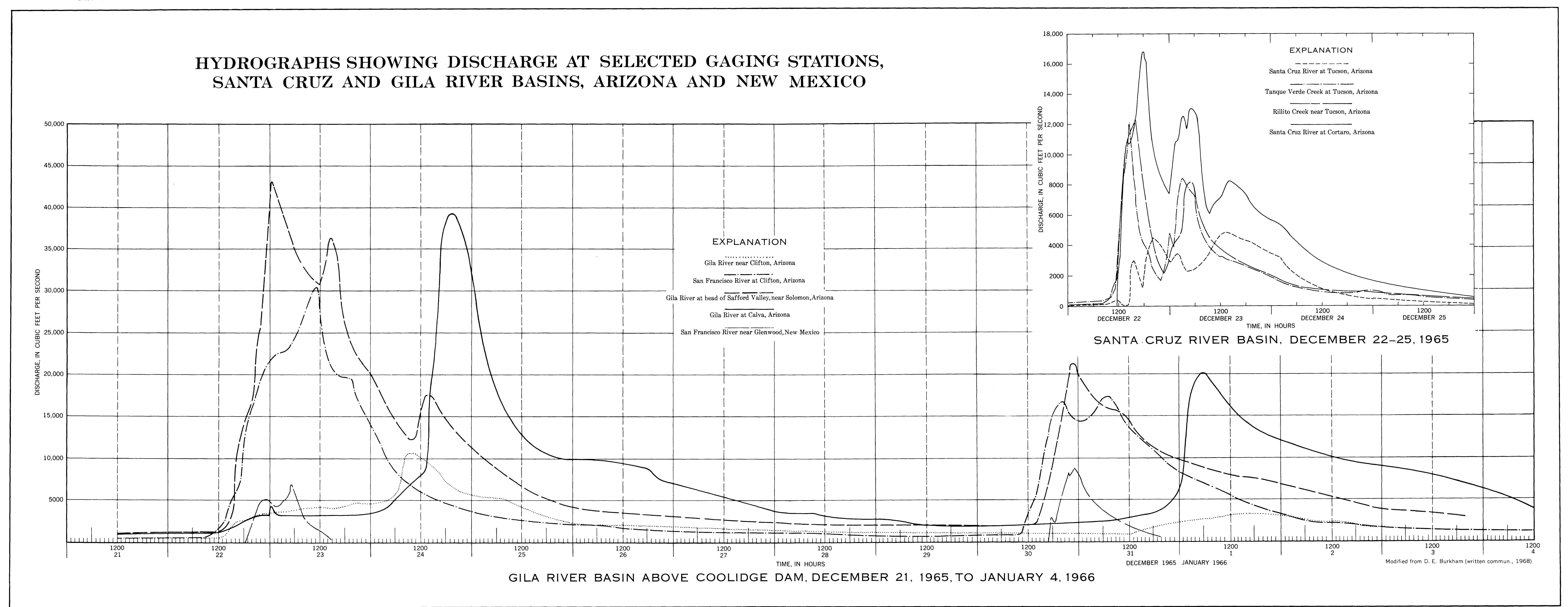




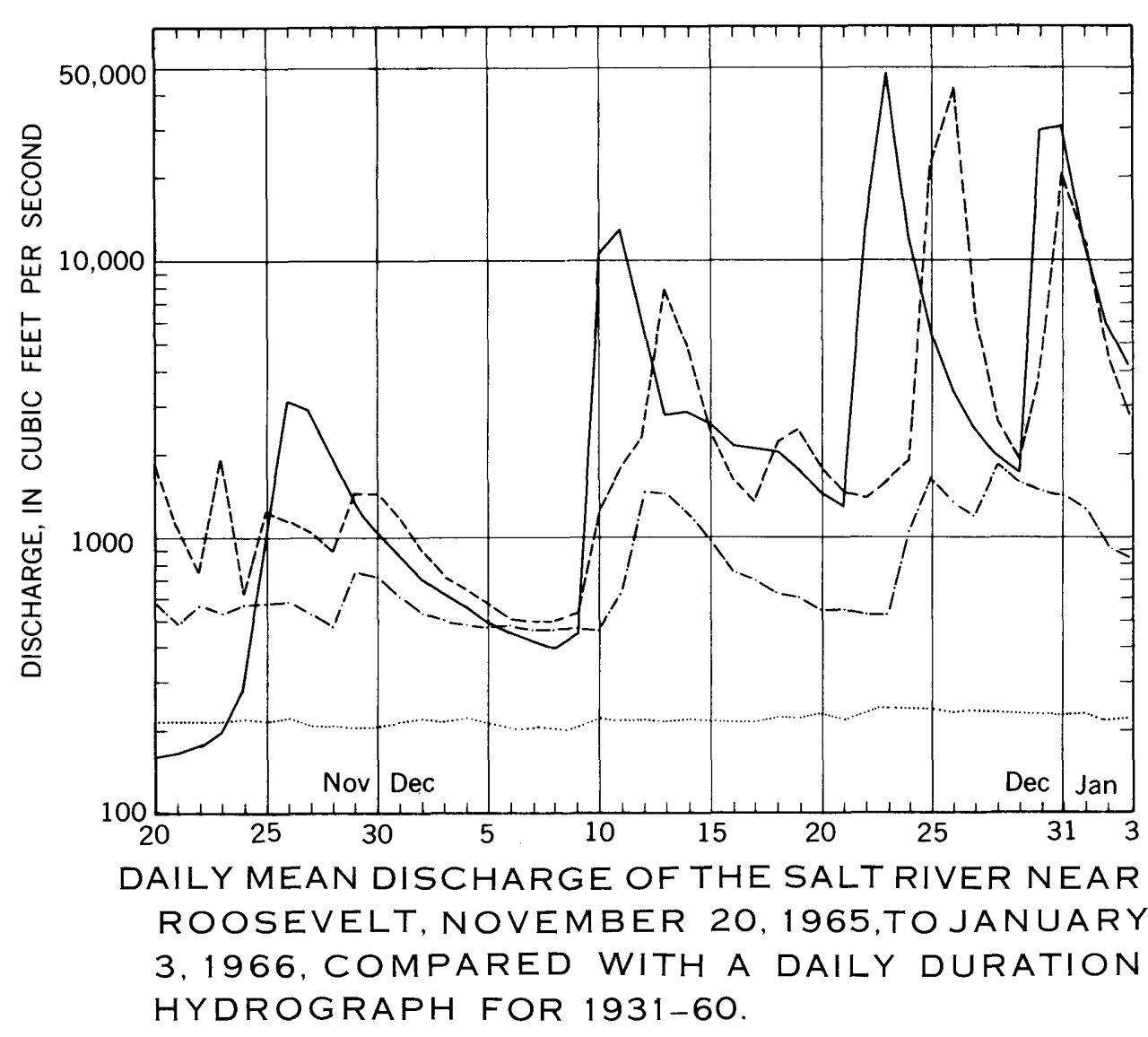

EXPLANATION

Daily mean discharge from November 20 ,

Maximum daily mean discharge for indicated
day during 1931-60 water years

Daily mean discharge that was exceeded 10

Median daily
mean discharge during
water years
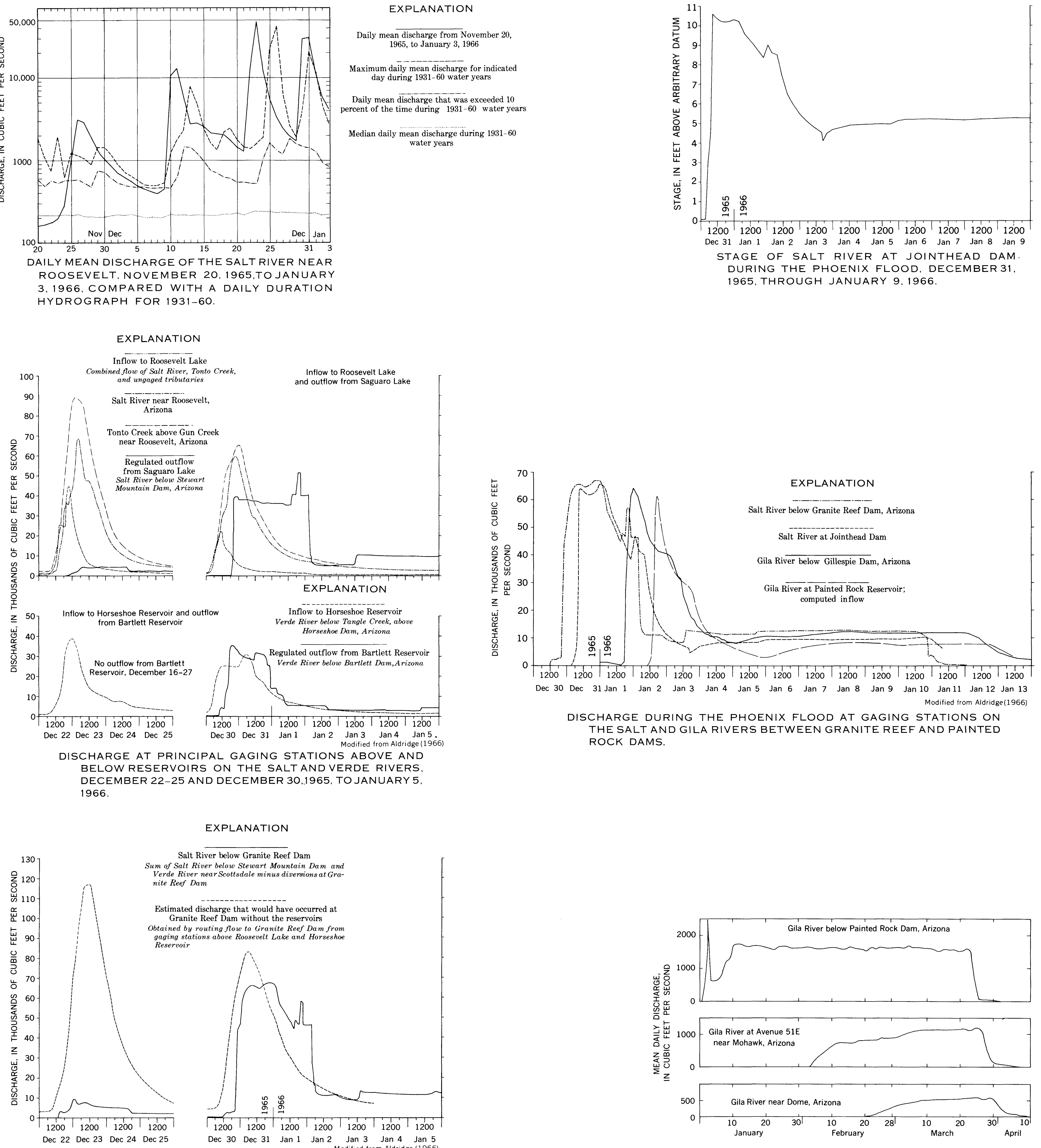

Salt River below Granite Reef Dam verde River near Scottsdale minus diversions at Gra-

ctimated discharge that would have occurred at

ed by routing flow to Granite Reef Dam froth

政

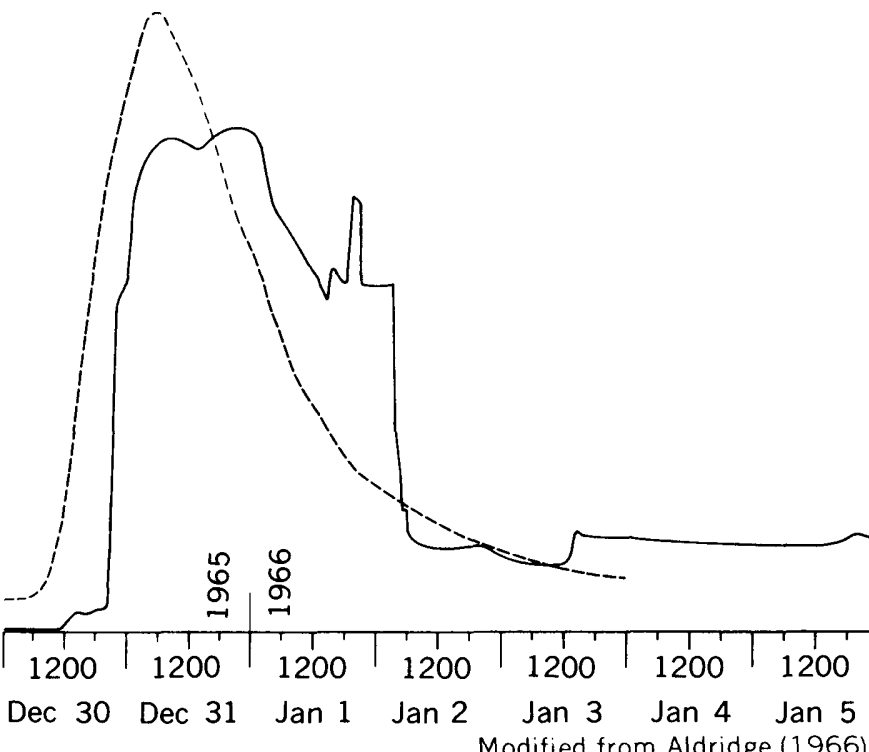

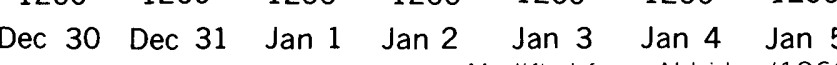

REGULATED DISCHARGE OF SALT RIVER BELOW GRANITE

REEF DAM AND ESTIMATED DISCHARGE THAT WOULD

HAVE OCCURRED WITHOUT THE RESERVOIRS, DECEM

1966

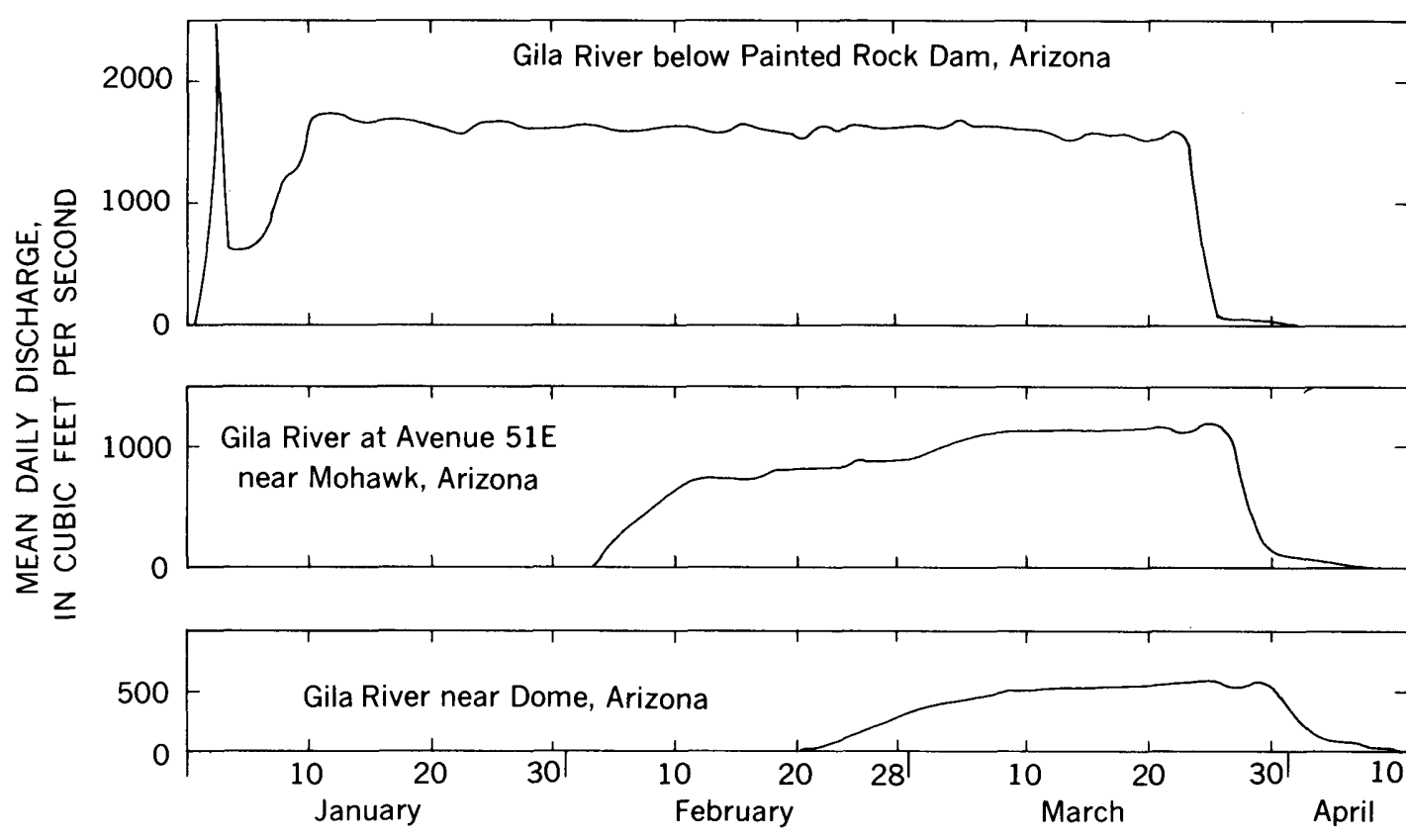

DISCHARGE AT GAGING STATIONS ON THE GILA RIVER DOWNSTREAM FROM PAINTED ROCK RESERVOIR, 1966 


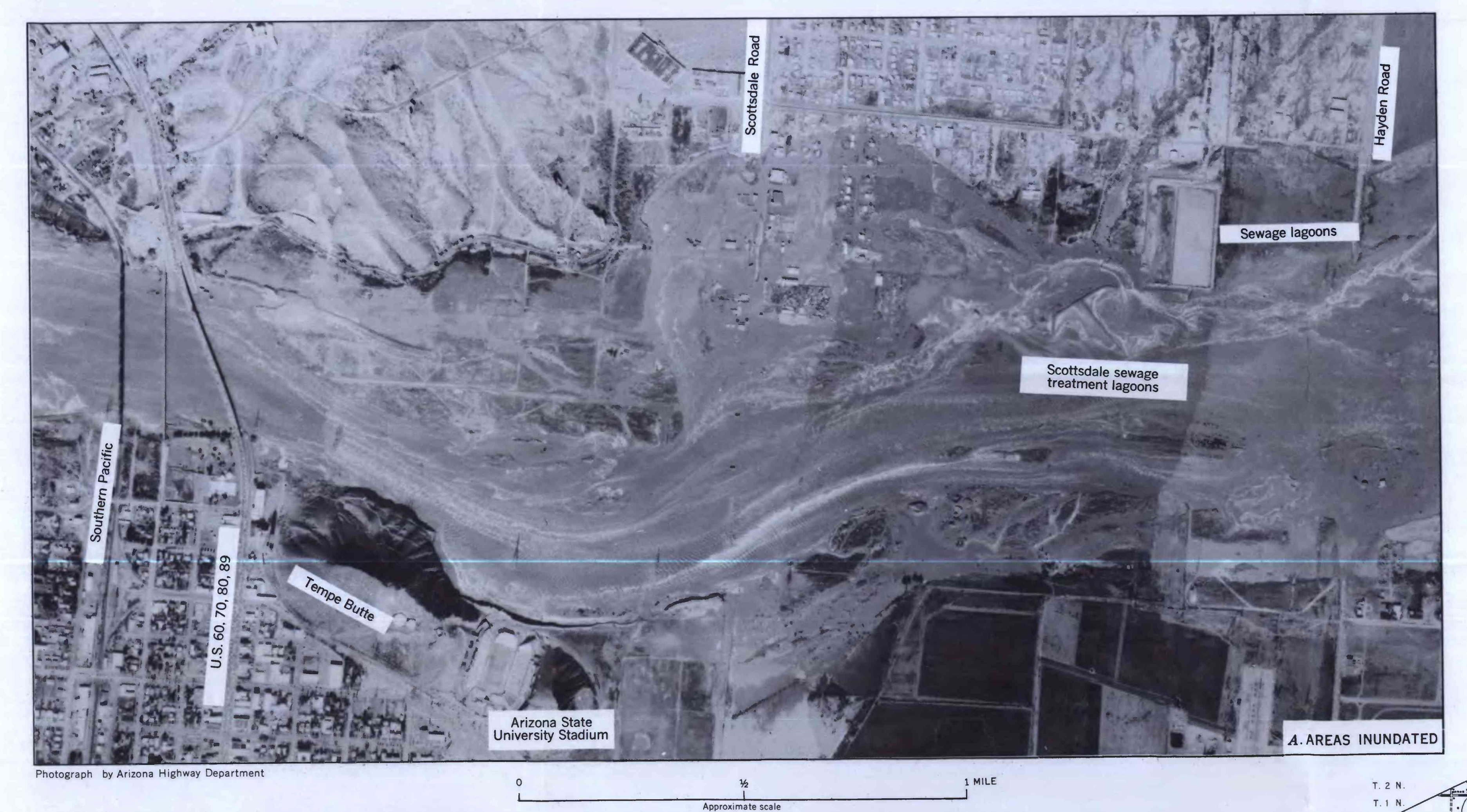

OF THE PHOENIX FLOOD ON THE SALT RIVER NEAR TEMPE, ARIZONA

(1)



N. $-5,100$

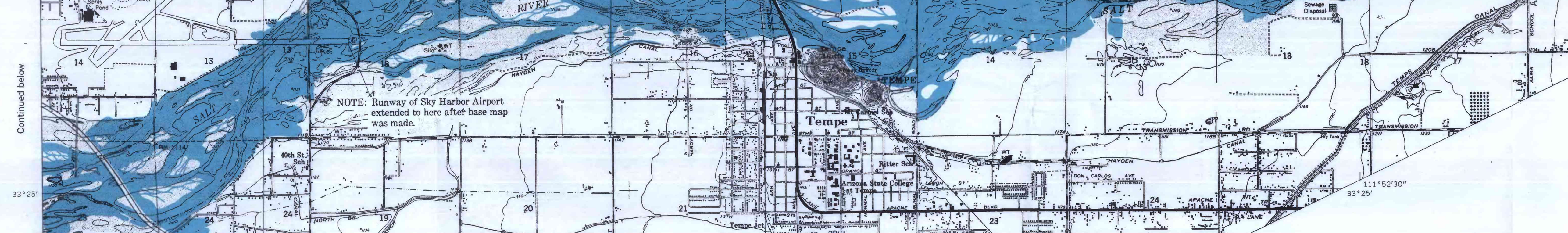

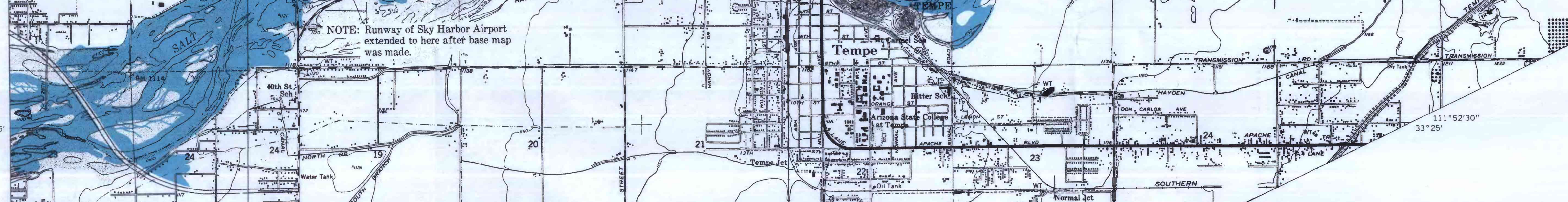

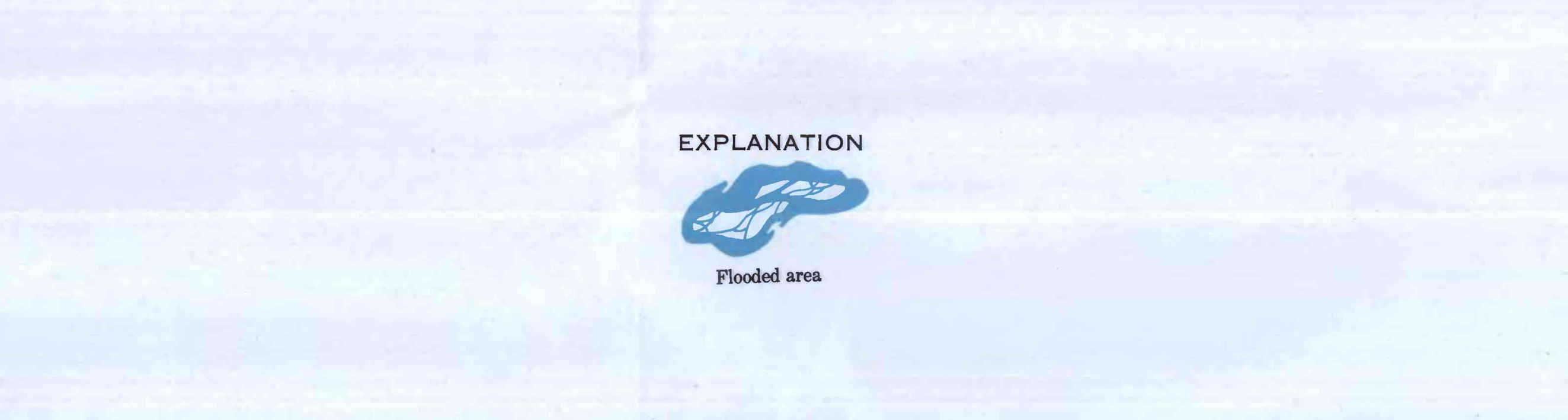

FLOOD MAP AND PHOTOGRAPHS OF THE SALT RIVER IN THE PHOENIX METROPOLITAN AREA, ARIZONA

UNITED STATES DEPARTMENT OF THE INTERIOR

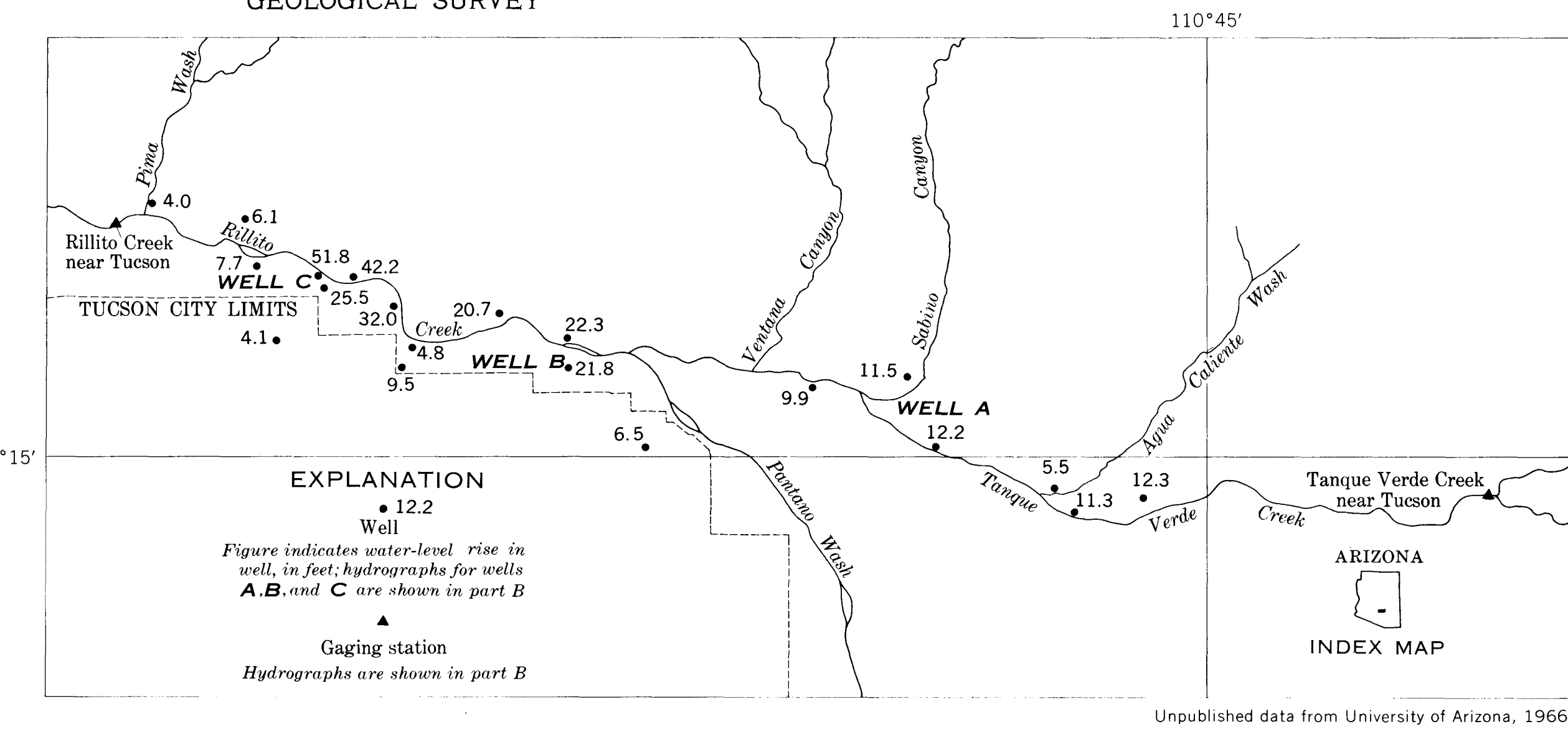

A.-RISES IN WATER LEVELS IN WELLS ALONG RILLITO AND TANQUE VERDE CREEKS, NOVEMBER 16, 1965, TO JANUARY 4,1966.

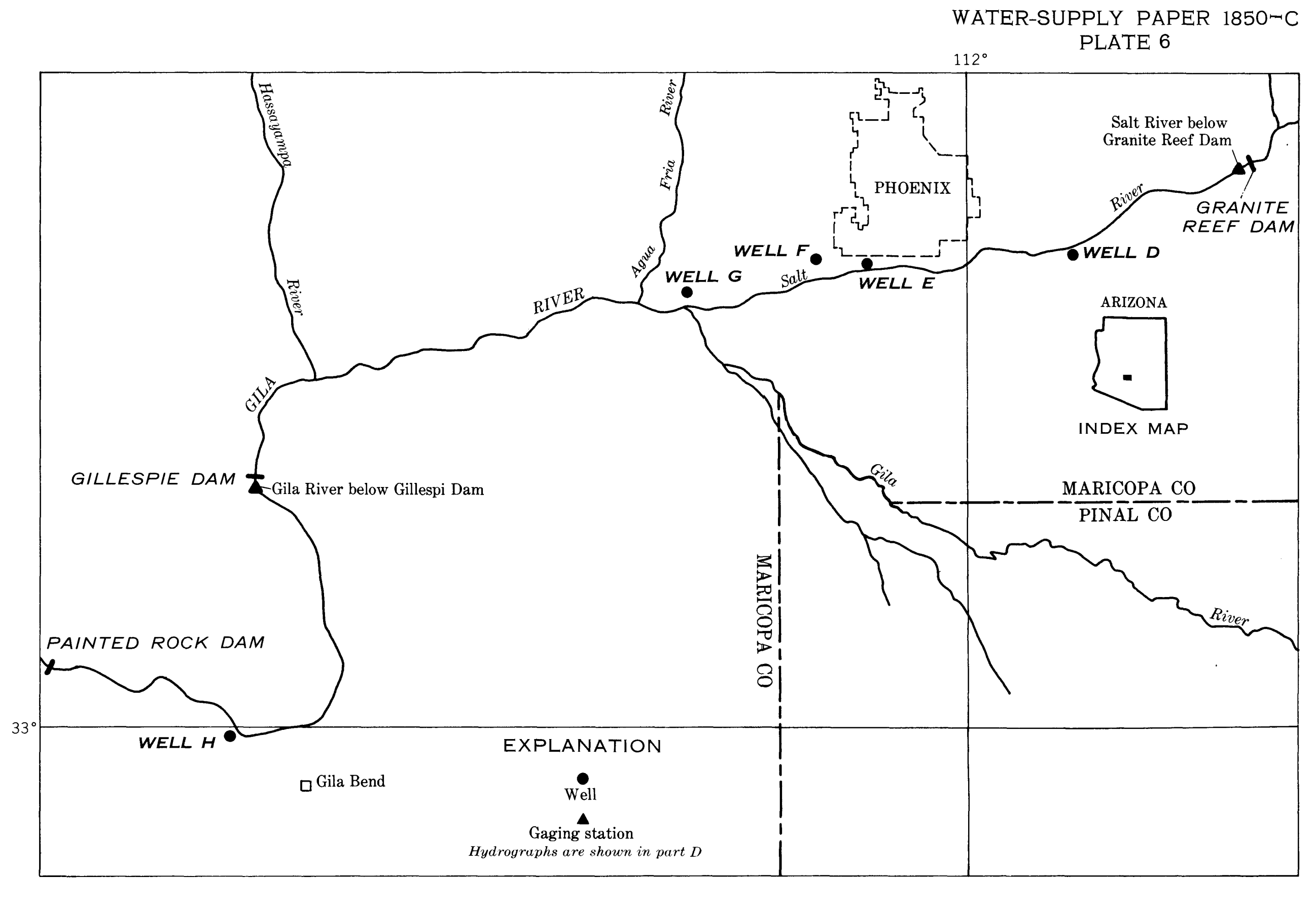

20 MILES

C.- LOCATION OF SELECTED WELLS ALONG THE SALT AND GILA Rivers.
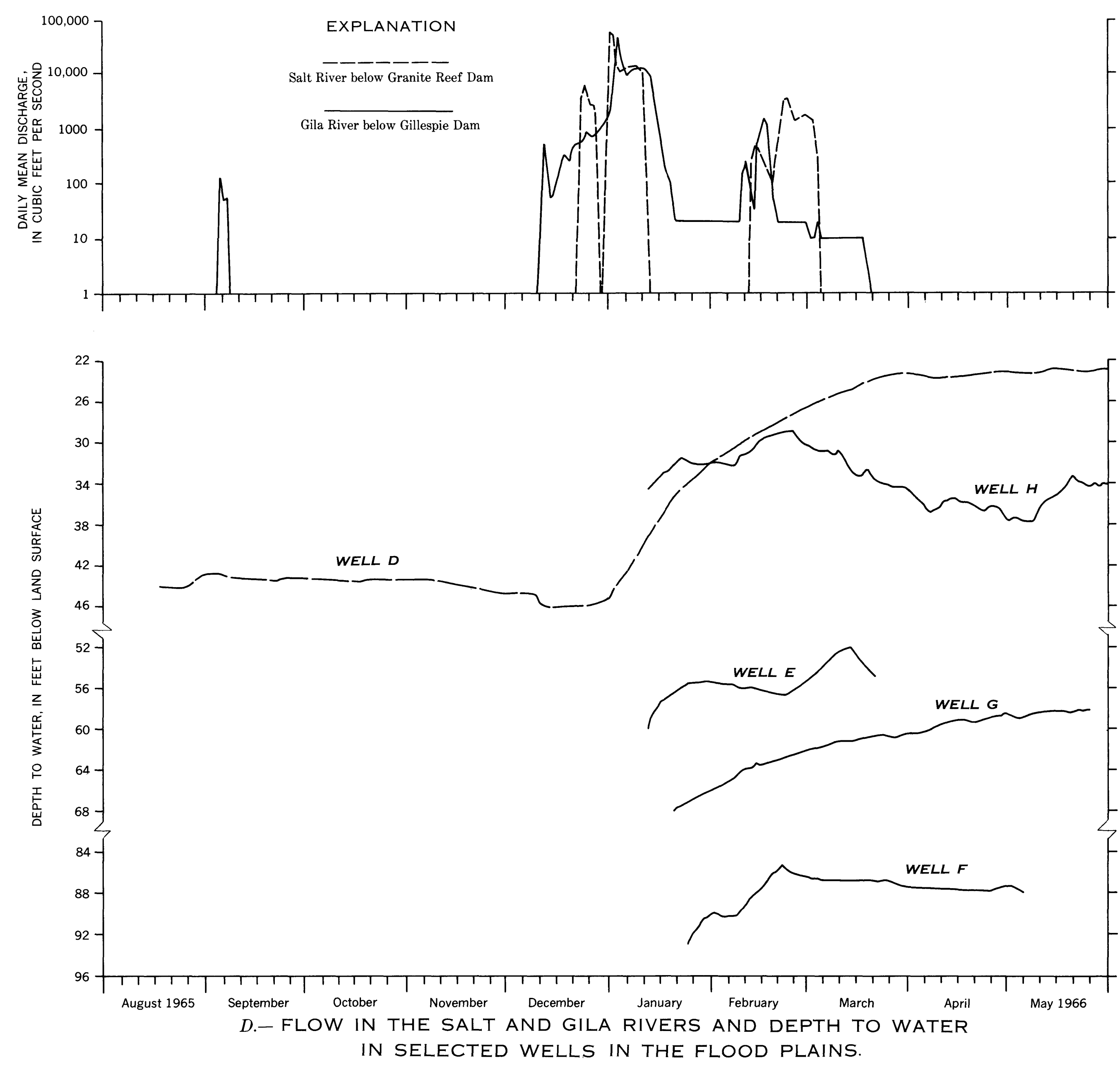